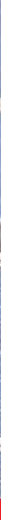

\title{
IntechOpen
}

\section{Perovskite and \\ Piezoelectric Materials}

Edited by Someshwar Pola, Neeraj Panwar and Indrani Coondoo

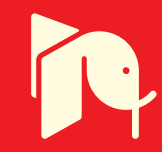





\section{Perovskite and Piezoelectric Materials \\ Edited by Someshwar Pola, Neeraj Panwar and Indrani Coondoo}



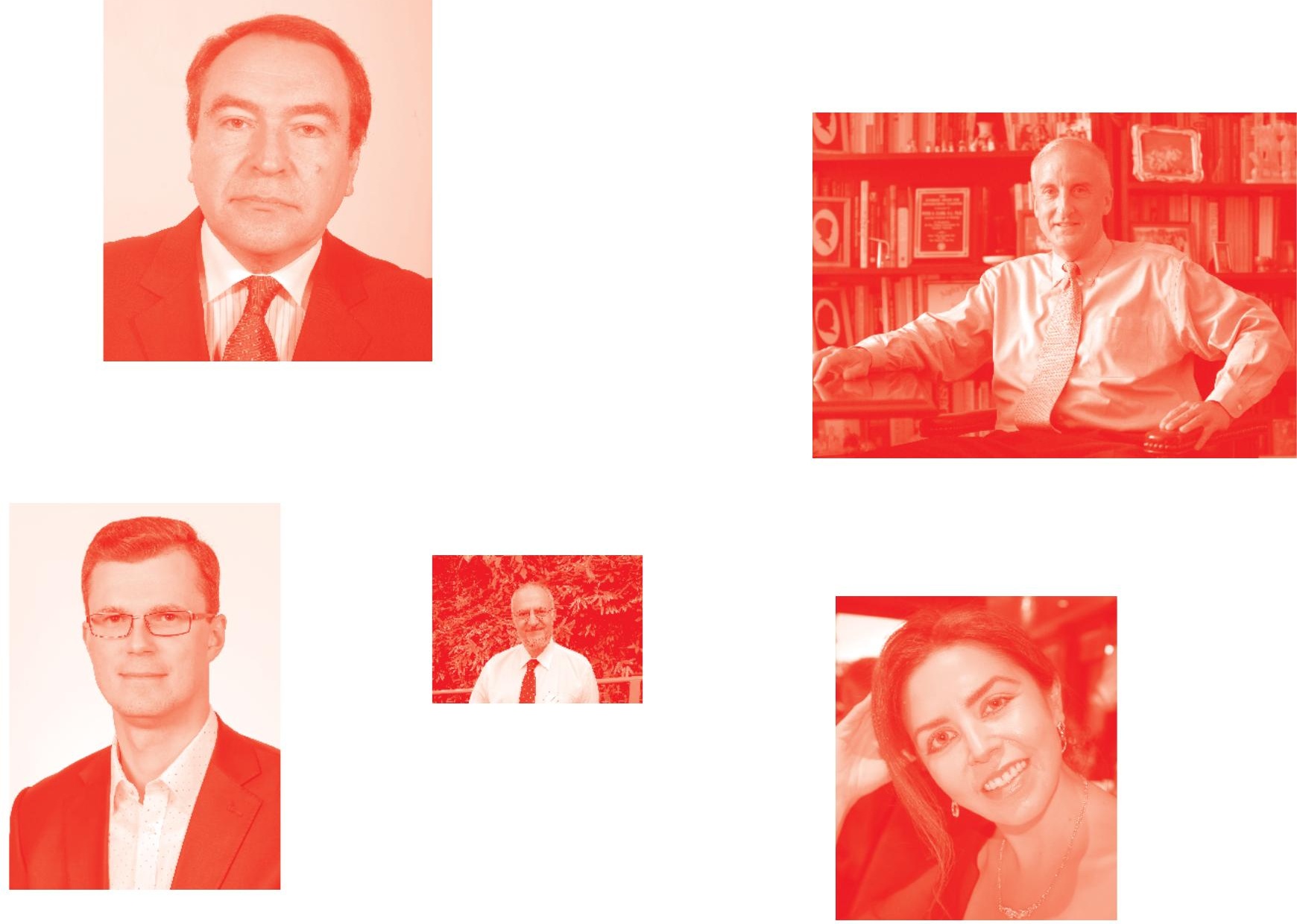

Supporting open minds since 2005
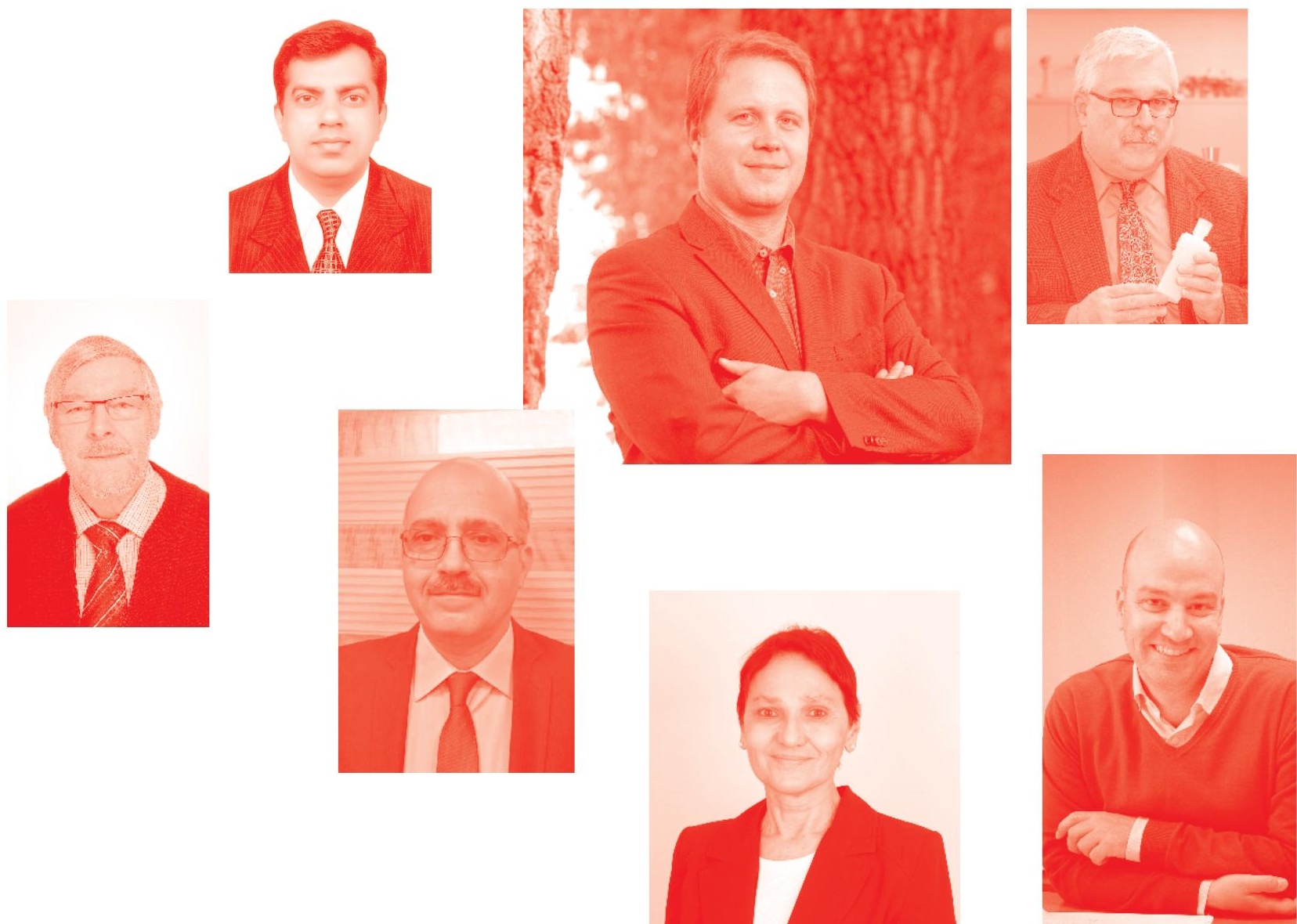
Perovskite and Piezoelectric Materials

http : //dx. doi. org/10.5772/intechopen. 87690

Edited by Someshwar Pola, Neeraj Panwar and Indrani Coondoo

\section{Contributors}

Vo Thanh Tung, Dang Anh Tuan, Badreddine Smiri, Faouzi Saidi, Hassen Maaref, Chung Nguyen Thai, Thuy Le Xuan, Thinh Tran Ich, Asma Bakkali, José Pelegrí Sebastia, Abdelouahid Lyhyaoui, Youssef Laghmich, Jose Antonio Alonso, C.A. Lopez, C. Alvarez-Galvan, C. Abia, M.T. Fernandez-Diaz, Someshwar Pola, Mustafa Can, Burak Gültekin, Ali Kemal Havare, Shirin Siyahjani, Halilibrahim Çiftçi, Sergey Shornikov, Tahira Mahmood, Madeeha Aslam, Abdul Naeem, Manojit De, Ramesh Gade, Le Tran Uyen Tu, Truong Van Chuong

(๑) The Editor(s) and the Author(s) 2021

The rights of the editor(s) and the author(s) have been asserted in accordance with the Copyright, Designs and Patents Act 1988. All rights to the book as a whole are reserved by INTECHOPEN LIMITED. The book as a whole (compilation) cannot be reproduced, distributed or used for commercial or non-commercial purposes without INTECHOPEN LIMITED's written permission. Enquiries concerning the use of the book should be directed to INTECHOPEN LIMITED rights and permissions department (permissions@intechopen.com).

Violations are liable to prosecution under the governing Copyright Law .

\section{(cc) BY}

Individual chapters of this publication are distributed under the terms of the Creative Commons Attribution 3.๑ Unported License which permits commercial use, distribution and reproduction of the individual chapters, provided the original author(s) and source publication are appropriately acknowledged. If so indicated, certain images may not be included under the Creative Commons license. In such cases users will need to obtain permission from the license holder to reproduce the material. More details and guidelines concerning content reuse and adaptation can be found at http : //www . intechopen . com/copyright-policy. html .

Notice

Statements and opinions expressed in the chapters are these of the individual contributors and not necessarily those of the editors or publisher. No responsibility is accepted for the accuracy of information contained in the published chapters. The publisher assumes no responsibility for any damage or injury to persons or property arising out of the use of any materials, instructions, methods or ideas contained in the book.

First published in London, United Kingdom, 2021 by IntechOpen

IntechOpen is the global imprint of INTECHOPEN LIMITED, registered in England and Wales, registration number: 11086078 , 5 Princes Gate Court, London, SW7 2QJ, United Kingdom Printed in Croatia

British Library Cataloguing-in-Publication Data

A catalogue record for this book is available from the British Library

Additional hard and PDF copies can be obtained from orders@intechopen.com

Perovskite and Piezoelectric Materials

Edited by Someshwar Pola, Neeraj Panwar and Indrani Coondoo

p. $\mathrm{cm}$.

Print ISBN 978-1-78985-665-1

Online ISBN 978-1-78985-666-8

eBook (PDF) ISBN 978-1-78985-678-1 


\section{We are IntechOpen, \\ the world's leading publisher of Open Access books}

Built by scientists, for scientists

\section{$5,200+$}

Open access books available

156

Countries delivered to
$127,000+$

International authors and editors
$150 \mathrm{M}+$

Downloads

Our authors are among the

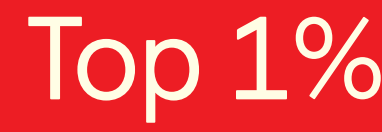

most cited scientists

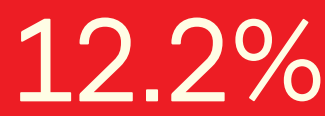

Contributors from top 500 universities

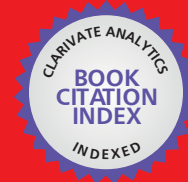

WEB OF SCIENCE ${ }^{\mathrm{TM}}$

Selection of our books indexed in the Book Citation Index in Web of Science ${ }^{\mathrm{TM}}$ Core Collection (BKCI)

Interested in publishing with us?

Contact book.department@intechopen.com

Numbers displayed above are based on latest data collected.

For more information visit www.intechopen.com

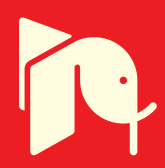





\section{Meet the editors}

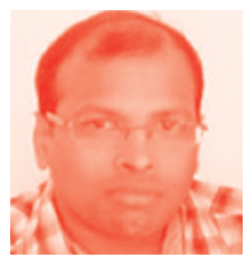

Dr. Someshwar Pola has been working as an Assistant Professor in the Department of Chemistry, University College of Science, Osmania University since 2018. He worked as an Assistant Professor in the Department of Chemistry, Nizam College, Osmania University from 2013 to 2017. He received his B.Sc. from Kakatiya University, M.Sc from P.G Center, Mirzapur, Osmania University, and Ph.D. in chemistry from Kakatiya University, Warangal, Telangana State, India. Dr. Pola worked as a visiting faculty member at the Institute of Chemistry, Academic Sinica, Taipei, Taiwan for two months in 2017. He also worked as a Postdoctoral Fellow (PDF) at the Institute of Chemistry, Academic Sinica, Taipei, Taiwan from August 2008 to April 2012. During this period, he focused on the synthesis and characterization of organic functional materials towards single-crystal field-effect transistors. During the period of his doctoral research work (2002 to 2006), he developed various methodologies in inorganic and analytical chemistry. He also has industrial experience in medicinal research and development (AR\&D), GVK Biosciences Pvt. Ltd., Hyderabad, India. Dr. Pola has published over 48 research papers in reputed international and national journals and has one book chapter to his credit. He has also presented research papers at 35 national and 25 international conferences. He has delivered guest/invited lectures in various colleges/conferences. He is a Life Member of the Society of Materials Chemistry (SMC), Materials Research Society of India (MRSI), Indian Science Congress Association (ISCA), India, and a member in the American Chemical Society (ACS). He is a reviewer for Elsevier, ACS, and RSC journals. He has 8 years of teaching and 20 years of research experience. His research focuses on supramolecular chemistry, solar cell device fabrication studies, organic field-effect transistors, photocatalysis of organic pollutants in the presence of titanates, perovskites and related semiconductor and metal-organic frameworks. Under his supervision, 6 postgraduate students have completed their dissertation work, 3 Ph.D. students have been awarded and 8 scholars are currently working for their Ph.D. degree. His research activities are supported by various funding agencies like NSC-Taiwan, UGC, DST and CSIR, New Delhi, India.

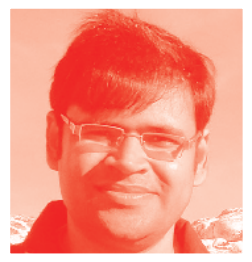

Neeraj Panwar, Ph.D., is presently a Senior Assistant Professor in the Department of Physics, Central University of Rajasthan India. He has postdoctoral research experience from the University of Puerto Rico, U.S.A. and the University of Aveiro, Portugal. He has had several research collaborations at the international and national levels. He has guided two Ph.D. theses and several master's and undergraduate students for their dissertation work. He has published more than seventy international research papers. His areas of interest include magnetism and lead-free piezoelectric and electroceramic materials. He is also interested in practicing yoga. 


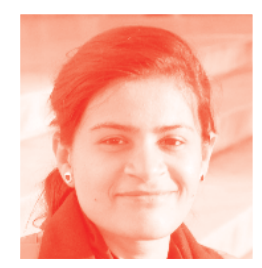

Dr. Indrani Coondoo has been working as a research scientist at the University of Aveiro, Portugal since 2019. She has worked as an FCT postdoctoral researcher at the same university (20102017). She was a visiting researcher at the University of Puerto Rico, U.S.A. (2010). She obtained her Doctorate degree from the Faculty of Applied Sciences, University of Delhi (2008). She served as a CSIR Research Associate and Project Fellow at the National Physical Laboratory, India (2008-2009). Her research interest lies in the study of lead-free piezoelectrics, layered ferroelectrics, and multiferroics. She has published more than fifty research papers in journals of international repute. She has one U.S.A. patent to her credit. She has edited a book entitled "Ferroelectrics" and written many book chapters, as well as review articles. 


\section{Contents}

Preface

Section 1

Photocatalysis

Chapter 1

Significant Role of Perovskite Materials for Degradation of Organic

Pollutants

by Someshwar Pola and Ramesh Gade

Chapter 2

Lead-Free Perovskite Nanocomposites: An Aspect for Environmental

Application

by Manojit De

Chapter 3

Perovskite Nanoparticles

by Burak Gultekin, Ali Kemal Havare, Shirin Siyahjani, Halil Ibrahim Ciftci and Mustafa Can

\section{Section 2}

Perovskite Solar Cells

Chapter 4

Organic Inorganic Perovskites: A Low-Cost-Efficient Photovoltaic Material by Madeeha Aslam, Tahira Mahmood and Abdul Naeem

Chapter 5

Structural Phase Transitions of Hybrid Perovskites $\mathrm{CH}_{3} \mathrm{NH}_{3} \mathrm{PbX}_{3}(\mathrm{X}=\mathrm{Br}, \mathrm{Cl})$ from Synchrotron and Neutron Diffraction Data by Carlos Alberto López, María Consuelo Alvarez-Galván, Carmen Abia, María Teresa Fernández-Díaz and José Antonio Alonso

Section 3

Thermal and Physical Properties of Perovskites

Chapter 6

Thermodynamics of Perovskite: Solid, Liquid, and Gas Phases by Sergey Shornikov 
Synthesis and Investigation of the Physical Properties of Lead-Free BCZT

Ceramics

by Dang Anh Tuan, Vo Thanh Tung, Le Tran Uyen Tu and Truong Van Chuong

Chapter 8

Optimal Temperature Sensor Based on a Sensitive Material

by Asma Bakkali, José Pelegri-Sebastia, Youssef Laghmich

and Abdelouahid Lyhyaoui

\section{Section 4}

Piezoelectric Effect of Perovskites

Chapter 9

Effect of Piezoelectric Filed on the Optical Properties of (311) A and (311) B Oriented InAlAs/InP Heterostructures

by Badreddine Smiri, Faouzi Saidi and Hassen Maaref

Chapter 10

Static and Dynamic Analysis of Piezoelectric Laminated Composite Beams and Plates

by Chung Nguyen Thai, Thinh Tran Ich and Thuy Le Xuan 


\section{Preface}

Perovskite materials are a one of a kind compound with structures associated with that of an inorganic rock deposit. The origin of perovskite material is $\mathrm{CaTiO} 3$, and this is resultant from a paternal phase with the universal formula ABO3. Perovskite materials have been fully investigated since the middle of the 12 th century due to their distinctive properties such as primarily dielectric, ferroelectric, and piezoelectric. This wide range of performance parameters has been adapted into various fields such as electronic conductivity, multiferroic properties, superconductivity, magnetic ordering, thermoelectric, optoelectrical, solar cells, photocatalysis, and piezo-photocatalysis properties. Independently, from these mainly physical characteristics, the phases exhibit an extensive array of chemical features. Perovskite materials are used as photocatalytic and electrode materials for degradation of industrial wastewater and solid oxide fuel cells where perovskite material with elevated oxide ion electronic conductivity, ionic conductivity, and thermal conductivity are required. Several perovskite phases exhibit suitable photocatalytic and photo-redox properties, frequently dependent upon the occurrence of chemical imperfections in the solid phase. This difficulty is an outcome of two key factors. Firstly, the crystal assemblies included in the term 'perovskite' include a wide range, from the basic cubic 'aristo-type' $\mathrm{BaTiO} 3$ to cation and anion short phases, integrated phases with the cuprates superconductor, and hexagonal perovskite materials associated with SrNiO3. Moreover, both the physical and chemical properties of any associate of these structural formulae can be modified over a broad series by comparatively basic ion-exchange into all or part of the A-, and B- positions. This extensive range of variability consists of the development of perovskite materials in which the A-cation is substituted by a simple organic compound, characterized by the perovskite material such as methylammonium lead iodide, now deeply investigated as the fundamental of 'perovskite' solar cells. Furthermore, the applications of superlattice thin films and nanomaterial exhibit novel and fully serendipitous results when related to the actions of the distinct bulk phases. The objective of this book is to deliver a compressed summary of this huge body of information. A framework of the systems of these phases is of key significance as a requirement of various chemical-physical properties. To present a complete outlook, crystal systems are typically signified as perfect forms. The book is systematized into nine chapters with four categories. In category one, we present a short overview of many fascinating results in photocatalytic perovskite materials and the outcomes of the research on lead-free perovskite nanocomposites (Chapter 1, 2 \& 3). Organic-inorganic perovskites and related aspects in perovskite solar cells and structural phase transitions of hybrid perovskites are described in category two (Chapter $4 \& 5$ ). In category three, the thermal and physical properties of perovskite materials with various phases are briefly mentioned (Chapter 6, 7, \& 8). Finally, category four describes the 
significance of the piezoelectric effect of perovskite materials (Chapter $9 \& 10$ ). I am thankful to the Department of Chemistry, Osmania University for providing literature facilities for this book work.

Someshwar Pola Osmania University,

India

Neeraj Panwar

Central University of Rajasthan, India

Indrani Coondoo University of Aveiro,

Portugal 
Section 1

Photocatalysis 



\title{
Chapter 1
}

\section{Significant Role of Perovskite Materials for Degradation of Organic Pollutants}

\author{
Someshwar Pola and Ramesh Gade
}

\begin{abstract}
The advancement and the use of visible energy in ecological reparation and photodegradation of organic pollutants are being extensively investigated worldwide. Through the last two decades, great exertions have been dedicated to emerging innocuous, economical, well-organized and photostable photocatalysts for ecofriendly reparation. So far, many photocatalysts mostly based on ternary metal oxides and doped with nonmetals and metals with various systems and structures have been described. Among them, perovskite materials and their analogs (layertype perovskites) include an emerged as semiconductor-based photocatalysts due to their flexibility and simple synthesis processes. This book chapter precisely concentrates on the overall of related perovskite materials and their associated systems; precisely on the current progress of perovskites that acts as photocatalysts and ecofriendly reparation; explores the synthesis methods and morphologies of perovskite materials; and reveals the significant tasks and outlooks on the investigation of perovskite photocatalytic applications.
\end{abstract}

Keywords: layered-type perovskite materials, photocatalysis, photodegradation, organic pollutants

\section{Introduction}

Solar energy is one of the primary sources in the field of green and pure energy that points to the power predicament and climate change task. Solar energy consumption is an ecological reconciliation, and then, the chemical change in solar is presence exhaustive, considered throughout global [1, 2]. In general, solar energy is renewed into a wide range of developments, such as degradation of organic pollutants as photocatalysis, splitting of water molecules for producing clean energy, and reduction of $\mathrm{CO}_{2}$ gas $[3,4]$. Consuming a similar perception, metal-oxide photocatalysis has also been widely examined for possible exertions in ecological restitution as well as the photodegradation and elimination of organic toxins in the aquatic system [5, 6], decrease of bacterial inactivation [7-9], and heavy metal ions [10-12]. Throughout the earlier few years, excellent applications have been dedicated to evolving well-organized, less expensive, and substantial photocatalysts, particularly those that can become active under visible light such as $\mathrm{NaLaTiO}_{6}, \mathrm{Ag}_{3} \mathrm{PO}_{4} / \mathrm{BaTiO}_{3}, \mathrm{Pt} / \mathrm{SrTiO}_{3}, \mathrm{SrTiO}_{3} \mathrm{TiN}$, noble-metal-SrTiO 3 composites, $\mathrm{GdCoO}_{3}$, orthorhombic perovskites $\mathrm{LnVO}_{3}$ and $\mathrm{Ln}_{1-\mathrm{x}} \mathrm{Ti}_{\mathrm{x}} \mathrm{VO}_{3}(\mathrm{Ln}=\mathrm{Ce}$, Pr, and $\mathrm{Nd}$ ), $\mathrm{Ca}_{0.6} \mathrm{Ho}_{0.4} \mathrm{MnO}_{3}$, Ce-doped $\mathrm{BaTiO}_{3}$, fluorinated $\mathrm{Bi}_{2} \mathrm{WO}_{6}$, graphitic 
carbon nitride- $\mathrm{Bi}_{2} \mathrm{WO}_{6}, \mathrm{BaZrO}_{3-\delta}, \mathrm{CaCu}_{3} \mathrm{Ti}_{4} \mathrm{O}_{12}$, [13-24], graphene-doped perovskite materials, and nonmetal-doped perovskites [25]. Furthermore, directed to years extended exhaustive investigation exertions on the pursuit of innovative photocatalytic systems, particularly that can produce the overall spectrum of visible-light. Out of a vast assemblage of photocatalysts, perovskite or layered-type perovskite systems and its analogs include a better candidate for capable semiconductor-based photocatalysts due to their framework easiness and versatility, excellent photostability, and systematic photocatalytic nature. In general, the ideal perovskite structure is cubic, and the formula is $\mathrm{ABO}_{3}$. Where $\mathrm{A}$ is different metal cations having charge +1 or +2 or +3 nature and $B$ site occupies with tri or tetra and pentavalent nature, which covers the whole family of perovskite oxide materials by sensibly various metal ions at A and B locations [26], aside from a perfect cubic perovskite system, basic alteration perhaps persuaded by several cations exchange. Such framework alteration could undoubtedly vary the photophysical, optical, and photocatalytic activities of primary oxides.

Moreover, a sequence of layered-type perovskite materials contains many 2D blocks of the $\mathrm{ABO}_{3}$ framework, which are parted by fixed blocks. The scope of formulating multicomponent perovskite systems by whichever fractional change of cations in A and B or both positions or injecting perovskite oxides into a layeredtype framework agrees scientists investigate and control the framework of crystals and the correlated electronic and photocatalytic activities of the perovskites. So far, hundreds of various types of perovskite or perovskite-based catalysts have been published, and more outstandingly, some $\mathrm{ABO}_{3}$-related materials became renowned with "referred" accomplishment for catalytic activities. Thus, these systems (perovskite materials) have exposed highly capable of upcoming applications on the source of applying more attempts to them. While several outstanding reviews mean that explained that perovskites performed as photocatalyst for degradation of organic pollutants [27-30], only an insufficient of them content consideration to inorganic perovskite (mostly $\mathrm{ABO}_{3}$-related) photocatalysts [31-33]. A wide range of tagging and complete attention of perovskite materials, for example, layered-type perovskite acting as photocatalysts, is relatively deficient. The purpose of this book chapter is to precise the current progress of perovskite-based photocatalysts for ecological reparation, deliberate current results, and development on perovskite oxides as catalysts, and allow a view on the upcoming investigation of perovskite materials. After a short outline on the wide-ranging structure of perovskite oxides, it was stated that perovskites act as a photocatalyst that are incorporated, arranged and explored based on preparation methods [29, 34], photophysical properties based on bandgap energies, morphology-based framework and the photocatalytic activities depends on either UV or visible light energy of the semiconducting materials. Finally, this chapter is based on the current advancement and expansion of perovskite photocatalytic applications under solar energy consumption. The potential utilization, new tasks, and the research pathway will be accounted for the final part of the chapter [35].

\section{Results and discussion}

\subsection{Details of perovskite oxide materials}

\subsubsection{Perovskite frameworks}

The standard system of perovskite-based materials could be designated as $\mathrm{ABO}_{3}$, where the $\mathrm{A}$ and $\mathrm{B}$ are cations with 12 -fold coordinated and 6 -fold 
coordinated to concerning oxygen anions. Figure 1a describes the typically coordinated basic of the $\mathrm{ABO}_{3}$ system, which consists of a 3D system, $\mathrm{BO}_{6}$ octahedra as located at corner, and at the center, $A$ cation are occupied. Within the $\mathrm{ABO}_{3}$ system, the A cation usually is group I and II or a lanthanide metal, whereas the B is commonly a transition metal ion. The tolerance factor $(t)=1$ calculated by using an equation $t=(r A+r O) / \sqrt{ } 2(r B+r O)$, where $r_{O}, r_{A}$, and $r_{B}$ are the radii of respective ions $A$ and $B$ and oxygen elements for a cubic crystal structure $\mathrm{ABO}_{3}$ perovskite system [36].

For constituting a stable perovskite, it is typically the range of $t$ value present in between 0.75 and 1.0. The lower value of $t$ builds a somewhat slanted perovskite framework with rhombohedral or orthorhombic symmetry. In the case of $t$, it is approximately 1 ; then, perovskite structure is an ideal cubic system at high temperatures. Even though the value of $t$, obtained by the size of metal ion, is a significant guide for the permanency of perovskite systems, the factor of octahedral $(u) u=r_{B} / r_{O}$ and the role of the metal ions composition of A and B atoms and the coordination number of respective metals are considered [37]. Given the account of those manipulating factors and the electro-neutrality, the $\mathrm{ABO}_{3}$ perovskite can hold a broad variety of sets of $A$ and $B$ by equal or dissimilar oxidation states and ionic radii. Moreover, the replacement of $\mathrm{A}$ or $\mathrm{B}$ as well as both the cations could be partly by the doping of various elements, to range the $\mathrm{ABO}_{3}$ perovskite into a wide-ranging family of $A_{m}^{1} A_{1-m}^{1} B_{n}^{1} B_{1-n}^{1} \mathrm{O}_{3 \pm \delta}$ [38]. The replacement of several cations into the either $A$ or $B$ positions could modify the structure of the original system and therefore improve the photocatalytic activities [23]. After various metal ions in perovskite oxide are doped, the optical and electronic band positions, which influence the high impact on the photocatalytic process, are modified [24].

a)

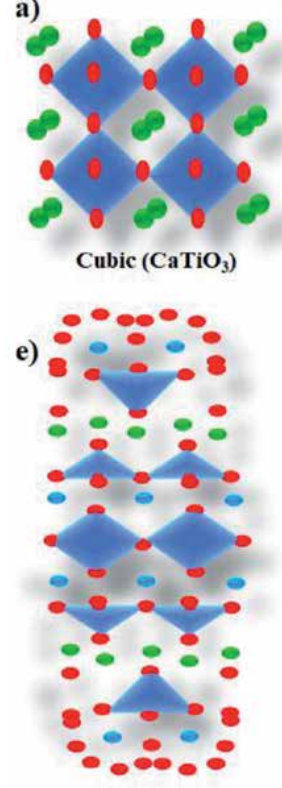

Dion-Jacobsen $\left(\mathrm{KCa}_{2} \mathrm{Nb}_{3} \mathrm{O}_{10}\right)$

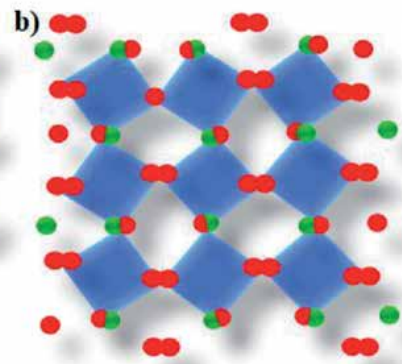

Orthorhombic $\left(\mathrm{CaTiO}_{3}\right)$

f)

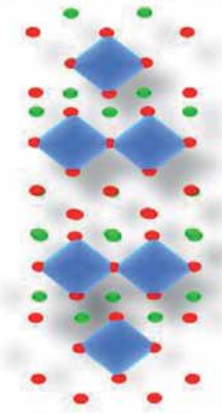

$\{111\}$ layered

$\left(\mathrm{Ba}_{5} \mathrm{Ta}_{4} \mathrm{O}_{15}\right)$ c)

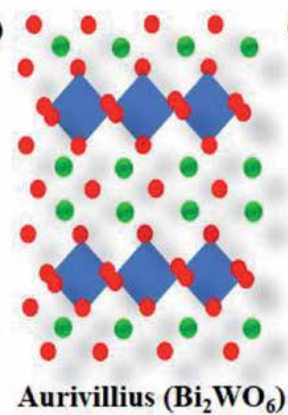

g)

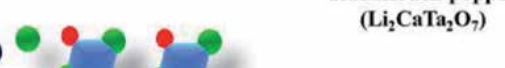

Figure 1.

Both crystal and layered type perovskite oxides (blue small balls: A-site element; dark blue squares: $\mathrm{BO}_{6}$ octahedra with green and red balls are oxygen). 


\subsubsection{Layered perovskite-related systems}

Moreover, to the overall $\mathrm{ABO}_{3}$ system, further characteristic polymorphs of the perovskite system are Brownmillerite (BM) $\left(\mathrm{A}_{2} \mathrm{~B}_{2} \mathrm{O}_{5}\right)$ framework [39]. BM is a type of oxygen-deficient perovskite, in which the unit cell is a system of wellorganized $\mathrm{BO}_{4}$ and $\mathrm{BO}_{6}$ units. The coordination number of cations occupied by A-site was decreased to eight because of the oxygen deficiency. Perovskite $\left(\mathrm{ABO}_{3}\right)$ oxides have three dissimilar ionic groups, construction for varied and possibly useful imperfection chemistry. Moreover, the partial replacement of A and B ions is permitted even though conserving the perovskite system and shortages of cations at the A-site or of oxygen anions are common [40]. The Ion-exchange method is used for the replacement of existing metal ions with similar sized or dissimilar oxidation states; then, imperfections can be announced into the system. The imperfection concentrations of perovskites could be led by doping of different cations [24]. Oxygen ion interstitials or vacancies could be formed by the replacement of $\mathrm{B}$-position cations with higher or lower valence, respectively, fabricating new compounds of $\mathrm{AB}(1-\mathrm{m}) \mathrm{B}_{\mathrm{m}} \mathrm{I}_{3-\delta}$ [41]. A typical oxygen-deficient perovskite system is Brownmillerite $\left(\mathrm{A}_{2} \mathrm{~B}_{2} \mathrm{O}_{5}\right)$, in which one part of six oxygen atoms is eliminated. Moreover, the replacement of exciting a site cation to new cation with higher oxidation state metal ions then the formed new materials with new framework with different stoichiometry is $\mathrm{A}_{1-\mathrm{m}} \mathrm{A}_{\mathrm{m}}{ }^{\mathrm{I}} \mathrm{BO}_{3}$ [41]. In the case of the replacement of $\mathrm{A}$-site ions with smaller oxidation state cations, consequences in oxygen-deficient materials with new framework such as $\mathrm{A}_{1-\mathrm{m}} \mathrm{A}_{\mathrm{m}}{ }^{\mathrm{I}} \mathrm{BO}_{3-\mathrm{x}}$ are developed. Thermodynamically, the replacement of $\mathrm{B}$-position vacancies in perovskite systems is not preferable due to the compact size and the high charge of $\mathrm{B}$ cations [42]. A-position vacancies are more detected due to the $\mathrm{BO}_{3}$ range in perovskite system forms a stable network [43]; the 12 coordinated sites can be partly absent due to bigger-size A cations. Lately, presenting suitable imperfections on top of the surface of perovskite oxides has been thoroughly examined as a means of varying the bands' position and optical properties of the starting materials. For this reason, perovskite materials afford a tremendous objective for imperfection originating to vary the photocatalytic activity of perovskite material-based photocatalysts [44].

The typical formula for the furthermost recognized layered perovskite materials is designated as $A_{n+1} B_{n} O_{3 n+1}$ or $A_{2}{ }^{I} A_{n-1} B_{n n} O_{3 n+1}$ (Ruddlesden-Popper (RP) phase), $A^{I}\left[A_{n-1} B_{n} O_{3 n+1}\right]$ (Dion-Jacobson (DJ) phase) for $\{100\}$ series, $\left(A_{n} B_{n} O_{3 n+2}\right)$ for $\{110\}$ series and $\left(A_{n+1} B_{n} O_{3 n+3}\right)$ for $\{111\}$, and $\left(\mathrm{Bi}_{2} \mathrm{O}_{2}\right)\left(\mathrm{A}_{n-1} \mathrm{~B}_{\mathrm{n}} \mathrm{O}_{3 \mathrm{n}+1}\right)$ (Aurivillius phase) series. In these systems, $\mathrm{n}$ represents the number of $\mathrm{BO}_{6}$ octahedra that duration a layer, which describes the width of the layer. Typical samples of these layered systems are revealed in Figure 1c-g. For RP phases, their frameworks consist of $A^{l} O$ as the spacing layer for the intergrowth $\mathrm{ABO}_{3}$ system. These materials hold fascinating properties such as ferroelectricity, superconductivity, magnetoresistance, and photocatalytic activity. $\mathrm{Sr}_{2} \mathrm{SnO}_{4}$ and $\mathrm{Li}_{2} \mathrm{CaTa}_{2} \mathrm{O}_{7}$ systems are materials of simple RP kind photocatalysts. A common formula for DJ phase is $A^{I}\left[A_{n-1} B_{n} O_{3 n+1}\right](n>1)$, where $\mathrm{A}^{\mathrm{I}}$ splits the perovskite-type slabs and is characteristically a monovalent alkali cation. The typical DJ kind photocatalysts are $\mathrm{RbLnTa}_{2} \mathrm{O}_{7}(\mathrm{n}=2)$ and $\mathrm{KCa}_{2} \mathrm{Nb}_{3} \mathrm{O}_{10}$ $(n=3)$. Associates of the $A_{n} B_{n} O_{3 n+2}$ and $A_{n+1} B_{n} O_{3 n+3}$ structural sequences with dissimilar layered alignments have also been recognized in some photocatalysts like $\mathrm{Sr}_{2} \mathrm{Ta}_{2} \mathrm{O}_{7}$ and $\mathrm{Sr}_{5} \mathrm{Ta}_{4} \mathrm{O}_{15}(\mathrm{n}=4)$. For Aurivillius phases, their frameworks are constructed by one after another fluctuating layers of $\left[\mathrm{Bi}_{2} \mathrm{O}_{2}\right]^{2+}$ and virtual perovskite blocks. $\mathrm{Bi}_{2} \mathrm{WO}_{6}$ and $\mathrm{BiMoO}_{6}(\mathrm{n}=1)$, found as the primary ferroelectric nature for Aurivillius materials, lately have been extensively investigated as visible light photocatalysts. 


\subsection{Perovskite systems for photocatalysis}

A broad array of perovskite photocatalysts have been advanced for organic pollutant degradation in the presence of ultraviolet or visible-light-driven through the last two decades [45]. These typical examples and brief investigational consequences on perovskites are concise giving to their systems, then perovskite materials categorized into six groups. Precisely, $\mathrm{ABO}_{3}$-type perovskites, $\mathrm{AA}^{\mathrm{I}} \mathrm{BO}_{3}$, $\mathrm{A}^{\mathrm{I}} \mathrm{ABO}_{3}, \mathrm{ABB}^{\mathrm{I}} \mathrm{O}_{3}$ and $\mathrm{AB}(\mathrm{ON})_{3}$-type perovskites, and $\mathrm{AA}^{\mathrm{I}} \mathrm{BB}^{\mathrm{II}} \mathrm{O}_{3}$-type perovskites are listed in Table 1.

\subsection{Photocatalytic properties perovskite oxides}

$\mathrm{NaTaO}_{3}$ has been a standard perovskite material for a well-organized UV-light photocatalyst for degradation of organic pollutants and production of $\mathrm{H}_{2}$ and $\mathrm{O}_{2}$ through water splitting [46-57]. It can be prepared by various methods such as solid-state [46-48, 53, 56], hydrothermal [49, 52, 54, 55], molten salt [57] and sol-gel $[50,51]$ and with wide bandgap of $4.0 \mathrm{eV}$. In order to enhance the surface area of $\mathrm{NaTaO}_{3}$ bulk material, many investigators tried to use further synthetic ways to make nanosized particles as an additional study on the $\mathrm{NaTaO}_{3}$ photocatalyst for degradation of organic pollutants. Kondo et al. prepared a colloidal range of $\mathrm{NaTaO}_{3}$ nanoparticles consuming three-dimensional mesoporous carbon as a pattern, which was pretend by the colloidal arrangement of silica nanospheres. After calcining the mesoporous carbon matrix, a colloidal arrangement of $\mathrm{NaTaO}_{3}$ nanoparticles with a range of $20 \mathrm{~nm}$ and a surface area of $34 \mathrm{~m}^{2} \mathrm{~g}^{-1}$ was attained. C-doped $\mathrm{NaTaO}_{3}$ material was tested for degradation of $\mathrm{NO}_{\mathrm{x}}$ under UV light [36]. Several titanates such as $\mathrm{BaTiO}_{3}[58-60]$, $\mathrm{Rh}$ or Fe-doped $\mathrm{BaTiO}_{3}[61,62], \mathrm{CaTiO}_{3}[63,64]$ and $\mathrm{Cu}[65]$, $\mathrm{Rh}$ [66], $\mathrm{Ag}$ and $\mathrm{La}$-doped $\mathrm{CaTiO}_{3}[67]$, and $\mathrm{PbTiO}_{3}[68,69]$ were also described as UV or visible light photocatalysts. Magnetic $\mathrm{BiFeO}_{3}$, recognized as the one of the multi-ferric perovskite materials in magnetoelectric properties, was also examined as a visible light photocatalyst for photodegradation of organic pollutants because of small bandgap energy (2.2 eV) [70-79]. In a previous account, $\mathrm{BiFeO}_{3}$ with a bandgap of around $2.18 \mathrm{eV}$ produced by a citric acid-supported sol-gel technique has revealed its visible-light-driven photocatalytic study by the disintegration of methyl orange dye [70]. The subsequent investigations on $\mathrm{BiFeO}_{3}$ are primarily concentrated on the synthesis of new framework $\mathrm{BiFeO}_{3}$ with various morphologies. For instance, Lin and Nan et al. prepared $\mathrm{BiFeO}_{3}$ unvarying microspheres and microcubes by a using hydrothermal technique as revealed in Figure 2 [73].

The bandgap energies of $\mathrm{BiFeO}_{3}$ compounds were found to be about $1.82 \mathrm{eV}$ for $\mathrm{BiFeO}_{3}$ microspheres and 2.12-2.27 eV for microcubes. This indicated that the absorption edge was moved toward the longer wavelength that is influenced by the crystal-field strength, particle size, and morphology. The microcube material showed the maximum photocatalytic degradation performance of congo red dye under visible-light irradiation due to the quite low bandgap energy. Further, a simplistic aerosol-spraying method was established for the synthesis of mesoporous $\mathrm{BiFeO}_{3}$ hollow spheres with improved activity for the photodegradation of RhB dye and 4-chlorophenol, because of improved light absorbance ensuing from various light reflections in a hollow chamber and a very high surface area [71]. Moreover, a unusually improved water oxidation property on $\mathrm{Au}$ nanoparticle-filled $\mathrm{BiFeO}_{3}$ nanowires under visible-light-driven was described [77]. The $\mathrm{Au}-\mathrm{BiFeO}_{3}$ hybrid system was encouraged by the electrostatic contact of negatively charged $\mathrm{Au}$ nanoparticles and positively charged $\mathrm{BiFeO}_{3}$ nanowires at $\mathrm{pH} 6.0$ giving to their various isoelectric points. An improved absorbance between 500 and $600 \mathrm{~nm}$ was found for $\mathrm{Au} / \mathrm{BiFeO}_{3}$ systems because of the characteristic Au surface plasmon band 
existing visible light region then which greater influenced in the photodegradation of organic pollutants. Also, the study of photoluminescence supported improvement of the photocatalytic property due to the effective charge transfer from $\mathrm{BiFeO}_{3}$ to $\mathrm{Au}$. Even though $\mathrm{Ba}, \mathrm{Ca}, \mathrm{Mn}$, and $\mathrm{Gd}$-doped $\mathrm{BiFeO}_{3}$ nanomaterials have exhibited noticeable photocatalytic property for the degradation of dyes [80-84], several nano-based $\mathrm{LaFeO}_{3}$ with various morphologies such as nanoparticles, nanorods, nanotubes, nanosheets, and nanospheres have also been synthesized for visible light photocatalysts for degradation of organic dyes [85-93]. Sodium bismuth titanate $\left(\mathrm{Bi}_{0.5} \mathrm{Na}_{0.5} \mathrm{TiO}_{3}\right)$ has been extensively used for ferroelectric and piezoelectric devices. It was also investigated as a UV-light photocatalyst with a bandgap energy of $3.0 \mathrm{eV}$ [94-97]. Hierarchical micro/nanostructured $\mathrm{Bi}_{0.5} \mathrm{Na}_{0.5} \mathrm{TiO}_{3}$ was produced by in situ self-assembly of $\mathrm{Bi}_{0.5} \mathrm{Na}_{0.5} \mathrm{TiO}_{3}$ nanocrystals under precise hydrothermal conditions, through the evolution mechanism was examined in aspect means that during which the growth mechanism was studied [95]. It was anticipated that the hierarchical nanostructure was assembled through a method of nucleation and growth and accumulation of nanoparticles and following in situ dissolution-recrystallization of the microsphere type nanoparticles with extended heating period and enhanced temperature or basic settings. The $3 \mathrm{D}$ hierarchical $\mathrm{Bi}_{0.5} \mathrm{Na}_{0.5} \mathrm{TiO}_{3}$ showed very high photocatalytic activity for the decomposition of methyl orange dye because of the adsorption of dye molecules and bigger surface area. The properties of $\mathrm{Bi}_{0.5} \mathrm{Na}_{0.5} \mathrm{TiO}_{3}$ were also assessed by photocatalytic degradation of nitric oxide in the gas phase [95]. $\mathrm{La}_{0.7} \mathrm{Sr}_{0.3} \mathrm{MnO}_{3}$, acting as a photocatalyst, was examined for solar light-based photocatalytic decomposition of methyl orange [96-98]. In addition, $\mathrm{La}_{0.5} \mathrm{Ca}_{0.5} \mathrm{NiO}_{3}$ [99], $\mathrm{La}_{0.5} \mathrm{Ca}_{0.5} \mathrm{CoO}_{3-\delta}$ [100], and $\mathrm{Sr}_{1-\mathrm{x}} \mathrm{Ba}_{\mathrm{x}} \mathrm{SnO}_{3}$ ( $\left.\mathrm{x}=0-1\right)$ [101] nanoparticles were synthesized for revealing improved photocatalytic degradation of dyes. A-site strontium-based perovskites such as $\mathrm{SrTi}_{1-\mathrm{x}} \mathrm{Fe}_{\mathrm{x}} \mathrm{O}_{3-\delta}, \mathrm{SrTi}_{0.1} \mathrm{Fe}_{0.9} \mathrm{O}_{3-\delta}, \mathrm{SrNb}_{0.5} \mathrm{Fe}_{0.5} \mathrm{O}_{3}$, and $\mathrm{SrCo}_{0.5} \mathrm{Fe}_{0.5} \mathrm{O}_{3-\delta}$ compounds were prepared through solid-state reaction and solgel approaches, and were examined for the degradation of organic pollutants under visible light irradiation [102-105]. Also, some other researchers modified A-site with lanthanum-based perovskites such as $\mathrm{LaNi}_{1-\mathrm{x}} \mathrm{Cu}_{\mathrm{x}} \mathrm{O}_{3}$ and $\mathrm{LaFe}_{0.5} \mathrm{Ti}_{0.5} \mathrm{O}_{3}$ were confirmed as effective visible light photocatalysts for the photodegradation of p-chlorophenol $[91,106,107]$. The other $\mathrm{ABB}^{\mathrm{I}} \mathrm{O}_{3}$ kind photocatalysts with $\mathrm{Ca}(\mathrm{TiZr}) \mathrm{O}_{3}$ [108], $\mathrm{Ba}(\mathrm{ZrSn}) \mathrm{O}_{3}$ [109], $\mathrm{Na}(\mathrm{BiTa}) \mathrm{O}_{3}$ [110], $\mathrm{Na}(\mathrm{TiCu}) \mathrm{O}_{3}$ [111], $\mathrm{Bi}(\mathrm{MgFeTi}) \mathrm{O}_{3}$ [112], and $\mathrm{Ag}(\mathrm{TaNb}) \mathrm{O}_{3}$ [113] have also been studied. Related to $\mathrm{AA}^{\mathrm{I}} \mathrm{BO}_{3}$-type perovskites, the $\mathrm{ABB}^{\mathrm{I}} \mathrm{O}_{3}$ kind system means that $\mathrm{BI}$-site substitution by a different cation is another option for tuning the physicochemical or photocatalytic properties of perovskites materials as photocatalyst, due to typically the $\mathrm{B}$-position cations in $\mathrm{ABO}_{3}$ mostly regulate the position of the conduction band, moreover to construct the structure of perovskite system with oxygen atoms. The band positions of photocatalyst can be magnificently modified by sensibly coalescing dual or ternary metal cations at the B-position, or changing the ratio of several cations, which has been fine verified by the various materials as mentioned above. More studies on $\mathrm{ABB}^{\mathrm{I}} \mathrm{O}_{3}$ kind of photocatalysts are projected to show their new exhilarating photocatalytic efficiency.

The mesoporous nature of $\mathrm{LaTiO}_{2} \mathrm{~N}$ of photocatalyst attended due to thermal ammonolysis process of $\mathrm{La}_{2} \mathrm{Ti}_{2} \mathrm{O}_{7}$ precursor from polymer complex obtained from the solid-state reaction. The oxynitride analysis revealed that the pore size and shape, lattice defects and local defects, and oxidation states' local analysis related between morphology and photocatalytic activity were reported by Pokrant et al. [114]. Due to the high capability of accommodating an extensive array of cations and valences at both $\mathrm{A}$ - and $\mathrm{B}$-sites, $\mathrm{ABO}_{3}$-kind perovskite materials are capable materials for fabricating solid-solution photocatalysts. On the other hand, equally the A and B cations can be changed by corresponding cations subsequent in a perovskite with the formula of $\left(\mathrm{ABO}_{3}\right)_{\mathrm{x}}\left(\mathrm{A}^{\mathrm{I}} \mathrm{B}^{\mathrm{I}} \mathrm{O}_{3}\right)_{1-\mathrm{x}}$. Additional solid solution examples with $\mathrm{CaZrO}_{3}-\mathrm{CaTaO}_{2} \mathrm{~N}$ 
[115], $\mathrm{SrTiO}_{3}-\mathrm{LaTiO}_{2} \mathrm{~N}$ [116], $\mathrm{La}_{0.8} \mathrm{Ba}_{0.2} \mathrm{Fe}_{0.9} \mathrm{Mn}_{0.1} \mathrm{O}_{3-\mathrm{x}}$ [117], $\mathrm{Na}_{1-\mathrm{x}} \mathrm{La}_{\mathrm{x}} \mathrm{Fe}_{1-\mathrm{x}} \mathrm{Ta}_{\mathrm{x}} \mathrm{O}_{3}$ [118], $\mathrm{Na}_{0.5} \mathrm{La}_{0.5} \mathrm{TiO}_{3}-\mathrm{LaCrO}_{3}$ [119], $\mathrm{Cu}-\left(\mathrm{Sr}_{1-\mathrm{y}} \mathrm{Na}_{\mathrm{y}}\right)-\left(\mathrm{Ti}_{1-\mathrm{x}} \mathrm{Mo}_{\mathrm{x}}\right) \mathrm{O}_{3}$ [120], $\mathrm{Na}_{1-\mathrm{x}} \mathrm{La}_{\mathrm{x}} \mathrm{Ta}_{1-\mathrm{x}}$ $\mathrm{Cr}_{\mathrm{x}} \mathrm{O}_{3}$ [121], $\mathrm{BiFeO}_{3}-\left(\mathrm{Na}_{0.5} \mathrm{Bi}_{0.5}\right) \mathrm{TiO}_{3}$ [122], and $\mathrm{Sr}_{1-\mathrm{x}} \mathrm{Bi}_{\mathrm{x}} \mathrm{Ti}_{1-\mathrm{x}} \mathrm{Cr}_{\mathrm{x}} \mathrm{O}_{3}$ [123] have been used as photocatalysts for splitting of water molecules under visible light.

\subsection{Photocatalytic activity of layered perovskite materials}

In the general formula of the RP phase, $\mathrm{A}_{n-1} \mathrm{~A}_{2}{ }^{\mathrm{I}} \mathrm{B}_{n} \mathrm{O}_{3 \mathrm{n}+1}, \mathrm{~A}$ and $\mathrm{A}^{\mathrm{I}}$ are alkali, alkaline earth, or rare earth metals, respectively, while $B$ states to transition metals. $A$ and $A^{I}$ cations are placed in the perovskite layer and boundary with 12 -fold cuboctahedral and 9-fold coordination to the anions, respectively, whereas B cations are sited inside the perovskite system with anionic squares, octahedra, and pyramids. The tantalum-based RP phase materials have been examined as photocatalysts for degradation of organic pollutants under UV light irradiation conditions; such materials are $\mathrm{K}_{2} \mathrm{Sr}_{1.5} \mathrm{Ta}_{3} \mathrm{O}_{10}$ [124], $\mathrm{Li}_{2} \mathrm{CaTa}_{2} \mathrm{O}_{7}$ [125], $\mathrm{H}_{1.81} \mathrm{Sr}_{0.81} \mathrm{Bi}_{0.19} \mathrm{Ta}_{2} \mathrm{O}_{7}$ [126], and $\mathrm{N}$-alkyl chain inserted $\mathrm{H}_{2} \mathrm{CaTa}_{2} \mathrm{O}_{7}$ [127]. A series of various metals and $\mathrm{N}$-doped perovskite materials were synthesized, such as $\mathrm{Sn}, \mathrm{Cr}, \mathrm{Zn}, \mathrm{V}, \mathrm{Fe}, \mathrm{Ni}, \mathrm{W}$, and $\mathrm{N}$-doped $\mathrm{K}_{2} \mathrm{La}_{2} \mathrm{Ti}_{3} \mathrm{O}_{10}$, for photocatalysis studies under UV and visible light irradiation [128-133]. Still, only Sn-doping efficiently decreased the bandgap energy of $\mathrm{K}_{2} \mathrm{La}_{2} \mathrm{Ti}_{3} \mathrm{O}_{10}$ from $3.6 \mathrm{eV}$ to $2.7 \mathrm{eV}$. The bandgap energy of $\mathrm{N}$-doped $\mathrm{K}_{2} \mathrm{La}_{2} \mathrm{Ti}_{3} \mathrm{O}_{10}$ was measured to be around $3.4 \mathrm{eV}$. Additional $\mathrm{RP}$ phase kind titanates like $\mathrm{Sr}_{2} \mathrm{SnO}_{4}$ [134], $\mathrm{Sr}_{3} \mathrm{Ti}_{2} \mathrm{O}_{7}$ [135], Cr-doped $\mathrm{Sr}_{2} \mathrm{TiO}_{4}$ [136], $\mathrm{Sr}_{4} \mathrm{Ti}_{3} \mathrm{O}_{10}$ [137], $\mathrm{Na}_{2} \mathrm{Ca}_{2} \mathrm{Nb}_{4} \mathrm{O}_{13}$ [138], and $\mathrm{Rh}$ - and $\mathrm{Ln}$-doped $\mathrm{Ca}_{3} \mathrm{Ti}_{2} \mathrm{O}_{7}$ [139] have also been examined. $\mathrm{Bi}_{2} \mathrm{WO}_{6}(2.8 \mathrm{eV})$ shows very high oxygen evolution efficacy than $\mathrm{Bi}_{2} \mathrm{MoO}_{6}(3.0 \mathrm{eV})$ from aqueous $\mathrm{AgNO}_{3}$ solution under visible-light-driven. Because of the appropriate bandgap energy, comparatively elevated photocatalytic performance, and good constancy, $\mathrm{Bi}_{2} \mathrm{MO}_{6}$ materials have been thoroughly examined as the Aurivillius phase kind that acts as photocatalysts under visible light. In this connection, hundreds of publications associated to the $\mathrm{Bi}_{2} \mathrm{MoO}_{6}$ and $\mathrm{Bi}_{2} \mathrm{WO}_{6}$ act as photocatalysts so far reported. Most of the investigations in the reports are concentrated on the synthesis of various nanostructured $\mathrm{Bi}_{2} \mathrm{MoO}_{6}$ and $\mathrm{Bi}_{2} \mathrm{WO}_{6}$ as well as nanofibers, nanosheets, ordered arrays, hollow spheres, hierarchical architectures, inverse opals, and nanoplates, etc., by various synthesis techniques like solvothermal, hydrothermal, electrospinning, molten salt, thermal evaporation deposition, and microwave. All these methods of hydrothermal process have been frequently working for the controlled sizes, shapes, and morphologies of the particles. The photocatalytic properties of these perovskite materials are mostly examined by the photodegradation of organic pollutants. Moreover, the investigations on the simple $\mathrm{Bi}_{2} \mathrm{MoO}_{6}$ and $\mathrm{Bi}_{2} \mathrm{WO}_{6}$, doped with various metals and nonmetals such as $\mathrm{Zn}, \mathrm{Er}, \mathrm{Mo}, \mathrm{Zr}, \mathrm{Gd}, \mathrm{W}, \mathrm{F}$, and N, into $\mathrm{Bi}_{2} \mathrm{MoO}_{6}$ and $\mathrm{Bi}_{2} \mathrm{WO}_{6}$ was studied for increasing the photocatalytic performance under visible light. Therefore, these $\mathrm{Bi}_{2} \mathrm{MO}_{6}$-based photocatalysts is not specified here, due to further full deliberations that can be shown in many reviews [140-142].

$\mathrm{ABi}_{2} \mathrm{Nb}_{2} \mathrm{O}_{9}$ where $\mathrm{A}$ is $\mathrm{Ca}, \mathrm{Sr}, \mathrm{Ba}$ and $\mathrm{Pb}$ is other type of the AL-like layered perovskite material [143-150]. The bandgap energy of $\mathrm{PbBi}_{2} \mathrm{Nb}_{2} \mathrm{O}_{9}$ is $2.88 \mathrm{eV}$ and originally described as an undoped with single-phase layered-type perovskite material used as photocatalyst employed under visible light irradiation [144]. $\mathrm{Bi}_{5} \mathrm{FeTi}_{3} \mathrm{O}_{15}$ is also Aurivillius (AL) type multi-layered nanostructured perovskite material with a low bandgap energy $(2.1 \mathrm{eV})$ and also shows photocatalytic activity under visible light $[151,152]$. Mostly, these materials were synthesized using the hydrothermal method that has been frequently working for the controlled shapes such as flower-like hierarchical morphology, nanoplate-based, and the complete advance process from nanonet-based to nanoplate-based micro-flowers was shown. The photocatalytic activity of $\mathrm{Bi}_{5} \mathrm{FeTi}_{3} \mathrm{O}_{15}$ was studied by the degradation of rhodamine 
$\mathrm{B}$ and acetaldehyde under visible light [151]. The La substituted $\mathrm{Bi}_{5-\mathrm{x}} \mathrm{La}_{\mathrm{x}} \mathrm{Ti}_{3} \mathrm{FeO}_{15}$ $(\mathrm{x}=1,2)$ Al-type layered materials were synthesized through hydrothermal method and these materials were used for photodegradation of rhodamine $\mathrm{B}$ under solarlight irradiation [153]. Among all AL-type perovskite materials, only $\mathrm{PbBi}_{2} \mathrm{Nb}_{2} \mathrm{O}_{9}$, $\mathrm{Bi}_{2} \mathrm{MO}_{6}(\mathrm{M}=\mathrm{W}$ or $\mathrm{Mo})$, and $\mathrm{Bi}_{5} \mathrm{Ti}_{3} \mathrm{FeO}_{15}$ are very high photocatalytic active under visible-light-driven due to low bandgap energy and photostability. Another type of layered perovskite material is Dion-Jacobson phase (DJ), a simple example is $\mathrm{CsBa}_{2} \mathrm{M}_{3} \mathrm{O}_{10}(\mathrm{M}=\mathrm{Ta}, \mathrm{Nb})$ and oxynitride crystals used for degradation of caffeine from wastewater under UVA- and visible-light-driven [154]. Similarly, another DJ phase material such means Dion-Jacobsen (DJ) as $\mathrm{CsM}_{2} \mathrm{Nb}_{3} \mathrm{O}_{10}(\mathrm{M}=\mathrm{Ba}$ and $\mathrm{Sr})$ and also doped with nitrogen used for photocatalysts for degradation of methylene blue [155]. Zhu et al. prepared tantalum-based \{111\}-layered type of perovskite material such as $\mathrm{Ba}_{5} \mathrm{Ta}_{4} \mathrm{O}_{15}$ from hydrothermal method, which has been frequently employed for the controlled shape like hexagonal structure with nanosheets and used as photocatalyst for photodegradation of rhodamine $\mathrm{B}$ and gaseous formaldehyde [156]. Pola et al. synthesized a layered-type perovskite material constructed on $\mathrm{A}^{\mathrm{I}} \mathrm{A}^{\mathrm{II}} \mathrm{Ti}_{2} \mathrm{O}_{6}$ $\left(\mathrm{A}^{\mathrm{I}}=\mathrm{Na}\right.$ or $\mathrm{Ag}$ or $\mathrm{Cu}$ and $\left.\mathrm{A}^{\mathrm{II}}=\mathrm{La}\right)$ structure for the photodegradation of several organic pollutants and industrial wastewater under visible-light-driven [157-162].

\begin{tabular}{|c|c|c|c|c|}
\hline Perovskite system & $\begin{array}{l}\text { Synthesis } \\
\text { process }\end{array}$ & $\begin{array}{l}\text { Light } \\
\text { source }\end{array}$ & Pollutants & References \\
\hline $\mathrm{NaTaO}_{3}$ & HT & UV & $\mathrm{CH}_{3} \mathrm{CHO}$ & {$[163]$} \\
\hline La-doped $\mathrm{NaTaO}_{3}$ & SG & UV & MB & {$[164]$} \\
\hline La-doped $\mathrm{NaTaO}_{3}$ & HT & UV & MB & [165] \\
\hline Cr-doped $\mathrm{NaTaO}_{3}$ & HT & UV & MB & {$[166]$} \\
\hline Eu-doped $\mathrm{NaTaO}_{3}$ & SS & UV & $\mathrm{MB}$ & {$[167]$} \\
\hline Bi-doped $\mathrm{NaTaO}_{3}$ & SS & UV & MB & {$[168]$} \\
\hline $\mathrm{N}$-doped $\mathrm{NaTaO}_{3}$ & SS & UV & $\mathrm{MB}$ & [169] \\
\hline C-doped $\mathrm{NaTaO}_{3}$ & HT & Visible & $\mathrm{NO}_{\mathrm{x}}$ & {$[36]$} \\
\hline N/F co-doped $\mathrm{NaTaO}_{3}$ & $\mathrm{HT}$ & UV & $\mathrm{RhB}$ & {$[170]$} \\
\hline $\mathrm{SrTiO}_{3}$ & $\mathrm{HT}$ & UV & $\mathrm{RhB}$ & {$[42,43,171]$} \\
\hline $\mathrm{Fe}$-doped $\mathrm{SrTiO}_{3}$ & SG & Visible & $\mathrm{RhB}$ & {$[172]$} \\
\hline $\mathrm{N}$-doped $\mathrm{SrTiO}_{3}$ & HT & Visible & $\mathrm{MB}, \mathrm{RhB}, \mathrm{MO}$ & {$[173]$} \\
\hline F-doped $\mathrm{SrTiO}_{3}$ & $\mathrm{BM}$ & Visible & NO & {$[174]$} \\
\hline $\mathrm{Ni} /$ La-doped $\mathrm{SrTiO}_{3}$ & SG & Visible & MG & {$[175]$} \\
\hline $\mathrm{S} / \mathrm{C}$ co-doped $\mathrm{SrTiO}_{3}$ & SS & Visible & 2-Propanol & {$[176]$} \\
\hline N/La-doped $\mathrm{SrTiO}_{3}$ & SG & Visible & 2-Propanol & {$[177]$} \\
\hline Fe-doped $\mathrm{SrTiO}_{3}$ & ST & $\begin{array}{l}\text { Visible } \\
\text { light }\end{array}$ & $\mathrm{TC}$ & {$[178]$} \\
\hline $\mathrm{SrTiO}_{3} / \mathrm{Fe}_{2} \mathrm{O}_{3}$ & HT & Visible & TC & [179] \\
\hline $\mathrm{BaTiO}_{3}$ & SG & UV & Pesticide & {$[36]$} \\
\hline $\mathrm{BaTiO}_{3}$ & SG & UV & Aromatics & {$[58]$} \\
\hline $\mathrm{BaTiO}_{3}$ & HT & UV & MO & {$[58]$} \\
\hline $\mathrm{KNbO}_{3}$ & $\mathrm{HT}$ & Visible & $\mathrm{RhB}$ & {$[180]$} \\
\hline $\mathrm{KNbO}_{3}$ & $\mathrm{HT}$ & UV & $\mathrm{RhB}$ & {$[181]$} \\
\hline $\mathrm{KNbO}_{3}$ & $\mathrm{HT}$ & Visible & $\mathrm{MB}$ & {$[182]$} \\
\hline $\mathrm{NaNbO}_{3}$ & SS & UV & $\mathrm{RhB}$ & [183] \\
\hline $\mathrm{NaNbO}_{3}$ & Imp. & UV & 2-Propanol & [184] \\
\hline $\mathrm{NaNbO}_{3}$ & SS & UV & MB & [185] \\
\hline
\end{tabular}


Significant Role of Perovskite Materials for Degradation of Organic Pollutants DOI: http://dx.doi.org/10.5772/intechopen.91680

\begin{tabular}{|c|c|c|c|c|}
\hline Perovskite system & $\begin{array}{l}\text { Synthesis } \\
\text { process }\end{array}$ & $\begin{array}{l}\text { Light } \\
\text { source }\end{array}$ & Pollutants & References \\
\hline $\mathrm{N}$-doped $\mathrm{NaNbO}_{3}$ & SS & UV & 2-Propanol & {$[186-188]$} \\
\hline $\mathrm{Ru}$-doped $\mathrm{NaNbO}_{3}$ & $\mathrm{HT}$ & Visible & Phenol & [189] \\
\hline $\mathrm{AgNbO}_{3}$ & SS & UV & $\mathrm{MB}$ & {$[190]$} \\
\hline La-doped $\mathrm{AgNbO}_{3}$ & SS & Visible & 2-Propanol & {$[191]$} \\
\hline $\mathrm{BiFeO}_{3}$ & SG & UV-Vis & $\begin{array}{l}\text { MO, RhB, } \\
4-\mathrm{CP}\end{array}$ & {$[69-77]$} \\
\hline Ba-doped $\mathrm{BiFeO}_{3}$ & ES & Visible & $\mathrm{CR}$ & [79] \\
\hline Ca-doped $\mathrm{BiFeO}_{3}$ & ES & Visible & $\mathrm{CR}$ & {$[80]$} \\
\hline $\mathrm{Ba}$ or $\mathrm{Mn}$-doped $\mathrm{BiFeO}_{3}$ & ES & Visible & $\mathrm{CR}$ & {$[82]$} \\
\hline $\mathrm{Ca}$ or $\mathrm{Mn}$-doped $\mathrm{BiFeO}_{3}$ & HT & $\begin{array}{l}\text { UV- } \\
\text { Visible }\end{array}$ & $\mathrm{RhB}$ & {$[82]$} \\
\hline Gd-doped $\mathrm{BiFeO}_{3}$ & SG & Visible & $\mathrm{RhB}$ & {$[83]$} \\
\hline $\mathrm{LaFeO}_{3}$ & Comb. & UV & Methyl phenol & {$[84]$} \\
\hline $\mathrm{LaFeO}_{3}$ & SG & Visible & $\mathrm{RhB}$ & {$[85]$} \\
\hline $\mathrm{LaFeO}_{3}$ & HT & Visible & $\begin{array}{l}\mathrm{RhB}, \mathrm{MB}, \\
\text { chlorophenol }\end{array}$ & {$[86,90,91,192]$} \\
\hline Ca-doped $\mathrm{LaFeO}_{3}$ & SS & Visible & $\mathrm{MB}$ & {$[92]$} \\
\hline $\mathrm{LnFeO}_{3}(\mathrm{Pr}, \mathrm{Y})$ & SG & Visible & $\mathrm{RhB}$ & {$[193]$} \\
\hline $\mathrm{SrFeO}_{3-\mathrm{x}}$ & US & Visible & Phenol & [194] \\
\hline $\mathrm{SrFeO}_{3}$ & SS & Visible & $\mathrm{MB}$ & {$[195]$} \\
\hline $\mathrm{BaZrO}_{3}$ & SG & UV & $\mathrm{MB}$ & {$[196]$} \\
\hline $\mathrm{BaZrO}_{3}$ & $\mathrm{HT}$ & UV & $\mathrm{MO}$ & [197] \\
\hline $\begin{array}{l}\mathrm{ATiO}_{3}(\mathrm{~A}=\mathrm{Fe}, \mathrm{Pb}) \text { and } \mathrm{AFeO}_{3} \\
(\mathrm{~A}=\mathrm{Bi}, \mathrm{La}, \mathrm{Y})\end{array}$ & SG & Visible & $\mathrm{MB}$ & [198] \\
\hline $\mathrm{Zn}_{0.9} \mathrm{Mg}_{0.1} \mathrm{TiO}_{3}$ & SG & Visible & $\mathrm{MB}$ & [199] \\
\hline $\begin{array}{l}\mathrm{SrTiO}_{3} \text { nanocube-coated CdS } \\
\text { microspheres }\end{array}$ & HT & Visible & $\begin{array}{l}\text { Antibiotic } \\
\text { pollutants }\end{array}$ & {$[200]$} \\
\hline $\mathrm{Ag} / \mathrm{AgCl} / \mathrm{CaTiO}_{3}$ & HT & Visible & $\mathrm{RhB}$ & [201] \\
\hline $\mathrm{TiO}_{2}$-coupled $\mathrm{NiTiO}_{3}$ & SS & Visible & $\mathrm{MB}$ & [202] \\
\hline $\mathrm{ZnTiO}_{3}$ & $\mathrm{HT}$ & UV & MO and PCP & [203] \\
\hline $\mathrm{Mg}$-doped $\mathrm{BaZrO}_{3}$ & SS & UV & $\mathrm{MB}$ & [204] \\
\hline $\mathrm{SrSnO}_{3}$ & MW & UV & $\mathrm{MO}$ & {$[205]$} \\
\hline $\mathrm{LaCoO}_{3}$ & MW & Visible & $\mathrm{MO}$ & [206] \\
\hline $\mathrm{LaCoO}_{3}$ & Ads. & UV & $\mathrm{MB}, \mathrm{MO}$ & [207] \\
\hline $\mathrm{LaCoO}_{3}$ & ES & UV & $\mathrm{RhB}$ & [208] \\
\hline $\mathrm{LaNiO}_{3}$ & SG & Visible & $\mathrm{MO}$ & [209] \\
\hline $\mathrm{Bi}_{0.5} \mathrm{Na}_{0.5} \mathrm{TiO}_{3}$ & HT & UV & $\mathrm{MO}$ & {$[93]$} \\
\hline $\mathrm{La}_{0.7} \mathrm{Sr}_{0.3} \mathrm{MnO}_{3}$ & SG & $\begin{array}{l}\text { Solar } \\
\text { light }\end{array}$ & $\mathrm{MO}$ & {$[97]$} \\
\hline $\mathrm{La}_{0.5} \mathrm{Ca}_{0.5} \mathrm{NiO}_{3}$ & SG & UV & RB5 & {$[98]$} \\
\hline $\mathrm{La}_{0.5} \mathrm{Ca}_{0.5} \mathrm{CoO}_{3}$ & SG & UV & $\mathrm{CR}$ & [99] \\
\hline $\mathrm{Sr}_{1-\mathrm{x}} \mathrm{Ba}_{\mathrm{x}} \mathrm{SnO}_{3}$ & SS & UV & Azo-dye & {$[100]$} \\
\hline $\mathrm{BaCo}_{1 / 2} \mathrm{Nb}_{1 / 2} \mathrm{O}_{3}$ & SG & Visible & $\mathrm{MB}$ & [210] \\
\hline $\mathrm{Ba}\left(\mathrm{In}_{1 / 3} \mathrm{~Pb}_{1 / 3} \mathrm{M}_{1 / 3}\right) \mathrm{O}_{3}(\mathrm{M}=\mathrm{Nb}$ and $\mathrm{Ta})$ & SS & Visible & $\mathrm{MB}, 4-\mathrm{CP}$ & {$[211]$} \\
\hline $\begin{array}{l}\mathrm{A}\left(\mathrm{In}_{1 / 3} \mathrm{Nb}_{1 / 3} \mathrm{~B}_{1 / 3}\right) \mathrm{O}_{3}(\mathrm{~A}=\mathrm{Sr}, \mathrm{Ba} \\
\mathrm{B}=\mathrm{Sn}, \mathrm{Pb})\end{array}$ & SS & Visible & $\mathrm{MB}, 4-\mathrm{CP}$ & {$[212]$} \\
\hline $\mathrm{SrTi}_{1-\mathrm{x}} \mathrm{Fe}_{\mathrm{x}} \mathrm{O}_{3-\delta}$ & SS & Visible & $\mathrm{MB}$ & {$[102]$} \\
\hline
\end{tabular}




\begin{tabular}{|c|c|c|c|c|}
\hline Perovskite system & $\begin{array}{l}\text { Synthesis } \\
\text { process }\end{array}$ & $\begin{array}{l}\text { Light } \\
\text { source }\end{array}$ & Pollutants & References \\
\hline $\mathrm{SrTi}_{0.1} \mathrm{Fe}_{0.9} \mathrm{O}_{3-\delta}$ & SG & $\begin{array}{l}\text { Solar } \\
\text { light }\end{array}$ & MO & [103] \\
\hline $\mathrm{SrFe}_{0.5} \mathrm{Co}_{0.5} \mathrm{O}_{3-\delta}$ & SG & $\begin{array}{l}\text { Solar } \\
\text { light }\end{array}$ & CR & [213] \\
\hline $\mathrm{LaFe}_{0.5} \mathrm{Ti}_{0.5} \mathrm{O}_{3}$ & SG & UV & Phenol & {$[90]$} \\
\hline $\mathrm{Bi}\left(\mathrm{Mg}_{3 / 8} \mathrm{Fe}_{2 / 8} \mathrm{Ti}_{3 / 8}\right) \mathrm{O}_{3}$ & MS & Visible & $\mathrm{MO}$ & {$[110]$} \\
\hline $\mathrm{LaTi}(\mathrm{ON})_{3}$ & SG & Visible & Acetone & [214] \\
\hline$\left(\mathrm{Ag}_{0.75} \mathrm{Sr}_{0.25}\right)\left(\mathrm{Nb}_{0.75} \mathrm{Ti}_{0.25}\right) \mathrm{O}_{3}$ & SS & Visible & $\mathrm{CH}_{3} \mathrm{CHO}$ & [215] \\
\hline $\mathrm{La}_{0.8} \mathrm{Ba}_{0.2} \mathrm{Fe}_{0.9} \mathrm{Mn}_{0.1} \mathrm{O}_{3-\mathrm{x}}$ & SG & $\begin{array}{l}\text { Solar } \\
\text { light }\end{array}$ & $\mathrm{MO}$ & {$[115]$} \\
\hline $\mathrm{Cu}-\left(\mathrm{Sr}_{1-\mathrm{y}} \mathrm{Na}_{\mathrm{y}}\right)\left(\mathrm{Ti}_{1-\mathrm{x}} \mathrm{Mo}_{\mathrm{x}}\right) \mathrm{O}_{3}$ & HT & Visible & Propanol & [118] \\
\hline $\mathrm{BiFeO}_{3}-\left(\mathrm{Na}_{0.5} \mathrm{Bi}_{0.5}\right) \mathrm{TiO}_{3}$ & SG & Visible & $\mathrm{RhB}$ & {$[120]$} \\
\hline $\mathrm{SrBi}_{2} \mathrm{Nb}_{2} \mathrm{O}_{9}$ & $\begin{array}{l}\text { SG } \\
\text { SS }\end{array}$ & UV & Aniline, RhB & {$[145,146]$} \\
\hline $\mathrm{ABi}_{2} \mathrm{Nb}_{2} \mathrm{O}_{9}(\mathrm{~A}=\mathrm{Sr}, \mathrm{Ba})$ & SG & UV & MO & {$[147]$} \\
\hline $\mathrm{Bi}_{5} \mathrm{Ti}_{3} \mathrm{FeO}_{15}$ & $\begin{array}{l}\mathrm{HT} \\
\mathrm{SS}\end{array}$ & Visible & $\begin{array}{l}\mathrm{RhB}, \mathrm{CH}_{3} \mathrm{CHO} \\
\mathrm{IPA}\end{array}$ & {$[149,150]$} \\
\hline $\mathrm{Bi}_{5-\mathrm{x}} \mathrm{La}_{\mathrm{x}} \mathrm{Ti}_{3} \mathrm{FeO}_{15}$ & SS & $\begin{array}{l}\text { Solar } \\
\text { light }\end{array}$ & $\mathrm{RhB}$ & {$[151]$} \\
\hline $\begin{array}{l}\mathrm{Bi}_{3} \mathrm{SrTi}_{2} \mathrm{TaO}_{12} \\
\mathrm{Bi}_{2} \mathrm{LaSrTi}_{2} \mathrm{TaO}_{12}\end{array}$ & SS & UV & $\mathrm{RhB}$ & [216] \\
\hline $\mathrm{Ba}_{5} \mathrm{Ta}_{4} \mathrm{O}_{15}$ & $\mathrm{HT}$ & UV & $\mathrm{RhB}$ & {$[154]$} \\
\hline $\mathrm{N}$-doped $\mathrm{Ln}_{2} \mathrm{Ti}_{2} \mathrm{O}_{7}(\mathrm{Ln}=\mathrm{La}, \mathrm{Pr}, \mathrm{Nd})$ & HT & Visible & MO & {$[217]$} \\
\hline $\mathrm{CdS} / \mathrm{Ag} / \mathrm{Bi}_{2} \mathrm{MoO}_{6}$ & SG & Visible & $\mathrm{RhB}$ & {$[218]$} \\
\hline
\end{tabular}

SS: solid state; HT: hydrothermal; SG: sol-gel; BM: ball-milling; ES: electronspun; MW: microwave; Comb.: combustion; US: ultrasonic; MS: molten salt; Imp.: impregnation; Ads.: adsorption; ST: solvothermal; RhB: rhodamine B; MO: methyl orange; MB: methylene blue; 4-cp: 4-chlorophenol; MG: malachite green; CR: congo red; NO: nitrogen monoxide; PA: isopropyl alcohol; TC: tetracycline; and PCP: pentachlorophenol.

Table 1.

Perovskite materials used as photocatalysts $\left(A B O_{3}, A A^{I} B O_{3}, A A^{I} B O_{3}, A B B^{I} O_{3}, A B(O N)_{3}\right.$, and $\left.A A^{I} B B^{I I} O_{3}\right)$ for degradation of pollutants.
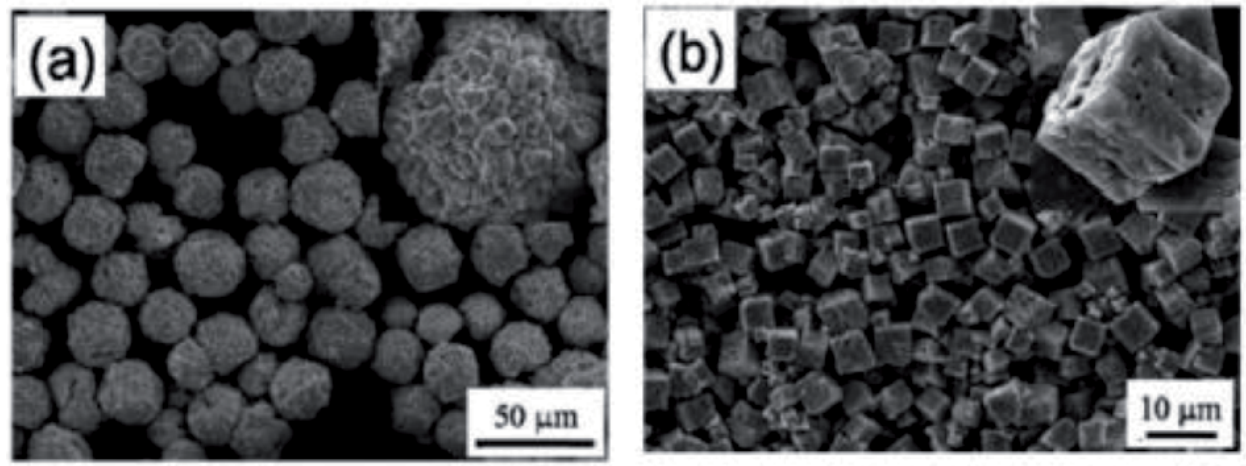

Figure 2.

SEM patterns of $\mathrm{BiFeO}_{3}$ : (a) microspheres and (b) microcubes. The intensified pictures are revealed in the upper part inserts. Recopied with consent from Ref. [147]. Copyright (C) 2010, American Chemical Society. 


\section{Acknowledgements}

Authors would like to thank DST-FIST schemes and CSIR, New Delhi. One of us (Ramesh Gade) thanks Council of Scientific \& Industrial Research (CSIR), New Delhi, for the award of Junior Research Fellowship.

\section{Thanks}

I am thankful to Department of Chemistry, University College of Science, Osmania University, for their continuous attention in this study and useful discussions, and to Prof. B. Manohar for her support in working on the chapter.

\section{Author details}

Someshwar Pola* and Ramesh Gade

Department of Chemistry, University College of Science, Osmania University, Hyderabad, Telangana, India

*Address all correspondence to: somesh.pola@gmail.com

\section{IntechOpen}

(C) 2020 The Author(s). Licensee IntechOpen. This chapter is distributed under the terms of the Creative Commons Attribution License (http://creativecommons.org/licenses/ by/3.0), which permits unrestricted use, distribution, and reproduction in any medium, provided the original work is properly cited. (cc) BY 


\section{References}

[1] Barbara DA, Jared JS, Tyson AB, William WA. Insights from placing photosynthetic light harvesting into context. Journal of Physical Chemistry Letters. 2014;5:2880-2889

[2] von Erika S, Paul SM, James DA, Lori B, Piers F, David F, et al. Chemistry and the linkages between air quality and climate change. Chemical Reviews. 2015;115:3856-3897

[3] Rowan JB, Andrew TH. Review of major design and scale-up considerations for solar photocatalytic reactors. Industrial and Engineering Chemistry Research. 2009;48:8890-8905

[4] Michael GW, Emily LW, James RM, Shannon WB, Qixi M, Elizabeth AS, et al. Solar water splitting cells.

Chemical Reviews. 2010;110:6446-6473

[5] Manvendra P, Rahul K, Kamal K, Todd M, Charles UP Jr, Dinesh M. Pharmaceuticals of emerging concern in aquatic systems: Chemistry, occurrence, effects, and removal methods. Chemical Reviews. 2019;119:3510-3673

[6] Marta M, Jolanta K, Iseult L, Marianne M, Jan K, Steve B, et al. Changing environments and biomolecule coronas: Consequences and challenges for the design of environmentally acceptable engineered nanoparticles. Green Chemistry. 2018;20:4133-4168

[7] Wanjun W, Guiying L, Dehua X, Taicheng A, Huijun Z, Po KW. Photocatalytic nanomaterials for solardriven bacterial inactivation: recent progress and challenges. Environmental Science: Nano. 2017;4:782-799

[8] Wanjun WYY, Taicheng A, Guiying L, Ho YY, Jimmy CY, Po KW. Visible-light-driven photocatalytic inactivation of E. coli $\mathrm{K}-12$ by bismuth vanadate nanotubes: bactericidal performance and mechanism. Environmental Science \& Technology. 2012;46:4599-4606

[9] Nadine C, Stefanie I, Elisabeth S, Marjan V, Karolin K, David D, et al. Inactivation of antibiotic resistant bacteria and resistance genes by ozone: From laboratory experiments to full-scale wastewater treatment. Environmental Science \& Technology. 2016;50:11862-11871

[10] Yidong Z, Xiangxue W, Ayub K, Pengyi W, Yunhai L, Ahmed A, et al. Environmental remediation and application of nanoscale zero-valent iron and its composites for the removal of heavy metal ions: A review. Environmental Science \& Technology. 2016;50:7290-7304

[11] Wenya H, Kelong A, Xiaoyan R, Shengyan W, Lehui L. Inorganic layered ion-exchangers for decontamination of toxic metal ions in aquatic systems. Journal of Materials Chemistry A. 2017;5:19593-19606

[12] Manolis JM, Mercouri GK. Metal sulfide ion exchangers: Superior sorbents for the capture of toxic and nuclear waste-related metal ions. Chemical Science. 2016;7:4804-4824

[13] Chunli K, Kunkun X, Yuhan W, Dongmei H, Ling Z, Fang L, et al. Synthesis of $\mathrm{SrTiO}_{3}-\mathrm{TiN}$ nanocomposites with enhanced photocatalytic activity under simulated solar irradiation. Industrial and Engineering Chemistry Research. 2018;57:11526-11534

[14] Vaidyanathan S, Ryan KR, Eduardo EW. Synthesis and UVvisible-light photoactivity of noblemetal-SrTiO 3 composites. Industrial and Engineering Chemistry Research. 2006;45:2187-2193 
[15] Partha M, Aarthi T,

Giridhar M, Srinivasan N.

Photocatalytic Degradation of Dyes and Organics with Nanosized $\mathrm{GdCoO}_{3}$. Journal of Physical Chemistry C. 2007;111(4):1665-1674

[16] Dipankar S, Sudarshan M, Guru Row TN, Giridhar M. Synthesis, structure, and photocatalytic activity in orthorhombic perovskites $\mathrm{LnVO}_{3}$ and $\mathrm{Ln}_{1-\mathrm{x}} \mathrm{Ti}_{\mathrm{x}} \mathrm{VO}_{3}(\mathrm{Ln}=\mathrm{Ce}, \mathrm{Pr}$, and $\mathrm{Nd})$. Industrial and Engineering Chemistry Research. 2009;48:7489-7497

[17] Barrocas B, Sério S, Rovisco A, Melo Jorge ME. Visible-light photocatalysis in $\mathrm{Ca}_{0.6} \mathrm{Ho}_{0.4} \mathrm{MnO}_{3}$ films deposited by RF-magnetron sputtering using nanosized powder compacted target. Journal of Physical Chemistry C. 2014;118:590-597

[18] Tokeer A, Umar F, RubyP. Fabrication and photocatalytic applications of perovskite materials with special emphasis on alkali-metal-based niobates and tantalates. Industrial and Engineering Chemistry Research. 2018;57:18-41

[19] Senthilkumar P, Arockiya Jency D, Kavinkumar T, Dhayanithi D, Dhanuskodi S, Umadevi M, et al. Built-in electric field assisted photocatalytic dye degradation and photoelectrochemical water splitting of ferroelectric $\mathrm{Ce}$ doped $\mathrm{BaTiO}_{3}$ nanoassemblies. ACS Sustainable Chemistry \& Engineering. 2019;7:12032-12043

[20] Hongbo F, Shicheng Z, Tongguang X, Yongfa Z, Jianmin C. Photocatalytic degradation of $\mathrm{RhB}$ by fluorinated $\mathrm{Bi}_{2} \mathrm{WO}_{6}$ and distributions of the intermediate products.

Environmental Science \& Technology. 2008;42:2085-2091

[21] Sandeep K, Sunita K, Meganathan T, Ashok KG. Achieving enhanced visiblelight-driven photocatalysis using type-II $\mathrm{NaNbO}_{3} / \mathrm{CdS}$ core/shell heterostructures. ACS Applied Materials \& Interfaces. 2014;6:13221-13233

[22] Yanlong T, Binbin C, Jiangli L, Jie F, Fengna X, Xiaoping D. Hydrothermal synthesis of graphitic carbon nitride$\mathrm{Bi}_{2} \mathrm{WO}_{6}$ heterojunctions with enhanced visible light photocatalytic activities. ACS Applied Materials \& Interfaces. 2013;5:7079-7085

[23] Anindya SP, Gaurangi G, Mohammad Q. Ordered-disordered $\mathrm{BaZrO}_{3-\delta}$ hollow nanosphere/carbon dot hybrid nanocomposite: A new visible-light-driven efficient composite photocatalyst for hydrogen production and dye degradation. ACS Omega. 2018;3:10980-10991

[24] Joanna HC, Matthew SD, Robert GP, Christopher PI, James RD, John BC, et al. Visible light photo-oxidation of model pollutants using $\mathrm{CaCu}_{3} \mathrm{Ti}_{4} \mathrm{O}_{12}$ : $\mathrm{An}$ experimental and theoretical study of optical properties, electronic structure, and selectivity. Journal of the American Chemical Society. 2011;133:1016-1032

[25] Rasel D, Chad DV, Agnes S, Bin C, Ahmad FI, Xianbo L, et al. Recent advances in nanomaterials for water protection and monitoring. Chemical Society Reviews. 2017;46:6946-7020

[26] Pena MA, Fierro JLG. Chemical structures and performance of perovskite oxides. Chemical Reviews. 2001;101:1981-2018

[27] Anna K, Marcos FG, Gerardo C. Advanced nanoarchitectures for solar photocatalytic applications. Chemical Reviews. 2012;112:1555-1614

[28] Wei W, Moses OT, Zongping S. Research progress of perovskite materials in photocatalysisand photovoltaics-related energy conversion and environmental treatment. Chemical Society Reviews. 2015;44:5371-5408 
[29] Guan Z, Gang L,

Lianzhou W, John TSI. Inorganic perovskite photocatalysts for solar energy utilization. Chemical Society Reviews. 2016;45:5951-5984

[30] Xiubing H, Guixia Z, Ge W, John TSI. Synthesis and applications of nanoporous perovskite metal oxides. Chemical Science. 2018;9:3623-3637

[31] Beata B, Ewa K, Joanna N, Zhishun W, Maya E, Bunsho O, et al. Preparation of $\mathrm{CdS}$ and $\mathrm{Bi}_{2} \mathrm{~S}_{3}$ quantum dots co-decorated perovskite-type $\mathrm{KNbO}_{3}$ ternary heterostructure with improved visible light photocatalytic activity and stability for phenol degradation. Dalton Transactions. 2018;47:15232-15245

[32] Hamidreza A, Yuan W, Hongyu S, Mehran R, Hongxing D. Ordered mesoand macroporous perovskite oxide catalysts for emerging applications. Chemical Communications. 2018;54:6484-6502

[33] Bin L, Gang L, Lianzhou W. Recent advances in 2D materials for photocatalysis. Nanoscale. 2016;8:6904-6920

[34] Chunping X, Prasaanth Ravi A, Cyril A, Rafael L, Samuel M. Nanostructured materials for photocatalysis. Chemical Society Reviews. 2019;48:3868-3902

[35] Xiaoyun C, Jun X, Yueshan X, Feng L, Yaping D. Rare earth double perovskites: a fertile soil in the field of perovskite oxides. Inorganic Chemistry Frontiers. 2019;6:2226-2238

[36] Xiaoyong W, Shu Y, Qiang D, Tsugio S. Preparation and visible light induced photocatalytic activity of $\mathrm{C}-\mathrm{NaTaO}_{3}$ and $\mathrm{C}-\mathrm{NaTaO}_{3}-\mathrm{Cl}_{-} \mathrm{TiO}_{2}$ composite. Physical Chemistry Chemical Physics. 2013;15:20633-20640

[37] Shaodong S, Xiaojing Y, Qing Y, Zhimao Y, Shuhua L. Mesocrystals for photocatalysis: A comprehensive review on synthesis engineering and functional modifications. Nanoscale Advances. 2019;1:34-63

[38] Ming Y, Xian LH, Shicheng Y, Zhaosheng L, Tao Y, Zhigang Z. Improved hydrogen evolution activities under visible light irradiation over $\mathrm{NaTaO}_{3}$ codoped with lanthanum and chromium. Materials Chemistry and Physics. 2010;121:506-510

[39] Shin S, Yonemura M. Orderdisorder transition of $\mathrm{Sr}_{2} \mathrm{Fe}_{2} \mathrm{O}_{5}$ from brownmillerite to perovskite structure at an elevated temperature. Materials Research Bulletin. 1978;13:1017-1021

[40] Wagner FT, Somorjai GA.

Photocatalytic and photoelectrochemical hydrogen production on strontium titanate single crystals. Journal of the American Chemical Society. 1980;102:5494-5502

[41] Guowei L, Graeme RB, Thomas TMP. Vacancies in functional materials for clean energy storage and harvesting: The perfect imperfection. Chemical Society Reviews. 2017;46:1693-1706

[42] Xiao W, Gang X, Zhaohui R, Chunxiao X, Ge S, Gaorong H. PVAassisted hydrothermal synthesis of $\mathrm{SrTiO}_{3}$ nanoparticles with enhanced photocatalytic activity for degradation of $\mathrm{RhB}$. Journal of the American Ceramic Society. 2008;91:3795-3799

[43] Xiao W, Gang X, Zhaohui R, Chunxiao X, Wenjian W, Ge S, et al. Single-crystal-like mesoporous $\mathrm{SrTiO}_{3}$ spheres with enhanced photocatalytic performance. Journal of the American Ceramic Society. 2010;93:1297-1305

[44] Bo W, Ming-Yu Q, Chuang H, Zi-Rong T, Yi-Jun X. Photocorrosion inhibition of semiconductor-based photocatalysts: Basic principle, current development, and future perspective. ACS Catalysis. 2019;9:4642-4687 
[45] Natalita MN, Xingdong W, Rachel AC. High-throughput synthesis and screening of titania-based photocatalysts. ACS Combinatorial Science. 2015;17:548-569

[46] Hideki K, Akihiko K. Highly efficient decomposition of pure water into $\mathrm{H}_{2}$ and $\mathrm{O}_{2}$ over $\mathrm{NaTaO}_{3}$ photocatalysts. Catalysis Letters. 1999;58:153-155

[47] Hideki K, Akihiko K. Water splitting into $\mathrm{H}_{2}$ and $\mathrm{O}_{2}$ on alkali tantalate photocatalysts $\mathrm{ATaO}_{3}(\mathrm{~A}=\mathrm{Li}, \mathrm{Na}$, and $\mathrm{K})$. Journal of Physical Chemistry $B$. 2001;105(19):4285-4292

[48] Hideki K, Akihiko K. Photocatalytic water splitting into $\mathrm{H}_{2}$ and $\mathrm{O}_{2}$ over various tantalate photocatalysts. Catalysis Today. 2003;78:561-569

[49] Liu JW, Chen G, Li ZH, Zhang ZG. Hydrothermal synthesis and photocatalytic properties of $\mathrm{ATaO}_{3}$ and $\mathrm{ANbO}_{3}(\mathrm{~A}=\mathrm{Na}$ and $\mathrm{K})$. International Journal of Hydrogen Energy. 2007;32:2269-2272

[50] Che-Chia H, Hsisheng T. Influence of structural features on the photocatalytic activity of $\mathrm{NaTaO}_{3}$ powders from different synthesis methods. Applied Catalysis A. 2007;331:44-50

[51] Che-Chia H, Chien-Cheng T, Hsisheng T. Structure characterization and tuning of perovskite-like $\mathrm{NaTaO}_{3}$ for applications in photoluminescence and photocatalysis. Journal of the American Ceramic Society. 2009;92:460-466

[52] Xia L, Jinling Z. Facile hydrothermal synthesis of sodium tantalate $\left(\mathrm{NaTaO}_{3}\right)$ nanocubes and high photocatalytic properties. Journal of Physical Chemistry C. 2009;113:19411-19418

[53] Fu X, Wang X, Leung DYC, Xue W, Ding Z, Huang H, et al. Photocatalytic reforming of glucose over La doped alkali tantalate photocatalysts for $\mathrm{H}_{2}$ production. Catalysis Communications. 2010;12:184-187

[54] Yokoi T, Sakuma J, Maeda K, Domen K, Tatsumi T, Kondo JN. Preparation of a colloidal array of $\mathrm{NaTaO}_{3}$ nanoparticles via a confined space synthesis route and its photocatalytic application. Physical Chemistry Chemical Physics. 2011;13:2563-2570

[55] Shi J, Liu G, Wang N, Li C. Microwave-assisted hydrothermal synthesis of perovskite $\mathrm{NaTaO}_{3}$ nanocrystals and their photocatalytic properties. Journal of Materials Chemistry. 2012;22:18808-18813

[56] Meyer T, Priebe JB, Silva RO, Peppel T, Junge H, Beller M, et al. Advanced charge utilization from $\mathrm{NaTaO}_{3}$ photocatalysts by multilayer reduced graphene oxide. Chemistry of Materials. 2014;26:4705-4711

[57] Li Y, Gou H, Lu J, Wang C. A twostep synthesis of $\mathrm{NaTaO}_{3}$ microspheres for photocatalytic water splitting. International Journal of Hydrogen Energy. 2014;39:13481-13485

[58] Gomathi Devi L, Krishnamurthy G. $\mathrm{TiO}_{2} / \mathrm{BaTiO}_{3}$-assisted photocatalytic mineralization of diclofop-methyl on UV-light irradiation in the presence of oxidizing agents. Journal of Hazardous Materials. 2009;162:899-905

[59] Gomathi Devi L, Krishnamurthy G. $\mathrm{TiO}_{2}$ - and $\mathrm{BaTiO}_{3}$-assisted photocatalytic degradation of selected chloroorganic compounds in aqueous medium: Correlation of reactivity/ orientation effects of substituent groups of the pollutant molecule on the degradation rate. The Journal of Physical Chemistry. A. 2011;115:460-469

[60] Liu J, Sun Y, Li Z. Ag loaded flowerlike $\mathrm{BaTiO}_{3}$ nanotube arrays: Fabrication 
and enhanced photocatalytic property. CrystEngComm. 2012;14:1473-1478

[61] Maeda K. Rhodium-doped barium titanate perovskite as a stable p-type semiconductor photocatalyst for hydrogen evolution under visible light. ACS Applied Materials \& Interfaces. 2014;6:2167-2173

[62] Upadhyay S, Shrivastava J, Solanki A, Choudhary S, Sharma V, Kumar P, et al. Enhanced photoelectrochemical response of $\mathrm{BaTiO}_{3}$ with Fe doping: Experiments and firstprinciples analysis. Journal of Physical Chemistry C. 2011;115:24373-24380

[63] Mizoguchi H, Ueda K, Orita M, Moon S-C, Kajihara K, Hirano M, et al. Decomposition of water by a $\mathrm{CaTiO}_{3}$ photocatalyst under UV light irradiation. Materials Research Bulletin. 2002;37:2401-2406

[64] Shimura K, Yoshida H. Hydrogen production from water and methane over Pt-loaded calcium titanate photocatalyst. Energy \& Environmental Science. 2010;3:615-617

[65] Zhang H, Chen G, Li Y, Teng Y. Electronic structure and photocatalytic properties of copper-doped $\mathrm{CaTiO}_{3}$. International Journal of Hydrogen Energy. 2010;35:2713-2716

[66] Nishimoto S, Matsuda M, Miyake M. Photocatalytic activities of $\mathrm{Rh}$-doped $\mathrm{CaTiO}_{3}$ under visible light irradiation. Chemistry Letters. 2006;35:308-309

[67] Zhang H, Chen G, He X, Xu J. Electronic structure and photocatalytic properties of $\mathrm{Ag}-\mathrm{La}$ codoped $\mathrm{CaTiO}_{3}$. Journal of Alloys and Compounds. 2012;516:91-95

[68] Arney D, Watkins T, Maggard PA. Effects of particle surface areas and microstructures on photocatalytic $\mathrm{H}_{2}$ and $\mathrm{O}_{2}$ production over $\mathrm{PbTiO}_{3}$. Journal of the American Ceramic Society. 2011;94:1483-1489

[69] Zhen C, Yu JC, Liu G, Cheng $\mathrm{H}-\mathrm{M}$. Selective deposition of redox co-catalyst(s) to improve the photocatalytic activity of single-domain ferroelectric $\mathrm{PbTiO}_{3}$ nanoplates. Chemical Communications. 2014;50:10416-10419

[70] Gao F, Chen X, Yin K, Dong S, Ren Z, Yuan F, et al. Visible-Light photocatalytic properties of weak magnetic $\mathrm{BiFeO}_{3}$ nanoparticles.

Advanced Materials. 2007;19:2889-2892

[71] Huo Y, Miao M, Zhang Y, Zhu J, $\mathrm{Li} \mathrm{H}$. Aerosol-spraying preparation of a mesoporous hollow spherical $\mathrm{BiFeO}_{3}$ visible photocatalyst with enhanced activity and durability. Chemical Communications. 2011;47:2089-2091

[72] Cho CM, Noh JH, Cho I-S, An J-S, Hong KS. Low-temperature hydrothermal synthesis of pure $\mathrm{BiFeO}_{3}$ nanopowders using triethanolamine and their applications as visible-light photocatalysts. Journal of the American Ceramic Society. 2008;91:3753-3755

[73] Li S, Lin Y-H, Zhang B-P, Wang Y, Nan C-W. Controlled fabrication of $\mathrm{BiFeO}_{3}$ uniform microcrystals and their magnetic and photocatalytic behaviors. Journal of Physical Chemistry C.

2010;114:2903-2908

[74] Xu X, Lin Y-H, Li P, Shu L, Nan C-W. Synthesis and photocatalytic behaviors of high surface area $\mathrm{BiFeO}_{3}$ thin films. Journal of the American Ceramic Society. 2011;94:2296-2299

[75] Ji W, Yao K, Lim Y-F, Liang YC, Suwardi A. Large modulation of perpendicular magnetic anisotropy in a $\mathrm{BiFeO}_{3} / \mathrm{Al}_{2} \mathrm{O}_{3} / \mathrm{Pt} / \mathrm{Co} / \mathrm{Pt}$ multiferroic heterostructure via spontaneous polarizations. Applied Physics Letters. 2013;103:062901 
[76] Chen XY, Yu T, Gao F, Zhang HT, Liu LF, Wang YM, et al. Application of weak ferromagnetic $\mathrm{BiFeO}_{3}$ films as the photoelectrode material under visible-light irradiation. Applied Physics Letters. 2007;91:022114

[77] Li S, Zhang J, Kibria MG, Mi Z, Chaker M, Ma D, et al. Remarkably enhanced photocatalytic activity of laser ablated $\mathrm{Au}$ nanoparticle decorated $\mathrm{BiFeO}_{3}$ nanowires under visiblelight. Chemical Communications. 2013;49:5856-5858

[78] Huo Y, Jin Y, Zhang Y. Citric acid assisted solvothermal synthesis of $\mathrm{BiFeO}_{3}$ microspheres with high visiblelight photocatalytic activity. Journal of Molecular Catalysis A: Chemical. 2010;331:15-20

[79] Schultz AM, Zhang Y, Salvador PA, Rohrer GS. Effect of crystal and domain orientation on the visible-light photochemical reduction of $\mathrm{Ag}$ on $\mathrm{BiFeO}_{3}$. ACS Applied Materials \& Interfaces. 2011;3:1562-1567

[80] Feng YN, Wang HC,

Shen Y, Lin YH, Nan CW. Magnetic and photocatalytic behaviors of Ba-doped $\mathrm{BiFeO}_{3}$ nanofibers. International Journal of Applied Ceramic Technology. 2014;11:676-680

[81] Feng YN, Wang HC, Luo YD, Shen Y, Lin YH. Ferromagnetic and photocatalytic behaviors observed in $\mathrm{Ca}$-doped $\mathrm{BiFeO}_{3}$ nanofibres. Journal of Applied Physics. 2013;113:146101

[82] Wang HC, Lin YH, Feng YN, Shen Y. Photocatalytic behaviors observed in $\mathrm{Ba}$ and $\mathrm{Mn}$ doped $\mathrm{BiFeO}_{3}$ nanofibers. Journal of Electroceramics. 2013;31:271

[83] Pei YL, Zhang C. Effect of ion doping in different sites on the morphology and photocatalytic activity of $\mathrm{BiFeO}_{3}$ microcrystals. Journal of Alloys and Compounds. 2013;570:57-60
[84] Guo R, Fang L, Dong W, Zheng F, Shen M. Enhanced photocatalytic activity and ferromagnetism in $\mathrm{Gd}$ doped $\mathrm{BiFeO}_{3}$ nanoparticles. Journal of Physical Chemistry C. 2010;114:21390-21396

[85] Deganello F, Tummino ML, Calabrese C, Testa ML, Avetta P, Fabbi D, et al. A new, sustainable $\mathrm{LaFeO}_{3}$ material prepared from biowastesourced soluble substances. New Journal of Chemistry. 2015;39:877-885

[86] Li L, Wang X, Zhang Y. Enhanced visible light-responsive photocatalytic activity of $\mathrm{LnFeO}_{3}(\mathrm{Ln}=\mathrm{La}$, $\mathrm{Sm}$ ) nanoparticles by synergistic catalysis. Materials Research Bulletin. 2014;50:18-22

[87] Thirumalairajan S, Girija K, Mastelaro VR, Ponpandian N. Photocatalytic degradation of organic dyes under visible light irradiation by floral-like $\mathrm{LaFeO}_{3}$ nanostructures comprised of nanosheet petals. New Journal of Chemistry. 2014;38:5480-5490

[88] Parida KM, Reddy KH, Martha S, Das DP, Biswal N. Fabrication of nanocrystalline $\mathrm{LaFeO}_{3}$ : An efficient sol-gel auto-combustion assisted visible light responsive photocatalyst for water decomposition. International Journal of Hydrogen Energy. 2010;35:12161-12168

[89] Tijare SN, Joshi MV, Padole PS, Mangrulkar PA, Rayalu SS, Labhsetwar NK. Photocatalytic hydrogen generation through water splitting on nano-crystalline $\mathrm{LaFeO}_{3}$ perovskite. International Journal of Hydrogen Energy. 2012;37:10451-10456

[90] Thirumalairajan S, Girija K, Ganesh I, Mangalaraj D, Viswanathan C, Balamurugan A. Controlled synthesis of perovskite $\mathrm{LaFeO}_{3}$ microsphere composed of nanoparticles via selfassembly process and their associated photocatalytic activity. Chemical Engineering Journal. 2012;209:420-428 
[91] Hu R, Li C, Wang X, Sun Y, Jia H, $\mathrm{Su} \mathrm{H}$, et al. Photocatalytic activities of $\mathrm{LaFeO}_{3}$ and $\mathrm{La}_{2} \mathrm{FeTiO}_{6}$ in p-chlorophenol degradation under visible light. Catalysis Communications. 2012;29:35-39

[92] Thirumalairajan S, Girija K, Hebalkar NY, Mangalaraj D, Viswanathan C, Ponpandian N. Shape evolution of perovskite $\mathrm{LaFeO}_{3}$ nanostructures: A systematic investigation of growth mechanism, properties and morphology dependent photocatalytic activities. RSC Advances. 2013;3:7549-7561

[93] Li FT, Liu Y, Liu RH, Sun ZM, Zhao DS, Kou CG. Preparation of Ca-doped $\mathrm{LaFeO}_{3}$ nanopowders in a reverse microemulsion and their visible light photocatalytic activity. Materials Letters. 2010;64:223-225

[94] Invalid reference

[95] Li J, Wang G, Wang H, Tang C, Wang Y, Liang C, et al. In situ selfassembly synthesis and photocatalytic performance of hierarchical $\mathrm{Bi} 0.5 \mathrm{Na} 0.5 \mathrm{TiO}_{3}$ micro/nanostructures. Journal of Materials Chemistry. 2009;19:2253-2258

[96] Wang L, Wang W. Photocatalytic hydrogen production from aqueous solutions over novel $\mathrm{Bi} 0.5 \mathrm{Na} 0.5 \mathrm{TiO}_{3}$ microspheres. International Journal of Hydrogen Energy. 2012;37:3041-3047

[97] Ai Z, Lu G, Lee S. Efficient photocatalytic removal of nitric oxide with hydrothermal synthesized $\mathrm{Na} 0.5 \mathrm{Bi0} .5 \mathrm{TiO}_{3}$ nanotubes. Journal of Alloys and Compounds. 2014;613:260-266

[98] Hu CC, Lee YL, Teng H. Efficient water splitting over $\mathrm{Na}_{1-\mathrm{x}} \mathrm{K}_{\mathrm{x}} \mathrm{TaO}_{3}$ photocatalysts with cubic perovskite structure. Journal of Materials Chemistry. 2011;21:3824-3830
[99] Ghiasi M, Malekzadeh MA. Solar photocatalytic degradation of methyl orange over $\mathrm{La}_{0.7} \mathrm{Sr}_{0.3} \mathrm{MnO}_{3}$ nanoperovskite. Separation and Purification Technology. 2014;134:12-19

[100] Yazdanbakhsh M, Tavakkoli H, Hosseini SM. Characterization and evaluation catalytic efficiency of $\mathrm{La}_{0.5} \mathrm{Ca}_{0.5} \mathrm{NiO}_{3}$ nanopowders in removal of reactive blue 5 from aqueous solution. Desalination. 2011;281:388-395

[101] Tavakkoli H, Beiknejad D, Tabari T. Fabrication of perovskite-type oxide $\mathrm{La}_{0.5} \mathrm{Ca}_{0.5} \mathrm{CoO}_{3-\delta}$ nanoparticles and its dye removal performance. Desalination and Water Treatment. 2014;52:7377-7388

[102] Sales HB, Bouquet V,

Deputier S, Ollivier S, Gouttefangeas F, Guilloux-Viry G, et al. $\mathrm{Sr}_{1-\mathrm{x}} \mathrm{Ba}_{\mathrm{x}} \mathrm{SnO}_{3}$ system applied in the photocatalytic discoloration of an azo-dye. Solid State Sciences. 2014;28:67-73

[103] Bae SW, Borse PH, Lee JS. Dopant dependent band gap tailoring of hydrothermally prepared cubic $\mathrm{SrTi}_{\mathrm{x}} \mathrm{M}_{1-\mathrm{x}} \mathrm{O}_{3}(\mathrm{M}=\mathrm{Ru}, \mathrm{Rh}, \mathrm{Ir}, \mathrm{Pt}$, $\mathrm{Pd})$ nanoparticles as visible light photocatalysts. Applied Physics Letters. 2008;92:104107

[104] Ghaffari M, Huang H, Tan PY, Tan OK. Synthesis and visible light photocatalytic properties of $\mathrm{SrTi}_{(1-\mathrm{x})} \mathrm{Fe}_{\mathrm{x}} \mathrm{O}_{(3-\delta)}$ powder for indoor decontamination. Powder Technology. 2012;225:221-226

[105] Chen HX, Wei ZX, Wang Y, Zeng WW, Xiao CM. Preparation of $\mathrm{SrTi}_{0.1} \mathrm{Fe}_{0.9} \mathrm{O}_{3-\delta}$ and its photocatalysis activity for degradation of methyl orange in water. Materials Chemistry and Physics. 2011;130:1387-1393

[106] Jeong ED, Yu SM, Yoon JY, Bae JS, Cho CR, Lim KT, et al. Efficient visible light photocatalysis in cubic $\mathrm{Sr}_{2} \mathrm{FeNbO}_{6}$. Journal of Ceramic Processing Research. 2012;13:305-309 
[107] Li J, Jia L, Fang W, Zeng J.

Enhancement of activity of

$\mathrm{LaNi}_{0.7} \mathrm{Cu}_{0.3} \mathrm{O}_{3}$ for photocatalytic water

splitting by reduction treatment at moderate temperature. International

Journal of Hydrogen Energy.

2010;35:5270-5275

[108] Li J, Zeng J, Jia L, Fang W.

Investigations on the effect of $\mathrm{Cu}^{2+}$ /

$\mathrm{Cu}^{1+}$ redox couples and oxygen

vacancies on photocatalytic activity

of treated $\mathrm{LaNi}_{1-\mathrm{x}} \mathrm{Cu}_{\mathrm{x}} \mathrm{O}_{3}(\mathrm{x}=0.1,0.4$,

0.5). International Journal of Hydrogen

Energy. 2010;35:12733-12740

[109] Li H, Liang Q, Gao LZ, Tang SH, Cheng ZY, Zhang BL, et al. Catalytic production of carbon nanotubes by decomposition of $\mathrm{CH}_{4}$ over the prereduced catalysts $\mathrm{LaNiO}_{3}, \mathrm{La}_{4} \mathrm{Ni}_{3} \mathrm{O}_{10}$, $\mathrm{La}_{3} \mathrm{Ni}_{2} \mathrm{O}_{7}$ and $\mathrm{La}_{2} \mathrm{NiO}_{4}$. Catalysis Letters. 2001;74:185-188

[110] Yuan Y, Zhao Z, Zheng J, Yang M, Qiu L, Li Z, et al. Polymerizable complex synthesis of $\mathrm{BaZr}_{1-\mathrm{x}} \mathrm{SnxO}_{3}$ photocatalysts: Role of $\mathrm{Sn}^{4+}$ in the band structure and their photocatalytic water splitting activities. Journal of Materials Chemistry. 2010;20:6772-6779

[111] Kang HW, Lim SN, Park SB. Photocatalytic $\mathrm{H}_{2}$ evolution under visible light from aqueous methanol solution on $\mathrm{NaBi}_{\mathrm{x}} \mathrm{Ta}_{1-\mathrm{x}} \mathrm{O}_{3}$ prepared by spray pyrolysis. International Journal of Hydrogen Energy. 2012;37:4026-4035

[112] Xu L, Li C, Shi W, Guan J, Sun Z. Visible light-response $\mathrm{NaTa}_{1-\mathrm{x}} \mathrm{Cu}_{\mathrm{x}} \mathrm{O}_{3}$ photocatalysts for hydrogen production from methanol aqueous solution. Journal of Molecular Catalysis A: Chemical. 2012;360:42-47

[113] Zhang W, Chen J, An X, Wang Q, Fan L, Wang F, et al. Rapid synthesis, structure and photocatalysis of pure bismuth A-site perovskite of $\mathrm{Bi}\left(\mathrm{Mg}_{3 / 8} \mathrm{Fe}_{2 / 8} \mathrm{Ti}_{3 / 8}\right) \mathrm{O}_{3}$. Dalton Transactions. 2014;43:9255-9259
[114] Ni L, Tanabe M, Irie H. A visiblelight-induced overall water-splitting photocatalyst: Conduction-bandcontrolled silver tantalite. Chemical Communications. 2013;49:10094-10096

[115] Simone P, Marie CC, Stephan I, Alexandra EM, Rolf E. Mesoporosity in photocatalytically active oxynitride single crystals. Journal of Physical Chemistry C. 2014;118(36):20940-20947

[116] Wu P, Shi J, Zhou Z, Tang W, Guo L. $\mathrm{CaTaO}_{2} \mathrm{~N}-\mathrm{CaZrO}_{3}$ solid solution: Band-structure engineering and visiblelight-driven photocatalytic hydrogen production. International Journal of Hydrogen Energy. 2012;37:13704-13710

[117] Luo W, Li Z, Jiang X, Yu T, Liu L, Chen X, et al. Correlation between the band positions of $\left(\mathrm{SrTiO}_{3}\right)_{1-\mathrm{x}} \cdot\left(\mathrm{LaTiO}_{2} \mathrm{~N}\right)_{\mathrm{x}}$ solid solutions and photocatalytic properties under visible light irradiation. Physical Chemistry Chemical Physics. 2008;10:6717-6723

[118] Wei ZX, Xiao CM, Zeng WW, Liu JP. Magnetic properties and photocatalytic activity of $\mathrm{La}_{0.8} \mathrm{Ba}_{0.2} \mathrm{Fe}_{0.9} \mathrm{Mn}_{0.1} \mathrm{O}_{3-\delta}$ and $\mathrm{LaFe}_{0.9} \mathrm{Mn}_{0.1} \mathrm{O}_{3-\delta}$. Journal of Molecular Catalysis A: Chemical. 2013;370:35-43

[119] Kanhere P, Nisar P, Tang Y, Pathak B, Ahuja R, Zheng J, et al. Electronic structure, optical properties, and photocatalytic activities of $\mathrm{LaFeO}_{3}-\mathrm{NaTaO}_{3}$ solid solution. Journal of Physical Chemistry C. 2012;116:22767-22773

[120] Shi J, Ye J, Zhou Z, Li M, Guo L. Hydrothermal synthesis of $\mathrm{Na}_{0.5} \mathrm{La}_{0.5} \mathrm{TiO}_{3}-\mathrm{LaCrO}_{3}$ solid-solution single-crystal nanocubes for visiblelight-driven photocatalytic $\mathrm{H}_{2}$ evolution. Chemistry-A European Journal. 2011;17:7858-7867

[121] Qiu X, Miyauchi M, Yu H, Irie H, Hashimoto K. Visible-light-driven 
$\mathrm{Cu}(\mathrm{II})-\left(\mathrm{Sr}_{1-\mathrm{y}} \mathrm{Na}_{\mathrm{y}}\right)\left(\mathrm{Ti}_{1-\mathrm{x}} \mathrm{Mo}_{\mathrm{x}}\right) \mathrm{O}_{3}$ photocatalysts based on conduction band control and surface ion modification. Journal of the American Chemical Society. 2010;132:15259-15267

[122] Yi ZG, Ye JH. Band gap tuning of $\mathrm{Na}_{1-\mathrm{x}} \mathrm{La}_{\mathrm{x}} \mathrm{Ta}_{1-\mathrm{x}} \mathrm{Cr}_{\mathrm{x}} \mathrm{O}_{3}$ for $\mathrm{H}_{2}$ generation from water under visible light irradiation. Journal of Applied Physics. 2009;106:074910

[123] Liu H, Guo Y, Guo B, Dong W, Zhang D. $\mathrm{BiFeO}_{3}-\left(\mathrm{Na}_{0.5} \mathrm{Bi}_{0.5}\right) \mathrm{TiO}_{3}$ butterfly wing scales: Synthesis, visiblelight photocatalytic and magnetic properties. Journal of the European Ceramic Society. 2012;32:4335-4340

[124] Lv M, Xie Y, Wang Y, Sun X, Wu F, $\mathrm{Chen} \mathrm{H}$, et al. Bismuth and chromium co-doped strontium titanates and their photocatalytic properties under visible light irradiation. Physical Chemistry Chemical Physics. 2015;17:26320-26329

[125] Yao W, Ye J. Photocatalytic properties of a new photocatalyst $\mathrm{K}_{2} \mathrm{Sr}_{1.5} \mathrm{Ta}_{3} \mathrm{O}_{10}$. Chemical Physics Letters. 2007;435:96-99

[126] Liang Z, Tang K, Shao Q, Li G, Zeng S, Zheng H. Synthesis, crystal structure, and photocatalytic activity of a new two-layer Ruddlesden-Popper phase, $\mathrm{Li}_{2} \mathrm{CaTa}_{2} \mathrm{O}_{7}$. Journal of Solid State Chemistry. 2008;181:964-970

[127] Li Y, Chen G, Zhou C, Li Z. Photocatalytic water splitting over a protonated layered perovskite tantalate $\mathrm{H}_{1.81} \mathrm{Sr}_{0.81} \mathrm{Bi}_{0.19} \mathrm{Ta}_{2} \mathrm{O}_{7}$. Catalysis Letters. 2008;123:80-83

[128] Wang Y, Wang C, Wang L, Hao Q, Zhu X, Chen X, et al. Preparation of interlayer surface tailored protonated double-layered perovskite $\mathrm{H}_{2} \mathrm{CaTa}_{2} \mathrm{O}_{7}$ with n-alcohols, and their photocatalytic activity. RSC Advances. 2014;4:4047-4054

[129] Kumar V, Uma GS. Investigation of cation $\left(\mathrm{Sn}^{2+}\right)$ and anion $\left(\mathrm{N}^{3-}\right)$ substitution in favor of visible light photocatalytic activity in the layered perovskite $\mathrm{K}_{2} \mathrm{La}_{2} \mathrm{Ti}_{3} \mathrm{O}_{10}$. Journal of Hazardous Materials. 2011;189:502-508

[130] Thaminimulla CTK, Takata T, Hara M, Knodo JN, Domen K. Effect of chromium addition for photocatalytic overall water splitting on $\mathrm{Ni}-$ $\mathrm{K}_{2} \mathrm{La}_{2} \mathrm{Ti}_{3} \mathrm{O}_{10}$. Journal of Catalysis. 2000;196:362-365

[131] Yang YH, Chen QY, Yin ZL, Li J. Study on the photocatalytic activity of $\mathrm{K}_{2} \mathrm{La}_{2} \mathrm{Ti}_{3} \mathrm{O}_{10}$ doped with zinc $(\mathrm{Zn})$. Applied Surface Science. 2009;255:8419-8424

[132] Yang Y, Chen Q, Yin Z, Li J. Study on the photocatalytic activity of $\mathrm{K}_{2} \mathrm{La}_{2} \mathrm{Ti}_{3} \mathrm{O}_{10}$ doped with vanadium (V). Journal of Alloys and Compounds. 2009;488:364

[133] Huang Y, Wei Y, Cheng S, Fan L, Li Y, Lin J, et al. Photocatalytic property of nitrogen-doped layered perovskite $\mathrm{K}_{2} \mathrm{La}_{2} \mathrm{Ti} 3 \mathrm{O} 10$. Solar Energy Materials and Solar Cells. 2010;94:761-766

[134] Wang B, Li C, Hirabayashi D, Suzuki K. Hydrogen evolution by photocatalytic decomposition of water under ultraviolet-visible irradiation over $\mathrm{K}_{2} \mathrm{La}_{2} \mathrm{Ti}_{3-\mathrm{x}} \mathrm{M}_{\mathrm{x}} \mathrm{O}_{10+\delta}$ perovskite. International Journal of Hydrogen Energy. 2010;35:3306-3312

[135] Sato J, Saito N, Nishiyama H, Inoue Y. New photocatalyst group for water decomposition of $\mathrm{RuO}_{2}-$ loaded p-block metal (In, Sn, and $\mathrm{Sb}$ ) oxides with d10 Configuration. The Journal of Physical Chemistry. B. 2001;105:6061-6063

[136] Jeong H, Kim T, Kim D, Kim K. Hydrogen production by the photocatalytic overall water splitting on $\mathrm{NiO} / \mathrm{Sr}_{3} \mathrm{Ti}_{2} \mathrm{O}_{7}$ : Effect of preparation method. International Journal of Hydrogen Energy. 2006;31:1142-1146 
[137] Sun X, Xie Y, Wu F, Chen H, Lv M, Ni S, et al. Photocatalytic hydrogen production over chromium doped layered perovskite $\mathrm{Sr}_{2} \mathrm{TiO}_{4}$. Inorganic Chemistry. 2015;54:7445-7453

[138] Ko YG, Lee WY. Effects of nickelloading method on the water-splitting activity of a layered $\mathrm{NiO}_{\mathrm{x}} / \mathrm{Sr}_{4} \mathrm{Ti}_{3} \mathrm{O}_{10}$ photocatalyst. Catalysis Letters. 2002;83:157-160

[139] Arney D, Fuoco L, Boltersdorf J, Maggard PA. Flux synthesis of $\mathrm{Na}_{2} \mathrm{Ca}_{2} \mathrm{Nb}_{4} \mathrm{O}_{13}$ : The influence of particle shapes, surface features, and surface areas on photocatalytic hydrogen production. Journal of the American Ceramic Society. 2012;96:1158-1162

[140] Nishimoto S, Okazaki Y, Matsuda M, Miyake M. Photocatalytic $\mathrm{H}_{2}$ evolution by layered perovskite $\mathrm{Ca}_{3} \mathrm{Ti}_{2} \mathrm{O}_{7}$ codoped with $\mathrm{Rh}$ and $\mathrm{Ln}$ $(\mathrm{Ln}=\mathrm{La}, \mathrm{Pr}, \mathrm{Nd}, \mathrm{Eu}, \mathrm{Gd}, \mathrm{Yb}$, and $\mathrm{Y})$ under visible light irradiation. Journal of the Ceramic Society of Japan. 2009;117:1175-1179

[141] Zhang L, Wang H, Chen Z, Wong PK, Liu J. $\mathrm{Bi}_{2} \mathrm{WO}_{6}$ micro/nanostructures: Synthesis, modifications and visible-light-driven photocatalytic applications. Applied Catalysis B. 2011;106:1-13

[142] Zhang L, Zhu Y. A review of controllable synthesis and enhancement of performances of bismuth tungstate visible-light-driven photocatalysts. Catalysis Science \& Technology. 2012;2:694-706

[143] Zhang N, Ciriminna R, Ragliaro M, $\mathrm{Xu} Y \mathrm{YJ}$. Nanochemistry-derived $\mathrm{Bi}_{2} \mathrm{WO}_{6}$ nanostructures: towards production of sustainable chemicals and fuels induced by visible light. Chemical Society Reviews. 2014;43:5276-5287

[144] Fu H, Pan C, Yao W, Zhu Y. Visible-light-induced degradation of rhodamine $\mathrm{B}$ by nanosized $\mathrm{Bi}_{2} \mathrm{WO}_{6}$.
The Journal of Physical Chemistry. B. 2005;109:22432-22439

[145] Kim HG, Hwang DW,

Lee JS. An undoped, single-phase oxide photocatalyst working under visible light. Journal of the American Chemical Society. 2004;126:8912-8913

[146] Kim HG, Becker OS, Jang JS, Ji SM, Borse PH, Lee JS. A generic method of visible light sensitization for perovskiterelated layered oxides: Substitution effect of lead. Journal of Solid State Chemistry. 2006;179:1214-1218

[147] Kim HG, Borse PH, Jang JS, Jeong ED, Lee JS. Enhanced photochemical properties of electron rich $\mathrm{W}$-doped $\mathrm{PbBi}_{2} \mathrm{Nb}_{2} \mathrm{O}_{9}$ layered perovskite material under visiblelight irradiation. Materials Letters. 2008;62:1427-1430

[148] Wu W, Liang S, Chen Y, Shen L, Zheng H, Wu L. High efficient photocatalytic reduction of 4-nitroaniline to $\mathrm{p}$-phenylenediamine over microcrystalline $\mathrm{SrBi}_{2} \mathrm{Nb}_{2} \mathrm{O}_{9}$. Catalysis Communications. 2012;17:39-42

[149] Kim JH, Hwang KT, Kim US, Kang YM. Photocatalytic characteristics of immobilized $\mathrm{SrBi}_{2} \mathrm{Nb}_{2} \mathrm{O}_{9}$ film for degradation of organic pollutants. Ceramics International. 2012;38:3901-3906

[150] Wu W, Liang S, Wang X, Bi J, Liu P, Wu L. Synthesis, structures and photocatalytic activities of microcrystalline $\mathrm{ABi}_{2} \mathrm{Nb}_{2} \mathrm{O}_{9}(\mathrm{~A}=\mathrm{Sr}$, $\mathrm{Ba})$ powders. Journal of Solid State Chemistry. 2011;184:81-88

[151] Li Y, Chen G, Zhang H, Lv G. Band structure and photocatalytic activities for $\mathrm{H}_{2}$ production of $\mathrm{ABi}_{2} \mathrm{Nb}_{2} \mathrm{O}_{9}(\mathrm{~A}=$ $\mathrm{Ca}, \mathrm{Sr}, \mathrm{Ba})$. International Journal of Hydrogen Energy. 2010;35:2652-2656

[152] Sun S, Wang W, Xu H, Zhou L, Shang M, Zhang L. $\mathrm{Bi}_{5} \mathrm{FeTi}_{3} \mathrm{O}_{15}$ 
hierarchical microflowers:

Hydrothermal synthesis, growth

mechanism, and associated visible-light-driven photocatalysis. Journal of Physical Chemistry C. 2008;112:17835-17843

[153] Jang JS, Yoon SS, Borse PH, Lim KT, Hong TE, Jeong ED, et al. Synthesis and characterization of aurivilius phase $\mathrm{Bi}_{5} \mathrm{Ti}_{3} \mathrm{FeO}_{15}$ layered perovskite for Visible light photocatalysis. Journal of the Ceramic Society of Japan. 2009;117:1268-1272

[154] Naresh G, Mandal TK. Excellent sun-light-driven photocatalytic activity by aurivillius layered perovskites, $\mathrm{Bi}_{5-\mathrm{x}} \mathrm{La}_{\mathrm{x}} \mathrm{Ti}_{3} \mathrm{FeO}_{15}(\mathrm{x}=1,2)$. ACS Applied Materials \& Interfaces. 2014;6:21000-21010

[155] Bożena C, Mirabbos H. UVA- and visible-light-driven photocatalytic activity of three-layer perovskite Dion-Jacobson phase $\mathrm{CsBa}_{2} \mathrm{M}_{3} \mathrm{O}_{10}$ $(\mathrm{M}=\mathrm{Ta}, \mathrm{Nb})$ and oxynitride crystals in the removal of caffeine from model wastewater. Journal of Photochemistry and Photobiology A: Chemistry. 2016;324:70-80

[156] Reddy JR, Kurra S, Guje R, Palla S, Veldurthi NK, Ravi G, et al. Photocatalytic degradation of methylene blue on nitrogen doped layered perovskites, $\mathrm{CsM}_{2} \mathrm{Nb}_{3} \mathrm{O}_{10}(\mathrm{M}=\mathrm{Ba}$ and $\mathrm{Sr})$. Ceramics International. 2015;41:2869-2875

[157] Xu TG, Zhang C, Shao X, Wu K, Zhu YF. Monomolecular-layer $\mathrm{Ba}_{5} \mathrm{Ta}_{4} \mathrm{O}_{15}$ nanosheets: Synthesis and investigation of photocatalytic properties.

Advanced Functional Materials. 2006;16:1599-1607

[158] Ramesh G, Jakeer A, Kalyana LY, Seid YA, Tao YT, Someshwar P.

Photodegradation of organic dyes and industrial wastewater in the presence of layer-type perovskite materials under visible light irradiation. Journal of
Environmental Chemical Engineering. 2018;6:4504-4513

[159] Friedmann D, Mendice C, Bahnemann D. $\mathrm{TiO}_{2}$ for water treatment: Parameters affecting the kinetics and mechanisms of photocatalysis. Applied Catalysis B: Environmental. 2010;99:398-406

[160] Rizzo L, Meric S, Kassino D, Guida M, Russo F, Belgiorno V.

Degradation of diclofenac by $\mathrm{TiO}_{2}$ photocatalysis: UV absorbance kinetics and process evaluation through a set of toxicity bioassays. Water Research. 2009;43:979-988

[161] Konstantinou T, Albanis A. $\mathrm{TiO}_{2-}$ assisted photocatalytic degradation of azo dyes in aqueous solution: Kinetic and mechanistic investigations. A review. Applied Catalysis B: Environmental. 2004;49:1-14

[162] Kim SH, Ngo HH, Shon HK, Vigneswaran S. Adsorption and photocatalysis kinetics of herbicide onto titanium oxide and powdered activated carbon. Separation and Purification Technology. 2008;58:335-342

[163] Liu JW, Chen G, Li ZH, Zhang ZG. Electronic structure and visible light photocatalysis water splitting property of chromium-doped $\mathrm{SrTiO}_{3}$. Journal of Solid State Chemistry. 2006;179:3704-3708

[164] Torres-Martínez LM, Cruz-López A, Juárez-Ramírez I, Elena Meza-de la Rosa M. Methylene blue degradation by $\mathrm{NaTaO}_{3}$ sol-gel doped with Sm and La. Journal of Hazardous Materials. 2009;165:774-779

[165] Li X, Zang J. Hydrothermal synthesis and characterization of lanthanum-doped $\mathrm{NaTaO}_{3}$ with high photocatalytic activity. Catalysis Communications. 2011;12:1380-1383

[166] Yiguo S, Wang S, Meng Y, Wang HHX. Dual substitutions of 
single dopant $\mathrm{Cr}^{3+}$ in perovskite $\mathrm{NaTaO}_{3}$ : synthesis, structure, and photocatalytic performance. RSC Advances.

2012;2:12932-12939

[167] Wang B, Kanhere PD, Chen Z, Nisar J, Biswarup PA. Anion-doped natao $_{3}$ for visible light photocatalysis. Journal of Physical Chemistry C. 2014;118:10728-10739

[168] Kanhere PD, Zheng J, Chen Z. Site specific optical and photocatalytic properties of $\mathrm{Bi}$-doped $\mathrm{NaTaO}_{3}$. Journal of Physical Chemistry C. 2011;115:11846-11853

[169] Liu D-R, Wei C-D, Xue B, Zhang X-G, Jiang Y-S. Synthesis and photocatalytic activity of $\mathrm{N}$-doped $\mathrm{NaTaO}_{3}$ compounds calcined at low temperature. Journal of Hazardous Materials. 2010;182:50-54

[170] Zhang J, Jiang Y, Gao W, Hao H. Synthesis and visible photocatalytic activity of new photocatalyst $\mathrm{MBI}_{2} \mathrm{O}_{4}$ $(\mathrm{M}=\mathrm{Cu}, \mathrm{Zn})$. Journal of Materials Science: Materials in Electronics. 2014;25:3807-3815

[171] Da Silva LF, Avansi W, Andres J, Ribeiro C. Long-range and short-range structures of cube-like shape $\mathrm{SrTiO}_{3}$ powders: Microwaveassisted hydrothermal synthesis and photocatalytic activity. Physical Chemistry Chemical Physics.

2013;15:12386-12393

[172] Xie T-H, Sun X, Lin J. Enhanced photocatalytic degradation of RhB driven by visible light-induced MMCT of $\mathrm{Ti}$ (IV)-O-Fe(II)formed in Fe-doped $\mathrm{SrTiO}_{3}$. Journal of Physical Chemistry C. 2008;112:9753-9759

[173] Zou F, Zheng J, Qin X, Zhao Y, Jiang L, Zhi J, et al. Chemical Communications. 2012;48:8514-8516

[174] Wang J, Yin S, Zhang Q, Saito F, Sato T. Mechanochemical synthesis of $\mathrm{SrTiO}_{3-\mathrm{x}} \mathrm{F}_{\mathrm{x}}$ with high visible light photocatalytic activities for nitrogen monoxide destruction. Journal of Materials Chemistry. 2003;13:2348-2352

[175] Jia A, Liang X, Su Z, Zhu T, Liu S. Synthesis and effect of calcinations temperature on the physical-chemical properties and photocatalytic activities of $\mathrm{Ni}$, La coded $\mathrm{SrTiO}_{3}$. Journal of Hazardous Materials. 2010;178:233-242

[176] Ohno T, Tsubota T, Nakamura Y, Sayama K. Preparation of S, C cationcodoped $\mathrm{SrTiO}_{3}$ and its photocatalytic activity under visible light.

Applied Catalysis A. 2005;288:74-79

[177] Miyauchi M, Takashio M, Tobimastu H. Photocatalytic activity of $\mathrm{SrTiO}_{3}$ codoped with nitrogen and lanthanum under visible light illumination. Langmuir. 2004;20:232-236

[178] Li P, Liu C, Wu G, Yang H, Lin S, Ren A, et al. Solvothermal synthesis and visible light-driven photocatalytic degradation for tetracycline of $\mathrm{Fe}$-doped $\mathrm{SrTiO}_{3}$. RSC Advances. 2014;4:47615-47624

[179] Liu C, Wu G, Chen J, Hang K, Shi W. Fabrication of a visible-lightdriven photocatalyst and degradation of tetracycline based on the photoinduced interfacial charge transfer of $\mathrm{SrTiO}_{3} /$ $\mathrm{Fe}_{2} \mathrm{O}_{3}$ nanowires. New Journal of Chemistry. 2016;40:5198-5208

[180] Lan J, Zhou X, Liu G, Yu J, Zhang J, Zhi L, et al. Enhancing photocatalytic activity of one-dimensional $\mathrm{KNbO}_{3}$ nanowires by $\mathrm{Au}$ nanoparticles under ultraviolet and visible-light. Nanoscale. 2011;3:5161-5167

[181] Jiang L, Qui Y, Yi Z. Potassium niobate nanostructures: controllable morphology, growth mechanism, and photocatalytic activity. Journal of Materials Chemistry A. 2013;1:2878-2885 
[182] Yan L, Zhang T, Lei W, Xu Q, Zhou X, Xu P, et al. Catalytic activity of gold nanoparticles supported on $\mathrm{KNbO}_{3}$ microcubes. Catalysis Today. 2014;224:140-146

[183] Li G, Yi Z, Bai Y, Zhang W, Zhang $\mathrm{H}$. Anisotropy in photocatalytic oxidization activity of $\mathrm{NaNbO}_{3}$ photocatalyst. Dalton Transactions. 2012;41:10194-10198

[184] Hard template synthesis of nanocrystalline $\mathrm{NaNbO}_{3}$ with enhanced photocatalytic performance. Catalysis Letters. 2012;142:901-906

[185] Katsumata K-I, Cordonier CEJ, Shichi T, FuJishima A. Photocatalytic activity of $\mathrm{NaNbO}_{3}$ thin films. Journal of the American Chemical Society. 2009;131:3856-3857

[186] Yu J, Yu Y, Zhou P, Xiao W, Cheng B. Morphology-dependent photocatalytic-production activity of CDS. Applied Catalysis B. 2014:156-157

[187] Pai MR, Majeed J, Banerjee AM, Arya A, Bhattacharya S, Rao R, et al. Role of $\mathrm{Nd} 3+$ ions in modifying the band structure and photocatalytic properties of substituted indium titanates, $\mathrm{In}_{2(1-\mathrm{x})} \mathrm{Nd}_{2 \mathrm{x}} \mathrm{TiO}_{5}$ oxides. Journal of Physical Chemistry C. 2012;116:1458-1471

[188] Haifeng S, Xiukai L, Hideol W, Zhingang Z, Jinhua Y. 3- Propanol photodegradation over nitrogen-doped $\mathrm{NaNbO}_{3}$ powders under visible-light irradiation. Journal of Physics and Chemistry of Solids. 2009;70:931-935

[189] Paul B, Choo KH. Visible light active $\mathrm{Ru}$-doped sodium niobate perovskite decorated with platinum nanoparticles via surface capping. Catalysis Today. 2014;230:138-144

[190] Shu H, Xie J, Xu H, Li H, Gu Z, Sun G, et al. Structural characterization and photocatalytic activity of $\mathrm{NiO} /$
$\mathrm{AgNbO}_{3}$. Journal of Alloys and

Compounds. 2010;496:633-637

[191] Li G, Kako T, Wamg D, Zou Z, Ye Y. Enhanced photocatalytic activity of La-doped $\mathrm{AgNbO}_{3}$ under visible light irradiation. Dalton Transactions. 2009:2423-2427

[192] Wang Y, Gao Y, Chen L, Zhang H. Geothite as an efficient heterogeneous fenton catalyst for the degradation of methyl orange. Chemical Engineering Journal. 2012;239:322-331

[193] Li L, Zhang M, Tan P, Gu W, Wang X. Synthetic photocatalytic activity of $\mathrm{LnFeO}_{3}(\mathrm{Ln}=\mathrm{Pr}, \mathrm{Y})$ perovskites under visible-light illumination. Ceramics International. 2014;40:13813-13817

[194] Jia L, Ding T, Li Q, Tang Y. Study of photocatalytic performance of $\mathrm{SrFeO}_{3-\mathrm{x}}$ by ultrasonic radiation. Catalysis Communications. 2007;8:963-966

[195] Gaffari M, Tan PY, Oruc ME, Tan OK, Tse MS. Effect of ball milling on the characteristics of nano structure $\mathrm{SrFeO}_{3}$ powder for photocatalytic degradation of methylene blue under visible light irradiation and its reaction kinetics. Catalysis Today. 2011;161:70-77

[196] Ye T-N, Xu M, Fu W, Cai Y-Y, Wei X, Wang K-X, et al. The crystallinity effect of mesocrystalline $\mathrm{BaZrO}_{3}$ hollow nanosheres on charge separation for photocatalysis. Chemical Communications. 2014;50:3021-3023

[197] Prastomo N, Zakaria NHB, Kawamura G, Muto H, Matsuda A. High surface area $\mathrm{BaZrO}_{3}$ photocatalyst prepared by base-hot-water treatment. Journal of the European Ceramic Society. 2011;31:2699-2705

[198] Li L, Liu X, Zhang Y, Nuhfer NT, Barmak K, Salvador PA, et al. Visiblelight photochemical activity of 
heterostructured core-shell materials composed of selected ternary titanates and ferrites coated by $\mathrm{TiO}_{2}$. ACS Applied Materials \& Interfaces. 2013;5:5064-5071

[199] Cai Z, Zhou H, Song J, Zhao F, Li J. Preparation and characterization of $\mathrm{Zn}_{0.9} \mathrm{Mg}_{0.1} \mathrm{TiO}_{3}$ via electrospinning. Dalton Transactions. 2011;40:8335-8339

[200] Wu G, Xiao L, Gu W, Shi W, Jiang D, Liu C. Fabrication and excellent visible-light-driven photodegradation activity for antibiotics of $\mathrm{SrTiO}_{3}$ nanocube coated $\mathrm{CdS}$ microsphere heterojunctions. RSC Advances. 2016;6:19878-19886

[201] Wang Y, Niu C-G, Liang W, Wang Y, Zhang X-G, Zeng G-M. Synthesis of fern-like $\mathrm{Ag} / \mathrm{AgCl} / \mathrm{CaTiO}_{3}$ plasmonic photocatalysts and their enhanced visible-light photocatalytic properties. RSC Advances.

2016;6:47873-47882

[202] Shu X, He J, Chen D. Visiblelight-induced photocatalyst based on nickel titanate nanoparticles. Industrial and Engineering Chemistry Research. 2008;47:4750-4753

[203] Ding N, Chen X, Wu C-ML, $\mathrm{Li} \mathrm{H}$. Adsorption of nucleobase pairs on hexagonal boron nitride sheet: hydrogen bonding versus stacking. Physical Chemistry Chemical Physics. 2013;15:20203-20209

[204] Ma X, Zhang J, Li H, Duan B, Guo L, Que M, et al. Violet blue longlasting phosphorescence properties of $\mathrm{Mg}$-doped $\mathrm{BaZrO}_{3}$ and its ability to assist photocatalysis. Journal of Alloys and Compounds. 2013;580:564-569

[205] Alammar T, Hamm I, Grasmik V, Wark M, Mudring A-V. Microwaveassisted synthesis of perovskite $\mathrm{SrSnO}_{3}$ nanocrystals in ionic liquids for photocatalytic applications. Inorganic Chemistry. 2017;56(12):6920-6932
[206] Jung WY, Hong S-S. Synthesis of $\mathrm{LaCoO}_{3}$ nanoparticles by microwave process and their photocatalytic activity under visible light irradiation. Journal of Industrial and Engineering Chemistry. 2013;19:157-160

[207] Dong B, Li Z, Li Z, Xu X, Song M, Zheng W, et al. Highly efficient $\mathrm{LaCoO}_{3}$ nanofibers catalysts for photocatalytic degradation of rhodamine B. Journal of the American Ceramic Society. 2010;93:3587-3590

[208] Shasha F, Niu H, Tao Z, Song J, Mao C, Zhang S, et al. Low temperature synthesis and photocatalytic property of perovskite-type $\mathrm{La}-\mathrm{CoO}_{3}$ hollow spheres. Journal of Alloys and Compounds. 2013;576:5-12

[209] Lia Y, Yaoa S, Wenb W, Xuea L, Yana Y. Sol-gel combustion synthesis and visible-light-driven photocatalytic property of perovskite $\mathrm{LaNiO}_{3}$. Journal of Alloys and Compounds. 2010;491:560-564

[210] Capon RJ, Rooney F, Murray LM, Collins E, Sim ATR, Rostas JAP, et al. Dragmacidins: New Protein Phosphatase Inhibitors from a Southern Australian Deep-Water Marine Sponge, Spongosorites sp. Materials Letters. 2007;61:3959-3962

[211] Hwang DW, Kim HG, Lee JS, Kim J, Li W, Se Hyuk O. Photocatalytic hydrogen production from water over M-Doped $\mathrm{La}_{2} \mathrm{Ti}_{2} \mathrm{O}_{7}(\mathrm{M}=\mathrm{Cr}, \mathrm{Fe})$ under visible light irradiation $(\lambda>420 \mathrm{~nm})$. The Journal of Physical Chemistry. B. 2005;109:15001-15007

[212] Hur SG, Kim TW, Hwang S-J, Choy J-H. Influences of A- and B-site cations on the physicochemical properties of perovskite-structured $\mathrm{A}(\mathrm{In} 1 / 3 \mathrm{Nb} 1 / 3 \mathrm{~B} 1 / 3) \mathrm{O} 3$ (A = Sr, Ba; $\mathrm{B}=\mathrm{Sn}, \mathrm{Pb})$ photocatalysts. Journal of Photochemistry and Photobiology A. 2006;183:176-181 
[213] Thirunavukkarasu SK, Abdul AR, Chidambaram J, Govindasamy R, Sampath M, Arivarasan VK, et al. Green synthesis of titanium dioxide nanoparticles using Psidium guajava extract and its antibacterial and antioxidant properties. Advances in Materials Research. 2014;58:968-976

[214] Aguiar R, Kalytta A, Reller A, Weidenkaff A, EbbinghauscSG. Photocatalytic decomposition of acetone using $\operatorname{LaTi}(\mathrm{O}, \mathrm{N}) 3$ nanoparticles under visible light irradiation. Journal of Materials Chemistry. 2008;18:4260-4265

[215] Wang D, Kako T, Ye J. Efficient photocatalytic decomposition of acetaldehyde over a solid-solution perovskite $\left(\mathrm{Ag}_{0.75} \mathrm{Sr}_{0.25}\right)\left(\mathrm{Nb}_{0.75} \mathrm{Ti}_{0.25}\right) \mathrm{O}_{3}$ under visible-light irradiation. Journal of the American Chemical Society. 2008;130:2724-2725

[216] Dong W, Kaibin T, Zhenhua L, Huagui Z. Synthesis, crystal structure, and photocatalytic activity of the new three-layer aurivillius phases, $\mathrm{Bi}_{2} \mathrm{ASrTi}_{2} \mathrm{TaO}_{12}(\mathrm{~A}=\mathrm{Bi}, \mathrm{La})$. Journal of Solid State Chemistry. 2010;183:361-366

[217] Fanke M, Zhanglian H, James A, Ming L, Mingjia Z, Feng Y, et al. Visible light photocatalytic activity of nitrogendoped $\mathrm{La}_{2} \mathrm{Ti}_{2} \mathrm{O}_{7}$ nanosheets originating from band gap narrowing. Nano Research. 2012;5:213-221

[218] Danjun W, Huidong S, Li G, Feng F, Yucang L. Design and construction of the sandwich-like z-scheme multicomponent cds/ag/ bi2moo6 heterostructure with enhanced photocatalytic performance in rhb photodegradation. New Journal of Chemistry. 2016;40:8614-8624 


\title{
Nanocomposites: An Aspect for Environmental Application
}

\author{
Manojit De
}

\begin{abstract}
Perovskites possess an interesting crystal structure and its structural properties allow us to achieve various applications. Beside its ferroelectric, piezoelectric, magnetic, multiferroic, etc., properties, these branches of materials are also useful to develop materials for various environmental applications. As the population is increasing nowadays, different type of environmental pollution is one of the growing worries for society. The effort of researchers and scientists focuses on developing new materials to get rid of these individual issues. With modern advances in synthesis methods, including the preparation of perovskite nanocomposites, there is a growing interest in perovskite-type materials for environmental application. Basically, this chapter concludes with a few of the major issues in the recent environment: green energy (solar cell), fuel cell, sensors (gas and for biomedical), and remediation of heavy metals from industrial wastewater.
\end{abstract}

Keywords: perovskite, nanocomposite, fuel cell, sensors, solar cell, heavy metals, wastewater treatment

\section{Introduction}

Perovskites possess a very interesting crystal structure; are basically a combination of three basic crystal structures (simple cubic structure, body center cubic structure, and face-centered cubic structure). The extraordinary range of structure and properties interplay of perovskites makes them an exceptional research field for different branches like materials science, physics and solid-state chemistry. A wide range of unique functional materials and device ideas can be predicted through a basic understanding of the correlation between structural and chemical compatibility.

The perovskite structure is shown to be the single most adaptable ceramic host. Inorganic perovskite-type oxides are attractive compounds for varied applications due to its large number of compounds, they exhibit both physical and biochemical characteristics and their nano-formulation have been utilized as catalysts in many reactions due to their sensitivity, unique long-term stability, and anti-interference ability. Some perovskite materials are very hopeful applicants for the improvement of effective anodic catalysts performance. Depending on perovskite-phase metal oxides' distinct variety of properties they became useful for various applications they are newly used in electrochemical sensing of alcohols, glucose, hydrogenperoxide, gases, and neurotransmitters. Perovskite organometallic halide showed efficient essential properties for photovoltaic solar cells. 


\subsection{The basic structure of perovskites}

Figure 1 depicts the ideal perovskite structure. In the ideal crystal structure of perovskite with general formula $\mathrm{ABX}_{3}$; where " $\mathrm{A}$ " and " $\mathrm{B}$ " are generally metal cations and " $\mathrm{X}$ " is an oxide or halide like $\mathrm{Cl}, \mathrm{Br}$, I, etc., " $\mathrm{A}$ " can be $\mathrm{Ca}, \mathrm{K}, \mathrm{Na}, \mathrm{Pb}, \mathrm{Sr}$, and other rare-earth metals which occupy the 12 -fold coordinated sites between the octahedra. "X" forms the $\mathrm{BX}_{6}$ octahedra where $\mathrm{B}$ located at the center of octahedra. Perovskite can be described as consisting of corner-sharing [ $\mathrm{BX}_{6}$ ] octahedra with the A-cation occupying the 12-fold coordination site formed in the middle of the cube of eight such octahedra. In an ideal cubic unit cell of perovskite, Wyckoff positions for $\mathrm{A}$ - ion is at cube-corner positions $(0,0,0)$; ion $\mathrm{B}$ sites at body center position $(1 / 2,1 / 2,1 / 2)$ and ion $X$ sits at face-centered positions $(1 / 2,1 / 2,0)$. Figure 2 shows the elements which can be in $\mathrm{A}$-site, $\mathrm{B}$-site from the periodic table.

In ideal perovskite such as $\mathrm{SrTiO}_{3}$ [3], $\mathrm{CsSnBr}_{3}$ [4], etc., there is no such distortion in the unit cell. There are many different types of lattice distortions that can happen due to the flexibility of bond angles within the ideal perovskite structure [5].

i. Distortion in $\mathrm{BX}_{6}$ octahedra, by the Jahn-Teller effect.

ii. Off-center displacement of $\mathrm{B}$-cation in $\mathrm{BX}_{6}$ octahedra, this one of the causes for ferroelectricity in these type of materials.

iii. So-called tilting in octahedra framework, usually occurring as a result of too small A-cations at cuboctahedral site.

iv. Ordering of more than one type of cations A or B, or of vacancies.

$\mathrm{v}$. Ordering of more than one kind of anions $\mathrm{X}$, or of vacancies.

The different physical properties (mainly electronic, magnetic, dielectric, and piezoelectric properties) of perovskite materials are crucially dependent on these distortions. The distortion as a consequence of cationic substitution can be used to fine-tune physical properties exhibited by perovskite.
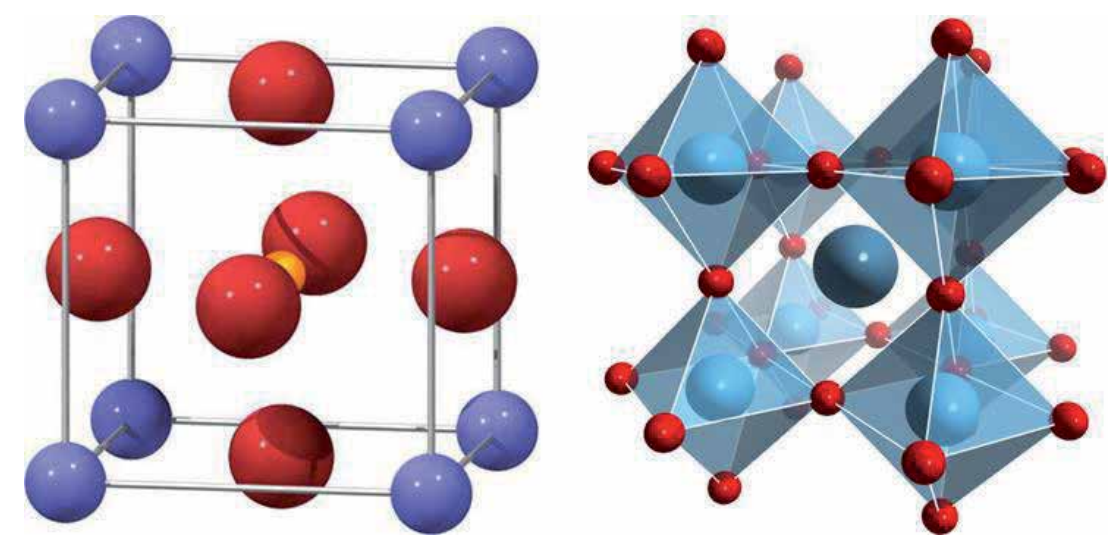

Figure 1.

The ideal structure for perovskite; blue balls represent the A-site, yellow ball shows the B-site, and magenta balls showing the position of $X$ anion (face center position) [1]. 


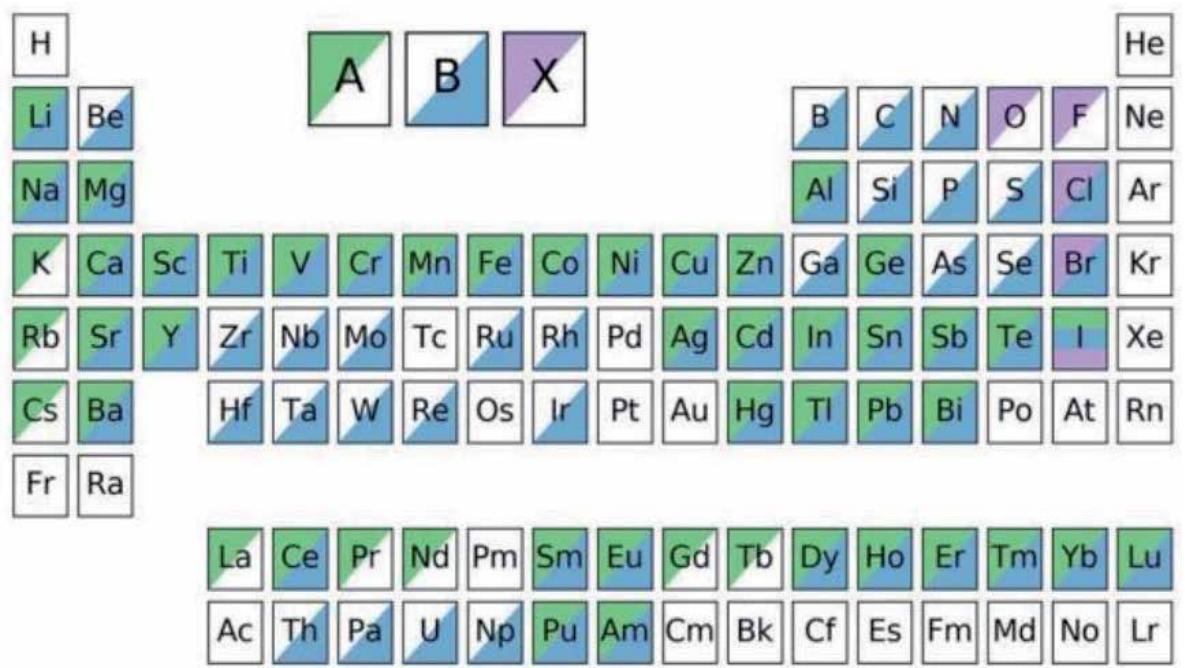

Figure 2.

A map of the elements in the periodic table which can occupy the A, B, and/or X sites [2].

In the case of perovskite structure (closed packed), A-cation must fit among four $\mathrm{BX}_{6}$ octahedra. Each A-cation is surrounded by 12 nearest $\mathrm{X}$-anions (12fold coordination). Therefore A-cations have limited space to accommodate itself in the interstitial position. In the case of ideal perovskite structure, the cell axis (a) is geometrically related to the ionic radii $\left(r_{A}, r_{B}\right.$, and $\left.r_{X}\right)$ as described in the following equation.

$$
a=\sqrt{2}\left(r_{A}+r_{X}\right)=2\left(r_{B}+r_{X}\right)
$$

The ratio of the two expressions for the cell length is called Goldschmidt's tolerance factor $(t)$ and it allows us for evaluating the degree of distortion in the unit cell. The expression for Goldschmidt's tolerance factor [6] is as follows.

$$
t=\frac{\left(r_{A}+r_{X}\right)}{\sqrt{2}\left(r_{B}+r_{X}\right)}
$$

where $r_{A}$ is the radius of A-cation is, $r_{B}$ is the radius for $\mathrm{B}$-cation and $r_{X}$ is the radius for $\mathrm{X}$-anion.

\subsection{Why lead-free?}

Lead (and its oxide form) is highly hazardous and its harmfulness is further improved due to its volatilization at high temperature mainly during calcination and sintering causing environmental pollution during different sample preparation techniques [7]. According to the European Union (EU), hazardous substances like lead and other heavy metals is planning to strictly prohibit $[8,9]$.

\subsubsection{Toxicity effects of lead}

The main indications of lead poisoning are tiredness, muscles and joints pain, abdominal uneasiness, etc. Sometimes the deposition of lead sulfide 
can be found out in the dental margin of the gums of the patients having poor dental hygiene. Lead harming has been considered as a health hazard, for its bad effects on neurological and cerebral development [10-12]. The main route of absorption in adults is the respiratory region where $30-70 \%$ of inhaled lead (typically the inorganic form like oxides and salts) goes into the cardiovascular system. The maximum tolerance of lead in blood ranges from 1.45 to $2.4 \mathrm{~mol} \mathrm{~L}^{-1}$ (30-50 g $100 \mathrm{~mL}^{-1}$ ) with a provision of 6 monthly observations [13]. Basically, lead has few significant biochemical properties that give toxic effects on the human biological system. (i) As lead is electropositive in nature, it shows a very high affinity for the enzymes, which are necessary for the synthesis of hemoglobin. (ii) The divalent lead behaves similarly to calcium preventing mitochondrial oxidative phosphorylation as a result intelligence quotient (IQ) got reducing. (iii) The transcription of DNA can also disturb by lead by interacting with binding protein and nucleic acids $[14,15]$. Figure 3 illustrated the adverse effect of lead on the human body.

Bearing in mind the hazardous effect of $\mathrm{Pb}$ in $\mathrm{Pb}$-based compounds, the research communities focused on designing the materials which are basically $\mathrm{Pb}$-free. Hence, this chapter concludes with some $\mathrm{Pb}$-free perovskite-type materials for the environmental application point of view.

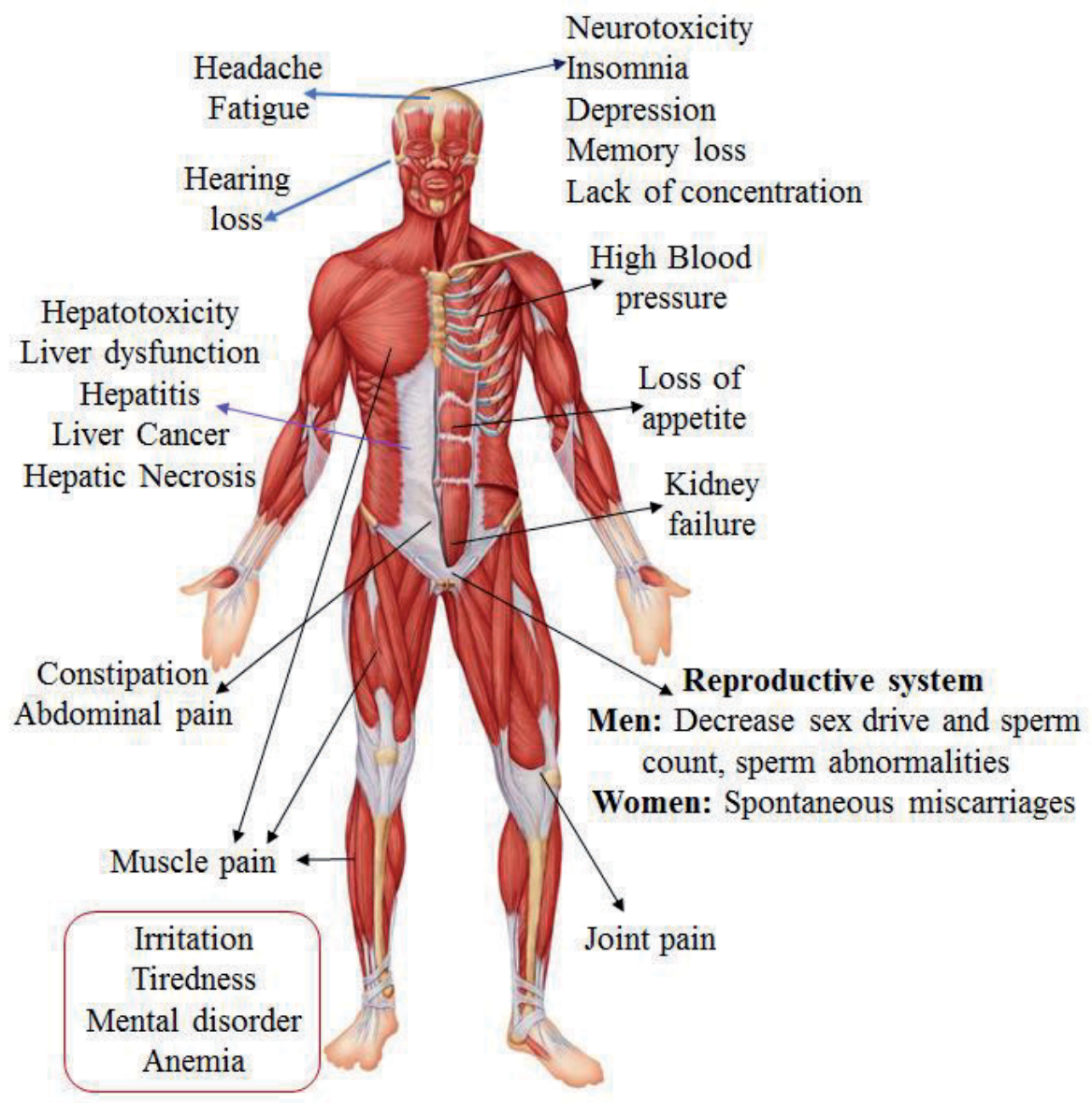

Figure 3

Schematic diagram for toxic effect of lead on human body. 


\section{Application of $\mathrm{Pb}$-free perovskites from a different aspect of environmental}

\subsection{Perovskites as solid oxide fuel cells}

Recently, the inorganic perovskite-type of oxide nanomaterials have been widely applied in the processing of chemically modified electrodes $[16,17]$. They have acknowledged considerable attention in the last few decades because of their catalytic activity in diverse processes like purification of waste gas and catalytic combustion.

In the fuel cell, there is a direct conversion of chemical energy into electrical energy similar to a battery. These are attractive because of their great efficiency, low emission, almost zero pollution (basically noise pollution). The solid oxide fuel cells (SOFCs) have come into the picture as effective substitutions to the combustion engines due to their prospective to minimize the environmental impact of the use of conventional fossil fuels. Perovskite oxides exhibited attractive properties like a high electrical and ionic conductivity similar to that of metals and the perfect mix of these two types [18]. This mixed conduction properties of perovskite oxides are advantageous for electrochemical reaction. The working principle of a SOFC is depicted by Figure 4 [19]. The perovskite $\mathrm{Ba}_{0.5} \mathrm{Sr}_{0.5} \mathrm{Co}_{0.8} \mathrm{Fe}_{0.2} \mathrm{O}_{3-\delta}$ used as an effective cathode for intermediary SOFC reported by Shao and Haile. This cathode unveiled the maximum power density of 402 and $1010 \mathrm{~mW} \mathrm{~cm}^{-2}$ at 500 and $600^{\circ} \mathrm{C}$, respectively [20]. The combination of single and double perovskite oxide $\mathrm{Ba}_{0.5} \mathrm{Sr}_{0.5}\left(\mathrm{Co}_{0.7} \mathrm{Fe}_{0.3}\right)_{0.6875} \mathrm{~W}_{0}$. ${ }_{3125} \mathrm{O}_{3-\delta}$ (B-SCFW) was investigated by Shin et al. [21] for self-assembled perovskite composites for SOFC. In contrast, Goodenough reported that the double perovskite $\mathrm{Sr}_{2} \mathrm{MgMnMoO}_{6-\delta}$ can act as an anode material for SOFC with dry methane as the fuel and it shows maximum power density of $438 \mathrm{~mW} \mathrm{~cm}^{-2}$ at $800^{\circ} \mathrm{C}$. This anode material exhibited long-term stability and having oxygen insufficiency, as well as some good environmental effects like tolerance to sulfur, stability in reducing atmosphere [22]. Table 1 enlisted with some perovskites used as anode and cathode for SOFCs.

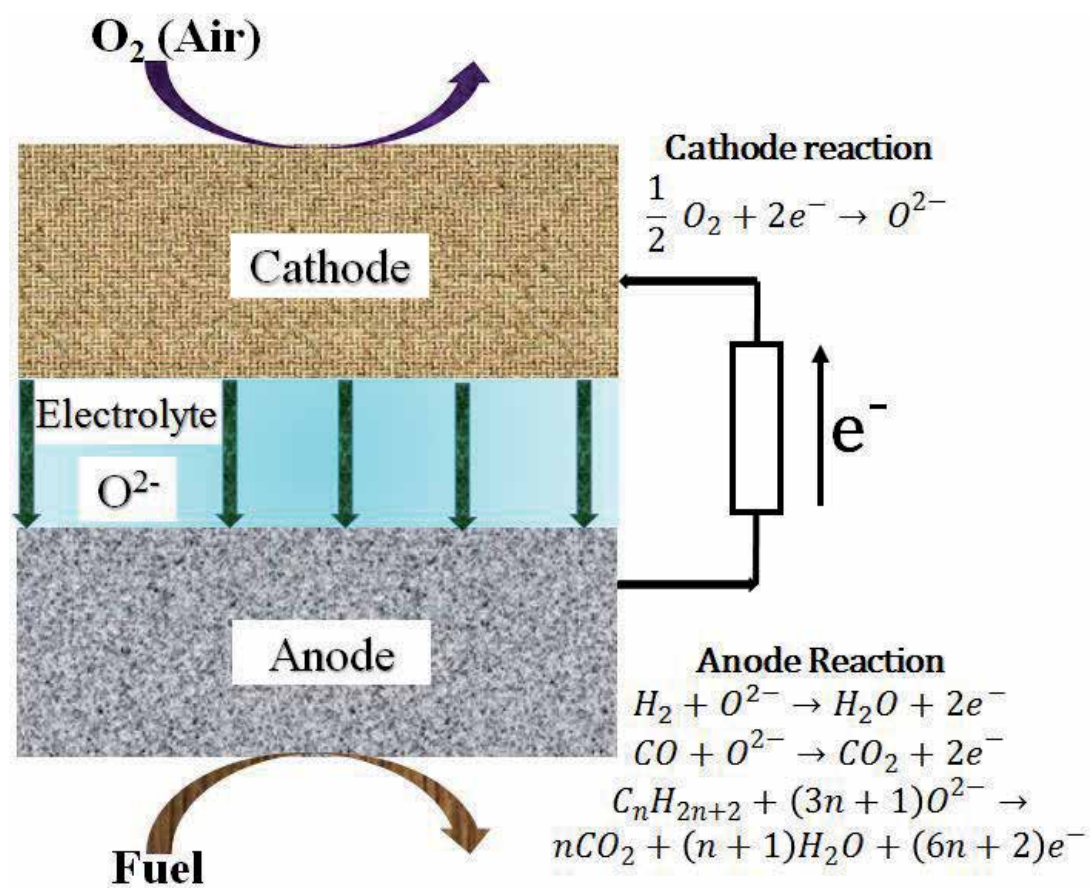

Figure 4.

Diagram illustrates the working principle of SOFC [19]. 


\begin{tabular}{|c|c|c|c|c|c|}
\hline $\begin{array}{l}\text { Perovslkite } \\
\text { compositions }\end{array}$ & $\begin{array}{l}\text { Anode/ } \\
\text { cathode } \\
\text { In cell }\end{array}$ & Fuel used & $\begin{array}{c}\text { Operating } \\
\text { temperature } \\
\left({ }^{\circ} \mathrm{C}\right)\end{array}$ & $\begin{array}{c}\text { Maximum } \\
\text { power } \\
\text { density } \\
\left(\mathrm{mW} \mathrm{cm}^{-2}\right)\end{array}$ & Reference \\
\hline \multirow[t]{2}{*}{$\mathrm{Ba}_{0.5} \mathrm{Sr}_{0.5} \mathrm{Co}_{0.8} \mathrm{Fe}_{0.2} \mathrm{O}_{3-\delta}$} & \multirow[t]{2}{*}{ Cathode } & \multirow{2}{*}{$\begin{array}{l}\text { Humidified } \mathrm{H}_{2} \\
\qquad\left(\sim 3 \% \mathrm{H}_{2} \mathrm{O}\right)\end{array}$} & 500 & 402 & \multirow[t]{2}{*}{ [20] } \\
\hline & & & 600 & 1010 & \\
\hline $\mathrm{Ba}_{0.5} \mathrm{Sr}_{0.5} \mathrm{Co}_{0.2} \mathrm{Fe}_{0.8} \mathrm{O}_{3-\delta}$ & Cathode & Humidified $\mathrm{H}_{2}$ & 800 & 266 & {$[23]$} \\
\hline \multirow[t]{2}{*}{$\mathrm{NdFeO}_{3}$} & \multirow[t]{2}{*}{ Anode } & \multirow{2}{*}{$\begin{array}{l}\text { Sulfur vapor } \\
\text { or } \mathrm{SO}_{2}\end{array}$} & 620 & 0.154 & \multirow[t]{2}{*}{ [24] } \\
\hline & & & 650 & 0.265 & \\
\hline $\mathrm{La}_{0.6} \mathrm{Sr}_{0.4} \mathrm{Fe}_{0.8} \mathrm{Co}_{0.2} \mathrm{O}_{3}$ & Cathode & Glycerol & 800 & Not reported & {$[25]$} \\
\hline $\mathrm{Sm}_{0.5} \mathrm{Sr}_{0.5} \mathrm{CoO}_{3-\delta}$ & Cathode & Not reported & 700 & 936 & {$[26]$} \\
\hline $\mathrm{La}_{0.8} \mathrm{Sr}_{0.2} \mathrm{Cr}_{0.97} \mathrm{~V}_{0.03} \mathrm{O}_{3}$ & Anode & Dry methane & 800 & Not reported & [27] \\
\hline $\mathrm{La}_{0.75} \mathrm{Sr}_{0.25} \mathrm{Cr}_{0.5} \mathrm{Mn}_{0.5} \mathrm{O}_{3}$ & Anode & Methane & Not reported & Not reported & [28] \\
\hline
\end{tabular}

Table 1.

Summary report of few Pb-free perovskites used in SOFCs.

\subsection{Perovskites as sensor}

\subsubsection{Perovskites as glucose sensor}

It is very essential to determine hydrogen peroxide $\left(\mathrm{H}_{2} \mathrm{O}_{2}\right)$ and glucose analytically in any aspect of our daily life. In environmental waste management, chemical and food industries, and medical diagnostics $\mathrm{H}_{2} \mathrm{O}_{2}$ widely used as one of the most important oxidizing agents [29]. On the other hand, glucose represents a fundamental component in human blood that delivers energy through the metabolic process. If in human blood, the glucose concentration fluctuates than the normal range of $80-120 \mathrm{mg} \mathrm{dL}^{-1}(4.4-6.6 \mathrm{mM})$ is related to the metabolic disorder from insulin insufficiency and hyperglycemia, the so-called diabetes mellitus [30]. To perform the diagnosis and supervision of such health issue it is necessary a tight observation of glucose level of blood. Hence, it is very significant to make the biosensors for the sensitive determination of glucose and $\mathrm{H}_{2} \mathrm{O}_{2}$. Basically, there are two types of glucose sensors available: enzymatic and non-enzymatic. Different types of enzymatic glucose sensors were constructed and used in the literature exhibiting the advantages of simplicity and sensitivity. However, enzymatic glucose sensors suffered from the lack of stability and the difficult procedures required for the effective immobilization of the enzyme on the electrode surface. The lack of enzyme stability was attributed to its intrinsic nature because the enzyme activity was highly affected by poisonous chemicals, $\mathrm{pH}$, temperature, humidity, etc. As a result, most attention was given for a sensitive, simple, stable, and selective nonenzymatic glucose sensor. Different novel materials were proposed for the electrocatalytic oxidation of glucose like noble nanometals, nanoalloys, metal oxides, and inorganic perovskite oxides. Inorganic perovskite oxides as nanomaterials exhibited fascinating properties for glucose sensing like ferroelectricity, superconductivity, charge ordering, high thermopower, good biocompatibility, catalytic.

Wang et al. utilized a carbon paste electrode (CPE) modified with $\mathrm{LaNi}_{0.5} \mathrm{Ti}_{0.5} \mathrm{O}_{3}$ (LNT) as a promising nonenzymatic glucose sensor. This glucose sensor displayed a perfect electrochemical activity and was used to quantify of glucose with great sensitivity of $1630.57 \mu \mathrm{A} \mathrm{mM}^{-1} \mathrm{~cm}^{-2}$ and a low detection limit of $0.07 \mu \mathrm{M}$. This glucose sensor also demonstrated an excellent reproducibility, long-term immovability, as well as outstanding selectivity with no interference from the common interfering 
substances such as dopamine, ascorbic acid, and uric acid [31]. The perovskitespinel type composite oxide $\mathrm{LaNi}_{0.5} \mathrm{Ti}_{0.5} \mathrm{O}_{3}-\mathrm{NiFe}_{2} \mathrm{O}_{4}$ with different compositions was demonstrated as the glucose sensor by Wang et al. This material also exhibits admirable reproducibility, stability and selectivity in glucose sensitivity with a linear signal-to-glucose concentration range of $0.5-10 \mathrm{mM}$ and a detection limit $(\mathrm{S} / \mathrm{N}=3)$ of $0.04 \mathrm{mM}$ [32]. Furthermore, $\mathrm{La}_{\mathrm{x}} \mathrm{Sr}_{1-\mathrm{x}} \mathrm{Co}_{\mathrm{y}} \mathrm{Fe}_{1-\mathrm{y}} \mathrm{O}_{3-\delta}(\mathrm{x}=0.6 ; \mathrm{y}=0.0$ and 0.2$)$ perovskites were studied as electro-catalytic materials for $\mathrm{H}_{2} \mathrm{O}_{2}$ and glucose electrochemical sensors by Liotta et al. [33]. The group of Atta et al. has reformed $\mathrm{SrPdO}_{3}$ perovskite with gold nanoparticles to be employed as a non-enzymatic voltammetric glucose sensor. This nanocomposite disclosed an excellent performance to glucose sensing in terms of highly reproducible response, high sensitivity, low detection limit, appreciable selectivity, long-standing stability [34]. He et al. depicted that the perovskite oxide $\mathrm{La}_{0.6} \mathrm{Sr}_{0.4} \mathrm{CoO}_{3-\delta}$ can provide superior electro-oxidation activities (to $\mathrm{H}_{2} \mathrm{O}_{2}$ and glucose) over $\mathrm{La}_{0.6} \mathrm{Sr}_{0.4} \mathrm{Co}_{0.2} \mathrm{Fe}_{0.8} \mathrm{O}_{3-\delta}$ and $\mathrm{LaNi}_{0.6} \mathrm{Co}_{0.4} \mathrm{O}_{3}$ that translates to good $\mathrm{H}_{2} \mathrm{O}_{2}$ or glucose detection performance. They have modified the sensor by making composite with reduced graphene oxide (RGO) and $\mathrm{La}_{0.6} \mathrm{Sr}_{0.4} \mathrm{CoO}_{3-\delta}$ for exhibiting higher detection properties and improved selectivity [35].

\subsubsection{Perovskites as a gas sensor}

The clean air is undoubtedly most necessary than water for human health, but unfortunately, human activities accompanying socioeconomic developments are the vital pollution sources. So, it is very important to closely observe the quality of the air, including the indoor air quality (IAQ) as we spent most of our time ( $90 \%$ ) of our time in the indoor climate, to prevent different unusual symptoms [35-37]. Thus, researchers and scientists across the whole globe have been developing new and advanced material based innovative methods for consistent and careful detection of gases and volatile organic compounds (VOCs) hazardous to human and environmental health $[38,39]$. The environmental worries about health hazards due to the existence of poisonous gases, for example, $\mathrm{CO}, \mathrm{CO}_{2}, \mathrm{NO}_{2}, \mathrm{O}_{3}$, etc., and subsequent safety regulations have demanded the enhanced use of sensors in various sceneries from the industrial sites to automobiles, the different workplaces and even homes. Among the several toxic gases, $\mathrm{CO}$ and $\mathrm{NO}_{2}$ are the most harmful air pollutants and are dangerous for animals, plants and as well as human beings. The Occupational Safety and Health Administration (OSHA) have also announced the limit lowest tolerance for these type of gases in a particular time period, for example, the limits for $\mathrm{CO}$ and $\mathrm{NO}_{2}$ gases are $\sim 20 \mathrm{ppm}$ and $\sim 5 \mathrm{ppm}$ over the period of $8 \mathrm{~h}$ respectively. Over-acquaintance to these gases could be the reason for diseases and in dangerous cases even loss of human life [40]. There are a number of features that the materials can have to be utilized as gas sensors, explicitly, an excellent similitude with the target gases, easy to synthesize, thermal stability, appropriate electronic structure, and adaptation with present technologies. The perovskite oxides are interesting materials as gas sensors because of their ideal bandgap, excellent thermal stability and the size difference between the Aand B-sites cations, tolerating different dopants addition for monitoring the catalytic properties and their semiconducting properties. Lots of perovskites were synthesized to utilize as gas sensors for detecting different hazardous gases. Table 2 enlisted with different perovskite oxides for various gas sensing applications.

\subsection{Perovskites as solar cell materials}

The consumption of energy has been continuously increasing globally and limitations of sources of fossil fuels leading to perform the research on sustainable, environment-friendly, and renewable energy sources. Due to the abundance of sun rays on 


\begin{tabular}{|c|c|c|c|}
\hline Perovskites & Sensing for & Response ratio \% & Reference \\
\hline $\mathrm{LaCoO}_{3}$ & $\mathrm{CO}$ & $\begin{array}{l}\text { Under } 5000 \mathrm{ppm} \mathrm{CO} \text { at } 500^{\circ} \mathrm{C} \text {, the thick film } \\
\text { sensor achieved a high sensing response of } \\
\sim 279.86\end{array}$ & {$[41]$} \\
\hline $\mathrm{La}_{0.9} \mathrm{Ce}_{0.1} \mathrm{CoO}_{3}$ & $\mathrm{CO}$ & $240 \%$ with respect to $100 \mathrm{ppm} \mathrm{CO}$ in air & {$[42]$} \\
\hline $\begin{array}{l}\text { Ca modified } \\
\mathrm{LaFeO}_{3}\end{array}$ & $\mathrm{SO}_{2}$ & $\begin{array}{l}\text { Maximum resistive response of } 3 \mathrm{ppm} \mathrm{SO}_{2} \text { was } \\
\text { detected at } \sim 275^{\circ} \mathrm{C} \text { by the } \mathrm{LaCaFeO}_{3} \text { samples, } \\
\text { and it shows } 15 \% \text { higher efficiency than } \mathrm{LaFeO}_{3}\end{array}$ & {$[43]$} \\
\hline $\mathrm{NdFe}_{1-\mathrm{x}} \mathrm{Co}_{\mathrm{x}} \mathrm{O}_{3}$ & $\mathrm{CO}$ & $1215 \%$ at $170^{\circ} \mathrm{C}$ for $0.03 \%$ CO gas & {$[44]$} \\
\hline $\mathrm{LaFeO}_{3}$ & Ethanol & Not reported & {$[45]$} \\
\hline $\mathrm{Ag}-\mathrm{LaFeO}_{3}$ & Methanol & $\begin{array}{l}\text { The maximum response to the other test gases } \\
\text { is } 8\end{array}$ & {$[46]$} \\
\hline $\mathrm{Ag}-\mathrm{LaFeO}{ }_{3}$ & Formaldehyde & $\begin{array}{l}\text { Best response to } 0.5 \mathrm{ppm} \text { formaldehyde (24.5) } \\
\text { at } 40^{\circ} \mathrm{C}\end{array}$ & {$[47]$} \\
\hline $\mathrm{SrFeO}_{3}$ & Ethanol & Not reported & {$[48]$} \\
\hline $\mathrm{LaFeO}_{3}$ & $\mathrm{NO}_{2}$ & Not reported & [49] \\
\hline $\mathrm{GaFeO}_{3}$ & Ethanol & Ethanol sensing down to $1 \mathrm{ppm}$ at $350^{\circ} \mathrm{C}$ & {$[50]$} \\
\hline $\mathrm{SrTi}_{1-\mathrm{x}} \mathrm{Fe}_{\mathrm{x}} \mathrm{O}_{3-\delta}$ & Hydrocarbons & Not reported & {$[51]$} \\
\hline$\alpha-\mathrm{Fe}_{2} \mathrm{O}_{3} / \mathrm{LaFeO}_{3}$ & Acetone & $\begin{array}{l}\text { Response } 48.3 \% \text { at } 100 \mathrm{ppm} \text { concentration at } \\
350^{\circ} \mathrm{C}\end{array}$ & {$[52]$} \\
\hline $\mathrm{YCo}_{1-\mathrm{x}} \mathrm{Pd}_{\mathrm{x}} \mathrm{O}_{3}$ & $\mathrm{CO}, \mathrm{NO}_{2}$ & Different for the different composition & {$[53]$} \\
\hline $\mathrm{ZnSnO}_{3}$ & n-Propanol gas & $\begin{array}{l}\text { The detection limit of } \mathrm{ZnSnO}_{3} \text { nanospheres to } \\
500 \mathrm{ppb} \text { n-propanol gas could reach } 1.7\end{array}$ & {$[54]$} \\
\hline $\begin{array}{l}\mathrm{LaFeO}_{3} \text { and } \\
\text { rGO- } \mathrm{LaFeO}_{3}\end{array}$ & $\mathrm{NO}_{2}$ and $\mathrm{CO}$ & $\begin{array}{l}\text { Response } 183.4 \% \text { for } 3 \mathrm{ppm} \text { concentration of } \\
\mathrm{NO}_{2} \text { at a } 250^{\circ} \mathrm{C}\end{array}$ & {$[55]$} \\
\hline $\begin{array}{l}\mathrm{BaTiO}_{3} / \mathrm{LaFeO}_{3} \\
\text { nanocomposite }\end{array}$ & Ethanol & Response $102.7 \%$ to $100 \mathrm{ppm}$ ethanol at $128^{\circ} \mathrm{C}$ & {$[56]$} \\
\hline $\mathrm{Ba}-\mathrm{BiFeO}_{3}$ & Ethanol & $\begin{array}{l}\text { Temperature dependent sensing performance } \\
\text { toward } 100 \mathrm{ppm} \text { ethanol gas; maximum } \\
\text { sensitivity at } 400^{\circ} \mathrm{C}\end{array}$ & {$[57]$} \\
\hline La doped $\mathrm{BiFeO}_{3}$ & Acetone & $\begin{array}{l}\text { The morphotropic phase boundary (MPB) phase } \\
\mathrm{Bi}_{0.9} \mathrm{La}_{0.1} \mathrm{FeO}_{3} \text { shows ultra-low concentration } \\
\text { detection of } 50 \mathrm{ppb} \text { acetone }\end{array}$ & {$[58]$} \\
\hline Pr doped $\mathrm{BiFeO}_{3}$ & Formaldehyde & $50 \mathrm{ppm}, 190^{\circ} \mathrm{C}, \mathrm{R}_{\mathrm{gas}} / \mathrm{R}_{\mathrm{air}}=17.6$ & {$[59]$} \\
\hline $\mathrm{BaTiO}_{3}$ thick films & $\mathrm{H}_{2} \mathrm{~S}$ & $\mathrm{BaTiO}_{3}$ sensor operated at $350^{\circ} \mathrm{C}$ & {$[60]$} \\
\hline Sr doped $\mathrm{BaTiO}_{3}$ & $\mathrm{NH}_{3}$ and $\mathrm{NO}_{2}$ & $\begin{array}{l}0.2 \mathrm{~mol} \% \text { doping of } \mathrm{Sr} \text { showed enhanced } \\
\text { performance for sensing of both } \mathrm{NO}_{2} \text { and } \mathrm{NH}_{3} \\
\text { gases at room temperature }\end{array}$ & {$[61]$} \\
\hline $\mathrm{BaSnO}_{3}$ & $\mathrm{SO}_{2}$ & $10 \mathrm{ppm}$ of $\mathrm{SO}_{2}$ & {$[62]$} \\
\hline $\mathrm{BaSnO}_{3}$ & LPG & $\begin{array}{l}\text { Addition of noble metal Pt, the operating } \\
\text { temperature decreases and the sensitivity } \\
\text { improved but also imparted partial selectivity } \\
\text { for the detection of LPG }\end{array}$ & {$[63]$} \\
\hline $\mathrm{LaBO}_{3}(\mathrm{~B}=\mathrm{Fe}, \mathrm{Co})$ & Acetone & $\begin{array}{l}\text { At a low operating temperature of } 120^{\circ} \mathrm{C} \text { showed } \\
\text { that the } \mathrm{LaFeO}_{3} \mathrm{NFs} \text { based sensor displayed high } \\
\text { stable and selective response toward } 40 \mathrm{ppm} \\
\text { acetone with fast response and recovery time of } \\
14 \text { and } 49 \mathrm{~s}\end{array}$ & {$[64]$} \\
\hline
\end{tabular}

Table 2.

Tabulated with different perovskite oxides for various gas sensing applications. 
our globe, the transformation of sunlight into electricity is one of the most favorable studies for increasing energy demands without having any adverse effect on the global climate. Solar cell technology offers an eco-friendly and renewable energy path to convert photon energy into electricity openly [65]. Nowadays a large effort has been put in the research to develop high efficiency, low-cost photovoltaic devices but regrettably did not succeed yet. During the last decade, research into perovskite solar cells (PSCs) has increased and it also been nominated as a runner-up for the top 10 breakthroughs research of 2013 by the editors of Science [66]. The organic-inorganic perovskite having the general formula $\mathrm{ABX}_{3}$ where $\mathrm{A}$ is cesium (Cs), methylammonium (MA), or formamidinium (FA); $\mathrm{B}$ is $\mathrm{Pb}$ or $\mathrm{Sn}$; and $\mathrm{X}$ is $\mathrm{Cl}, \mathrm{Br}$, or I, have recently appeared as an exciting class of semiconductors which can act as solar cell materials [67]. These organic-inorganic halide perovskite solar cells have shown substantial improvement of power conversion efficiency (PCE) from the preliminary efficiency of 3.8\% [68] to about 22.1\% [69]. The maximum theoretical power conversion efficiency accomplished by perovskite $\left(\mathrm{CH}_{3} \mathrm{NH}_{3} \mathrm{PbI}_{3}\right)$ is about 31.4\% [70]. The organic-halide perovskites owing extraordinary performance because of some unique properties like (i) high absorbing coefficient, (ii) high charge carrier mobility, (iii) long diffusion length, (iv) direct bandgap which can be engineered easily and (v) moreover easy to fabricate [71]. The normally used $\mathrm{Pb}$-based perovskites have numerous advantages such as (i) large diffusion length, (ii) absorption range, (iii) low exciton binding energy, and (iv) high carrier mobility. Conversely, the $\mathrm{Pb}$-based perovskite solar cell has a serious toxic issue on both humans and the environment [72]. Generally, PV panels are positioned on the roof of houses or in the open field, their exposure to rainfall is inescapable. In the manifestation of rain and moisture the degradation of $\mathrm{PbI}_{2}$ may cause mild to acute health issues, like effects on cardiovascular, neurological, reproductive system [73] mainly it is carcinogenic [72,74] Additionally, lead pollution has severe effects on water and soil resources and emission of greenhouse gasses $[75,76]$. Hence, in PSCs, it is essential to replace $\mathrm{Pb}$ for economical green energy conversion devices which may use mankind in future endeavors [77].

To develop Pb-free PSCs, $\mathrm{Sn}^{2+}$ metal cation was the first another candidate to replace $\mathrm{Pb}^{2+}$ as of its comparable electronic configuration and effective ionic radius $\left(\mathrm{Sn}^{2+}: 115 \mathrm{pm}\right)$ to lead $\left(\mathrm{Pb}^{2+}: 119 \mathrm{pm}\right)$ [78]. The hybrid organic-inorganic halide perovskites having the chemical formula of $\mathrm{AM}^{\mathrm{IV}} \mathrm{X}^{\mathrm{VII}}$, where $\mathrm{A}$ represents a small monovalent organic molecule, $\mathrm{M}^{\mathrm{IV}}$ is a divalent group-IVA cation and $\mathrm{X}^{\mathrm{VII}}$ is a halogen anion, have recently attracted remarkable attention in the photovoltaic community $\mathrm{MASnI}_{3}$ and MASn- $\left(\mathrm{I}_{3-\mathrm{x}} \mathrm{Br}_{\mathrm{x}}\right)$ have been shown to the efficiencies of 6.4\% [79] and 5.73\% [80] respectively. $\mathrm{CH}_{3} \mathrm{NH}_{3} \mathrm{SnI}_{3}, \mathrm{HC}\left(\mathrm{NH}_{2}\right)_{2} \mathrm{SnI}_{3}, \mathrm{NH}_{2} \mathrm{NH}_{3} \mathrm{SnI}_{3}$, and $\mathrm{NH}_{2}\left(\mathrm{CH}_{2}\right)_{3} \mathrm{SnI}_{3}$ is the promising candidate to be a light sensitizer with suitable inorganic hole-transport material to achieve cost-effective and efficient lead-free perovskite solar cell $[81,82]$. Gagandeep et al. uses the graphene as the layer for charge transport and is demonstrate the structure like $\mathrm{n}$-Graphene $/ \mathrm{CH}_{3} \mathrm{NH}_{3} \mathrm{SnI}_{3} / \mathrm{p}$ Graphene which shows the efficiency of 10.67-13.28\% [83]. Giorgi et al. substituted $\mathrm{Pb}$ by TlBi $\left(\mathrm{MATl}_{0.5} \mathrm{Bi}_{0.5} \mathrm{I}_{3}\right)$ and $\operatorname{InBi}\left(\mathrm{MAIn}_{0.5} \mathrm{Bi}_{0.5} \mathrm{I}_{3}\right)$ and depicted that these systems are quietly equivalent to $\mathrm{MAPbI}_{3}$ and can be good replacements for PSCs [84]. Germanium is also assumed as a possible candidate for $\mathrm{Pb}$ substitution in halide perovskites. The theoretical structure and electronic properties of $\mathrm{AGeI}_{3}(\mathrm{~A}=\mathrm{MA}$, FA, Cs) were investigated by Krishnamoorthy et al. [85]. There are several elements like: $\mathrm{Bi}$ [86-88], $\mathrm{Sb}$ [89-91], Ti [92], $\mathrm{Cu}[93,94]$ that use to substitute $\mathrm{Pb}$ to decrease the lead pollution. The group of Song et al. has reported the photovoltaic application of $\mathrm{Sn}$-based halide perovskite materials having the general formula $\mathrm{ASnI}_{3}(\mathrm{~A}=\mathrm{Cs}$, methylammonium and formamidinium tin iodide as the representative light absorbers). Among all the perovskites, $\mathrm{CsSnI}_{3}$ devices accomplished a maximum power conversion efficiency of $4.81 \%$ [95]. Nishimura et al. synthesized 
$\mathrm{GeI}_{2}$ doped $\mathrm{FA}_{0.98} \mathrm{EDA}_{0.01} \mathrm{SnI}_{3}$ and $\mathrm{GeI}_{2}$ doped $\mathrm{EA}_{0.98} \mathrm{EDA}_{0.01} \mathrm{SnI}_{3}$ PSCs and shows the power conversion efficiency of $13.24 \%$ for lead-free perovskite solar cell has been demonstrated with mixed cation and surface passivation [96].

\subsection{Removal of heavy metals from wastewater}

To survive on this planet the clean air, water, and foods are essential to all forms of life. The surface and the groundwaters are only the sources of clean water which help to all living systems as well as human activities such as consuming, irrigation of crops, industrial application, etc. [97]. Water pollution is one of the most worldwide common issues as the population outbursts and industrial evolutions are there. Day by day, the heavy metals (maybe in the form of ions) are released into water bodies by various industries [98] and are exceedingly water-soluble, non-decomposable, oncogenic agents and cause adverse health complications on the animals as well as human beings. Wastewaters coming out from various industries contain many heavy metal ions, for example, $\mathrm{Cu}^{2+}, \mathrm{As}^{5+}, \mathrm{Ni}^{2+}, \mathrm{Sb}^{5+}, \mathrm{Zn}^{2+}, \mathrm{Cd}^{2+}$, and $\mathrm{Pb}^{2+}$ [99]. In addition to heavy metal ions, the different organic and inorganic dyes are alternative pollutant releases from different industries for example papers, textiles, and plastics where the dyes are used for coloring their product and also generate significant volumes of wastewater. Many of these dyes containing heavy metal ions have a tendency to store in the living entities causing a different type of diseases and disorders [100-102]. Hence, it is essential to purify the metal-contaminated water before its discharge to the environment. Among all compare to current methods to remove heavy metal from the contaminated water $[100,101]$ adsorption method is the most likely one because it low cost-effective, high efficiency, and simple to run.

\subsubsection{Heavy metals from contaminated water}

The group of Zhang et al. synthesized the porous nano-calcium titanate microspheres via a citric acid assisted modified sol-gel method and used for absorption of heavy metals like lead, cadmium, and zinc [103]. Haron et al. reported that the nano-crystalline $\mathrm{LaGdO}_{3}$ perovskite was synthesized by the co-precipitation method could adsorb heavy metal ions $\left(\mathrm{Cd}^{2+}\right.$ and $\left.\mathrm{Pb}^{2+}\right)$ which should be the attention in an application such as wastewater treatment [104]. Zhang et al. synthesized porous nano-barium-strontium titanate via sol-gel method using sorghum straw as a template and investigate about adsorption mechanism of $\mathrm{Pb}, \mathrm{Zn}$, and $\mathrm{Cd}$ from contaminated water [105]. $\mathrm{LaFeO}_{3}$ nanoparticles were synthesized by Rao et al. by the sol-gel method in presence of different chelating agents and these nanoparticles utilized for an adsorbent of the removal of heavy metal ions in particular cadmium ion. The $\mathrm{LaFeO}_{3}$ sample prepared with succinic acid (SA) as a chelating agent shows a higher removal efficiency of $\mathrm{Cd}^{2+}$ ions from aqueous systems [106]. Zhang et al. investigated Sr modified $\mathrm{LaFeO}_{3}$ and its structural and catalytic activity. $\mathrm{La}_{0.8} \mathrm{Sr}_{0.2} \mathrm{FeO}_{3}$ contributed significantly enhanced activity in methane combustion and $\mathrm{CO}$ oxidation because the oxygen vacancies accelerated the dissociation of gaseous oxygen on the surface in $\mathrm{CO}$ oxidation and facilitated the diffusion of lattice oxygen from the bulk to the surface during $\mathrm{CH}_{4}$ combustion [107]. The perovskite $\mathrm{LaAlO}_{3}$ was manufactured using the co-precipitation method by Haron et al. The structural and efficiency of removal of heavy metal $\left(\mathrm{Cd}^{2+}\right.$ and $\left.\mathrm{Pb}^{2+}\right)$ were extremely investigated by them. The adsorption performance was studied which fit with the Langmuir isotherm. The results disclosed that $\mathrm{LaAlO}_{3}$ perovskite showed high efficiency as heavy metal ions remover from the contaminated water. This adsorbent could be recycled with an EDTA solution and reprocessed with only slightly less efficient than that of the fresh sample [108]. The group of Chen et al. synthesized ternary photocatalyst $\mathrm{ZnTiO}_{3} / \mathrm{Zn}_{2} \mathrm{Ti}_{3} \mathrm{O}_{8} / \mathrm{ZnO}$ 
heterojunction which displays excellent performance for the degradation of organic pollutants as well as reduction of heavy metal $\mathrm{Cr}(\mathrm{VI})$ ions from wastewater [109]. Figure 5 schematically represent the heavy metal ion $\left(\mathrm{Cd}^{2+}\right)$ adsorb with $\mathrm{LaFeO}_{3}$ perovskite prepared with the different chelating agent.

\subsubsection{Heavy metals in wastewater from the textile industry}

The growing population in our globe, demands to clothe and increase with the taming sense of fashion and lifestyle thus textiles are contrived to meet the growing demands. In several countries such as India and Sri Lanka; the production of textile becomes their source of income that subsidizes their gross domestic product (GDP). However, this has brought both significances to such countries either in a positive way which is an enhancement of the economy or in a negative way indorsed to environmental pollution. The textile industries have been adapted as the worst reprobates of pollution contributors [110]. Especially, in India, according to the Central Pollution Control Board [111], a total of 2324 textile industries are set up. The textile industries employ different types of dyes for the manufacturing of various fabric materials. In reality, about 1 million different dyes are found in the market [112] and roughly 700,000 tons of artificial dyes are produced per year [113]. The disposal of dyes in waters exemplifies a severe environmental issue due to the coinciding presence of various types of pollutants [114-116]. All traditional methods used for the treatment of dyes and/or heavy metals have limitations because of cost, efficiency and operational complications. Among all of them, adsorption was exposed as one of the most effective methods due to its simplicity in operation, adaptability, high-treatment efficiency and low cost, and hence it is extensively applied for wastewaters treatment [117-121].

The perovskite oxide $\mathrm{La}_{0.9} \mathrm{Sr}_{0.1} \mathrm{FeO}_{3}$, capped with cetyl trimethyl ammonium bromide (CTAB) cationic surfactant, and used as a sorbent for the removal of the anionic Congo red (CR) dye from aqueous solutions was reported by Ali et al. [122]. The group of Chu et al. demonstrated the efficiency of $\mathrm{Ag}-\mathrm{La}_{0.8} \mathrm{Ca}_{0.2} \mathrm{Fe}_{0.94} \mathrm{O}_{3-\delta}$ for the removal of organic and bacterial pollutants by catalytic peroxymonosulfate (PMS) activation. The oxygen vacancies in the B-site of perovskite enhances PMS activation and $\mathrm{The} \mathrm{SO}_{4} \bullet$ and $\bullet \mathrm{OH}$ radicals enhance the biocidal activity [123]. Nanocrystalline $\mathrm{LaAlO}_{3}: \mathrm{Sm}^{3+}: \mathrm{Bi}^{3+}$ composites are used to adsorb Direct Blue-53 (DB53) dye was reported by Pratibha et al. [124]. This adsorbent is good and promising in the adsorption capacity and is advantageous in the elimination of toxic and non-biodegradable pollutants from water. The group of Dong et al. hydrothermally synthesized perovskite $\mathrm{BaZrO}_{3}$ in the form of hollow micro- and nano-sphere. This size-tunable $\mathrm{BaZrO}_{3}$ hollow nanospheres exhibited an excellent adsorption performance for reactive dyes in acidic conditions and can be used as excellent circular adsorbents for removing reactive dyes. They show the adsorption capacities are over

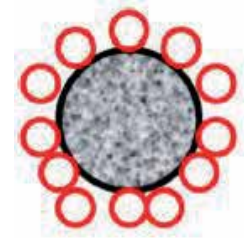

SA

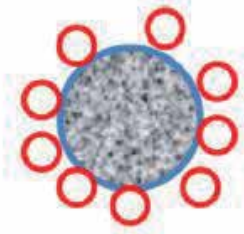

$\mathrm{CA}$

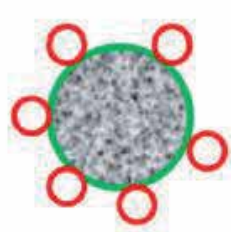

$\mathrm{OA}$

Heavy metal ions $\left(\mathrm{Cd}^{2+}\right)$

Figure 5.

Representation of the adsorption process over $\mathrm{LaFeO}_{3}$ nanoparticles surfaces prepared using succinic acid (SA), citric acid (CA), and oxalic acid (OA) [106]. 
$160 \mathrm{mg} \mathrm{g}^{-1}$ for different investigated dyes at a $\mathrm{pH}$ value of 2 . The adsorbents were easily recovered by using a basic solution with the adsorption performance persistent and the desorption rate is more than $97 \mathrm{wt} \%$ [125]. Siddharth et al. synthesized the perovskite structure of Ti-doped $\mathrm{BaMnO}_{3}\left(\mathrm{BaMn}_{0.85} \mathrm{Ti}_{0.15} \mathrm{O}_{2.93}\right)$ and its enhanced photocatalytic degradation ( $99 \%$ ) as compared to $\mathrm{BaMnO}_{3}$ toward toxic water impurities like RhB and MB dyes within 270 and 150 min under sunlight [126].

\section{Conclusion}

Nowadays, because of the increasing population and demand for natural resources is a global concern, hence, it is one of the origins of environmental pollution. Among all the pollutants, lead is one of the most hazardous materials. For the reason that the toxic effect of lead in lead-based materials, the researcher communities and scientists concentrated on synthesized lead-free materials possessing the same properties. Since the last two decades, the investigation on such materials enhances unexpectedly. Lead-free perovskite materials with excellent dielectric and piezoelectric properties belonging to the ferroelectric family and these reduce the adverse effect on the human body as well as the environment. Here this chapter concludes with different applications like SOFCs, sensors, solar cells, wastewater treatment of lead-free perovskite materials.

\section{Thanks}

I am whole heartily thankful to Dr. Sushrisangita Sahoo from Bhubaneshwar for continuous support during working in this chapter. I am thankful to Dr. S. Suganya from CSIR-Central Salt and Marine Chemicals Research Institute (CSMCRI), Bhavnagar, Gujrat, and Ms. Ananya Rout from NIT-Raipur, for their encouragement to start writing this chapter.

\section{Author details}

Manojit De $\mathrm{e}^{1,2}$

1 Department of Pure and Applied Physics, Guru Ghasidas Vishwavidyalaya, Bilaspur, India

2 Department of Applied Physics, Chouksey Engineering College, Bilaspur, India

*Address all correspondence to: manojit.manojit.de1@gmail.com

IntechOpen

(C) 2020 The Author(s). Licensee IntechOpen. This chapter is distributed under the terms of the Creative Commons Attribution License (http://creativecommons.org/licenses/ by/3.0), which permits unrestricted use, distribution, and reproduction in any medium, provided the original work is properly cited. (cc) BY 


\section{References}

[1] Levy MR. Crystal Structure and Defect Property Predictions in Ceramic Materials. London: Department of Materials, Imperial College of Science, Technology and Medicine. January 2005. Available from: https://spiral.imperial. ac.uk/bitstream/10044/1/11804/2/LevyMR-2005-PhD-Thesis.pdf

[2] New Tolerance Factor to Predict the Stability of Perovskite Oxides and Halides. Available from: https://arxiv. org/ftp/arxiv/papers/1801/1801.07700. pdf

[3] Glazer AM. The classification of tilted octahedra in perovskites. Acta Crystallographica. Section B: Structural Crystallography \& Crystal Chemistry. 1972;28:3384-3392

[4] Scaife DE, Weller PF, Fisher WG. Crystal preparation and properties of cesium tin(II) trihalides. Journal of Solid State Chemistry. 1974;9:308-314

[5] Aleksandrov KS. The sequences of structural phase transitions in perovskites. Ferroelectrics. 1976;14:801-805

[6] Goldschmidt VM. Die Gesetze der Krystallochemie. Die Naturwissenschaften. 1926;21:477-485

[7] Yugong W, Zhang H, Zhang Y, Jinyi M, Daohua X. Lead-free piezoelectric ceramics with composition of (0.97-x) $\mathrm{Na}_{1 / 2} \mathrm{Bi}_{1 / 2} \mathrm{TiO}_{3}-0.03 \mathrm{NaNbO}_{3}-\mathrm{xBaTiO}_{3}$. Journal of Materials Science. 2003;38:987

[8] United Nations Environment Programme. Nairobi; 2019. Available from: https://www.unenvironment.org/ explore-topics/transport/what-we-do/ partnership-clean-fuels-and-vehicles/ lead-campaign

[9] Takenaka T, Nagata H. Current status and prospects of lead-free piezoelectric ceramics. Journal of the European Ceramic Society. 2005;25:2693-2700
[10] Gordon JN, Taylor A, Bennett PN. Lead poisoning: Case studies. British Journal of Clinical Pharmacology. 2002;53:451

[11] Barltrop D, Smith AM. Kinetics of lead interaction with human erythrocytes. Postgraduate Medical Journal. 1985;51:770

[12] Rabinowitz MB, Wetherill GW, Kopple JD. Kinetic analysis of lead metabolism in healthy humans. The Journal of Clinical Investigation. 1976;58:260

[13] Courtney D, Meekin SR. Changes in blood lead levels of solderers following the introduction of the control of lead at work regulations. Occupational Medicine. 1985;35:128

[14] Goering PL. Lead-protein interactions as a basis for lead toxicity. Neurotoxicology. 1993;14:45

[15] Chisolm JJ Jr. Treatment of lead poisoning. Modern Treatment. 1971;8:593-611

[16] Galal A, Atta NF, Ali SM. Optimization of the synthesis conditions for $\mathrm{LaNiO}_{3}$ catalyst by microwave assisted citrate method for hydrogen production. Applied Catalysis, A: General. 2011;409-410:202-208

[17] Giang HT, Duy HT, Ngan PQ, Thai GH, Thu DTA, Thu DT, et al. Hydrocarbon gas sensing of nanocrystalline perovskite oxides $\mathrm{LnFeO}_{3}$ $(\mathrm{Ln}=\mathrm{La}, \mathrm{Nd}$ and $\mathrm{Sm})$. Sensors and Actuators B: Chemical. 2011;158:246

[18] Ishihara T. In: Ishihara T, editor. Perovskite Oxide for Solid Oxide Fuel Cells, Fuel Cells and Hydrogen Energy. Springer Science Business Media, LLC; 2009. pp. 1-16 Chapter 1 
[19] Atta NF, Galal A, El-Ads EH. Perovskite nanomaterials-Synthesis, characterization, and applications. In: Pan L, Zhu G, editors. Perovskite Materials-Synthesis, Characterisation, Properties, and Applications. Rijeka: IntechOpen, DOI: 10.5772/61280. Available from: https://www. intechopen.com/books/perovskitematerials-synthesis-characterisationproperties-and-applications/ perovskite-nanomaterials-synthesischaracterization-and-applications

[20] Shao Z, Haile SM. A highperformance cathode for the next generation of solid-oxide fuel cells. Nature. 2004;431:170-173

[21] Shin J, Xu W, Zanella M, Dawson K, Savvin SN, Claridge JB, et al. Self-assembled dynamic perovskite composite cathodes for intermediate temperature solid oxide fuel cells. Nature Energy. 2017;2:16214

[22] Huang YH, Dass RI, Xing ZL, Goodenough JB. Double perovskites as anode materials for solid-oxide fuel cells. Science. 2006;312:254-257

[23] Song K, Lee K. Characterization of $\mathrm{Ba}_{0.5} \mathrm{Sr}_{0.5} \mathrm{M}_{1-\mathrm{x}} \mathrm{Fe}_{\mathrm{x}} \mathrm{O}_{3-\delta}(\mathrm{M}=\mathrm{Co}$ and $\mathrm{Cu})$ perovskite oxide cathode materials for intermediate temperature solid oxide fuel cells. Ceramics International. 2012;38:5123-5131

[24] Tongyun C, Liming S, Feng L, Weichang Z, Qianfeng Z, Xiangfeng C. $\mathrm{NdFeO}_{3}$ as anode material for $\mathrm{S} / \mathrm{O}_{2}$ solid oxide fuel cells. Journal of Rare Earths. 2012;30(11):1138-1141

[25] Conceiçăo LD, Silva AM, Ribeiro NFP, Souza MMVM. Combustion synthesis of $\mathrm{La}_{0.7} \mathrm{Sr}_{0.3} \mathrm{Co}_{0.5} \mathrm{Fe}_{0.5} \mathrm{O}_{3}$ (LSCF) porous materials for application as cathode in IT-SOFC. Materials Research Bulletin. 2011;46:308-314

[26] Wang F, Chen D, Shao Z. $\mathrm{Sm}_{0.5} \mathrm{Sr}_{0.5} \mathrm{CoO}_{3-\delta}$-infiltrated cathodes for solid oxide fuel cells with improved oxygen reduction activity and stability. Journal of Power Sources. 2012;216:208-215

[27] Vernoux P, Guillodo M, Fouletier J, Hammou A. Alternative anode material for gradual methane reforming in solid oxide fuel cells. Solid State Ionics. 2000;135:425-431

[28] Tao SW, Irvine JTS. A redox-stable efficient anode for solid-oxide fuel cells. Nature Materials. 2003;2:320-323

[29] Deganello F, Liotta LF, Leonardi SG, Neri G. Electrochemical properties of Ce-doped $\mathrm{SrFeO}_{3}$ perovskites-modified electrodes towards hydrogen peroxide oxidation. Electrochimica Acta.

2016;190:939-947

[30] Wang J. Electrochemical glucose biosensors. Chemical Reviews. 2008;108:814-825

[31] Wang Y, Xu Y, Luo L, Dinga Y, Liu X, Huang A. A novel sensitive nonenzymatic glucose sensor based on perovskite $\mathrm{LaNi}_{0.5} \mathrm{Ti}_{0.5} \mathrm{O}_{3}$-modified carbon paste electrode. Sensors and Actuators B. 2010;151:65-70

[32] Wang Y, Xu Y, Luo L, Ding Y, Liu X. Preparation of perovskite-type composite oxide $\mathrm{LaNi}_{0.5} \mathrm{Ti}_{0.5} \mathrm{O}_{3}-\mathrm{NiFe}_{2} \mathrm{O}_{4}$ and its application in glucose biosensor. Journal of Electroanalytical Chemistry. 2010;642:35-40

[33] Liotta LF, Puleo F, La Parola V, Leonardi SG, Donato N, Aloisio D, et al. $\mathrm{La}_{0.6} \mathrm{Sr}_{0.4} \mathrm{FeO}_{3-\delta}$ and $\mathrm{La}_{0.6} \mathrm{Sr}_{0.4} \mathrm{Co}_{0.2} \mathrm{Fe}_{0.8} \mathrm{O}_{3-\delta}$ perovskite materials for $\mathrm{H}_{2} \mathrm{O}_{2}$ and glucose electrochemical sensors. Electroanalysis. 2014;27:1-10

[34] El-Ads EH, Galal A, Atta NF. Electrochemistry of glucose at gold nanoparticles modified graphite/ $\mathrm{SrPdO}_{3}$ electrode-Towards a novel non-enzymatic glucose sensor. Journal 
of Electroanalytical Chemistry. 2015;749:42-52

[35] Wolkoff P. Volatile organic compounds sources, measurements, emissions, and the impact on indoor air quality. Indoor Air. 1995;5:5

[36] Boor BE, Spilak MP, Laverge J, Novoselac A, Xu Y. Human exposure to indoor air pollutants in sleep microenvironments: A literature review. Building and Environment. 2017; 125:528

[37] Joshi N, Hayasaka T, Liu Y, Liu H, Oliveira ON, Lin L. A review on chemiresistive room temperature gas sensors based on metal oxide nanostructures, graphene and 2D transition metal dichalcogenides. Microchimica Acta. 2018;185:213

[38] Zheng H, Ou JZ, Strano MS, Kaner RB, Mitchell A, Kalantarzadeh K. Nanostructured tungsten oxideProperties, synthesis, and applications. Advanced Functional Materials. 2011;21:2175

[39] Bai J, Zhou B. Titanium dioxide nanomaterials for sensor applications. Chemical Reviews. 2014;114:10131

[40] Thirumalairajan S, Girija K, Mastelaro VR, Ponpandian N. Surface morphology-dependent roomtemperature $\mathrm{LaFeO}_{3}$ nanostructure thin films as selective $\mathrm{NO}_{2}$ gas sensor prepared by radio frequency magnetron sputtering. ACS Applied Materials \& Interfaces. 2014;6:13917-13927

[41] Jun-Chao D, Hua-Yao L, Ze-Xing C, Xiao-Dong Z, Guo X. $\mathrm{LaCoO}_{3}$-based sensors with high sensitivity to carbon monoxide. RSC Advances. 2015;5:65668-65673

[42] Ghasdi M, Alamdari H, Royer S, Adnot A. Electrical and $\mathrm{CO}$ gas sensing properties of nanostructured $\mathrm{La}_{1-\mathrm{x}} \mathrm{Ce}_{\mathrm{x}} \mathrm{CoO}_{3}$ perovskite prepared by activated reactive synthesis. Sensors and Actuators B. 2011;156:147-155

[43] Sowmya P, Kaushik SD, Siruguri V, Diptikanta S, Viegas AE, Chandrabhas $\mathrm{N}$, et al. Investigation of Ca substitution on the gas sensing potential of $\mathrm{LaFeO}_{3}$ nanoparticles towards low concentration $\mathrm{SO}_{2}$ gas. Dalton Transactions. 2016;45(34):13547-13555

[44] Zhang R, Jifan H, Zhouxiang H, Ma Z, Zhanlei W, Yongjia Z, et al. Electrical and $\mathrm{CO}$-sensing properties of $\mathrm{NdFe}_{1-\mathrm{x}} \mathrm{Co}_{\mathrm{x}} \mathrm{O}_{3}$ perovskite system. Journal of Rare Earths. 2010;28(4):591-595

[45] Benali A, Azizi S, Bejar M, Dhahri E, Graça MFP. Structural, electrical and ethanol sensing properties of double-doping $\mathrm{LaFeO}_{3}$ perovskite oxides. Ceramics International. 2014;40(9):14367-14373

[46] Rong O, Zhang Y, Hu J, Li K, Wang H, Chen M, et al. Design of ultrasensitive $\mathrm{Ag}-\mathrm{LaFeO}_{3}$ methanol gas sensor based on quasi molecular imprinting technology. Scientific Reports. 2018;8:14220

[47] Yumin Z, Qingju L, Jin Z, Qin Z, Zhu Z. High sensitive and selective formaldehyde gas sensor using molecular imprinting technique based on $\mathrm{Ag}-\mathrm{LaFeO}$. Journal of Materials Chemistry C. 2014;2:10067

[48] Wang Y, Chen J, Wu X. Preparation and gas-sensing properties of perovskite-type $\mathrm{SrFeO}_{3}$ oxide. Materials Letters. 2001;49:361-364

[49] Carotta MC, Butturi MA, Martinelli G, Sadaoka Y, Nunziante P, Traversa E. Microstructural evolution of nanosized $\mathrm{LaFeO}_{3}$ powders from the thermal decomposition of a cyano-complex for thick film gas sensors. Sensors and Actuators B. 1997;44:590-594 
[50] Sen S, Chakraborty N, Rana P, Narjinary M, Mursalin SD, Tripathy S, et al. Nanocrystalline gallium ferrite: A novel material for sensing very low concentration of alcohol vapour. Ceramics International. 2015;41:10110

[51] Sahner K, Moos R, Matam M, Tunney JJ, Post M. Hydrocarbon sensing with thick and thin film p-type conducting perovskite materials. Sensors and Actuators B. 2005;108:102-112

[52] Zhang D et al. Microwave-assisted synthesis of porous and hollow $\alpha-\mathrm{Fe}_{2} \mathrm{O}_{3} /$ $\mathrm{LaFeO}_{3}$ nanostructures for acetone gas sensing as well as photocatalytic degradation of methylene blue. Nanotechnology. 2020;31:215601

[53] Francesco B, Ada F, Valerio V, Marco M, Rossella B. Optimization of perovskite gas sensor performance: Characterization, measurement and experimental design. Sensors. 2017;17:1352

[54] Yaoyu Y, Yanbai S, Pengfei Z, Rui L, Ang L, Sikai Z, et al. Fabrication, characterization and n-propanol sensing properties of perovskite-type $\mathrm{ZnSnO}_{3}$ nanospheres based gas sensor. Applied Surface Science. 2020;509:145335

[55] Neeru S, Himmat KS, Sharma SK, Sachdev K. Fabrication of $\mathrm{LaFeO}_{3}$ and rGO- $\mathrm{LaFeO}_{3}$ microspheres based gas sensors for detection of $\mathrm{NO}_{2}$ and $\mathrm{CO}$. RSC Advances. 2020;10:1297-1308

[56] Wang H, Guo Z, Wentao H, Li S, Yongjia Z, Ensi C. Ethanol sensing characteristics of $\mathrm{BaTiO}_{3} / \mathrm{LaFeO}_{3}$ nanocomposite. Materials Letters. 2019;234:40-44

[57] Dong G, Huiqing F, Hailin T, Jiawen F, Qiang L. Gas-sensing and electrical properties of perovskite structure p-type barium-substituted bismuth ferrite. RSC Advances. 2015;5:29618-29623
[58] Silu P, Min M, Weijie Y, Ziqian W, Zihan W, Jian B, et al. Acetone sensing with parts-per-billion limit of detection using a $\mathrm{BiFeO}_{3}$-based solid solution sensor at the morphotropic phase boundary. Sensors and Actuators B: Chemical. 2020;313:128060

[59] Tie Y, Ma SY, Pei ST, Zhang QX, Zhu KM, Zhang R, et al. Pr doped $\mathrm{BiFeO}_{3}$ hollow nanofibers via electrospinning method as a formaldehyde sensor. Sensors and Actuators B: Chemical. 2020;308:127689

[60] Jain GH, Patil LA, Wagh MS, Patil DR, Patil SA, Amalnerkar DP. Surface modified $\mathrm{BaTiO}_{3}$ thick film resistors as $\mathrm{H}_{2} \mathrm{~S}$ gas sensors. Sensors and Actuators B. 2006;117:159-165

[61] Patil RP, Gaikwad SS, Karanjekar AN, Khanna PK, Jain GH, Gaikwad VB, et al. Optimization of strontium- doping concentration in $\mathrm{BaTiO}_{3}$ nanostructures for room temperature $\mathrm{NH}_{3}$ and $\mathrm{NO}_{2}$ gas sensing. Materials Today Chemistry. 2020;16:100240

[62] Artem M, Marina R, Alexander B, Alexander G. Nanocrystalline $\mathrm{BaSnO}_{3}$ as an alternative gas sensor material: Surface reactivity and high sensitivity to $\mathrm{SO}_{2}$. Materials. 2015;8:6437-6454

[63] Gopal Reddy CV, Manorama SV, Rao VJ. Preparation and characterization of barium stannate: Application as a liquefied petroleum gas sensor. Journal of Materials Science: Materials in Electronics. 2001;12:137-142

[64] Katekani S, Swart HC, Mhlongo GH. $\mathrm{LaBO}_{3}$ (B = Fe, Co) nanofibers and their structural, luminescence and gas sensing characteristics. Physica B: Physics of Condensed Matter. 2020;578:411883

[65] Shaikh JS, Shaikh NS, Sheikh AD, Mali SS, Kale AJ, Kanjanaboos P, et al. 
Perovskite solar cells: In pursuit of efficiency and stability. Materials and Design. 2017;136:54

[66] Newcomer juices up the race to harness sunlight. Science. 2013;342:1438-1439. DOI: 10.1126/ science.342.6165.1438-b

[67] Gholipour S, Saliba M. From exceptional properties to stability challenges of perovskite solar cells. Small. 2018;14:1802385

[68] Kojima A, Teshima K, Shirai Y, Miyasaka T. Organometal halide perovskite as visible-light sensitizer for photovoltaic cells. Journal of the American Chemical Society. 2009;131:6050-6051

[69] Yang WS, Noh JH, Jeon NJ, Kim YC, Ryu S, Seo J, et al. High-performance photovoltaic perovskite layers fabricated through intramolecular exchange. Science. 2015;348:1234-1237

[70] Yin WJ, Yang JH, Kang J, Yan Y, Wie SH. Halide perovskite materials for solar cells: A theoretical review. Journal of Materials Chemistry. 2015;3:8926-8942

[71] Green MA, Baillie AH, Snaith HJ. The emergence of perovskite solar cells. Nature Photonics. 2014;8:506-514

[72] Babayigit A, Ethirajan A, Muller M, Conings B. Toxicity of organometal halide perovskite solar cells. Nature Materials. 2016;15:247-251

[73] Flora G, Gupta D, Tiwari A. Toxicity of lead: A review with recent updates. Interdisciplinary Toxicology. 2012;5:47-58

[74] Benmessaoud IR, Mahul-Mellier A-L, Horv'ath E, Maco B, Spina M, Lashuel HA, et al. Health hazards of methylammonium lead iodide based perovskites: Cytotoxicity studies. Toxicological Research. 2016;5:407-419
[75] Gong J, Darling SB, You F.

Perovskite photovoltaics:

Lifecycle assessment of energy and environmental impacts.

Energy \& Environmental Science. 2015;8:1953-1968

[76] Fthenakis VM, Kim HC, Alsema E. Emissions from photovoltaic life cycles. Environmental Science \& Technology. 2008;42:2168-2174

[77] Antonio A. Perovskite solar cells go lead free. Joule. 2017;1:659-664

[78] Hoefler SF, Trimmel G, Rath T. Progress on lead-free metal halide perovskite for photovoltaic applications: A review. Monatshefte fuer Chemie. 2017;148:795-826

[79] Noel NK, Stranks SD, Abate A, Wehrenfennig C, Guarnera S, Haghighirad AB, et al. Lead-free organic-inorganic tin halide perovskites for photovoltaic applications. Energy \& Environmental Science. 2014;7:3061-3068

[80] Hao F, Stoumpos CC, Cao DH, Chang RP, Kanatzidis H. Lead-free solid-state organic-inorganic halide perovskite solar cells. Nature Photonics. 2014;8(8):489-494

[81] Mandapu U, Vedanayakam SV, Thyagarajan K, Reddy MR, Babu BJ. Design and simulation of high efficiency tin halide perovskite solar cells. International Journal of Renewable Energy Research. 2017;7:1603-1612

[82] Ngoc TV, Doan HT. Lead-free hybrid organic-inorganic perovskites or solar cell applications. Journal of Chemical Physics. 2020;152:014104

[83] Gagandeep, Mukhtiyar S, Ramesh K, Vinamrita S. Graphene as charge transport layers in lead free perovskite solar cell. Materials Research Express. 2019;6:115611 
[84] Giorgi G, Yamashita K. Alternative, lead-free, hybrid organic-inorganic perovskites for solar applications: A DFT analysis. Chemistry Letters. 2015;44:826-828

[85] Krishnamoorthy T, Ding H, Yan C, Leong WL, Baikie T, Zhang Z, et al. Lead-free germanium iodide perovskite materials for photovoltaic applications. Journal of Materials Chemistry A. 2015;3:23829-23832

[86] Yang B, Chen J, Hong F, Mao X, Zheng K, Yang S, et al. Lead-free, air-stable all-inorganic cesium bismuth halide perovskite nanocrystals. Angewandte Chemie International Edition. 2017;56:12471-12475

[87] Lou Y, Fang M, Chen J, Zhao Y. Formation of highly luminescent cesium bismuth halide perovskite quantum dots tuned by anion exchange. Chemical Communications. 2018;54:3779-3782

[88] Nelson RD, Santra K, Wang Y, Hadi A, Petrich JW, Panthani MG. Synthesis and optical properties of ordered-vacancy perovskite cesium bismuth halide nanocrystals. Chemical Communications. 2018;54: 3640-3643

[89] Zuo C, Ding L. Lead-free perovskite materials $\left(\mathrm{NH}_{4}\right)_{3} \mathrm{Sb}_{2} \mathrm{I}_{\mathrm{x}} \mathrm{Br}_{9-\mathrm{x}}$. Angewandte Chemie International Edition. 2017;56:6528-6532

[90] Saparov B, Hong F, Sun J-P, Duan H-S, Meng W, Cameron S, et al. Thinfilm preparation and characterization of $\mathrm{Cs}_{3} \mathrm{Sb}_{2} \mathrm{I}_{9}$ : A lead-free layered perovskite semiconductor. Chemistry of Materials. 2015;27:5622-5632

[91] Hebig J-C, Kühn I, Flohre J, Kirchartz T. Optoelectronic properties of $\left(\mathrm{CH}_{3} \mathrm{NH}_{3}\right)_{3} \mathrm{Sb}_{2} \mathrm{I}_{9}$ thin films for photovoltaic applications. ACS Energy Letters. 2016;1:309-314

[92] Chen M, Ju M-G, Carl AD, Zong Y, Grimm RL, Gu J, et al. Cesium
titanium(IV) bromide thin films based stable lead-free perovskite solar cells. Joule. 2018;2:558-570

[93] Li X, Zhong X, Hu Y, Li B, Sheng Y, Zhang Y, et al. Organic-inorganic copper(II)-based material: A lowtoxic, highly stable light absorber for photovoltaic application. Journal of Physical Chemistry Letters. 2017;8:1804-1809

[94] Yang P, Liu G, Liu B, Liu X, Lou $\mathrm{Y}$, Chen J, et al. All-inorganic $\mathrm{Cs}_{2} \mathrm{CuX}_{4}(\mathrm{X}=\mathrm{Cl}, \mathrm{Br}$, and $\mathrm{Br} / \mathrm{I})$ perovskite quantum dots with blue-green luminescence. Chemical Communications. 2018;54:11638-11641

[95] Tze-Bin S, Takamichi Y, Shinji A, Kanatzidis Mercouri G. Performance enhancement of lead-free tin-based perovskite solar cells with reducing atmosphere-assisted dispersible additive. ACS Energy Letters. 2017;2(4):897-903

[96] Nishimura K, Kamarudin MA, Hirotani D, Hamada K, Shen Q, Iikubo S, et al. Lead-free tin-halide perovskite solar cells with $13 \%$ efficiency. Nano Energy. 2020;74:104858

[97] World Health Organization. Guidelines for Drinking Water Quality. 2nd ed. Geneva, Switzerland: Author; 1997

[98] Fu F, Wang Q. Removal of heavy metal ions from wastewaters: A review. Journal of Environmental Management. 2011;92:407-418

[99] Biswas AK, Tortajada C. Water quality management: An introductory frame work. Water Resources Management. 2011;27:5-11

[100] Taseidifar M, Makavipour F, Pashley RM, Rahman AM. Removal of heavy metal ions from water using ion flotation. Environmental Technology and Innovation. 2017;8:182-190 
[101] Mohammed AA, Ebrahim SE, Alwared AI. Flotation and sorptiveflotation methods for removal of lead ions from wastewater using SDS as surfactant and barley husk as biosorbent. Journal of Chemistry. 2013:1-6

[102] Rauf MA, Qadri SM, Ashraf S, Al-Mansoori KM. Sorption and desorption of $\mathrm{Pb}^{2+}$ ions by dead Sargassum sp. biomass. Chemical Engineering Journal. 2009;150:90-95

[103] Zhanga D, Chun-li Z, Zhou P. Preparation of porous nano-calcium titanate microspheres and its adsorption behavior for heavy metal ion in water. Journal of Hazardous Materials. 2011;186:971-977

[104] Haron W, Sirimahachai U, Wisitsoraat A, Wongnawa S. Removal of $\mathrm{Cd}^{2+}$ and $\mathrm{Pb}^{2+}$ from water by $\mathrm{LaGdO}_{3}$ perovskite. SNRU Journal of Science and Technology. 2017;9(3):544-551

[105] Zhang D, Wang M, Tan Y. Preparation of porous nano-bariumstrontium titanate by sorghum straw template method and its adsorption capability for heavy metal ions. Acta Chimica Sinica: Chinese Edition. 2010;68(16):1641-1648

[106] Rao MP, Musthafa S, Wu JJ, Anandan S. Facile synthesis of perovskite $\mathrm{LaFeO}_{3}$ ferroelectric nanostructures for heavy metal ion removal applications.

Materials Chemistry and Physics. 2019;232:200-204

[107] Zhang X, Li H, Li Y, Shen W. Structural properties and catalytic activity of Sr-substituted $\mathrm{LaFeO}_{3}$ perovskite. Chinese Journal of Catalysis. 2012;33:1109-1114

[108] Haron W, Wisitsoraat A, Sirimahachai U, Wongnawa S. Removal of toxic heavy metal ions from water with $\mathrm{LaAlO}_{3}$ perovskite. Songklanakarin
Journal of Science and Technology. 2018;40(5):993-1001

[109] Chen F, Yu C, Wei L, Fan Q, Ma F, Zeng J, et al. Fabrication and characterization of $\mathrm{ZnTiO}_{3} /$ $\mathrm{Zn}_{2} \mathrm{Ti}_{3} \mathrm{O}_{8} / \mathrm{ZnO}$ ternary photocatalyst for synergetic removal of aqueous organic pollutants and $\mathrm{Cr}(\mathrm{VI})$ ions. Science of The Total Environment. 2020;706:136026

[110] Normala H, Soo YRG. Removal of heavy metals from textile wastewater using zeolite. Environment Asia. 2010;3:124-130

[111] CPCB (Central Pollution Control Board). Advance Methods for Treatment of Textile Industry Effluents. Publication No. RERES/7/2007. New Delhi: Government of India; 2007. Available from: https://www.researchgate.net/ publication/280572135_advance_ methods_for_treatment_of_textile_ industry_effluents

[112] Asad S, Amoozegar MA, Pourbabaee AA, Sarbolouki MN, Dastgheib SMM. Decolorization of textile azo dyes by newly isolated halophilic and halotolerant bacteria. Bioresource Technology. 2007;98:2082-2088

[113] Ananthanarayanan Y, Natchimuthu K, Sudipta T, Soundarapandian K, Ramasundaram T. Environment-friendly management of textile mill wastewater sludge using epigeic earthworms: Bioaccumulation of heavy metals and metallothionein production. Journal of Environmental Management. 2020;254:109813

[114] Toledano Garcia D, Ozer LY, Parrino F, Ahmed M, Brudecki GP, Hasan SW, et al. Photocatalytic ozonation under visible light for the remediation of water effluents and its integration with an electromembrane bioreactor. Chemosphere. 2018;209:534-541 
[115] Matei E, Covaliu CI, Predescu A, Berbecaru A, Tarcea C, Predescu C. Remediation of wastewater with ultraviolet irradiation using a novel titanium (IV) oxide photocatalyst. Analytical Letters. 2019;52:2180-2187

[116] Neagu S, Cojoc R, Enache M, Mocioiu OC, Precupas A, Popa VT, et al. Biotransformation of waste glycerol from biodiesel industry in carotenoids compounds by halophilic microorganisms. Waste and Biomass Valorization. 2019;10:45-52

[117] Kamińska G, Dudziak M, Kudlek E, Bohdziewicz J. Preparation, characterization and adsorption potential of grainy halloysite-CNT composites for anthracene removal from aqueous solution. Nanomaterials. 2019;9:890

[118] Wang J, Zhuang S. Removal of various pollutants from water and wastewater by modified chitosan adsorbents. Critical Reviews in Environmental Science and Technology. 2017;47:2331-2386

[119] Xu S, Zhang S, Chen K, Han J, Liu H, Wu K. Biosorption of $\mathrm{La}^{3+}$ and $\mathrm{Ce}^{3+}$ by Agrobacterium sp. HN1. Journal of Rare Earths. 2011;29:265-270

[120] Yagub MT, Sen TK, Afroze S, Ang HM. Dye and its removal from aqueous solution by adsorption: A review. Advances in Colloid and Interface Science. 2014;209:172-184

[121] Almasri DA, Saleh NB, Atieh MA, McKay G, Ahzi S. Adsorption of phosphate on iron oxide doped halloysite nanotubes. Scientific Reports. 2019;9:3232

[122] Ali SM, Eskandrani AA. The sorption performance of cetyl trimethyl ammonium bromide-capped $\mathrm{La}_{0.9} \mathrm{Sr}_{0.1} \mathrm{FeO}_{3}$ perovskite for organic pollutants from industrial processes. Molecules. 2020;25(7):1640
[123] Chu Y, Tan X, Shen Z, Liu P, Han N, Kang J, et al. Efficient removal of organic and bacterial pollutants by Ag- $\mathrm{La}_{0.8} \mathrm{Ca}_{0.2} \mathrm{Fe}_{0.94} \mathrm{O}_{3-\delta}$ perovskite via catalytic peroxymonosulfate activation. Journal of Hazardous Materials. 2018;356:53-60

[124] Pratibha S, Dhananjaya N, Manjunatha CR, Narayana A. Fast adsorptive removal of direct blue-53 dye on rare-earth doped Lanthanum aluminate nanoparticles: Equilibrium and kinetic studies. Materials Research Express. 2019;6:1250i5

[125] Dong Z, Ye T, Zhao Y, Yu J, Wang F, et al. Perovskite $\mathrm{BaZrO}_{3}$ hollow micro- and nanospheres: Controllable fabrication, photoluminescence and adsorption of reactive dyes. Journal of Materials Chemistry. 2011;21:5978

[126] Siddharth S, Soumitra M, Sonia R, Hari R, Bisht RS, Panigrahi SK, et al. Ti doped $\mathrm{BaMnO}_{3}$ perovskite structure as photocatalytic agent for the degradation of noxious air and water pollutants. SN Applied Sciences. 2020;2:310 


\title{
Perovskite Nanoparticles
}

\author{
Burak Gultekin, Ali Kemal Havare, Shirin Siyahjani, \\ Halil Ibrahim Ciftci and Mustafa Can
}

\begin{abstract}
2D perovskite nanoparticles have a great potential for using in optoelectronic devices such as Solar Cells and Light Emitting Diodes within their tuneable optic and structural properties. In this chapter, it is aimed to express "relation between chemical structures and photo-physical behaviours of perovskite nanoparticles and milestones for their electronic applications". Initially, general synthesis methods of perovskite nanoparticles have been explained. Furthermore, advantages and disadvantages of the methods have been discussed. After the synthesis, formation of 2D perovskite crystal and effects on shape factor, particle size and uniformity of perovskite have been explained in detail. Beside these, optic properties of luminescent perovskite nanoparticles have been summarized a long with spectral band tuning via size and composition changes. In addition, since their different optical properties and relatively more stable chemical structure under ambient conditions, a comprehensive compilation of opto-electronic applications of $2 \mathrm{D}$ perovskite nanoparticles have been prepared.
\end{abstract}

Keywords: perovskite, nanoparticle, nanocrystal, opto-electronics, solar cells, OLEDs, fluorescence

\section{Introduction}

Inorganic and/or hybrid perovskites have become prominent in solutionprocessed optoelectronics. Thousands of reports have been proposed about photovoltaics [1-3], light-emitting diodes (LEDs) [4-6], lasers [6, 7] and photodetectors [8-12] containing perovskite semiconductors per annum over the past decade. Halide perovskite nanostructures exhibit tremendous optic and electrical properties such as strong absorption and/or emission, higher photoluminescence quantum yields (PLQYs) [13-15], higher exciton binding energies and tuneable bandgaps than those of bulk perovskites and other nanomaterials. With these outstanding properties, they may offer many scopes for optoelectronics. Particularly, hybrid lead halide perovskite nanostructures (PNSs) offer unique opportunities for lightemitting diode (LED) applications. PNSs represent larger exciton binding energies and longer carrier decay times than those of bulk crystals. In addition to this, their narrow emission band makes them good candidates for LED [16] and laser [17]. Since Schmidt et al. reported the synthesis of first colloidal PNS by a simple procedure under ambient conditions in 2014 [18], many research groups have started working on these materials. In the synthesis of PNSs, it is possible to use organic ligands for capping to stop crystals ' growth in the nanometer scale. Furthermore, 
capping ligands can reduce surface defects in the same way of the traditional NC preparation. Thus, size and shape of PNSs can be tune finely from a single perovskite layer or below the exciton Bohr radius for using the effect to quantum confinement to multilayers which exhibits bulk-like properties. With this method, it is possible to prepare nanostructures like quantum dots (QDs), nanoplatelets (NPLs), nanosheets (NSs), and nanowires (NWs) [19, 20].

In a simple PNS growth process, the methylammonium cations are embedded in the voids of the corner-sharing PbX6 octahedra, the long alkyl chain cations only at the periphery of the octahedra with their chains hanging it. Therefore, long alkylammonium ions can be used as the capping agents to limit the growth of the PNSs in three-dimension (3D). There are five common methods proposed in the literature which exhibits good prospects for obtaining various uniform and defect free PNSs. These are named as solvent-induced precipitation, hot injection, template assisted, ligand assisted reprecipitation and emulsion methods $[13,14]$.

In order to determine the structural and optic properties of PNSs, some characterization techniques such as small angle x-ray scattering (SAXS), scanning electron microscope (SEM), absorption and emission spectroscopy have been used extensively. It is crucial to know the shape and optical response of the nanocrystals for the further opto-electronic application of them.

As it is mentioned above, well-defined PNSs were used in many applications such as optical lasing and LEDs. Some of those applications have been investigated intensively by many groups all around the world. Some milestone studies have been presented comparatively.

\section{Synthesis of nano-crystalline perovskites}

There are many proposed methods for synthesis of PNSs by many groups in the literatures. Here we would like to express five of most common and essential methods (Figure 1) [21-23].

\subsection{Solvent-induced precipitation}

The physical properties of perovskite cluster such as size of NPs can be arranged by using long alkyl chain amine derivatives while oleic acid ensures the colloid stability via preventing the aggregation. In order to initiate the solvent-induced precipitation, and obtain colloidal $\mathrm{MAPbBr}_{3} \mathrm{NPs}$, lead bromide $\left(\mathrm{PbBr}_{2}\right)$ and methyl ammonium bromide $\left(\mathrm{CH}_{3} \mathrm{NH}_{3} \mathrm{Br}\right)$ were mixed with Octylammoniumbromide $(\mathrm{OABr})$ in acetone with oleic acid (OAc) and octylamine and the solution were kept at $80^{\circ} \mathrm{C}$. The PLQY of obtained NPSs was about $20 \%$ as well as stable over three months. After this first attempt, PLQY of NPSs was increased up to $83 \%$ by optimization of the molar ratios of starting materials [22, 24].

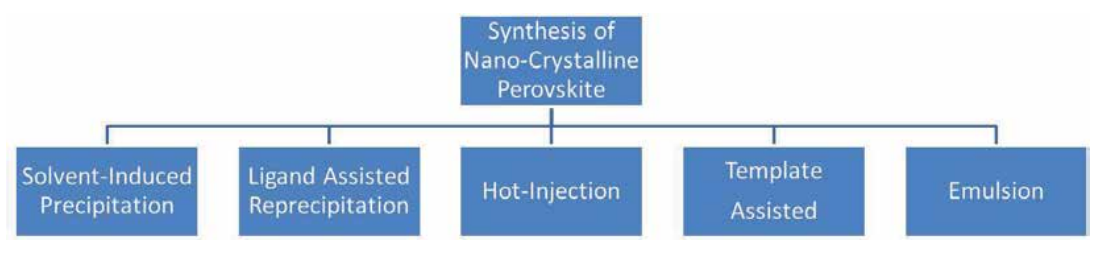

Figure 1.

Common synthesis methods for perovskite nano-crystals. 


\subsection{Ligand assisted reprecipitation (LARP) technique}

In this method, a polar solvent such as dimethylformamide (DMF) which dissolve all starting materials and capping ligands have been used to prepare the precursor solution $[25,26]$. This solution is added dropwise into vigorously stirred toluene which is not a good solvent for starting materials and perovskite crystal. Zhang et al. has demonstrated the synthesis of colour- tuneable PNSs (average particle size of $3.3 \pm 0.7 \mathrm{~nm}$ ) with a PLQYs of 50-70\% (Figure 2) [25]. It was claimed that a slight descending of PLQY was observed by the ascending of the size of the perovskite crystal $(2-8 \mu \mathrm{m}, \mathrm{PLQY}<0.1 \%)$. In the nanometer scales, surface defects of the crystals can easily be passivated by ligands. Thus, most of the photo-generated charges can recombine before there are trapped by defects on the surface. However, due to the less ligand passivation, the number of defects on the bulk perovskite structure's surface, which increased the number of trapped charges, are significantly high, resulting very low PLQY. In another study, well-defined cubic and thermally stable $\mathrm{FAPbX}_{3}$ nanocrystals (about $10 \mathrm{~m}$ ) has been prepared by LARP method. The reported PLQY for the NPS was 75\% [27]. A new procedure, which was used to obtain a core-shell shape by using the LARP approach has been recently demonstrated. With this proposed method, a solutionprocessed, stable core-shell-type Methyl ammonium $\left(\mathrm{MA}^{+}\right)+$Octyl ammonium $\left(\mathrm{OA}^{+}\right)$lead bromide perovskite NPs $(\approx 5-12 \mathrm{~nm})$ with good PLQY was prepared. In addition to this, Core-shell-type NPs was accomplished by systematically changing the molar ratio of capping ligands, $\mathrm{OABr}$, and $\mathrm{MABr}$ without altering total amount of alkylammonium bromide and synthesis conditions. The color tunability of NPs in the blue to green spectral region (438-521 nm), high PLQY, and reasonable stability under ambient condition are credited to the quantum confinement imparted by the crystal engineering associated with core-shell NP formation [28-30].

\subsection{Hot injection method}

The stability of inorganic perovskites is significantly higher than that of hybrid perovskite crystals. By changing the organic cation with an inorganic one (e.g., Cs),

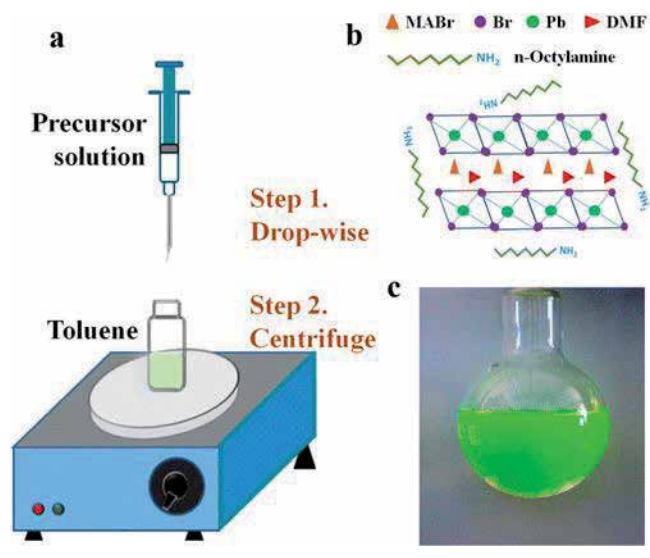

Figure 2.

(a) Schematic illustration of Set up for LARP; (b) starting materials and shape of perovskite nano-crystals; (c) image of typical solution containing $\mathrm{CH}_{3} \mathrm{NH}_{3} \mathrm{PbBr} 3$ nano-structures (reproduced with permission of Ref. [26]). 
chemical and thermal stability of Perovskite material can be increased greatly (Figure 3) [31, 32]. It is possible to obtain various crystal phases by changing the temperature resulting a shifting in the optical responses. The hot injection method is a widespread method for synthesizing inorganic PNSs. In a typical synthesis procedure, a solution of $\mathrm{PbX}_{2}(\mathrm{X}=\mathrm{I} / \mathrm{Br} / \mathrm{Cl})$, in octadecene (ODE) along with oleic acid and oleyl amine is prepared. During stirring, Cs-oleate is injected into that solution quickly under dry condition at $140-200^{\circ} \mathrm{C}$ [32]. To quench the reaction (after 5-10 sec), the reactor is cooled with an ice bath. With this method, it is easy to obtain uniform nanocubes with the size of 4-15 nm edge length representing high PLQY, up to 50-90\% and a very narrow emission band $(12-42 \mathrm{~nm})$ in the visible region $(410-700 \mathrm{~nm})$. As it was mentioned before, the optical properties of the NPSs are related to shape, size,

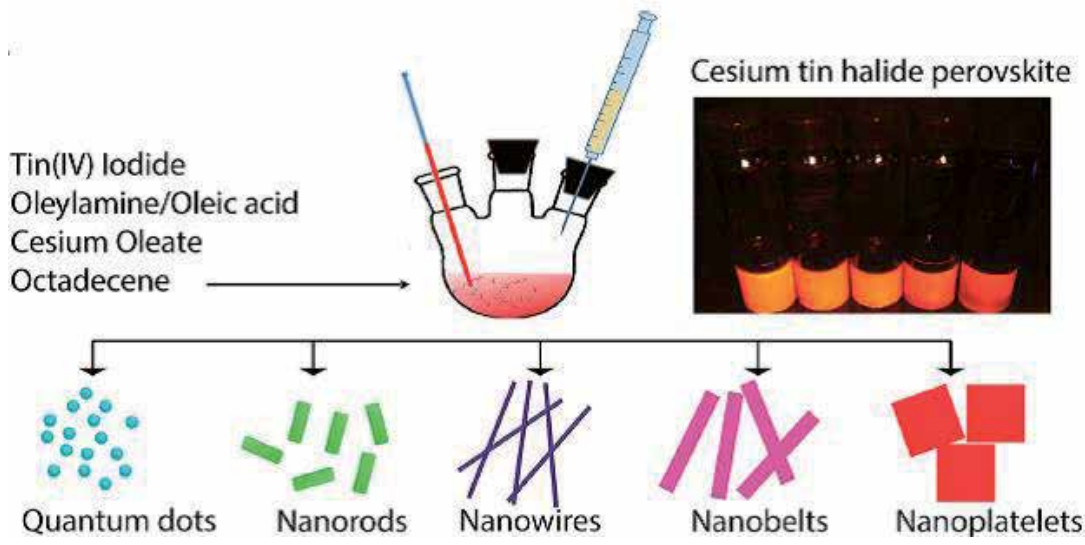

Figure 3.

Schematic illustration of Hot-injection method (left-hand), image of Cs2SnI6 samples under UV-light (righthand) and possible crystal shapes obtained by hot-injection method (reproduced with permission of Ref. [33]).

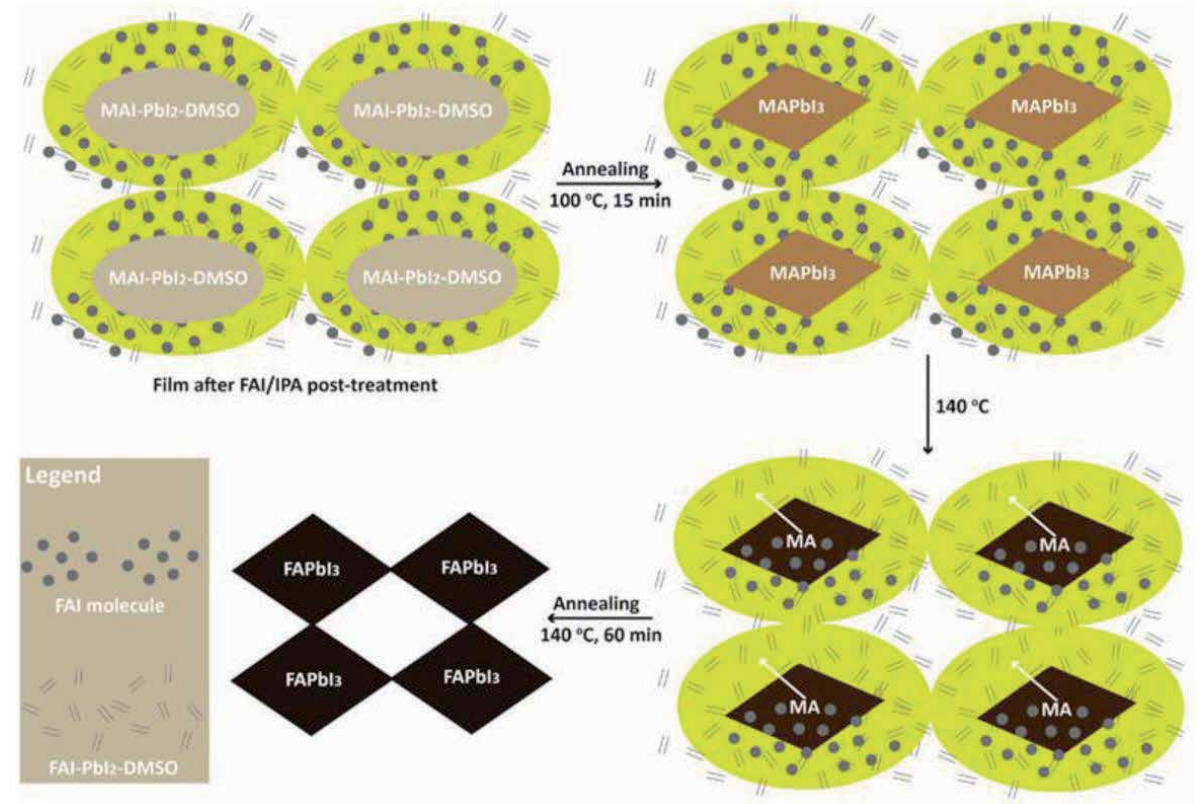

Figure 4.

Schematic illustration of perovskite nano-crystal preparation by the template-assisted method (reproduced with permission of Ref. [36]). 
and surface chemistry of them due to the significant changes in the band structures. Therefore, by the variation of the surfactants, ligands, reaction temperature and time, the perovskite precursor composition can be adjust [33] to realize the formation of nanowires, nanoplatelets, spherical dots, and nanorods (Figure 3) [33-35]. A critical point for the hot injection is the reaction temperature effecting the size of the NPSs. To overcome this disadvantage, new methods have been developed for hybrid perovskites.

\subsection{Template-assisted method}

In this synthesis approach, NP formation is induced by a specific substrate such as mesoporous silica and aluminum oxide film as a template [36-38]. NPSs is obtained on the films which exhibits intensive light. This technique is suitable for formation of mono disperse NPSs with various narrow emissions (full wind of half maximum (FWHM) is less than $40 \mathrm{~nm}$ ) bands from green (FWHM: 22 $\mathrm{nm}$ ) to near infrared (FWHM: $36 \mathrm{~nm}$ ) by template optimization [37-39]. In a recent study, perovskite nanocrystals have been prepared in a nano-porous structure. By this kind of strategies, it is possible to confine PNSs $(<10 \mathrm{~nm})$ without any capping agents (Figure 4). In other words, the emission wavelength of perovskite nanoparticles can be arranged precisely for sophisticated photonic applications such as lasers $[36,37]$.

\subsection{Emulsion method}

In order to control the crystallization of perovskite and obtain uniform NPs, emulsion synthesis method was modified [40]. By using this method, it is possible to tune the size of PNSs under $10 \mathrm{~nm}$ with an PLQY up to 92\% [41]. In this method, an emulsion is prepared with two immiscible solvents. After that, a demulsifier is added into this for initiating solvent mixing and start crystallization (Figure 5). For this procedure, DMF and n-hexane are very good candidates as immiscible solvents while tert-butanol or acetone is used as demulsifier solvent [41]. This method is suitable to obtain PNSs in solid state which can be used in another solvent matrix for an application later. Furthermore, long alkyl ammonium halides are used as capping agent.
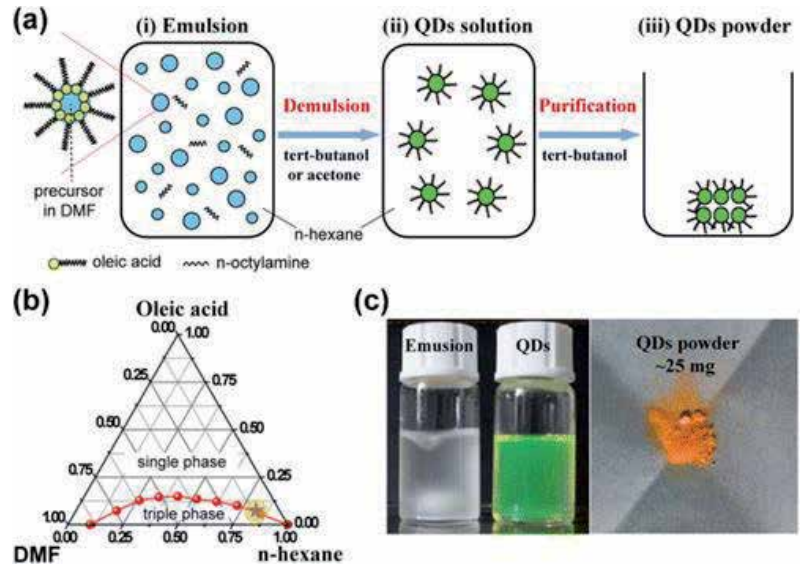

Figure 5.

(a) Schematic illustration of preparation of nano-crystals by emulsion method; (b) ternary phase diagram (DMF/OA/n-hexane); (c) image of a typical $\mathrm{CH}_{3} \mathrm{NH}_{3} \mathrm{PbBr}_{3}$ emulsion, colloidal NP solution and solid-state powder of $\mathrm{CH}_{3} \mathrm{NH}_{3} \mathrm{PbBr} 3 \mathrm{NPs}$ (reproduced with permission of Ref. [41]). 


\section{Form factor of perovskite nano-crystals}

\subsection{Crystal shape of perovskite nano structures}

As it is well known, semiconductor perovskite crystalline structure has a general formula of $\mathrm{ABX}_{3}$ where $\mathrm{A}$ is an organic or inorganic cation, organic methylammonium $\left(\mathrm{MA}^{+}\right)$, formamidinium $\left(\mathrm{FA}^{+}\right)$or inorganic cesium $\left(\mathrm{Cs}^{+}\right)$; $\mathrm{B}$ is a metal cation typically lead $\left(\mathrm{Pb}^{2+}\right)$ or tin cations $\left(\mathrm{Sn}^{2+}\right)$; and $\mathrm{X}$ is a halide anion. Cation $\mathrm{A}$ is slightly larger than centred cation $\mathrm{B}$ having 6 co-ordination number. There is a significant relationship, which is called "Goldschimidt Tolerance Factor" between the size of the ions and the formation and the shape of the crystal structure:

$$
t^{\prime}=\frac{r_{A}+r_{X}}{\sqrt{2}\left(r_{B}+r_{X}\right)}
$$

Where, $r_{A}$ and $r_{B}$ are cationic diameters of $\mathrm{A}$ and $\mathrm{B}$ respectively, and $r_{X}$ is the anionic diameter of halide in $\AA$. When cation $\mathrm{A}$ is too big or cation $\mathrm{B}$ is too small $(>1)$, hexagonal perovskite crystal is formed. If cation $A$ and has the ideal size $(0.9<t<1)$, cubic perovskite crystal is formed. Finally, if cation is too small to fit into the space between metal cations (B) $(0.7<t<0.9)$, orthorhombic perovskite structure is formed [42].

\subsection{Geometry of perovskite nano-crystals}

A typical "bulk" perovskite structure is called three-dimensional (3D) which is usually comply to the $\mathrm{ABX}_{3}$ formula completely [43-45]. In this situation, "3D” refers to the growth in every dimension without any confinement. $\mathrm{X}$ anions are combined through corner sharing to form a 3D network. Beside this, the cation A occupies the site in the middle of eight octahedra, and each element needs to owe the proper valence state to keep a whole charge balance [43]. To obtain nano crystalline perovskites, crystal growth must be restricted with at least one dimension by a capping agent or a matrix. Thus, It is possible to prepare various dimensioned PNSs such as zero, one or two dimension (0D, 1D or 2D respectively) (Figure 6) [1, 46].

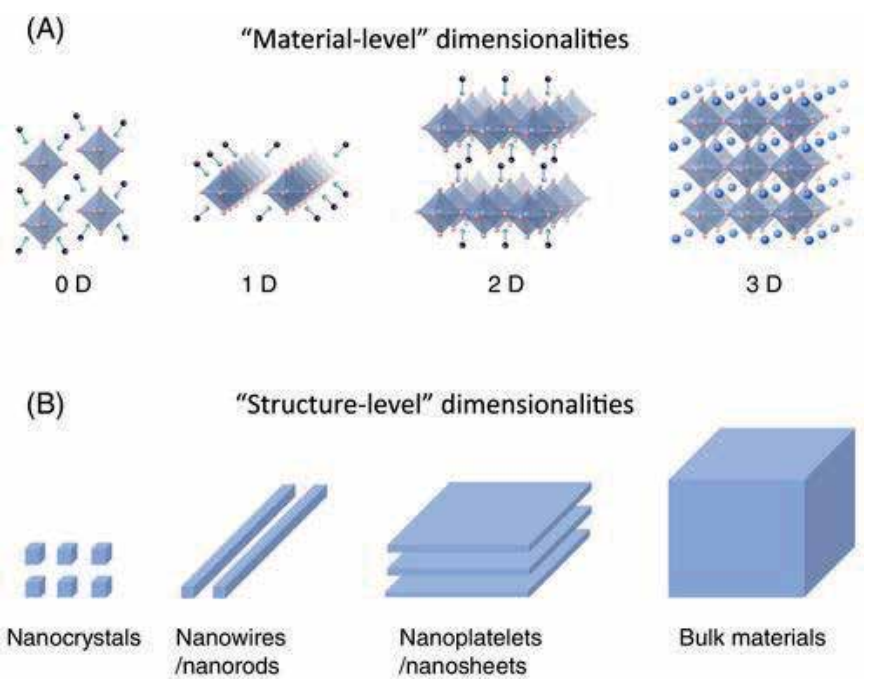

Figure 6.

Schematic illustration of low-dimensional perovskites and $3 D$ perovskite crystal structures (reproduced with permission of Ref. [47]). 


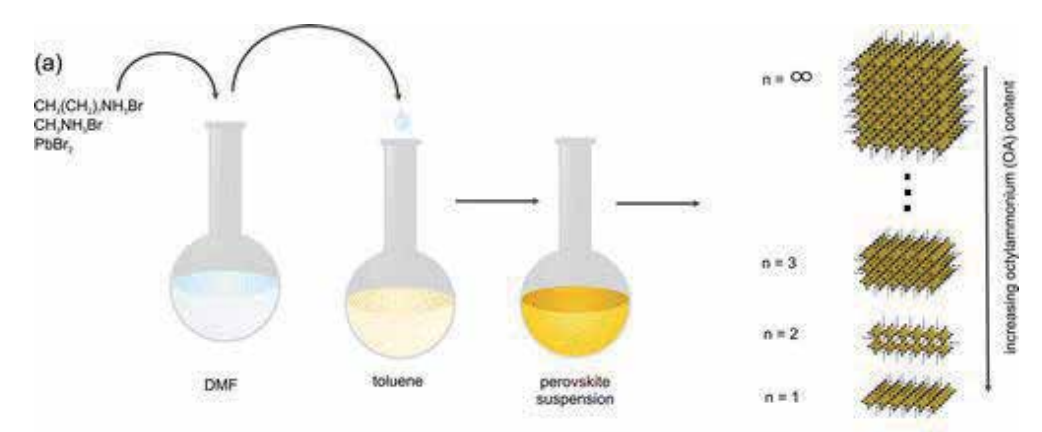

(b)

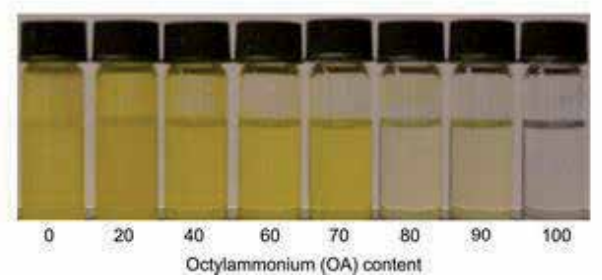

Figure 7.

(a) Schematic illustration of the perovskite nanopelets preparation. (b) Image of OA/MA perovskite suspensions in toluene under ambient light (reproduced with permission of Ref. [49]).

OD PNSs have been synthesized, by LARP [25], hot-injection [32] and template-assisted method [36] by different groups. Crystal growth is prevented in all dimensions by a ligand or a metal-oxide matrix to obtained quantum-dot-like NPs representing good Photoluminescence (PL). Prepared nanostructures have been used in many opto-electronic applications such as solar cells, LEDs and laser.

Perovskite nanowires or nanorods are basically $1 D$ PNSs which represent outstanding anisotropic optical and electrochemical properties with very high PLQFs [41]. 1D PNSs have been proposed by Horvath et al. for the first time in the literature. Nanowires with 50-400 nm wide and $10 \mu \mathrm{m}$ length has been prepared in DMF solution phase. Obtained NPSs have been used in Solar Cells [48], LED [4] and photodetector [8] applications.

Consequently, 2D PNSs are nano pellet or nano sheet shaped materials which consist of several unit cells leading larger binding energy for the exciton and more intense PL. These types of NPs have similar networks with corresponded 3D perovskite, however the general formula of $\mathrm{ABX}_{3}$ is changed when the NP is very thin. Number of sheets in a nano pellet NPS can be determine by using absorption and emission spectroscopy. Differences of Absorption and emission maximum of nanosheets ( $\mathrm{n}=1,2,3$ and 4$)$ are very significant. Maximum absorption peak is red shifted with the increasing of number of unit cell (nano- pellet) (Figure 7).

\section{Applications of perovskite nanoparticles}

\subsection{Optical properties of perovskite nanoparticles}

In recent years, perovskite is a very important milestone in solar cell research, thanks to its perfect exciton and charge carrier properties. This excellent performance has allowed perovskite to be used as outstanding light emitters in Light Emitting Diodes (LEDs) and other optoelectronic applications [4, 7, 25, 50-56]. One of the most attractive features of perovskites is their emissions, which can be easily adjusted in the visible range compared to traditional III-V and II-VI groups. All inorganic-perovskite $\mathrm{ABX}_{3}$ emissions, including quantum dots and nanoplatelets, 
can cover the entire visible area, even close to the infrared or ultraviolet region, by substituting halide elements from chloride to iodine $[26,34,51,57,58]$. Another way to adjust the emission is to insert other organic molecules into it or replace anions/ cations.

\subsection{High quantum efficiency}

Perovskites are considered superior light emitters owing to their large absorption coefficients and high quantum yields $[59,60]$. The high quantum yield generally indicates that most of the absorbed photons are transformed by radiative recombination processes. High quantum yields (90\%) have been reported in inorganic $\mathrm{ABX}_{3}$ and organic-inorganic methyl-ammonium halide perovskite nanocrystals without further surface treatment $[26,34]$. However, the main reason for reduced quantum efficiency in conventional III -V and II -VI groups is that these nanocrystalline structures are often affected by surface defects or donor-receptor levels. In perovskite, where there are very few electrical charge trapping conditions, high quantum efficiency is the result of the formation of a clear band gap that greatly supports exciton radial recombination efficiency [61].

\subsection{Quantum confinement effect}

Optical absorption and emission characteristics of a semiconductor can be adjusted by changing the size of the semiconductor. If the size of such materials is in the nanocrystal range, changes in band gaps can be observed. The reduction of the crystal size causes the quantum capture effect to be observed and the bandwidth to shift to blue. If the semiconductor size is too small to compare with the Bohr radius of excitons, quantum trapping can be seen in the optical properties of the semiconductors. For example, the quantum capture effect is quite evident in completely inorganic $\mathrm{CsPbr}_{3}$ perovskite nanocrystals and in organic-inorganic methyl ammonium lead halide perovskite nanocrystals. This can usually be observed when the nanocrystalline size is comparable to the exciton Bohr radius. Figure $8 \mathbf{a}$ and $\mathbf{b}$ demonstrate that the emission of $\mathrm{Cs} \mathrm{PbBr} 3$ perovskite nanocrystals can actually be adjusted from $2.7 \mathrm{eV}$ to $2.4 \mathrm{eV}$, with a size ranging from $4 \mathrm{~nm}$ to $12 \mathrm{~nm}$, which is compatible with the theoretical calculation [34].

\subsection{Linear absorption and emission}

Many groups have focused on improving the band spacing, excitonic characteristic and optical properties of photoluminescent quantum yields of halide perovskite nanocrystals for optoelectronic applications in recent years [18, 62]. The most interesting of these features is that bandwidth is adjustable. It is possible to adjust the bandwidth by changing the individual components of the metal halide perovskites (MHP). Optical properties of bulk perovskite thin films could be changed across the entire visible spectrum. Thus, it has been shown that the optical properties of $\mathrm{MAPbBr}_{3}$ nanocrystals, which have an emission of approximately 529 $\mathrm{nm}$, can also be altered throughout the entire visible spectrum $[63,64]$. For $\mathrm{CsPb}(\mathrm{X}$ $=\mathrm{Cl}, \mathrm{Br}$ or $\mathrm{I})_{3}$ nanocrystals, using halide components, the emission wavelength is from $410 \mathrm{~nm}(\mathrm{X}=\mathrm{Cl}),(\mathrm{X}=\mathrm{Br})$ to $512 \mathrm{~nm},(\mathrm{X}=\mathrm{I})$ It has been shown that it can be shifted to $685 \mathrm{~nm}$ (Figure 9).

Adjustable optical features of perovskite nanocrystals are based on the electronic structure of these materials (Figure 10). The conduction band consists of external $\mathrm{p}$ orbitals of halid and antibonding orbitals of hybridization of $\mathrm{Pb} 6 \mathrm{p}$ orbitals. The valence band consists of antibonding of the hybridization of $\mathrm{Pb} 6 \mathrm{~s}$ 


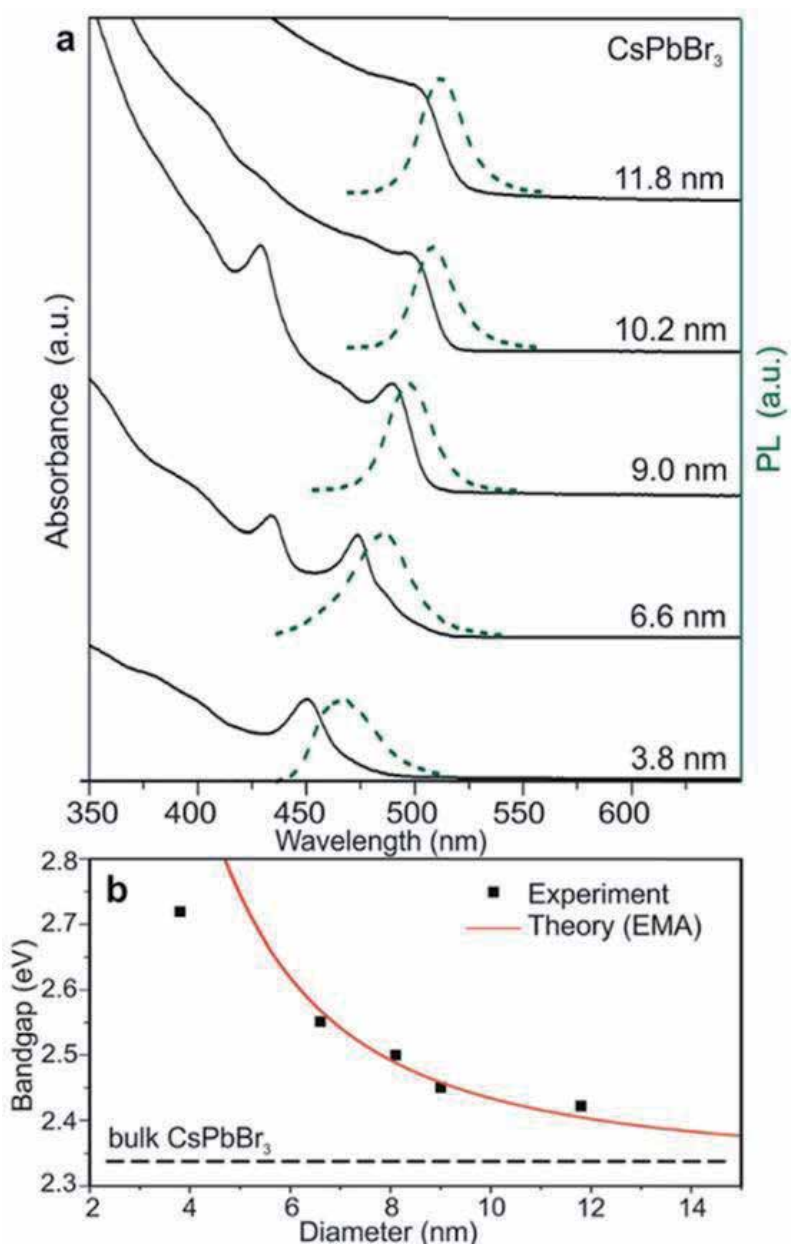

Figure 8.

(a) The emission spectra of $\mathrm{CsPbBr} 3$ NCs Quantum-size effects in the absorption and (b) experimental versus effective mass approximation size (theoretical technique) with respect to the band gap energy range (reproduced with permission of Ref. [34]).

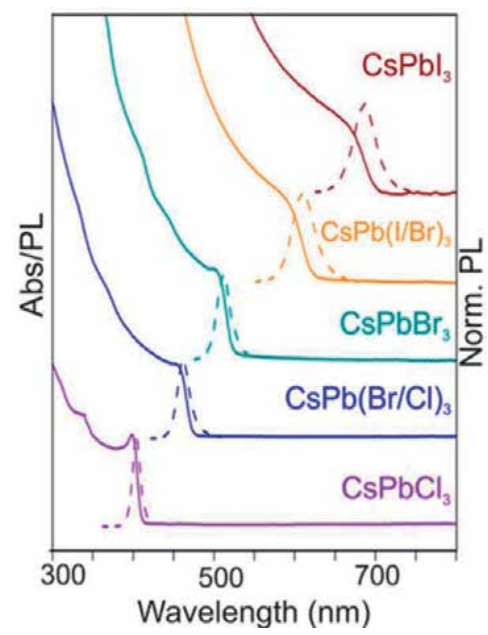

Figure 9.

UV-vis and photoluminescence spectra shows that the band gap could be tuned by controlling of $\mathrm{Cs} P b \mathrm{X}_{3} \mathrm{NCs}$ as a function of halide (reproduced with permission of Ref. [34]). 


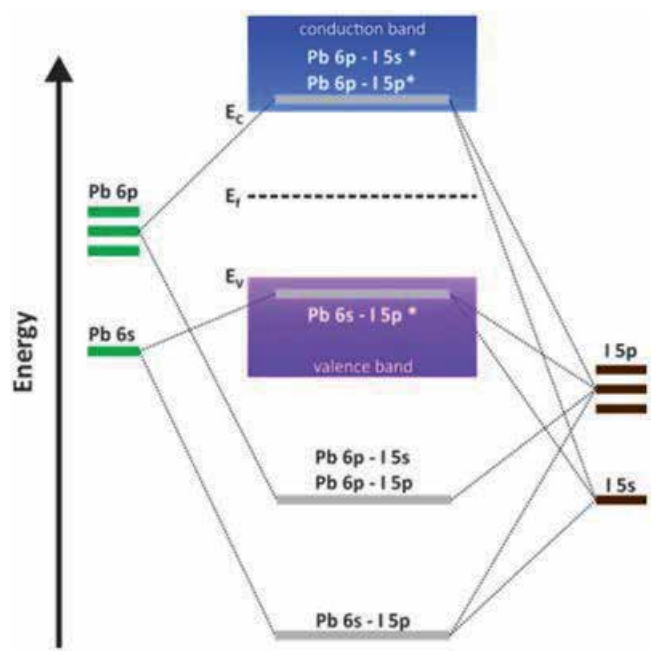

Figure 10.

The energy bands forms in a lead iodide perovskite by the crossing of lead and iodide orbitals (reproduced with permission of Ref. [23]).

and the same halide p-orbitals. The conduction band is generally p-like owing to the high density energy bands lead density contribution [65]. However, as the opposite of this situation, the band gap of gallium arsenide occurs between bonding and antibonding orbitals. As the halide component changes, the valence energy band shifts at the limit value, while only small changes occur in the energy limit value of the transmission band [66]. The cation A does not contribute considerably to the conduction and valence orbitals, but has an important effect on the band gap of perovskite [67]. As a result, emission energies of MA-based perovskite nanocrystals have been demonstrated to range from halide to 407 to $734 \mathrm{~nm}$. It is understood that the FA emissions of nanocrystalline FAs shifted to $408 \mathrm{~nm}$ with $\mathrm{Cl}$, to $535 \mathrm{~nm}$ with $\mathrm{Br}$ and to I and $784 \mathrm{~nm}$, that is, to red [68-70]. In addition, the $\mathrm{B}$ cation has a significant mission in changing the optical properties of metal halide perovskites nanocrystals. As is known, the lead is a harmful element for the nature, instead of using the band [71], the band gap and PL emissions of the metal halide perovskites nanocrystals shifted from $\mathrm{Cl}$ to $443 \mathrm{~nm}$ and from I to $953 \mathrm{~nm}$. The reason is probably a result of the higher electronegativity of $\mathrm{Sn}_{2}$ than $\mathrm{Pb}_{2}$ [72]. But, the stability of $\mathrm{Sn}^{2+}$ and similarly $\mathrm{Ge}^{2+}$ based on perovskite compounds is too weak owing to the reduction of non-interacting electron pair effects corresponding to a decrease in the stability of the divalent oxidation state [73]. As a result, PL emissions or energy band gaps of nanocrystalline structures obtained by various methods depend only on stoichiometry [74]. Stokes shift is an important parameter at the absorption and emission spectra that LHP nanocrystals show typically small, ranging from 20 to $85 \mathrm{meV}$ [75-77]. Stokes shift increases as nanocrystals decrease in size. This is clarified by the creation of a compatible hole state that can be delocalized across the whole nanocrystal [78]. PL line widths of metal halide perovskites are another important point, particularly for LED applications. In fact, the line widths are commonly in the range of 70-110 $\mathrm{meV}$ and have been found to vary significantly with respect to the halide content. In many articles, the halide component greatly varies the PL spectra in terms of wavelength, for instance for $\mathrm{Cl}$-perovskites reaches to 10-12 $\mathrm{nm}$ and for I-perovskites $40 \mathrm{~nm}$. In terms of photoluminescent quantum yields, LHP nanocrystals display high values with the more epitaxial shell range the more chalcogenide QDs without electronic passivation [69]. Some article abot MA based halide perovskites have been reached to $80 \%$ to $95 \%$ for photoluminescent 

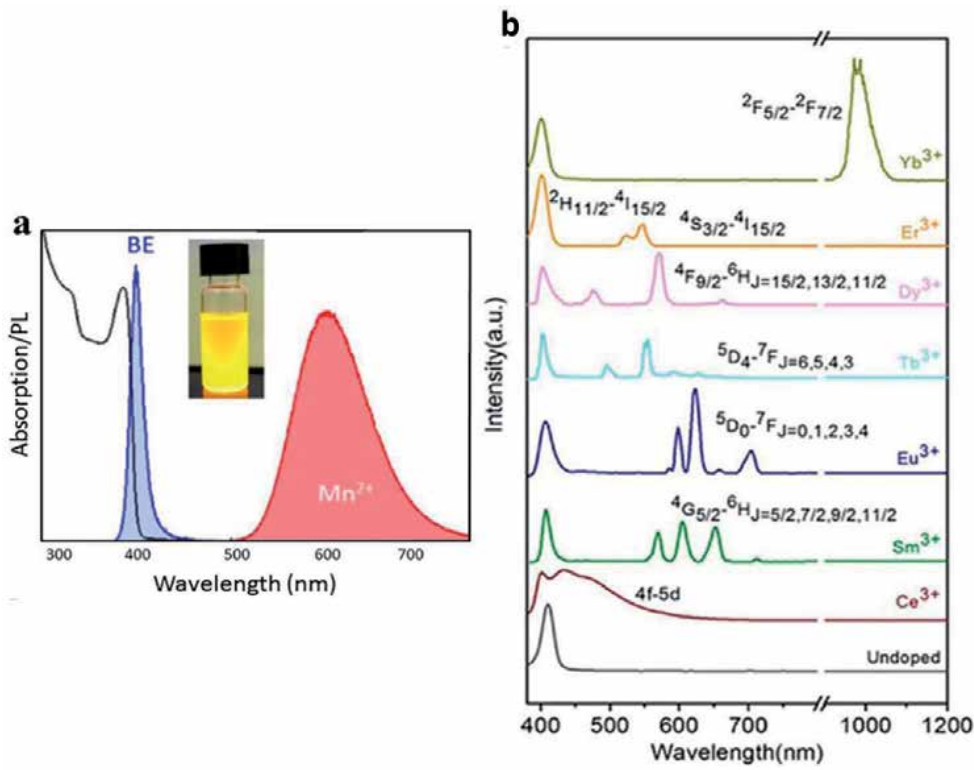

Figure 11.

(a) Photoluminescence emission \& absorption of $\mathrm{Mn}$-doped $\mathrm{CsPbCl} 3 \mathrm{NCs}$, (b) photoluminescence curves of $\mathrm{Cs} \mathrm{PbCl}_{3} \mathrm{NCs}$ doped with different lanthanide ions (reproduced with permission of Refs. [23, 87]).

quantum yields, bromide and iodides, respectively [79-82]. The defect tolerance of halide perovskite gives new properties due to orbital structure and the bandgap that forms between two antibonding orbitals. FA-based nanocrystals also reach to $70-90 \%$ photoluminescent quantum yield. There is alternative method that doping with additional ions to handle the optical emission of LHP nanocrystals. The addition of $\mathrm{Mn}^{2+}$ ions leads to a strong Stokes shift emission by the band gap given by the perovskite matrix and the emission from the atomic states of the $\mathrm{Mn}^{2+}$ ions (Figure 11a) [83]. $\mathrm{Mn}^{2+}$ doping can result in a pair of controllable emissions from both localized $\mathrm{Mn}^{2+}$ states and band gap recombination $[84,85]$. In contrary, $\mathrm{Cs} \mathrm{PbBr}_{3}$ nanocrystals have been shown to cause blue shift of doping, band boundary and PL emission with other divalent cations such as $\mathrm{Sn}^{2+}, \mathrm{Cd}^{2+}$ and $\mathrm{Zn}^{2+}$. In these cases, an important portion (0.2-0.7\%) of the original $\mathrm{Pb}$ ions have been exchanged by new metal cations that produce alloy nanocrystals. $\mathrm{Cs} \mathrm{PbBr} 3$ alloy nanocrystals with $0.2 \%$ content of $\mathrm{AI}^{3+}$ ions have a blue shear PL emission with a centre of $456 \mathrm{~nm}$ and a relatively high $42 \%$ photoluminescence quantum yield [86]. In all these cases, perovskite nanocrystals act as an absorbent host that stimulates dopants through energy transfer. In addition, by selecting specific dopant atoms, the emission wavelengths of the nanocrystals obtained can be easily adjusted. When lanthanide ions are doped, the emission of $\mathrm{CsPCl}_{3}$ nanocubes ranges from $400 \mathrm{~nm}$ to $1000 \mathrm{~nm}$ and their quantum yields are $15 \%$ and $35 \%$, respectively (Figure 11b) [87].

\section{Optoelectronics applications of perovskite}

\subsection{Optical lasing}

High absorption coefficient and strong photoluminescence is the most powerful side of metal halide perovskite. It is possible to obtain a laser with a high quantum efficiency material and a suitable optical band spacing. With the understanding 
that amplified spontaneous emission can be obtained from perovskite material, research has focused on this subject. Especially, laser obtaining studies were carried out by using perovskite crystal. It has also been shown that perovskite nanowires in high crystalline feature can have a Fabry-Perot gap to obtain a laser in the material [88]. By manipulating the content of the halide in the composition of the perovskite lead to control the emission wavelength and obtain the laser in the entire visible spectrum (Figure 12a and b) [57]. Zhu et al. showed that laser exposure in $\mathrm{CH}_{3} \mathrm{NH}_{3}$ $\mathrm{PbX}_{3}$ with an exponentially low current threshold $\left(220 \mathrm{~nJ} \mathrm{~cm}{ }^{-2}\right)$ and a corresponding carrier density as low as $1.5 \times 10^{16} \mathrm{~cm}^{-3}$.

\subsection{Light emitting diodes}

Metal halide perovskite has great potential due to its easy preparation, low cost and high-performance light emitting diodes. Perovskites in LED applications show well a high colour purity, usually 15-25 nm full width half-colour purity for electroluminescence spectra. The point is here that the colour adjustment can cover the entire visible part of the spectrum by changing the content of different halides within the compounds. Therefore, many researchers have achieved high performance in perovskite quantum dot LED because of their quantum dots, strong luminescence, and high external quantum yields (Figure 13a) [25]. The stability problem of organic-inorganic hybrid perovskite can be eliminated by synthesizing the inorganic perovskites (ie $\mathrm{CsPB}_{3}$ ) as quantum dots for LED applications (Figure 13b) [89]. The performance of perovskite nanoplatelets in LED applications was lower than the quantum dots (Figure 13c). As a result, perovskite has a great potential for lighting and display applications as a new generation LED material.

\subsection{Alternative applications}

FETs, photodetectors, and single photon emitters are the other potential optoelectronic application devices. Liu et al. [90] used perovskite nanoplatelets to produce a FET on a $\mathrm{Si} / \mathrm{SiO}_{2}$. For this device, the current-voltage curve showed ohmic contact between the perovskite nanoplatelets and electrodes, and a linear dependency was noted. In that study, they revealed a strong light-material interaction and broadband light harvesting capability of perovskite. Many photodetectors were fabricated based on a horizontal $\mathrm{CH}_{3} \mathrm{NH}_{3} \mathrm{PbI}_{3}$ nanowire array [49]. The
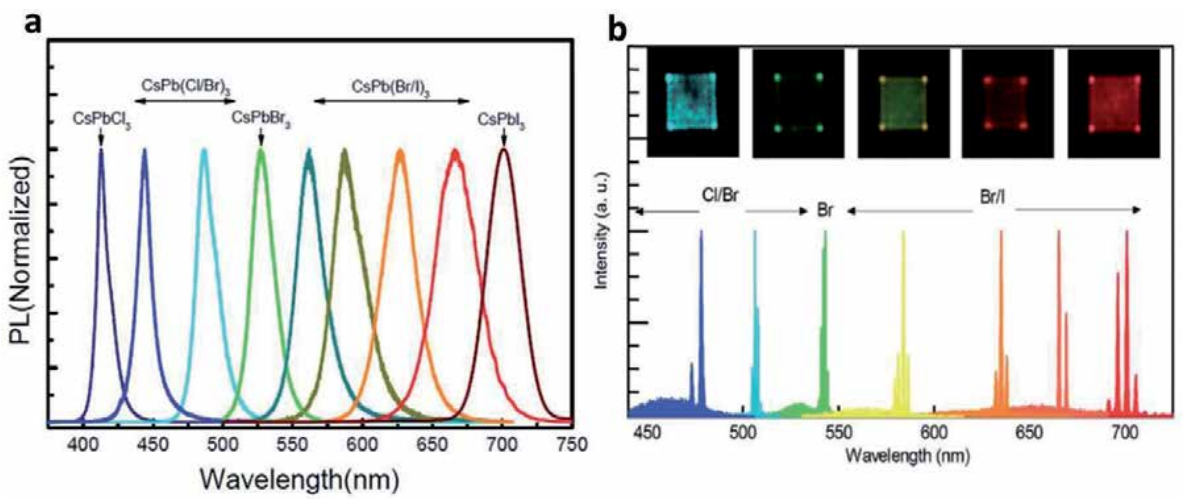

Figure 12.

(a) Photoluminescence spectra of $\mathrm{Cs} P b(X=C l, B r, I) 3$ nanoplatelets and $(b)$ wavelength adjustment of perovskite lasing by controlling the content of halide in $C s P b X_{3}$ perovskite $[6,7,57]$. There is really an extraordinary point about nanowires is that they have very little carrier capture area and the laser quantum efficiency reaches to $100 \%$ (reproduced with permission of Ref. [6]). 


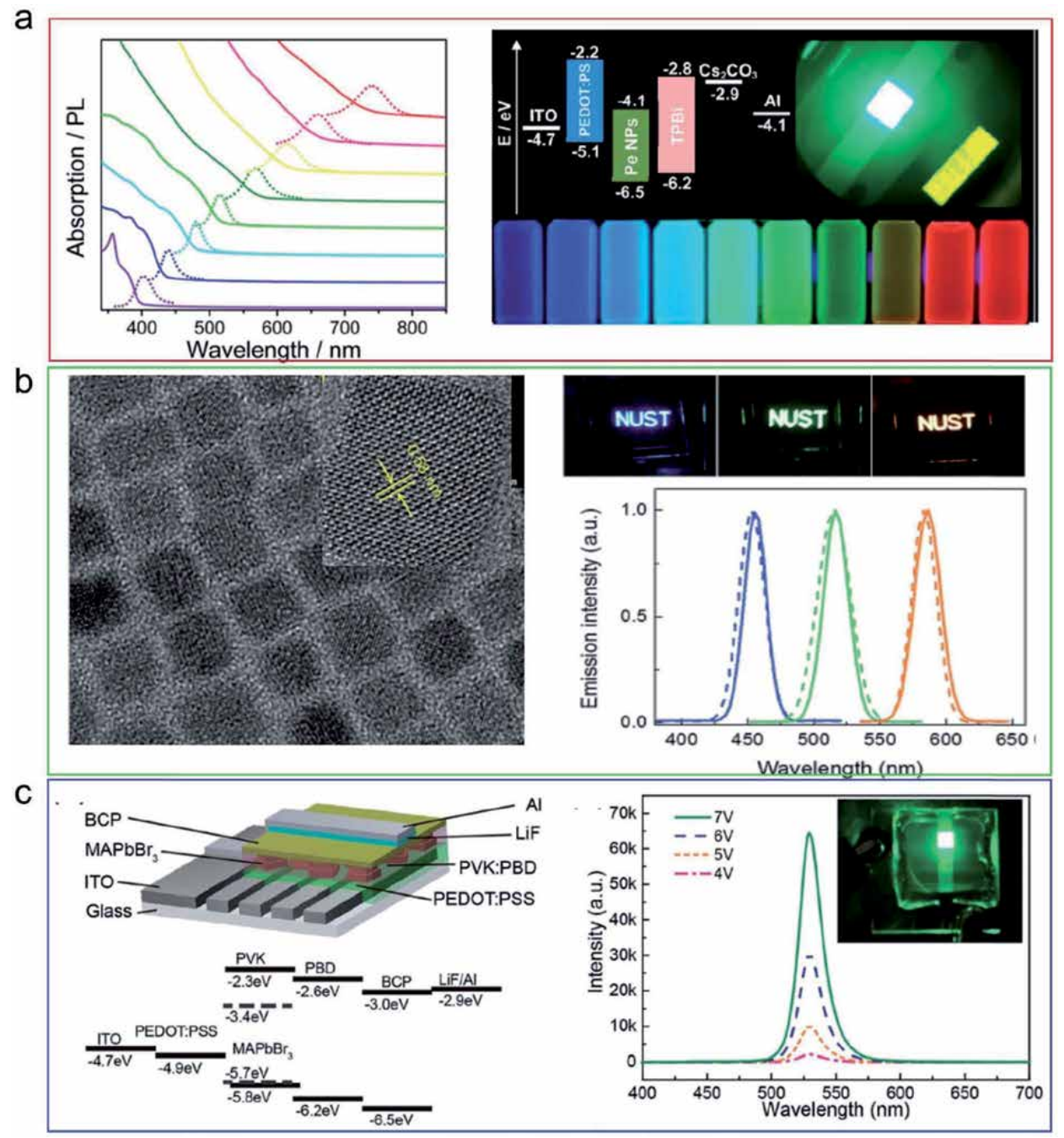

Figure 13.

In LED applications are generally used a low dimensional perovskite. (a) $\mathrm{CH}_{3} \mathrm{NH}_{3} \mathrm{PbX} 3$ quantum dots are applied to fabricate $\mathrm{LED}$, (b) $\mathrm{Cs} P b X_{3}$ quantum dots are used to form $\mathrm{LED}$, (c) $\mathrm{CH}_{3} \mathrm{NH}_{3} \mathrm{PbX} 3$ nanoplatelets are performed to obtain LED (reproduced with permission of Refs. [25, 89, 91]).

response time compared to the obtained photodetectors bulk perovskite and other inorganic nanowire photodetectors is higher in terms of response time of $0.3 \mathrm{~ms}$, $1.3 \mathrm{~A} \mathrm{~W}^{-1}$ response and a detectivity of $2.5 \times 10^{12}$ Jones. Park et al. show a remarkable perovskite nanomaterials application that Quantum dots were used as a single photon emitter at standard conditions. $\mathrm{CsPb}_{3}$ perovskite was used as the leading material to synthesize cubic shapes and quantum dots with an average size of $10 \mathrm{~nm}$. Perovskite quantum dots displayed an excellent photon beam of emitted light and photoluminescence (PL) intensity fluctuations associated with PL life. It is defined that phenomenon as "A-type flashing" that is popular in the quantum dot system.

\section{Conclusion}

In this chapter, preparation methods and applications of 2D perovskite nanoparticles were reviewed. The most crucial points for synthesis method are uniformity 
and form factor of synthesised nanoparticles. Furthermore, structural, optic, and electrochemical properties of $2 \mathrm{D}$ perovskites have been introduced in detail. Due to the low ionic interaction in the crystal structures, organo-halide perovskites exhibit low stability under ambient conditions. However, 2D perovskite nanoparticles still offer a great potential due to the structure-dependent optic and electronic properties.

\section{Author details}

Burak Gultekin $^{1 *}$, Ali Kemal Havare ${ }^{2}$, Shirin Siyahjani ${ }^{1}$, Halil Ibrahim Ciftci $^{3}$ and Mustafa $\mathrm{Can}^{4}$

1 Ege University, Solar Energy Institute, Izmir, Turkey

2 Faculty of Engineering, Electric and Electronics Engineering, Photoelectronic Lab. (PEL), Toros University, Mersin, Turkey

3 Faculty of Life Sciences, Medicinal and Biological Chemistry Science Farm Joint Research Laboratory, Kumamoto University, Kumamoto, Japan

4 Faculty of Engineering and Architecture, Izmir Katip Celebi University, Izmir, Turkey

*Address all correspondence to: burakgultekin@gmail.com

\section{IntechOpen}

(C) 2020 The Author(s). Licensee IntechOpen. This chapter is distributed under the terms of the Creative Commons Attribution License (http://creativecommons.org/licenses/ by/3.0), which permits unrestricted use, distribution, and reproduction in any medium, provided the original work is properly cited. (cc) BY 


\section{References}

[1] Tsai $\mathrm{H}$ et al. High-efficiency twodimensional Ruddlesden-Popper perovskite solar cells. Nature. 2016;536(7616):312-316

[2] Liu M, Johnston MB, Snaith HJ. Efficient planar heterojunction perovskite solar cells by vapour deposition. Nature. 2013;501(7467):395-398

[3] Burschka J et al. Sequential deposition as a route to high-performance perovskite-sensitized solar cells. Nature. 2013;499(7458):316-319

[4] Yuan M et al. Perovskite energy funnels for efficient light-emitting diodes. Nature nanotechnology. 2016;11(10):872-877

[5] Tan Z-K et al. Bright light-emitting diodes based on organometal halide perovskite. Nature nanotechnology. 2014;9(9):687-692

[6] Zhu $\mathrm{H}$ et al. Lead halide perovskite nanowire lasers with low lasing thresholds and high quality factors. Nature materials. 2015;14(6):636-642

[7] Zhang Q et al. Room-temperature near-infrared high-Q perovskite whispering-gallery planar nanolasers. Nano letters. 2014;14(10):5995-6001

[8] Miao J et al. Highly Sensitive Organic Photodetectors with Tunable Spectral Response under Bi-Directional Bias. Advanced Optical Materials. 2016;4(11):1711-1717

[9] Miao J, Zhang F. Recent progress on highly sensitive perouskite photodetectors. Journal of Materials Chemistry C. 2019;7(7):1741-1791

[10] Miao J et al. Photomultiplication type organic photodetectors with broadband and narrowband response ability. Advanced Optical Materials. 2018;6(8):1800001
[11] Li L et al. Achieving EQE of 16,700\% in P3HT: PC 71 BM based photodetectors by trap-assisted photomultiplication. Scientific reports. 2015;5(1):1-7

[12] Li L et al. Revealing the working mechanism of polymer photodetectors with ultra-high external quantum efficiency. Physical Chemistry Chemical Physics. 2015;17(45):30712-30720

[13] Jung S et al. Enhancement of Photoluminescence Quantum Yield and Stability in CsPbBr3 Perovskite Quantum Dots by Trivalent Doping. Nanomaterials. 2020;10(4):710

[14] Zhang D et al. Increasing Photoluminescence Quantum Yield by Nanophotonic Design of Quantum-Confined Halide Perovskite Nanowire Arrays. Nano letters. 2019;19(5):2850-2857

[15] Khan $\mathrm{Y}$ et al. Waterproof perovskites: high fluorescence quantum yield and stability from a methylammonium lead bromide/formate mixture in water. Journal of Materials Chemistry C. 2020

[16] Dong, Q., et al., Operational stability of perovskite light emitting diodes. Journal of Physics: Materials, 2020. 3(1): p. 012002.

[17] Stylianakis MM et al. Inorganic and hybrid perovskite based laser devices: a review. Materials. 2019;12(6):859

[18] Schmidt LC et al. Nontemplate synthesis of $\mathrm{CH} 3 \mathrm{NH} 3 \mathrm{PbBr} 3$ perovskite nanoparticles. Journal of the American Chemical Society. 2014;136(3):850-853

[19] Chen D, Chen X. Luminescent perovskite quantum dots: synthesis, microstructures, optical properties and applications. Journal of Materials Chemistry C. 2019;7(6):1413-1446

[20] Zhang C, Kuang DB, Wu WQ. A Review of Diverse Halide Perovskite 
Morphologies for Efficient Optoelectronic Applications. Small Methods. 2020;4(2):1900662

[21] Ha S-T et al. Metal halide perovskite nanomaterials: synthesis and applications. Chemical science. 2017;8(4):2522-2536

[22] Gopinathan N et al. Solvents driven structural, morphological, optical and dielectric properties of lead free perouskite $\mathrm{CH} 3 \mathrm{NH} 3 \mathrm{SnCl} 3$ for optoelectronic applications: Experimental and DFT study. Materials Research Express. 2020

[23] Shamsi J et al. Metal halide perovskite nanocrystals: synthesis, post-synthesis modifications, and their optical properties. Chemical reviews. 2019;119(5):3296-3348

[24] Shen D et al. Understanding the solvent-assisted crystallization mechanism inherent in efficient organicinorganic halide perovskite solar cells. Journal of Materials Chemistry A. 2014;2(48):20454-20461

[25] Xing J et al. High-efficiency lightemitting diodes of organometal halide perovskite amorphous nanoparticles. ACS nano. 2016;10(7):6623-6630

[26] Zhang F et al. Brightly luminescent and color-tunable colloidal $\mathrm{CH} 3 \mathrm{NH} 3 \mathrm{PbX} 3$ $(X=B r, I, C l)$ quantum dots: potential alternatives for display technology. ACS nano. 2015;9(4):4533-4542

[27] Pathak S et al. Perovskite crystals for tunable white light emission. Chemistry of Materials. 2015;27(23):8066-8075

[28] Tyagi P, Arveson SM, Tisdale WA. Colloidal organohalide perovskite nanoplatelets exhibiting quantum confinement. The journal of physical chemistry letters. 2015;6(10):1911-1916

[29] Hassan Y et al. Structure-Tuned Lead Halide Perovskite Nanocrystals. Advanced materials. 2016;28(3):566-573
[30] Luo B et al. Synthesis, optical properties, and exciton dynamics of organolead bromide perovskite nanocrystals. The Journal of Physical Chemistry C. 2015;119(47):26672-26682

[31] Wang Y et al. Thermodynamically stabilized $\beta$-CsPbI3-based perovskite solar cells with efficiencies $>18 \%$. Science. 2019;365(6453):591-595

[32] Shi J et al. Efficient and stable CsPbI 3 perovskite quantum dots enabled by in situ ytterbium doping for photovoltaic applications. Journal of Materials Chemistry A. 2019;7(36):20936-20944

[33] Wang A et al. Controlled synthesis of lead-free and stable perovskite derivative Cs2SnI6 nanocrystals via a facile hotinjection process. Chemistry of Materials. 2016;28(22):8132-8140

[34] Protesescu L et al. Nanocrystals of cesium lead halide perovskites ( $\mathrm{Cs} P b \mathrm{X}$, $X=C l, B r$, and $I$ ): novel optoelectronic materials showing bright emission with wide color gamut. Nano letters. 2015;15(6):3692-3696

[35] Lim S-C et al. Binary halide, ternary perovskite-like, and perovskite-derivative nanostructures: hot injection synthesis and optical and photocatalytic properties. Nanoscale. 2017;9(11):3747-3751

[36] Shi, P., et al., Template-Assisted Formation of High-Quality $\alpha$-Phase HC (NH2) 2PbI3 Perovskite Solar Cells. Advanced Science, 2019. 6(21): p. 1901591.

[37] Ashley MJ et al. Templated synthesis of uniform perovskite nanowire arrays. Journal of the American Chemical Society. 2016;138(32):10096-10099

[38] Malgras V et al. Hybrid methylammonium lead halide perovskite nanocrystals confined in gyroidal silica templates. Chemical Communications. 2017;53(15):2359-2362 
[39] Ananthakumar S, Babu SM.

Progress on synthesis and applications of hybrid perovskite semiconductor nanomaterials - A review. Synthetic Metals. 2018;246:64-95

[40] Pileni M-P. The role of soft colloidal templates in controlling the size and shape of inorganic nanocrystals. Nature materials. 2003;2(3):145-150

[41] Huang $\mathrm{H}$ et al. Emulsion synthesis of size-tunable $\mathrm{CH} 3 \mathrm{NH} 3 \mathrm{PbBr} 3$ quantum dots: an alternative route toward efficient light-emitting diodes. ACS applied materials \& interfaces. 2015;7(51):28128-28133

[42] Wali Q et al. Advances in stability of perovskite solar cells. Organic Electronics. 2020;78:105590

[43] Wang, R., et al., A review of perovskites solar cell stability. Advanced Functional Materials, 2019. 29(47): p. 1808843.

[44] Heo JH et al. Efficient inorganicorganic hybrid heterojunction solar cells containing perovskite compound and polymeric hole conductors. Nature photonics. 2013;7(6):486

[45] Etgar L. The merit of perovskite's dimensionality; can this replace the 3D halide perovskite? Energy \& Environmental Science.

2018;11(2):234-242

[46] Ma C et al. High performance lowdimensional perouskite solar cells based on a one dimensional lead iodide perovskite. Journal of Materials Chemistry A. 2019;7(15):8811-8817

[47] Zhu P, Zhu J. Low-dimensional metal halide perovskites and related optoelectronic applications. InfoMat. 2020;2(2):341-378

[48] Kulkarni SA et al. Perovskite Nanoparticles: Synthesis, Properties, and Novel Applications in Photovoltaics and LEDs. Small Methods. 2019;3(1):1800231

[49] Sichert JA et al. Quantum size effect in organometal halide perovskite nanoplatelets. Nano letters. 2015;15(10):6521-6527

[50] Wang J et al. Interfacial control toward efficient and lowvoltage perovskite light-emitting diodes. Advanced Materials. 2015;27(14):2311-2316

[51] Hong WL et al. Efficient lowtemperature solution-processed lead-free perovskite infrared lightemitting diodes. Advanced Materials. 2016;28(36):8029-8036

[52] Bade SGR et al. Fully printed halide perovskite light-emitting diodes with silver nanowire electrodes. ACS nano. 2016;10(2):1795-1801

[53] Li G et al. Highly efficient perovskite nanocrystal light-emitting diodes enabled by a universal crosslinking method. Advanced Materials. 2016;28(18):3528-3534

[54] Veldhuis SA et al. Perovskite materials for light-emitting diodes and lasers. Advanced Materials. 2016;28(32):6804-6834

[55] Liang D et al. Color-pure violet-lightemitting diodes based on layered lead halide perouskite nanoplates. ACS nano. 2016;10(7):6897-6904

[56] Yantara N et al. Inorganic halide perouskites for efficient light-emitting diodes. The journal of physical chemistry letters. 2015;6(21):4360-4364

[57] Zhang Q et al. High-quality whispering-gallery-mode lasing from cesium lead halide perovskite nanoplatelets. Advanced Functional Materials. 2016;26(34):6238-6245 
[58] Fu Y et al. Nanowire lasers of formamidinium lead halide perovskites and their stabilized alloys with improved stability. Nano letters. 2016;16(2):1000-1008

[59] Nedelcu G et al. Fast anion-exchange in highly luminescent nanocrystals of cesium lead halide perouskites ( $C s P b X 3, X=C l, B r, I)$. Nano letters. 2015;15(8):5635-5640

[60] Akkerman QA et al. Tuning the optical properties of cesium lead halide perovskite nanocrystals by anion exchange reactions. Journal of the American Chemical Society. 2015;137(32):10276-10281

[61] Wu K et al. Ultrafast interfacial electron and hole transfer from $\mathrm{CsPbBr} 3$ perovskite quantum dots. Journal of the American Chemical Society. 2015;137(40):12792-12795

[62] Huang $\mathrm{H}$ et al. Colloidal lead halide perovskite nanocrystals: synthesis, optical properties and applications. NPG Asia Materials. 2016;8(11):e328-e328

[63] Noh JH et al. Chemical management for colorful, efficient, and stable inorganic-organic hybrid nanostructured solar cells. Nano letters. 2013;13(4):1764-1769

[64] Tanaka $\mathrm{K}$ et al. Comparative study on the excitons in lead-halidebased perovskite-type crystals $\mathrm{CH} 3 \mathrm{NH} 3 \mathrm{PbBr} 3 \mathrm{CH} 3 \mathrm{NH} 3 \mathrm{PbI} 3$. Solid state communications. 2003;127(9-10):619-623

[65] Umebayashi T et al. Electronic structures of lead iodide based lowdimensional crystals. Physical Review B. 2003;67(15):155405

[66] Schulz P et al. Interface energetics in organo-metal halide perovskite-based photovoltaic cells. Energy \& Environmental Science. 2014;7(4):1377-1381
[67] Eperon GE et al. Formamidinium lead trihalide: a broadly tunable perovskite for efficient planar heterojunction solar cells. Energy \& Environmental Science. 2014;7(3):982-988

[68] Protesescu L et al. Monodisperse formamidinium lead bromide nanocrystals with bright and stable green photoluminescence. Journal of the American Chemical Society. 2016;138(43):14202-14205

[69] Lignos I et al. Unveiling the shape evolution and halide-ion-segregation in blue-emitting formamidinium lead halide perovskite nanocrystals using an automated microfluidic platform. Nano letters. 2018;18(2):1246-1252

[70] Minh DN et al. Roomtemperature synthesis of widely tunable formamidinium lead halide perovskite nanocrystals. Chemistry of Materials. 2017;29(13):5713-5719

[71] Hao F et al. Lead-free solid-state organic-inorganic halide perovskite solar cells. Nature photonics. 2014;8(6):489

[72] Jellicoe TC et al. Synthesis and optical properties of lead-free cesium tin halide perovskite nanocrystals. Journal of the American Chemical Society. 2016;138(9):2941-2944

[73] Chen Q et al. Under the spotlight: The organic-inorganic hybrid halide perovskite for optoelectronic applications. Nano Today. 2015;10(3):355-396

[74] Zhang D et al. Synthesis of composition tunable and highly luminescent cesium lead halide nanowires through anion-exchange reactions. Journal of the American Chemical Society. 2016;138(23):7236-7239

[75] Tong Y et al. From precursor powders to $\mathrm{CsPbX} 3$ perovskite nanowires: onepot synthesis, growth mechanism, and oriented self-assembly. Angewandte 
Chemie International Edition. 2017;56(44):13887-13892

[76] Brennan MC et al. Origin of the size-dependent stokes shift in $\mathrm{Cs} \mathrm{PbBr} 3$ perovskite nanocrystals. Journal of the American Chemical Society. 2017;139(35):12201-12208

[77] Brennan MC, Zinna J, Kuno M. Existence of a size-dependent Stokes shift in $\mathrm{Cs} \mathrm{PbBr} 3$ perovskite nanocrystals. ACS Energy Letters. 2017;2(7):1487-1488

[78] Huang G et al. Postsynthetic Doping of MnCl2 Molecules into Preformed $\mathrm{Cs} \mathrm{PbBr} 3$ Perovskite Nanocrystals via a Halide Exchange-Driven Cation Exchange. Advanced materials. 2017;29(29):1700095

[79] Gonzalez-Carrero S et al. The luminescence of $\mathrm{CH} 3 \mathrm{NH} 3 \mathrm{PbBr} 3$ perovskite nanoparticles crests the summit and their photostability under wet conditions is enhanced. Small. 2016;12(38):5245-5250

[80] Liu F et al. Highly luminescent phase-stable CsPbI3 perovskite quantum dots achieving near 100\% absolute photoluminescence quantum yield. ACS nano. 2017;11(10):10373-10383

[81] Tong Y et al. Highly luminescent cesium lead halide perovskite nanocrystals with tunable composition and thickness by ultrasonication. Angewandte Chemie International Edition. 2016;55(44):13887-13892

[82] Koscher BA et al. Essentially trapfree $\mathrm{CsPbBr} 3$ colloidal nanocrystals by postsynthetic thiocyanate surface treatment. Journal of the American Chemical Society. 2017;139(19):6566-6569

[83] Meinardi F et al. Doped halide perovskite nanocrystals for reabsorptionfree luminescent solar concentrators. ACS Energy Letters. 2017;2(10):2368-2377
[84] Giansante C, Infante I. Surface traps in colloidal quantum dots: a combined experimental and theoretical perspective. The journal of physical chemistry letters. 2017;8(20):5209-5215

[85] He M et al. Mn-Doped cesium lead halide perovskite nanocrystals with dual-color emission for WLED. Dyes and Pigments. 2018;152:146-154

[86] Liu M et al. Aluminum-Doped Cesium Lead Bromide Perovskite Nanocrystals with Stable Blue Photoluminescence Used for Display Backlight. Advanced Science. 2017;4(11):1700335

[87] Pan G et al. Doping lanthanide into perovskite nanocrystals: highly improved and expanded optical properties. Nano letters. 2017;17(12):8005-8011

[88] Xing J et al. Vapor phase synthesis of organometal halide perovskite nanowires for tunable room-temperature nanolasers. Nano letters. 2015;15(7):4571-4577

[89] Song J et al. Quantum dot lightemitting diodes based on inorganic perovskite cesium lead halides (CsPbX3). Advanced materials. 2015;27(44):7162-7167

[90] Liu J et al. Two-dimensional CH3NH3PbI3 perovskite: Synthesis and optoelectronic application. ACS nano. 2016;10(3):3536-3542

[91] Ling Y et al. Bright light-emitting diodes based on organometal halide perovskite nanoplatelets. Advanced materials. 2016;28(2):305-311 

Section 2

Perovskite Solar Cells 



\title{
Organic Inorganic Perovskites: A Low-Cost-Efficient Photovoltaic Material
}

\author{
Madeeha Aslam, Tahira Mahmood and Abdul Naeem
}

\begin{abstract}
Organic-inorganic perovskite materials, due to the simultaneous possession of various properties like optical, electronic and magnetic beside with their structural tunability and good processability, has concerned the attention of researchers from the field of science and technology since long back. Recently, the emergence of efficient solar cells based on organic-inorganic perovskite absorbers promises to alter the fields of thin film, dye-sensitized and organic solar cells. Solution processed photovoltaics based on organic-inorganic perovskite absorbers $\mathrm{CH}_{3} \mathrm{NH}_{3} \mathrm{PbI}_{3}$ have attained efficiencies of over $25 \%$. The increase in popularity and considerable enhancement in the efficiency of perovskites since their discovery in 2009 is determined by over 6000 publications in 2018. However, although there are broad development prospects for perovskite solar cells (PSCs), but the use of $\mathrm{CH}_{3} \mathrm{NH}_{3} \mathrm{PbI}_{3}$ results in lead toxicity and instability which limit their application. Therefore, the development of environmental-friendly, stable and efficient perovskite materials for future photovoltaic applications has long-term practical significance, which can eventually be commercialized.
\end{abstract}

Keywords: organic-inorganic, Perovskite, photovoltaic material, optical properties, solar cells

\section{Introduction}

Organic-inorganic perovskites materials have emerged as a promising material for high-efficiency nanostructured devices such as light-emitting diodes (LEDs), detectors, field-effect transistors, and photovoltaic devices etc. [1, 2]. Organicinorganic perovskites have attracted extensive attention due to their promising optical and electronic properties, excellent crystallinity, adjustable bandgap, long charge diffusion length, electroluminescence, and conductivity $[3,4]$. As the most fascinating new-generation photovoltaic materials, organic-inorganic perovskite due to their facile synthesis, low temperature deposition, and capability to make flexible devices has been considered as a vigorous component of the efficient, low-cost, lightweight and flexible Perovskite solar cells [3, 4]. Perovskite solar cells (PSCs) have rapidly become the leading edge of third generation $3 \mathrm{G}$ photovoltaic technologies $[5,6]$. PSCs based on the organic inorganic perovskite materials have fascinated great consideration, with their power conversion efficiencies (PCEs) reaching $25.2 \%$ certified $[7,8]$. Over the past several months, it has been observed 
a surprising revolution and rapid progress in the field of emerging photovoltaic, with the understanding of highly efficient solar cells based on organic inorganic perovskite materials. This perovskite technology is now well-matched with the $1 \mathrm{G}$ and $2 \mathrm{G}$ technologies and is thus probably be embraced by the conventional photovoltaic community and industry $[9,10]$.

\subsection{Organic inorganic perovskite materials in solar cells}

The advent of organic inorganic perovskite based solar cells has resulted in rapid growth in photovoltaic history. Organic inorganic perovskite materials have recently, fascinated greater attention due to its outstanding light-harvesting features [7].

Organic-inorganic perovskite absorbers have appeared in the field of DSSCs since 2009. The first perovskite-sensitized DSSCs were developed by Kojima et al. [11] which obtained PCE of $3.13 \%$ using liquid electrolytes. However, continuous irradiation produced a photocurrent decay in an open cell when exposed to air. Later, the electron transporting layer (Titania) surface and perovskite processing were optimized, and in 2011, Im et al. [12] developed first stable PSC, using $\mathrm{CH}_{3} \mathrm{NH}_{3} \mathrm{PbI}_{3}$-based iodide liquid electrolyte offered a PCE of $6.5 \%$. However, the perovskite nanocrystals dissolved in the liquid iodide electrolyte solution, and the cell degraded within 10 minutes. To avoid the problem of perovskite dissolution in an electrolytic solution, the liquid electrolyte was replaced by a solid in 2012, and a PCE of $9 \%$ was achieved showing good stability up to $500 \mathrm{~h}$ without significant losses $[13,14]$. Afterward, $\mathrm{Al}_{2} \mathrm{O}_{3}$ an insulating network was used to substitute conducting nano porous $\mathrm{TiO}_{2}$. By using mixed $\mathrm{MAPbI}_{3-\mathrm{x}} \mathrm{Cl}_{\mathrm{X}}$ as the sensitizer, an enhanced open-circuit voltage $\left(\mathrm{V}_{\mathrm{OC}}\right)$ and PCE (10.9\%) was achieved [15]. In 2013, a successive deposition method for the perovskite layer within the porous metal oxide film was developed. The fabrication technique for solid-state mesoscopic solar cells greatly improved the reproducibility of cell performance and produced a high PCE of 15\%. Many PSC devices are now attaining PCE > 20\% since 2015 and 25\% in 2019 [4, 16]. National Renewable Energy Laboratory (NREL), on 3rd August 2019 declared a new world record PCE of $25.2 \%$ for PSCs. This PCE value is improved up to $\sim 28 \%$ for perovskite-silicon tandem structures [4].

\subsection{Device architecture of perovskite solar cells}

PSC consists of a perovskite absorbing material sandwiched between electron transporting layer (ETL) and hole-transporting layer (HTL) along with the transparent conducting oxide substrate (FTO) and a top electrode such as gold, silver [17]. In PSCs, the effective charge separation and the light harvesting efficiency are significantly affected by the properties like particle size, porosity, surface area, surface morphology, band gap, thickness of semiconductor materials, and the nature of organometal halide perovskites [18].

The primary function of an ETL is to extract a photo generated electron from perovskite and then transfer to electrodes. The basic criterion for an ideal ETL is high optical transmittance, excellent electron mobility, high conductivity, and an appropriate work function. ETL also performs as hole-blocking layer (HBL) [19]. The configuration and the choice of ETL are essential factors to understand the electronic mechanisms in PSCs, which control processes such as carrier separation, extraction, transport, and the recombination. Hence, the configuration of device structure is critical to alter different materials for ETL, electrode contacts, and the barrier layer of insight these processes and mechanisms [18]. $\mathrm{TiO}_{2}$, which has a wide band gap, has been extensively studied as an efficient electron transport material 
(ETM). Moreover, $\mathrm{ZnO}$ and other $n$-type semiconductors such as $\mathrm{SnO}_{2}, \mathrm{Nb}_{2} \mathrm{O}_{5}$ and $\mathrm{BaSnO}_{3}$ are frequently used as ETMs and are used in flexible perovskite solar cells $[20,21]$.

The HTL lies in the heart i.e. in between the metal electrode and perovskite of device. It plays a central-role in the PSC and extracts holes from the perovskite and transfer them to top-electrode. It avoids the direct contact of perovskite and top electrode [22]. For efficient hole transport, the highest occupied molecular orbit (HOMO) must match the valence band (VB) of perovskite materials. According to the chemical composition, HTMs in PSCs can be divided into two types: organic and inorganic HTMs. Spiro-OMeTAD is the most used organic HTM, which displays good penetration in perovskite and is an appropriate match with the VB energy of perovskite, though its hole mobility is not as superior as that of other organic HTMs. $[21,23]$. Hence, in order to improve the hole mobility, polymers are doped with $p$-type (i.e., cobalt or Lithium salts) or some additives (i.e., bis (trifluoromethane) sulfonimide lithium, LiTFSI, and 4-tert-butyl pyridine, TBP) [21, 24]. Other organic materials reported as HTMs in PSCs are PTAA, PEDOT:PSS, $\mathrm{P}_{3} \mathrm{HT}$ etc. [25]. Inorganic $p$-type semiconductor materials, due to their advantages such as high hole mobility, wide band gap, and easy solvent treatment process as compared with organic HTMs exhibit the possibility to replace organic HTMs. The reported inorganic HTMs for PSCs are $\mathrm{NiO}, \mathrm{CuSCN}, \mathrm{CuI}, \mathrm{CsSnI}_{3}$ etc. [21, 25].

A PSC includes an organic-inorganic perovskite material as the light-harvesting active layer. Amongst the component's PSCs, perovskite materials perform a key role. Perovskite is comprised of earth abundant and inexpensive materials. It is processed at lower temperature rather via the printing techniques [26]. The organic-inorganic perovskites can exhibit appropriately good ambipolar charge transport and the primary functions of photovoltaic operation comprising light absorption, generation of charges, and transport of both electrons and holes. They perform both as efficient light absorbers and charge carriers [21]. The commonly used perovskites are Methylammonium lead triiodide $\left(\mathrm{CH}_{3} \mathrm{NH}_{3} \mathrm{PbI}_{3}\right)$ and formamidinium lead triiodide $\left(\mathrm{CH}_{3}\left(\mathrm{NH}_{2}\right)_{2} \mathrm{PbI}_{3}\right)$ [27]. Moreover, The PSC architecture is represented in Figure 1.

The two main device architectures of PSC are

1. mesoscopic

2. planar

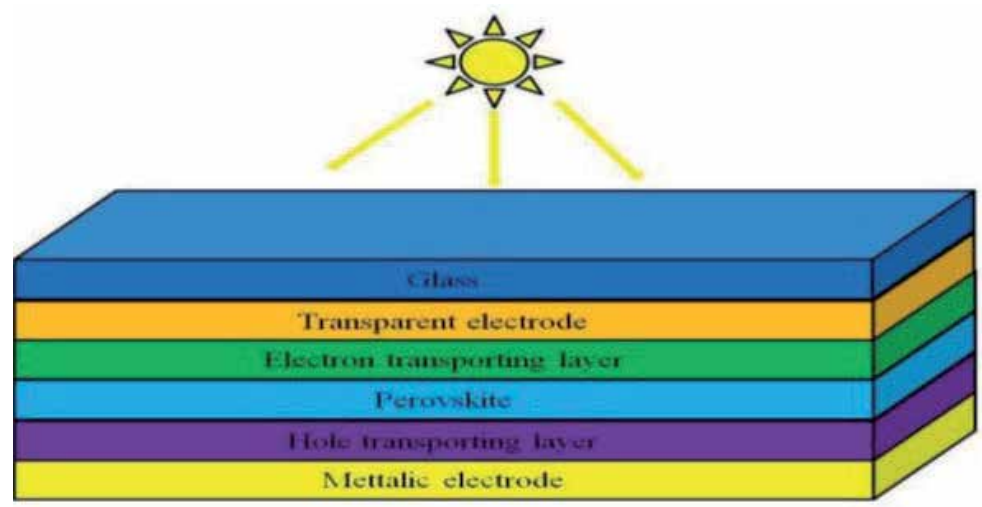

Figure 1.

Representative architecture of PSC. 
The conventional PSC consists of mesoscopic n-i-p structure and is the novel architecture of PSC devices which consists of an FTO, an electron transport layer (ETL), a mesoporous oxide layer such as $\mathrm{TiO}_{2}$, or $\mathrm{SnO}_{2}$, perovskite (light absorbing) layer, a hole transport layer (HTL), and an electrode layer. The mesoporous $\mathrm{TiO}_{2}$ layer played a significant role in the electron transfer process and as a scaffold providing mechanical support of the perovskite crystal. The use of mesoporous materials in PSC permit the perovskite material to adhere to the mesoporous metal oxide framework to increase the light-receiving area of the photosensitive material and results in improving the efficiency of the device. The mesoporous layer was usually less than $300 \mathrm{~nm}$. The presently mesoporous structure of PSCs is one of the most common structures with a power conversion efficiency (PCE) greater than $20 \%$ [28]. The mesoscopic structure due to the fabrication ease and outstanding best efficiencies is the most extensively adopted in research labs. However, high temperature $\left({ }^{>} 450^{\circ} \mathrm{C}\right)$ sintering is required for mesoporous layer-based devices, which prevents the use of plastic substrates [29-31]. To overcome this problem, the planar perovskite solar cell was developed that showed comparable performance for mesoporous perovskite solar cell. Planar heterojunction PSCs have been reported by several researchers in which only compact layers of ETM and HTM is used without a mesoporous layer at a temperature lower than $200^{\circ} \mathrm{C}[19,21,32]$. Hence the planar structure turns out to be very attractive for basic research purposes. The mesoscopic and planar structures of PSC are represented in Figure 2.

\subsection{Structure of perovskite materials}

Perovskites materials are designated by the formula $\mathrm{ABX}_{3}$, where $\mathrm{A}$ and $\mathrm{B}$ are cations of different sizes (A being larger than B) and X is an anion [7]. The crystal structure of perovskites is depicted in Figure 3 and it has a cubic crystal structure with three-dimensional (3D) framework sharing BX6 octahedron with the A ion placed at the octahedral interstices $[33,34]$. In organic-inorganic materials, the $A$ is organic cations generally methylammonium, ethylammonium and formamidinium and $\mathrm{B}$ is usually metal ions of group IV such as $\mathrm{Pb}^{2+}, \mathrm{Sn}^{2+}$ and $\mathrm{Ge}^{2+}$ whereas the $\mathrm{X}$ are VII group anions $\mathrm{I}^{-}, \mathrm{Cl}^{-}$and $\mathrm{Br}^{-}[2,7,34]$.

The crystallographic stability and probable structure of perovskite can be inferred by studying a "tolerance factor" $t$ and an "octahedral factor" $\mu$. A "tolerance factor" is defined as the "ratio of the A-X distance to the B-X distance in an idealized solid-sphere model" and is represented by the formula:

$$
t=\frac{\left(R_{A}+R_{X}\right)}{\left[\sqrt{2\left(R_{B}+R_{X}\right)}\right]}
$$

where $R_{A}, R_{B}$ and $R_{X}$ are the ionic radii of the corresponding ions.

An "octahedral factor" is defined as "the ratio $\frac{R_{B}}{R_{X}}$ ".

For halide $(\mathrm{X}=\mathrm{F}, \mathrm{Cl}, \mathrm{Br}, \mathrm{I})$ perovskites, generally $0.81<\mathrm{t}<1.11$ and $0.44<\mu<0.90$ [35]. If $t$ value lies in the narrow range $0.89-1.0$, the structure is cubic, but, if it is lower, symmetric tetragonal or orthorhombic structures is expected [2]. Regardless of these limitations, conversions between these structures are common on heating, at the high-temperature cubic phase is generally obtained.

For the organic-inorganic perovskites, organic cation A usually methylammonium $\left(\mathrm{CH}_{3} \mathrm{NH}_{3}{ }^{+}\right)$with $\mathrm{R}_{\mathrm{A}}=0.18 \mathrm{~nm}$, ethylammonium $\left(\mathrm{CH}_{3} \mathrm{CH}_{2} \mathrm{NH}_{3}{ }^{+}\right)$ $\left(\mathrm{R}_{\mathrm{A}}=0.23 \mathrm{~nm}\right)$ and formamidinium $\left(\mathrm{NH}_{2} \mathrm{CHNH}_{2}{ }^{+}\right)\left(\mathrm{R}_{\mathrm{A}}=0.19-0.22 \mathrm{~nm}\right)$ are used. The cation $B$ is commonly $\mathrm{Pb}\left(\mathrm{R}_{\mathrm{B}}=0.119 \mathrm{~nm}\right)$; however, $\mathrm{Sn}\left(\mathrm{R}_{\mathrm{B}}=0.110 \mathrm{~nm}\right)$ forms similar compounds with more ideal bandgap but exhibits lower stability 

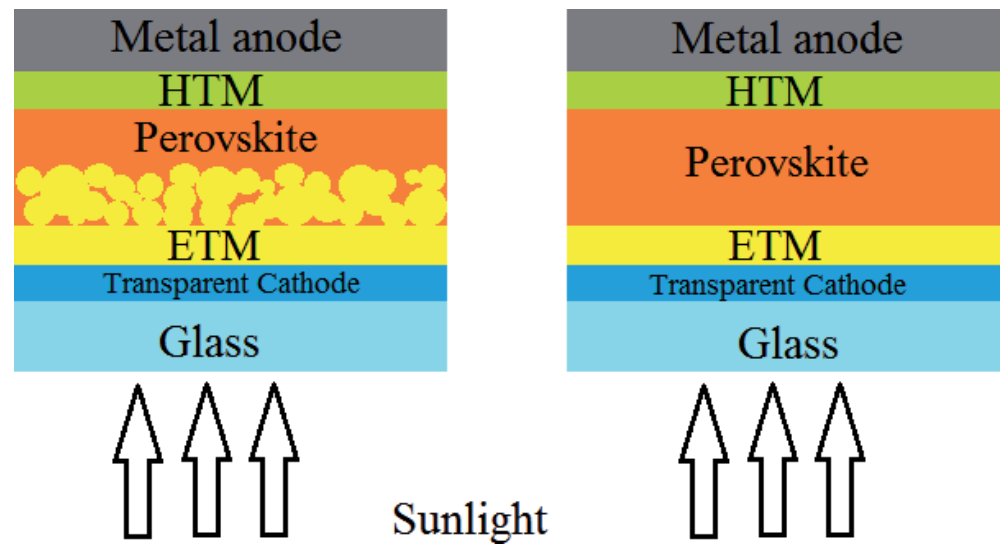

Figure 2.

Representative scheme of a mesoporous (right) and planar PSC (left).
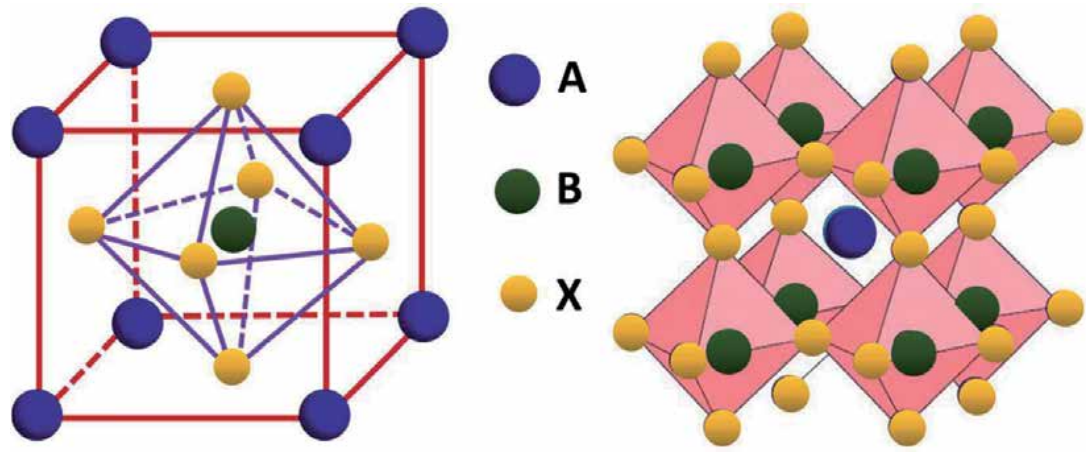

Figure 3.

Structure of perovskite.

(ascribed to the oxidation of $\mathrm{Sn}$ to $\mathrm{SnI}_{4}$ in the iodide perovskite). The anion $\mathrm{X}$ is a halogen, generally iodine $\left(\mathrm{R}_{\mathrm{X}}=0.220 \mathrm{~nm}\right)$ is used, however $\mathrm{Br}$ and $\mathrm{Cl}$ are also used $\left(\mathrm{R}_{\mathrm{X}}=0.196 \mathrm{~nm}\right.$ and $\left.0.181 \mathrm{~nm}\right)[35,36]$. The commonly used organic inorganic perovskite material is methylammonium lead triiodide $\left(\mathrm{CH}_{3} \mathrm{NH}_{3} \mathrm{PbI}_{3}\right)$.

$\mathrm{MAPbX}_{3}$ perovskite show multiple phases as a function of composition and temperature. These different phases have markedly different optical and electrical properties as well as stability. $\mathrm{MAPbI}_{3}$ displayed $\alpha$-phase, $\delta$-phase, and $\gamma$-phases with transition temperatures of $400 \mathrm{~K}, 333 \mathrm{~K}$, and $180 \mathrm{~K}$, respectively. Generally, the $\delta$-phase $\mathrm{MAPbI}_{3}$ is used as absorber in solar cell due to its thermodynamically stable nature at room temperature and its increased conductivity and absorption coefficient $\left(>26 \mathrm{~mm}^{-1}\right)$ in contrast to the $\alpha$-phase. Though, a phase transition from $\delta$-phase to $\alpha$-phase may occur under continuous 1 sun illumination [15].

\section{Synthesis of inorganic-organic solar cells materials}

The deposition technique of organic-inorganic perovskites films is quite an important issue for perovskite studies, because the possible use of perovskite materials depends on the availability of simple and perfect thin film deposition method. As concerns the preparation methods of organometallic halide perovskite $\mathrm{CH}_{3} \mathrm{NH}_{3} \mathrm{PbX}_{3}$ thin films, solution-based procedures have been proposed to manufacture thin films. 
However, deposition of organic-inorganic perovskite materials is often challenging due to different physical and chemical properties of the organic and inorganic parts of perovskite materials [15]. Despite of this, several significant methods are used for thin film deposition of organic-inorganic hybrid perovskites. Various methods used for perovskite deposition are solution-processed (one-step and two-step) deposition, evaporation method, and vapor assisted solution process (VASP) are the typically adopted methods for film deposition [15, 37, 38].

1. one-step precursor solution deposition (Spin-coating technique)

2.two-step sequential deposition (including the vapor-assisted solution process)

3.thermal evaporation technique

\subsection{One-step precursor solution deposition (spin-coating technique)}

One-step processing (spin-coating) is a suitable technique extensively applied for uniform thin film deposition and is based on the co-deposition of both the inorganic and organic components either through solution processing or thermal evaporation. In solution processing, a mixture of both $\mathrm{MX} 2\left(\mathrm{M} 1 / 4 \mathrm{~Pb}, \mathrm{Sn} ; \mathrm{X}^{1 / 4} \mathrm{Cl}-, \mathrm{Br}-\right.$, I-) and $\mathrm{AX}$ (A $1 / 4$ methylammonium MA); formamidinium, $\mathrm{FA}$ ) is dissolved in an organic solvent and deposited through the spin coating to form a film (Figure 4), followed by annealing to produce the perovskite layer [15]. The post deposition annealing of the films at low temperature $\left(\mathrm{T}<250^{\circ} \mathrm{C}\right)$ is sometimes used to increase phase purity and crystallinity [6]. Spin-coating allows deposition of hybrid perovskites on various substrates, containing glass, quartz, plastic, and silicon. Selection of suitable parameters such as substrate, spin speed and the substrate temperature are essential for this technique and can be selected accordingly. The wetting properties of the solution on the chosen substrate can be improved by pre-treating the substrate with a suitable adhesion agent. The spin-coating technique does not involve cumbersome equipment and it gives high-quality films in quite short time at room temperature. It is considered as a distinct case of solution crystal growth, which results in the formation of highly oriented perovskites layer on a substrate. In order to obtain a layer with the desired thickness, optimization of various parameters such as concentration of perovskites solution, and spin-coating parameters (spin speed, acceleration and spin duration) can be carried out. Generally, 2D homogeneous perovskites films with a thickness ranging $10 \mathrm{~nm}$ to $100 \mathrm{~nm}$ can be obtained by carefully choosing the parameters. The selection of solvent is also important by considering the solubility for both the organic ammonium and the inorganic lead halide. The usually used solvents for spin coating technique are Dimethylformamide (DMF) or Dimethyl sulfoxide (DMSO) [39]. These spin-coated perovskites films are very reproducible, and this technique is suitable for all PSC structures (mesoporous vs. planar) [39].

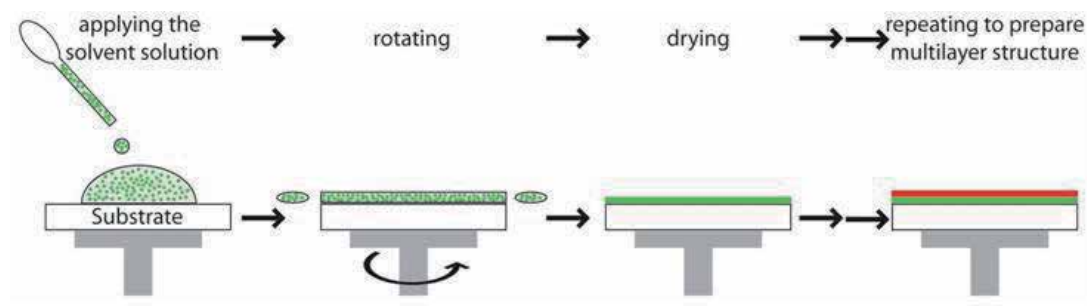

Figure 4.

Schematic of the spin-coating process [4]. 


\subsection{Two-step dip-coating}

Mitzi [40] first time reported the two-step dipping technique in 1998, and later by Burschka et al. [41] in 2013. In a two-step dip-coating deposition process, a metal halide $\mathrm{PbI}_{2}$ layer is first deposited by vacuum evaporation or spin-coated on a substrate. Then this coated film is altered into the perovskite by dipping into an organic MAI solution as it is shown in Figure 4. This method offered PCE of 15\% and certified 14.14\% [39]. Suitable selection of solvent is important for the dipping process. The solvent is selected such that can dissolve organic salt but cannot metal halide and the final organic-inorganic perovskite, toluene/2-propanol mixture is an appropriate solvent for the organic salt. The organic cations in solution intercalate into and react with metal halide on the substrate and form a crystalline film [6]. The dipping times are quite short: several seconds to minutes, depending on the system. This method is a suitable method for a variety of inorganic and organics, even if they have an incompatibility in solubility. This process effectively reduces the chemical reaction between the perovskite and the underlying ETL. The development of successive deposition methods has offered a variety of ETL options, though allowing for perovskite films to be prepared successfully at room temperature [15]. In addition, Chen et al. [42] developed a vapor assisted solution processing (VASP) method that used the reaction between MAI vapor and pre-deposited $\mathrm{PbI}_{2}$ to form the completed perovskite film. The resulting $\mathrm{MAPbI}_{3}$ exhibits excellent film quality.

\subsection{Thermal evaporation technique}

M. Era et al. [43] first used thermal evaporation method. They used the dual source vapor deposition by using ammonium iodide $\mathrm{RNH}_{3} \mathrm{I}$ and lead iodide $\mathrm{PbI}_{2}$, organic and inorganic source were co-evaporated and deposited on quartz. The pressure of evaporation chamber was about $10^{-6}$ Torr. By using this method, it is possible to precisely control the smoothness and thickness of the films. However, it is often hard to balance the organic and inorganic rates, which is important in attaining the correct composition of the resultant perovskite films. Furthermore, Mitzi et al. [40] developed another method, by using a single evaporation source to deposit perovskites thin films called single source thermal ablation (SSTA) technique. This consists of a vacuum chamber, with an electrical feed-through to a thin tantalum sheet heater. A suspension of insoluble powders in a drying solvent is placed on the heater. Under a suitable vacuum, the temperature goes to approximately $1000^{\circ} \mathrm{C}$ in 1-2 second, the whole starting charge ablates from the heater. After ablation, the organic and inorganic parts reassemble on the substrates to yield films of the chosen product. Liu et al. [44] in 2013, improved this technique as a dual-source vapor deposition method for pinhole-free $\mathrm{MAPbI}_{\mathrm{x}}^{1-} \mathrm{Cl}_{\mathrm{x}}$ perovskite films with a thickness of hundreds of nanometers for planar PSCs.

Later on, the chemical vapor deposition (CVD) method was reported by Leyden et al. [45], which precisely control the crystallization process. Vapor deposition methods are appropriate for multi-layered thin-film and a variety of substrates, though needs high vacuum [39]. However, this method has drawbacks of yield and therefore is not very effectively employed at industrial scale [46]. Though great achievements have been attained, researchers still meet some challenges, involving reproducibility and grain boundaries of perovskite films which are considered as a defect region initiates carrier recombination and accelerates device degradation. Hence, efforts to increasing grain size and reducing grain boundary of films are critical for stable and highly efficient PSCs. 


\section{Advances in perovskite solar cells}

The possibility of merging the properties of inorganic with those of organic solids has inspired intensive research into the versatile properties. Organicinorganic perovskite materials have been widely used in PSCs using different ETLs and HTLs. The optimization of materials and structures is one of the solutions to improve the PCE. Table 1 shows some representative devices and their architectures and performance.

Methylammonium Lead halide perovskites $\left(\mathrm{MAPbX}_{3}\right)$ are mostly regarded as promising light absorbers owing their many advantages comprising high absorption coefficients, optimal bandgaps, and long-range exciton diffusion lengths. These perovskites have led to solar cells with PCEs upto15\% in combination with meso-structure metal oxides and deposition methods (such as sequential and vapor deposition) [34]. There were few attempts to synthesize new perovskites by changing halide anions $(\mathrm{X})$ in the $\mathrm{MAPbX}_{3}$ structure, but these materials did not result too much improvement in device efficiency. Optical and electronic properties of organo lead halide perovskites have been considered by replacing MA cation with other organic cations such as ethyl ammonium and formamidinium [55].

Dkhissi et al. [32] fabricated an efficiently $\mathrm{CH}_{3} \mathrm{NH}_{3} \mathrm{PbI}_{3}$-based planar perovskite solar cells on polymer substrates at $150^{\circ} \mathrm{C}$ or below. The hole blocking layer employed is $\mathrm{a} \mathrm{TiO}_{2}$ layer. The devices showed an average efficiency of $10.6 \pm 1.2 \%$, and a maximum efficiency of $12.3 \%$ for flexible perovskite solar cells, presenting great potential for further enhancement of the low-cost, low-temperature processing solar technology.

In 2014, Choi et al. [55] modified perovskite material with Cesium (Cs) by doping methyl ammonium lead iodide perovskites by Cesium to improve the performance of inverted-type perovskite/fullerene planar heterojunction hybrid solar cells. $\mathrm{Cs}_{\mathrm{x}} \mathrm{MA}_{1-\mathrm{x}} \mathrm{PbI}_{3}$ perovskite devices achieved improvement in device efficiency from $5.51-6.8 \%$ with an optimized $10 \%$ Cs doping concentration. The devices exhibited an outstanding increase in efficiency due to increases in short-circuit current density and open-circuit voltage.

\begin{tabular}{|c|c|c|c|c|}
\hline ETL & HTL & Perovskite & PCE & References \\
\hline $\mathrm{Gr} / \mathrm{ZnO}-\mathrm{QDs}$ & Spiro-OMeTAD & $\mathrm{CH}_{3} \mathrm{NH}_{3} \mathrm{PbI}_{3}$ & 9.73 & {$[18]$} \\
\hline $\mathrm{TiO}_{2}-\mathrm{Al}_{2} \mathrm{O}_{3}$ & $\begin{array}{c}\text { Spiro-OMeTAD } \\
\text {-Li-TFSI }\end{array}$ & - & 10 & [24] \\
\hline ZnO-NPs & Spiro-OMeTAD & $\mathrm{CH}_{3} \mathrm{NH}_{3} \mathrm{PbI}_{3}$ & 10.2 & {$[34]$} \\
\hline $\mathrm{TiO}_{2}$ & Spiro-OMeTAD & $\mathrm{CH}_{3} \mathrm{NH}_{3} \mathrm{PbI}_{3-\mathrm{x}} \mathrm{Cl}_{\mathrm{x}}$ & 11.7 & [47] \\
\hline $\mathrm{TiO}_{2}$ & Spiro-OMeTAD & $\mathrm{CH}_{3} \mathrm{NH}_{3} \mathrm{PbI}_{3}$ & 15.4 & {$[48]$} \\
\hline $\mathrm{TiO}_{2}$ & - & $\mathrm{CH}_{3} \mathrm{NH}_{3} \mathrm{~Pb} \mathrm{Br}_{3}-\mathrm{n}$ & 8.54 & {$[49]$} \\
\hline $\mathrm{SnO}_{2} \mathrm{QD}$ & $\begin{array}{c}\text { Spiro-OMeTAD- } \\
\text { Li-TFSI }\end{array}$ & $\begin{array}{c}\mathrm{Cs}_{0.05}\left(\mathrm{MA}_{0.17} \mathrm{FA}_{0.83}\right) 0.95 \mathrm{~Pb} \\
\left(\mathrm{I}_{0.83} \mathrm{Br}_{0.17}\right)_{3}\end{array}$ & 20.79 & [50] \\
\hline $\mathrm{SnO}_{2} \mathrm{QD}$ & $\begin{array}{l}\text { Spiro-OMeTAD- } \\
\text { Li-TFSI }\end{array}$ & $\mathrm{CH}_{3} \mathrm{NH}_{3} \mathrm{PbI}_{3}$ & 19.73 & [50] \\
\hline $\mathrm{SnO}_{2}$ & Spiro-OMeTAD & Cs/MA/FA perovskite & 20.7 & [51] \\
\hline $\mathrm{SnO}_{2} \mathrm{QD}$ & Spiro-OMeTAD & $\mathrm{CH}_{3} \mathrm{NH}_{3} \mathrm{PbI}_{3}$ & 19.12 & [52] \\
\hline $\mathrm{TiO}_{2}$ & Spiro-OMeTAD & $(\mathrm{FAI})_{0.81}\left(\mathrm{PbI}_{2}\right)_{0.85}\left(\mathrm{MAPbBr}_{3}\right)_{0.15}$ & 21.02 & {$[53,54]$} \\
\hline
\end{tabular}

Table 1.

Comparison of different organic inorganic perovskite materials with different hole and electron TLs in PSCs. 
$\mathrm{CH}_{3} \mathrm{NH}_{3} \mathrm{PbBr}_{3}$ and $\mathrm{CH}_{3} \mathrm{NH}_{3} \mathrm{PbI}_{3}$, were used as sensitizers for $\mathrm{TiO}_{2}$ in a liquid junction solar cell, with open-circuit voltages of 0.61 and $0.71 \mathrm{~V}$ were achieved. $\mathrm{CH}_{3} \mathrm{NH}_{3} \mathrm{PbI}_{3}$ on mesoporous $\mathrm{TiO}_{2}$ showed good charge transport properties, where the perovskite is both the absorber and the hole conductor. Further $\mathrm{CH}_{3} \mathrm{NH}_{3} \mathrm{PbX}_{3}$ $(\mathrm{X}=\mathrm{Br}, \mathrm{I})$, mixed perovskite lead halides i.e., $\mathrm{CH}_{3} \mathrm{NH}_{3} \mathrm{PbI}_{2} \mathrm{Cl}, \mathrm{CH}_{3} \mathrm{NH}_{3} \mathrm{PbBr}_{3}-\mathrm{xCl}$, and $\mathrm{CH}_{3} \mathrm{NH}_{3} \mathrm{PbI}_{3}-\mathrm{xCl}$, were studied [27].

Giacomo et al. [47] fabricated PSCs using $\mathrm{CH}_{3} \mathrm{NH}_{3} \mathrm{PbI}_{3}-\mathrm{xCl}$ x with different holetransporting materials. The mostly used Spiro-OMeTAD has been compared to the P3HT. By changing the energy level of $\mathrm{P}_{3} \mathrm{HT}$ and optimizing the device fabrication, PCE reached to $9.3 \%$. They showed that $\mathrm{P}_{3} \mathrm{HT}$ can be used a suitable low-cost hole transport material for efficient perovskite based solar cells.

$\mathrm{NiO}$ has been tried as a substitute for organic molecular or polymeric HTMs (spiro-MeOTAD), displaying encouraging results in the $\mathrm{TiO}_{2} / \mathrm{CH}_{3} \mathrm{NH}_{3} \mathrm{PbI}_{3}$ configuration, a PCE of $9.5 \%$ was attained with nanocrystalline $\mathrm{NiO}$ layer. As the valence band edge $(5.4 \mathrm{eV})$ for $\mathrm{NiO}$ is near to that of iodide perovskite $(5.3 \mathrm{eV})$, so posttreatment of $\mathrm{NiO}$ film by means of UV light or oxygen plasma is vital to progress hole injection efficiency due to an increase in the work function of $\mathrm{NiO}$ by such post treatments. UV-ozone post-treated $\mathrm{NiO}$ usually has a greater photovoltaic performance than untreated $\mathrm{NiO}$, due to change in work function and an enhancement in wettability indicating a better chemical interaction between perovskite and $\mathrm{NiO}[36]$.

The use of perovskites with mixed cations and halides has become significant for $\mathrm{PV}$ applications which are mainly $\mathrm{MAPbX}_{3}, \mathrm{FAPbX}_{3}$ and $\mathrm{CsPbX}_{3}(\mathrm{X}=\mathrm{Br}$ or I). on the introduction of MA into FA brings the crystallization of FA perovskite (because MA is slightly smaller than FA) which allows a large fraction of the yellow phase to continue. MA/FA compounds show notable PCEs and therefore the development of these compounds is an opportunity in the advancement of PSCs. Saliba et al. [56] introduces an innovative approach using a triple Cs/MA/FA cation mixture where Cs is used to progress MA/FA perovskite compounds. A small amount of Cs is enough to efficiently suppress yellow phase impurities allowing the preparation of pure, defect-free perovskite films.

Song et al. [57] reported that the combination of FA decreases the release of organic species but does not stop the formation of I/HI. Though, the addition of Cs successfully overcomes the release of all volatile gases. The best photostability is found with FA/Cs mixed perovskites, presenting the complete removal of MA from mixed-cation perovskite is favored for more photostable perovskites.

As $\mathrm{CH}_{3} \mathrm{NH}_{3} \mathrm{PbI}_{3}$ has ambipolar properties and is slightly more p-type than $\mathrm{n}$-type and is satisfactory to develop p-n junction-like devices without an HTM, known as HTM-free photovoltaic cells. $\mathrm{CH}_{3} \mathrm{NH}_{3} \mathrm{PbI}_{3}$ could act both as light absorber and hole transporter in a $\mathrm{CH}_{3} \mathrm{NH}_{3} \mathrm{PbI}_{3} /$ mesoporous $\mathrm{TiO}_{2}$ heterojunction device with a $\mathrm{PCE}$ of $5.5 \%$. It was observed that HTM-free perovskite solar cells had a poor FF and a low Voc as compared to those with an HTM, which is related to the larger shunt current along with a lower IPCE for these devices [2].

Different proportions of inorganic $(\mathrm{Pb}, \mathrm{Sn})$ cations, organic cations and halide anions ( $\mathrm{I}, \mathrm{Br}, \mathrm{Cl})$ can be combined in mixed perovskites, permitting their properties to be fine-tuned [35]. Tuning of bandgap of $\mathrm{MAPbX}_{3}$ has been attained through the substitution of I with $\mathrm{Cl} / \mathrm{Br}$ ions, which occurs from a dependence of electronic energies on the effective exciton mass. The optical absorption can be tuned by bandgap engineering to comprise the whole visible spectrum. In the meantime, the combination of $\mathrm{Cl} / \mathrm{Br}$ into iodide-based structure has markedly advanced the charge transport and the separation kinetics within the perovskite layer. Hence, by tuning the composition of perovskite resulted in improved efficiency and the stability of PSCs. It was observed that an increase in the size of perovskite cation materials 
resulted in a reduction in the bandgap. A tunable bandgap can be obtained (between 1.48 and $2.23 \mathrm{eV}$ ) by replacing the methylammonium with a slightly larger formamidinium cation. Significantly, the reduced bandgap led to a PCE of up to $14.2 \%$ and high short circuit currents $\left(>23 \mathrm{~mA} \mathrm{~cm}^{-2}\right)$ [31].

$\mathrm{CH}_{3} \mathrm{NH}_{3} \mathrm{SnI}_{3}$ is demanded to be a low-carrier-density p-type metal. Theoretical calculations on perovskite recommended that their electronic properties intensely depend on the structure of the inorganic cage and formation of the perovskite octahedral network. By changing the inorganic and organic components and their stoichiometric ratio, it is probable to control the system dimensionality and electronic and optical properties. Furthermore, the presence of weak bonds in the perovskite structures ensures malleability and flexibility that could permit the deposition of thin films on flexible substrates [26].

\section{Toxicity and stability issue}

\subsection{Lead: the toxicity problem}

Regardless of the excellent properties and high efficiencies, the poor stability of organic-inorganic perovskite materials are yet a serious challenge, inhibiting PSCs from being commercialized. To be marketable for commercial purposes, PSCs need to be capable of work constantly for over 20 years under outdoor conditions. Thus, large consideration has recently been centered to overcome barriers associated with stability and environmental compatibility of perovskite materials [10].

Presently there is a debate on the use of lead (main component) in PSCs, which causes toxicity problems during device manufacture, placement, and disposal. Hence, the toxicity of lead-based perovskites is an obvious problem due to leaching of lead into the environment [9]. Lead toxicity has been pointed out as one of the most challenging barriers towards the commercialization of solar cells, as compared to stability issues and cost-effective production ways. The environmental impact benefits of lead-free (or lead-reduced) solar cells have been analyzed by Life Cycle Assessment (LCA) [15].

\subsubsection{Lead-free perovskite solar cells}

Up to now, several research groups have ambiguously proven their solution to this challenge. Thus, it is critical to test alternatives to attain similar optical and photovoltaic performances for the commercialization of PSCs. Several research groups have tried to replace lead with other elements ( $\mathrm{Sn}, \mathrm{Ge}$ ) and organic cations with inorganic cations to form new appropriate non-toxic and stable perovskite materials, which may be a long journey before the final commercialization of PSCs [9].

It is worth studying alternatives using lead-free PSCs, but Lead-free PSCs reached a PCE of only 6\% at a time when lead-based PSCs produced efficiency of $17 \%$. Moreover, the $\mathrm{Sn}$-based solar cells display poorer stability than $\mathrm{Pb}$-based solar cells. [14]. Bivalent $\mathrm{Sn}$ is the most favorable choice for replacing $\mathrm{Pb}$ as they both are in the same group and possess analogous lone-pair s orbitals [10]. Both $\mathrm{Sn}$ - and $\mathrm{Pb}$-based materials have a tetragonal structure under ambient conditions; however, Sn-based perovskite have a higher symmetrized $\alpha$ phase as compared to the $\mathrm{Pb}$-based materials lower symmetrized $ß$ phase [47]. Chung et al. [54] first demonstrated $\mathrm{CsSnI}_{3}$ as a solid electrolyte in DSSCs. Afterward Chen et al. [58] fabricated a photovoltaic device, ITO/CsSnI $3 / \mathrm{Au} / \mathrm{Ti}$, attaining very low PCE of $0.88 \%$. However, $\mathrm{Sn}^{2+}$ based perovskite undergoes oxidation from $\mathrm{Sn}^{2+}$ to $\mathrm{Sn}^{4+}$, which is destructive 
for the charge transport properties, and PCE. Recently, Lv 2019 [59] reported the replacement of spiro-OMeTAD by a $\mathrm{Zn}$-derivative porphyrin in a lead-free solar cell has resulted in stability up to $60 \mathrm{~h}$ for water and $100 \mathrm{~h}$ for thermal stability.

There is another approach of mixed $\mathrm{Pb} / \mathrm{Sn}$ perovskite Solar cell have also been reported. Lead and tin were revealed to be arbitrarily spread in the [MX6] octahedra in the perovskite and percentage of tin could be altered from 0 to 1 [15]. These devices presented the best photocurrent at a 50\% mixing ratio. SnO used as ETL has also resulted with good PCE of (13\%) and stability ( $>700$ h storage) $[15,60]$. LCA showed the replacement of lead did not decrease the environmental impacts, meanwhile the loss of PCE and stability generates an environmental burden. However, those studies are also interesting because they draw consideration to other toxicity problems occurring from the solvent use during processing of charge transport layers (ETLs) [15].

For lead-free inorganic perovskites Tetravalent cations have also been thought to replace $\mathrm{Pb}$. A new chemical formula of $\mathrm{A}_{2} \mathrm{BX}_{6}$ structure is designed by eliminating half of the $\mathrm{B}$-site ions in the $\mathrm{ABX}_{3}$ perovskite for adjusting the heterovalent cation substitution as shown in Figure 1. Due to the lack of connectivity in the [BX6] octahedral structure, the $\mathrm{A}_{2} \mathrm{BX}_{6}$ can be considered as a $0 \mathrm{D}$ non-perovskite which results in different optical and optoelectronic properties of the A2BX6 from those of the $\mathrm{ABX}_{3}$. $[3,9,10]$. Amongst the $\mathrm{A}_{2} \mathrm{BX}_{6}$ perovskites, $\mathrm{Cs}_{2} \mathrm{SnI}_{6}, \mathrm{Cs}_{2} \mathrm{TiBr}_{6}$ and $\mathrm{Cs}_{2} \mathrm{PdBr}_{6}$ have been employed in photovoltaic devices $[9,10]$. Chung et al. [54] first utilized this material as a solid hole transport material in DSSCs.

Furthermore, a special concern for toxicity must be upraised during experimental work in the laboratory, since hazards arise primarily by the absorption of the toxic lead when used in solution, which is significantly higher, particularly through the dermal and respiratory routes; some of the lead derivatives are soluble both in water and fat, posing a high risk. Solvents such as dimethylformamide (DMF) and dimethylsulfoxide (DMSO) are not only toxic, but also raise the risk of bio incorporation as they are miscible in all ratios with water. Thus, these solvents have also been considered as a major contributor to environmental impact [61].

\subsection{Stability}

Perovskite solar cells (PSCs) have been established with promising PCEs. Regardless of the great potential as PV material in terms PCE, the instability of the PSCs is one of the core barriers for larger scale applications [8, 9, 39]. At present, PSCs can only perform for several months under active conditions, whereas traditional silicon cells can operate for more than 25 years. Therefore, stability issues must be reasonably dealt with before its actual use and commercialization [62] Poor stability of PSCs is due to several affiliated factors resulting from exposure to moisture, oxygen, light, and heat $[63,64]$.

Nevertheless, the importance of stability has been highlighted and recognized as the foremost problem, in the past five years to solve for the perovskite solar cells (PSCs) to be able to challenge in the market arena. So, how to increase the stability of perovskite solar cells is the most significant issue in this field [15].

In this section, the effect of environmental factors will be discussed on PSCs along with approaches developed to improve stability of perovskite solar cells.

\subsubsection{Moisture}

The first environmental factor observed to degrade perovskites was Moisture/ water. The instability of perovskite at high humidity is the serious issue that needs to be focused. Solar cells when exposed to moisture (water), due to the hygroscopic 
nature of the organic components of perovskite materials are spontaneously affected by moisture access and then degrade [62]. It has basically been supposed that moisture-induced degradation is the leading issue, imitating $\mathrm{MNH}_{3} \mathrm{PbI}_{3}$ stability under ambient conditions.

Prolonged exposure of perovskite material to water vapor activates an irreversible degradation which eventually leads to transformation of the perovskite back to the initial precursors (such as $\mathrm{PbI}_{2}$ ). In detail, perovskite forms hydrate complexes with water such as $\left(\mathrm{CH}_{3} \mathrm{NH}_{3}\right)_{4} \mathrm{PbI}_{6} \cdot 2 \mathrm{H}_{2} \mathrm{O}$ and leaves out $\mathrm{PbI}_{2}$, which tend to crystallize, forcing the forward reaction. Moreover, $\mathrm{MA}^{+}$is slightly acidic and reacts with water to form volatile methylamine $\left(\mathrm{CH}_{3} \mathrm{NH}_{3}\right)$ and hydroiodic acid (HI), according to the following reaction (1): [63].

$$
\left(\mathrm{CH}_{3} \mathrm{NH}_{3}\right)_{4} \mathrm{PbI}_{6} \cdot 2 \mathrm{H}_{2} \mathrm{O} \rightarrow \mathrm{PbI}_{2}+4 \mathrm{CH}_{3} \mathrm{NH}_{3}+4 \mathrm{HI}+2 \mathrm{H}_{2} \mathrm{O}
$$

Some researchers have reported that the compositions, microstructures (such as grain size) also affected the moisture stability of perovskite devices and concluded that larger grains resulted in a smaller area density of grain boundaries, which can be correlated with the improved stability [65].

In demand to progress the chemical stability of $\mathrm{MAPbI}_{3}$-based PSCs against moisture, scientists have proposed replacing the organic cation $\mathrm{MA}^{+}$with alternative components at the A position. For example, $\mathrm{FAPbI}_{3}$ has been presented to be further thermally stable than $\mathrm{MAPbI}_{3}$ because of its larger tolerance factor. Though, $\mathrm{FAPbI}_{3}$ suffers a phase transition from the a-FAPbI 3 (black triangular) phase to the d-FAPbI 3 (yellow hexagonal) phase due to the presence of moisture. Furthermore, degradation of $\mathrm{FA}_{0.9} \mathrm{Cs}_{0.1} \mathrm{PbI}$ is prevented by adding a small amount of cesium (Cs) into orbital lead-iodine to form $\mathrm{FA}_{0.9} \mathrm{Cs}_{0.1} \mathrm{PbI}$ in high humidity environment [33].

Smith et al. [66] discussed that Low-dimensional 2-D perovskites exhibited better moisture stability than 3D perovskites due to the hydrophobic nature of organic cations. Though, the insulating aspect of the organic cations with poorer charge transport resulted in lower PCE as compared to 3D perovskites. Therefore, various efforts have been made to form a quasi-2D (or 2D-3D mixture) and 2D on top of 3D (2D@3D) to use the benefits of both 2D and 3D perovskites. The use of $2 \mathrm{D}$ perovskite is mostly to improve the moisture stability, a thin 2D layer was deposited on top of 3D $\mathrm{MAPbI}_{3}$ perovskite to cover it fully and shield the 3D perovskite from moisture. The highest PCE for 2D@3D perovskite solar cell was observed to be of $18.0 \%$, with an enhanced device stability under both inert $(90 \%$ of initial PCE for $32 \mathrm{~d}$ ) and ambient conditions (72\% of initial PCE for $20 \mathrm{~d}$ ) without encapsulation.

Polymers, such as poly(4-vinylpyridine) (PVP), poly (methyl methacrylate) (PMMA) covering p-type and n-type semiconductors, or insulators, were also reported to improve stability. These long chain polymer acts as defect passivator and a moisture blocker by forming a network along perovskite grains and resulted in improved device efficiency and stability $[58,60,64]$.

\subsubsection{Light}

Light-induced perovskite solar cell degradation and environmental stability are the most frequently cited villains. Early on, stability of PSC was a big issue. But just as there were quick improvements in efficiency of PSCs, there has also been similar quick progresses in stability. Ultraviolet light (UV) can also cause the degradation of $\mathrm{MAPbI} 3$ perovskite. For e.g. the commonly used $\mathrm{TiO}_{2}$ electron transport layer 
(ETL) for these PSCs is responsible for UV-induced degradation. According to the international standards for climate chamber tests (IEC 61646), solar cells need to tolerate long-term stability at $85^{\circ} \mathrm{C}$.

Bryant et al. [67] demonstrated that contact of $\mathrm{MeNH}_{3} \mathrm{PbI}_{3}$ films to both light and molecular oxygen can initiate quick degradation. Particularly, this reaction is started by the deprotonation of the methylammonium cation of the perovskite by a photogenerated reactive oxygen species (superoxide, $\mathrm{O}_{2}{ }^{-}$). The stability of $\mathrm{MeNH}_{3} \mathrm{PbI}_{3}$ based devices was checked under different operating (e.g. light and dark) and environmental conditions and infer that oxygen induced degradation, is relatively dominant than moisture induced degradation and limits the working stability of $\mathrm{MeNH}_{3} \mathrm{PbI}_{3}$ containing devices under ambient conditions. Moreover, they pointed out that this fall in device performance can be reduced by the addition of electron acceptor layers within device architecture. Such layers are exposed to augment electron extraction from the absorber (perovskite material) before they react with oxygen, hence decreasing the amount of superoxide $\mathrm{O}_{2}{ }^{-}$and increasing the device stability.

It was noticed that by replacing MA with Cs and FA resulted in improved photostability of the PSCs. By systematically monitoring the development of PL intensity of perovskites, light-induced formation and annihilation of defects were reported to induce photo-instability [68]. Photostability can be improved through defect control by passivating which acted as a defect reservoir on the surface and grain boundaries. To stabilize surface defects, polyethylene oxide was applied and thus improved photostability was achieved. By substituting MA with FA, the degradation became slow with small pores forming on the surface after exposure to light. Moreover, Addition of Cs into the MAFA (forming CsMAFA) further lessen the degradation. XPS, XRD, Fourier transform infrared (FT-IR) spectrometry, and ultraviolet-visible absorption spectrometry were used to investigate the variation of MAPbI3 films under illumination. The result showed that light induced degradation is the main cause of degradation. Using polymer such as PTAA (Poly(triarylamine)) as the HTM, it was observed that pure $\mathrm{MAPbI}_{3}$ devices retained nearly $100 \%$ of their initial efficiency after $1000 \mathrm{~h}$ aging under constant illumination at room temperature. PTAA which act as a protection layer, inhibited the discharge of gaseous degradation products enhanced stability. However, for devices using Spiro as the HTM, their stability under illumination was lesser than that using PTAA $[69,70]$.

\subsubsection{Heat}

Heat is also another factor that influences stability due to the inherent matter with low formation energies, and perovskites thus have a great response to a small increase in external temperature [71]. Organic-inorganic perovskites tend to decompose due to the instability of organic $\mathrm{A}^{+}$cations under thermal atmosphere. Commercial solar cells should be able to work efficiently above $85^{\circ} \mathrm{C}$, to have any influence in the market.

$\mathrm{MAPbI}_{3}$ is basically unstable upon thermal stress which produces a discharge of $\mathrm{I}_{2}$ and the presence of metallic $\mathrm{Pb}$ at $40^{\circ} \mathrm{C}$ in the dark [63]. This is produced by the decomposition reactions (2) and (3):

$$
\begin{gathered}
\mathrm{CH}_{3} \mathrm{NH}_{3} \mathrm{PbI}_{3} \rightarrow \mathrm{CH}_{3} \mathrm{NH}_{3} \mathrm{I}+\mathrm{PbI}_{2} \\
\mathrm{PbI}_{2} \rightarrow \mathrm{PbO}+\mathrm{I}_{2}
\end{gathered}
$$


Although reaction (3) is reversible at just $80-85^{\circ} \mathrm{C}$, methylammonium iodide decomposes into more volatile compounds as represented by reactions (4) and (5):

$$
\begin{aligned}
& \mathrm{CH}_{3} \mathrm{NH}_{3} \mathrm{I} \rightarrow \mathrm{CH}_{3} \mathrm{I}+\mathrm{NH}_{3} \\
& \mathrm{CH}_{3} \mathrm{NH}_{3} \mathrm{I} \rightarrow \mathrm{CH}_{3} \mathrm{NH}_{2}+\mathrm{HI}
\end{aligned}
$$

It was found that $\mathrm{HI}(\mathrm{g})$ and $\mathrm{CH}_{3} \mathrm{NH}_{2}(\mathrm{~g})$ were dominant products during the decomposition of $\mathrm{MAPbI}_{3}$ and only trace amounts of $\mathrm{CH}_{3} \mathrm{I}$ and $\mathrm{NH}_{3}$ were found. Though, the ratio of $\mathrm{CH}_{3} \mathrm{I}$ and $\mathrm{NH}_{3}$ increased at higher temperature and lesser than $\mathrm{HI}_{(\mathrm{g})}$ and $\mathrm{CH}_{3} \mathrm{NH}_{2(\mathrm{~g})}$. In short, $\mathrm{HI}_{(\mathrm{g})}$ and $\mathrm{CH}_{3} \mathrm{NH}_{2}(\mathrm{~g})$ were the dominant decomposition products at ambient temperature under vacuum while $\mathrm{CH}_{3} \mathrm{I}$ and $\mathrm{NH}_{3}$ gases were obtained at high temperature. Both processes occurred simultaneously near ambient temperature in vacuum and the later was favored at high temperature.

To find out the decomposition temperature of perovskites, Thermogravimetric analysis (TGA) was used. From the mass loss of TGA curve for $\mathrm{MAPbI}_{3}$, the decomposition onset temperature was found to be $234^{\circ} \mathrm{C}$ [62]. This indicates that as the practical application temperature usually is less than $100^{\circ} \mathrm{C}$, so this high decomposition temperature made the stability of $\mathrm{MAPbI}_{3}$ not a big issue. The as prepared film did not show any changes in XRD patterns when stayed inside the vacuum for up to three days. This might be owing to the purer perovskite films without any exposure to the ambient atmosphere. Though, the commonly degradation of the perovskite solar cell was apparent even with encapsulation. This could be inadequate to estimate the long-term stability of a photovoltaic material, which is essential to work for a long time at temperatures lower than the decomposition temperature [72]. The fact that inert condition and encapsulation cannot completely avoid $\mathrm{MAPbI}_{3}$ perovskite degradation. At low temperature, the degradation of $\mathrm{MAPbBr}_{3}$ was found by only releasing $\mathrm{HBr}$ and $\mathrm{CH}_{3} \mathrm{NH}_{2}$ gases [69]. The encapsulation of devices is essential not only to prevent exposure to oxygen and moisture, but also to avoid leakage of volatile decomposition products. Photostability can also be increased by replacing MA cation with more stable Cs/FA combination.

Substituting organic cations with inorganic $\mathrm{Cs}^{+}$or $\mathrm{Rb}^{+}$cations is also valuable to stabilize perovskite solar cells [73, 74]. Grancini et al. [74] stated an ultra-stable $2 \mathrm{D} / 3 \mathrm{D}\left(\mathrm{HOOC}\left(\mathrm{CH}_{2}\right)_{4} \mathrm{NH}_{3}\right)_{2} \mathrm{PbI}_{4} / \mathrm{CH}_{3} \mathrm{NH}_{3} \mathrm{PbI}_{3}$ perovskite, presenting a $\mathrm{PCE}$ of $12.9 \%$ with carbon electrodes and $14.6 \%$ with the normal mesoporous structure and stability of one-year.

By introducing n-butylammonium iodide (BAI) to $\mathrm{MAPbI}_{3}$ perovskite, a mixed $2 \mathrm{D}(\mathrm{BA})_{2} \mathrm{PbI}_{4}$ structure is formed, which probably provide an improved protection for the $3 \mathrm{D}$ perovskite against heat stress [75]. Octylammonium (OA) cation has also been reported to enhance the thermal stability of perovskites and keep $80 \%$ of their initial efficiency for $760 \mathrm{~h}$ aged at $85^{\circ} \mathrm{C}$ in ambient atmosphere without encapsulation [76]. Other additives, such as $\pi$-conjugated polymer, nonvolatile ionic liquids, bifunctional hydroxylamine hydrochloride guanidinium isothiocyanate, have also been reported to improve the thermal stability of various perovskites [77-79].

\section{Conclusion and perspective}

The discovery and development of organic inorganic perovskite materials have become a hot research topic in the field of photovoltaics. This chapter deals with a comprehensive discussion on the properties and applications of organic inorganic perovskites materials in PSCs. The extraordinarily outstanding performances of organic inorganic perovskites result of their excellent properties. Solar cells based 
on organic inorganic perovskite materials have achieved much advancement, both in PCE and stability, in a short very time. To PSCs, though great progress has been attained, there are still various obstacles in terms of stability and toxicity until its practical usage in the PV market. However, for large-scale performance, are required to be overcome. So far, great research has been made to overcome these issues by changing the composition of organic inorganic perovskite material either by replacing $\mathrm{Pb}$ with $\mathrm{Sn}$ or Ge or organic methyl with other organic or inorganic cations. However, commercialization of an organic inorganic perovskite solar cell needs further development in both efficiency and long-term stability, with low-cost photovoltaic materials and ease of printability. To increase stability, various methods such as the use of buffer layers, varying the composition of organic inorganic perovskite materials, and better techniques of encapsulation. In inference, the research which has been enduring for the past five years has attained significant results. Future research needs to endeavor for longer stability with high efficiency.

\section{Author details}

Madeeha Aslam, Tahira Mahmood* and Abdul Naeem

National Centre of Excellence in Physical Chemistry, University of Peshawar, Peshawar, Pakistan

*Address all correspondence to: tahiramahmood@uop.edu.pk

\section{IntechOpen}

(C) 2020 The Author(s). Licensee IntechOpen. This chapter is distributed under the terms of the Creative Commons Attribution License (http://creativecommons.org/licenses/ by/3.0), which permits unrestricted use, distribution, and reproduction in any medium, provided the original work is properly cited. (cc) BY 


\section{References}

[1] Shi PP, Lu SQ, Song XJ, Chen XG, Liao WQ Li PF, Tang YY, Xiong RG. Two-Dimensional Organic-Inorganic Perovskite Ferroelectric Semiconductors with Fluorinated Aromatic Spacers. J Am Chem Soc. 2019; 141: 18334-18340. DOI: $10.1021 /$ jacs.9b10048.

[2] Yang Z, Zhang WH. Organolead halide perovskite: A rising player in high-efficiency solar cells. Chin J Catalysis. 2014; 35: 983-988. DOI: 10.1016/S1872-2067(14)60162-5.

[3] Zhang C, Arumugam GM, Liu C, Hu J, Yang Y, Schropp REI, Mai Y. Inorganic halide perovskite materials and solar cells. APL Mater 2019;7. DOI: 10.1063/1.5117306.

[4] Mali SS, Patil JV, Hong CK. Making air-stable all-inorganic perovskite solar cells through dynamic hot-air. Nano Today. 2020; 33. DOI: 10.1016/j. nantod.2020.100880.

[5] Ji C, Liang C, Zhang H, Sun M, Song Q, Sun F, Feng X, Liu N, Gong H, Li D, You F, He Z. Secondary grain growth in organic-inorganic perovskite films with ethylamine hydrochloride additives for highly efficient solar cells. ACS Appl Mater Interfaces. 2020;1-30. DOI:10.1021/acsami.9b23468.

[6] Kandjani SA, Mirershad S, Nikniaz A. Inorganic-Organic Perovskite Solar Cells. In: editor. Solar Cells - New Approaches and Reviews. 10.5772/58970.

[7] Wang L, Fan B, Zheng B, Yang Z, Yin P, Huo L. Organic functional materials: recent advances in allinorganic perovskite solar cells. Sustainable Energy Fuels. 2020. DOI: 10.1039/DOSE00214C.

[8] Hadadian M, Smått J-H, CorreaBaena J-P. The role of carbon-based materials in enhancing the stability of perovskite solar cells. Energy Environ Sci. 2020; 13: 1377-1407.

[9] Yi Z, Ladi NH, Shai X, Li H, Shen Y, Wang M. Will organic-inorganic hybrid halide lead perovskites be eliminated from optoelectronic applications? Nanoscale Adv. 2019; 1:1276-1289. DOI: 10.1039/C8NA00416A.

[10] Bo Li, Fu L, Li S, Li H, Pan L, Wang L, Chang B, Yin L. Pathways toward high-performance inorganic perovskite solar cells: challenges and strategies. J Mater Chem A. 2019. DOI: 10.1039/C9TA04114A.

[11] Kojima A, Teshima K, Shirai Y, Miyasaka T. Organometal Halide Perovskites as Visible-Light Sensitizers for Photovoltaic Cells. J Am Chem Soc 2009; 131: 6050-6051. DOI:10.1021/ja809598r.

[12] Im JH, Lee CR, Lee JW, Park SW, Park NG. 6.5\% efficient perovskite quantum-dot-sensitized solar cell. Nanoscale. 2011; 3: 4088-4093.

DOI:10.1039/c1nr10867k.

[13] Kim H-S, Lee C-R, Im J-H, Lee K-B, Moehl T, Marchioro A, Moon S-J, Humphry-Baker R, Yum J-H, Moser JE, Gratzel M, Park N-G. Lead Iodide Perovskite Sensitized All-Solid-State Submicron Thin Film Mesoscopic Solar Cell with Efficiency Exceeding 9\%. Sci Rep. 2012; DOI: 2. 10.1038/srep00591.

[14] Venugopal G, Krishnamoorthy K, Mohan R, Kim S-J. An investigation of the electrical properties of graphene oxide films. Mater. Chem. Phys. 2012; 132: 29-33. DOI: 10.1016/j. matchemphys.2011.10.040.

[15] Song T-B, Chen Q, Zhou H, Jiang C, Wang H-H, Yang YM, Liu Y, You J, Yang Y. Perovskite solar cells: 
film formation and properties. J

Mater Chem A. 2015. DOI: 10.1039/

C4TA05246C.

[16] Urbina A. The balance between efficiency, stability and environmental impacts in perovskite solar cells: a review. J Phys Energy 2020;2. DOI: 10.1088/2515-7655/ab5eee.

[17] Zhou H, Chen Q, Li G, Luo S, Song T-b, Duan H-S, Hong Z, You J, Liu Y, Yang Y. Interface engineering of highly efficient perovskite solar cells. New York; 2014. Contract No: 6196.

[18] Ameen S, Akhtar MS, Seo H-K, Nazeeruddin MK, Shin H-S. An Insight into Atmospheric Plasma Jet Modified ZnO Quantum Dots Thin Film for Flexible Perovskite Solar Cell: Optoelectronic Transient and Charge Trapping Studies. J Phys Chem C 2015;119: 10379-10390.

[19] Thuat NT, Thoa BB, Tran NB, Tu Nm, Minh Nn, Huong Hnl, Trang PT, Van P.V.T, Tu TT, Linh DT. Fabrication of organolead iodide perovskite solar cells with niobium-doped titanium dioxide as compact layer. Comm. Phys. 2017; 27,121-130. DOI: 10.15625/0868-3166/27/2/9811.

[20] Mali SS, Patil JV, Kim H, Hong CK. Synthesis of $\mathrm{SnO}_{2}$ nanofibers and nanobelts electron transporting layer for efficient perovskite solar cells. Nanoscale. 2018. DOI:10.1039/ C8NR00695D.

[21] Zhou D, Zhou T, Tian Y, Zhu X, Tu Y. Perovskite-Based Solar Cells: Materials, Methods, and Future Perspectives. J Nanometer. 2018;2018. DOI:10.1155/2018/8148072.

[22] Vivo P, Salunke JK, Priimagi A. Hole-Transporting Materials for Printable Perovskite Solar Cells. Materials 2017;10: 1087. DOI:10.3390/ ma10091087.
[23] Mahmood T, Aslam M, Naeem A. Graphene/Metal Oxide Nanocomposite Usage as Photoanode in Dye-Sensitized and Perovskite Solar Cells. In: editor.

Reconfigurable Materials. United Kingdom: intechopen; 2020.

[24] Habisreutinger SN, Leijtens T, Eperon GE, Stranks SD, Nicholas RJ, Snaith HJ. Carbon Nanotube/Polymer Composites as a Highly Stable Hole Collection Layer in Perovskite Solar Cells. Nano Lett. 2014;14: 5561-5568.

[25] Chen Y, Zhang L, Zhang Y, Gao H, Yan H. Large-area perovskite solar cells - a review of recent progress and issues RSC Adv. 2018; 8: 10489-10508 DOI:10.1039/C8RA00384J.

[26] Fan J, Jia B, Gu M. Perovskite-based low-cost and high-efficiency hybrid halide solar cells. Photon Res. 2014; 2: 111-120. DOI: 10.1364/PRJ.2.000111.

[27] Schoonman J. Organic-inorganic lead halide perovskite solar cell materials: A possible stability problem. Chem Phys Lett. 2015; 619: 193-195.

DOI: 10.1016/j.cplett.2014.11.063.

[28] Shi Z, Jayatissa AH. PerovskitesBased Solar Cells: A Review of Recent Progress, Materials and Processing Methods. Materials (Basel). 2018; 11: 1-34. DOI:10.3390/ma11050729.

[29] Ava T, Al Mamun A, Marsillac S, Namkoong G. A Review: Thermal Stability of Methylammonium Lead Halide Based Perovskite Solar Cells. Applied Sciences. 2019; 9:188. DOI: 10.3390/app9010188

[30] Jiang Q Zhang X, You J. $\mathrm{SnO}_{2}$ : A Wonderful Electron Transport Layer for Perovskite Solar Cells. 2018. DOI: 10.1002/smll.201801154.

[31] Tan H, Jain A, Voznyy O, Lan X, Arquer FPGd, Fan JZ, Quintero-Bermudez R, 
Yuan M, Zhang B, Zhao Y, Fan F, Li P, Quan LN, Zhao Y, Lu Z-H, Yang Z, Hoogland S, Sargent EH. Efficient and stable solution-processed planar perovskite solar cells via contact passivation. Science. 2017; 355: 722-726.

[32] Dkhissi Y, Huang F, Rubanov S, Xiao M, Bach U, Spiccia L, Caruso RA, Cheng Y-B. Low temperature processing of flexible planar perovskite solar cells with efficiency over 10\%. J Power Sour. 2015;278: 325-331. DOI: 10.1016/j. jpowsour.2014.12.104.

[33] Diao X-F, Tang Y-l, Tang T-Y, Xie Q, Xiang K, Liu G-f. Study on the stability of organic-inorganic perovskite solar cell materials based on first principle. Molecul Phys. 2019. DOI:10.1080/00268 976.2019.1665200

[34] Boix PP, Nonomura K, Mathews N, Mhaisalkar SG. Current progress and future perspectives for organic/inorganic perovskite solar cells. Mater Today 2014; 17: 16-23. DOI: 10.1016/j.mattod.2013.12.002.

[35] Green MA, Ho-Baillie A, Snaith HJ. The emergence of perovskite solar cells Nat Photon. 2014 DOI:10.1038/ nphoton.2014.134

[36] Park N-G. Perovskite solar cells: an emerging photovoltaic technology. Mater Today. 2015;18 DOI: 10.1016/j. mattod. 2014.07.007

[37] Song Z, Watthage SC, Phillips AB, Heben MJ. Pathways toward highperformance perovskite solar cells: review of recent advances in organometal halide perovskites for photovoltaic applications. J Photon Energy. 2016; 6. DOI: 10.1117/1.JPE.6.022001

[38] Dymshits A, Henning A, Segev G, Rosenwaks Y, Etgar L. The electronic structure of metal oxide/ organo metal halide perovskite junctions in perovskite based solar cells. Sci Rep. 2015;5 1-6. DOI:10.1038/srep08704
[39] Li D, Shi J, Xu Y, Luo Y, Wu H, Meng Q. Inorganic-organic halide perovskites for new photovoltaic technology. Nat Sci Review 2018; 5: 559576. DOI: $10.1093 / \mathrm{nsr} / \mathrm{nw} x 100$

[40] Mitzi DB, Prikas MT, Chondroudis K. Thin Film Deposition of Organic-Inorganic Hybrid Materials Using a Single Source Thermal Ablation Technique. Chem Mater 1999; 11: 542-544.

[41] Burschka J, Pellet N, Moon SJ, Humphry-Baker R, Gao P, Nazeeruddin MK, Gratzel M. Sequential deposition as a route to highperformance perovskite-sensitized solar cells. Nature. 2013; 499: 316-319. DOI: 10.1038/nature12340

[42] Chen Q, Zhou H, Hong Z, Luo S, Duan H-S, Wang H-H, Liu Y, Li G, Yang Y. Planar Heterojunction Perovskite Solar Cells via Vapor-Assisted Solution Process. J Am Chem Soc. 2014; 136: 622-625. DOI:10.1021/ja411509g

[43] Era M, Morimoto S, Tsutsui T, Saito S. Organic inorganic heterostructure electroluminescent device using a layered perovskite semiconductor $\left(\mathrm{C}_{6} \mathrm{H}_{5} \mathrm{C}_{2} \mathrm{H}_{4} \mathrm{NH}_{3}\right)_{2}$ $\mathrm{PbI}_{4}$ Appl Phys Lett. 1994; 65. DOI: 10.1063/1.112265

[44] Liu M, Johnston MB, Snaith HJ. Efficient planar heterojunction perovskite solar cells by vapour deposition. Nature. 2013; 501: 395-398. DOI: $10.1038 /$ nature12509

[45] Leyden MR, Jiang Y, Qi Y. Chemical Vapor Deposition Grown Formamidinium Perovskite Solar Modules with High Steady State Power and Thermal Stability J Mater Chem A. 2016. DOI: 10.1039/C6TA04267H

[46] Banerjee D, Chattopadhyay KK. Hybrid Inorganic Organic Perovskites: A Low-Cost-Efficient Optoelectronic Material In: editor. Perovskite 
Photovoltaics. Elsevier;

2018. 123-162. DOI: $10.1016 /$

B978-0-12-812915-9.00005-8.

[47] Giacomo FD, Razza S, Matteocci F, D'Epifanio A, Licoccia S, Brown TM, Carlo AD. High efficiency CH3NH3PbI $\left.{ }_{3 \mathrm{x}}\right) \mathrm{Cl}_{\mathrm{x}}$ perovskite solar cells with poly(3-hexylthiophene) hole transport layer. J Power Sour. 2014; 251: 152-156.

[48] He M, Zheng D, Wang M, Lin C, Lin Z. High Efficiency Perovskite Solar Cells: From Complex Nanostructure to Planar Heterojunction. J Mater Chem A, 2013. DOI: $10.1039 / \mathrm{C} 3 \mathrm{TA} 14160 \mathrm{H}$

[49] Aharon S, Cohen BE, Etgar L. Hybrid Lead Halide Iodide and Lead Halide Bromide in Efficient Hole Conductor Free Perovskite Solar Cell. J Phys Chem C 2014; 118:17160-17165.

[50] Yang G, Chen C, Yao F, Chen Z, Zhang Q Zheng X, Ma J, Lei H, Qin P, Xiong L, Ke W, Li G, Yan Y, Fang G. Effective Carrier Concentration Tuning of $\mathrm{SnO}_{2}$ Quantum Dot Electron Selective Layers for High-Performance Planar Perovskite Solar Cells. Adv Mater. 2018. DOI:10.1002/adma.201706023.

[51] Anaraki EH, Kermanpur A, Steier L, Domanski K, Matsui T, Tress W, Saliba M, Abate A, Gratzel M, Hagfeldt A, Correa-Baena J-P. Highly efficient and stable planar perovskite solar cells by solution-processed tin oxide. Energy Environ Sci. 2016. DOI:10.1039/C6EE02390H.

[52] Xiong L, Qin M, Chen C, Wen J, Yang G, Guo Y, Ma J, Zhang Q, Qin P, Li S, Fang G. Fully High-TemperatureProcessed $\mathrm{SnO}_{2}$ as Blocking Layer and Scaffold for Efficient, Stable, and Hysteresis-Free Mesoporous Perovskite Solar Cells Adv Funct Mater. 2018. DOI:10.1002/adfm.201706276.

[53] Bi D, Yi C, Luo J, Décoppet J-D, Zhang F, Zakeeruddin SM, Li X, Hagfeldt A, Grätzel M. Polymertemplated nucleation and crystal growth of perovskite films for solar cells with efficiency greater than 21\%. Nat Energy. 2016. DOI: 10.1038/nenergy.2016.142.

[54] Chung I, Lee B, He J, Chang RPH, Kanatzidis MG. All-solidstate dye-sensitized solar cells with high efficiency. Nature. 2012; 486-490. DOI:10.1038/nature11067.

[55] Choi H, Jeong J, Kim H-B, Kim S, Walker B, Kim G-H, Kim JY. Cesiumdoped methylammonium lead iodide perovskite light absorber for hybrid solar cells. Nano Energy. 2014; 7: 80-85.

[56] Saliba M, Matsui T, Seo J-Y, Domanski K, Correa-Baena J-P, Nazeeruddin MK, Zakeeruddin SM, Tress W, Abate A, Hagfeldt A, Gratzel M. Cesium-containing triple cation perovskite solar cells: improved stability, reproducibility and high efficiency. Energy Environ Sci. 2016; 9: 1989-1997. DOI:10.1039/C5EE03874J

[57] Song Z, Wang C, Phillips AB, Grice CR, Zhao D, Yu Y,

Chen C, Li C, Yin X, Ellingson RJ, Heben MJ, Yan Y. Probing the Origins of Photodegradation in Organic-Inorganic Metal Halide Perovskites with TimeResolved Mass Spectrometry. Sustain Energy Fuel. 2018. DOI:10.1039/ C8SE00358K.

[58] Chen Z, Wang JJ, Ren Y, Yu C, Shum K. Schottky solar cells based on $\mathrm{CsSnI}_{3}$ thin-films. Phys Lett. 2012; 101. DOI:10.1063/1.4748888.

[59] Lv X, Xiao G, Feng X, Liu J, Yao X, Cao J. Acyl hydrazone-based porphyrin derivative as hole transport material for efficient and thermally stable perovskite solar cells Dyes and Pigments. 2018. DOI: 10.1016/j.dyepig.2018.08.056.

[60] Jiang J, Wang Q, Jin Z, Zhang X, Lei J, Bin H, Zhang Z-G, Li Y, Liu SF. Polymer Doping for High-Efficiency Perovskite Solar Cells with Improved 
Moisture Stability. Adv Energy Mater. 2017. DOI: 10.1002/aenm.201701757.

[61] Zhang J, Gao X, Deng Y, Zha Y, Yuan C. Comparison of life cycle environmental impacts of different perovskite solar cell systems. Sol Energy Mater \& Solar Cell 2017;166: 9-17. DOI: 10.1016/j.solmat.2017.03.008.

[62] Zhang S, Han G. Intrinsic and environmental stability issues of perovskite photovoltaics. Prog Energy 2020 2:1-44. DOI:10.1088/2516-1083/ ab70d9.

[63] Schileo G, Grancini G. Halide perovskites: current issues and new strategies to push material and device stability. J Phys: Energy 021005.

2020; 2: 1-12. DOI:10.1088/2515-7655/ ab6cc4

[64] Szostak R, Silva JC, Turren-Cruz S-H, Soares MM, Freitas Ro, Hagfeldt A, Tolentino HCN, Nogueira AF. Nanoscale mapping of chemical composition in organic-inorganic hybrid perovskite films. Sci Adv. 2019; 5: 1-7.

[65] Wang Q, Chen B, Liu Y, Deng Y, Bai Y, Dong Q, Huang J. Scaling Behavior of Moisture-induced Grain Degradation in Polycrystalline Hybrid Perovskite Thin Films. Energy Environ Sci. 2016. DOI:10.1039/C6EE02941H.

[66] Smith IC, Hoke ET, SolisIbarra D, McGehee MD, Karunadasa HI. A Layered Hybrid Perovskite Solar-Cell Absorber with Enhanced Moisture Stability. Angew Chem Int Ed. 2014;53: 1-5. DOI: 10.1002/anie.201406466

[67] Bryant D, Aristidou N, Pont S, Sanchez-Molina I, Chotchunangatchaval T, Wheeler S, Durrant JR, Haque SA. Light and oxygen induced degradation limits the operationalstabilityof methylammonium lead triiodide perovskite solar cells. Energy Environ Sci. 2016. DOI:10.1039/ C6EE00409A
[68] Motti SG, Meggiolaro D,

Barker AJ, Perini CAR, Ball JM, Angelis FD, Petrozza A, Gandini M, MosconiE, KimM.Controllingcompeting photochemical reactions stabilizes perovskite solar cells. Nature Photonics. 2019. DOI: $10.1038 / \mathrm{s} 41566-019-0435-1$

[69] Juarez-Perez EJ, Hawash Z, Raga SR, Ono LK, Qi Y. Thermal degradation of $\mathrm{CH} 3 \mathrm{NH} 3 \mathrm{PbI} 3$ perovskite into $\mathrm{NH} 3$ and $\mathrm{CH} 3 \mathrm{I}$ gases observed by coupled thermogravimetry-mass spectrometry analysis $\uparrow$ Energy Environ Sci. 2016;9. DOI: 10.1039/c6ee02016j

[70] JuarezPerez EJ, Ono LK, Maeda M, Jiang Y, Hawash Z, Qi Y. Photo-, Thermal-decomposition in Methylammonium Halide Lead Perovskites and inferred design principles to increase photovoltaic device stability. J Mater Chem A. 2018. DOI: 10.1039/C8TA03501F

[71] Yang J, Hong Q, Yuan Z, Xu R, Guo X, Xiong S, Liu X, Braun S, Li Y, Tang J, Duan C, Fahlman M, Bao Q. Unraveling Photostability of Mixed Cation Perovskite Films in Extreme Environment. Adv Optical Mater 2018. DOI: 10.1002/adom.201800262.

[72] Conings B, Drijkoningen J, Gauquelin N, Babayigit A, D’Haen J, D'Olieslaeger L, Ethirajan A, Verbeeck J, Manca J, Mosconi E, Angelis FD, Boyen H-G. Intrinsic Thermal Instability of Methylammonium Lead Trihalide Perovskite. Adv Energy Mater. 2015; 5. DOI: 10.1002/aenm.201500477.

[73] Saliba M, Matsui T, Domanski K, Seo J-Y, Ummadisingu A, Zakeeruddin SM, Correa-Baena J-P, Tress WR, Abate A, Hagfeldt A, Grätzel M. Incorporation of rubidium cations into perovskite solar cells improves photovoltaic performance. Science. 2016; 354: 206-209.

[74] Grancini G, Rolda'n-Carmona C, Zimmermann I, Mosconi E, X.Lee, 
Martineau D, Narbey S, Oswald F, AngelisFD, GraetzelM, NazeeruddinMK. One-Year stable perovskite solar cells by 2D/3D interface engineering. Nature Communications 2017; 8. DOI:10.1038/ ncomms15684.

[75] Lin Y, Bai Y, Fang Y, Chen Z, Yang S, Zheng X, Shi Tang, Liu Y, Zhao J, Huang J. Enhanced Thermal Stability in Perovskite Solar Cells by Assembling 2D/3D Stacking Structures. J Phys Chem Lett 2018; 9: 654-658. DOI: 10.1021/acs.jpclett.7b02679.

[76] Jung M, Shin TJ, Seo J, Kim G, Seok SI. Structural features and their functions in surfactant-armoured methylammonium lead iodide perovskites for highly efficient and stable solar cells. Energy Environ Sci. 2018. DOI: 10.1039/C8EE00995C.

[77] Du J, Wang Y, Zhang Y, Zhao G, Jia Y, Zhang X, Liu Y. Ionic LiquidAssisted Improvements in the Thermal Stability of CH3NH3PbI3 Perovskite Photovoltaics. Phys Status Solidi RRL 2018. DOI:10.1002/pssr.201800130

[78] Xia R, Fei Z, Drigo N, Bobbink FD, Huang Z, Jasiu-nas R, Franckevicius M, Gulbinas V, Mensi M, Fang X, Roldán-Carmona C, Nazeeruddin MK, Dyson PJ. Retarding Thermal Degradation in Hybrid Perovskites by Ionic Liquid Additives. Adv Funct. Mater. 2019. DOI:10.1002/ adfm.201902021

[79] Zou J, Liu W, Deng W, Lei G, Zeng S, Xiong J, Gu H, Hu Z, Wang X, Li J. An efficient guanidinium isothiocyanate additive for improving the photovoltaic performances and thermal stability of perovskite solar cells. Electrochim. Acta 2018. DOI: 10.1016/j.electacta.2018.08.117. 



\title{
Structural Phase Transitions of Hybrid Perovskites $\mathrm{CH}_{3} \mathrm{NH}_{3} \mathrm{PbX}_{3}$ $(\mathrm{X}=\mathrm{Br}, \mathrm{Cl})$ from Synchrotron and Neutron Diffraction Data
}

\author{
Carlos Alberto López, María Consuelo Alvarez-Galván, \\ Carmen Abia, María Teresa Fernández-Díaz and \\ José Antonio Alonso
}

\begin{abstract}
Methylammonium (MA) lead trihalide perovskites, that is, $\mathrm{CH}_{3} \mathrm{NH}_{3} \mathrm{PbX}_{3}$ (X = I, $\mathrm{Br}, \mathrm{Cl}$ ), have emerged as a new class of light-absorbing materials for photovoltaic applications. Indeed, since their implementation in solar-cell heterojunctions, they reached efficiencies above $23 \%$. From a crystallographic point of view, there are many open questions that should be addressed, including the role of the internal motion of methylammonium groups within $\mathrm{PbX}_{6}$ lattice under extreme conditions, such as low/high temperature or high pressure. For instance, in $\mathrm{MAPbBr}_{3}$ perovskites, the octahedral tilting can be induced upon cooling, lowering the space group from the aristotype $P m \overline{3} m$ to $I 4 / m c m$ and Pnma. The band gap engineering brought about by the chemical management of $\mathrm{MAPb}(\mathrm{Br}, \mathrm{Cl})_{3}$ perovskites has been controllably tuned: the gap progressively increases with the concentration of $\mathrm{Cl}$ ions from 2.1 to $2.9 \mathrm{eV}$. In this chapter, we review recent structural studies by state-ofthe-art techniques, relevant to the crystallographic characterization of these materials, in close relationship with their light-absorption properties.
\end{abstract}

Keywords: methyl ammonium (MA) lead trihalide perovskite, phase transition, octahedral tilting, MA orientation, Fourier synthesis, H location

\section{Introduction}

Organic-inorganic hybrid perovskites have risen as promising materials for a new generation of solar cells because of their ease of manufacturing and good performance, competing with the best photovoltaic devices based on silicon [1-5]. The introduction of $\mathrm{CH}_{3} \mathrm{NH}_{3} \mathrm{PbX}_{3}(\mathrm{X}=\mathrm{Br}$ and $\mathrm{I})$ as the absorber material in an electrolyte-based solar cell structure established the beginning of perovskitebased photovoltaics [2]. However, the power conversion efficiency (PCE) and cell stability were low due to the corrosion of the perovskites by the liquid electrolyte. The replacement of the liquid electrolyte with a solid hole-transporting material led to a key progress in 2012, resulting in both higher PCE and cell stability [3] . Subsequently, great efforts have been devoted to the improvement of these hybrid 
perovskite-based cells, including the development of a wide range of architectures, which allowed the PCEs to increase up to $23 \%$ within the past years [6].

These halide perovskites have been shown to be finer and to exhibit more suitable optical and electrical properties, like the adaptability of the band gap by introducing different halides (i.e., bromide, chloride, iodide) and its relative proportion in the perovskite [7-9], the high absorption coefficient [10], and the long lifetime of the photogenerated species [11].

$\mathrm{CH}_{3} \mathrm{NH}_{3} \mathrm{PbI}_{3}$-based perovskite solar cells have been more extensively investigated, due to their fast charge extraction rates and their near-complete visible light absorption, related to a relatively low band gap, around $1.6 \mathrm{eV}[11,12]$. However, the poor stability of $\mathrm{CH}_{3} \mathrm{NH}_{3} \mathrm{PbI}_{3}$ and rapid degradation in humidity has remained a drawback for its practical application, as a commercialized product [13-16]. $\mathrm{CH}_{3} \mathrm{NH}_{3} \mathrm{PbBr}_{3}$ constitutes a promising alternative, which presents a good charge transport in devices due to its long exciton diffusion length [17]. In addition, its cubic phase and low ionic mobility lead to a better stability under air and moist conditions, compared to the pseudo-cubic $\mathrm{CH}_{3} \mathrm{NH}_{3} \mathrm{PbI}_{3}$ phase, [7, 17-19]. Nevertheless, there are some undesirable features to be concerned about in these bromide-based perovskites; it is the case of a larger band gap $(2.2 \mathrm{eV})$, which decreases the solar light absorption $[20,21]$, the relatively large exciton binding energy and the reduced light absorption beyond its band edge at $550 \mathrm{~nm}$ (linked to its, previously indicated, larger band gap), associated with more limited efficiencies of $\mathrm{CH}_{3} \mathrm{NH}_{3} \mathrm{PbBr}_{3}$ solar cells [7, 18, 19, 22-24].

In parallel with the evaluation of the influence of a particular chemical composition of the perovskite, it is mandatory to determine the crystallographic structure under conditions in which the sample will be used. As other perovskite structures, $\mathrm{CH}_{3} \mathrm{NH}_{3} \mathrm{PbBr}_{3}$ consists of a framework constituted by corner-sharing $\mathrm{PbBr}_{6}$ octahedra, determining large cages where $\mathrm{CH}_{3} \mathrm{NH}_{3}{ }^{+}$units are located. In order to correlate the crystal structure of the perovskites with their electro-optical properties, it is necessary to exhaustively study the structural details, including the orientation of the methylammonium (MA) units within the perovskite cage in the course of phase transitions. Although synchrotron XRD data are essential, this can only be fully accomplished using neutrons as a probe due to the presence of protons. Usually, the MA configuration is linked to the rotation or tilting of the $\mathrm{PbX}_{6}$ octahedra; the more symmetric the octahedral framework is, the more delocalized appears the organic unit inside the inorganic cage. This information is essential to establish relationships between these structures and the macroscopic phenomenology (optical and physical properties when these compounds are used as optoelectronic materials).

$\mathrm{MAPbBr}_{3}$ was previously studied by diffraction techniques in single crystal form by $\mathrm{X}$-ray or in deuterated samples by neutron beams [25-27]. On the other hand, $\mathrm{CH}_{3} \mathrm{NH}_{3} \mathrm{PbCl}_{3}$ is also an alternative material that presents a wider band gap $(3.1 \mathrm{eV})$, being also sensitive to the UV region. Furthermore, this compound exhibits a fast photoresponse and long-term photostability, having a charge carrier concentration, mobility, and diffusion length comparable with the best-developed crystal structures of $\mathrm{CH}_{3} \mathrm{NH}_{3} \mathrm{PbI}_{3}$ and $\mathrm{CH}_{3} \mathrm{NH}_{3} \mathrm{PbBr}_{3}$ [28-30]. However, both $\mathrm{CH}_{3} \mathrm{NH}_{3} \mathrm{PbCl}_{3}$ and the mixed anion $\mathrm{CH}_{3} \mathrm{NH}_{3} \mathrm{~Pb}\left(\mathrm{Br}_{1-\mathrm{x}} \mathrm{Cl}_{\mathrm{x}}\right)_{3}$ have been less investigated [31].

In this chapter, we review our previous work on the $\mathrm{CH}_{3} \mathrm{NH}_{3} \mathrm{~Pb}\left(\mathrm{Br}_{1-\mathrm{x}} \mathrm{Cl}_{\mathrm{x}}\right)_{3}$ system, where we have investigated the crystallographic features in powdered, non-deuterated samples, using the mentioned state of the art techniques, neutron and synchrotron $\mathrm{X}$-ray diffraction [32-34]. The crystal structure of $\mathrm{MAPbBr}_{3}$ has been determined and refined at different temperatures, describing the evolution of the orientation of MA group in the 120-295 K temperature range. We found a partial delocalization in the cubic phase (at room temperature), where $\mathrm{C}$ and $\mathrm{N}$ atoms present large multiplicity positions, becoming progressively localized across the sequence cubic-tetragonal 
and finally orthorhombic, at $120 \mathrm{~K}$, with MA units fully oriented in the (101) plane [32]. Regarding to Cl-containing phases, a systematic study of the structural properties of hybrid mixed perovskites $\mathrm{CH}_{3} \mathrm{NH}_{3} \mathrm{~Pb}\left(\mathrm{Br}_{1-\mathrm{x}} \mathrm{Cl}_{\mathrm{x}}\right)_{3}$ by combining synchrotron $\mathrm{X}$-ray diffraction and UV-vis spectroscopic analyses reveals that the band gap can be chemically tuned according to the $\mathrm{Br} / \mathrm{Cl}$ ratio. The orientation of the organic MA units may also play an important role in the optoelectronic properties of these materials. By neutron powder diffraction, we found at RT three different orientations depending on the chlorine content and, therefore, on the unit-cell size. At lower temperatures, we unveiled that the halide disorder prevents the cooperative rearrangements needed to drive the octahedral $\mathrm{PbX}_{6}$ tiltings in intermediate $\mathrm{Br} / \mathrm{Cl}$ ratios; only $\mathrm{CH}_{3} \mathrm{NH}_{3} \mathrm{PbCl}_{3}$ underwent conspicuous phase transitions (cubic at room temperature, evolving to tetragonal and orthorhombic at $120 \mathrm{~K}$ ) [33]. H-bond interactions with the halide ions stabilize these conformations, in accordance to reported theoretical calculations [34].

\section{Crystal growth}

The crystal growth of $\mathrm{CH}_{3} \mathrm{NH}_{3} \mathrm{~Pb}\left(\mathrm{Br}_{1-\mathrm{x}} \mathrm{Cl}_{\mathrm{x}}\right)_{3}(\mathrm{x}=0,0.33,0.5,0.67$ and 1) [32-34] was made from stoichiometric amounts of $\mathrm{CH}_{3} \mathrm{NH}_{3} \mathrm{X}$ and $\mathrm{PbX}_{2}(\mathrm{X}=\mathrm{Cl}, \mathrm{Br})$. Previously, the methyl ammonium bromide and methyl ammonium chloride were synthesized from methyl amine $\left(\mathrm{CH}_{3} \mathrm{NH}_{2}\right)$ and the corresponding acid $\mathrm{HBr}$ and $\mathrm{HCl}$, respectively, according to the following reaction:

$$
\mathrm{CH}_{3} \mathrm{NH}_{2}+\mathrm{HX} \rightarrow \mathrm{CH}_{3} \mathrm{NH}_{3} \mathrm{X} \text { (from } \mathrm{X}=\mathrm{Br} \text { to } \mathrm{Cl} \text { ) }
$$

Then, the obtained methyl ammonium halides were reacted with the lead halide in stoichiometric amounts in dimethyl formamide (DMF) according to the following reaction:

$$
\mathrm{CH}_{3} \mathrm{NH}_{3} \mathrm{X}+\mathrm{PbX}_{2} \rightarrow \mathrm{CH}_{3} \mathrm{NH}_{3} \mathrm{PbX}_{3} \text { (from } \mathrm{X}=\mathrm{Br} \text { to } \mathrm{Cl} \text { ) }
$$

From this procedure, the mixed halide perovskites were obtained as well crystallized materials, showing crystals of variable sizes and colors, varying from orange for $\mathrm{CH}_{3} \mathrm{NH}_{3} \mathrm{PbBr}_{3}$ to white for $\mathrm{CH}_{3} \mathrm{NH}_{3} \mathrm{PbCl}_{3}$, adopting progressively paler hues of yellow as $\mathrm{Cl}$ content increases, as shown in the optical microscope images included in Figure 1. The effect of halide composition on the morphology and structure of

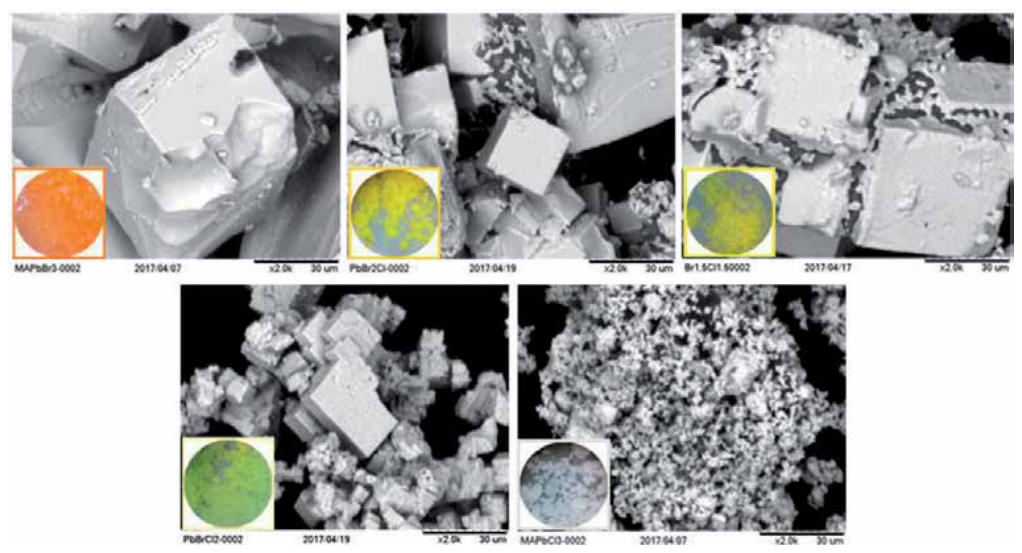

Figure 1.

SEM images of the mixed perovskites and optical microscope images of as-grown $\mathrm{CH}_{3} \mathrm{NH}_{3} \mathrm{PbX}$ (from $\mathrm{X}=\mathrm{Br}$ to $\mathrm{Cl}$ ) perovskites. The insets show the color variation as $\mathrm{Cl}$ is introduced. 
crystals was observed in SEM images as is also shown in Figure 1. In all cases, the obtained perovskites show cuboid-type microcrystals. The content of chloride induces a decrease in the size of the crystals of the mixed perovskites.

\section{Synchrotron X-ray diffraction studies}

Synchrotron X-ray diffraction (SXRD) technique provides an extreme angular resolution of the patterns, useful to define the symmetry of the different phases and to determine its evolution below room temperature (RT). The crystal structures at RT are cubic, and they are well defined in the space group $P m \overline{3} m$. In this model, the lead and bromine atoms are placed in $1 a(0,0,0)$ and $3 d(1 / 2,0,0)$ Wyckoff sites, respectively; and the organic unit is positioned in $1 b(1 / 2,1 / 2,1 / 2)$. Figure 2a shows selected reflections of the Rietveld refinements corresponding to $\mathrm{x}=0$ in comparison with $\mathrm{x}=0.33,0.5,0.67$, and 1 members at RT. Figure $\mathbf{2 b}$ and $\mathbf{c}$ plot the unit-cell parameter variation and the anisotropic atomic displacement parameters (ADPs) of $\mathrm{X}$ site, respectively. The unit-cell parameters exhibit an expected reduction as the amount of $\mathrm{Cl}$ increases, but this change is not linear. As shown in the Figure 2c, the disks perpendicular to the $\mathrm{Pb}-\mathrm{X}-\mathrm{Pb}$ bonds exhibit an oblate shape, meaning the ADPs of X atoms are considerably anisotropic. Since the thermal vibrations in this direction are allowed in perovskites, this behavior is not surprising. However, for the intermediate mixed halide phases $(\mathrm{x}=0.33,0.5$, and 0.67$)$, the ADPs show a nonmonotonic variation compared to both end members $(x=0$ and 1$)$, although the difference does not overcome two times the standard deviations and is less significant.

These anomalies were assigned to the structural disorder introduced by the mixture of halides, for $\mathrm{x}=0.33,0.5$, and 0.67 . The ADPs should account for the structural
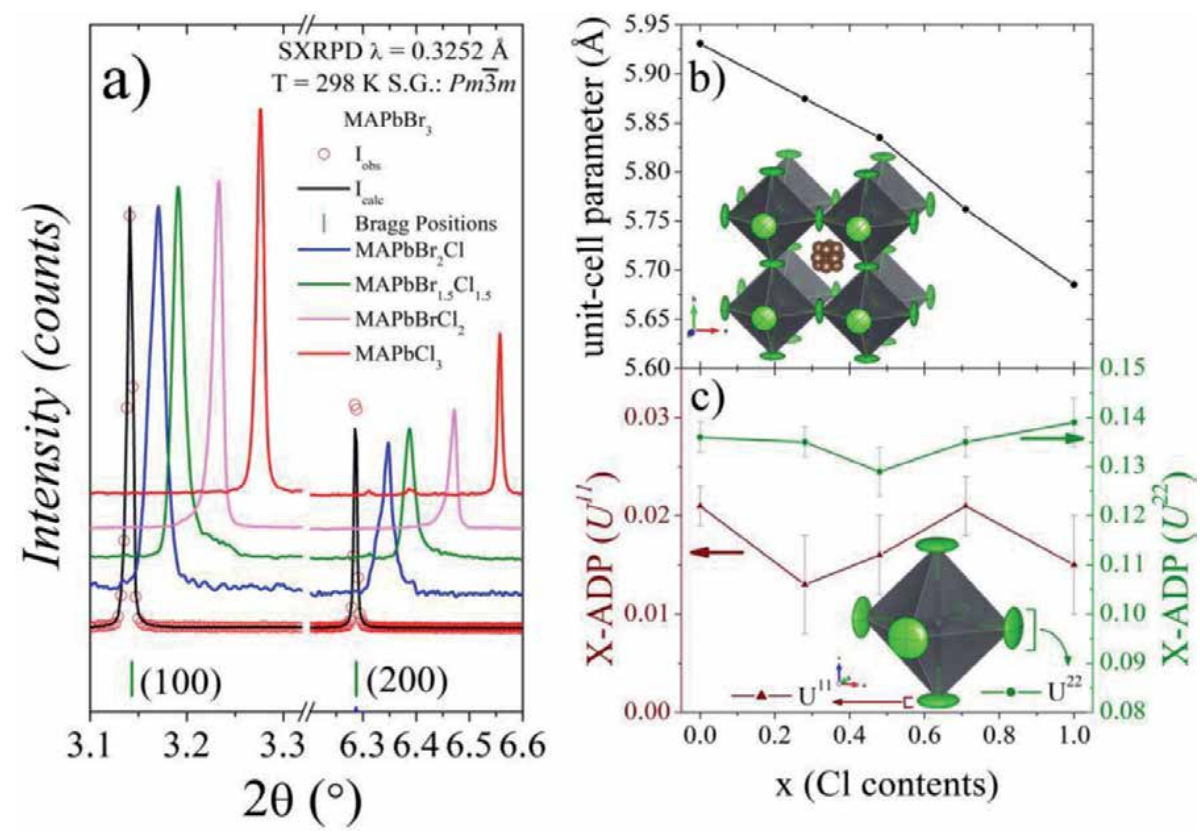

Figure 2.

(a) SXRD profiles for $\mathrm{CH}_{3} \mathrm{NH}_{3} \mathrm{PbBr}_{3}$ at RT, after a pattern matching showing the characteristic perovskite peaks and the absence of impurities. Red circles are the experimental points, the black full line is the calculated profile. The green vertical marks represent the allowed Bragg positions in the Pm ${ }^{-} 3 \mathrm{~m}$ space group. The $\mathrm{Cl}$-doped patterns are added in this plot to compare with the bromide parent. (b) Unit-cell parameters evolution and $(c)$ variation of the anisotropic atomic displacement parameters $(A D P)$ of $X$ site with the $C l$ contents. 


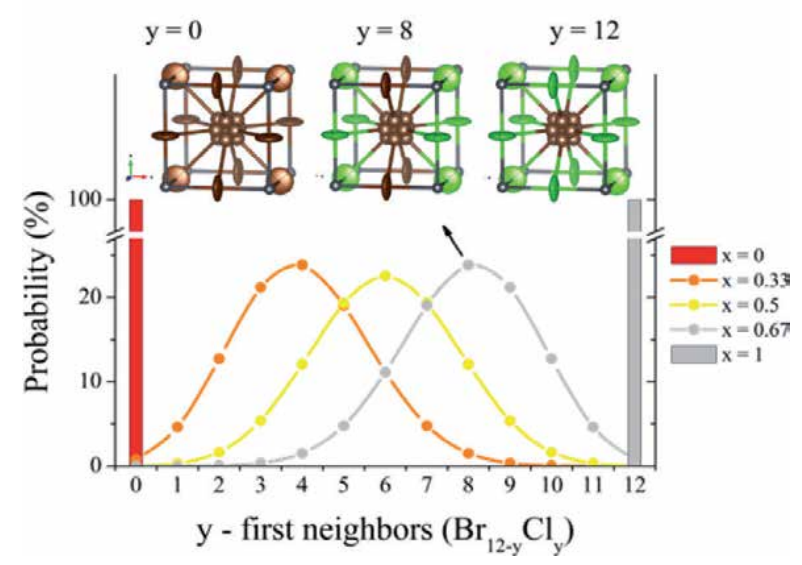

Figure 3.

Statistical probability distribution of the halide environment in $\mathrm{CH}_{3} \mathrm{NH}_{3} \mathrm{~Pb}\left(\mathrm{Cl}_{1-x} \mathrm{Br}_{x}\right)_{3}(x=0,0.33,0.5,0.67$, and 1).

disorder, which can also generate a perturbation in the interactions between the inorganic $\mathrm{PbX}_{6}$ skeleton and the methyl-ammonium units. This perturbation is absent in $\mathrm{MAPbBr}_{3}$ and $\mathrm{MAPbCl}_{3}$, containing single halide ions. In Figure 3, a statistical probability distribution of the halide environment of MA groups is plotted, showing this behavior for the different samples $\left(\mathrm{CH}_{3} \mathrm{NH}_{3} \mathrm{~Pb}\left(\mathrm{Cl}_{1-\mathrm{x}} \mathrm{Br}_{\mathrm{x}}\right)_{3}, \mathrm{x}=0,0.33,0.5,0.67\right.$, and 1). It is remarkable that for $\mathrm{MAPbBr}_{3}$ and $\mathrm{MAPbCl}_{3}$ the probability is $100 \%$, because all of their MA units are coordinated to $12 \mathrm{Br}$ or $\mathrm{Cl}$ atoms; this state contrasts with the mixed halide situations $(\mathrm{x}=0.33,0.5$, and 0.67$)$. These distributions reveal the high structural disorder given in mixed situations, in contrast to both end members. These probably induce tensions in the lattice preventing a linear behavior between the pure bromine and chlorine compounds. The inserts in Figure 3 include illustrative schemes of the extreme situations in comparison with an intermediate case where the MA is coordinated to eight chlorides and four bromides, $y=8$.

The thermal variation of the crystallographic structures was followed between $120 \mathrm{~K}$ and RT. Figure 4 shows the temperature evolution of selected diffraction

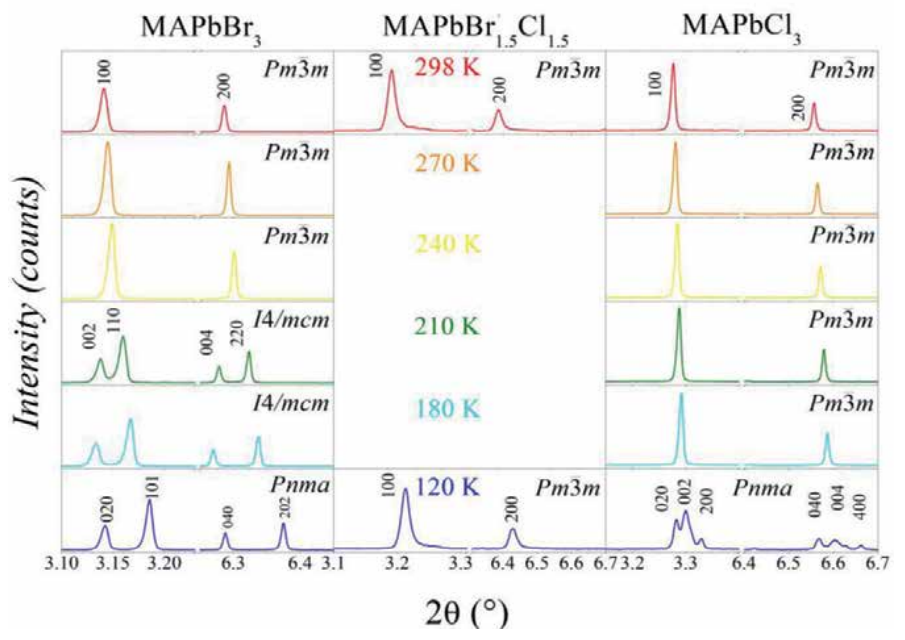

Figure 4 .

Thermal evolution of selected diffraction lines in which the phase transitions are evidenced, from SXRD data, collected at MSPD diffractometer at ALBA synchrotron (Spain) [32-34]. 
lines for all members of the series. In this temperature range, only both end members of the series ( $x=0$ and 1 ) exhibit phase transitions; in contrast, for the mixed halide compositions, no phase transitions have been detected, observing cubic structures $(P m \overline{3} m)$ either at RT or down to $120 \mathrm{~K}$, as illustrated in Figure 4.

Previous works also report on the polymorphic evolution of $\mathrm{Br}$ and $\mathrm{Cl}$ phases. Swainson et al. report on two phase transitions for $\mathrm{MAPbBr}_{3}$ : Pnma $\leftarrow(\approx 150 \mathrm{~K}) \rightarrow I 4 / m c m \leftarrow(\approx 223 \mathrm{~K}) \rightarrow P m \overline{3} m[25]$. These phases were also described by other authors from single crystal data [26, 27]. It was reported by Poglitsch et al. that the $\mathrm{MAPbCl}_{3}$ perovskite goes through two phase transitions: $P 222_{1} \leftarrow(173 \mathrm{~K}) \rightarrow P 4 / m m m \leftarrow(179 \mathrm{~K}) \rightarrow P m \overline{3} m$ [35]. Afterward, Chi et al. stated that the orthorhombic polymorph corresponds to the Pnma space group, but with a unit-cell twice the size of the cubic aristotype $\left(\mathrm{a} \approx \mathrm{b} \approx \mathrm{c} \approx 2 \mathrm{a}_{\mathrm{p}}\right)$ [36].

Our work on $\mathrm{CH}_{3} \mathrm{NH}_{3} \mathrm{PbBr}_{3}$ shows that the SXRD patterns collected at RT, 270 and $240 \mathrm{~K}$ correspond to cubic symmetry, defined in the $P m \overline{3} m$ space group; at 210 and $180 \mathrm{~K}$ to tetragonal symmetry in the $I 4 / \mathrm{mcm}$ space group; and at $120 \mathrm{~K}$ to orthorhombic symmetry, defined in the Pnma space group [32]. On the other hand, $\mathrm{CH}_{3} \mathrm{NH}_{3} \mathrm{PbCl}_{3}$ remains stable as cubic down to $180 \mathrm{~K}$; however, the SXRD patterns at 120 and $150 \mathrm{~K}$ exhibit a conspicuous splitting of some reflections [33]. According to the model proposed by Chi et al. [36]; this splitting (at 120 and $150 \mathrm{~K}$ ) is possible. Nevertheless, some extra lines during the preliminary refinements seem to indicate that there is another phase, orthorhombic (Pnma), resembling the one observed in $\operatorname{MAPbBr}_{3}\left(a \approx \sqrt{ } 2 a_{\mathrm{p}} ; b \approx 2 a_{\mathrm{p}} ; c \approx \sqrt{2} a_{\mathrm{p}}\right)$. If the coexistence of both phases mentioned is taken into consideration, a satisfactory refinement at 150 and $120 \mathrm{~K}$ can be completed, with only slight differences in the peak width; being wider for the case of the conventional Pnma phase compared to the doubled Pnma structure. A possible explanation could be that microstructural features cause this phase mixture.

For $\mathrm{MAPbCl}_{3}$, the mechanism or transient state from cubic to orthorhombic symmetry has been until now only partially known. As indicated previously, the tetragonal phase $(P 4 / \mathrm{mmm})$ was observed in a very narrow range of temperature, 172.9-178.8 K. Up to now, no new reports have appeared on this transient tetragonal phase. Several patterns were collected sequentially [33] in this narrow temperature range, with intervals of $2.5 \mathrm{~K}$, as illustrated in Figure 5a, where a different phase is evidenced between 169 and $164 \mathrm{~K}$. This phase, corresponding to the pattern at $167.2 \mathrm{~K}$, was initially fitted to the tetragonal model; however, additional diffraction lines were observed evidencing an orthorhombic symmetry. Matching the patterns observed at 120,150 , and $160 \mathrm{~K}$, Pnma $\left(a \approx \sqrt{ } 2 a_{\mathrm{p}} ; b \approx 2 a_{\mathrm{p}} ; c \approx \sqrt{ } 2 a_{\mathrm{p}}\right)$ provided a satisfactory fit at $167.2 \mathrm{~K}$. In this last case, at low temperatures the unit-cell
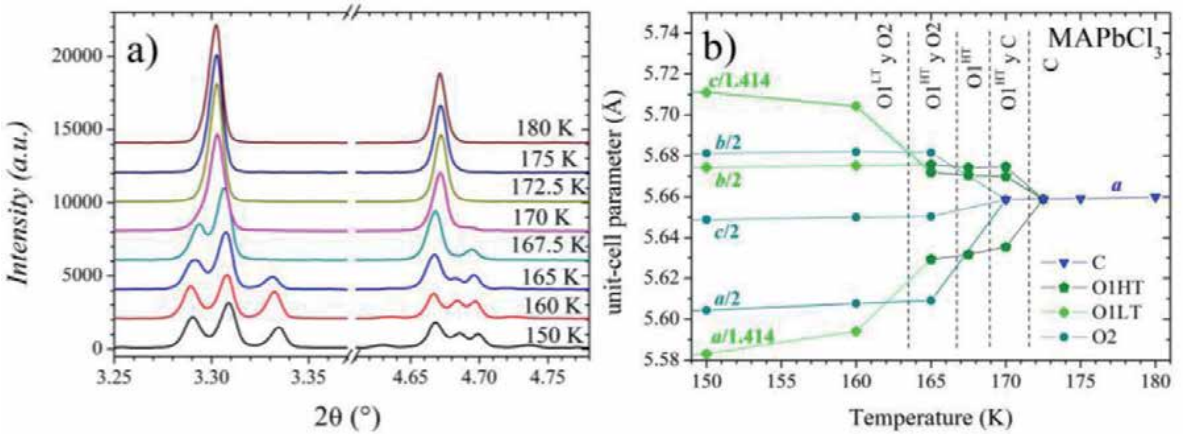

Figure 5.

(a) Selected angular region of the SXRD patterns of $\mathrm{MAPbCl}_{3}$ at increasing temperatures (from 150 to $180 \mathrm{~K}$ ), illustrating the evolution and splitting of some characteristic reflections. (b) Thermal evolution of the unit-cell parameters of the $\mathrm{MAPbCl}_{3}$ perovskite in the same temperature range [33]. 


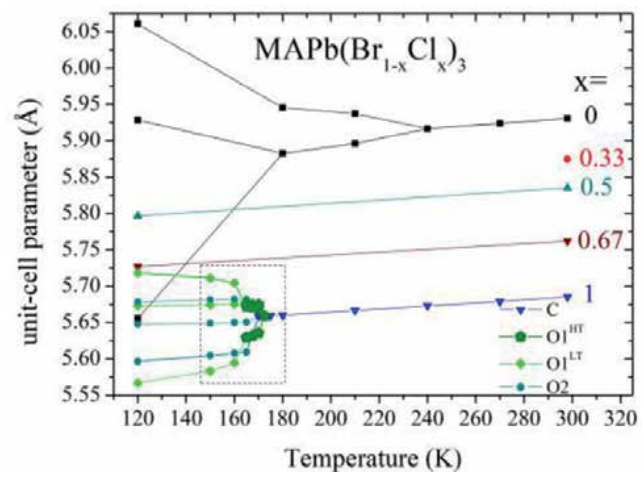

Figure 6.

Thermal evolution of the unit-cell parameters of $M A P b\left(B r_{1-x} C l_{x}\right)_{3}$ perovskites in the 120-300 K temperature range [33].

parameters tend to be considerably less split. In Figure 5b, the unit-cell parameters variation as a function of temperature is displayed in this narrow temperature range.

From these facts, it is clear that $\mathrm{MAPbCl}_{3}$ goes through a more complex transition compared to the ones already reported. To summarize, there are three phases: cubic, orthorhombic $\left(a \approx \sqrt{ } 2 a_{\mathrm{p}} ; b \approx 2 a_{\mathrm{p}} ; c \approx \sqrt{ } 2 a_{\mathrm{p}}\right)$, and a second orthorhombic $\left(a \approx 2 a_{\mathrm{p}} ; b \approx 2 a_{\mathrm{p}} ; c \approx 2 a_{\mathrm{p}}\right.$ ), as indicated in Figure 5b as C, O1, and O2, respectively. Moreover, the $\mathrm{O} 1$ phase can be separated into two states with different distorted degrees: $\mathrm{O} 1^{\mathrm{HT}}$ and $\mathrm{O} 1^{\mathrm{LT}}$. In the first case, the unit-cell parameters splitting with respect to cubic $a_{\mathrm{p}}$ is substantially lower than in the second case. Moreover, an inversion between $b / 2$ and $c / \sqrt{ } 2$ values is observed in Figure $5 \mathbf{b}$. Additionally, a high tetragonality is observed in $\mathrm{O}^{\mathrm{HT}}$ where the following relationship between the unit-cell parameters is observed: $a / \sqrt{ } 2<b / 2 \approx c / \sqrt{ } 2$. This fact can explain the previous description in the tetragonal symmetry in this short temperature range.

As reported in [32, 33], $\mathrm{MAPbCl}_{3}$ and $\mathrm{MAPbBr}_{3}$ are stable above 179 and $237 \mathrm{~K}$ respectively; therefore, it is relevant to mention the lack of any other phase transition down to $120 \mathrm{~K}$ in mixed halide perovskites. The anion disorder in the mixed-halide phases could explain this surprising behavior. As it was previously mentioned, the interactions between the organic and inorganic parts differ from one sample to another within the present series (see Figure 3). The phase transitions are prevented due to the halide disorder, because it does not allow the rearrangements that are required in terms of octahedral tilting. Finally, SXRD measurements and their analysis (Rietveld refinements) provide new evidence that allows to complete the polymorphic evolution in the $\operatorname{MAPb}\left(\mathrm{Br}_{1-\mathrm{x}} \mathrm{Cl}_{\mathrm{x}}\right)_{3}$ family, as displayed in Figure 6.

\section{Neutron diffraction studies}

The neutron powder diffraction (NPD) investigation is essential to obtain a detailed description of these hybrid materials. The NPD data were collected at the D2B diffractometer (ILL, France) $[32,34]$. In particular, the distribution of MA groups can be elucidated taking advantage of the high contrast in the coherent scattering lengths of $\mathrm{Pb}, \mathrm{Br}, \mathrm{Cl}, \mathrm{C}, \mathrm{N}$, and $\mathrm{H}$, of $9.405,6.795,9.577,6.646,9.36$, and $-3.739 \mathrm{fm}$, respectively.

However, there is an issue in the resolution of the structure that needs to be considered. The incoherent background in a powder experiment is considerably large due to the presence of hydrogen, which has an important inelastic component 
(25,274 barns) that gives rise to a significant incoherent background. This is not an obstacle for the crystallographic Rietveld refinement if sufficient statistic is achieved during the measurement.

On the other hand, due to the negative contribution of hydrogen to the scattering, some strategies are necessary to find the adequate distribution of MA in the inorganic $\mathrm{PbX}_{6}$ framework. First, the instrumental and the unit-cell parameters were refined using the Le Bail method. The structure of the octahedral $\mathrm{PbX}_{6}$ framework was eventually considered in the model, placing the lead atoms at $1 a(0,0,0)$ sites and bromine/chlorine atoms at $3 d(1 / 2,0,0)$ positions in the $P m \overline{3} m$ space group. Later on, Difference Fourier Maps (DFM) were acquired from the observed and calculated patterns, unveiling the missing negative and positive nuclear density (in scattering length) around A site of the perovskite structure $(1 b(1 / 2,1 / 2,1 / 2))$.

This analysis in $\mathrm{MAPbBr}_{3}$ [32] yields the positive (represented in yellow) and negative (represented in blue) isosurfaces shown in Figure 7a, which correspond to the $\mathrm{C} / \mathrm{N}$ and $\mathrm{H}$ positions, respectively. The negative scattering regions are due to the negative scattering of protons while the positive zone corresponds to $\mathrm{C} / \mathrm{N}$ atoms. These nuclear densities support that the MA units are delocalized in the A site of the perovskite. Considering the positive density, the $\mathrm{C} / \mathrm{N}$ atoms are located at $24 i(1 / 2, y, y)$ positions. Then, the $\mathrm{H}$ positions can be elucidated from the geometric shape of methylammonium group. The observed geometry can be satisfied with two hydrogen atoms located at $24 l(1 / 2, y, z)$ and $24 m(x, x, z)$ Wyckoff sites. This analysis in $\mathrm{MAPbBr}_{3}$ reveals that the MA group is delocalized at room temperature along the [110] direction, involving six possible orientations.

In contrast, the $\mathrm{DFM}$ for $\mathrm{MAPbBr}_{2} \mathrm{Cl}$ show that the positive and negative densities match exactly with those expected for the MA cations oriented along [111] directions. It is possible to deduce that the $\mathrm{C} / \mathrm{N}$ and $\mathrm{H}$ atoms are placed at $8 g(x, x, x)$ and $24 m(x, x, z)$ Wyckoff positions, respectively (Figure 7b). $\mathrm{MAPbBr}_{1.5} \mathrm{Cl}_{1.5}$ exhibits a peculiar negative distribution (see Figure 7c), where there are four negative zones along the [001] directions, suggesting that the MA units are along this direction. However, along [111] directions, there also appears a non-negligible density, which is unrealistic. This dichotomy is resolved by analyzing the $\mathrm{C} / \mathrm{N}$ density from the positive surface; it unveils that the $\mathrm{C} / \mathrm{N}$ atoms are indeed delocalized along the [100]
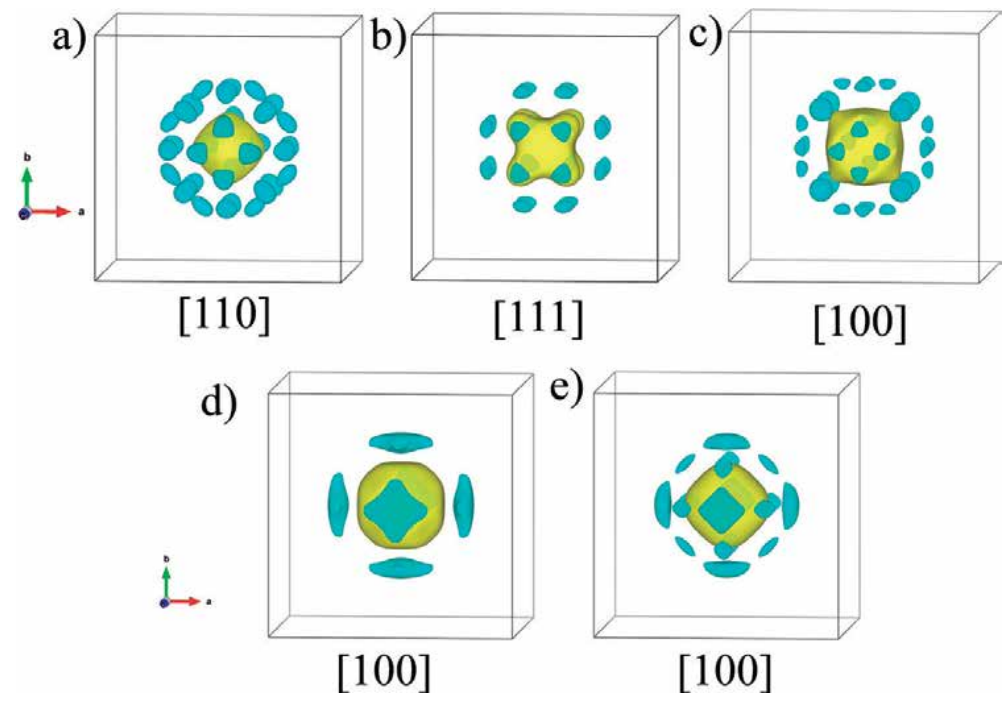

Figure 7.

DFM isosurfaces of $M A P b\left(B r_{1-x} C l_{x}\right)_{3}$ series for $x=o(a), 0.33(b), 0.5(c), 0.67(d)$, and $1(e)$, from NPD data $[32,34]$. 
directions. The same procedure in $\mathrm{MAPbBrCl}_{2}$ and $\mathrm{MAPbCl}_{3}$, see Figure $7 \mathbf{d}$ and $\mathbf{e}$, shows that the MA groups are oriented along [100] directions, in the same way as $\mathrm{MAPbBr}_{1.5} \mathrm{Cl}_{1.5}$. From this result, which only can be obtained from NPD data, a clear restriction in the MA delocalization is unveiled, evolving from [110] to [111] and finally to [100] directions, while $\mathrm{X}$ is progressively enriched in $\mathrm{Cl}^{-}$anions.

From the Fourier synthesis maps, a crystallographic model can be built with the MA configurations, to start the structural Rietveld refinements from the NPD data. Additionally, the MA displacement toward $-\mathrm{NH}_{3}$ group also can be considered, as it was proposed from theoretical calculations $[37,38]$. This last approach corresponds to the plausible chemical interactions between the organic cation and inorganic framework; the greater electronegativity of nitrogen produces $\mathrm{H}$-bond interactions shorter than $\mathrm{C}-\mathrm{H} \cdots \mathrm{X}$. Considering this last fact and the DFM results, the Rietveld refinement of NPD data was made. Minor amounts of MACl were identified and included in the refinements as second phase in the chlorine phase. The Rietveld fits for $\mathrm{MAPbBr}_{3}$ and $\mathrm{MAPbCl}_{3}$ are illustrated in Figure 8. The crystal structure data after these refinements are listed in Table 1 for all the compositions. This table shows the three different combinations of $\mathrm{C} / \mathrm{N}$ and $\mathrm{H}$ positions considering three possibilities for the MA delocalization in the $\mathrm{PbX}_{6}$ framework observed in the $\mathrm{MAPb}\left(\mathrm{Br}_{1-\mathrm{x}} \mathrm{Cl}_{\mathrm{x}}\right)_{3}$ series. The variation of the unit-cell parameter with the $\mathrm{Cl}$-doping is illustrated in Figure 9; here, a change of slope is observed in the perovskite with an equimolar amount of $\mathrm{Br}$ and $\mathrm{Cl}$ atoms, according to the anomaly obtained from synchrotron data.

For $\mathrm{x}=0.5,0.67$, and 1 , the unit cell experiences a contraction, keeping a constant slope as $\mathrm{Cl}$ is introduced, with the MA oriented in the same direction. On the other hand, for $\mathrm{x}=0$ and 0.33 , the unit cell parameters do not follow this trend, the MA orientation is different.

A feature to highlight is the difference in negative density (arising from $\mathrm{H}$ positions) observed between [111] and [100] orientations. While along the [111] direction, the three terminal $\mathrm{H}$ atoms of the $\mathrm{H}_{3} \mathrm{C}-\mathrm{NH}_{3}{ }^{+}$units are fixed, as it is shown in Figure $7 \mathbf{b}$. From the negative density displayed in Figure 7c-e, it is noticeable that four $\mathrm{H}$ atoms are comprised in the (100) plane, suggesting that the MA units occupy equivalent positions, so that each $\mathrm{H}$ points to each of the four halides in the edges of the perovskite unit cell, thus manifesting the importance of $\mathrm{H} \cdots \mathrm{X}$ hydrogen bonds. This bonded $\mathrm{H}$ is located in $\mathrm{H} 11$ site and the other two $\mathrm{H}$ of the amine group are located in the $\mathrm{H} 12$ site. Similarly, the methyl group is formed by C2, H21, and H22 atoms.
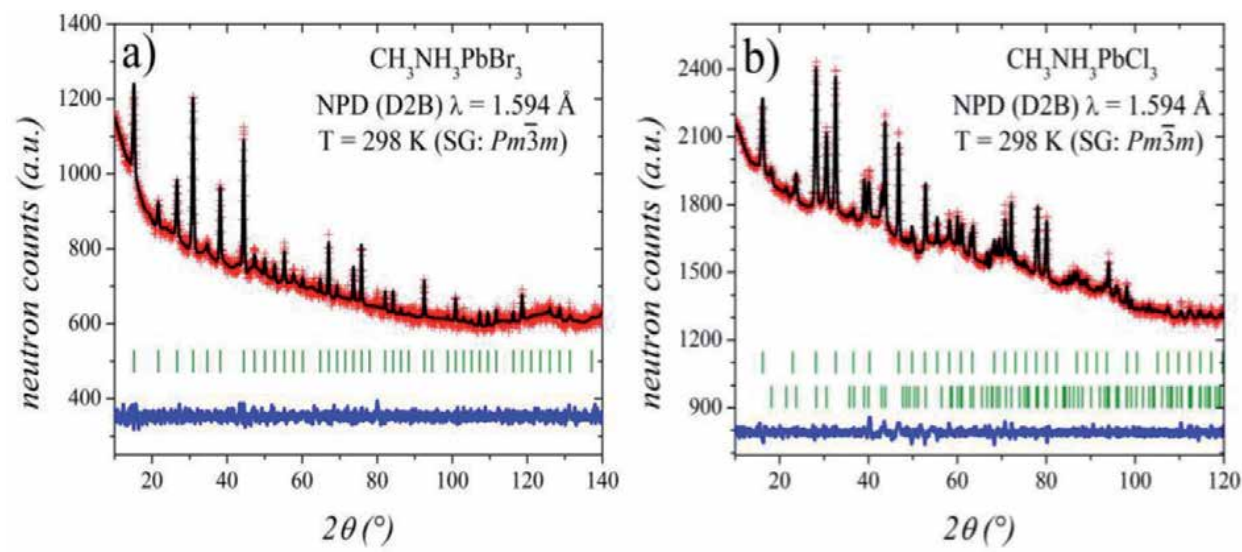

Figure 8.

Rietveld plots of a) $\mathrm{MAPbBr}_{3}$ and b) $\mathrm{PbPbCl}_{3}$ from NPD data, at RT. 


\begin{tabular}{|c|c|c|c|c|c|}
\hline $\operatorname{MAPb}\left(\mathrm{Br}_{1-\mathrm{x}} \mathrm{Cl}_{\mathrm{x}}\right)_{3}$ & $x=0$ [32] & $\begin{array}{c}x=0.33 \\
{[34]}\end{array}$ & $x=0.5[34]$ & $x=0.67[34]$ & $x=1[34]$ \\
\hline$a(\AA)$ & $5.9259(4)$ & $5.8454(8)$ & $5.80459(6)$ & $5.76955(7)$ & $5.6813(5)$ \\
\hline \multicolumn{6}{|l|}{$\begin{array}{l}\mathrm{Pb}(1 a) \\
(0,0,0)\end{array}$} \\
\hline $\mathrm{U}^{11}=\mathrm{U}^{22}=\mathrm{U}^{33}$ & $0.025(2)$ & $0.048(3)$ & $0.0313(3)$ & $0.031(2)$ & $0.0239(4)$ \\
\hline \multicolumn{6}{|l|}{$\begin{array}{l}\mathrm{Br}_{1-\mathrm{x}} \mathrm{Cl}_{\mathrm{x}}(3 d) \\
(0.5,0,0)\end{array}$} \\
\hline$x$ & 0 & $0.38(3)$ & $0.55(5)$ & $0.67(8)$ & 1 \\
\hline $\mathrm{U}^{11}$ & $0.018(5)$ & $0.028(5)$ & $0.0147(5)$ & $0.026(4)$ & $0.0471(8)$ \\
\hline $\mathrm{U}^{22}=\mathrm{U}^{33}$ & $0.138(5)$ & $0.130(5)$ & $0.1318(6)$ & $0.104(4)$ & $0.1151(7)$ \\
\hline \multirow[t]{2}{*}{$\mathrm{N} / \mathrm{C}$} & $\begin{array}{c}\mathrm{C} / \mathrm{N} \\
(0.5, y, y) \\
0.407(2)\end{array}$ & $\begin{array}{l}\mathrm{N} 1(x, x, x) \\
0.5809(5)\end{array}$ & $\begin{array}{c}\mathrm{N} 1(0.5,0.5, z) \\
0.6290(4)\end{array}$ & $\begin{array}{c}\mathrm{N} 1(0.5,0.5, z) \\
0.6294(5)\end{array}$ & $\begin{array}{c}\mathrm{N} 1(0.5,0.5, z) \\
0.6594(5)\end{array}$ \\
\hline & & $\begin{array}{l}\mathrm{C} 2(x, x, x) \\
0.4378(5)\end{array}$ & $\begin{array}{c}\mathrm{C} 2(0.5,0.5, z) \\
0.3777(6)\end{array}$ & $\begin{array}{c}\mathrm{C} 2(0.5,0.5, z) \\
0.3782(6)\end{array}$ & $\begin{array}{c}\mathrm{C} 2(0.5,0.5, z) \\
0.4113(5)\end{array}$ \\
\hline $\mathrm{U}_{\text {iso }}{ }^{*} / \mathrm{U}_{\text {eq }}$ & $0.039(7)$ & $0.061(1)^{*}$ & $0.051^{*}$ & $0.051^{*}$ & $0.039(1)^{*}$ \\
\hline \multirow[t]{4}{*}{$\mathrm{H}$} & $\begin{array}{c}\mathrm{H} 1(0.5, y, x) \\
0.414(9) \\
0.247(9)\end{array}$ & $\begin{array}{c}\mathrm{H} 11(x, y, x) \\
0.5839(6) \\
0.7363(9)\end{array}$ & $\begin{array}{c}\mathrm{H} 11(0.5, y, z) \\
0.664(1) \\
0.702(3)\end{array}$ & $\begin{array}{c}\mathrm{H} 11(0.5, y, z) \\
0.665(1) \\
0.702(3)\end{array}$ & $\begin{array}{c}\mathrm{H} 11(0.5, y, z) \\
0.670(1) \\
0.720(2)\end{array}$ \\
\hline & $\begin{array}{c}\mathrm{H} 2(x, x, z) \\
0.326(3) \\
0.448(4)\end{array}$ & $\begin{array}{c}\mathrm{H} 12(x, y, x) \\
0.4416(7) \\
0.2841(8)\end{array}$ & $\begin{array}{c}\mathrm{H} 12(x, y, z) \\
0.640(1) \\
0.419(1) \\
0.702(3)\end{array}$ & $\begin{array}{c}\mathrm{H} 12(x, y, z) \\
0.641(1) \\
0.418(1) \\
0.702(3)\end{array}$ & $\begin{array}{c}\mathrm{H} 12(x, y, z) \\
0.6445(9) \\
0.4166(9) \\
0.720(2)\end{array}$ \\
\hline & & & $\begin{array}{c}\mathrm{H} 21(0.5, y, z) \\
0.331(1) \\
0.305(3)\end{array}$ & $\begin{array}{c}\mathrm{H} 21(0.5, y, z) \\
0.331(1) \\
0.306(1)\end{array}$ & $\begin{array}{c}\mathrm{H} 21(0.5, y, z) \\
0.326(1) \\
0.351(2)\end{array}$ \\
\hline & & & $\begin{array}{c}\mathrm{H} 22(x, y, z) \\
0.357(1) \\
0.583(1) \\
0.305(3)\end{array}$ & $\begin{array}{c}\mathrm{H} 22(x, y, z) \\
0.356(1) \\
0.583(1) \\
0.306(3)\end{array}$ & $\begin{array}{c}\mathrm{H} 22(x, y, z) \\
0.354(1) \\
0.585(1) \\
0.351(2)\end{array}$ \\
\hline $\mathrm{U}_{\text {iso }}{ }^{*} / \mathrm{U}_{\text {eq }}$ & $0.06(1)$ & $0.063^{*}$ & $0.063^{*}$ & $0.063^{*}$ & $0.059(2)^{*}$ \\
\hline $\mathrm{R}_{\mathrm{p}}(\%)$ & 1.3 & 1.3 & 1.0 & 1.2 & 0.8 \\
\hline $\mathrm{R}_{\mathrm{wp}}(\%)$ & 1.6 & 1.7 & 1.3 & 1.5 & 0.9 \\
\hline$\chi^{2}$ & 1.1 & 1.1 & 1.3 & 1.1 & 1.2 \\
\hline $\mathrm{R}_{\mathrm{Bragg}}(\%)$ & 3.9 & 10.8 & 6.5 & 6.5 & 6.4 \\
\hline
\end{tabular}

Table 1.

Crystallographic data for $M A P b\left(B r_{1-x} C l_{x}\right)_{3}$ with $x=0,0.33,0.5,0.67$, and 1 at room temperature from NPD.

Looking back at the unit-cell variation, the contraction of the $\mathrm{PbX}_{6}$ octahedron as $\mathrm{Cl}$ is introduced leads to a localization of the MA units, limiting their orientation. This decrease has been deeply studied below room temperature, as the crystal goes through the phase transitions: cubic-tetragonal-orthorhombic [37, 39, 40]; however, in this case, $\mathrm{CH}_{3} \mathrm{NH}_{3} \mathrm{~Pb}\left(\mathrm{Br}_{1-\mathrm{x}} \mathrm{Cl}_{\mathrm{x}}\right)_{3}$, the behavior is observed in the same crystal system at RT [34]. This finding shows that the MA freedom degree reduction can occur either by a decrease in the symmetry or by size reduction (keeping the symmetry) in the $\mathrm{PbX}_{6}$ network. These discoveries renew the question whether the inorganic framework deformation occurs and then the MA units accommodate in the preferred directions, or the reduction in the MA freedom degree enables the 


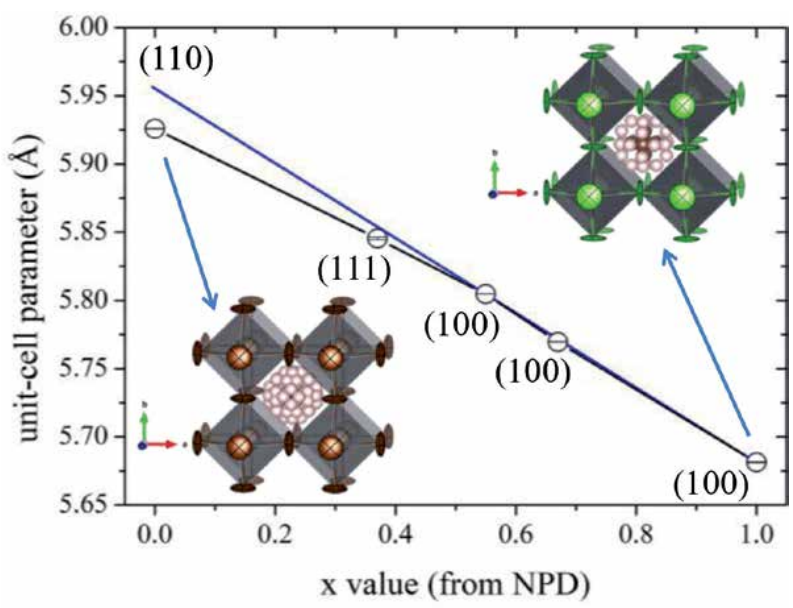

Figure 9.

Unit-cell parameters variation for $M A P b\left(B r_{1-x} C l_{x}\right)_{3}$ from NPD data, at RT.

inorganic framework to adopt a low symmetry. This dichotomy is as the chicken and egg paradox, which has previously been analyzed from DFT calculations, concluding that phase transitions occur as a synergic effect between both organic and inorganic components [38]. In the present case, the MA restrictions occur without symmetry reduction in the inorganic framework, but as a consequence of a contraction in the unit-cell size, so it is possible to think that the $\mathrm{PbX}_{6}$ lattice drives the MA freedom degree. However, it is only an appreciation and it is not enough to resolve this dichotomy.

On the other hand, the role of $\mathrm{H} \cdots \mathrm{X}$ interactions in the MA conformations is not unknown [41, 42] but shows some controversy among the different results. Theoretical studies from DFT indicate that the energy difference between the low index [100], [110], and [111] orientations of the MA units is similar, but with a subtle preference for the [100] orientation in the tetragonal $\mathrm{MAPbI}_{3}$ [40]. However, the ab initio simulations performed by Shimamura et al. [43] unveiled that [110] was the preferred orientation due to the $\mathrm{H} \cdots \mathrm{X}$ interactions in $\mathrm{MAPbI}_{3}$. Differently, $\mathrm{Li}$ et al. identify [111] and [100] for the $\mathrm{MAPbI}_{3}$ and $\mathrm{MAPbCl}_{3}$ perovskites as the favorable orientations [38]. Afterward, Varadwaj et al. reinforced this last idea by also stating that these orientations were the most energetically favorable [37]. Although there is significant theoretical work regarding the MA units orientation in cubic symmetry, experimental evidence is lacking $[25,27,36]$. It is the case of the Baikie et al. [27] report that focuses on the three structures for $\mathrm{X}=\mathrm{I}, \mathrm{Br}$, and $\mathrm{Cl}$ from X-ray and neutron diffraction data. In all the cases, the MA components were refined in the [110] direction. In this context of theoretical results, the considerations made in the $\mathrm{CH}_{3} \mathrm{NH}_{3} \mathrm{~Pb}\left(\mathrm{Br}_{1-\mathrm{x}} \mathrm{Cl}_{\mathrm{x}}\right)_{3}$ refinements offer new possibilities to achieve a detailed analysis of the crystal studies in comparison with these theoretical works. Once the atomic positions are refined, a "deconvolution" of the MA units orientation can be done for the directions [111] and [100]. The H...X interactions obtained can be compared with those predicted theoretically.

The MA units within the cubic unit cell are presented in Figure 10, the three alignment possibilities ([110], [111], and [100]) are shown. The MA in $\mathrm{MAPbBr}_{3}$ is aligned along [110] and, as can be seen in Figure 10a and d, in this orientation the $\mathrm{N}-\mathrm{H}$ bonds point toward face centers or cube corners forming smalls angles $\mathrm{N}-\mathrm{H} \cdots \mathrm{Br}$; hence, this configuration is not suitable for hydrogen bonding. On the other hand, for $\mathrm{x}=0.33$ (along [111] direction), the $\mathrm{H}-\mathrm{C}$ and $\mathrm{H}-\mathrm{N}$ interact with a 

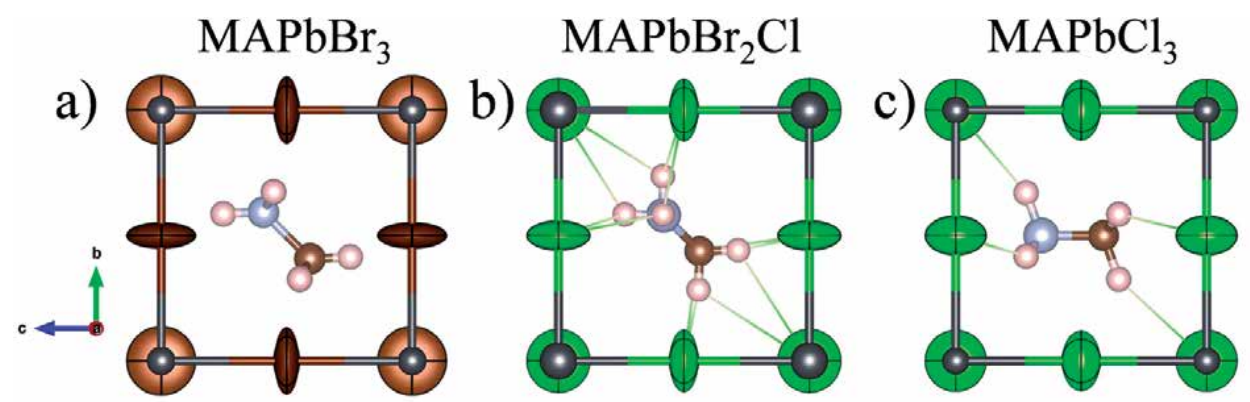

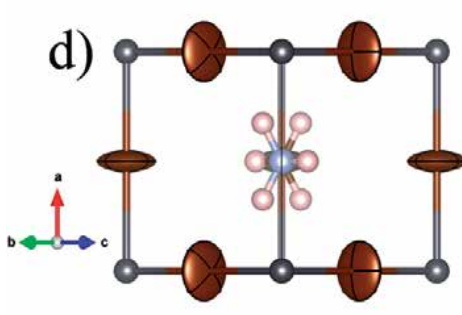

[110]

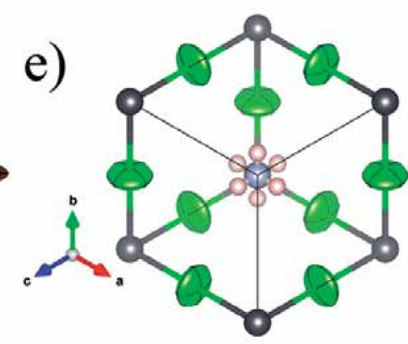

[111]

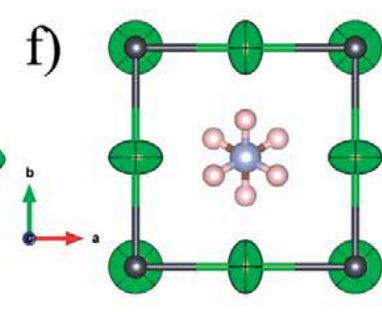

$[100]$

Figure 10.

Methyl-ammonium conformations in the $\mathrm{MAPbBr}_{3}(a)$ and (d), $\mathrm{MAPbBr} 2 \mathrm{Cl}(b)$ and $(e)$ and $\mathrm{MAPbCl}_{3}$ $(c)$ and $(f)$ perovskites, from NPD data at RT. The lowest figures $(d),(e)$ and $(f)$ are shown along the MA direction.

unique halide $\left(\mathrm{X}=\mathrm{Cl}_{0.33} \mathrm{Br}_{0.67}\right)$ with a distance of 2.921(5) and 3.088(5) $\AA$, respectively (Figure 10b and e). As mentioned, the MA conformation is slightly displaced, the $(\mathrm{C}) \mathrm{H} \cdots \mathrm{X}$ distances are larger than $(\mathrm{N}) \mathrm{H} \cdots \mathrm{X}$, which could be explained by the bigger electronegativity of $\mathrm{N}$ compared to $\mathrm{C}$.

Varadwaj et al. reported on DFT calculations for the $\mathrm{MAPbBr}_{3}$ perovskite [37]; the distances were determined, being $\approx 2.44$ and $\approx 3.47 \AA$ for $(\mathrm{N}) \mathrm{H} \cdots \mathrm{X}$ and $(\mathrm{C})$ $\mathrm{H} \cdots \mathrm{X}$, respectively, in agreement with the NPD experimental results. Carrying out the same procedure for $\mathrm{x}=0.5,0.67$ and 1 , we can determine the orientation of the MA units, which resulted to be [100]. The MA units can be rotated in four positions along the three [100] directions giving 12 possible conformations. With the help of DFT calculations, the orientation of the MA unit for bromine and chlorine phases can be estimated, with the additional fact that the $(\mathrm{N}) \mathrm{H} \cdots \mathrm{X}$ distances are shorter that the $(\mathrm{C}) \mathrm{H} \cdots \mathrm{X}$ ones $[37,38]$. Figure $10 \mathbf{c}$ and $\mathbf{f}$ illustrate a MA unit from the obtained $\mathrm{C}, \mathrm{N}$, and $\mathrm{H}$ atomic positions for $\mathrm{MAPbCl}_{3}$; two different types of $\mathrm{H}$-bonds

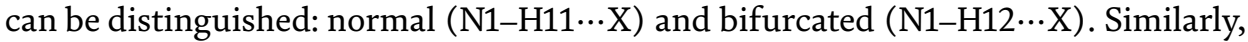
the methyl group shows the same interactions through $\mathrm{H} 21$ and $\mathrm{H} 22$ atoms. The obtained distances for $\mathrm{x}=1$ phase are 2.459(8) and 2.615(8) $\AA$ for N1-H11 ... X and N1-H12 ‥X, respectively [38].

The structural refinement from NPD data provide, for the first time, experimental evidence of the MA alignments, confirming the theoretical results reported up to now.

\section{Optoelectronic properties}

The optoelectronic properties are characterized using different techniques such as diffuse reflectance UV-vis spectra and building a photodetector device where the current-voltage (IV) curves are measured under illuminations. This device was fabricated by drop-casting the perovskite solution onto $\mathrm{Au} / \mathrm{Cr}$ pre-patterned electrodes. 


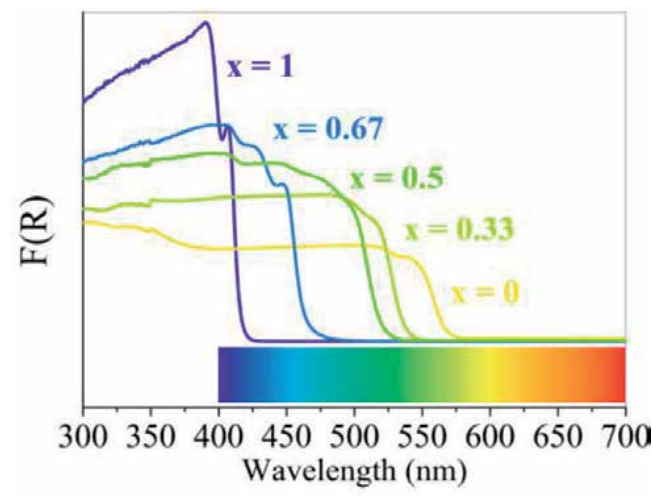

Figure 11.

UV-vis absorption spectra for $M A P b\left(B r_{1-x} C l_{x}\right)_{3}$. The colored line corresponds to the band gap energy.

The UV-vis spectra, illustrated in Figure 11, are used to calculate the optical absorption coefficient $(\alpha)$ according to the Kubelka-Munk equation:

$$
\mathrm{F}(\mathrm{R})=\alpha=(1-\mathrm{R}) 2 /(2 \mathrm{R})
$$

where $\mathrm{R}$ is the reflectance (\%). The optical band gap is determined from the extrapolation of the linear part of the transformed Kubelka-Munk spectrum with the $h \nu$ axis. Absorbance vs. wavelength of the incident radiation for $\mathrm{MAPb}\left(\mathrm{Br}_{1-}\right.$ $\left.{ }_{\mathrm{x}} \mathrm{Cl}_{\mathrm{x}}\right)_{3}$ series is illustrated in Figure 11. In this plot, the included color chart allows accounting for the sample color shown in Figure 1.

The absorption edge presents a gradual evolution, which is consistent with the continuous structural changes, mainly with the unit-cell parameter evolution. The energy of the gap experiences an increment as $\mathrm{Br}$ is progressively replaced with $\mathrm{Cl}$. This is, in principle, expected as a consequence of the smaller covalency of the leadhalide bonds within the $\mathrm{PbX}_{6}(\mathrm{X}=\mathrm{Cl}, \mathrm{Br})$ octahedra [31]. Recent studies by density functional theory (DFT) show that the edges of the valence band and conduction band of $\mathrm{CH}_{3} \mathrm{NH}_{3} \mathrm{X}_{3}$ perovskites are principally made up of $p_{\mathrm{X}}$ and $p_{\mathrm{Pb}}$ states, respectively [44]. It is shown that near the gap, the predominant contribution is brought about by $\mathrm{Pb}-\mathrm{Pb}$ transitions, involving $s$-type orbitals. The characteristic absorption band above $3 \mathrm{eV}$ is fundamentally driven by $s_{\mathrm{Pb}}-p_{\mathrm{Pb}}$ interactions, tuned by the unit-cell

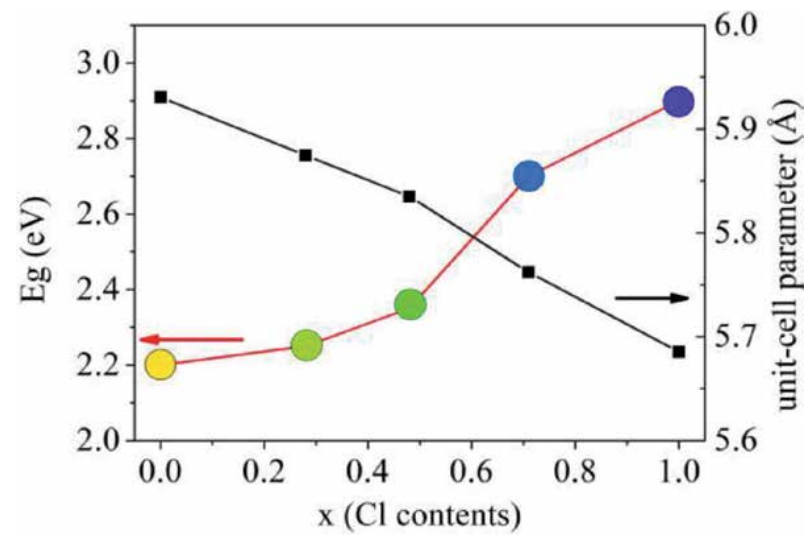

Figure 12.

Comparison between the band gap energy and unit-cell parameter in $M A P b\left(B r_{1-x} C l_{x}\right)_{3}$, as a function of the $C l$ content, $x$. The color in the circles corresponds to the band gap energy. 
size, with a lower contribution by $p_{\mathrm{Pb}}-p_{\mathrm{X}}$ and $s_{\mathrm{X}}-p_{\mathrm{X}}$ transitions. These latter contributions decrease from iodine to bromine and then to chlorine, that is, when increasing the electronegativity of the halide, which accounts for the variation of the gap with the chemical nature of X, as shown in Figures 11 and 12. Endres et al. [45] found a remarkably low $\mathrm{DOS}$ at the valence band of $\mathrm{MAPbX}_{3}$, from either ultraviolet and inverse photoemission spectroscopies or from theoretical densities of states (DOS), calculated via density functional theory. They found a strong band dispersion at the valence band due to the coupling between halide $p$ and $\mathrm{Pb} s$ antibonding orbitals, observing the absence of significant densities of states tailing into the perovskite gaps.

As already mentioned, an interesting feature is that the compositional evolution of the band gap energy (Eg) does not possesses a linear behavior; it seems that the deviation from linearity is reminiscent of that unveiled for the variation of the unit-cell parameters at RT (see Figure 12). By additionally contrasting the Eg value with the $\mathrm{Pb}-\mathrm{X}$ bond lengths, a non-linear behavior is apparent, suggesting that the evolution of Eg is not only related to the inorganic features. From this point of view, it is interesting to consider that, although all these studies are based on the nominally simple cubic perovskite structure, these compounds are in fact very complex. For example, in $\left(\mathrm{CH}_{3} \mathrm{NH}_{3}\right) \mathrm{PbI}_{3}$, the dynamics associated with the $\left(\mathrm{CH}_{3} \mathrm{NH}_{3}\right)^{+}$ions are still not fully understood, although ab initio calculations show [46] that at room and higher temperature, the rotation of $\mathrm{CH}_{3} \mathrm{NH}_{3}$ molecules can be viewed as effectively giving local structures that are cubic- and tetragonal-like from the point of view of the $\mathrm{PbI}_{3}$ framework, though
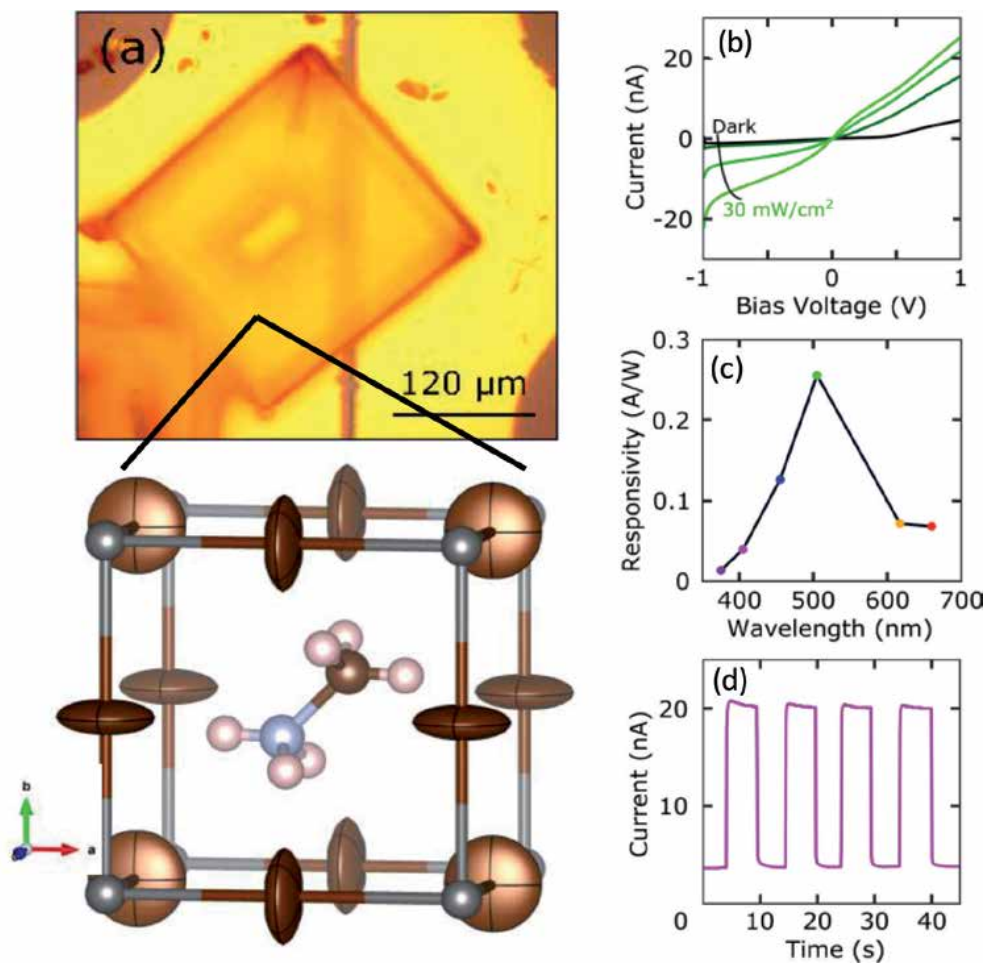

Figure 13.

(a) View of the photodetector device, including a crystal of $M A P b B r_{3}$ grown between two pre-patterned electrodes, separated apart by $10 \mu \mathrm{m}$. (b) Current-voltage (I-V) curves obtained with 505-nm illumination, by changing the incident optical power densities. (c) Spectrum displaying the responsivity for different wavelengths. (d) Generated photocurrent vs. time, when a modulated illumination is applied with a fixed voltage [32]. 
in fact having lower symmetry. These arrangements are locally polar, with sizable polarization, $\sim 10 \mu \mathrm{C} / \mathrm{cm}^{2}$ due to the dipoles on the organic part [46]. The structural transitions are thus analogous to the transitions between two ferroelectric structures, where there is strong screening of charged defects that can lead to enhanced mobility and charge collection.

This observation seems to confirm that the increase in the Eg with the amount of $\mathrm{Cl}^{-}$is closely correlated with the effect of the MA and its freedom degree in the inorganic framework. The change in the MA delocalization upon the incorporation of smaller $\mathrm{Cl}^{-}$anions is an additional parameter to be considered in the tuning of the optical properties of these hybrid materials.

Furthermore, the potential of these materials in optoelectronic applications can be exemplified for $\mathrm{MAPbBr}_{3}$ measuring the current-voltage (I-V) in a photodetector device that is illustrated in Figure 13a [32]. The effect of the optical power densities under illumination at $505 \mathrm{~nm}$ in the I-V curves is plotted in Figure 13b. This device shows a photoresponse among the best ones reported for other perovskite-based photodetectors, even considering different geometries: $2 \mathrm{D}-\mathrm{MAPbI}_{3}[47,48]$, thin films [49, 50], nanowires [51, 52], and networks [53]. Starting from I-V measurements using different wavelengths from alternative light sources, always keeping the same illumination power of $1 \mathrm{~mW} / \mathrm{cm}^{2}$, it is possible to calculate the wavelength responsivity spectrum of the photodetector, by using the next formula:

$$
\mathrm{R}=\mathrm{I}_{\mathrm{ph}} / \mathrm{P}
$$

with $\mathrm{R}$ being the responsivity, $\mathrm{I}_{\mathrm{ph}}$ the generated photocurrent, and $\mathrm{P}$ the illumination power on the device. These data are illustrated in Figure 13c, they show a maximum in the responsivity $(0.26 \mathrm{~A} / \mathrm{W})$ for light with wavelength of $505 \mathrm{~nm}$, which is near the wavelength with maximum solar irradiance. Figure 13d plots the current through the device as a function of time with a fixed voltage under pulsed illumination. This illumination mode allows to characterize the response time of the photodetector, in this device $<100 \mathrm{~ms}$ (limited by the experimental setup), which is comparable with values previously reported $[47,48,54,55]$.

\section{Conclusions}

This chapter reviews recent structural results on the $\mathrm{CH}_{3} \mathrm{NH}_{3}\left(\mathrm{Br}_{1-\mathrm{x}} \mathrm{Cl}_{\mathrm{x}}\right)_{3}$ series. The combination of SXRD and NPD has permitted to address issues related to phase transitions below RT, and the conformation of the $\mathrm{CH}_{3} \mathrm{NH}_{3}{ }^{+}$units within the inorganic cages, formed by corner-sharing $\mathrm{PBX}_{6}$ octahedra. Interestingly, it was observed that the progressive localization of the organic units is not only achieved through a reduction in symmetry (from cubic to tetragonal, and finally to orthorhombic), but also simply as a consequence of the contraction of the cubic unit cells as the $\mathrm{Cl}$ contents increase. This can be interpreted as an evolution of the $\mathrm{X}-\mathrm{H}$ hydrogen bonds, as previously observed from DFT calculations. In summary, we have contributed with novel experimental evidence that facilitates a more reasonable design of hybrid halide perovskites with tunable properties for solar cell technologies.

\section{Acknowledgements}

This work was supported by the Spanish MINECO for funding MAT201784496-R. The authors thank ALBA and ILL for making the facilities available. CAL acknowledges ANPCyT and UNSL for financial support (projects PICT2017-1842 and PROICO 2-2016), Argentine. 
We acknowledge support for the publication fee by the CSIC Open Access Publication Support Initiative through its Unit of Information Resources for Research (URICI).

\section{Conflict of interest}

The authors declare no conflict of interest.

\section{Author details}

Carlos Alberto López ${ }^{1,2 *}$, María Consuelo Alvarez-Galván ${ }^{3}$, Carmen Abia ${ }^{1,4}$, María Teresa Fernández-Díaz ${ }^{4}$ and José Antonio Alonso ${ }^{1 *}$

1 Instituto de Ciencia de Materiales de Madrid, CSIC, Madrid, Spain

2 Instituto de Investigaciones en Tecnología Química (INTEQUI), UNSL, CONICET, Facultad de Química, Bioquímica y Farmacia, UNSL, San Luis, Argentina

3 Instituto de Catálisis y Petroleoquímica, CSIC, Madrid, Spain

4 Institut Laue Langevin, Grenoble, France

*Address all correspondence to: calopez@unsl.edu.ar and ja.alonso@icmm.csic.es

\section{IntechOpen}

(C) 2020 The Author(s). Licensee IntechOpen. This chapter is distributed under the terms of the Creative Commons Attribution License (http://creativecommons.org/licenses/ by/3.0), which permits unrestricted use, distribution, and reproduction in any medium, provided the original work is properly cited. (cc) BY 
Structural Phase Transitions of Hybrid Perovskites $\mathrm{CH}_{3} \mathrm{NH}_{3} \mathrm{PbX}(\mathrm{X}=\mathrm{Br}, \mathrm{Cl})$ from Synchrotron... DOI: http://dx.doi.org/10.5772/intechopen.91421

\section{References}

[1] Lee MM, Teuscher J, Miyasaka T, et al. Efficient hybrid solar cells based on meso-superstructured organometal halide perovskites. Science (80-). 2012;338:643-647

[2] Kojima A, Teshima K, Shirai Y, et al. Organometal halide perovskites as visible-light sensitizers for photovoltaic cells. Journal of the American Chemical Society. 2009;131:6050-6051

[3] Kim H-S, Lee C-R, Im J-H, et al. Lead iodide perovskite sensitized all-solidstate submicron thin film mesoscopic solar cell with efficiency exceeding $9 \%$. Scientific Reports. 2012;2:591

[4] Ye M, Hong X, Zhang F, et al. Recent advancements in perovskite solar cells: Flexibility, stability and large scale. Journal of Materials Chemistry A. 2016;4:6755-6771

[5] Fan Z, Sun K, Wang J. Perovskites for photovoltaics: A combined review of organic-inorganic halide perovskites and ferroelectric oxide perovskites. Journal of Materials Chemistry A. 2015;3:18809-18828

[6] Green MA, Emery K, Hishikawa Y, et al. Solar cell efficiency tables (version 48). Progress in Photovoltaics: Research and Applications. 2016;24:905-913

[7] Noh JH, Im SH, Heo JH, et al. Chemical management for colorful, efficient, and stable inorganic-organic hybrid nanostructured solar cells. Nano Letters. 2013;13:1764-1769

[8] Sadhanala A, Deschler F, Thomas TH, et al. Preparation of singlephase films of $\mathrm{CH} 3 \mathrm{NH} 3 \mathrm{~Pb}(\mathrm{I} 1-\mathrm{xBrx}) 3$ with sharp optical band edges. Journal of Physical Chemistry Letters. 2014;5:2501-2505

[9] Eperon GE, Stranks SD, Menelaou C, et al. Formamidinium lead trihalide: $\mathrm{A}$ broadly tunable perovskite for efficient planar heterojunction solar cells. Energy \& Environmental Science. 2014;7:982

[10] De Wolf S, Holovsky J, Moon S-J, et al. Organometallic halide perovskites: Sharp optical absorption edge and its relation to photovoltaic performance. Journal of Physical Chemistry Letters. 2014;5:1035-1039

[11] Stranks SD, Eperon GE, Grancini G, et al. Electron-hole diffusion lengths exceeding 1 micrometer in an organometal trihalide perovskite absorber. Science (80-). 2013;342: 341-344

[12] Xing G, Mathews N, Sun S, et al. Long-range balanced electron- and hole-transport lengths in organicinorganic $\mathrm{CH} 3 \mathrm{NH} 3 \mathrm{PbI} 3$. Science (80-). 2013;342:344-347

[13] Han Y, Meyer S, Dkhissi Y, et al. Degradation observations of encapsulated planar $\mathrm{CH}_{3} \mathrm{NH}_{3} \mathrm{PbI}_{3}$ perovskite solar cells at high temperatures and humidity. Journal of Materials Chemistry A. 2015;3:8139-8147

[14] Yang J, Siempelkamp BD, Liu D, et al. Investigation of $\mathrm{CH}_{3} \mathrm{NH}_{3} \mathrm{PbI}_{3}$ degradation rates and mechanisms in controlled humidity environments using in situ techniques. ACS Nano. 2015;9:1955-1963

[15] Akbulatov AF, Frolova LA, Dremova NN, et al. Light or heat: What is killing lead halide perovskites under solar cell operation conditions? Journal of Physical Chemistry Letters. 2020;11:333-339

[16] Senocrate A, Kim GY, Grätzel M, et al. Thermochemical stability of hybrid halide perovskites. ACS Energy Letters. 2019;4:2859-2870

[17] Kedem N, Brenner TM, Kulbak M, et al. Light-induced increase of electron 
diffusion length in a $\mathrm{p}-\mathrm{n}$ junction type $\mathrm{CH} 3 \mathrm{NH} 3 \mathrm{PbBr} 3$ perovskite solar cell. Journal of Physical Chemistry Letters. 2015;6:2469-2476

[18] Talbert EM, Zarick HF, Orfield NJ, et al. Interplay of structural and compositional effects on carrier recombination in mixedhalide perovskites. RSC Advances. 2016;6:86947-86954

[19] Sheng R, Ho-Baillie A, Huang S, et al. Methylammonium lead bromide perovskite-based solar cells by vaporassisted deposition. Journal of Physical Chemistry C. 2015;119:3545-3549

[20] Zheng X, Chen B, Wu C, et al. Room temperature fabrication of $\mathrm{CH} 3 \mathrm{NH} 3 \mathrm{PbBr} 3$ by anti-solvent assisted crystallization approach for perovskite solar cells with fast response and small J-V hysteresis. Nano Energy. 2015;17:269-278

[21] Arora N, Orlandi S, Dar MI, et al. High open-circuit voltage: Fabrication of formamidinium lead bromide perovskite solar cells using fluorenedithiophene derivatives as holetransporting materials. ACS Energy Letters. 2016;1:107-112

[22] Edri E, Kirmayer S, Cahen D, et al. High open-circuit voltage solar cells based on organic-inorganic lead bromide perovskite. Journal of Physical Chemistry Letters. 2013;4:897-902

[23] Heo JH, Song DH, Im SH. Planar $\mathrm{CH}_{3} \mathrm{NH}_{3} \mathrm{PbBr}_{3}$ hybrid solar cells with $10.4 \%$ power conversion efficiency, fabricated by controlled crystallization in the spin-coating process. Advanced Materials. 2014;26:8179-8183

[24] Zarick HF, Boulesbaa A, Puretzky AA, et al. Ultrafast carrier dynamics in bimetallic nanostructureenhanced methylammonium lead bromide perovskites. Nanoscale. 2017;9:1475-1483
[25] Swainson IP, Hammond RP, Soullière C, et al. Phase transitions in the perovskite methylammonium lead bromide, $\mathrm{CH}_{3} \mathrm{ND}_{3} \mathrm{PbBr}_{3}$. Journal of Solid State Chemistry. 2003;176:97-104

[26] Mashiyama H, Kawamura Y, Kasano H, et al. Disordered configuration of methylammonium of $\mathrm{CH}_{3} \mathrm{NH}_{3} \mathrm{PbBr}_{3}$ determined by single crystal neutron diffractometry. Ferroelectrics. 2007;348: 182-186

[27] Baikie T, Barrow NS, Fang Y, et al. A combined single crystal neutron/Xray diffraction and solid-state nuclear magnetic resonance study of the hybrid perovskites $\mathrm{CH}_{3} \mathrm{NH}_{3} \mathrm{PbX}_{3}(\mathrm{X}=\mathrm{I}, \mathrm{Br}$ and $\mathrm{Cl}$ ). Journal of Materials Chemistry A. 2015;3:9298-9307

[28] Maculan G, Sheikh AD, Abdelhady AL, et al. $\mathrm{CH}_{3} \mathrm{NH}_{3} \mathrm{PbCl}_{3}$ single crystals: Inverse temperature crystallization and visible-blind UV-photodetector. Journal of Physical Chemistry Letters. 2015;6:3781-3786

[29] Rana S, Awasthi K, Bhosale SS, et al. Temperature-dependent electroabsorption and electrophotoluminescence and exciton binding energy in $\mathrm{MAPbBr}_{3}$ perovskite quantum dots. Journal of Physical Chemistry C. 2019;123:19927-19937

[30] Gao Z-R, Sun X-F, Wu Y-Y, et al. Ferroelectricity of the orthorhombic and tetragonal $\mathrm{MAPbBr}_{3}$ single crystal. Journal of Physical Chemistry Letters. 2019;10:2522-2527

[31] Comin R, Walters G, Thibau ES, et al. Structural, optical, and electronic studies of wide-bandgap lead halide perovskites. Journal of Materials Chemistry C. 2015;3:8839-8843

[32] López CA, Martínez-Huerta MV, Alvarez-Galván MC, et al. Elucidating the methylammonium (MA) conformation in $\mathrm{MAPbBr}_{3}$ perovskite with application in solar cells. Inorganic Chemistry. 2017;56:14214-14219 
[33] Alvarez-Galván MC, Alonso JA, López CA, et al. Crystal growth, structural phase transitions, and optical gap evolution of $\mathrm{CH}_{3} \mathrm{NH}_{3} \mathrm{~Pb}\left(\mathrm{Br}_{1}-\mathrm{xClx}\right)_{3}$ perovskites. Crystal Growth \& Design. 2018;19(2):918-924. DOI: 10.1021/acs. cgd.8b01463

[34] López CA, Álvarez-Galván MC, Martínez-Huerta MV, et al.

Dynamic disorder restriction of methylammonium (MA) groups in chloride-doped MAPbBr3 hybrid perovskites: A neutron powder diffraction study. Chemistry: A European Journal. 2019;25:4496-4500

[35] Poglitsch A, Weber D.

Dynamic disorder in methylammoniumtrihalogenoplumbates (II) observed by millimeter-wave spectroscopy. The Journal of Chemical Physics. 1987;87:6373-6378

[36] Chi L, Swainson I, Cranswick L, et al. The ordered phase of methylammonium lead chloride $\mathrm{CH}_{3} \mathrm{ND}_{3} \mathrm{PbCl}_{3}$. Journal of Solid State Chemistry. 2005;178:1376-1385

[37] Varadwaj A, Varadwaj PR, Marques HM, et al. Halogen in materials design: Revealing the nature of hydrogen bonding and other noncovalent interactions in the polymorphic transformations of methylammonium lead tribromide perovskite. Materials Today Chemistry. 2018;9:1-16

[38] Li J, Rinke P. Atomic structure of metal-halide perovskites from first principles: The chicken-and-egg paradox of the organic-inorganic interaction. Physical Review B. 2016;94: 045201

[39] Leguy AMA, Goñi AR, Frost JM, et al. Dynamic disorder, phonon lifetimes, and the assignment of modes to the vibrational spectra of methylammonium lead halide perovskites. Physical Chemistry Chemical Physics. 2016;18:27051-27066
[40] Frost JM, Walsh A. What is moving in hybrid halide perovskite solar cells? Accounts of Chemical Research. 2016;49:528-535

[41] Yin T, Fang Y, Fan X, et al. Hydrogen-bonding evolution during the polymorphic transformations in $\mathrm{CH}_{3} \mathrm{NH}_{3} \mathrm{PbBr}_{3}$ : Experiment and theory. Chemistry of Materials. 2017;29:5974-5981

[42] Bernasconi A, Page K, Dai Z, et al. Ubiquitous short-range distortion of hybrid perovskites and hydrogenbonding role: The $\mathrm{MAPbCl}_{3}$ case. Journal of Physical Chemistry C. 2018;122:28265-28272

[43] Shimamura K, Hakamata T, Shimojo F, et al. Rotation mechanism of methylammonium molecules in organometal halide perovskite in cubic phase: An ab initio molecular dynamics study. The Journal of Chemical Physics. 2016;145:224503

[44] Tablero CC. The effect of the halide anion on the optical properties of lead halide perovskites. Solar Energy Materials \& Solar Cells. 2019;195: 269-273

[45] Endres J, Egger DA, Kulbak M, et al. Valence and conduction band densities of states of metal halide perovskites: A combined experimental-theoretical study. Journal of Physical Chemistry Letters. 2016;7:2722-2729

[46] Ong KP, Wu S, Nguyen TH, et al. Multi band gap electronic structure in $\mathrm{CH}_{3} \mathrm{NH}_{3} \mathrm{PbI}_{3}$. Scientific Reports. 2019;9:2144

[47] Wang G, Li D, Cheng H-C, et al. Wafer-scale growth of large arrays of perovskite microplate crystals for functional electronics and optoelectronics. Science Advances. 2015;1:e1500613

[48] Liu J, Xue Y, Wang Z, et al. Two-dimensional $\mathrm{CH}_{3} \mathrm{NH}_{3} \mathrm{PbI}_{3}$ 
perovskite: Synthesis and

optoelectronic application. ACS Nano.

2016;10:3536-3542

[49] Zhang Y, Du J, Wu X, et al.

Ultrasensitive photodetectors based

on island-structured $\mathrm{CH}_{3} \mathrm{NH}_{3} \mathrm{PbI}_{3}$

thin films. ACS Applied Materials \&

Interfaces. 2015;7:21634-21638

[50] Lu H, Tian W, Cao F, et al. A

self-powered and stable all-perovskite photodetector-solar cell nanosystem. Advanced Functional Materials. 2016;26:1296-1302

[51] Deng H, Dong D, Qiao K, et al. Growth, patterning and alignment of organolead iodide perovskite nanowires for optoelectronic devices. Nanoscale. 2015;7:4163-4170

[52] Zhuo S, Zhang J, Shi Y, et al.

Self-template-directed synthesis of porous perovskite nanowires at room temperature for high-performance visible-light photodetectors.

Angewandte Chemie International Edition. 2015;54:5693-5696

[53] Deng H, Yang X, Dong D, et al. Flexible and semitransparent organolead triiodide perovskite network photodetector arrays with high stability. Nano Letters. 2015;15:7963-7969

[54] Li D, Dong G, Li W, et al. High performance organic-inorganic perovskite-optocoupler based on lowvoltage and fast response perovskite compound photodetector. Scientific Reports. 2015;5:7902

[55] Dong R, Fang Y, Chae J, et al. High-gain and low-driving-voltage photodetectors based on organolead triiodide perovskites. Advanced Materials. 2015;27:1912-1918 
Section 3

\section{Thermal and Physical Properties of Perovskites}





\title{
Thermodynamics of Perovskite: Solid, Liquid, and Gas Phases
}

\author{
Sergey Shornikov
}

\begin{abstract}
The present work is devoted to the review of experimental data on thermodynamic properties of perovskite in the condensed state, as well as the gas phase components over perovskite and its melts at high temperatures.
\end{abstract}

Keywords: perovskite, thermodynamics

\section{Introduction}

Calcium titanate $\left(\mathrm{CaTiO}_{3}\right)$ or perovskite was found by Rose [1] in the Ural Mountains in 1839 and named after the Russian Statesman Count Lev Perovski. Perovskite is a relatively rare mineral, which is a promising material for use as matrices for safe long-term storage of actinides and their rare earth analogs that are present in radioactive waste [2]. It is of particular interest for petrology and cosmochemical research as a mineral which is a part of refractory $\mathrm{Ca}-\mathrm{Al}$ inclusions often found in carbonaceous chondrites, which are the earliest objects of the solar system with unusual isotopic characteristics [3-5].

In addition to perovskite, two more calcium titanates, $\mathrm{Ca}_{3} \mathrm{Ti}_{2} \mathrm{O}_{7}[6]$ and $\mathrm{Ca}_{4} \mathrm{Ti}_{3} \mathrm{O}_{10}$ [7], melting incongruently, were found in the $\mathrm{CaO}-\mathrm{TiO}_{2}$ system. The other calcium titanates, $\mathrm{Ca}_{4} \mathrm{TiO}_{6}[8], \mathrm{Ca}_{3} \mathrm{TiO}_{5}$ [9], $\mathrm{Ca}_{8} \mathrm{Ti}_{3} \mathrm{O}_{14}[8,10], \mathrm{Ca}_{2} \mathrm{TiO}_{4}$ [9], $\mathrm{Ca}_{5} \mathrm{Ti}_{4} \mathrm{O}_{13}$ [11], $\mathrm{Ca}_{2} \mathrm{Ti}_{3} \mathrm{O}_{8}[6], \mathrm{CaTi}_{2} \mathrm{O}_{5}$ [12, 13], $\mathrm{CaTi}_{3} \mathrm{O}_{7}$ [14], $\mathrm{Ca}_{2} \mathrm{Ti}_{5} \mathrm{O}_{12}$ [15], and $\mathrm{CaTi}_{4} \mathrm{O}_{9}[16]$, are mentioned in the literature. They also seem to be unstable, which does not exclude their possible existence [17]. Compiled in this paper on the basis of data $[7,18]$, as well as the results of recent studies by Gong et al. [19], the phase diagram of the $\mathrm{CaO}-\mathrm{TiO}_{2}$ system in the high-temperature region is shown in Figure 1.

\section{The thermodynamic properties of perovskite solid phase}

Thermochemical data on perovskite [20-24] are based on calorimetric measurements of entropy of perovskite formation $\Delta S_{298}\left(\mathrm{CaTiO}_{3}\right)$, obtained by Shomate [25], and high-temperature heat capacity of perovskite $C_{p}\left(\mathrm{CaTiO}_{3}\right)$ in the temperature ranges of $15-398 \mathrm{~K}$ [26], 293-773 K [27], 376-1184 K [28], 383-1794 K [29], and $413-1825 \mathrm{~K}[30]$. The differences between the data do not exceed $5 \mathrm{~J} /(\mathrm{mol} \mathrm{K})$ up to a temperature of $1200 \mathrm{~K}$, but they are quite contradictory at higher temperatures (Figure 2). 


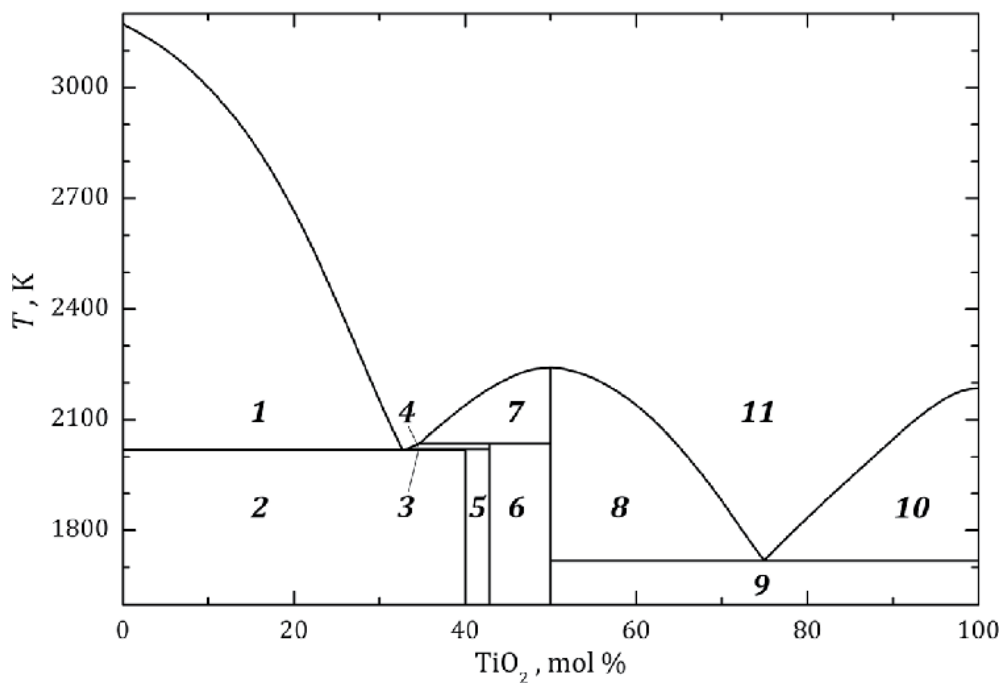

Figure 1.

The phase diagram of the $\mathrm{CaO}-\mathrm{TiO}_{2}$ system $[7,18,19]:$ (1) $\mathrm{CaO}+$ liquid; (2) $\mathrm{CaO}+\mathrm{Ca}_{3} \mathrm{Ti}_{2} \mathrm{O}_{7}$;

(3) $\mathrm{Ca}_{3} \mathrm{Ti}_{2} \mathrm{O}_{7}+$ liquid; (4) $\mathrm{Ca}_{4} \mathrm{Ti}_{3} \mathrm{O}_{10}+$ liquid; (5) $\mathrm{Ca}_{3} \mathrm{Ti}_{2} \mathrm{O}_{7}+\mathrm{Ca}_{4} \mathrm{Ti}_{3} \mathrm{O}_{10}$; (6) $\mathrm{Ca}_{4} \mathrm{Ti}_{3} \mathrm{O}_{10}+\mathrm{CaTiO}$;

$(7,8) \mathrm{CaTiO}_{3}+$ liquid; (9) $\mathrm{CaTiO}_{3}+\mathrm{TiO}_{2} ;(10) \mathrm{TiO}_{2}+$ liquid; (11) liquid.

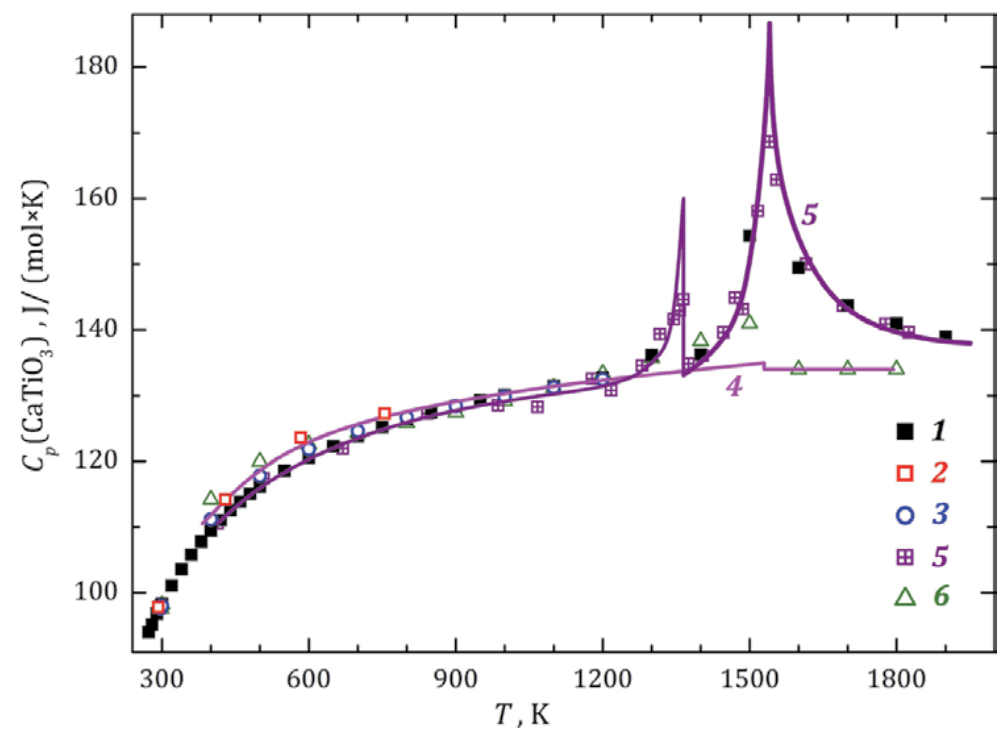

Figure 2.

Heat capacity of perovskite: (1-5) determined via high-temperature calorimetry [26-30], respectively, and (6) taken from [22].

Naylor and Cook [29] determined the enthalpy of perovskite phase transition: $2.30 \pm 0.07 \mathrm{~kJ} / \mathrm{mol}$ at $1530 \pm 1 \mathrm{~K}$. The overlapping phase transitions in perovskite observed by Guyot et al. [30] (Figure 2) were explained as consequences of a structural change, i.e., a transition from orthorhombic $(\mathrm{Pbnm})$ to orthorhombic $(\mathrm{Cmcm})$ structure at $1384 \pm 10 \mathrm{~K}$ and the overlapping of transitions from orthorhombic to tetragonal $(I 4 / \mathrm{mcm})$ and tetragonal to cubic phase $(P m \overline{3} \mathrm{~m})$ at $1520 \pm 10 \mathrm{~K}$ with heat effects of $1.0 \pm 0.5$ and $5.5 \pm 0.5 \mathrm{~kJ} / \mathrm{mol}$, respectively. The considerable anomaly of perovskite heat capacity above $1520 \mathrm{~K}$ could be caused by strong disordering of the cubic phase up to the temperature of perovskite melting. However, based on diffraction data about perovskite structure obtained in the 
Thermodynamics of Perovskite: Solid, Liquid, and Gas Phases

DOI: http://dx.doi.org/10.5772/intechopen.91309

\begin{tabular}{|c|c|c|c|c|}
\hline Experimental approach & $T, \mathrm{~K}$ & $\Delta H_{T}, \mathrm{~kJ} / \mathrm{mol}$ & $\Delta S_{T}, \mathrm{~J} /(\mathrm{mol} \mathrm{K})$ & Refs. \\
\hline $\mathrm{HF} / \mathrm{HCl}$ solution calorimetry & 298 & & $1.05 \pm 0.21$ & {$[25]$} \\
\hline $\mathrm{HF} / \mathrm{HCl}$ solution calorimetry & 298 & $-40.48 \pm 0.42$ & $1.86 \pm 0.71$ & [33] \\
\hline Bomb calorimetry & 298 & $-41.84 \pm 1.88$ & & [32] \\
\hline Adiabatic calorimetry & 298 & $-40.48 \pm 1.65$ & $2.40 \pm 0.35$ & {$[26]$} \\
\hline Adiabatic calorimetry & 298 & $-47.11 \pm 1.41$ & $2.72 \pm 0.23$ & {$[28]$} \\
\hline $\mathrm{Na}_{6} \mathrm{Mo}_{4} \mathrm{O}_{15}$ solution calorimetry & 298 & $-42.98 \pm 1.96$ & & {$[34]$} \\
\hline EMF & $888-972$ & $-37.05 \pm 3.28$ & $4.62 \pm 3.54$ & [35] \\
\hline EMF & $900-1250$ & $-40.07 \pm 0.05$ & $3.15 \pm 0.05$ & {$[36]$} \\
\hline $\mathrm{Pb}_{2} \mathrm{~B}_{2} \mathrm{O}_{5}$ solution calorimetry & 973 & $-38.73 \pm 1.34$ & & [37] \\
\hline $\mathrm{Na}_{6} \mathrm{Mo}_{4} \mathrm{O}_{15}$ solution calorimetry & 975 & $-42.25 \pm 1.05$ & & [38] \\
\hline $\mathrm{Na}_{6} \mathrm{Mo}_{4} \mathrm{O}_{15}$ solution calorimetry & 975 & $-41.88 \pm 1.36$ & & [39] \\
\hline $\mathrm{Na}_{6} \mathrm{Mo}_{4} \mathrm{O}_{15}$ solution calorimetry & 976 & $-42.86 \pm 1.71$ & & {$[40]$} \\
\hline $\mathrm{Pb}_{2} \mathrm{~B}_{2} \mathrm{O}_{5}$ solution calorimetry & 1046 & $-42.38 \pm 1.82$ & & {$[37]$} \\
\hline$(\mathrm{Li}, \mathrm{Na}) \mathrm{BO}_{2}$ solution calorimetry & $1068 \pm 2$ & $-40.45 \pm 1.15$ & & {$[41]$} \\
\hline $\mathrm{Pb}_{2} \mathrm{~B}_{2} \mathrm{O}_{5}$ solution calorimetry & 1073 & $-40.43 \pm 1.87$ & $4.37 \pm 1.23$ & {$[42]$} \\
\hline $\mathrm{Pb}_{2} \mathrm{~B}_{2} \mathrm{O}_{5}$ solution calorimetry & 1074 & $-43.78 \pm 1.73$ & & {$[43]$} \\
\hline $\mathrm{Pb}_{2} \mathrm{~B}_{2} \mathrm{O}_{5}$ solution calorimetry & 1078 & $-42.83 \pm 3.12$ & & {$[44]$} \\
\hline EMF & $1180-1290$ & $-26.88 \pm 8.05$ & $11.07 \pm 6.44$ & {$[45]$} \\
\hline Raman spectroscopy & 1300 & & $2.82 \pm 1.29$ & [46] \\
\hline Thermochemical calculations & $1600-1800$ & $-37.19 \pm 0.14$ & $5.85 \pm 0.09$ & {$[22]$} \\
\hline Thermochemical calculations & $1600-2100$ & $-38.23 \pm 0.04$ & $5.00 \pm 0.02$ & [23] \\
\hline Thermochemical calculations & $1600-2100$ & $-37.47 \pm 0.03$ & $5.60 \pm 0.02$ & {$[24]$} \\
\hline DTA & $1740 \pm 20$ & $-37.55 \pm 3.76$ & & {$[47]$} \\
\hline Knudsen mass spectrometry & $1791-2241$ & $-39.98 \pm 0.54$ & $3.15 \pm 0.28$ & {$[48]$} \\
\hline Knudsen mass spectrometry & $2241-2398$ & $7.73 \pm 1.76$ & $24.39 \pm 0.76$ & [48] \\
\hline
\end{tabular}

Table 1.

Enthalpy and entropy of perovskite formation from simple oxides (calculated per 1 mol of compound).

temperature range of 296-1720 K, Yashima and Ali [31] concluded that the Cmcm phase does not exist and claimed that the first transition is the $(\mathrm{Pbnm}) \rightarrow(\mathrm{I} / \mathrm{mcm})$ at $1512 \pm 13 \mathrm{~K}$, followed by the $(I 4 / \mathrm{mcm}) \rightarrow(P m \overline{3} m)$ transition at $1635 \pm 2 \mathrm{~K}$.

Panfilov and Fedos'ev [32] determined the enthalpy of the reaction with a calorimetric bomb by burning stoichiometric mixtures of rutile $\mathrm{TiO}_{2}$ and calcium carbonate $\mathrm{CaCO}_{3}$ (here and below, the square brackets denote the condensed phase; the parentheses denote the gas phase):

$$
\left[\mathrm{CaCO}_{3}\right]+\left[\mathrm{TiO}_{2}\right]=\left[\mathrm{CaTiO}_{3}\right]+\left(\mathrm{CO}_{2}\right) .
$$

They then calculated the enthalpy of perovskite formation $\Delta \mathrm{H}_{298}\left(\mathrm{CaTiO}_{3}\right)$ : $-41.84 \pm 1.88 \mathrm{~kJ} / \mathrm{mol}$ (Table 1). Although the obtained value was determined with poor accuracy, due to the difficulty of determining the amounts of substances in the reaction products (1), it corresponded satisfactorily to the more accurate results of Kelley et al. [33], who determined this value according to the reaction:

$$
[\mathrm{CaO}]+\left[\mathrm{TiO}_{2}\right]=\left[\mathrm{CaTiO}_{3}\right]
$$


by solution calorimetry in a mixture of hydrofluoric and hydrochloric acids. The reactions of dissolution were more complete than combustion reaction (1).

Navrotsky et al. [19, 26, 34, 37-43] performed a number of studies by various calorimetric methods, using adiabatic calorimetry [26] and solution calorimetry in the ( $\mathrm{Li}, \mathrm{Na}) \mathrm{BO}_{2}$ [41], $\mathrm{Pb}_{2} \mathrm{~B}_{2} \mathrm{O}_{5}[37,42,43]$, and $\mathrm{Na}_{6} \mathrm{Mo}_{4} \mathrm{O}_{15}[19,34,38-40]$ salts in the temperature range $973-1074 \mathrm{~K}$. They determined the value of $\Delta H_{T}\left(\mathrm{CaTiO}_{3}\right)$, which lies in the range of -44 to $-39 \mathrm{~kJ} / \mathrm{mol}$; the accuracy of measurements was $2 \mathrm{~kJ} / \mathrm{mol}$ (Table 1). The differences could be due to the properties of the solvents that were used. Perovskite and its oxides are poorly soluble in oxide solvent $\mathrm{Pb}_{2} \mathrm{~B}_{2} \mathrm{O}_{5} ; \mathrm{Na}_{6} \mathrm{Mo}_{4} \mathrm{O}_{15}$ liquid alloy is quite volatile and cannot be used at temperatures above $1000 \mathrm{~K}$; ( $\mathrm{Li}, \mathrm{Na}) \mathrm{BO}_{2}$ solution is hygroscopic, which creates difficulties in synthesizing [49]. The $\Delta H_{1078}\left(\mathrm{CaTiO}_{3}\right)$ value determined by Koito et al. [44] by similar method (solution calorimetry in $\mathrm{Pb}_{2} \mathrm{~B}_{2} \mathrm{O}_{5}$ salt) is less accurate but close to the results obtained by Navrotsky et al. [19, 34, 37-43].

The data obtained by Sato et al. [28] using adiabatic calorimetry deviate negligibly (by as much as $7 \mathrm{~kJ} / \mathrm{mol}$ ) in the enthalpy values of $\left(\mathrm{H}_{T}-\mathrm{H}_{298}\right)$ in the temperature range above $1000 \mathrm{~K}$. Approximately the same systematic deviation is observed in determination of enthalpy of perovskite formation from oxides (per 1 mole of the compound) due to its use in calculations of the rough semiempirical approximation proposed in [50]. At the same time, the entropy of perovskite formation determined by Sato et al. [28] is in satisfactory agreement with the results obtained by Kelley and Mah [20], Woodfield et al. [26], and Prasanna and Navrotsky [42] and calculated by Gillet et al. [46] based on information obtained on Raman spectra (Table 1).

Golubenko and Rezukhina [45] studied the heterogeneous reaction using a solid electrolyte galvanic cell (EMF method) in the temperature range of 1180-1290 K:

$$
[\mathrm{CaO}]+1 / 2\left[\mathrm{Ti}_{2} \mathrm{O}\right]+3 / 4\left(\mathrm{O}_{2}\right)=\left[\mathrm{CaTiO}_{3}\right]
$$

A mixture of $\mathrm{FeO}$ and $\mathrm{Fe}$ (or $\mathrm{NbO}$ and $\mathrm{Nb}$ ) was used as the reference electrode, and a mixture of $\mathrm{La}_{2} \mathrm{O}_{3}-\mathrm{ThO}_{2}$ crystals was used as the solid electrolyte. The Gibbs energy of perovskite $\Delta G_{T}\left(\mathrm{CaTiO}_{3}\right)$ was calculated based on a compilation of fairly approximate literature data and their own estimates of the thermodynamic properties of the $\left[\mathrm{Ti}_{2} \mathrm{O}\right]$ compound. This produced a considerable error in determining this value (Figure 3). Rezukhina et al. [35] later made more precise measurements of $\Delta G_{T}\left(\mathrm{CaTiO}_{3}\right)$ in the temperature range of 888-972 K, inside a galvanic cell with $\mathrm{CaF}_{2}$ as the electrolyte (Figure 3 ). However, the non-systematic errors in determining $\Delta H_{T}\left(\mathrm{CaTiO}_{3}\right)$ and $\Delta S_{T}\left(\mathrm{CaTiO}_{3}\right)$ values were considerable (Table 1).

Taylor and Schmalzried [51] (at $873 \mathrm{~K}$ ) and Jacob and Abraham [36] (at 900$1250 \mathrm{~K}$ ) also determined the perovskite Gibbs energy via EMF using the same solid electrolyte. The obtained $\Delta G_{T}\left(\mathrm{CaTiO}_{3}\right)$ values were close to the results of Rezukhina et al. [35].

Klimm et al. [47] used differential thermal analysis (DTA) to determine the enthalpy of reaction (2) at $1740 \pm 20 \mathrm{~K}$. The obtained value is consistent with the results of thermochemical calculations, although it has a significant error.

Suito et al. $[54,55]$ has studied the equilibrium at $1873 \mathrm{~K}$ :

$$
[\mathrm{Ti}]+2(\mathrm{O})=\left[\mathrm{TiO}_{2}\right]
$$

in $\mathrm{CaO}-\mathrm{TiO}_{x}$ (or $\mathrm{CaO}-\mathrm{TiO}_{x}-\mathrm{Al}_{2} \mathrm{O}_{3}$ ) slags with liquid nickel, relative to oxygen and nitrogen, depending on the content of $\mathrm{Ti}$ (or $\mathrm{Al}$ ) in the metal. Crucibles made of $\mathrm{CaO}$ or $\mathrm{Al}_{2} \mathrm{O}_{3}$ were used. The activity $\left(a_{i}\right)$ of titanium oxide was estimated indirectly, depending on the content of $\mathrm{Al}, \mathrm{Ti}$, and $\mathrm{O}$ in the slag, and using data on the Gibbs energies of oxide formation (Figure 4). 


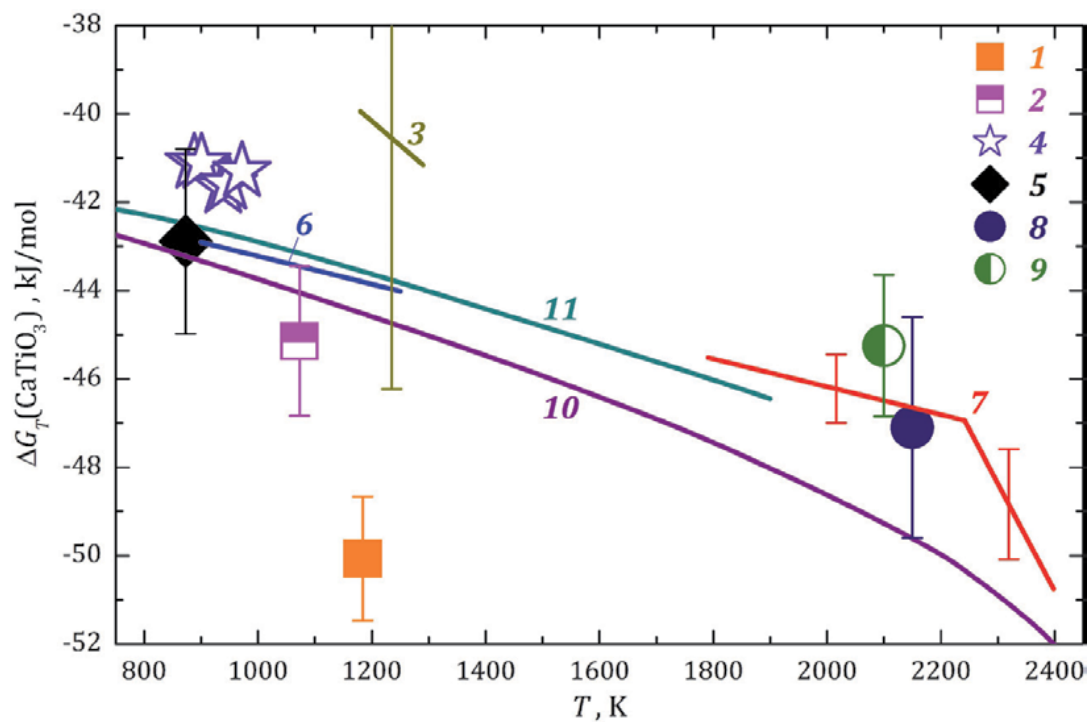

Figure 3.

The Gibbs energy of the formation of perovskite, determined via $(1,2)$ calorimetry $[28,42]$, respectively; (3-6) $E M F[35,36,45,51]$, respectively; (7-9) Knudsen effusion mass spectrometric method in $[48,52,53]$, respectively; $(10,11)$ calculated from thermochemical data in $[24,26]$, respectively.

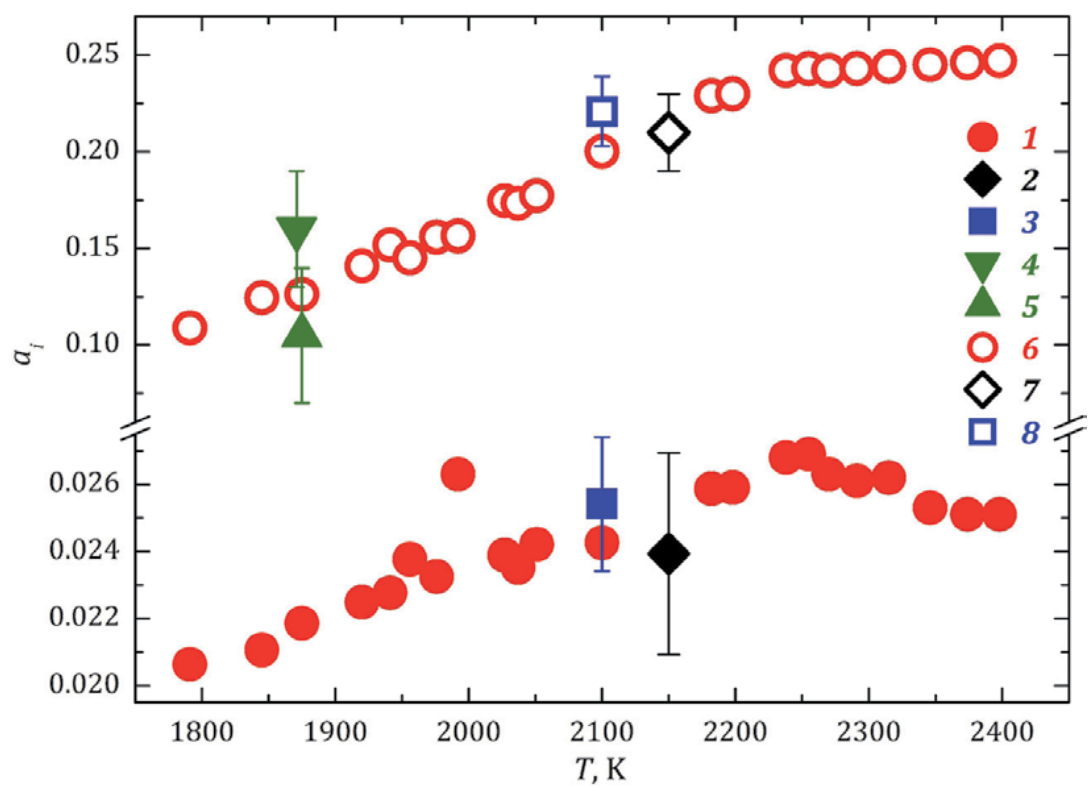

Figure 4.

Activities of $\mathrm{CaO}(1-3)$ and $\mathrm{TiO}_{2}(4-8)$ in perovskite, determined (1-3, 6-8) via Knudsen effusion mass spectrometry $[48,52,53]$ and $(4,5)$ in studying the heterogeneous equilibria of multicomponent melts $[54,55]$, respectively.

Banon et al. [52] (at $2150 \mathrm{~K}$ ) and Shornikov et al. [48, 53, 56] (at 1791-2398 K) determined the values of $\mathrm{CaO}$ and $\mathrm{TiO}_{2}$ activities (Figure 4) and $\Delta G_{T}\left(\mathrm{CaTiO}_{3}\right)$ via Knudsen effusion mass spectrometric method. The resulting values correlated with one another within the experimental error (up to $2.5 \mathrm{~kJ} / \mathrm{mol}$ ); however, the $\Delta G_{T}\left(\mathrm{CaTiO}_{3}\right)$ was obtained at a temperature $\sim 1000 \mathrm{~K}$ higher than in the earlier results (Figure 3). 
As it is seen in Figure 4, the values of oxide activities in perovskite determined via Knudsen effusion mass spectrometric method agree with one another in the investigated temperature range. As the temperature grows, there is a slight trend toward the higher activities of calcium and titanium oxides in the crystalline perovskite phase. This trend is less noticeable in the area of the liquid phase. The activities of titanium oxide calculated based on studying of equilibria in slags $[54,55]$ are fairly approximate but not inconsistent with the results in $[48,52,53]$.

The values of Gibbs energy of perovskite formation determined at 800-1200 K via EMF [35, 36, 51] and solution calorimetry [42] correlate satisfactorily with the obtained via Knudsen effusion mass spectrometric method [48, 52, 53, 56]. Figure 3 shows a good agreement between these data and the results from thermochemical calculations performed by Woodfield et al. [26]. The difference between our findings and the thermochemical data calculated by Bale et al. [24] is large in the perovskite melting area, but is still less than $3 \mathrm{~kJ} / \mathrm{mol}$.

The $\Delta H_{T}\left(\mathrm{CaTiO}_{3}\right)$ and $\Delta S_{T}\left(\mathrm{CaTiO}_{3}\right)$ values determined in [48] correlate with the results from studies performed via solution calorimetry [37, 41, 42, 44] and EMF [35, 36] at lower temperatures and by Raman spectroscopy [46] and DTA [47] at similar temperatures (Table 1).

\section{Melting of perovskite}

The data characterizing the melting of simple oxides [23, 24, 57-60] are quite rough (Table 2 ). According to different thermochemical data, perovskite's melting temperature lies in the range of 2188 to $2243 \mathrm{~K}$.

Klimm et al. [47] estimated the perovskite melting enthalpy as $56.65 \pm 11.33 \mathrm{~kJ} / \mathrm{mol}$ at $2220 \pm 20 \mathrm{~K}$ (Table 2) which is close to earlier thermochemical estimates [24, 59].

Shornikov [48] based on his own data (Table 1) has obtained more accurate values, characterizing the perovskite melting (Table 2 ). They coincide satisfactorily with the experimental data obtained by Klimm et al. [47] and the thermochemical

\begin{tabular}{lcccc}
\hline Compound & $\boldsymbol{T}, \mathbf{K}$ & $\boldsymbol{\Delta} \boldsymbol{H}_{\text {melt }}, \mathbf{~ k J} / \mathbf{m o l}$ & $\Delta \boldsymbol{S}_{\text {melt }}, \mathbf{J} /(\mathbf{m o l ~ K})$ & References \\
\hline $\mathrm{CaO}$ & 2843 & 52.00 & 18.29 & {$[59]$} \\
\hline 2845 & 79.50 & 27.94 & {$[24]$} \\
\hline $\mathrm{CaTiO}_{3}$ & $3200 \pm 50$ & 79.50 & 24.84 & {$[58]$} \\
\hline & $3210 \pm 10$ & 55.20 & 17.20 & {$[60]$} \\
\hline & 2188 & 41.84 & 19.12 & {$[61]$} \\
\hline & $222 \pm \pm 20$ & $56.65 \pm 11.33$ & $25.52 \pm 5.10$ & {$[47]$} \\
\hline $\mathrm{TiO}_{2}$ & 2233 & 53.32 & 23.88 & {$[24]$} \\
\hline & $2241 \pm 10$ & $47.61 \pm 1.84$ & $21.24 \pm 0.81$ & {$[48]$} \\
\hline & 2243 & 63.65 & 28.38 & {$[59]$} \\
\hline & 2103 & 66.90 & 31.81 & {$[23,58]$} \\
\hline & 2130 & $66.94 \pm 16.70$ & $31.43 \pm 7.84$ & {$[24]$} \\
\hline
\end{tabular}

Table 2.

Temperatures, enthalpies, and entropies of the melting of compounds in the $\mathrm{CaO}-\mathrm{TiO}_{2}$ system (calculated for 1 mol of compound) 
estimates made by Bale et al. [24]. The enthalpy of perovskite melting estimated using Walden's empirical rule [62] is also close to the result obtained by Shornikov [48]: $\Delta H_{\text {melt }}=8.8\left[\mathrm{~J} /(\mathrm{g}\right.$-at K) $] T_{\text {melt }}=49.39 \mathrm{~kJ} / \mathrm{mol}$.

\section{The thermodynamic properties of perovskite melts}

Thermodynamic information about the $\mathrm{CaO}-\mathrm{TiO}_{2}$ melts is quite scarce and limited by the results of only a few experimental studies. Consider the available experimental data, obtained by the Knudsen effusion mass spectrometry.

Banon et al. [52] investigated the evaporation of 24 compositions of the $\mathrm{CaTiO}_{3}$ $\mathrm{Ti}_{2} \mathrm{O}_{3}-\mathrm{TiO}_{2}$ system from molybdenum containers at $1900-2200 \mathrm{~K}$. The synthesized compositions contained up to $90.2 \mathrm{~mol} \% \mathrm{Ti}_{2} \mathrm{O}_{3}$ and up to $42 \mathrm{~mol} \% \mathrm{TiO}_{2}$ as well as $\mathrm{CaTiO}_{3}$ compound. Based on the partial vapor pressures $(\mathrm{Ca}),(\mathrm{TiO})$, and $\left(\mathrm{TiO}_{2}\right)$ over melts at $2150 \mathrm{~K}$, the authors calculated the $\mathrm{Ti}, \mathrm{TiO}, \mathrm{Ti}_{2} \mathrm{O}_{3}, \mathrm{TiO}_{2}$, and $\mathrm{CaTiO}_{3}$ activities, as well as mixing energies in the melts. In the case of the $\mathrm{CaTiO}_{3}-\mathrm{TiO}_{2}$ melts, the $\mathrm{TiO}_{2}$ and $\mathrm{CaTiO}_{3}$ activities were calculated by extrapolation from the data relating to the $\mathrm{CaTiO}_{3}-\mathrm{Ti}_{2} \mathrm{O}_{3}-\mathrm{TiO}_{2}$ system and thus had, according to the authors themselves, low accuracy, which apparently was caused by inconsistency with different versions of the $\mathrm{CaO}-\mathrm{TiO}_{2}$ phase diagram $[7,9,11,18]$. Nevertheless Banon et al. [52], interpreting the obtained high values of $\mathrm{TiO}_{2}$ activities in the region close to titanium dioxide (Figure 5), assumed the presence of immiscibility of the $\mathrm{CaO}$ $\mathrm{TiO}_{2}$ melts in this region.

Stolyarova et al. [63] investigated the properties of the gas phase over 14 compositions of the $\mathrm{CaO}-\mathrm{TiO}_{2}-\mathrm{SiO}_{2}$ system and also determined the values of oxide activity and melt mixing energy by high-temperature mass spectrometry during the evaporation of melts from tungsten effusion containers at 1800-2200 K. The synthesized compositions contained up to $70 \mathrm{~mol} \% \mathrm{CaO}$, up to $69 \mathrm{~mol} \% \mathrm{SiO}_{2}$, and up to $40 \mathrm{~mol} \% \mathrm{TiO}_{2}$. As it is shown in Figure 5, one of the two studied compositions of the $\mathrm{CaO}-\mathrm{TiO}_{2}$ system at $2057 \mathrm{~K}$ was in the " $\mathrm{CaO}+$ liquid" region, and thus its value

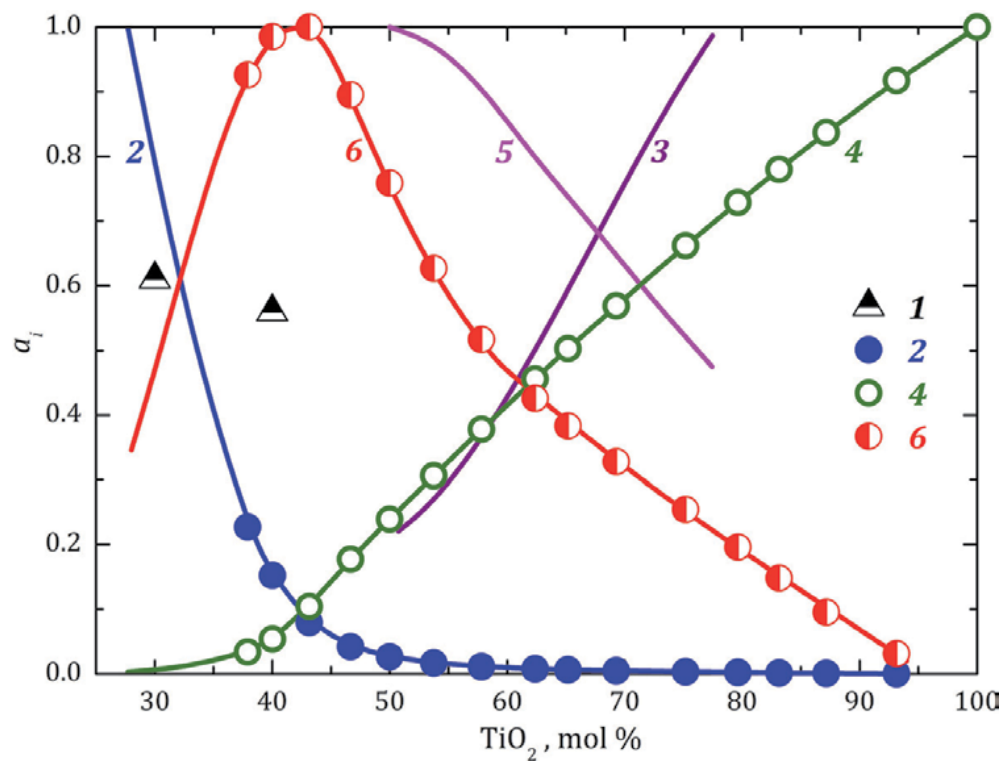

Figure 5.

The activities of $\mathrm{CaO}(1,2), \mathrm{TiO}_{2}(3,4)$, and $\mathrm{CaTiO}_{3}(5,6)$ in the $\mathrm{CaO}-\mathrm{TiO}_{2}$ melts, determined at $2057 \mathrm{~K}$ (1) in [63], at $2150 \mathrm{~K}(3,5)$ in [52], and at $2250 \mathrm{~K}(2,4,6)$ in [64]. 
should be close to 1 . The second composition was in the region of " $\mathrm{Ca}_{4} \mathrm{Ti}_{3} \mathrm{O}_{10}+$ liquid," according to the information presented in $[7,18]$, or in the region of " $\mathrm{Ca}_{3} \mathrm{Ti}_{2} \mathrm{O}_{7}+$ liquid," as follows from the data presented by Tulgar [11]. However, the calculated values are quite close (Figure 5), which contradicts the $\mathrm{CaO}-\mathrm{TiO}_{2}$ phase diagram (Figure 1). A possible reason for the discrepancies seems to be a significant error in the measurements of $\mathrm{CaO}$ activities in the melt, which may be, in our opinion, more than $50 \%$.

Shornikov [64] investigated the evaporation from molybdenum containers of more than 200 compositions of the $\mathrm{CaO}-\mathrm{TiO}_{2}$ system containing from 34 to $98 \mathrm{~mol}$ $\% \mathrm{TiO}_{2}$ at $2241-2441 \mathrm{~K}$. The studied compositions were the $\mathrm{CaO}-\mathrm{TiO}_{2}-\mathrm{SiO}_{2}$ residual melts containing up to $1 \mathrm{~mol} \% \mathrm{SiO}_{2}$ that was lost during high-temperature evaporation. The determined composition of the gas phase over the $\mathrm{CaO}-\mathrm{TiO}_{2}$ melts allowed to conclude that evaporation reactions are typical for individual oxides predominate.

The oxide activities in the $\mathrm{CaO}-\mathrm{TiO}_{2}$ melts were calculated according to Lewis equations [65]:

$$
a_{i}=p_{i} / p_{i}^{\circ}
$$

where $p_{i}^{\circ}$ and $p_{i}$ are the partial pressures of vapor species over individual oxide and melt, respectively. However, it is preferable to calculate the values of oxide activities using the Belton-Fruehan approach [66] via the following equation:

$$
\ln a_{i}=-\int x_{j} d \ln \left(a_{j} / a_{i}\right),
$$

in which the ratio of the oxide activities in the melt could be easily converted to the ratio of the partial pressures, proportional to the ion currents $\left(I_{i}\right)$ :

$$
\begin{aligned}
\ln a_{\mathrm{TiO}_{2}} & =-\int x_{\mathrm{CaO}} d \ln \left(p_{\mathrm{CaO}} / p_{\mathrm{TiO}_{2}}\right)=-\int x_{\mathrm{CaO}} d \ln \left(p_{\mathrm{Ca}} p_{\mathrm{O}} / p_{\mathrm{TiO}} p_{\mathrm{O}}\right) \\
& =-\int x_{\mathrm{CaO}} d \ln \left(I_{\mathrm{Ca}} / I_{\mathrm{TiO}}\right),
\end{aligned}
$$

and thus to evade the needs in additional thermochemical data, used in Eq. (5).

The consistency of the values of $\mathrm{TiO}_{2}$ activities calculated by relation (7) was verified using the Gibbs-Duhem equation [67]:

$$
\ln a_{\mathrm{CaO}}=-\int \frac{x_{\mathrm{TiO}_{2}}}{x_{\mathrm{CaO}}} d \ln a_{\mathrm{TiO}_{2}},
$$

Values of chemical potentials $\left(\Delta \mu_{i}\right)$, partial enthalpy $\left(\Delta H_{i}\right)$, and entropy $\left(\Delta S_{i}\right)$ of oxides in the $\mathrm{CaO}-\mathrm{TiO}_{2}$ melts were calculated by known equations [67]:

$$
\begin{gathered}
\Delta \mu_{i}=R T \ln a_{i} \\
\Delta \mu_{i}=\Delta H_{i}-T \Delta S_{i} \\
\Delta H_{i}=\frac{d\left(\Delta \mu_{i} / T\right)}{d(1 / T)}=R \frac{d \ln a_{i}}{d(1 / T)} \\
\Delta S_{i}=-d \Delta \mu_{i} / d T,
\end{gathered}
$$

which are related to the corresponding integral thermodynamic mixing functions: 


$$
\begin{gathered}
\Delta G_{T}^{m}=\sum_{i} x_{i} \Delta \mu_{i} \\
\Delta H_{T}=\sum_{i} x_{i} \Delta H_{i} \\
\Delta S_{T}=\sum_{i} x_{i} \Delta S_{i} \\
\Delta G_{T}^{m}=\Delta H_{T}-T \Delta S_{T}
\end{gathered}
$$

and are represented in Figure 6.

The results presented by Banon et al. [52] correlate with the data found in [64]. Some difference in values, as mentioned above, is probably due to the procedures for extrapolating information obtained by Banon et al. [52] for compositions of the $\mathrm{CaTiO}_{3}-\mathrm{Ti}_{2} \mathrm{O}_{3}-\mathrm{TiO}_{2}$ triple system, which could reduce their accuracy. The observed behavior of $\mathrm{TiO}_{2}$ activity in melts in the concentration region close to rutile may indicate some immiscibility of the melt, which follows from the observed inflection of the concentration dependence (Figure 5, line 3). However, in our opinion, the behavior of $\mathrm{TiO}_{2}$ and $\mathrm{CaTiO}_{3}$ activities (Figure 5, lines 4 and 6) are close to the ideal. The maximum value corresponds to the area of compositions close to

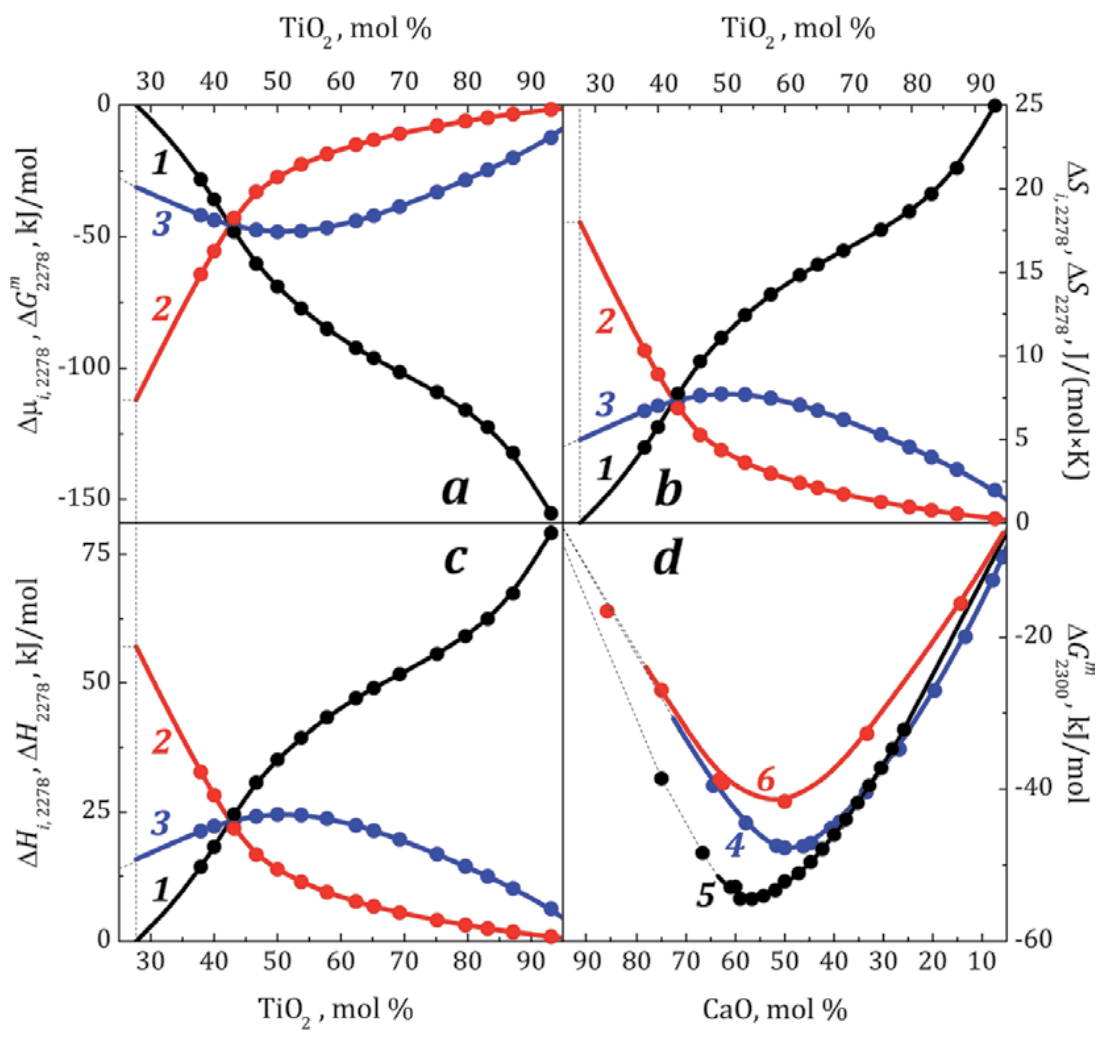

Figure 6.

The thermodynamic properties of the $\mathrm{CaO}-\mathrm{TiO}_{2}$ melts at $2278 \mathrm{~K}$ [64] (the chemical potentials of oxides and the mixing energy ( $a)$, the partial enthalpies of oxides and the enthalpy of formation $(b)$, and the partial entropies of oxides and the entropy of formation (c)); symbols: (1) $\mathrm{CaO},(2) \mathrm{TiO}_{2}$, (3) integral thermodynamic characteristics (mixing energy, enthalpy, and entropy of formation of the melts, respectively; the vertical dashed line marks the boundary of the " $\mathrm{CaO}+$ liquid" region and the melt) and the comparison of mixing energies (d) in the $\mathrm{CaO}-\mathrm{TiO}_{2}(4), \mathrm{CaO}-\mathrm{SiO}_{2}(5)$, and $\mathrm{CaO}-\mathrm{Al}_{2} \mathrm{O}_{3}$ (6) melts determined by the Knudsen effusion mass spectrometric method in $[64,68,69]$, respectively (the dashed lines correspond to heterogeneous areas). 
perovskite (Figure 5, lines 5 and 6). Differences with values obtained by Stolyarova et al. [63] (Figure 5, points 1), are caused, apparently, by the low accuracy of the latter.

The partial and integral thermodynamic regularities presented in Figure 6 characterizing the $\mathrm{CaO}-\mathrm{TiO}_{2}$ melts are symbate. The enthalpy and entropy of melt formation are positive. The extreme values of the integral thermodynamic properties of the melts are in the concentration ranges close to perovskite, which confirms its stability in the melt. Some displacement of the extremum of integral thermodynamic functions can be caused by the presence of oxide compounds with a large amount of $\mathrm{CaO}$ in comparison with perovskite $\mathrm{CaTiO}_{3}$ in the melt. A comparison of mixing energies in the $\mathrm{CaO}-\mathrm{TiO}_{2}$ melts at $2300 \mathrm{~K}$ with those for the $\mathrm{CaO}-\mathrm{SiO}_{2}$ [68] and $\mathrm{CaO}-\mathrm{Al}_{2} \mathrm{O}_{3}$ [69] melts (Figure 6d) indicates a stronger chemical interaction in the $\mathrm{CaO}-\mathrm{TiO}_{2}$ melts than the $\mathrm{CaO}-\mathrm{Al}_{2} \mathrm{O}_{3}$ melts, but smaller than in the $\mathrm{CaO}-\mathrm{SiO}_{2}$ melts. It manifests in more positive values of the mixing energy of the melts.

\section{The gas phase over perovskite}

The evaporation processes and the thermodynamic properties of simple oxides $\mathrm{CaO}$ and $\mathrm{TiO}_{2}$ were considered in detail in reference books $[23,24,57,70,71]$.

The gas phase over calcium oxide consists of the molecular components $(\mathrm{O})$, $\left(\mathrm{O}_{2}\right),\left(\mathrm{O}_{3}\right),\left(\mathrm{O}_{4}\right),(\mathrm{Ca}),\left(\mathrm{Ca}_{2}\right)$, and $(\mathrm{CaO})$ possibly formed by the following reactions:

$$
\begin{gathered}
{[\mathrm{CaO}]=(\mathrm{CaO})} \\
(\mathrm{CaO})=(\mathrm{Ca})+(\mathrm{O}) \\
2(\mathrm{Ca})=\left(\mathrm{Ca}_{2}\right) \\
2(\mathrm{O})=\left(\mathrm{O}_{2}\right) \\
3(\mathrm{O})=\left(\mathrm{O}_{3}\right) \\
4(\mathrm{O})=\left(\mathrm{O}_{4}\right) .
\end{gathered}
$$

The gas phase over titanium oxide contains similar vapor molecular forms (O), $\left(\mathrm{O}_{2}\right),\left(\mathrm{O}_{3}\right),\left(\mathrm{O}_{4}\right),(\mathrm{Ti}),\left(\mathrm{Ti}_{2}\right),\left(\mathrm{Ti}_{3}\right),(\mathrm{TiO})$, and $\left(\mathrm{TiO}_{2}\right)$ formed by similar reactions:

$$
\begin{gathered}
{\left[\mathrm{TiO}_{2}\right]=\left(\mathrm{TiO}_{2}\right)} \\
\left(\mathrm{TiO}_{2}\right)=(\mathrm{TiO})+(\mathrm{O}) \\
(\mathrm{TiO})=(\mathrm{Ti})+(\mathrm{O}) \\
2(\mathrm{Ti})=\left(\mathrm{Ti}_{2}\right) \\
3(\mathrm{Ti})=\left(\mathrm{Ti}_{3}\right) .
\end{gathered}
$$

Balducci et al. [72] detected $\left(\mathrm{Ti}_{2} \mathrm{O}_{3}\right)$ and $\left(\mathrm{Ti}_{2} \mathrm{O}_{4}\right)$ molecules in the gas phase over cobalt titanate $\mathrm{CoTiO}_{3}$ at 2210-2393 K by the Knudsen effusion mass spectrometric method, which can be involved in the following equilibria:

$$
\begin{gathered}
2\left(\mathrm{TiO}_{2}\right)=\left(\mathrm{Ti}_{2} \mathrm{O}_{4}\right), \\
\left(\mathrm{Ti}_{2} \mathrm{O}_{4}\right)=\left(\mathrm{Ti}_{2} \mathrm{O}_{3}\right)+(\mathrm{O}) .
\end{gathered}
$$


Note that the predominant components of the gas phase over these oxides are (Ca), $(\mathrm{CaO}),(\mathrm{TiO}),\left(\mathrm{TiO}_{2}\right),(\mathrm{O})$, and $\left(\mathrm{O}_{2}\right)$; the content of other vapor species does not exceed $1 \%$ of the total concentration at $1700-2200 \mathrm{~K}$.

The properties of the gas phase over perovskite were studied in less detail. The experimental conditions and results of high-temperature studies of perovskite evaporation we will consider below.

Zakharov and Protas [73] studied ion emission from the perovskite surface under the action of laser radiation and identified the ion of a complex molecule $\left(\mathrm{CaTiO}_{3}\right)$ in addition to the ions of simple oxides $\left(\mathrm{O}^{+}, \mathrm{Ca}^{+}, \mathrm{CaO}^{+}, \mathrm{Ti}^{+}, \mathrm{TiO}^{+}\right)$in the mass spectra of the vapor. They explained the presence of this ion by the similarity of high-temperature evaporation of alkaline-earth oxide titanates, which is confirmed by the composition of the observed condensates $\left(\mathrm{BaTiO}_{3}, \mathrm{SrTiO}_{3}\right.$, and $\mathrm{CaTiO}_{3}$ ) formed under similar conditions $[74,75]$. The intensity ratio of ion currents in the mass spectra of vapor over perovskite obtained at laser pulse duration of $800-1000 \mu$ s at a wavelength of $6943 \AA$ and energy of 3-5 J was as follows: $I_{\mathrm{O}}: I_{\mathrm{Ca}}$ : $I_{\mathrm{CaO}}: I_{\mathrm{Ti}}: I_{\mathrm{TiO}}: I_{\mathrm{CaTiO}_{3}}=0.41: 100: 0.17: 4.32: 0.54: 0.05$. Note that the $\mathrm{TiO}_{2}{ }^{+}$ion was not observed in the mass spectrum of vapor over both perovskite $\mathrm{CaTiO}_{3}$ and rutile $\mathrm{TiO}_{2}$. This is explained by the peculiarity of the mass spectrometric experiment using a laser, in which the easily ionizable molecular species dominate the mass spectra of vapor and the not readily ionizable molecules are discriminated. This selectivity of detected ions in the mass spectra of vapor significantly limits the accuracy and applicability of this method [76]. According to the data of [57], the estimated temperature of heating of perovskite under the action of laser radiation based on the possible equilibrium in the gas phase.

$$
(\mathrm{Ca})+(\mathrm{TiO})=(\mathrm{CaO})+(\mathrm{Ti})
$$

is $4890 \pm 70 \mathrm{~K}$, which is approximate, but does not contradict the conditions of similar laser-impact mass spectrometry experiments in the range 4000-6000 K.

Banon et al. [52] studied the evaporation of the $\mathrm{CaTiO}_{3}-\mathrm{Ti}_{2} \mathrm{O}_{3}-\mathrm{TiO}_{2}$ melts from molybdenum Knudsen effusion cells at $1900-2200 \mathrm{~K}$ by differential mass spectrometry. The mass spectra were recorded at a low ionizing voltage of $13 \mathrm{eV}$ in order to avoid possible fragmentation of the $\mathrm{TiO}_{2}{ }^{+}$molecular ion into $\mathrm{Ti}^{+}$and $\mathrm{TiO}^{+}$ fragmentation ions, which were also molecular ions.

Atomic calcium was the dominant component of the gas phase over the composites. The complex gaseous oxide $\left(\mathrm{CaTiO}_{3}\right)$ was not detected. The partial pressures of the $(\mathrm{Ca}),(\mathrm{TiO}),\left(\mathrm{TiO}_{2}\right)$, and $(\mathrm{O})$ vapor over perovskite at $2150 \mathrm{~K}$ were calculated using the thermochemical data of [57] and are shown in Figure 7 as a function of the inverse temperature (for easily understanding, the temperature scale was scaled appropriately).

Gaseous perovskite was also not detected in the mass spectrometric studies of high-temperature evaporation of various compositions of the $\mathrm{CaO}-\mathrm{TiO}_{2}-\mathrm{SiO}_{2}$ system from molybdenum and tungsten Knudsen effusion cells at 1700-2500 K $[53,63,82,83]$ presumably because the sensitivity of the equipment used in $[63,83]$ was insufficient for determining the $\mathrm{CaTiO}_{3}{ }^{+}$ion or because this was not the purpose of the study [53, 82].

Lopatin and Semenov [84] studied the evaporation of a mixture of calcium carbonate and titanium dioxide from tungsten cells by the Knudsen effusion mass spectrometry method in the temperature range $2100-2500 \mathrm{~K}$. The following ions were detected in the mass spectra of vapor over the mixture: $\mathrm{Ca}^{+}, \mathrm{CaO}^{+}, \mathrm{Ti}^{+}, \mathrm{TiO}^{+}$, $\mathrm{TiO}_{2}{ }^{+}$, and $\mathrm{CaTiO}_{3}{ }^{+}$. The energies of ion appearance in the mass spectra allowed the authors to determine the molecular origin of the $\mathrm{Ca}^{+}, \mathrm{CaO}^{+}, \mathrm{TiO}^{+}, \mathrm{TiO}_{2}{ }^{+}$, and $\mathrm{CaTiO}_{3}{ }^{+}$ions. The $\mathrm{TiO}^{+}$ion also contained a fragment component of the $\mathrm{TiO}_{2}{ }^{+}$ion, 


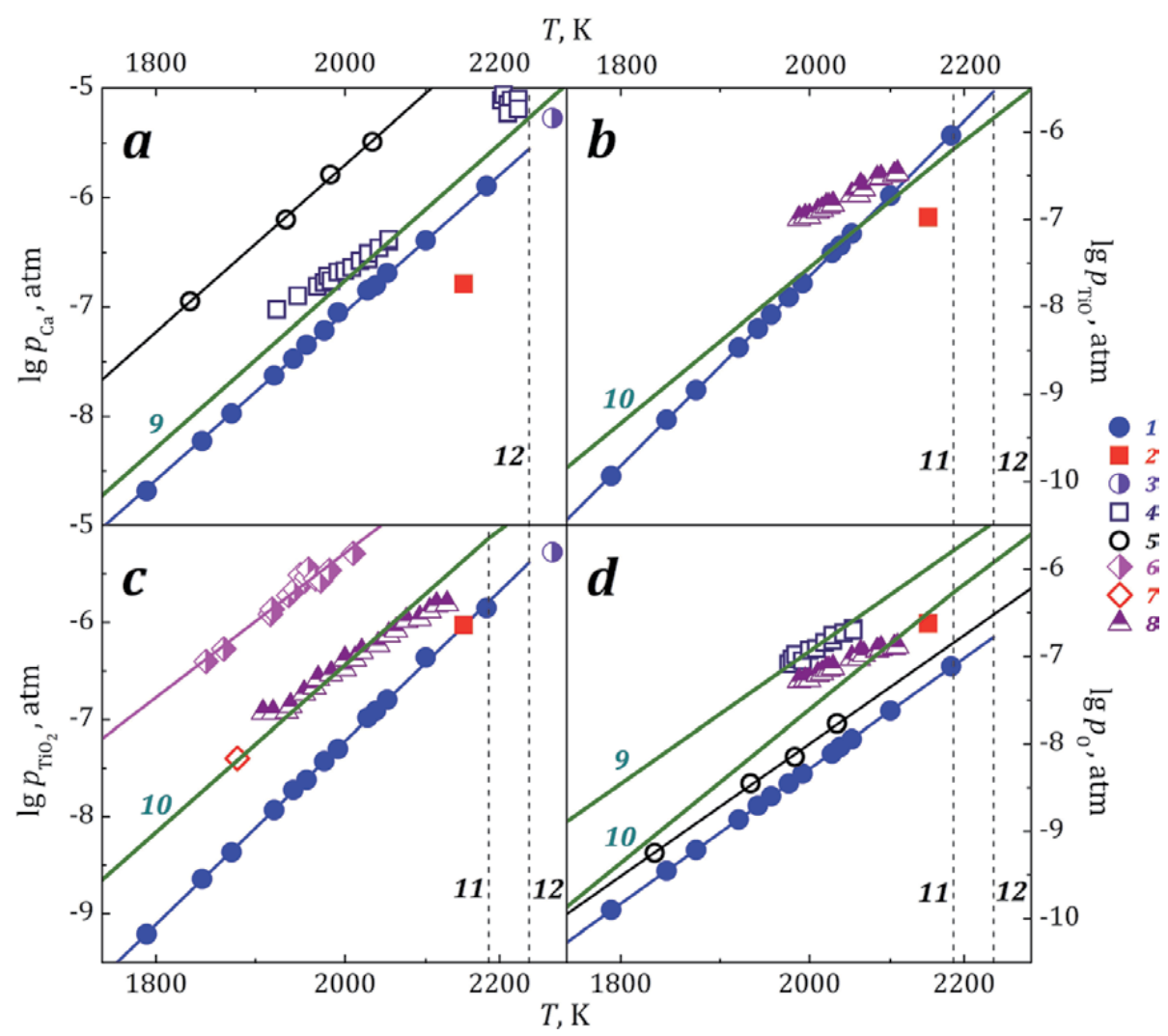

Figure 7.

The partial pressure of $\mathrm{Ca}(a), \mathrm{TiO}(b), \mathrm{TiO}_{2}(c)$, and $\mathrm{O}(d)$ over perovskite (1-3) and oxides of calcium (4, $5,9)$ and titanium $(6-8,10)$ vs. the inverse temperature, determined via Knudsen mass spectrometry: (1) in [77], (2) in [52], (4) in [78], (5) in [79], (6) in [80], (7) in [81], and (8) in [71]; using the vacuum furnace (according to Langmuir), (3) in [4]; and calculated, (9) and (10), according to the thermochemical data [57]; the vertical dashed lines (11) and (12) indicate melting points of titanium oxide and perovskite, respectively.

and the $\mathrm{Ti}^{+}$ion was completely fragmentary. The energy of appearance of the $\mathrm{CaTiO}_{3}{ }^{+}$molecular ion was determined to be $9 \pm 1 \mathrm{eV}$ (the energy of appearance of the gold ion was used as a standard). The partial pressures of vapor species $\left(p_{i}\right)$ were calculated by comparison with the accepted partial vapor pressures of gold taken as standard pressures $\left(p_{s}\right)$ by the equation:

$$
p_{i}=\frac{I_{i} T_{i}}{I_{s} T_{s}} p_{s} \times \frac{\sigma_{s} \gamma_{s} \eta_{s}}{\sigma_{i} \gamma_{i} \eta_{i}}
$$

where $I_{i}\left(I_{s}\right)$ is the intensity of the ion current of the $i$ th component of vapor (standard substance) recorded at a temperature $T_{i}\left(T_{s}\right)$. The calculation should also include the ratios of the effective ionization cross sections of the $i$ th molecular form and the standard substance $\left(\sigma_{i} / \sigma_{s}\right)$, isotope distributions $\left(\eta_{i} / \eta_{s}\right)$, and individual ion efficiencies $\left(\gamma_{i} / \gamma_{s}\right)$, which depend on various parameters of ion current recording devices. The pressures $p_{\mathrm{CaO}}, p_{\mathrm{TiO}_{2}}$, and $p_{\mathrm{CaTiO}_{3}}$ calculated by (31) were used to determine the temperature dependence of the equilibrium constant in the gas phase:

$$
\left(\mathrm{CaTiO}_{3}\right)=(\mathrm{CaO})+\left(\mathrm{TiO}_{2}\right)
$$




\begin{tabular}{|c|c|c|c|c|c|}
\hline Reaction & $T, \mathrm{~K}$ & $\begin{array}{l}\Delta_{r} H_{298} \\
\mathrm{~kJ} / \mathrm{mol}\end{array}$ & $\begin{array}{c}\Delta_{r} H_{T} \\
\mathrm{~kJ} / \mathrm{mol}\end{array}$ & $\begin{array}{c}\Delta_{r} S_{T} \\
\mathrm{~J} /(\mathbf{m o l ~ K})\end{array}$ & Refs. \\
\hline \multirow[t]{3}{*}{$\left(\mathrm{CaTiO}_{3}\right)=(\mathrm{CaO})+\left(\mathrm{TiO}_{2}\right)$} & $2287-2466$ & $545 \pm 8$ & $284 \pm 44$ & $28 \pm 19$ & {$[84]$} \\
\hline & 2000 & - & $298 \pm 30$ & - & {$[85]$} \\
\hline & 1956-2182 & - & $287 \pm 12$ & $18 \pm 6$ & {$[77]$} \\
\hline \multirow[t]{2}{*}{$\begin{array}{l}{[\mathrm{Ca}]+[\mathrm{Ti}]+3 / 2} \\
\left(\mathrm{O}_{2}\right)=\left(\mathrm{CaTiO}_{3}\right)\end{array}$} & $2287-2466$ & $-826 \pm 26$ & - & - & {$[84]$} \\
\hline & 1956-2182 & - & $-760 \pm 10$ & $-242 \pm 5$ & {$[77]$} \\
\hline \multirow[t]{3}{*}{$\left(\mathrm{CaTiO}_{3}\right)=(\mathrm{Ca})+(\mathrm{Ti})+3(\mathrm{O})$} & $2287-2466$ & $2225 \pm 26$ & - & - & {$[84]$} \\
\hline & 2000 & - & $1983 \pm 81$ & - & {$[85]$} \\
\hline & $1956-2182$ & - & $1993 \pm 15$ & $396 \pm 7$ & {$[77]$} \\
\hline \multirow[t]{2}{*}[\mathrm{CaTiO}_{3}]{$=\left(\mathrm{CaTiO}_{3}\right)$} & 2000 & - & $1030 \pm 22$ & - & {$[85]$} \\
\hline & 1956-2182 & - & $1027 \pm 10$ & $297 \pm 5$ & [77] \\
\hline
\end{tabular}

Table 3.

Enthalpies and entropies of the reactions involving the $\mathrm{CaTiO}_{3}$ molecule.

and subsequently calculate the enthalpies of formation $\left(\Delta_{f} H_{298}\right)$ and atomization $\left(\Delta_{a t} \mathrm{H}_{298}\right)$ of the $\mathrm{CaTiO}_{3}$ molecule (Table 3).

Zhang et al. [4] studied the isotope fractionation of calcium and titanium during the evaporation of a perovskite melt suspended on an iridium wire in a vacuum furnace at a temperature of $2278 \mathrm{~K}$ (according to Langmuir method). The change in the composition of the residual perovskite melt during evaporation suggested that the component that evaporated predominantly from the melt was its calcium component. The total vapor pressure over perovskite could be evaluated from the data obtained (Figure 7).

Shornikov [64, 77] investigated the evaporation of perovskite at 1791-2182 K and its melts at 2241-2441 K from molybdenum Knudsen effusion cells by hightemperature mass spectrometric method.

The $\mathrm{TiO}_{2}{ }^{+}, \mathrm{Ca}^{+}, \mathrm{TiO}^{+}$, and $\mathrm{O}^{+}$ions prevailed in the mass spectra of vapor over perovskite and its melts at the ionizing electron energy of $20 \mathrm{eV}$, as well as other ions characteristic of the mass spectra over individual oxides [57, 58, 71]. A small amount of $\mathrm{CaTiO}_{3}{ }^{+}$ion was observed, which was fragmented into $\mathrm{CaTi}^{+}, \mathrm{CaTiO}^{+}$, and $\mathrm{CaTiO}_{2}{ }^{+}$ions $\left(I_{\mathrm{CaTi}}: I_{\mathrm{CaTiO}}: I_{\mathrm{CaTiO} 2}: I_{\mathrm{CaTiO} 3}=6: 10: 13: 100\right)$. The ratio of the ion current intensities in the mass spectra of vapor over perovskite at $2182 \mathrm{~K}$ was the following: $I_{\mathrm{Ca}}: I_{\mathrm{CaO}}: I_{\mathrm{Ti}}: I_{\mathrm{TiO}}: I_{\mathrm{TiO} 2}: I_{\mathrm{CaTiO}}: I_{\mathrm{O}}: I_{\mathrm{O} 2}=80: 0.04: 0.1: 75: 100: 0.02: 40: 0.3$. It corresponded to that observed by Samoilova and Kazenas [78] in the same temperature range at evaporation of $\mathrm{CaO}$ from alundum cell and by Semenov [86] at evaporation of $\mathrm{TiO}_{2}$ from a tungsten cell.

The ratio of the ion current intensities in the mass spectra of vapor over perovskite melt containing $57.81 \pm 0.15 \mathrm{~mol} \% \mathrm{TiO}_{2}$ at $2278 \mathrm{~K}$ was the following: $I_{\mathrm{Ca}}: I_{\mathrm{CaO}}$ : $I_{\mathrm{Ti}}: I_{\mathrm{TiO}}: I_{\mathrm{TiO} 2}: I_{\mathrm{CaTiO}}: I_{\mathrm{O}}: I_{\mathrm{O} 2}=25: 0.02: 0.1: 56: 100: 0.13: 0.44: 0.012$, which is different from that for the case of perovskite [77].

The presence of $\mathrm{MoO}_{i}^{+}(i=0-3)$ ions in the mass spectra of vapor over perovskite was due to the evaporation of molybdenum cell at high temperature:

$$
[\mathrm{Mo}]=(\mathrm{Mo}) \text {, }
$$

as well as the interaction of perovskite with the cell material $\left(I_{\mathrm{TiO} 2}: I_{\mathrm{Mo}}: I_{\mathrm{MoO}}: I_{\mathrm{MoO} 2}\right.$ : $\left.I_{\mathrm{MoO} 3}=100: 0.8: 2.6: 7.3: 0.6\right)$ that was detected according to the following equilibria: 


$$
\begin{gathered}
(\mathrm{Mo})+(\mathrm{O})=(\mathrm{MoO}) \\
(\mathrm{MoO})+(\mathrm{O})=\left(\mathrm{MoO}_{2}\right) \\
\left(\mathrm{MoO}_{2}\right)+(\mathrm{O})=\left(\mathrm{MoO}_{3}\right) .
\end{gathered}
$$

Note that Berkowitz et al. [81] found that during evaporation of titanium oxide from a molybdenum liner inserted into a tantalum crucible at $1881 \mathrm{~K}, p_{\mathrm{MoO} 2}$ was initially $10-10^{2}$ times higher than $p_{\mathrm{TiO} 2}$. The $p_{\mathrm{MoO} 2}$ value gradually decreased and became comparable with the $p_{\mathrm{TiO} 2}$ value, which is significantly different from the other results $[52,53,56,64,77,82]$. The high $p_{\mathrm{MoO} 2}$ observed in [81] probably was due to poor quality of the molybdenum liner material (or its alloy). Possibly it was made using powder technology from $\mathrm{MoO}_{3}$ reduced to metal molybdenum at $\sim 1300 \mathrm{~K}$. It could lead to such an excess of partial pressure of $\left(\mathrm{MoO}_{3}\right)$ and its decrease as it evaporates from the surface layers of the liner material.

The appearance energies of ions in the mass spectra of vapor over perovskite were determined by the Warren method [87] and corresponded to the accepted values of the ionization energies of atoms and molecules [88]. The appearance energy of $\mathrm{CaTiO}_{3}{ }^{+}$ion in the mass spectra of vapor over perovskite was equal to $8.5 \pm 0.6 \mathrm{eV}$ (the appearance energy of silver ion was used as a standard) and corresponded to obtained by Lopatin and Semenov [84].

The established molecular composition of the gas phase over perovskite allowed us to draw a conclusion on the predominant evaporation of perovskite according to the reactions (17), (18), (20), (23), (24), and (25), typical for evaporation of simple oxides $[57,71,78,86]$. The presence of a small amount of $\left(\mathrm{CaTiO}_{3}\right)$ molecules in the gas phase over perovskite is probably due to the reaction:

$$
\left[\mathrm{CaTiO}_{3}\right]=\left(\mathrm{CaTiO}_{3}\right) .
$$

The partial pressure values of vapor species in the gas phase over perovskite were determined by the Hertz-Knudsen equation, written in the following form [89]:

$$
p_{i}=K_{\alpha} \frac{q_{i}}{s_{o r} C_{o r} t} \sqrt{\frac{2 \pi R T}{M_{i}}}
$$

where $q_{i}$ is the amount of $i$ th substance component evaporated from the effusion cell, $M_{i}$ is the molecular weight, $t$ is time of evaporation, $T$ is temperature, $C_{o r}$ is the Clausing coefficient characterized the effusion hole, and $s_{o r}$ is the hole area.

The $K_{\alpha}$ constant value was calculated taking into account the evaporation coefficient $\left(\alpha_{i}\right)$ of substance component associated with the molecule changing during its transition to the gas phase from the surface with an $S_{v}$ area, using the Komlev equation [90]:

$$
K_{\alpha}=\frac{1}{C_{o r}}+s_{o r} \frac{1-C_{c} \alpha_{i}}{S_{\mathrm{v}} \alpha_{i} C_{c}}
$$

where $C_{c}$ is the Clausing coefficient characterized effusion cell.

The Clausing coefficient is associated with the collision of vapor species inside the effusion orifice channel of effusion cell and their reverse reflection from the channel walls. Its value does not exceed 1 and depends on the ratio of the diameter of the effusion hole to its thickness. 
Taking into account predominance of typical for $\mathrm{CaO}$ and $\mathrm{TiO}_{2}$ vapor species in the gas phase over perovskite and small amounts of $\mathrm{CaTiO}_{3}$, the $\alpha_{i}$ values were used from [91].

The partial pressures of vapor species over perovskite at 1791-2182 K and its melts at $2278 \mathrm{~K}$ calculated using the relationships (38) and (39) with an error not exceeding 8\% are shown in Figure 8.

The partial pressure of atomic oxygen determined using the relationships (38) and (39) agrees satisfactorily with those calculated using the thermochemical data [57] on $K_{r}(T)$ equilibrium constants of possible reactions (18), (20), (24), (25), and (36) in the gas phase over perovskite in the following relations:

$$
\begin{gathered}
p_{\mathrm{O}}=\frac{p_{\mathrm{CaO}}}{p_{\mathrm{Ca}}} K_{18}(T) \\
p_{\mathrm{O}}=\frac{p_{\mathrm{TiO}_{2}}}{p_{\mathrm{TiO}}} K_{24}(T) \\
p_{\mathrm{O}}=\frac{p_{\mathrm{TiO}}}{p_{\mathrm{Ti}}} K_{25}(T) \\
p_{\mathrm{O}}=\sqrt{\frac{p_{\mathrm{O}_{2}}^{2}}{K_{20}(T)}} \\
p_{\mathrm{O}}=\frac{p_{\mathrm{MoO}_{3}}}{p_{\mathrm{MoO}_{2}}} K_{36}(T) .
\end{gathered}
$$

It should be noted that the $p_{\mathrm{O}}$ values calculated according to the independent reactions in the gas phase over perovskite (40)-(45) were the same. It confirmed the assumption about the molecular origin of the identified ions in the mass spectrum of vapor over perovskite.

As it follows from Figure 8a, the defined partial pressures of vapor species over perovskite can be represented as linear logarithmic dependence vs. the inverse temperature:

$$
\lg p_{i}=a_{i} / T+b_{i}
$$

Note that the relationship (45) is the same as the expression for the reaction constant [57]:

$$
R \ln K_{r}(T)=-\frac{\Delta_{r} G_{T}}{T}=-\frac{\Delta_{r} H_{T}}{T}+\Delta_{r} S_{T},
$$

which allows to determine the enthalpy $\left(\Delta_{r} H_{T}\right)$ and entropy $\left(\Delta_{r} S_{T}\right)$ of a reaction.

The partial pressures of the predominant vapor species of the gas phase over perovskite ( $\mathrm{Ca}, \mathrm{TiO}, \mathrm{TiO}_{2}$ and $\mathrm{O}$ ) are compared in Figure 7 with the results on evaporation of simple oxides $\left(\mathrm{CaO}\right.$ and $\left.\mathrm{TiO}_{2}\right)$ under similar redox conditions caused by the interaction of oxygen with molybdenum [79, 81], tungsten [71], and tantalum [80] effusion cells or in chemically neutral conditions (in the absence of this interaction) for alundum cell [78].

We used the $\mathrm{TiO}$ and $\mathrm{TiO}_{2}$ activities as well as the Gibbs energy of perovskite obtained by Banon et al. [52] and thermochemical data [52] on equilibriums (17), (18), (23), and (24) to estimate the partial pressure of vapor species over the perovskite at $2150 \mathrm{~K}$. Therefore, the obtained values characterized by the evaporation of perovskite were not under reducing conditions (from molybdenum cell), 

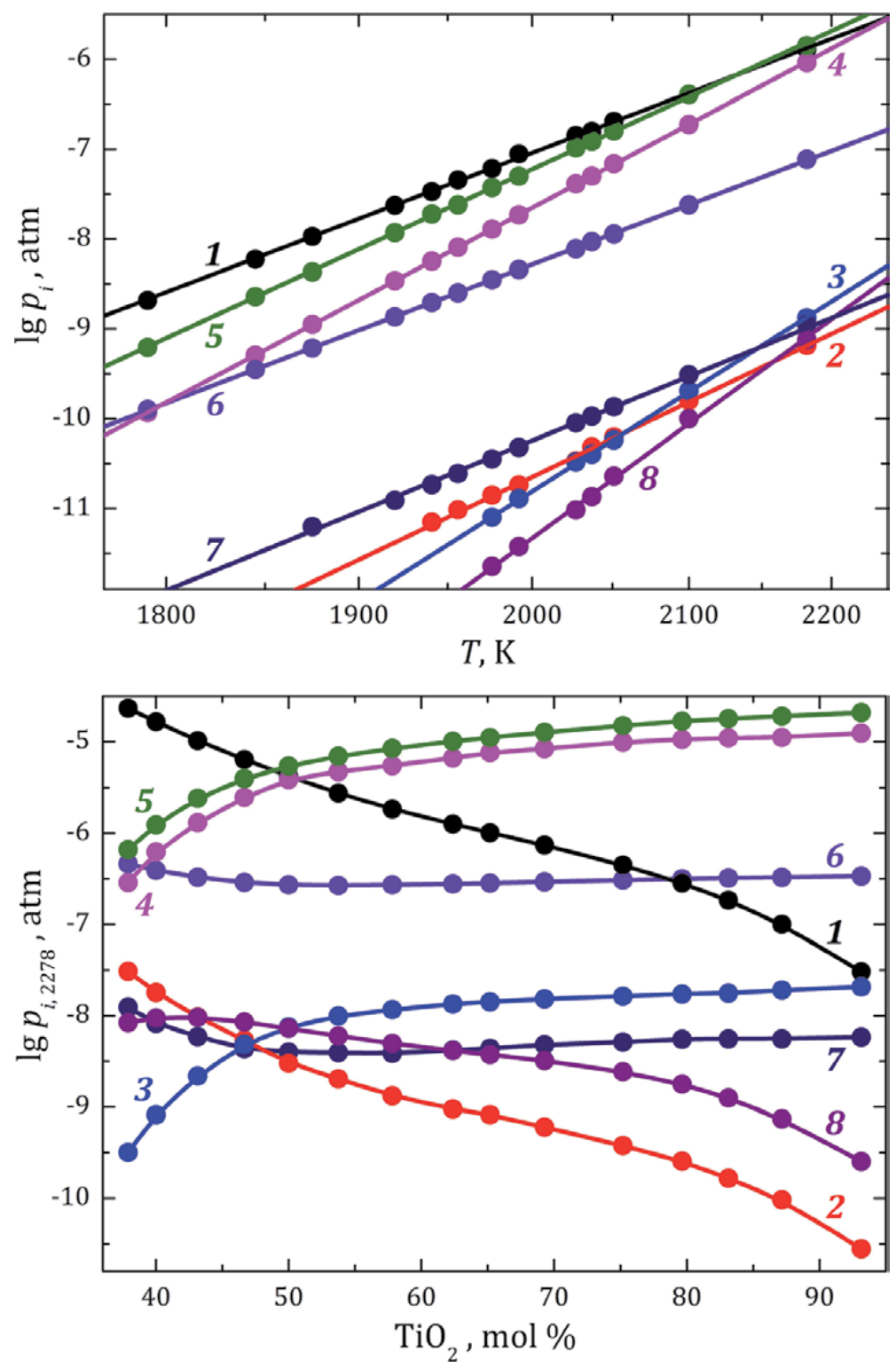

Figure 8.

The partial pressure values of vapor species over perovskite (a) [77] and over the $\mathrm{CaO}-\mathrm{TiO}_{2}$ melts at $2278 \mathrm{~K}$ (b) [64]: (1) $\mathrm{Ca}$, (2) $\mathrm{CaO}$, (3) $\mathrm{Ti}$, (4) $\mathrm{TiO}$, (5) $\mathrm{TiO}_{2}$, (6) O, (7) $\mathrm{O}_{2}$, and (8) $\mathrm{CaTiO}_{3}$.

but, in contrary, under chemically neutral conditions (in the absence of interaction of perovskite with the cell material).

Figure 7 also shows the partial pressures of vapor species over calcium and titanium oxides calculated using thermochemical data [57]. By comparison of the experimental data obtained in [71,77-81] and the calculated results, we can see the effect of reducing properties of cell materials on gas phase composition: tantalum [80], molybdenum [79, 81], tungsten [71], and alundum [78]. As we noted earlier [85], the greatest effect of cell materials on the vapor composition are observed with the oxygen-"deficient" species such as atomic calcium (Figure 7a), titanium monoxide (Figure 7b), and atomic oxygen itself (Figure 7d). There are no differences in the partial pressure of gaseous titanium dioxide (Figure 7c) determined in evaporation experiments using molybdenum [81] and tungsten [71] cells. 
The total vapor pressure over the perovskite melt at $2278 \mathrm{~K}$ obtained by Zhang et al. [4] is consistent with the extrapolated values of partial pressures of the predominant vapor species of the gas phase-atomic calcium and titanium dioxide (Figure 7a and c) found in [77].

Similar slopes of $\lg p_{\mathrm{Ca}}$ and $\lg p_{\mathrm{O}}$ vs. the inverse temperature for calcium oxide and perovskite in Figure 7a and d (lines 1, 5 and 9, respectively) indicate a predominant effect of calcium component on evaporation of calcium from perovskite. This also can explain the difference in the slope of $\lg p_{\text {TiO }}$ in Figure $7 \mathbf{b}$ in the case of perovskite (line 1) and rutile (line 10) according to the equilibriums (18) and (24).

The enthalpy and entropy of reactions involving $\mathrm{CaTiO}_{3}$ gaseous complex oxide calculated by the relationship (46) are given in Table 3. They are in a good agreement with those found by Lopatin and Semenov [84] and our earlier estimates [85].

The concentration dependences of partial pressures of vapor species over perovskite melts show a sharp decrease in $p_{\mathrm{Ca}}$ and $p_{\mathrm{CaO}}$ (Figure $\mathbf{8 b}$, lines 1 and 2) with increasing of $\mathrm{TiO}_{2}$ content in the melt. The vapor species containing titanium - $(\mathrm{Ti})$, ( $\mathrm{TiO})$, and $\left(\mathrm{TiO}_{2}\right)$ - are increased with increasing $\mathrm{TiO}_{2}$ concentration up to $65-70 \mathrm{~mol} \% \mathrm{TiO}_{2}$, and further they are almost constant in the region of 75$100 \mathrm{~mol} \% \mathrm{TiO}_{2}$ (Figure 8b, lines 3-5), which may indicate the immiscibility of the melt observed by Banon et al. [52]. The partial pressures of $(\mathrm{O})$ and $\left(\mathrm{O}_{2}\right)$ slightly vary throughout the concentration range under consideration, showing a minimum in the perovskite concentration (Figure 8b, lines 6 and 7). The $\left(\mathrm{CaTiO}_{3}\right)$ partial pressures have maximum values in the melt region with a high calcium content compared to the perovskite concentration (Figure $\mathbf{8 b}$, line 8 ), as it was noted earlier.

\section{Conclusions}

The thermodynamic properties of perovskite determined by different calorimetric approaches and EMF method agree with the results obtained via Knudsen effusion mass spectrometry at high temperatures. The resulting values of oxide activities in perovskite, as well as the Gibbs energy, the entropy and enthalpy of the formation of perovskite from simple oxides, and the melting enthalpy of perovskite are consistent with each other. The enthalpy of perovskite formation is constant throughout the temperature range, and the entropy of perovskite formation tends to increase slightly.

The oxide activities in perovskite melts were determined by mass spectrometric Knudsen effusion method. The thermodynamic properties of melts (chemical potentials of oxides and mixing energies, as well as partial and integral enthalpies and entropies of melt's formation) were calculated based on the experimental data. The obtained experimental information testifies to the symbate behavior of thermodynamic functions characterizing the melts. The extreme values of the integral thermodynamic properties of melts are in the concentration region close to perovskite, which confirms its stability in the melt. The displacement of the extremum of the integral thermodynamic functions in the $\mathrm{CaO}-\mathrm{TiO}_{2}$ melts can be caused by the presence in the melt of oxide compounds with a large amount of $\mathrm{CaO}$ compared to perovskite. A comparison of mixing energies in the $\mathrm{CaO}-\mathrm{TiO}_{2}$ melts with those for the $\mathrm{CaO}-\mathrm{SiO}_{2}$ and $\mathrm{CaO}-\mathrm{Al}_{2} \mathrm{O}_{3}$ melts indicates a stronger chemical interaction in the $\mathrm{CaO}-\mathrm{TiO}_{2}$ melts than the similar $\mathrm{CaO}-\mathrm{Al}_{2} \mathrm{O}_{3}$ melts, but smaller than in the $\mathrm{CaO}-\mathrm{SiO}_{2}$ melts.

The evaporation of perovskite and its melts from a molybdenum cell at high temperature was studied by the Knudsen effusion mass spectrometric method. The molecular components typical of simple oxides and the $\left(\mathrm{CaTiO}_{3}\right)$ gaseous complex 
oxide were identified in the gas phase over perovskite. The partial vapor pressures of the molecular components of the gas phase over perovskite were determined. A comparison of these values with the available experimental data and with the values corresponding to simple oxides showed that the character of perovskite evaporation is mainly affected by the calcium component of perovskite. The observed concentration dependences of the partial pressures of vapor species over the perovskite melts correspond to those characterizing the condensed phase.

\title{
Acknowledgements
}

This study was financially supported by the Presidium of the Russian Academy of Sciences (Program No. 7 "Experimental and Theoretical Studies of Solar System and Star Planetary System Objects. Transition Processes in Astrophysics”) and by the Russian Foundation for Basic Research (Grant No. 19-05-00801A "Thermodynamics of Formation Processes of Substance of Refractory Inclusions in Chondrites").

\section{Thanks}

I am grateful to Oleg Yakovlev (Vernadsky Institute of Geochemistry and Analytical Chemistry of the Russian Academy of Sciences) for his constant interest in this study and useful discussions and to Mikhail Nazarov (Vernadsky Institute of Geochemistry and Analytical Chemistry of the Russian Academy of Sciences) for his support during this work. I express my special gratitude to Marina Ivanova (Vernadsky Institute of Geochemistry and Analytical Chemistry of the Russian Academy of Sciences; National Museum of Natural History of Smithsonian Institution) for her help in working on the manuscript.

\section{Author details}

\author{
Sergey Shornikov \\ Vernadsky Institute of Geochemistry and Analytical Chemistry of Russian Academy \\ of Sciences, Moscow, Russia
}

*Address all correspondence to: sergey.shornikov@gmail.com

\section{IntechOpen}

(C) 2020 The Author(s). Licensee IntechOpen. This chapter is distributed under the terms of the Creative Commons Attribution License (http://creativecommons.org/licenses/ by/3.0), which permits unrestricted use, distribution, and reproduction in any medium, provided the original work is properly cited. (c) BY 


\section{References}

[1] Rose G. Uber einige neue mineralien des Urals. Journal für Praktische

Chemie. 1840;19:459-468

[2] Stefanovsky SV, Yudintsev SV. Titanates, zirconates, aluminates and ferrites as waste forms for actinide immobilization. Russian Chemical Reviews. 2016;85:962-994

[3] Wark D, Boynton WV. The formation of rims on calciumaluminum-rich inclusions: Step I-Flash heating. Meteoritics \& Planetary Science. 2001;36:1135-1166

[4] Zhang J, Huang S, Davis AM, Dauphas N, Hashimoto A, Jacobsen SB. Calcium and titanium isotopic fractionations during evaporation. Geochimica et Cosmochimica Acta. 2014;140:365-380

[5] Ivanova MA. Ca-Al-rich inclusions in carbonaceous chondrites: The oldest solar system objects.

Geochemistry International. 2016;54: 387-402

[6] Parga Pondal I, Bergt K. Sobre la combinacion de la cal en los sistemas $\mathrm{CaO}-\mathrm{TiO}_{2}$ y $\mathrm{CaO}-\mathrm{SiO}_{2}-\mathrm{TiO}_{2}$. Anales de la Sociedad Española de Física y Química. 1933;31:623-637

[7] Roth RS. Revision of the phase equilibrium diagram of the binary system calcia-titania, showing the compound $\mathrm{Ca}_{4} \mathrm{Ti}_{3} \mathrm{O}_{10}$. Journal of Research of NBS. 1958;61:437-440

[8] Lazaro SR. Estudo teoricoexperimental do titanato de calcio- $-\mathrm{CaTiO}_{3}$ [thesis]. Sao Carlos: Universidade Estadual Paulista, Intituto de Quimica; 2002. p. 57

[9] Wartenberg HV, Reusch HJ, Saran E. Schmelzpunktsdiagramme hochstfeuerfester oxyde. VII. Systeme mit $\mathrm{CaO}$ und $\mathrm{BeO}$. Zeitschrift für
Anorganische und Allgemeine Chemie. 1937;230:257-276

[10] Pontes FM, Pinheiro CD, Longo E, Leite ER, Lazaro SR, Varela JA, et al. The role of network modifiers in the creation of photoluminescence in $\mathrm{CaTiO}_{3}$. Materials Chemistry and Physics. 2003;78:227-233

[11] Tulgar HE. Solid state relationships in the system calcium oxide-Titanium dioxide. Istanbul Teknik Üniversitesi Bülteni. 1976;29:111-129

[12] Kisel NG, Limar TF, Cherednichenko IF. On calcium dititanate. Russian Journal of Inorganic Chemistry. 1972;8: 1782-1785

[13] Pfaff G. Peroxide route to synthesize calcium titanate powders of different composition. Journal of the European Ceramic Society. 1992;9:293-299

[14] Seko A, Hayashi H, Kashima H, Tanaka I. Matrix- and tensor-based recommender systems for the discovery of currently unknown inorganic compounds. Physical Review Materials. 2018;2:13805-1-13805-9

[15] Savenko VG, Sakharov VV.

Formation of calcium titanate $\mathrm{Ca}_{2} \mathrm{Ti}_{5} \mathrm{O}_{12}$ on thermolysis of mixed titanium and calcium hydroxides. Russian Journal of Inorganic Chemistry. 1979;24:1389-1391

[16] Limar TF, Kisel NG, Cherednichenko IF, Savos'kina AI. On calcium tetratitanate. Russian Journal of Inorganic Chemistry. 1972;17:559-561

[17] Oganov AR, Ma Y, Lyakhov AO, Valle M, Gatti C. Evolutionary crystal structure prediction as a method for the discovery of minerals and materials. Reviews in Mineralogy and Geochemistry. 2010;71:271-298 
[18] DeVries RC, Roy R, Osborn EF. Phase equilibria in the system $\mathrm{CaO}-$ $\mathrm{TiO}_{2}$. The Journal of Physical Chemistry. 1954;58:1069-1073

[19] Gong W, Wu L, Navrotsky A. Combined experimental and computational investigation of thermodynamics and phase equilibria in the $\mathrm{CaO}-\mathrm{TiO}_{2}$ system. Journal of the American Ceramic Society. 2018;101: 1361-1370

[20] Kelley KK, Mah AD. Metallurgical thermochemistry of titanium. U. S. Bur. Min. Repts. 1959. No. 5490. 48 p

[21] Reznitskii LA, Guzei AS.

Thermodynamic properties of alkaline earth titanates, zirconates, and hafnates. Russian Chemical Reviews. 1978;47: 99-119

[22] Robie RA, Hemingway BS. Thermodynamic properties of mineral and related substances at $298.15 \mathrm{~K}$ and 1 bar (105 Pascals) pressure and high temperatures. U. S. Geol. Surv. Bull. 1995. No. 2131. p. 461

[23] Barin I. Thermochemical Data of Pure Substances. VCH: Weinheim; 1995. p. 2003

[24] Bale CW, Belisle E, Chartrand P, Decterov SA, Eriksson G, Gheribi AE, et al. FactSage thermochemical software and databases, 2010-2016. Calphad. 2016;54:35-53

[25] Shomate CH. Heat capacities at low temperatures of the metatitanates of iron, calcium and magnesium. Journal of the American Chemical Society. 1946; 68:964-966

[26] Woodfield BF, Shapiro JL, Stevens R, Boerio-Goates J, Putnam RL, Helean KB, et al. Molar heat capacity and thermodynamic functions for $\mathrm{CaTiO}_{3}$. The Journal of Chemical Thermodynamics. 1999;31:1573-1583
[27] Buykx WJ. Specific heat, thermal diffusivity and thermal conductivity of synroc, perovskite, zirconolite and barium hollandite. Journal of Nuclear Materials. 1982;107:78-82

[28] Sato T, Yamazaki S, Yamashita T, Matsui T, Nagasaki T. Enthalpy and heat capacity of $\left(\mathrm{Ca}_{1-x} \mathrm{Pu}_{x}\right) \mathrm{TiO}_{3}(x=0$ and $0.20)$. Journal of Nuclear Materials. 2001;294:135-140

[29] Naylor BF, Cook OA. Hightemperature contents of the metatitanates of calcium, iron and magnesium. Journal of the American Chemical Society. 1946;68:1003-1005

[30] Guyot F, Richet P, Courtial P, Gillet P. High-temperature heat capacity and phase transitions of $\mathrm{CaTiO}_{3}$ perovskite. Physics and Chemistry of Minerals. 1993;20:141-146

[31] Yashima M, Ali R. Structural phase transition and octahedral tilting in the calcium titanate perovskite $\mathrm{CaTiO}_{3}$. Solid State Ionics. 2009;180:120-126

[32] Panfilov BI, Fedos'ev NN. Heat of formation of metatitanate calcium, strontium and barium. Russian Journal of Inorganic Chemistry. 1964;9: 2685-2692

[33] Kelley KK, Todd SS, King EG. Heat and free energy data for titanates of iron and the alkaline-earth metals. U. S. Bur. Min. Repts. 1954. No. 5059. p. 37

[34] Feng D, Shivaramaiah R, Navrotsky A. Rare-earth perovskites along the $\mathrm{CaTiO}_{3}-\mathrm{Na}_{0.5} \mathrm{La}_{0.5} \mathrm{TiO}_{3}$ join: Phase transitions, formation enthalpies, and implications for loparite minerals. American Mineralogist. 2016;101: 2051-2056

[35] Rezukhina TN, Levitskii VA, Frenkel' MY. Thermodynamic properties of barium and calcium tungstates. Russian Inorganic Materials. 1966;2:325-331 
[36] Jacob KT, Abraham KP.

Thermodynamic properties of calcium titanates: $\mathrm{CaTiO}_{3}, \mathrm{Ca}_{4} \mathrm{Ti}_{3} \mathrm{O}_{10}$, and

$\mathrm{Ca}_{3} \mathrm{Ti}_{2} \mathrm{O}_{7}$. The Journal of Chemical

Thermodynamics. 2009;41:816-820

[37] Putnam RL, Navrotsky A, Woodfield BF, Boerio-Goates J, Shapiro JL. Thermodynamics of formation for zirconolite $\left(\mathrm{CaZrTi}_{2} \mathrm{O}_{7}\right)$ from $\mathrm{T}=298.15 \mathrm{~K}$ to $\mathrm{T}=1500 \mathrm{~K}$. The Journal of Chemical Thermodynamics. 1999;31:229-243

[38] Navi NU, Shneck RZ, Shvareva TY, Kimmel G, Zabicky J, Mintz MH, et al. Thermochemistry of $\left(\mathrm{Ca}_{x} \mathrm{Sr}_{1-x}\right) \mathrm{TiO}_{3}$, $\left(\mathrm{Ba}_{x} \mathrm{Sr}_{1-x}\right) \mathrm{TiO}_{3}$, and $\left(\mathrm{Ba}_{x} \mathrm{Ca}_{1-x}\right) \mathrm{TiO}_{3}$ perovskite solid solutions. Journal of the American Ceramic Society. 2012;95: 1717-1726

[39] Sahu SK, Maram PS, Navrotsky A. Thermodynamics of nanoscale calcium and strontium titanate perovskites. Journal of the American Ceramic Society. 2013;96:3670-3676

[40] Helean KB, Navrotsky A, Vance ER, Carter ML, Ebbinghaus B, Krikorian O, et al. Enthalpies of formation of Cepyrochlore, $\mathrm{Ca}_{0.93} \mathrm{Ce}_{1.00} \mathrm{Ti}_{2.035} \mathrm{O}_{7.00}$, Upyrochlore, $\mathrm{Ca}_{1.46} \mathrm{U}_{0.23}^{4+} \mathrm{U}_{0.46}^{6+} \mathrm{Ti}_{1.85} \mathrm{O}_{7.00}$ and Gd-pyrochlore, $\mathrm{Gd}_{2} \mathrm{Ti}_{2} \mathrm{O}_{7}$ : Three materials relevant to the proposed waste form for excess weapons plutonium. Journal of Nuclear Materials. 2002;303: 226-239

[41] Takayama-Muromachi E, Navrotsky A. Energetics of compounds $\left(\mathrm{A}^{2+} \mathrm{B}^{4+} \mathrm{O}_{3}\right)$ with the perovskite structure. Journal of Solid State Chemistry. 1988;72:244-256

[42] Prasanna TRS, Navrotsky A. Energetics in the brownmilleriteperovskite pseudobinary $\mathrm{Ca}_{2} \mathrm{Fe}_{2} \mathrm{O}_{5-}$ $\mathrm{CaTiO}_{3}$. Journal of Materials Research. 1994;9:3121-3124

[43] Linton J, Navrotsky A, Fei Y. The thermodynamics of ordered perovskites on the $\mathrm{CaTiO}_{3}-\mathrm{FeTiO}_{3}$ join. Physics and Chemistry of Minerals. 1998;25: 591-596

[44] Koito S, Akaogi M, Kubota O, Suzuki T. Calorimetric measurements of perovskites in the system $\mathrm{CaTiO}_{3}-$ $\mathrm{CaSiO}_{3}$ and experimental and calculated phase equilibria for high-pressure dissociation of diopside. Physics of the Earth and Planetary Interiors. 2000;120: 1-10

[45] Golubenko AN, Rezukhina TN. Thermodynamic properties of calcium titanate from electrochemical measurements at elevated temperatures. Russian Journal of Physical Chemistry. 1964;38:2920-2923

[46] Gillet P, Guyot F, Price GD, Tournerie B, LeCleach A. Phase changes and thermodynamic properties of $\mathrm{CaTiO}_{3}$. Spectroscopic data, vibrational modelling and some insights on the properties of $\mathrm{MgSiO}_{3}$ perovskite. Physics and Chemistry of Minerals. 1993;20:159-170

[47] Klimm D, Schmidt M, Wolff N, Guguschev C, Ganschow S. On melt solutions for the growth of $\mathrm{CaTiO}_{3}$ crystals. Journal of Crystal Growth. 2018;486:117-121

[48] Shornikov SI. High-temperature mass spectrometric study of the thermodynamic properties of $\mathrm{CaTiO}_{3}$ perovskite. Russian Journal of Physical Chemistry A. 2019;93:1428-1434

[49] Topor ND, Suponitskii YL. The high-temperature microcalorimetry of inorganic substances. Russian Chemical Reviews. 1984;53:827-850

[50] Aronson S. Estimation of the heat of formation of refractory mixed oxides. Journal of Nuclear Materials. 1982;107: 343-346

[51] Taylor RW, Schmalzried H. The free energy of formation of some titanates, 
silicates and magnesium aluminate from measurements made with galvanic cells involving solid electrolyte. The Journal of Physical Chemistry. 1964;68:2444-2449

[52] Banon S, Chatillon C, Allibert M. Free energy of mixing in $\mathrm{CaTiO}_{3}-\mathrm{Ti}_{2} \mathrm{O}_{3}-$ $\mathrm{TiO}_{2}$ melts by mass spectrometry. Canadian Metallurgical Quarterly. 1981; 20:79-84

[53] Shornikov SI, Archakov IY. Mass spectrometric study of thermodynamic properties of the $\mathrm{SiO}_{2}-\mathrm{CaTiO}_{3}$ melts. In: Kudin L, Butman M, Smirnov A, editors. Proc. II Intern. Symp. on High Temperature Mass Spectrometry. Ivanovo: ISUCST; 2003. pp. 112-116

[54] Cho SW, Suito H. Thermodynamics of oxygen and nitrogen in liquid nickel equilibrated with $\mathrm{CaO}-\mathrm{TiO}_{x}$ and $\mathrm{CaO}-$ $\mathrm{TiO}_{x}-\mathrm{Al}_{2} \mathrm{O}_{3}$ melts. Metallurgical and Materials Transactions A. 1994;25:5-13

[55] Kishi M, Suito H. Thermodynamics of oxygen, nitrogen and sulfur in liquid iron equilibrated with $\mathrm{CaO}-\mathrm{TiO}_{x}$ and $\mathrm{CaO}-\mathrm{TiO}_{x}-\mathrm{Al}_{2} \mathrm{O}_{3}$ melts. Steel Research. 1994;65:261-266

[56] Shornikov SI, Archakov IY, Shultz MM. Thermodynamic properties of the melts, containing titanium dioxide. In: Gorynin IV, Ushkov SS, editors. Proc. 9th World Conf. on Titanium. Vol. 3. Saint-Petersburg: Prometey; 2000. pp. 1469-1473

[57] Glushko VP, Gurvich LV, Bergman GA, Veitz IV, Medvedev VA, Khachkuruzov GA, et al. Thermodynamic Properties of Individual Substances. Moscow: Nauka; 1978-1982 [in Russian]

[58] Chase MW. NIST-JANAF themochemical tables. Journal of Physical and Chemical Reference Data. 1998. p. 1951

[59] Nerad I, Danek V. Thermodynamic analysis of pseudobinary subsystems of the system $\mathrm{CaO}-\mathrm{TiO}_{2}-\mathrm{SiO}_{2}$. Chemical Papers. 2002;56:77-83

[60] Seo W-G, Zhou D, Tsukihashi F. Calculation of thermodynamic properties and phase diagrams for the $\mathrm{CaO}-\mathrm{CaF}_{2}, \mathrm{BaO}-\mathrm{CaO}$ and $\mathrm{BaO}-\mathrm{CaF}_{2}$ systems by molecular dynamics simulation. Materials Transactions. 2005;46:643-650

[61] Bottinga Y, Richet P.

Thermodynamics of liquid silicates, a preliminary report. Earth and Planetary Science Letters. 1978;40:382-400

[62] Kulikov IS. Thermal Dissociation of Compounds. 2nd ed. Metallurgiya: Moscow; 1969. p. 576 [in Russian]

[63] Stolyarova VL, Zhegalin DO, Stolyar SV. Mass spectrometric study of the thermodynamic properties of melts in the $\mathrm{CaO}-\mathrm{TiO}_{2}-\mathrm{SiO}_{2}$ system. Glass Physics and Chemistry. 2004;30: 142-150

[64] Shornikov SI. Thermodynamic properties of $\mathrm{CaO}-\mathrm{TiO}_{2}$ system: Experimental data and calculations. In: Gladyshev PP, editor. Physical and Analytical Chemistry of Natural and Technogenic Systems, New Technologies and MaterialsKhodakovsky's Readings. Dubna: Dubna State University; 2019. pp. 185-190 [in Russian]

[65] Lewis GN, Randall M.

Thermodynamics and the Free Energy of Chemical Substances. N. Y. \& London: McGrow-Hill Book Comp.; 1923. p. 676

[66] Belton GR, Fruehan RJ. The determination of activities of mass spectrometry: Some additional methods. Metallurgical and Materials

Transactions B. 1971;2:291-296

[67] Prigogine I, Defay R. Chemical Thermodynamics. London: Longman; 1954. p. 543 
[68] Shornikov SI, Archakov IY. Mass spectrometric study of phase relations and vaporization processes in the $\mathrm{CaO}-\mathrm{SiO}_{2}$ system. Glass Science and Technology: International Journal of the German Society of Glass Technology. 2000;73C2:51-57

[69] Shornikov SI, Stolyarova VL, Shultz MM. A mass-spectrometric study of vapor composition and thermodynamic properties of $\mathrm{CaO}-$ $\mathrm{Al}_{2} \mathrm{O}_{3}$ melts. Russian Journal of Physical Chemistry. 1997;71:19-22

[70] Kulikov IS. Thermodynamics of Oxides. Metallurgiya: Moscow; 1986. p. 344 [in Russian]

[71] Kazenas EK, Tsvetkov YV. Evaporation of Oxides. Moscow: Nauka; 1997. p. 543 [in Russian]

[72] Balducci G, Gigli G, Guido M. Identification and stability determinations for the gaseous titanium oxide molecules $\mathrm{Ti}_{2} \mathrm{O}_{3}$ and $\mathrm{Ti}_{2} \mathrm{O}_{4}$. The Journal of Chemical Physics. 1985;83:1913-1916

[73] Zakharov VP, Protas IM. Mass spectrometric study of ion emission during evaporation of complex substances under the influence of laser radiation. Izvestiya Akademii Nauk SSSR. Seriya Fizicheskaya (Bulletin of the Russian Academy of Sciences: Physics) 1974;38:238-243

[74] Schwarz H, Tourltellotte HA. Vacuum deposition by high-energy laser with emphasis on barium titanate films. Journal of Vacuum Science and Technology B. 1969;6:373-378

[75] Hao J, Si W, Xia XX, Guo R, Bhalla AS, Cross LE. Dielectric properties of pulsed-laser-deposited calcium titanate thin films. Applied Physics Letters. 2000;76:3100-3102

[76] Knox BE. Laser ion source analysis of solids. In: Ahearn AJ, editor. Trace Analysis by Mass Spectrometry. N. Y. \&
London: Academic Press; 1972. pp. 423-444

[77] Shornikov SI. Mass-spectrometric study of perovskite evaporation. Russian Journal of Physical Chemistry A. 2019;93:866-873

[78] Samoilova IO, Kazenas EK. Thermodynamics of dissociation and sublimation of calcium oxide. Russel Metals. 1995:33-35

[79] Shornikov SI. Evaporation processes and thermodynamic properties of the $\mathrm{CaO}-\mathrm{Al}_{2} \mathrm{O}_{3}-\mathrm{SiO}_{2}$ system and the materials based on this system [thesis]. Institute of Silicate Chemistry of RAS: Saint-Petersburg; 1993. p. 21 [in Russian]

[80] Groves WO, Hoch M, Johnston HL. Vapor-solid equilibria in the titaniumoxygen system. The Journal of Physical Chemistry. 1955;59:127-131

[81] Berkowitz J, Chupka WA, Inghram MG. Thermodynamics of the $\mathrm{Ti}-\mathrm{Ti}_{2} \mathrm{O}_{3}$ system and the dissociation energy of $\mathrm{TiO}$ and $\mathrm{TiO}_{2}$. The Journal of Physical Chemistry. 1957;61:1569-1572

[82] Archakov IY, Shornikov SI, Tchemekova TY, Shultz MM. The behavior of titanium dioxide in the slag melts. In: Gorynin IV, Ushkov SS, editors. Proc. 9th World Conf. on Titanium. Vol. 3. Saint-Petersburg: Prometey; 2000. pp. 1464-1468

[83] Ostrovski O, Tranell G, Stolyarova VL, Shultz MM, Shornikov SI, Ishkildin AI. Hightemperature mass spectrometric study of the $\mathrm{CaO}-\mathrm{TiO}_{2}-\mathrm{SiO}_{2}$ system. High Temperature Materials and Processes. 2000;19:345-356

[84] Lopatin SI, Semenov GA. Thermochemical study of gaseous salts of oxygen-containing acids: XI. Alkaline-earth metal titanates. Russ. J. General Chemistry. 2001;71:1522-1526 
[85] Shornikov SI, Yakovlev OI. Study of complex molecular species in the gas phase over the $\mathrm{CaO}-\mathrm{MgO}-\mathrm{Al}_{2} \mathrm{O}_{3}-\mathrm{TiO}_{2}-$ $\mathrm{SiO}_{2}$ system. Geochemistry International. 2015;53:690-699

[86] Semenov GA. The evaporation of titanium dioxide. Russian Inorganic Materials. 1969;5:67-73

[87] Warren JW. Measurement of appearance potentials of ions produced by electron impact, using a mass spectrometer. Nature. 1950;165:810-811

[88] Gurvich LV, Karachevtsev GV, Kondratev VN, Lebedev YA, Medvedev VA, Potapov VK, et al. Bond Dissociation Energies, Ionization Potentials, and Electron Affinity. Moscow: Nauka; 1974. p. 351

[in Russian]

[89] Shornikov SI. Thermodynamic study of the mullite solid solution region in the $\mathrm{Al}_{2} \mathrm{O}_{3}-\mathrm{SiO}_{2}$ system by mass spectrometric techniques. Geochemistry International. 2002;40:S46-S60

[90] Komlev GA. On determination of saturated steam pressure by effusion method. Russian Journal of Physical Chemistry. 1964;38:2747-2748

[91] Shornikov SI. Vaporization coefficients of oxides contained in the melts of Ca-Al-inclusions in chondrites. Geochemistry International. 2015;53:

1080-1089 


\title{
Synthesis and Investigation of the Physical Properties of Lead-Free BCZT Ceramics
}

\author{
Dang Anh Tuan, Vo Thanh Tung, Le Tran Uyen Tu \\ and Truong Van Chuong
}

\begin{abstract}
This work presents the structure, microstructure, and physical properties of low sintering temperature lead-free ceramics $0.52\left(\mathrm{Ba}_{0.7} \mathrm{Ca}_{0.3}\right) \mathrm{TiO}_{3}-0.48 \mathrm{Ba}\left(\mathrm{Zr}_{0.2} \mathrm{Ti}_{0.8}\right)$ $\mathrm{O}_{3}$ doped with nano-sized $\mathrm{ZnO}$ particles (noted as $\mathrm{BCZT} / \mathrm{x}$, $\mathrm{x}$ is the content of $\mathrm{ZnO}$ nanoparticles in wt.\%, $\mathrm{x}=0.00,0.05,0.10,0.15,0.20$, and 0.25 ). The obtained results of Raman scattering and dielectric measurements have confirmed that $\mathrm{Zn}^{2+}$ has occupied $\mathrm{B}$-site, to cause a deformation in the $\mathrm{ABO}_{3}$-type lattice of the $\mathrm{BCZT} / \mathrm{x}$ specimens. The $0.15 \mathrm{wt} . \% \mathrm{ZnO}$-modified ceramic sintered at $1350^{\circ} \mathrm{C}$ exhibited excellent piezoelectric parameters: $\mathrm{d}_{33}=420 \mathrm{pC} / \mathrm{N}, \mathrm{d}_{31}=-174 \mathrm{pC} / \mathrm{N}, \mathrm{k}_{\mathrm{p}}=0.483$, $k_{t}=0.423$, and $k_{33}=0.571$. The obtained results indicate that the high-quality leadfree BCZT ceramic could be successfully synthesized at a low sintering temperature of $1350^{\circ} \mathrm{C}$ with an addition of appropriated amount of $\mathrm{ZnO}$ nanoparticles. This work also reports the influence of the sintering temperature on structure, microstructure, and piezoelectric properties of BCZT/0.15 compound. By rising sintering temperature, the piezoelectric behaviors were improved and rose up to the best parameters at a sintering temperature of $1450^{\circ} \mathrm{C}\left(\mathrm{d}_{33}=576 \mathrm{pC} / \mathrm{N}\right.$ and $\left.\mathrm{k}_{\mathrm{p}}=0.55\right)$. The corresponding properties of undoped BCZT ceramics were investigated as a comparison. It also presented that the sintering behavior and piezo-parameters of doped BCZT samples are better than the undoped BCZT samples at each sintering temperature.
\end{abstract}

Keywords: lead-free, BZT-BCT, ceramics, nanoparticle, $\mathrm{ZnO}$

\section{Introduction}

Perovskite $\mathrm{ABO}_{3}$-type compounds with high flexibility in symmetry play an important role in materials science. Typical materials such as lead zirconate titanate (PZT) based on family $\mathrm{BaTiO}_{3}$ have received a lot of attention due to their outstanding dielectric, ferroelectric, and piezoelectric performance.

Nevertheless, PZT systems are globally restricted due to evaporating toxic lead oxide to the environment during preparation. With the recent growing demand of global environmental and human health protection, many non-lead materials have been systematically studied to replace the lead-based ceramics [1, 2].

In 2009, based on alternating with $\mathrm{A}$ - and/or B-sites in perovskite $\mathrm{BaTiO}_{3}$, Liu and Ren established a new lead-free ferroelectric system $\mathrm{Ba}\left(\mathrm{Zr}_{0.2} \mathrm{Ti}_{0.8}\right) \mathrm{O}_{3}-\mathrm{x}\left(\mathrm{Ba}_{0.7} \mathrm{Ca}_{0.3}\right)$ 
$\mathrm{TiO}_{3}$ (abbreviated as BZT-BCT) that has excellent piezoelectricity $\left(\mathrm{d}_{33}=620 \mathrm{pC} / \mathrm{N}\right.$ at $\mathrm{x}=50$, i.e., morphotropic phase boundary or MPB composition) [3]. After that the BZT-BCT materials have been widely studied [4-6]. It is noted that based $\mathrm{BaTiO}_{3}$ ceramics have been usually sintered at a very high temperature to obtain the desired properties [7-9] which causes many difficulties in the preparation and application of these materials. It is well-known that sintering behavior can be improved by using nanostructured raw materials and/or sintering aids [10,11]. Among commonly used dopants, $\mathrm{ZnO}$ (in nano- or microscale) is known as an effective sintering aid for enhancing density and electric properties of piezoceramics [12-14].

In this work, the effects of $\mathrm{ZnO}$ nanoparticles as well as sintering temperature on structure, microstructure, and some electric properties of $0.48 \mathrm{Ba}\left(\mathrm{Zr}_{0.2} \mathrm{Ti}_{0.8}\right)$ $\mathrm{O}_{3}-0.52\left(\mathrm{Ba}_{0.7} \mathrm{Ca}_{0.3}\right) \mathrm{TiO}_{3}$ or $\mathrm{BCZT}$ composition were detailedly presented.

\section{Experimental produces}

\subsection{Preparing ZnO nanoparticles}

$\mathrm{ZnO}$ nanoparticles were synthesized using zinc acetate and ammonia solutions as initial materials. Accordingly, zinc acetate was dissolved in distilled water to form solution. Then $\mathrm{NH}_{4} \mathrm{OH}$ solution was gradually dropped into zinc acetate solution and stirred until a white precipitate was received. The amount of $\mathrm{NH}_{4} \mathrm{OH}$ was enough so that the overall reaction to form $\mathrm{ZnO}$ is as follows [15]:

$$
\left(\mathrm{CH}_{3} \mathrm{COO}\right)_{2} \mathrm{Zn}+2 \mathrm{NH}_{4} \mathrm{OH} \rightarrow \mathrm{ZnO}+2\left(\mathrm{CH}_{3} \mathrm{COO}\right) \mathrm{NH}_{4}+\mathrm{H}_{2} \mathrm{O} .
$$

The obtained white precipitate was filtered and washed several times with the aid of vacuum filter machine and then annealed at a temperature of $250^{\circ} \mathrm{C}$ for $1 \mathrm{~h}$ to remove unwanted products.

Figure 1 shows XRD and microstructure image (measured by D8 Advance, Bruker AXS, and Nova NanoSEM 450-FEI, respectively) of the obtained ZnO powder after annealing at $250^{\circ} \mathrm{C}$ for $1 \mathrm{~h}$.

Detailed structural characterization demonstrated that the synthesized product possesses pure hexagonal symmetry [16]. The obtained $\mathrm{ZnO}$ particles are spherical in shape with their average diameter of $59 \mathrm{~nm}$ (according to Scherrer equation). The nanostructured $\mathrm{ZnO}$ powder was used as a sintering aid in fabrication of BCZT ceramics.
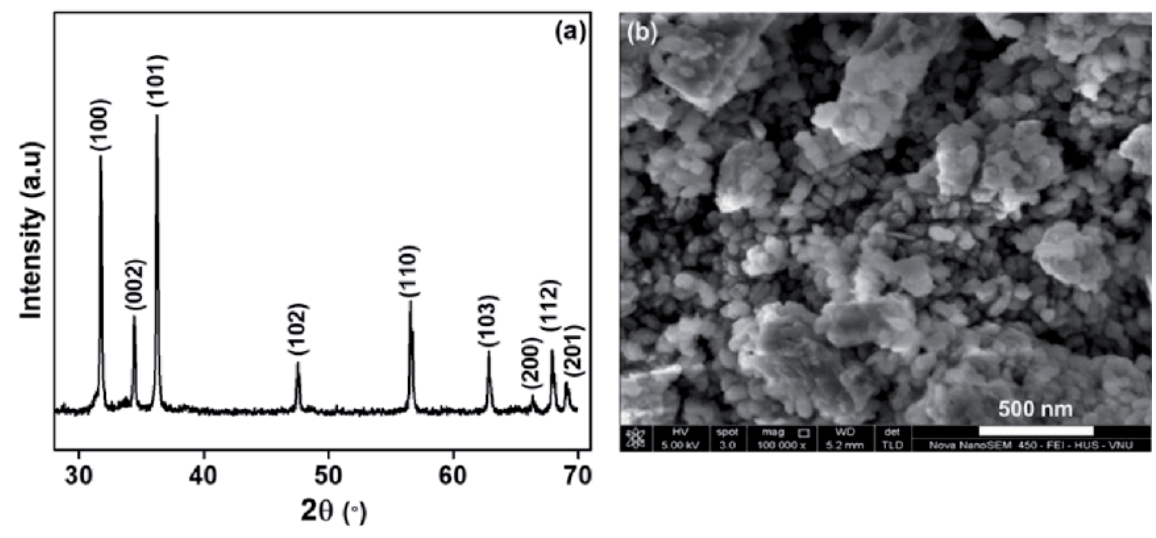

Figure 1.

(a) X-ray diffraction (XRD) and (b) micrograph of as-prepared $\mathrm{ZnO}$ powder. 


\subsection{Fabrication of $\mathrm{ZnO}$ nanoparticles doped BCZT ceramics at low sintering temperature}

A conventional ceramic fabrication technique was used to prepare lead-free ceramics $0.52\left(\mathrm{Ba}_{0.7} \mathrm{Ca}_{0.3}\right) \mathrm{TiO}_{3}-0.48 \mathrm{Ba}\left(\mathrm{Zr}_{0.2} \mathrm{Ti}_{0.8}\right) \mathrm{O}_{3}$ doped with $\mathrm{ZnO}$ nanoparticles (abbreviated as BCZT/x, $\mathrm{x}$ is the content of $\mathrm{ZnO}$ in wt.\%, $\mathrm{x}=0.00,0.05,0.10$, $0.15,0.20,0.25)$. The raw materials with high purity (>99\%) of $\mathrm{BaCO}_{3}, \mathrm{CaCO}_{3}$, $\mathrm{ZrO}_{2}$, and $\mathrm{TiO}_{2}$ (Merck) were weighed and mixed in a planetary milling machine (PM400/2-MA-Type) using ethanol as a medium for $20 \mathrm{~h}$. The obtained powders were calcined at $1250^{\circ} \mathrm{C}$ for $3 \mathrm{~h}$. The calcined powder was milled again in ethanol for $20 \mathrm{~h}$; after that the $\mathrm{x}$ wt.\% of $\mathrm{ZnO}$ nanoparticles were added, finely mixed, and then pressed into desired-shape specimens by uniaxial pressing with a pressure of $100 \mathrm{MPa}$. Sintering was carried out at various temperatures for $4 \mathrm{~h}$. The X-ray diffraction patterns were recorded at room temperature by a D8 Advance, Bruker AXS. The tetragonal and rhombohedral volume fractions, $\tau_{\mathrm{T}}$ and $\tau_{\mathrm{R}}$, were, respectively, evaluated using the equations below [17]:

$$
\begin{gathered}
\tau_{\mathrm{T}}=\frac{\mathrm{I}_{200}^{\mathrm{T}}+\mathrm{I}_{002}^{\mathrm{T}}}{\mathrm{I}_{200}^{\mathrm{T}}+\mathrm{I}_{200}^{\mathrm{R}}+\mathrm{I}_{002}^{\mathrm{T}}}, \\
\tau_{\mathrm{R}}=\frac{\mathrm{I}_{200}^{\mathrm{R}}}{\mathrm{I}_{200}^{\mathrm{T}}+\mathrm{I}_{200}^{\mathrm{R}}+\mathrm{I}_{002}^{\mathrm{T}}},
\end{gathered}
$$

where $\mathrm{I}_{200 / 002}^{\mathrm{T} / \mathrm{R}}$ are the corresponding tetragonal $(\mathrm{T})$ and rhombohedral $(\mathrm{R})$ peak intensities.

The crystalline structure and lattice parameters of all samples were estimated from fitting results of the XRD data by using the Powder Cell software [18]. The surface of the sintered samples was processed and cleaned by an ultrasonic cleaner and then observed by scanning electron microscopy (SEM, Nova NanoSEM 450FEI). Particle size is the mean linear intercept length that was determined using an intercept method with the assistance of Lince software [19]. The silver pastes were fired at $450^{\circ} \mathrm{C}$ for 30 minutes on both sides of these sintered bulks as electrodes for electrical measurements. Dielectric properties of the materials were determined together using an impedance analyzer (Agilent 4396B, Agilent Technologies, America, HIOKI3532) by measuring the capacitance of the specimens from room temperature to $120^{\circ} \mathrm{C}$. Raman scattering spectra was measured using LabRAM-1B (Horiba Jobin Yvon). Ferroelectricity was studied by using the Sawyer-Tower circuit method. In order to study piezoelectric properties, the samples were polled in silicon oil bath by applying the DC electric field of $2 \mathrm{kV} / \mathrm{mm}$ for 60 minutes at room temperature. The main piezoelectric parameters were calculated when using a resonance method (Agilent 4396B, HP4193A) and all formulas in the IEEE standard for piezoelectric ceramic characterization [20].

\section{Results and discussion}

\subsection{Structure, microstructure, and electric properties of $\mathrm{BCZT} / \mathrm{x}$ ceramics sintered at temperature of $1350^{\circ} \mathrm{C}$}

Figure 2(a) shows X-ray diffraction patterns of the BCZT/x ceramics at various contents of $\mathrm{ZnO}$ nanoparticles measured at room temperature. All the compositions have demonstrated pure perovskite phases, and no trace of secondary phase was 
detected in the investigated region. Figure 2(b) plots the enlarged XRD patterns in the range of $(44-46)^{\circ}$ of BCZT/x ceramics. As shown, all BCZT/x ceramics have tetragonal symmetry (space group P4mm) characterized by splitting (002)/(200) peaks at around $2 \theta$ of $45^{\circ}$ with the intensity changing between samples. Moreover, the position of these diffraction peaks shifted to lower angles as increasing $\mathrm{x}$.

Figure 3 illustrates the variation in the lattice parameters, (a, c), and tetragonality, $\mathrm{c} / \mathrm{a}$, as a function of the addition of $\mathrm{ZnO}$ nanoparticles, $\mathrm{x}$, for BCZT/x ceramics sintered at $1350^{\circ} \mathrm{C}$. As increasing $\mathrm{x}$, constant (a) increases significantly, whereas constant (c) and tetragonality (c/a) reach their maximum values at $\mathrm{x}=0.15 \mathrm{wt} . \%$. It likely indicated that $\mathrm{Zn}^{2+}$ ions were incorporated into the BCZT lattices, and a stable solid solution was formed in the ceramics. However, $\mathrm{Zn}^{2+}$ ions did not change crystal symmetry of the materials that only varied the size of unit cells. Considering the radii of $\mathrm{Ba}^{2+}, \mathrm{Ca}^{2+}, \mathrm{Ti}^{4+}, \mathrm{Zr}^{4+}, \mathrm{O}^{2-}$, and $\mathrm{Zn}^{2+}$ of $1.44,1.34,0.605,0.79,1.4$, and $0.74 \AA$, respectively, $\mathrm{Zn}^{2+}$ is possibly substituted for the $\mathrm{B}$-site at $\left(\mathrm{Ti}^{4+}, \mathrm{Zr}^{4+}\right)$ positions within $\mathrm{ABO}_{3}$ perovskite structure that induced a lattice distortion in the $\mathrm{BCZT} / \mathrm{x}$ ceramics. This result would cause the diffuse transition behavior in the materials and will be detailedly discussed in following section.

Surface morphologies of the BCZT/x ceramics sintered at $1350^{\circ} \mathrm{C}$ are shown in Figure 4.

It is evident that addition of nanostructured $\mathrm{ZnO}$ has strongly influenced the microstructure. Clean surfaces were observed for BCZT/x samples with

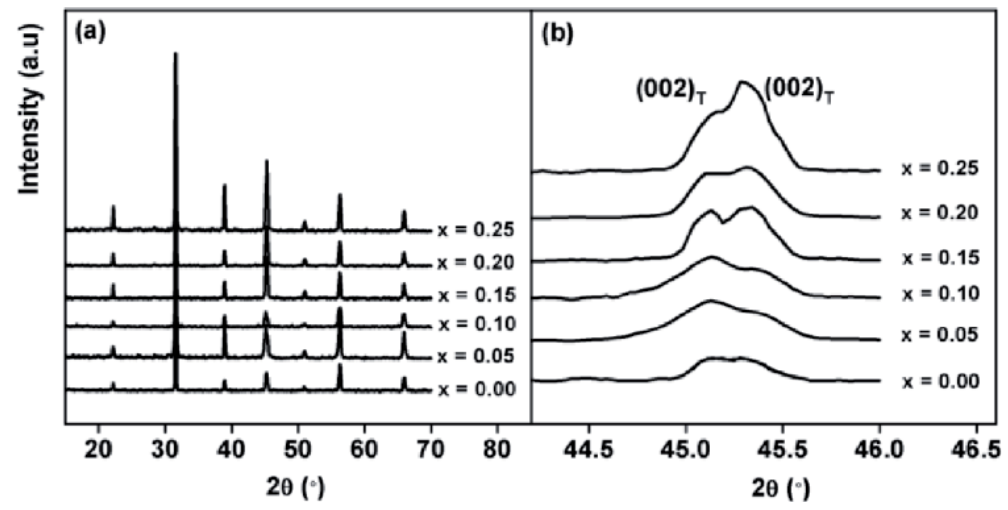

Figure 2.

$X R D$ patterns $(a)$ and expanded XRD patterns in the $2 \theta$ range of $(44-46)^{\circ}$ and $(b)$ of the $B C Z T / x$ ceramics sintered at temperature of $1350^{\circ} \mathrm{C}$.

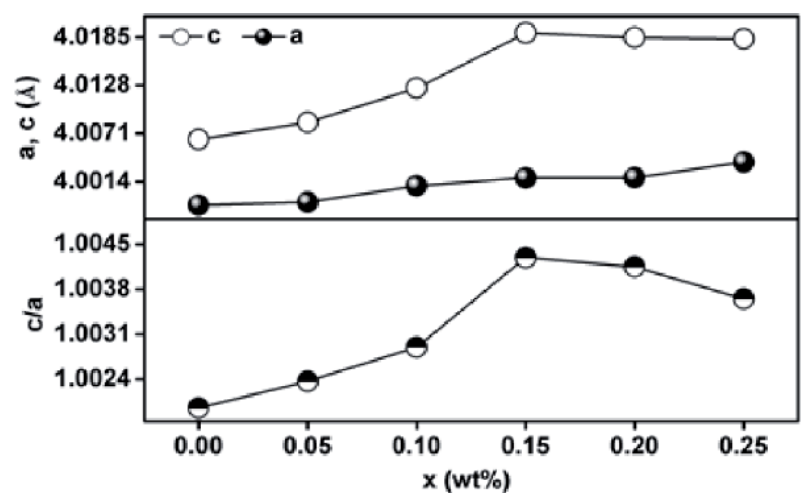

Figure 3.

Lattice parameters and tetragonality for the BCZT/x ceramics. 


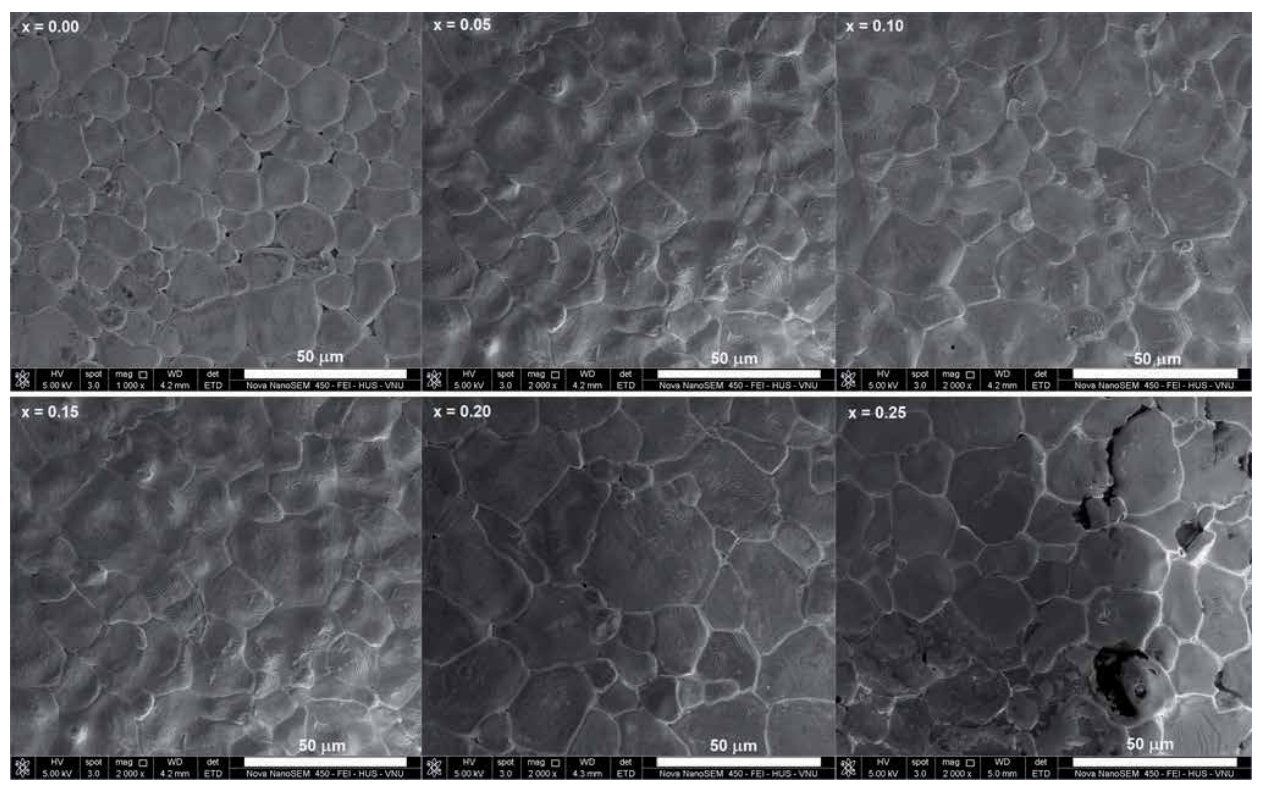

Figure 4 .

SEM images of the BCZT/x ceramics sintered at $1350^{\circ} \mathrm{C}$.

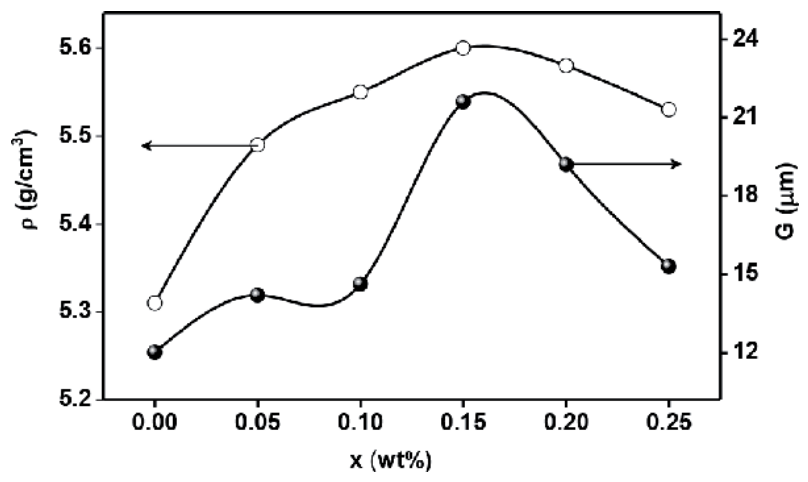

Figure 5.

Dependence of grain size, $G$, and density, $\rho$, on the $Z n O$ nanoparticle content.

$x=0.00-0.15$, and the grain size raised and reached the maximum value of $21.6 \mu \mathrm{m}$ at $\mathrm{x}=0.15$ compound. The liquid phase, however, appeared on the grain surface and boundary as $\mathrm{x}>0.15$. It may be an excess amount of nano-sized $\mathrm{ZnO}$ particles during sintering, accumulating at the surface and grain boundary to restrict grain size evolution. Therefore, the experimental results indicate that solubility limit of $\mathrm{ZnO}$ nanoparticles in BCZT substrate is below $0.15 \mathrm{wt} . \%$ at a sintered temperature of $1350^{\circ} \mathrm{C}$. Dependence of grain size and density on the $\mathrm{ZnO}$ nanoparticle content is in the same manner (Figure 5). Thus, the BCZT/0.15 composition was expected to possess excellent electric properties.

Figure 6 illustrates temperature dependences of permittivity, $\varepsilon(\mathrm{T})$, of the $\mathrm{BCZT} / \mathrm{x}$ ceramics measured with frequency of $1 \mathrm{kHz}$ at room temperature. It is clear to see that the samples with $\mathrm{ZnO}$ addition exhibit a typical temperature dependence of permittivity. A wide cubic-tetragonal phase transition at the temperature around $70^{\circ} \mathrm{C}$ was observed for all samples. Furthermore, another phase transition was found around $40^{\circ} \mathrm{C}$ for $\mathrm{BCZT} / 0.00$ composition (without $\mathrm{ZnO}$ nanoparticles) that is in the MPB region of BCZT and seems to be related to a tetragonal-rhombohedral 


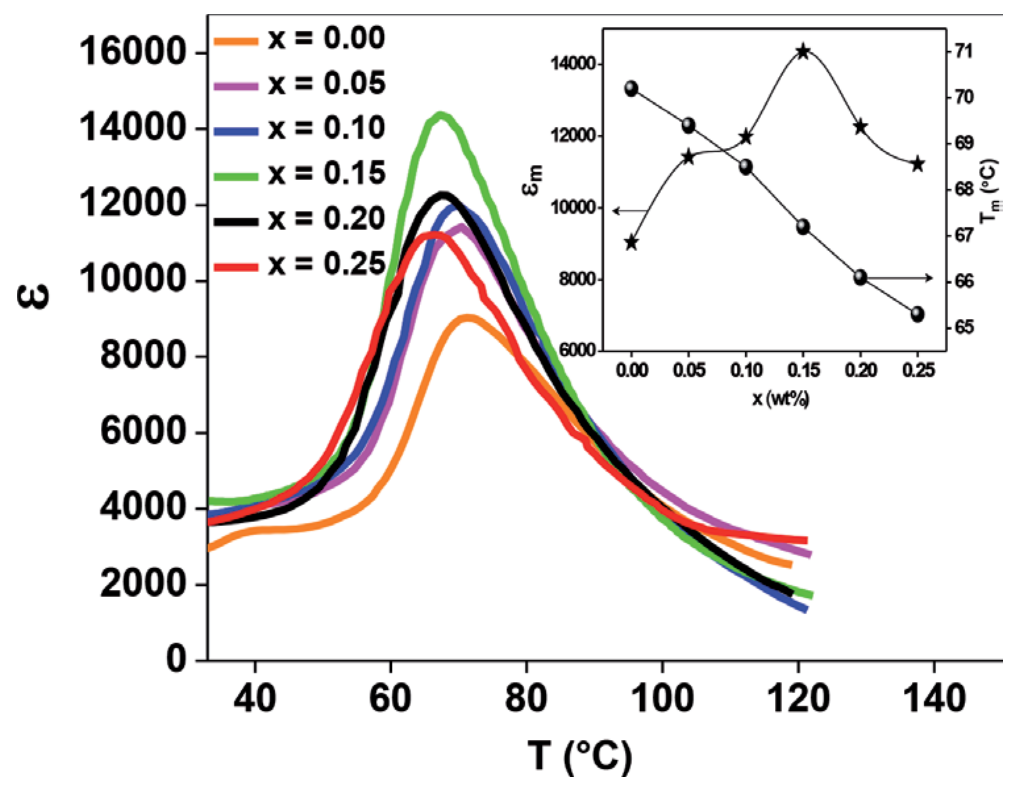

Figure 6.

Plot of permittivity versus temperature measured at $1 \mathrm{kHz}$ for BCZT/x systems.

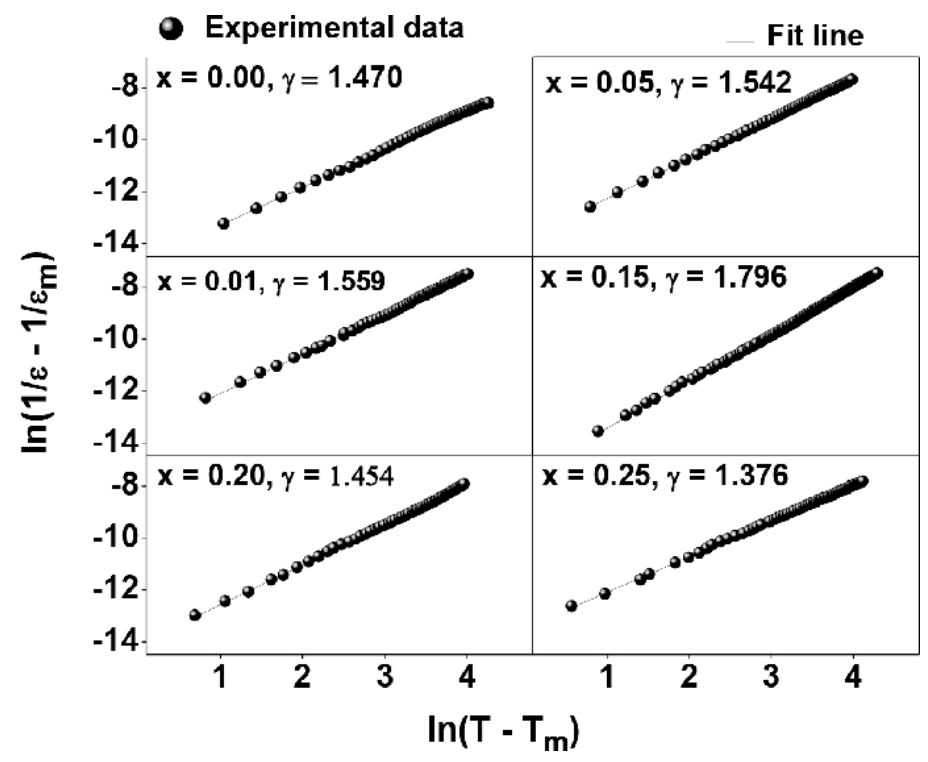

Figure 7.

$\ln \left(1 / \varepsilon-1 / \varepsilon_{m}\right)$ versus $\ln \left(T-T_{m}\right)$ for $B C Z T / x$ systems.

phase transition as reported in the literatures $[3,21]$. It is supposed that a part of the material has changed into rhombohedral phase with small amount so that this phase was not identified in X-ray patterns but can be observed in $\varepsilon(\mathrm{T})$ curve. The mentioned phase transition was disappeared as raising content of $\mathrm{ZnO}$ nanoparticles. It may be shifted to lower temperature. It can be seen that $\mathrm{ZnO}$ nanoparticles have strongly affected dielectric properties. First, the shape of permittivity-temperature curves of the $\mathrm{ZnO}$-added samples is broadened and shifted toward the lowertemperature region. This is due to the lattice distortion and indicates the ferroelectric diffuse transitions as reported in the literature [22]. The highest permittivity of 
the samples, $\varepsilon_{\mathrm{m}}$, nonlinearly depends on $\mathrm{ZnO}$ content. It increases with increasing $\mathrm{ZnO}$ content, reaches a maximal value of 14,361 for the BCZT/0.15 composition, and decreases monotonously after that. The temperature $\mathrm{T}_{\mathrm{m}}$ corresponding to the maximum permittivity, $\varepsilon_{\mathrm{m}}$, reduces with the increase of $\mathrm{ZnO}$ concentration due to the lattice distortion, as shown in XRD patterns. Figure 7 presents the plots of $\ln \left(1 / \varepsilon-1 / \varepsilon_{m}\right)$ versus $\ln \left(T-T_{m}\right)$ measured at $1 \mathrm{kHz}$ for the $\mathrm{BCZT} / \mathrm{x}$ ceramic that was fitted with modified Curie-Weiss law to obtain diffuseness degree parameter, $\gamma$. It can be observed that $\gamma$ was changed as a function of $\mathrm{x}$ and reached the highest value of 1.796 at $\mathrm{x}=0.15$ composition.

As mentioned above, the diffuse characterization of BCZT/x ceramics may be a result of replacing $\mathrm{Zn}^{2+}$ for B-site ion $\left(\mathrm{Ti}^{4+}, \mathrm{Zr}^{4+}\right)$. To put it more clearly, the room temperature Raman spectrum of the $\mathrm{BCZT} / \mathrm{x}$ ceramics was recorded and analyzed (Figure 8). As shown, Raman modes of $\mathrm{BaTiO}_{3}$-based systems are named as $\mathrm{A}_{1}$ (TO1), $\mathrm{A}_{1}(\mathrm{TO} 2), \mathrm{E}(\mathrm{TO} 2), \mathrm{A}_{1}(\mathrm{TO} 3)$, and $\mathrm{A}_{1}(\mathrm{LO} 3) / \mathrm{E}(\mathrm{LO} 3)$ in the range of $150-1000 \mathrm{~cm}^{-1}[6]$. The position and half-width of these modes were determined by fitting Raman data with Lorentzian function. E(TO2) vibration mode that has been associated with the tetragonal-cubic phase transition shifted to a lower wavenumber (Figure 9(a)). It means that substitution for B-site by $\mathrm{Zn}^{2+}$ results in reducing average B-O bonding energies. Thus, tetragonal-cubic

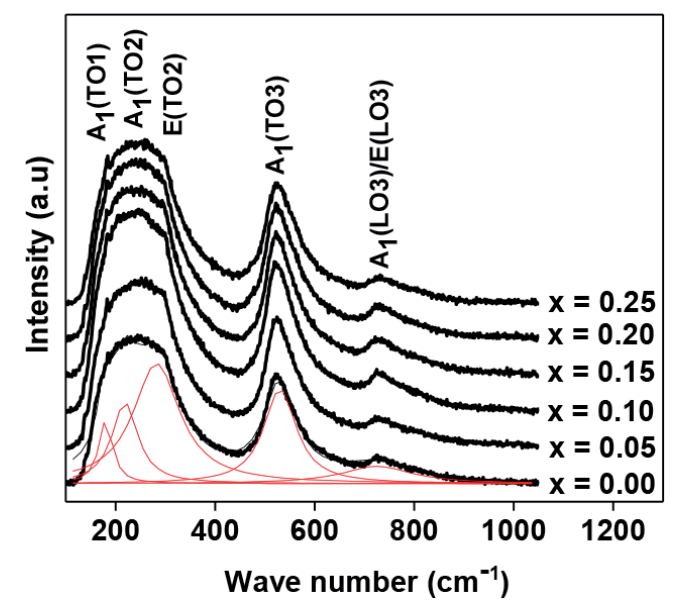

Figure 8.

Raman spectrum of $B C Z T / x$ systems recorded at room temperature.

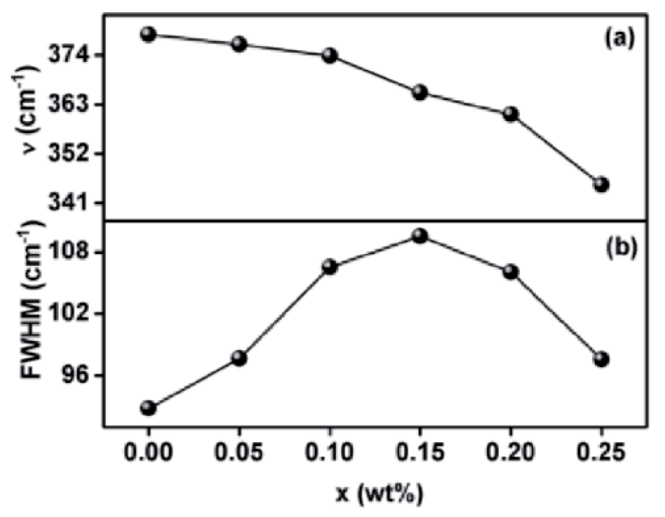

Figure 9.

(a) Raman shift, $\nu$, of E(TO2) and (b) half-width, FWHM, of $A_{1}\left(\mathrm{TO}_{2}\right)$ as a function of $Z n O$ nanoparticles, $x$. 


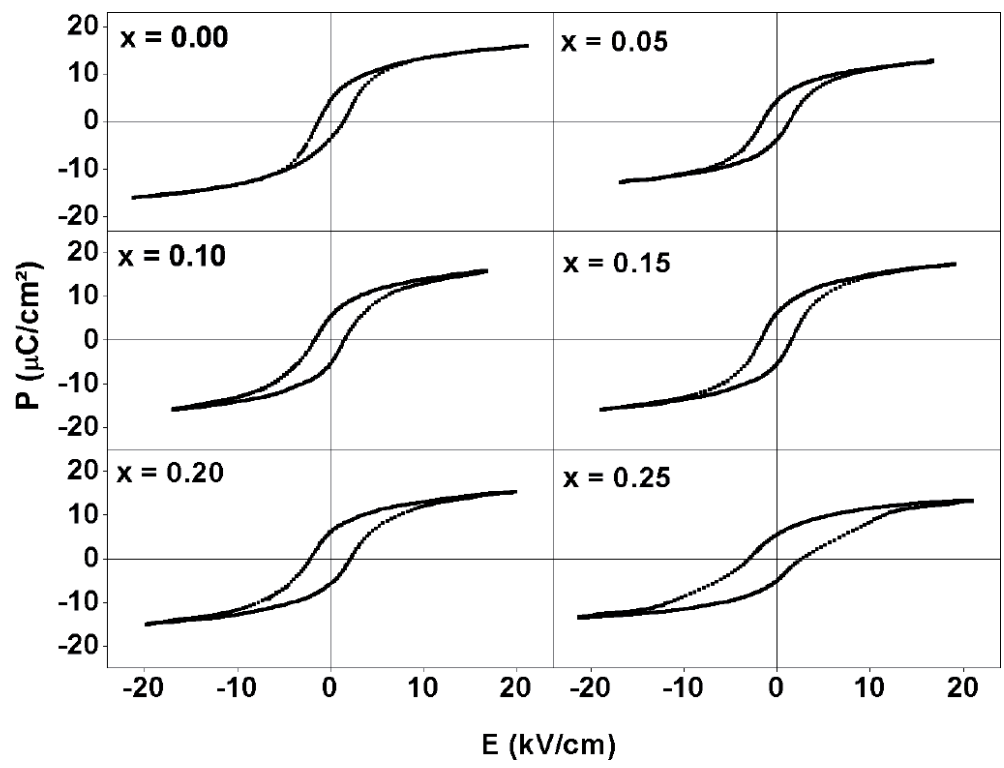

Figure 10.

Ferroelectric (P-E) hysteresis loops of BCZT/x systems.

phase transition temperature was diminished (see inset in Figure 6). The $A_{1}(T O 2)$ vibration mode was considered as the most sensitive to the lattice distortion that can be evaluated by FWHM of $\mathrm{A}_{1}$ (TO2) as presented in Figure 9(b). It has been shown that FWHM depends on $\mathrm{ZnO}$ content and reached a maximum value at $\mathrm{x}=0.15$. It means the material sample added with $0.15 \mathrm{wt} . \%$ of $\mathrm{ZnO}$ has shown the highest disorder [23]. In other words, FWHM of the Raman mode $\mathrm{A}_{1}$ (TO2) also reflects the diffuseness of the ferroelectric-paraelectric phase transition as diffuseness degree. This obtained result is well appropriated with the result given from the temperature dependence of permittivity as presented in Figure 6 where we can see that the diffuseness degree, $\gamma$, reached a maximum value for the same $\mathrm{ZnO}$ content $\mathrm{x}=0.15$ (see Figure 7).

Figure 10 presents P-E relationships for BCZT/x ceramics measured at room temperature. Received hysteresis loops were well saturated and fairly slim for all samples that assert again diffuse ferroelectric nature in BCZT/x ceramics. The characterized values of remanent polarization, $\mathrm{P}_{\mathrm{r}}$, and coercive field, $\mathrm{E}_{\mathrm{c}}$, depended on $\mathrm{ZnO}$ nanoparticles concentration as illustrated in Figure 11. As shown, when $\mathrm{x}$ content varied in the region of $0.00-0.25, \mathrm{P}_{\mathrm{r}}$ increased and reached a maximum value of $6.19 \mu \mathrm{C} / \mathrm{cm}^{2}$ at $\mathrm{x}=0.15$ and then decreased monotonously. This result could be explained based on an amelioration in microstructure [24]. According to that, poor ferroelectricity was received at grain boundary. Thus, polarization of grain boundary may be very small or zero. Alternatively, space charges eliminate polarization charge from grain surface that depletion layer can be established. That caused polarization interruption on particle surface to form depolarization field which lowers polarization. The reduced number of grain boundary is due to increasing grain size that could be the reason for raising remanent polarization and vice versa. In this study, the grain size, $G$, of these ceramics was controlled by varying doping concentration of nano-sized $\mathrm{ZnO}$ particles as shown in Figure 5. The dependence of coercive field on $\mathrm{ZnO}$ nanoparticles content shows that the parameter continuously intensified in the range of (1.36-2.72) $\mathrm{kV} / \mathrm{cm}$ as raising $\mathrm{x}$. In other words, $\mathrm{ZnO}$ nanoparticles made the ceramics harder. The enhancement of $E_{c}$ value could be due to the increase of charged oxygen vacancies as doping that pinned to the movement 


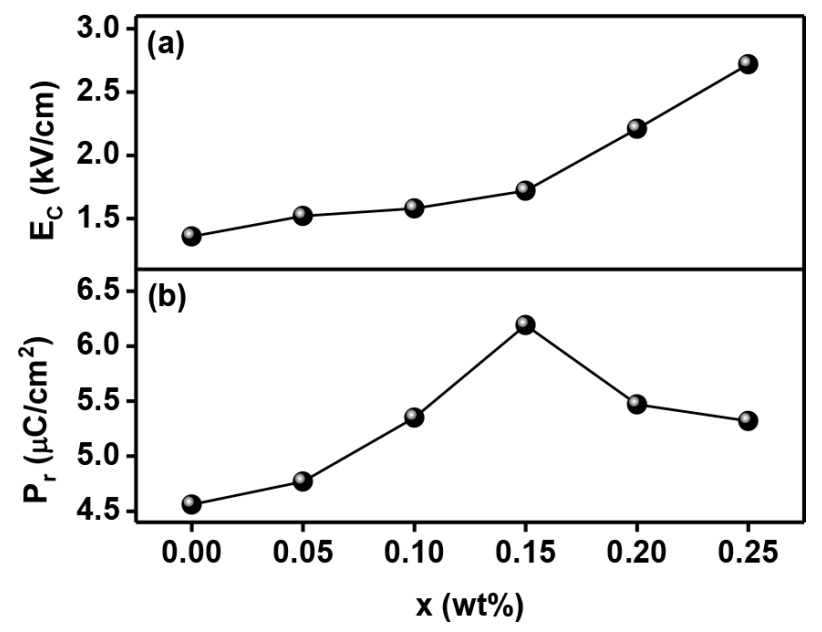

Figure 11.

Values of $E_{c}$ and $P_{r}$ as a function of $x$.

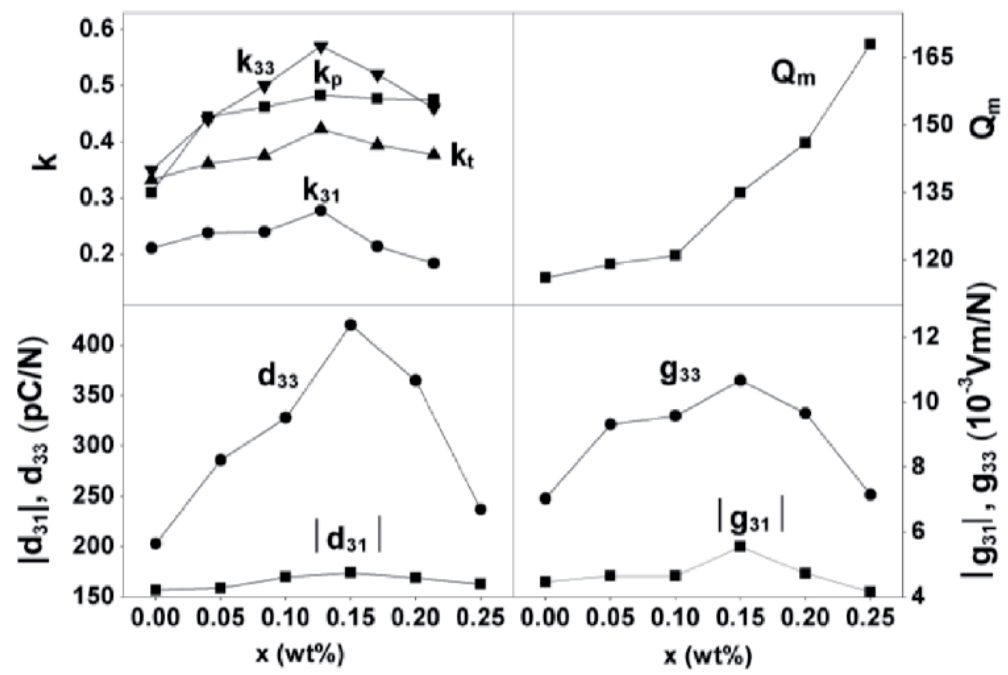

Figure 12.

Nano-sized $\mathrm{ZnO}$ content dependence of some piezo-parameters for BCZT/x ceramics.

of ferroelectric domain walls. The obtained values of $E_{c}$ demonstrated that the $\mathrm{BCZT} / \mathrm{x}$ materials are typically soft compared to electric properties.

Figure 12 displays the electromechanical coupling factor $(k)$, piezoelectric constant $\left(d_{33}, d_{31}, g_{33}, g_{31}\right)$, and mechanical quality factor $\left(Q_{m}\right)$ with various amounts of $\mathrm{ZnO}$ nanoparticles.

It can be observed that $\mathrm{k}, \mathrm{g}_{33}, \mathrm{~g}_{31}, \mathrm{~d}_{33}$, and $\mathrm{d}_{31}$ curves possess maximum values for the BCZT/0.15 compound as presented in Table 1.

As mentioned above, the comprehensive analysis of X-ray diffraction, SEM images, and dielectric properties have proven the $\mathrm{ZnO}$ addition induced lattice distortion. The degree of this local lattice distortion increased up to the maximal value as $\mathrm{ZnO}$ concentration raised up to $0.15 \mathrm{wt} . \%$. It is supposed the spontaneous polarization in each nano-domain has contributed to overall spontaneous polarization that enhanced piezoelectric characteristics of the material samples. Beyond the value of 0.15 , the piezoelectric parameters decrease due to residual amount of $\mathrm{ZnO}$ nanoparticles agglomerating at the surface and grain boundary restricting 


\begin{tabular}{lccccccc}
\hline $\mathbf{k}_{\mathbf{p}}$ & $\mathbf{k}_{\mathbf{t}}$ & $\mathbf{k}_{31}$ & $\mathbf{k}_{33}$ & $\begin{array}{c}\mathbf{d}_{33} \\
(\mathrm{pC} / \mathrm{N})\end{array}$ & $\begin{array}{c}\mathbf{d}_{31} \\
(\mathrm{pC} / \mathrm{N})\end{array}$ & $\mathbf{g}_{33}\left(\mathbf{1 0}^{-3} \mathrm{~V} \mathrm{~m} / \mathrm{N}\right)$ & $\mathbf{g}_{31}\left(\mathbf{1 0}^{-3} \mathrm{~V} \mathrm{~m} / \mathrm{N}\right)$ \\
\hline 0.483 & 0.423 & 0.278 & 0.571 & 420 & -174 & 10.68 & -5.55 \\
\hline
\end{tabular}

Table 1.

Values of coupling factors $\left(k_{p}, k_{t}, k_{31}, k_{33}\right)$ and piezoelectric constants $\left(d_{31}, d_{33}, g_{31}, g_{33}\right)$ of BCZT/o.15 sample sintered at $1350^{\circ} \mathrm{C}$.

grain size growth. According to Ying-Chieh Lee et al., $\mathrm{Zn}^{2+}$ is substituted into the $\mathrm{B}$-site to generate a doubly oxygen vacancy for compensation [12]. The presence of charged oxygen vacancies would be pinned by the movement of ferroelectric domain walls and consequently to enhance the $\mathrm{Q}_{\mathrm{m}}$ value. The result is similar to that reported by Jiagang Wu et al., who used micro-size $\mathrm{ZnO}$ as an acceptor for the $\mathrm{Ba}_{0.85} \mathrm{Ca}_{0.15} \mathrm{Ti}_{0.90} \mathrm{Zr}_{0.10} \mathrm{O}_{3}$ ceramic sintered at $1450^{\circ} \mathrm{C}[25]$.

\subsection{Influence of sintering temperature on structure, microstructure, and piezoelectric properties of doped BCZT ceramics}

In this section, effects of sintering temperature on the structure, microstructure, and piezoelectric properties of $0.15 \mathrm{wt} . \%$ modified BCZT or BCZT/0.15 compound are presented.

Figure 13 shows XRD patterns of BCZT/0.15 ceramic sintered at various temperatures of $1300,1350,1400$, and $1450^{\circ} \mathrm{C}$. All samples exhibited a pure perovskite phase, and no second-phase trace was detected in the investigated region. To study the effect of the sintering temperatures on the structure of BCZT/0.15 material, enlarged XRD patterns in the range of (44-46) ${ }^{\circ}$ corresponded to each sintering temperature were analyzed by fitting XRD data with Gaussian function (inset in Figure 13). As shown, there were splitting (200)/(002) diffraction peaks at around $2 \theta$ of $45^{\circ}$ for specimens sintered at 1300,1350 , and $1400^{\circ} \mathrm{C}$, which means these samples have tetragonal symmetry. However, coexistence of rhombohedral and tetragonal phases was observed for the sample sintered at $1450^{\circ} \mathrm{C}$ in which tetragonal volume fraction, calculated by using Eq. (1), was $67.3 \%$. In other words, $\mathrm{BCZT} / 0.15$ sample sintered at $1450^{\circ} \mathrm{C}$ could be MPB composition. Mixture of tetragonal-rhombohedral phases was also observed for BCZT/0.00 specimen (without $\mathrm{ZnO}$ nanoparticles) sintered at $1450^{\circ} \mathrm{C}$ (Figure 14) in which tetragonal volume

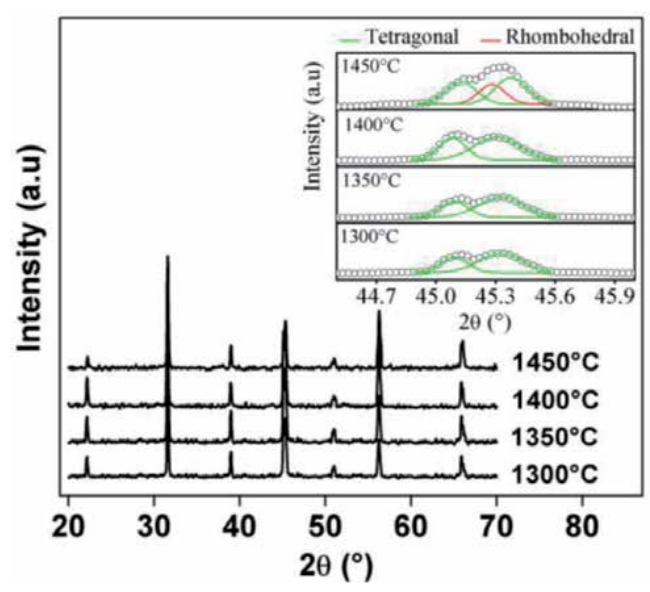

Figure 13.

XRD patterns of BCZT/0.15 sintered at different temperatures. 


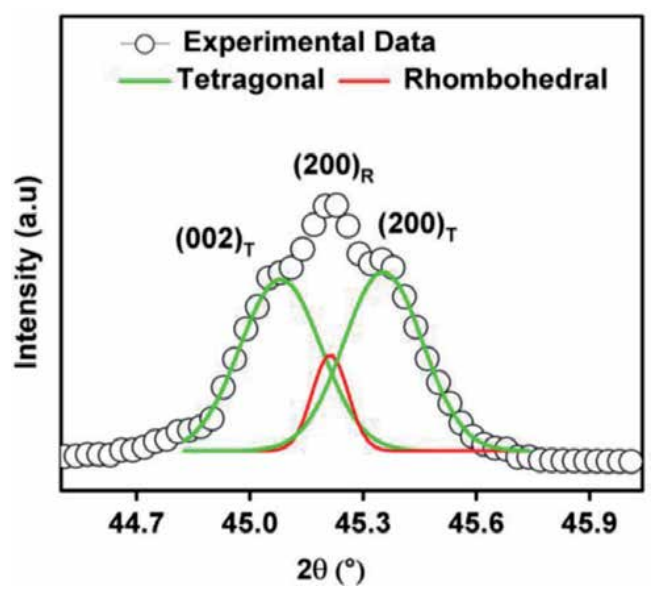

Figure 14.

Expanded XRD in the region of $(44-46)^{\circ}$ for BCZT/o.oo composition [26].

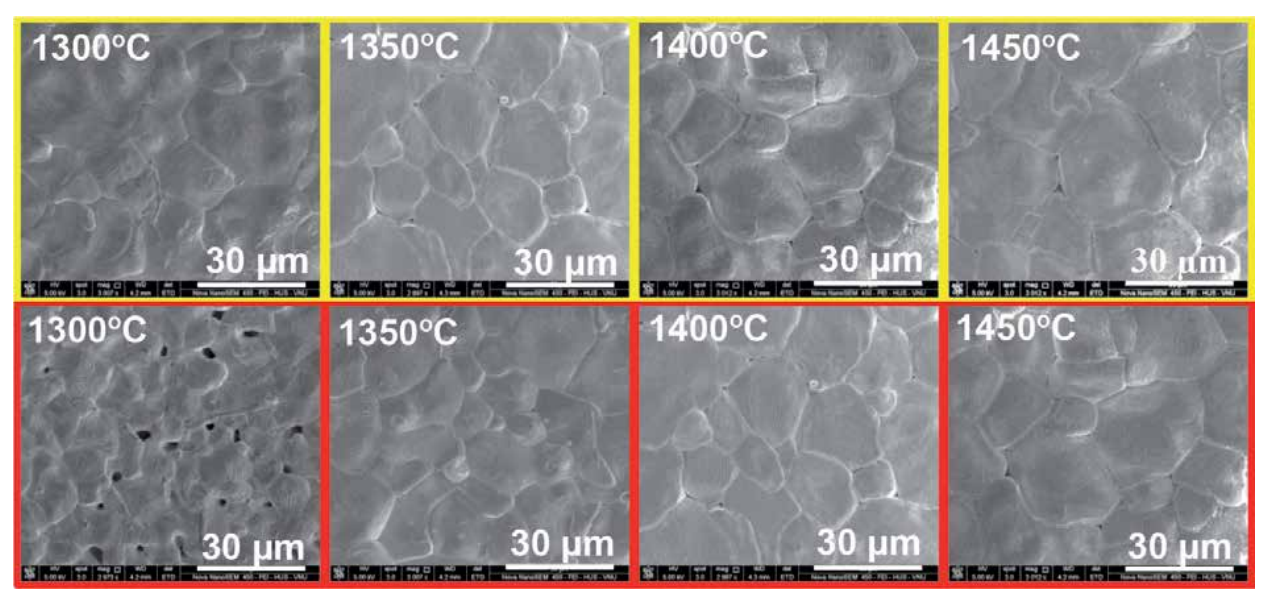

Figure 15.

SEM images of BCZT/0.15 (yellow border) and BCZT/o.oo (red border) ceramics sintered at various temperatures.

fraction was $71.7 \%$ [26]. It could be concluded that $\mathrm{ZnO}$ nanoparticle content of $0.15 \mathrm{wt} . \%$ did not change crystalline symmetry but only varied the fraction of phases in the samples. This could affect the piezoelectric properties of BCZT/0.00 and BCZT $/ 0.15$ samples sintered at $1450^{\circ} \mathrm{C}$.

Figure 15 illustrates surface morphologies of BCZT/0.00 and BCZT/0.15 materials sintered at various temperatures. Grain size and density were calculated and listed in Table 2.

Porous microstructure with small grains was received for BCZT/0.00 ceramic sintered at $1300^{\circ} \mathrm{C}$ (Figure 15). However, when $0.15 \mathrm{wt} . \%$ of $\mathrm{ZnO}$ nanoparticle content was added, a denser microstructure with larger particles is viewed at the same sintering temperature. Moreover, both grain size and density of BCZT/0.00 and BCZT/0.15 samples were raised as sintering temperature increased (Figure 16). Especially, grain size and density of BCZT/0.15 ceramics are larger than that of $\mathrm{BCZT} / 0.00$ ceramics for each sintering temperature. It could be concluded that a small amount of $\mathrm{ZnO}$ nanoparticles can be improved sintering behavior.

Figure 17 presents the comparison of the piezoelectric parameters for $\mathrm{BCZT} / 0.00$ and BCZT/0.15 materials. As the sintering temperature rises, the 


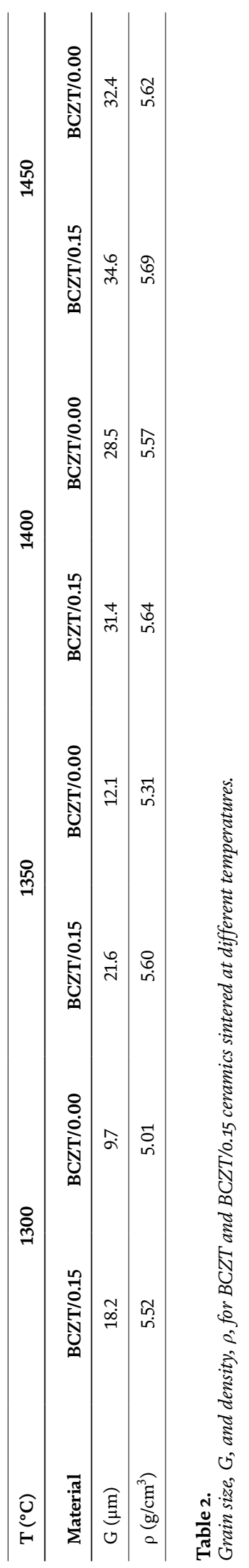




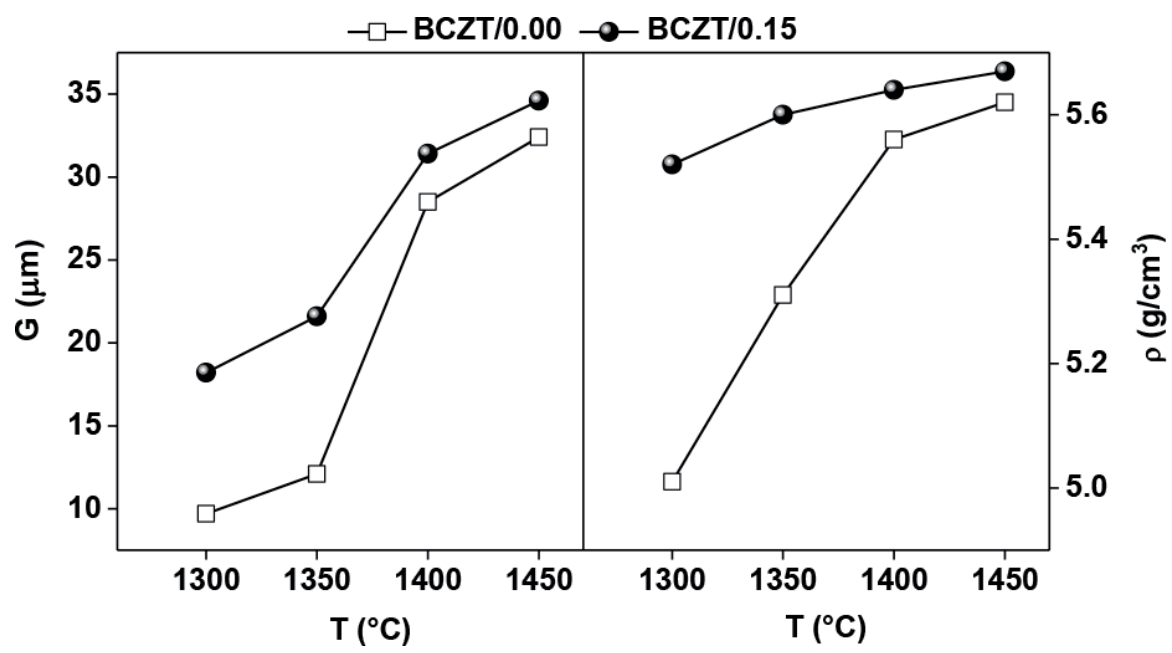

Figure 16.

Grain size and density of BCZT/0.0o and BCZT/0.15 materials as a function sintering temperature.

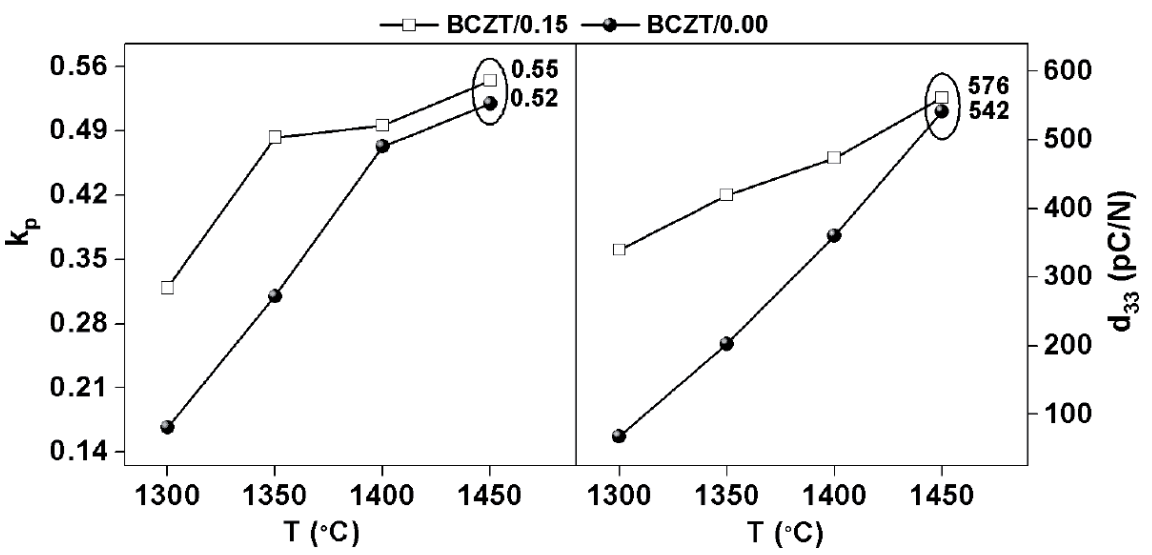

Figure 17.

Electromechanical factor, $k_{p}$, and piezoelectric coefficient, $d_{33}$, of BCZT/o.oo and BCZT/0.15 materials as a function sintering temperature.

piezoelectric parameters of both samples increase. Moreover, the piezoelectric parameters of BCZT/0.15 samples are higher than the ones of BCZT materials. These results may be due to the improvement in microstructure of the materials.

For the materials sintered at $1450^{\circ} \mathrm{C}, \mathrm{d}_{33}=576 \mathrm{pC} / \mathrm{N}$ and $\mathrm{k}_{\mathrm{p}}=0.55$ were obtained for BCZT/0.15 system, whereas these parameters for BCZT/0.00 system were $542 \mathrm{pC} / \mathrm{N}$ and 0.52 , respectively [9]. As known, in the MPB region, there are 14 possible polar directions with the low potential energy barrier that includes 6 in tetragonal phase and 8 in rhombohedral phase [27]. These polar directions are the optimum orientation during polarization process leading to the excellent piezoelectric properties. It could be supposed that there are only tetragonal and rhombohedral phases in two mentioning compositions and then their volume fractions are quantified as in Table 3. It can be seen that the nanostructured $\mathrm{ZnO}$ addition has heightened concentration of rhombohedral phase leading to enhance directional ability for polarization vectors. It is believed that it was the reason for the higher piezoelectric properties of BCZT/0.15 material than the BCZT/0.00 one. In other words, a competition between 


\begin{tabular}{lcc}
\hline Material & Tetragonal volume fraction & Rhombohedral volume fraction \\
\hline BCZT/0.15 & $63.7 \%$ & $36.3 \%$ \\
\hline BCZT/0.00 & $71.7 \%$ & $29.3 \%$ \\
\hline
\end{tabular}

Table 3.

Volume fraction of tetragonal and rhombohedral phases for BCZT and BCZT/o.15 materials sintered at $1450^{\circ} \mathrm{C}$ calculated by Eqs. (1) and (2).

structure phases with addition of $\mathrm{ZnO}$ nanoparticles could induce the difference in piezoelectric response for BCZT/0.00 and BCZT/0.15 samples.

\section{Conclusion}

The addition of $\mathrm{ZnO}$ nanoparticles with grain size of $59 \mathrm{~nm}$ has aided to successfully synthesize the BCZT/x ceramics at a relatively low sintering temperature of $1350^{\circ} \mathrm{C}$. The added $\mathrm{ZnO}$ particles in nanoscale influenced the relaxor ferroelectric phase change of the materials. As a result, BCZT/0.15 composition possessed the highest diffuseness characteristic. Remanent polarization was improved and reached to a maximum value of $6.19 \mu \mathrm{C} / \mathrm{cm}^{2}$ at $\mathrm{x}=0.15$, whereas the coercive field went up continuously under increasing doping concentration. The $\mathrm{ZnO}$ addition has also improved the quality of the piezoelectric material, and best quality was observed for BCZT/0.15 composition, given that the values of $\mathrm{d}_{33}, \mathrm{~d}_{31}, \mathrm{k}_{\mathrm{p}}, \mathrm{k}_{\mathrm{t}}$, and $\mathrm{k}_{33}$ are $420,-174,0.483,0.423$, and $0.571 \mathrm{pC} / \mathrm{N}$, respectively. The obtained results suggested that the lead-free BCZT/x material could be an expected lead-free piezoelectric ceramic for applications.

Besides, the influence of sintering temperature on structure, microstructure, and some piezoelectric parameters of BCZT/0.15 sample was examined. As the sintering temperature increased, improved sintering behavior and very high piezoelectric properties of $d_{33}=576 \mathrm{pC} / \mathrm{N}$ and $\mathrm{k}_{\mathrm{p}}=0.55$ were obtained for the sample sintered at $1450^{\circ} \mathrm{C}$. As a comparison, corresponded properties of $\mathrm{BCZT}$ without $\mathrm{ZnO}$ nanoparticles or BCZT/0.00 specimen were investigated. The received results show that sintering behavior and some piezo-parameters of BCZT/0.15 samples are better than that of BCZT/0.00 samples at each sintering temperature. Especially, the difference in properties for samples sintered at $1450^{\circ} \mathrm{C}$ is attributed to competition between structure phases occurred in materials.

\section{Acknowledgements}

This work was carried out in the framework of the National Project in Physics Program until 2020 under no. ĐTĐLCN.10/18. 


\section{Author details}

Dang Anh Tuan ${ }^{1}$, Vo Thanh Tung ${ }^{2 *}$, Le Tran Uyen $\mathrm{Tu}^{2}$ and Truong Van Chuong ${ }^{2}$ 1 Ha Nam Provincial Department of Science and Technology, Vietnam

2 University of Sciences, Hue University, Vietnam

*Address all correspondence to: vttung@hueuni.edu.vn

\section{IntechOpen}

(C) 2019 The Author(s). Licensee IntechOpen. This chapter is distributed under the terms of the Creative Commons Attribution License (http://creativecommons.org/licenses/ by/3.0), which permits unrestricted use, distribution, and reproduction in any medium, provided the original work is properly cited. (cc) BY 


\section{References}

[1] Panda PK. Review: Environmental friendly lead-free piezoelectric materials. Journal of Materials Science. 2009;44:5049-5062. DOI: 10.1007/ s10853-009-3643-0

\section{[2] Rodel J, Wook J, Seifert KTP, Eva-} Maria A, Granzow T, Damjanovic D. Perspective on the development of lead free piezoceramics. Journal of the American Ceramic Society. 2009;92(6):1153-1177. DOI: 10.1111/j.1551-2916.2009.03061.x

[3] Wenfeng L, Xiaobing R. Large piezoelectric effect in $\mathrm{Pb}$-free ceramics. Physical Review Letters. 2009;103:257602-257605. DOI: 10.1103/ PhysRevLett.103.257602

[4] Xue D, Zhou Y, Bao H, Zhou C, Gao J, et al. Elastic, piezoelectric, and dielectric proper ties of $\mathrm{Ba}\left(\mathrm{Zr}_{0.2} \mathrm{Ti}_{0.8}\right) \mathrm{O}_{3}-50\left(\mathrm{Ba}_{0.7} \mathrm{Ca}_{0.3}\right) \mathrm{TiO}_{3}$ $\mathrm{Pb}$-free ceramic at the morphotropic phase boundary. Journal of Applied Physics. 2011;109:054110. DOI: 10.1063/1.3549173

[5] Damjanovic D, Biancoli A, Batooli L, Vahabzadeh A, Trodahl J. Elastic, dielectric, and piezoelectric anomalies and Raman spectroscopy of $0.5 \mathrm{Ba}\left(\mathrm{Ti}_{0.8} \mathrm{Zr}_{0.2}\right)$ $\mathrm{O}_{3}-0.5\left(\mathrm{Ba}_{0.7} \mathrm{Ca}_{0.3}\right) \mathrm{TiO}_{3}$. Applied Physics Letters. 2012;100:192907. DOI: 10.1063/1.4714703

[6] Wu J, Xiao D, Wu W, Chen Q, Zhu J, Yang Z, et al. Composition and poling condition-induced electrical behavior of $\left(\mathrm{Ba}_{0.85} \mathrm{Ca}_{0.15}\right)\left(\mathrm{Ti}_{1-\mathrm{x}} \mathrm{Zr}_{\mathrm{x}}\right)$ $\mathrm{O}_{3}$ lead-free piezoelectric ceramics. Journal of the European Ceramic Society. 2012;32:891-898. DOI: 10.1016/j. jeurceramsoc.2011.11.003

[7] Wang P, Li Y, Lu Y. Enhanced piezoelectric properties of $\left(\mathrm{Ba}_{0.85} \mathrm{Ca}_{0.15}\right)$ $\left(\mathrm{Ti}_{0.9} \mathrm{Zr}_{0.1}\right) \mathrm{O}_{3}$ lead-free ceramics by optimizing calcinations and sintering temperature. Journal of the European Ceramic Society. 2011;31:2005-2012. DOI: 10.1016/j. jeurceramsoc.2011.04.023

[8] Su S, Zuo R, Lu S, Xu Z, Wang X, Li L. Poling dependence and stability of piezoelectric properties of $\mathrm{Ba}\left(\mathrm{Zr}_{0.2} \mathrm{Ti}_{0.8}\right)$ $\mathrm{O}_{3}-\left(\mathrm{Ba}_{0.7} \mathrm{Ca}_{0.3}\right) \mathrm{TiO}_{3}$ ceramics with huge piezoelectric coefficients. Current Applied Physics. 2011;11:S120-S123. DOI: 10.1016/j.cap.2011.01.034

[9] Tuan DA, Tinh NT, Tung VT, Chuong TV. Ferroelectric and piezoelectric properties of lead-free BCT- xBZT solid solutions. Materials Transactions. 2015;56(9):1370-1373. DOI: 10.2320/matertrans.MA201511

[10] Dung QTL, Chuong VT, Anh PD. The effect of $\mathrm{TiO}_{2}$ nanotubes on the sintering behavior and properties of PZT ceramics. Advances in Natural Sciences: Nanoscience and Nanotechnology. 2011;2:025013. DOI: 10.1088/2043-6262/2/2/025013

[11] Hayati R, Barzegar A.

Microstructure and electrical properties of lead free potassium sodium niobate piezoceramics with nano additive. Materials Science and Engineering B. 2010;172:121-126. DOI: 10.1016/j. mseb.2010.04.033

[12] Ying-Chieh L, Tai-Kuang L, Jhen-Hau J. Piezoelectric properties and microstructures of $\mathrm{ZnO}$-doped $\mathrm{Bi}_{0.5} \mathrm{Na}_{0.5} \mathrm{TiO}_{3}$ ceramics. Journal of the European Ceramic Society. 2011;31:3145-3152. DOI: 10.1016/j. jeurceramsoc.2011.05.010

[13] Ramajo LA, Taub J, Castro MS. Effect of $\mathrm{ZnO}$ addition on the structure, microstructure and dielectric and piezoelectric properties of $\mathrm{K}_{0.5} \mathrm{Na}_{0.5} \mathrm{NbO}_{3}$ ceramics. Materials Research. 2014;17(3):728-733. DOI: 10.1590/S1516-14392014005000048 
[14] Saeri MR, Barzegar A, Moghadama HA. Investigation of nano particle additives on lithium doped KNN lead free piezoelectric ceramics. Ceramics International. 2011;37(8):3083-3087. DOI: $10.1016 / j$. ceramint.2011.05.044

[15] Bari AR et al. Effect of solvent on the particle morphology of nanostructured $\mathrm{ZnO}$. Indian Journal of Pure and Applied Physics. 2009;47:24-27

[16] Yingying L, Leshu Y, Heyong H, Yuying F, Dongzhen C, Xin X. Application of the soluble saltassisted route to scalable synthesis of $\mathrm{ZnO}$ nanopowder with repeated photocatalytic activity. Nanotechnology. 2012;23(6):065402-065409. DOI: $10.1088 / 0957-4484 / 23 / 6 / 065402$

[17] Quintana-Nedelcos A, Fundora A, Amorín H, Siqueiros JM. Effects of $\mathrm{Mg}$ addition on phase transition and dielectric properties of $\mathrm{Ba}\left(\mathrm{Zr}_{0.05} \mathrm{Ti}_{0.95}\right)$ $\mathrm{O}_{3}$ system. The Open Condensed Matter Physics Journal. 2009;2:1-8. DOI: 10.2174/1874186X00902010001

[18] Kraus W, Nolze G. POWDER CELL - a program for the representation and manipulation of crystal structures and calculation of the resulting X-ray powder patterns. Journal of Applied Crystallography. 1996;29:301-303. DOI: 10.1107/S0021889895014920

[19] Tuan DA, Tung VT, Phuong LV. Analyzing 2D structure images of piezoelectric ceramics using ImageJ. International Journal of Materials and Chemistry. 2014;4(4): 88-91. DOI: 10.5923/j.ijmc.20140404.02

[20] The Institute of Electrical and Electronics Engineers, Inc. IEEE Standard on Piezoelectricity, ANSI/ IEEE Std 176-1987

[21] Gao J, Xue D, Wang Y, Wang D, Zhang L, Wu H, et al. Microstructure basis for strong piezoelectricity in $\mathrm{Pb}$-free $\mathrm{Ba}\left(\mathrm{Zr}_{0.2} \mathrm{Ti}_{0.8}\right) \mathrm{O}_{3}-\left(\mathrm{Ba}_{0.7} \mathrm{Ca}_{0.3}\right)$ $\mathrm{TiO}_{3}$ ceramics. Applied Physics Letters. 2011;99:092901. DOI: $10.1063 / 1.3629784$

[22] Hao J, Bai W, Li W, Zhai J. Correlation between the microstructure and electrical properties in highperformance $\left(\mathrm{Ba}_{0.85} \mathrm{Ca}_{0.15}\right)\left(\mathrm{Zr}_{0.1} \mathrm{Ti}_{0.9}\right)$ $\mathrm{O}_{3}$ lead-free piezoelectric ceramics. Journal of the American Ceramic Society. 2012;95(6):1998. DOI: 10.1111/j.1551-2916.2012.05146.x

[23] Dobal PS, Dixit A, Katiyar RS. Effect of lanthanum substitution on the Raman spectra of barium titanate thin films. Journal of Raman Spectroscopy. 2007;38:142-146. DOI: 10.1002/jrs.1600

[24] Mudinepalli VR, Feng L, Wen-Chin L, Murty BS. Effect of grain size on dielectric and ferroelectric properties of nanostructured $\mathrm{Ba}_{0.8} \mathrm{Sr}_{0.2} \mathrm{TiO}_{3}$ ceramics. Journal of Advanced Ceramics. 2015;4(1):46-53. DOI: $10.1007 / \mathrm{s} 40145-015-0130-8$

[25] Wu J, Xiao D, Wu W, Chen Q, Zhu J, Yang Z, et al. Role of room-temperature phase transition in the electrical properties of (Ba, Ca)

(Ti, $\mathrm{Zr}) \mathrm{O}_{3}$ ceramics. Scripta Materialia. 2011;65:771-774. DOI: 10.1016/j. scriptamat.2011.07.028

[26] Tuan AD, Tung TV, Chuong VT, Tinh TN, Huong TMN. Structure, microstructure and dielectric properties of lead-free BCT-xBZT ceramics near the morphotropic phase boundary. Indian Journal of Pure and Applied Physics. 2015;53:409-415

[27] Shrout TR, Zhang SJ. Lead-free piezoelectric ceramics: Alternatives for PZT? Journal of Electroceramics. 2007;19:111-124. DOI: 10.1007/ s10832-007-9047-0 



\title{
Chapter 8
}

\section{Optimal Temperature Sensor Based on a Sensitive Material}

\author{
Asma Bakkali, José Pelegri-Sebastia, Youssef Laghmich \\ and Abdelouahid Lyhyaoui
}

\begin{abstract}
The context of this chapter is the development of passive sensors for temperature sensing applications. The purpose is to successfully reduce the energy consumption in wireless sensor networks. The sensor is based on the electromagnetic transduction principle, and its originality is based on the integration of a high temperature-sensitive material into passive structure. Variation in temperature makes the dielectric constant of this material changing, and such modification induces variation in the resonant frequencies of high- $Q$ whispering-gallery modes in the millimeter-wave frequency range. In this way, the proposed device shows a linear response to the increasing temperature, and these variations can be remotely detected from a radar interrogation of an antenna loaded by the whispering-gallery mode resonator. Proposed device is a powerful tool for many interesting applications since it offers very low power consumption and provides environmentally friendly temperature measurement. The sensor is simulated in order to outline its performance and to show the benefit of the batteryless sensing device.
\end{abstract}

Keywords: energy consumption, wireless sensor networks, lifetime, energy conservation, whispering gallery mode, PLZT material, temperature, dielectric resonator

\section{Introduction}

From sensing motion to identifying a gas and measuring temperature, sensors are a key element in our daily lives for analytical, monitoring, and diagnostic applications [1-3]. Following the progress of technology and current concerns for the protection of the environment and people, the development of these devices is expanding significantly, to transform chemical, mechanical, and thermal phenomena into a measurable quantity: electrical signal. Nowadays, we are facing an explosion in the sensor market, and the number of applications is expanding in parallel with advances in electronics and wireless communication technologies.

Temperature detection is currently one of the most expected needs, as it is generally not well controlled at a low cost. Temperature sensors have been one of the first fields of application of micro-systems, and they now represent a very important part of this market due to the increasing demand in the consumer and domestic application sectors but also in production, aeronautics, and health. The main characteristics currently required of these components are most often to be miniature, efficient, and economical and can be integrated into complex electronic systems. 
Several research projects focus on optimizing the energy consumption of sensors by using innovative conservation techniques to improve the network's performance, including maximizing its lifespan. The sensor proposed in this chapter presents an interesting technological solution for temperature detection, thus allowing an extremely low consumption compared to conventional techniques. This new, highly integrated device requires no onboard power supply and uses electromagnetic transduction for temperature measurement.

\section{Passive and wireless temperature sensor design}

For decades, dielectric resonators (RDs) have been very important in the microwave field for many applications, such as oscillators and filtering devices [4]. The significant progress in the development of dielectric materials, both in terms of reliability and in improving the loss tangent at microwave frequencies, makes it possible to use them from microwave frequencies to millimetric frequencies. As the dimensions of these resonators can nowadays be small, they can be integrated into many telecommunication systems, in particular filtering systems where dielectric resonators have made it possible to maintain very good characteristics while reducing their size. These fields have led to a mature technology that allows the realization of reliable devices.

We have included the device proposed by Guillon et al. [5], composed of a silicon platform with coplanar lines on membrane. The second part is a dielectric resonator mounted on a support between the two coplanar lines. The entire device was coupled to an Monolithic Microwave Integrated Circuit (MMIC) amplifier, which subsequently made it possible to design a millimetric oscillator [6]. We can see that the device designed by Guillon was intended for the realization of an oscillator, which is not the case for us. The objective was to explore the interest that this device can provide in measuring small fluctuations in the dielectric permittivity of a material sensitive to temperature change. Therefore, the proposed structure is designed with more powerful simulation tools than those that existed in the 1990s, since they allow us to simulate the entire structure: coplanar lines, sensitive material, and dielectric resonator. In addition, we have resized the device to have the best possible transmission signal.

The temperature sensor shown in Figure 1 consists of two parts: the micromachined coplanar lines and the dielectric resonator covered with the sensitive material maintained by a support between and above these two lines. In the rest of this chapter, we will detail the design of this device before presenting the simulation results of the optimized structure.

\subsection{Presentation of the gallery modes}

Dielectric resonators operating in conventional electric (TE) or magnetic (TM) transverse modes radiate a significant portion of energy at millimeter wavelength frequencies [7]. In order to avoid dimensional problems and radiation losses at millimeter frequencies, we have chosen to use an excited dielectric resonator on gallery modes (whispering gallery modes).

From an electromagnetic point of view, one of the essential characteristics of WGMs is the distribution of energy in the resonator. The energy of the gallery modes has the particularity of being confined in a region close to the air-dielectric interface. Moreover, one of the main advantages offered by this type of mode is the possibility of exciting a dielectric resonator with an oversized geometry while remaining at the millimetric frequencies $[1,2]$. The dimensions of a resonator 


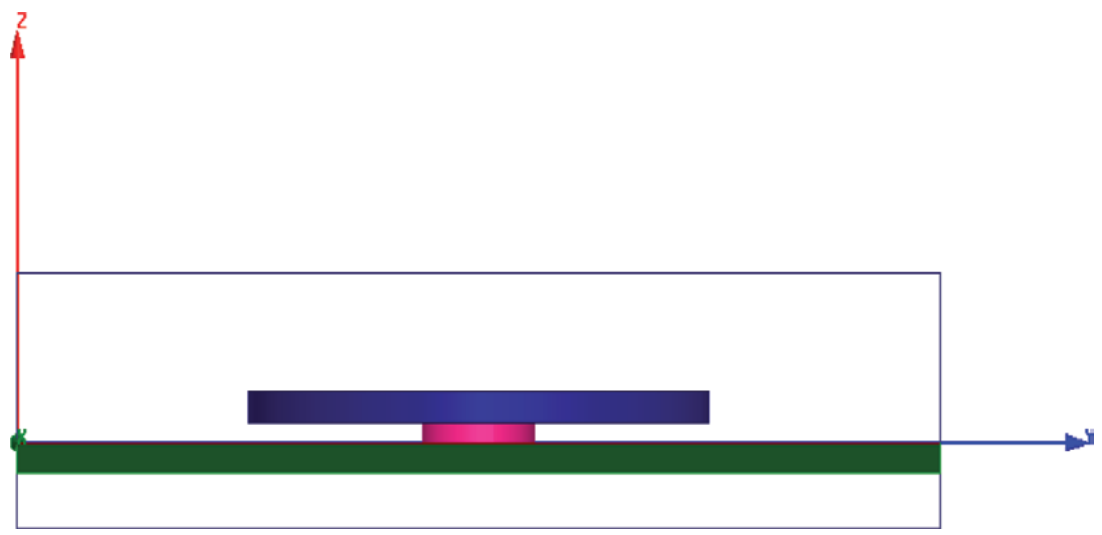

Figure 1.

Design of the temperature sensor: cross-section view.

excited in a gallery mode are much larger than those of the resonators used in conventional TE or TM modes. This makes it easier to use them at high frequencies since, given the dimensions of the resonator, it can be handled more easily.

The gallery modes of dielectric resonators are classified into two families: $\mathrm{WGH}_{\mathrm{n}, \mathrm{m}, \mathrm{l}}$ (magnetic field gallery modes) and $\mathrm{WGE}_{\mathrm{n}, \mathrm{m}, \mathrm{l}}$ (electric field gallery modes). This nomenclature makes it possible to identify each mode by taking into account the state of polarization and the importance of the transverse components of the electromagnetic field [3]. Thus, we are able to distinguish, on the one hand, WGE modes where the axial component of the field is essentially magnetic, while the transverse components are mainly electric. On the other hand, we distinguish the WGH modes, which correspond to the dual modes of the WGE. The three integers $\mathrm{n}, \mathrm{m}$, and $\mathrm{l}$ indicate the spatial configuration of the electromagnetic field inside the resonator (number of variations of the field in the three directions of the cylindrical reference mark):

- $\mathrm{n}$ : number of variations along the azimuthal direction

- $\mathrm{m}$ : number of variations according to radial direction

- 1: number of variations according to the axial direction

It is important to mention that the azimuth number has an influence on the caustic radius. Indeed, a high azimuth number results in a higher caustic radius and therefore in a confinement of electromagnetic energy closer to the lateral surface of the resonator.

\subsection{Advantages of gallery modes for temperature detection}

Among the main advantages of gallery modes, we will note here the most important for our study. First of all, the dimensions of the resonator excited on a gallery mode are much larger than in the case of conventional TE or TM modes. This oversizing makes it possible to consider the use of this resonator at millimeter frequencies by facilitating temperature detection. On the other hand, thanks to the high-energy confinement in the dielectric, the vacuum quality factors are practically limited only to the loss tangent of the material used [3]. The latter will thus be very sensitive to the presence of a variation in the dielectric properties of the sensitive material, which will improve its detection thanks to an offset in the resonance frequency of a gallery mode. 
In addition, it is important to note that the gallery modes have the particularity of not having any energy at the center of the resonator. As mentioned earlier, the larger the azimuth number, the larger the area. The central part can therefore be used to fix the resonator; we can add a shim to the circuit in order to maintain it or adjust its position in relation to the coupling circuit (lines) without disturbing its operation. Finally, these modes are no longer stationary but progressive when the resonator is excited by a progressive wave source, whose propagation constant is close to that of the gallery mode. The mode then propagates in the azimuthal direction. This type of excitation for gallery modes results in a directional coupling with the line. Therefore, this coupling will make it possible to consider the design of a directional filter with a narrow bandwidth, which in turn will allow temperature measurement thanks to the shift in its resonance frequency.

\section{Sensor based on millimeter band resonant gallery modes}

In order to define an innovative temperature sensor with high RF performance, we will take advantage of the dielectric properties of the sensitive material, the machining of coplanar membrane lines, and the characteristics of gallery modes. This new detection device is based on the modification of the resonance frequency of the dielectric resonator at the presence of a temperature variation. This is done by means of a perovskite material deposited above this resonator whose dielectric constant varies with a change in temperature.

The general concept chosen is to modify the electromagnetic coupling that exists in the device by temperature (see Figure 2). This concept allows for a very high sensitivity. Indeed, variable coupling can be achieved by modifying (globally or locally) the environment around the electromagnetic field lines by directly modifying the electromagnetic properties of the sensitive material.

In other words, a change in temperature leads to a change in the permittivity of the sensitive material and therefore a change in the electromagnetic field. This change affects the electrical parameters of the resonator, namely, the resonance frequency and the quality factor. Thus, a resonator designed to operate at a center frequency $\mathrm{f}_{0}$ for a particular gallery mode sees this shift as a function of the variation in the dielectric constant of the sensitive material. The electromagnetic field is therefore used to measure the temperature variation. The detection principle is then based on the shift in the resonance frequency of the gallery modes in the dielectric resonator. The latter and the sensitive material are used here to measure the influence of the permittivity of this material and to deduce the temperature variation.

In addition, this type of detection technique based on electromagnetic transduction has shown interesting results for the realization of an oscillator in the first place and subsequently for specific gas and pressure detection applications. These passive microwave sensors have been designed using the relaxation phenomenon present in sensitive materials. In particular, the installation of a functional $\mathrm{TiO}_{2}$-based

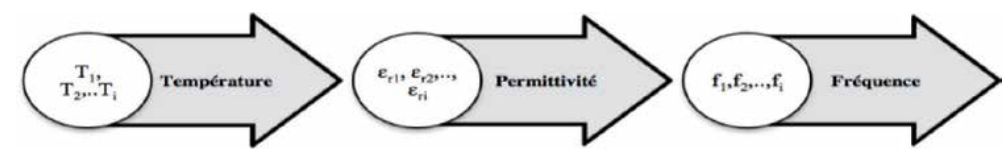

Figure 2.

Principle of electromagnetic transduction. 
microwave sensor in the Ka-band has been proposed for gas concentration detection. This detector is based on the direct variation of the dielectric properties of the resonator at the presence of a gas.

\section{Study of the geometric parameters of the sensor}

There are parameters that can modify the dielectric characteristics of a material such as temperature, for example. In our case, the aim is for this modification to generate a frequency variation in the microwave domain. The basic element is the sensing material whose dielectric constant varies with temperature. The choice of the sensitive material is not obvious because of the requirements imposed, which complicate the integration of dielectric materials into microelectronic circuits or their use in the manufacture of the sensor. To achieve this, we have carried out an in-depth bibliographical study in order to find the appropriate material that meets our specifications. Among the materials proposed in the literature, we chose lead-lanthanum-zirconatetitanate (Abbreviated in PLZT). As a result, and as part of our collaboration with the Faculty of Computer Science and Materials Sciences, Silesian University of Poland (see Figure 3) $[8,9]$, we have several samples taken in his laboratory.

Also referred to as lanthanum-doped lead zirconate titanate, this material meets our needs in terms of temperature range and operating frequency. In particular, it has a dielectric property that depends on the change in temperature (see Figure 4) $[10,11]$. Indeed, the variation in temperature has an impact on the properties of this material since it causes a relatively large change in its dielectric permittivity [12]. This PLZT will then detect the temperature change through its integration into the microwave circuit described in Figure 1, with a radius of RPLZT $=3.25 \mathrm{~mm}$ and a thickness of HPLZT $=10 \mu \mathrm{m}$.

The system is based relatively on the use of a dielectric resonator and two coplanar membrane lines. These lines serve as an excitation support for the RD gallery modes. The study of the mechanism of operation of these two components was widely discussed in the literature [13-15]. Thus, the RD has a radius of $\mathrm{R}_{\mathrm{RD}}=3.25 \mathrm{~mm}$, a thickness of HRD $360 \mu \mathrm{m}$, and a relative dielectric permittivity of 80. This dielectric resonator is held on the line plane by an Alumina $\left(\mathrm{Al}_{2} \mathrm{O}_{3}\right)$ wedge with $\mathrm{R}_{\text {Support }}=0.8 \mathrm{~mm}$ radius and $\mathrm{H}_{\text {Support }}=230 \mu \mathrm{m}$ height.

The RD's gallery mode excitation mechanism and sensitive material were selected, and a radar interrogation was carried out to transmit temperature information.

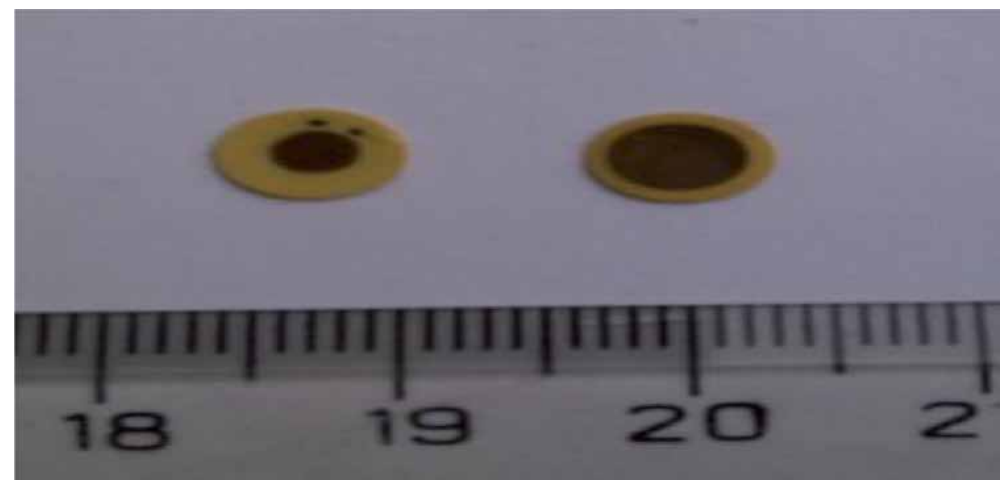

Figure 3.

Samples of PLZT material taken by Wawrzata and Korzekwa at the Silesian University of Poland. 


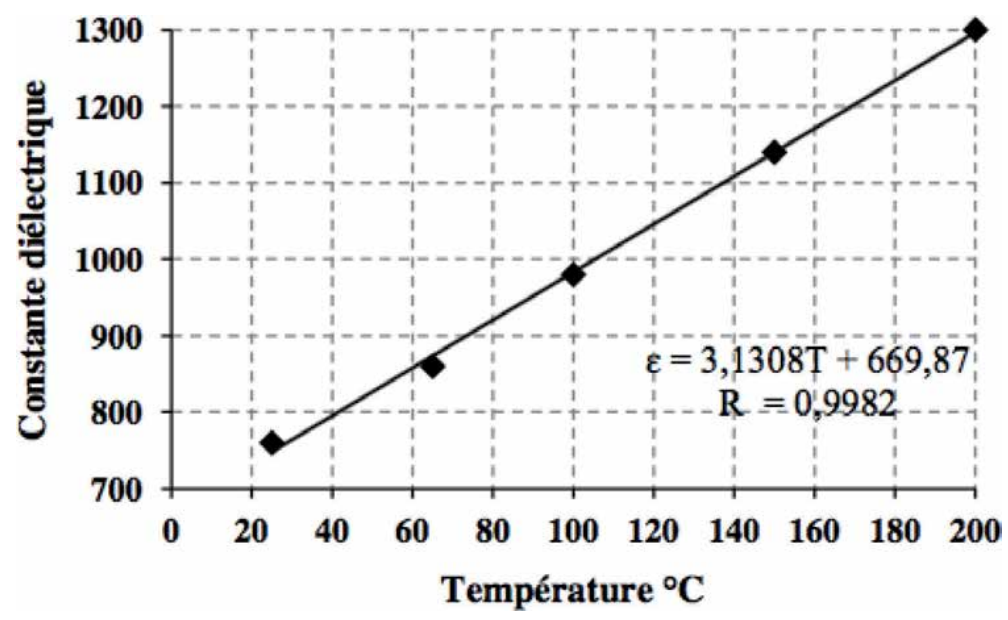

Figure 4.

Dielectric constant of PLZT as a function of temperature.

\section{Sensor interrogation method}

The transduction mode, size, and frequency of operation of this sensor are important characteristics that represent a technological break with the existing systems of passive wireless temperature sensors RFID and SAW.

To be remotely accessible, the sensor requires a reader that is compatible with its operating characteristics. Technical criteria for the use of a reader must be defined to satisfy the detection but also to ensure that the interrogation range is as long as possible. The existing readers for passive sensor interrogation, present in RFID and SAW technologies, do not meet the problems imposed on our study in terms of high frequencies of use and a range greater than $10 \mathrm{~m}$.

As a result, the characteristics of our sensor (wide range of detection, analysis, and processing of high frequency signals) guide us to consider a radar technology reader. Its operating principle, as with any radar, is to send a flow of electromagnetic waves to the sensor, which will return an echo whose power amplitude and will depend on the measured temperature. Indeed, radar is used in many applications such as level measurement, obstacle detection for automobiles, meteorology, or the military [16]. Its use for passive sensor network interrogation with RF transduction presents an innovative solution.

The proposed temperature sensor uses a millimeter radiofrequency transduction. The resonant frequencies of the sensor are included in the Ka band and shift from a bandwidth of a few hundred $\mathrm{MHz}$ to a few $\mathrm{GHz}$. An antenna to communicate remotely with the reader will connect the sensor. To interrogate this sensor, we turned to a radar technology reader developed during Chebila's thesis [17], according to precise technical criteria in terms of operating frequency satisfying wireless communication over a range greater than $20 \mathrm{~m}$. This distance remains a key point because many applications in the aeronautics, construction, and nuclear sectors refer to it for the installation of sensor networks. The modulation technique of this radar and its architecture based around a voltage-controlled oscillator (VCO) facilitated its realization and adjustment (see Figure 5).

The radar developed in 2011 is a frequency-modulated continuous radar (FMCW), used in the Ka band around $30 \mathrm{GHz}$ (see Figure 6) [17]. This HF radar will be used to remotely detect the temperature sensor measurements. The signals received by the reader must therefore inform us about the distance between the radar and the sensor but also about the temperature value coming from the 


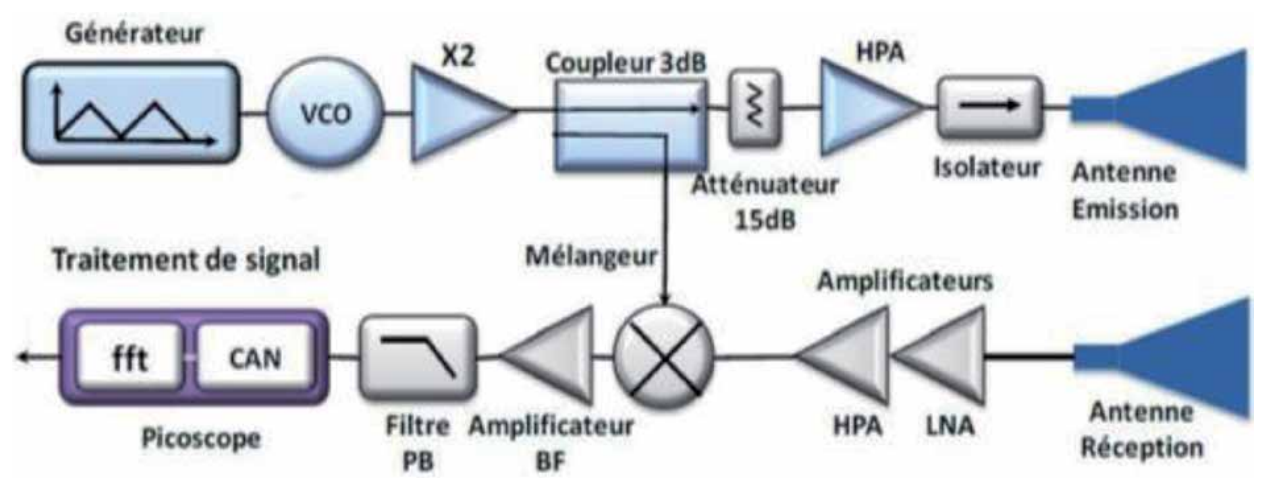

Figure 5.

Synoptic diagram of the $30 \mathrm{GHz}$ radar.

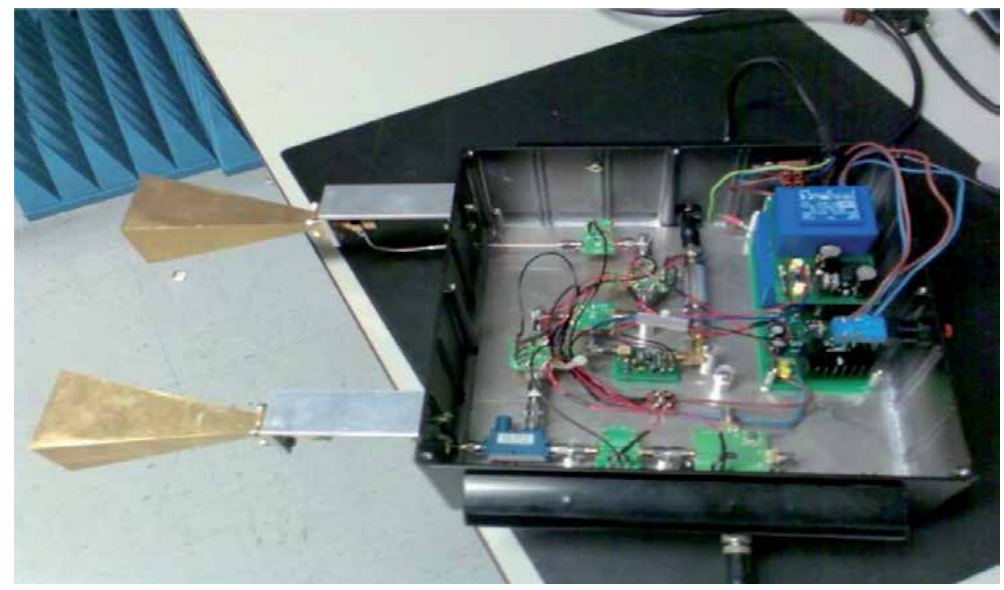

Figure 6.

Picture of the radar.

questioned measuring cell. In conclusion, the radar in question satisfies three important parameters for remote reading: its range is greater than $20 \mathrm{~m}$, works at a frequency compatible with the proposed sensor, and contains a system for identifying cells within a network.

The potential advantages of this type of transducer are:

- A significant reduction in signal losses thanks to the direct modulation of the microwave signal by the quantity to be measured

- A high sensitivity of the electromagnetic propagation to the environment used to perform the sensor function

- High spatial and temporal resolution due to the high operating frequency

- A more flexible choice of operating frequency that can be adapted to the different operating constraints of the sensor

- Easy integration into a measurement chain (radar and antennas)

The following section is devoted to the results of microwave measurements made using a high-performance simulator, allowing the frequency offset to be 
monitored and a direct relationship to be established between the temperature variation and the observed frequency offset. In this way, a temperature measurement is carried out via an electromagnetic transduction.

\section{Simulation results of the complete sensor}

Series of free oscillation simulations (eigenmode) using the HFSSTM software, applied to the dielectric resonators, determine the diameter, thickness, permittivity of the dielectric resonator, as well as the distribution of the electromagnetic field necessary to define the caustic and the optimal coupling with the coplanar lines (see Figure 7). The determination of caustic makes it possible to establish the distribution of the electromagnetic field in the RD and leads to the definition of the position of the coplanar lines. In addition, the diameter of the resonator and the confinement of the electromagnetic field in it impose the distance between these two micro-machined lines.

In this study, we studied a dielectric resonator with a relative permittivity of 80 and no losses. The thickness and diameter of the dielectric resonator are used to determine the resonance modes and frequencies associated with them. Subsequently, we are interested in the modification of the physical properties of the PLZT material in the presence of a change in temperature, more precisely the variation of its dielectric permittivity. As shown in Figure 8, we observe the distribution of the electric field of the $\mathrm{WGE}_{8.0 .0}$ mode in the dielectric resonator; it is thus isolated around $30 \mathrm{GHz}$.

We should also mention that the overall circuit of our detection system represents a directional filter consisting of two parts:

- Coplanar lines (CPWs) used for RD excitation and field propagation electromagnetic

- Dielectric resonator used for coupling and excited in WGM as well as the material PLZT as an element of recognition

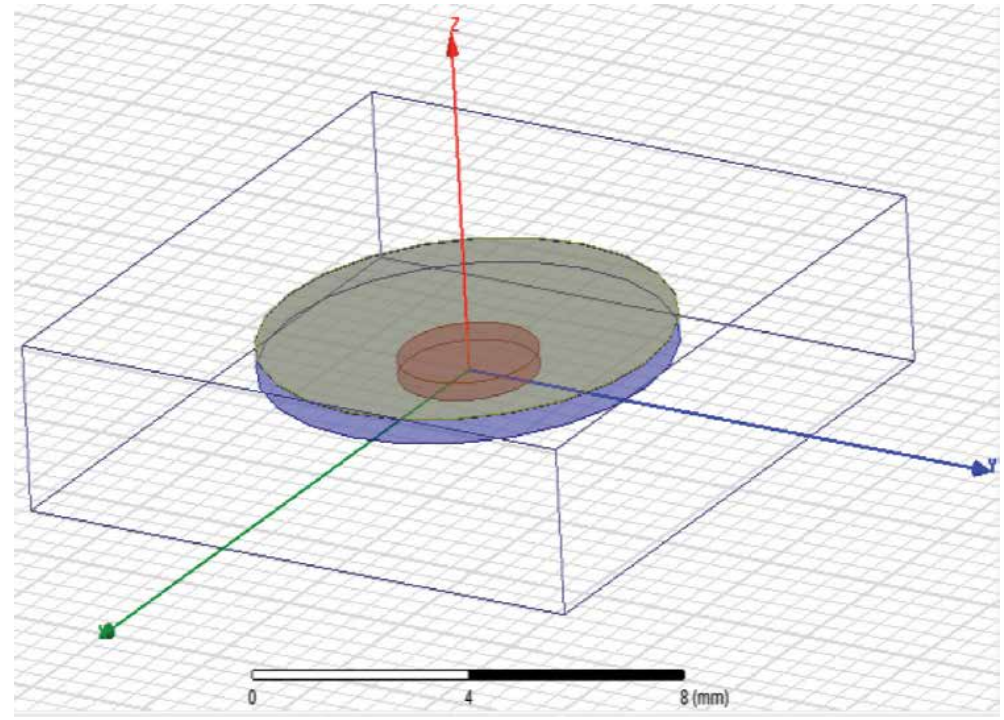

Figure 7.

The sensitive material and the resonator in the cavity with a holder. 


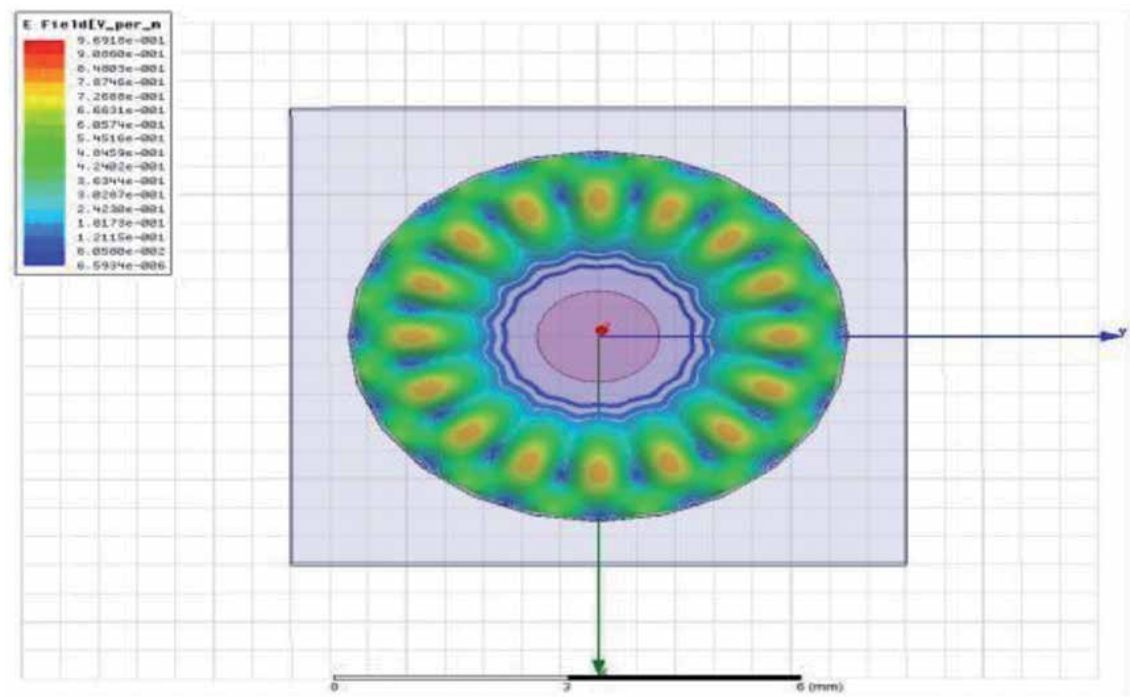

Figure 8.

The field distribution of the WGE $E_{8.0 .0}$ to $30 \mathrm{GHz}$ mode for eigenmode calculation (HFSS ${ }^{T M}$ ).

From the coupling coefficient $S_{12}$ between access 1 and 2 (simulated) given as a function of frequency, the gallery modes WGE and WGH were identified over a frequency range of 25-40 GHz. Figure 9 shows the look of the transmission parameter between access 1 and 2 in the Ka-band.

Figure 10(a) and (b) shows examples of simulation results corresponding to amplitudes of the magnetic field of the gallery modes at their resonant frequencies.

Based on the results obtained as well as those in the literature, PLZT appears to be the right candidate for frequency temperature transduction. Indeed, it has been previously demonstrated that the dielectric permittivity of this material can be modified in the presence of a temperature variation. We therefore aim to analyze the impact of such a modification on the resonance frequency of a gallery mode. For this purpose, the dielectric constant $\varepsilon_{\mathrm{r}}$ was varied between 700 and 900 with a

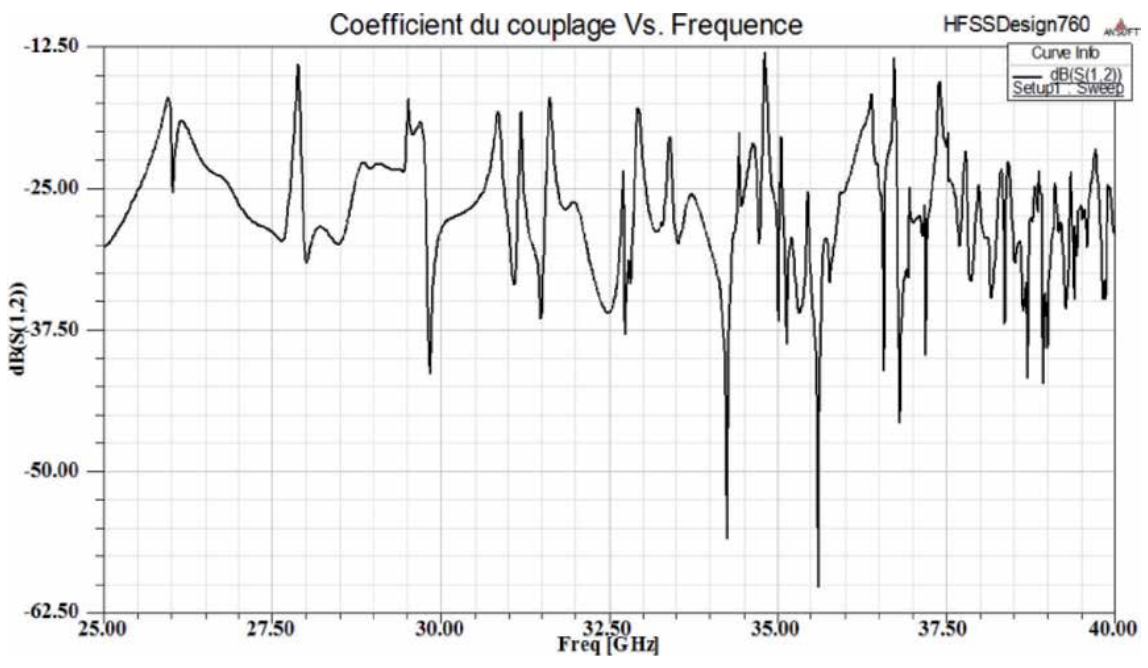

Figure 9.

Coupling coefficient $S_{12}$ as a function of frequency for $\varepsilon_{P L Z T}=760$. 


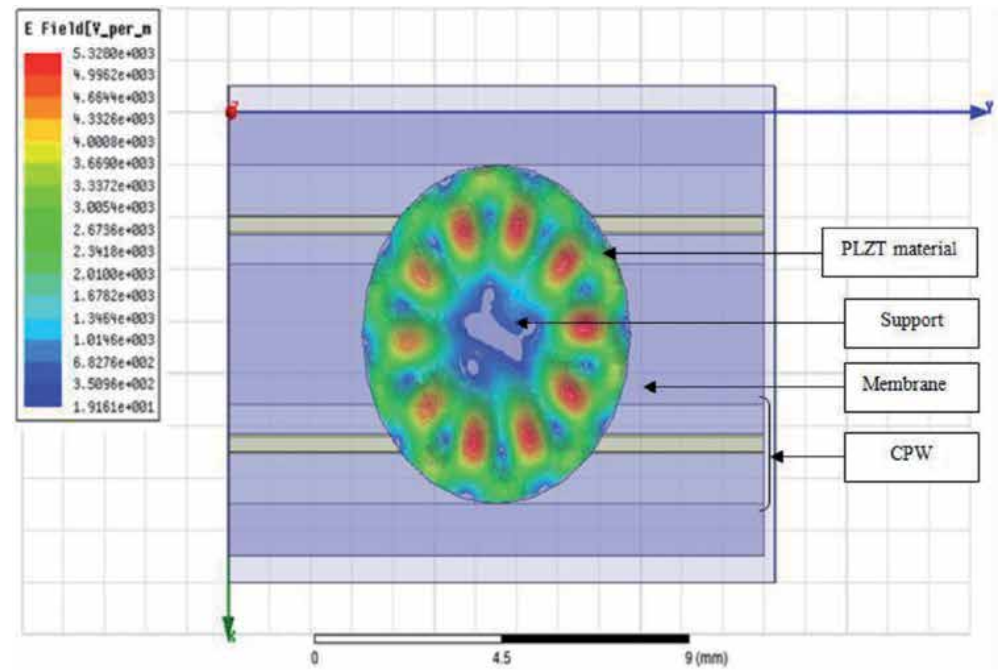

(a)

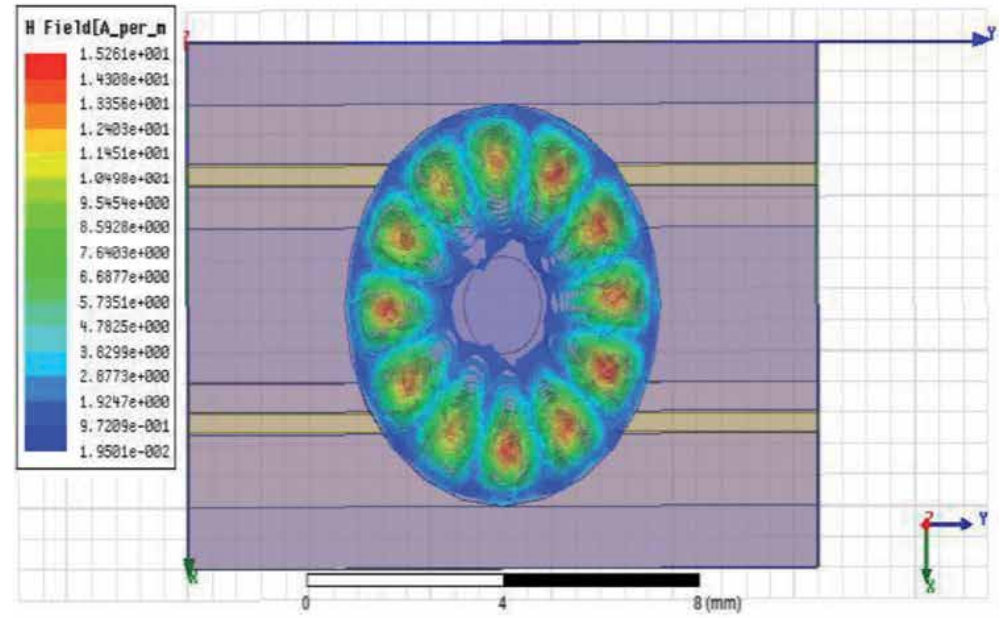

(b)

Figure 10.

Amplitude of the magnetic field of the gallery modes: (a) $W G H_{6,0,0}$ and (b) $W G E_{5,0,0}$.

$14 \%$ variation. As shown in Figure 11, the variation in PLZT permittivity produces measurable changes in resonance frequency, reflected in a shift to low frequencies of about $1 \mathrm{GHz}$, for example, in the case of $\mathrm{WGE}_{4.0 .0}$ mode.

These modifications on the resonance frequency for variations in the permittivity of the PLZT material highlight the high sensitivity of this type of device. This sensitivity represents that of the electromagnetic transducer, which transforms a variation in permittivity into a variation in the resonance frequency of a WGM. In order to evaluate this sensitivity, we have shown in Figure 12(a) and (b) the resonance frequencies of the $\mathrm{WGH}_{7.0 .0}$ and $\mathrm{WGE}_{9.0 .0}$ modes, respectively, as a function of the permittivity of the PLZT when there is a temperature variation. The results obtained with a cylindrical dielectric resonator have shown that the resonance frequencies of the gallery modes are very sensitive to the change in the permittivity of the sensitive material. The relationship between permittivity and resonance frequencies is approximately linear.

The sensor sensitivity is the combination of the transducer sensitivity with the variation in PLZT permittivity as a function of temperature. 


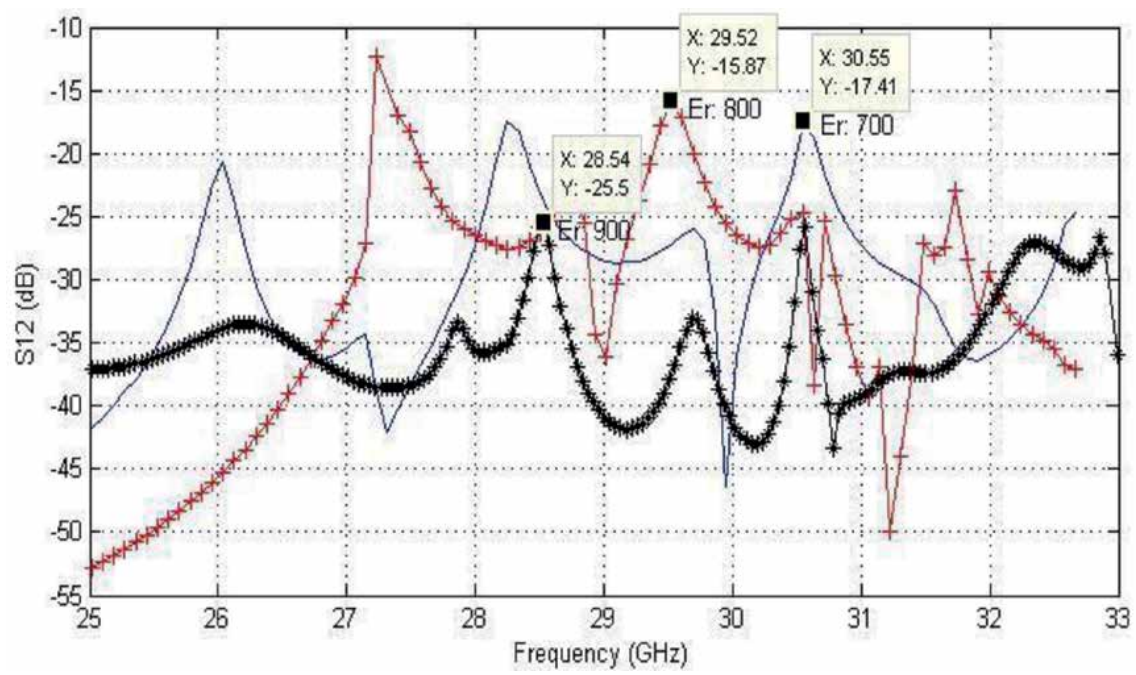

Figure 11.

Transmission coefficient $\left(S_{12}\right)$ as a function of frequency for variations in PLZT permittivity $\left(\varepsilon_{r}=700,800\right.$, and 900).

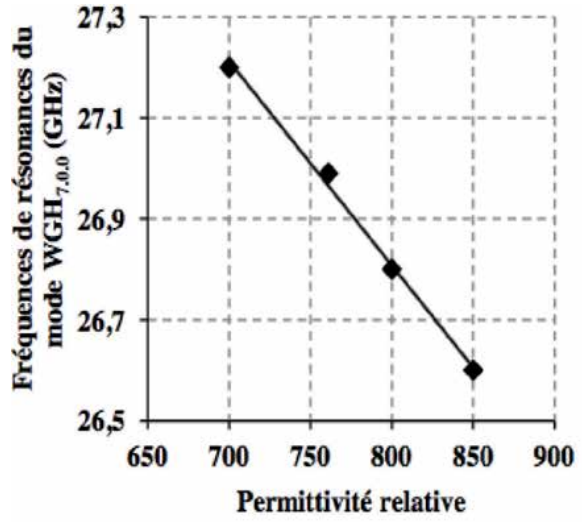

(a)

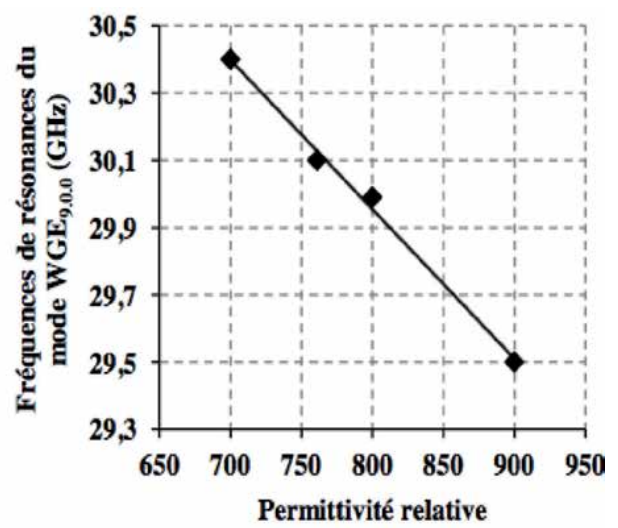

(b)

Figure 12.

Resonance frequencies as a function of PLZT permittivity: (a) $W G H_{7.0 .0}$ mode and (b) WGE $E_{9.0 .0}$ modes.

The gallery modes in the dielectric resonator and the PLZT material are evidently used here to measure the permittivity change and deduce the temperature change. From rigorous electromagnetic numerical simulations $\left(\mathrm{HFSS}^{\mathrm{TM}}\right)$, we show here that a small change in the permittivity of the sensitive material induces a large variation in the resonance frequency of the dielectric resonator gallery modes.

Based on the characteristics of the sensitive material used and the previous results, it is therefore possible to deduce the relationship between the resonance frequency of the detected gallery modes and the temperature. As a result, a linear dependence between frequency and temperature is clearly observed for all modes detected.

In other words, the relatively linear dependence between temperature and dielectric constant of the sensitive material leads to a change in the coupling coefficient between the two coplanar waveguides, and this is subsequently reflected in a shift in the resonance frequency of the system. As a result, a temperature variation usually results in a shift in the resonance frequency of the excited mode in the dielectric resonator. 


\section{Application of the sensor for marine fire detection}

In terms of application areas, sensor networks have a potential that is revolving around many sectors of our economy and our daily lives; from environmental monitoring and preservation to industrial manufacturing, automation in the transport and health sectors, and the modernization of medicine, agriculture, telematics, and logistics. This new technology promises to revolutionize the way we live, work, and interact with the physical environment around us. Wirelessly communicating sensors with computing capabilities facilitate a series of applications that were impossible or too expensive a few years ago. Today, these tiny and inexpensive devices can be literally scattered over roads, structures, walls, or machines, capable of detecting a variety of physical phenomena. Many fields of application are then considered, such as disaster detection and monitoring, environmental monitoring and biodiversity mapping, intelligent buildings, precision agriculture, machine monitoring and preventive maintenance, medicine and health, logistics, and intelligent transport.

Today, the use of these sensors is increasingly required for supervision and safety. Industrial companies then propose wireless sensors that can inform the user about the evolution of different physical quantities, so they constitute a very fertile research axis. In addition, the development of temperature sensors has several advantages, the most important of which is safety. The current trend, given the new applications that are emerging, is to oversize sensors and make them compatible with signal processing systems in order to obtain fully integrated systems. Environmental objectives and firefighting are the most targeted applications today. The lists of applications (safety, control, analysis, comfort, etc.) and fields of application (environment, safety, medical, automotive, home automation, etc.) are very long, reflecting the great interest in the development of temperature sensors.

The development of these systems generally includes a miniature, low-cost, and high-performance sensor. This is what drives our current research. Indeed, miniaturization is important to be able to easily embed autonomous systems that are increasingly distributed in networks. The cost price is of course an important factor and will be decisive for the marketing development of these sensors. The quest for performance is to make the information obtained by these sensors more reliable and affordable. This is practically interesting for the intended application: improving firefighting in marine applications.

Obviously, of all the disasters that can happen to a ship, fire is certainly the most horrible. Ashore, occupants of burning buildings can rely on fire pumps and ladders that can be on site within minutes of the first alarm signal. A ship at sea, on the other hand, must rely solely on itself in fighting the fire as in everything else, and from the first fire signal to retirement or hard-won victory, there may be no chance of a canoe rescue in bad weather.

The consequences of a fire on board a ship are always costly and sometimes tragic. It is therefore essential to have effective firefighting systems, but it is now known that conventional temperature sensors, which were widely used in the past, have significant disadvantages. The present system aims to solve this problem by using simple, autonomous, and inexpensive means. As a result, this design greatly reduces the overall energy consumption of the temperature sensor network on board ships.

To effectively control ship temperature, the use of this passive temperature sensor makes it possible to keep an eye on the temperature at all times, for long periods of time, and to alert staff in the event of a problem. If the temperature in a monitoring zone suddenly exceeds the threshold value, the sensor detects it in real time and transmits the information to the supervisor for intervention. This avoids 
the risk that the fire will remain ignored for a long time and therefore take on such a magnitude that any action to fight it will be too late and therefore futile.

This marine fire protection system therefore makes it possible to detect any fire risk in time, before or quickly after it is triggered, and to manage alarms in real time throughout the journey. Clearly, the deployment of such a network can provide an alarm system to detect intrusions, and it has a great advantage for long-term use on board ships without the need to charge or change the battery.

\section{Conclusion}

The results presented in this chapter are very encouraging: the simulation of a passive temperature sensor based on an electromagnetic transduction gave very good performances. These simulation results obtained, using previous work, allow us to consider the realization of a new high-performance temperature sensor. The optimization of this type of device was done using global electromagnetic simulations on HFSSTM including all system elements. The combination of PLZT material, dielectric resonator, and coplanar lines makes it possible to produce a narrowband filter that we excite with an electromagnetic wave at microwaves in gallery modes.

The key point of our application is based on the performance of the perovskite material and the properties of the RD gallery modes. Indeed, the dielectric resonator, covered with the sensitive material, is excited in a gallery mode that allows its oversizing with millimeter waves and its association with transmission lines in order to have a band-pass directional filter. Thus, we designed the entire 3D device on $\mathrm{HFSS}^{\mathrm{TM}}$. This software has the advantage of being rigorous and allows, a priori, taking into account all the physical and geometric characteristics of the device.

In the second part of this chapter, we have presented some of the results obtained. Several gallery modes have been observed over a wide frequency band; the Ka-band. We have therefore shown through these simulations that the measurement of the resonance frequency of a gallery mode in the dielectric resonator translates, in principle, a temperature variation with a remarkable sensitivity.

The implementation of such a device, which offers passive temperature detection, makes it possible to consider the design of a temperature sensor with high sensitivity electromagnetic transduction. The characterization of such a sensor makes it possible to evaluate the frequency in terms of geometric dispersions such as the influence of material thicknesses, and diameters will be presented in the next chapter.

Finally, our contribution provides innovative technology to meet the need to improve fire protection in maritime transport. Temperature control and traceability still require manual procedures dependent on onboard energy. The proposed system simplifies and automates relatively all these manual interventions and particularly does not require any onboard power supply. Thereafter, this firefighting device has a sensitivity of about $10 \mathrm{MHz} /{ }^{\circ} \mathrm{C}$ and allows the temperature to be controlled at any time and to react quickly in the event of a problem. 


\section{Author details}

Asma Bakkali1 ${ }^{1,2}$, José Pelegri-Sebastia ${ }^{2 *}$, Youssef Laghmich ${ }^{3}$ and Abdelouahid Lyhyaoui ${ }^{1}$

1 Laboratory of Innovative Technologies, Abdelmalek Essaadi University, Tangier, Morocco

2 Research Institute for Integrated Management of Coastal Areas, Universitat Politècnica de Valencia, Valencia, Spain

3 Polydisplinary Faculty, Hassan 1st University, Khouribga, Morocco

*Address all correspondence to: jpelegri@eln.upv.es

\section{IntechOpen}

(C) 2020 The Author(s). Licensee IntechOpen. This chapter is distributed under the terms of the Creative Commons Attribution License (http://creativecommons.org/licenses/ by/3.0), which permits unrestricted use, distribution, and reproduction in any medium, provided the original work is properly cited. (cc) BY 


\section{References}

[1] Peng H. Study of whispering gallery modes in double disk sapphire resonators. IEEE Transactions on Microwave Theory and Techniques. 1996;44:848-853

[2] Boriskina VS, Nosich IA. Radiation and absorption losses of the whisperinggallery-mode dielectric resonators excited by a dielectric waveguide. IEEE Transactions on Microwave Theory and Techniques. 1999;47:223-231

[3] Mouneyrac D. Utilisation des modes de galerie pour la caractérisation des semi-conducteurs et la réalisation d'un oscillateur ultra faible bruit de phase. Thèse de doctorat de l'Université de Limoges; 2011

[4] Guillon B. Conception et réalisation de circuits millimétriques micro-usines sur silicium: Application à la réalisation d'un oscillateur à résonateur diélectrique en bande Ka. Thèse de doctorat de l'Université Paul Sabatier de Toulouse; 1999

[5] Guillon B, Cros D, Pons P, Cazaux JL, Lalaurie JC, Plana R, et al. Ka band micromachined dielectric resonator oscillator. IEEE Electronics Letters. 1999;35(11):909-910

[6] Guillon B, Grenier K, Pons P, Cazaux JL, Lalaurie JC, Cros D, et al. Silicon micromachining for millimeterwave applications. Journal of Vacuum Science \& Technology, A: Vacuum, Surfaces, and Films. 2000;18(2):743-745

[7] Krupka J, Derzakowski K, Abramowicz A, Tobar ME, Geyer RG. Use of whispering-gallery modes for complex permittivity determinations of ultra-low-loss dielectric materials. IEEE Transactions on Microwave Theory and Techniques. 1999;47(6):752-759

[8] Wawrzała P, Korzekwa J. PLZT type ceramics as a material for applications in power pulse capacitors. IEEE Proceedings. 2012;10(1109):1-4
[9] Kuscer D, Korzekwa J, Kosec M, Skulski R. A- and B-compensated PLZT $\mathrm{x} / 90 / 10$ : Sintering and microstructural analysis. Journal of the European Ceramic Society. 2007;27:4499-4507

[10] Lee FY, Jo HR, Lynch CS, Pilon L. Pyroelectric energy conversion using PLZT ceramics and the ferroelectricergodic relaxor phase transition. Smart Materials and Structures. 2013;22:1-16

[11] Kozielski L, Plonska M, Bucko MM. Electrical and mechanical examination of PLZT/PZT graded structure for photovoltaic driven piezoelectric transformers. Materials Science Forum. 2010;636-637:396-373

[12] Trivijitkasem S, Koyvanich K. Characterization of lead lanthanum zirconate titanate (PLZT) ceramics sintered at various temperatures. Kasetsart Journal (Natural Science). 2007;41:192-197

[13] Jatlaoui MM. Capteurs passifs a transduction électromagnétique pour la mesure sans fil de la pression. Thèse de doctorat de l'INPT de Toulouse; 2009

[14] Saadaoui M. Optimisation des circuits passifs micro-ondes suspendus sur membrane diélectrique. Thèse de doctorat de l'Université Paul Sabatier de Toulouse; 2005

[15] Hallil H. Conception et réalisation d'un nouveau capteur de gaz passif communicant à transduction RF. Thèse de doctorat de l'Université Paul Sabatier de Toulouse; 2010

[16] LacommeP,HardangeJP, MarchaisJC, Normant E. Radar applications and roles. Air and Spaceborne Radar Systems. 2001;PM108:347-369

[17] Chebila F. Lecteur Radar pour Capteurs Passifs à Transduction Radio Fréquence. Thèse de doctorat de l'INPT de Toulouse; 2011 

Section 4

\section{Piezoelectric Effect of Perovskites}





\title{
Effect of Piezoelectric Filed on the Optical Properties of (311) A and (311) B Oriented InAlAs/InP Heterostructures
}

\author{
Badreddine Smiri, Faouzi Saidi and Hassen Maaref
}

\begin{abstract}
InAlAs alloy was grown by MOCVD on an InP (311) substrate with different polarities. Measurements of photoluminescence (PL) and photoreflectance (PR) were performed to study the impact of the V/III flux ratio. It is discovered that the PL line was shifted to a greater energy side with the increasing excitation power density, and no saturation was observed of its related PL intensity. It is a fingerprint of type II transition emission. However, the recombination of the type II interface showed a powerful dependence on $\mathrm{AsH} 3$ overpressure and substrate polarity. In fact, we have noted an opposite behavior of type II energy transition shift from A to B polarity substrate in respect to V/III ratio variation. PR signals corresponding to Franz-Keldysh Oscillation (FKO) were observed. The analysis of their period has allowed one to assess the value of the PZ field in the samples. PL-luminescence measurements were performed out as a function of temperature. PL peak energy, PL intensity, and half maximum full width show anomalous behaviors. Indicating the existence of localized carriers, they were ascribed to the energy potential modulation associated with the indium cluster formation and PZ field.
\end{abstract}

Keywords: V/III flux ratio, substrate polarity, piezoelectric field, Franz-Keldysh oscillation, photoreflectance, photoluminescence

\section{Introduction}

Recently, scientists have focused their interest in InAlAs/InP grown on nonconventional (n11) planes. For example, (311) A and (311) B are not acquired as compared to the used (001) surface due to their remarkable characteristics [1]. In addition, InAlAs semiconductor layers grown on (311) A/B-oriented InP substrates give several unique characteristics compared to those grown on $\operatorname{InP}(100)$. Indeed, in (311) plane, the strain and hydrostatic deformations are discovered to be improved compared to those on (100) plane [1-3]. The primary reasons for this are: (i) the presence of a built-in electric field, produced through the piezoelectric effect in the layer $[1,4,5]$ and (ii) the difference in arsenic segregation at the inverse interface. It is expected that these factors will be heavily dependent on growth conditions such as substrate orientation, V/III ratio.

InAlAs-InP materials have attracted tremendous interest over the past decades due to a variety of potential applications such as optical, optoelectronic and 
electronic devices $[6,7]$ due to its large direct band-gap energy, high electron mobility and the type II nature of the interface [8]. These advancement efforts were appointed by the fabrication and commercialization of a variety of devices such as Quantum cascade lasers (QCLs) [9, 10], Avalanche Photodiodes (APDs) [11] and high-electron-mobility transistor (HEMT) [12-14]. The heterostructures of $\mathrm{In}_{\mathrm{x}} \mathrm{Al}_{1-\mathrm{x}} \mathrm{As} / \mathrm{InP}$ have a type II transition $[1,15]$ which becomes a promising contender for the optical telecommunication light source. Different techniques such as Molecular Beam Epitaxy (MBE) and Metal-Organic Chemical Deposition (MOCVD) have developed this type of structure. However, the InAlAs material itself suffered from a large density of hetero-epitaxy-inherent defects [1, 6]: I Al content, (ii) phase separation, and (iii) InAlAs growth spinodal decomposition. Despite all this, the full potential of devices based on InAlAs/InP has still been obtained.

These issues are expected to be highly dependent on growth conditions (substrate polarity, V/III ratio, etc.) due to the large difference in bond energy between Al-As and In-As [15]. To date, most study work on the optical and electrical properties of InAlAs was performed on the conventional (100) planes, but little is known for the non-conventional (n11) planes. Different substrate orientations show various surface states, which are expected to influence the growth mode and even the optical and electrical properties of epilayers.

The existence of aluminum in the InAlAs layer, therefore, prompts the existence of the In- and Al-rich clusters, which is the consequence of the non-uniformity in the alloy composition. As a result, it contributes to the undulation of the InAlAs bandgap from which the localized energy level is present. In addition, the substrate polarity [A or B] in our nanostructures alters the containment of electrons-holes by changing the strain and the existence of piezoelectric (PZ) field within the structure. Similar heterostructures such as In0.21Ga0.79As/GaAs (311) A MQWs [16] and heterostructures AlGaAs/GaAs grown on (100), (311) A and (311) B-oriented substrates [17] have seen the carrier localization phenomenon. The previous investigation will be constrained to those samples implanted at high index $(11 \mathrm{~N})$. We have shown in our latest study [18] that the presence of localized carriers has been attributed to the energy potential modulation related to the existence of Indium clusters and PZ-field.

The aim of our chapter is to study the effect of PZ-field on the optical properties of InAlAs/InP (311) with different substrate polarity, elaborated by MOCVD. The research of their optical characteristics by PR and PL spectroscopy is a significant step to demonstrate the possibility of incorporating our structure into optoelectronic applications such as $1.55 \mu \mathrm{m}$ devices.

\section{Experimental details}

The studies are conducted on InP/InAlAs/InP (311) double heterostructures, marked as S1, S1', S1", S2, S2' and S2", which are cultivated at different V/III ratios by low-pressure metal-organic chemical vapor deposition (MOCVD). More information about development is summarized in Table 1. The source materials for the growth process are trimethylindium (TMIn), trimethylaluminum (TMAl) and (AsH3). At a substrate temperature of $600^{\circ} \mathrm{C}$, an InP layer of $100 \mathrm{~nm}$ thickness was developed. The growth rate of $\mathrm{InP}$ is approximately $0.17 \mathrm{~nm} / \mathrm{s}$. A $270 \mathrm{~nm}$ thick layer of $\operatorname{In}_{\mathrm{x}} \mathrm{Al}_{1-\mathrm{x}} \mathrm{As}$ was subsequently deposed. Each sample was finally capped with an InP layer of $10 \mathrm{~nm}$.

The source of excitation is the $514.5 \mathrm{~nm}$ line of the continuous-wave $\mathrm{Ar}^{+}$laser with an excitation density of $80 \mathrm{~W} / \mathrm{cm}^{2}$ in PL measurements. Spectral analysis of 
Effect of Piezoelectric Filed on the Optical Properties of (311) A and (311) B Oriented... DOI: http://dx.doi.org/10.5772/intechopen.89441

\begin{tabular}{lcc}
\hline Samples & Substrate orientation & V/III ratio molar \\
\hline S1 & (311) B & 25 \\
\hline$S 1^{\prime}$ & $(311) \mathrm{B}$ & 50 \\
\hline$S 1^{\prime \prime}$ & $(311) \mathrm{B}$ & 125 \\
\hline $\mathrm{S} 2$ & $(311) \mathrm{A}$ & 25 \\
\hline $\mathrm{S} 2^{\prime}$ & $(311) \mathrm{A}$ & 50 \\
\hline $\mathrm{S} 2^{\prime \prime}$ & $(311) \mathrm{B}$ & 125 \\
\hline
\end{tabular}

Table 1.

Growth conditions of the samples $S_{1}, S_{1}{ }^{\prime}, S_{1}{ }^{\prime \prime}, S_{2}, S_{2}{ }^{\prime}$ and $S_{2}{ }^{\prime \prime}$.

the luminescence measurements was performed out using JOBIN YVON HRD1 monochromatic and identified by a cooled Ge diode detector with a built-in amplifier. PR measurements were performed using a standard setup with the $514.5 \mathrm{~nm}$ line of $\mathrm{Ar}^{+}$laser as the pump light, which was mechanically chopped at $970 \mathrm{~Hz}$. The probe light was acquired from a $250 \mathrm{~W}$ tungsten halogen lamp dispersed with a 275 $\mathrm{mm}$ focal length monochromatic. The reflectance signal is detected by an InGaAs and silicon photodiodes.

\section{Results and discussions}

\subsection{Photoluminescence study}

\subsubsection{Effect of substrate polarity: PZ-field}

PL spectra were registered at low temperature to confirm the effect of PZ-field on the optical properties of InAlAs/InP (311) A/B:

Figure 1 illustrates the 10K-PL spectra of InAlAs/InP samples grown on (311) B and (311) A, respectively denoted S1 and S2. A higher energy side, both emissions at around 1.13 and $1.23 \mathrm{eV}$, for the samples $\mathrm{S} 1$ and $\mathrm{S} 2$, can be related to the interfacial defects between the InAlAs and InP layers $[1,19]$. Both PL bands occur at about 0.8 and $1.03 \mathrm{eV}$ for S1 and S2, respectively, on the lower energy side. A gradual InAs $\mathrm{s}_{\mathrm{x}} \mathrm{P}_{1-\mathrm{x}}$ layer formation at the interface between InP and InAlAs (see Figure 2) was explained by Hallara et al. [6]. For sample S1, an emission situated at around $1.27 \mathrm{eV}$ may be related to acceptor-band recombination [1].

To analyze the origin of the inverted interface (InP/InAlAs), we suggested a model based on arsenic segregation (some atomic monolayers) and linked the theoretical calculations with the experimental results.

We can conclude that the radiative recombination around the inverse interface with emission $1.03 \mathrm{eV}$ for the polarity $\mathrm{A}$ is due to the appearance of a gradual layer for $3 \mathrm{ML}$ of $\operatorname{InAs} \mathrm{s}_{\mathrm{x}} \mathrm{P}_{1-\mathrm{x}}$. In this case, the arsenic content of $\mathrm{xAs}$ is about $40 \%$, but in the inverted transition for polarity $\mathrm{B}$, it is in the order of $70 \%$. It is possible to estimate the band offset between InP and In0.513Al0.487As layers based on this reference [20]. In fact, the interface between $\operatorname{InP}$ and $\operatorname{In} A s_{x} P_{1-x}$ layers is type I, although it is type II for In0.513Al0.487As and $\operatorname{In} A s_{x} P_{1-x}$, where the $x A s$ content ranged from 0 to 0.78 .

There are two Gaussian peaks in the PL spectrum (see Figure 1). For the type II transition, an asymmetric band tail appeared in both S1 and S2 samples, resulting from unintentional thin strained InAs layers created at the InAlAs-InP interface $[1,18]$. To explain more, this layer's smaller band gap can conduct a quantum well 


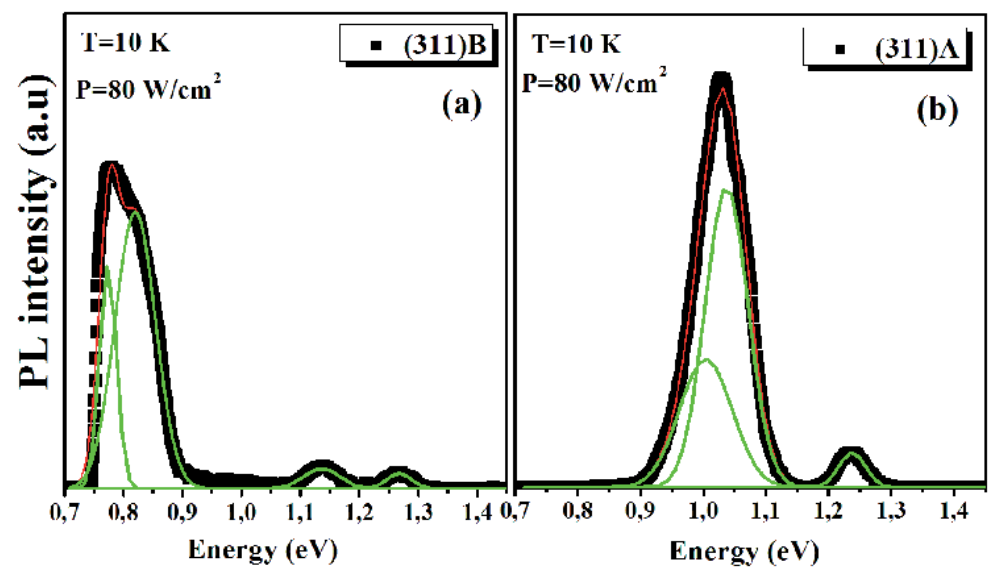

Figure 1.

(a) and (b) Low temperature PL spectra of InAlAs on InP (311) A and on InP (311) B, respectively. The green solid line is the Gaussian-fitting curve.

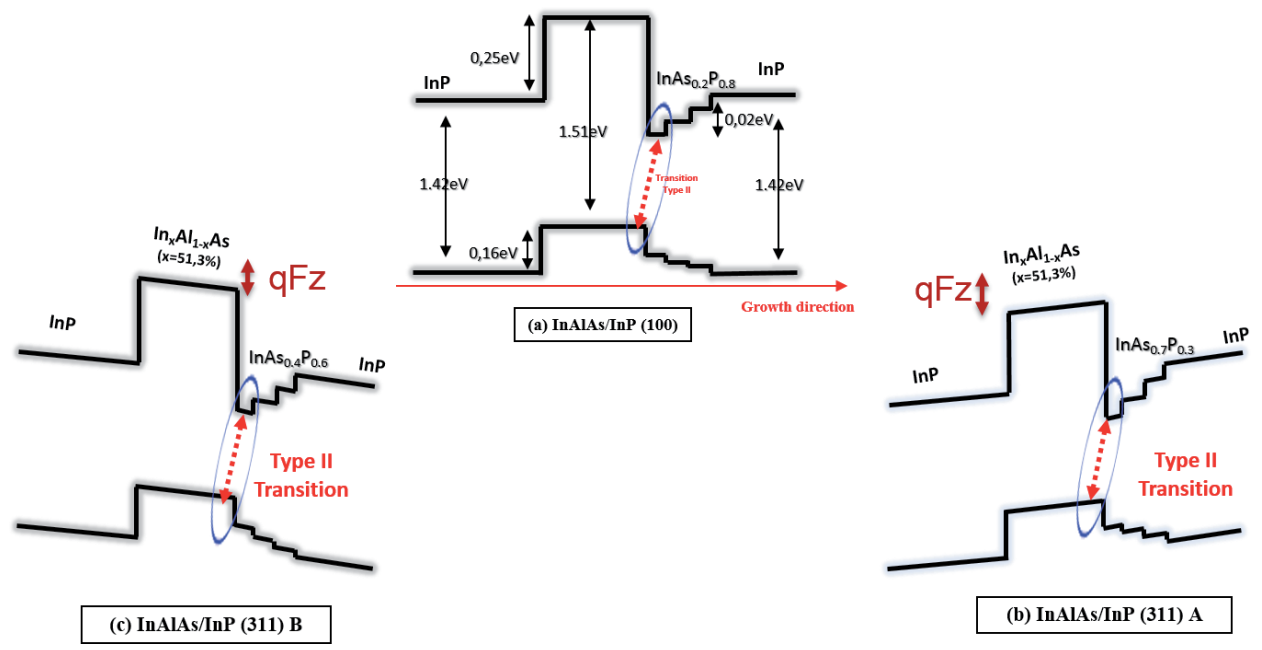

Figure 2.

(a) Schematic band diagrams for sample of InAlAs on InP (100), (b) on InP (311) A, and (c) on $\operatorname{InP}(311) B$.

at the interface, resulting in a mixed type I-II transition [21]. We notice that the difference in energy between the two transitions type II corresponds to the difference in arsenic atoms surface segregation. Thus, depending on the growth axis, this energy shift can be attributed to the piezoelectric field $[1,5]$. In addition, the existence of defect states may function as non-radiative centers resulting in a decrease in PL intensity. We should note that as mentioned at the beginning of this chapter, these defects originated from the development process as well as doping. Added to this, the sample cultivated on the (311) B surface has a greater residual impurity concentration than the samples cultivated on the (311) A surface [17]. For the (311) A sample, therefore, the interface quality is considered better than that for the (311) B sample.

\subsubsection{Effect of V/III flux ratio}

To verify the impact of V/III ratio on the optical properties of InAlAs/InP (311) $\mathrm{A} / \mathrm{B}, \mathrm{PL}$ spectra were recorded at low temperature. 
Effect of Piezoelectric Filed on the Optical Properties of (311) A and (311) B Oriented... DOI: http://dx.doi.org/10.5772/intechopen.89441

Figure 3 shows PL spectra at $10 \mathrm{~K}$ of the studied samples on different oriented InP substrate and at various V/III molar ratio. PL spectra are normalized and deconvoluted into various Gaussian curves for the convenience of comparison and to

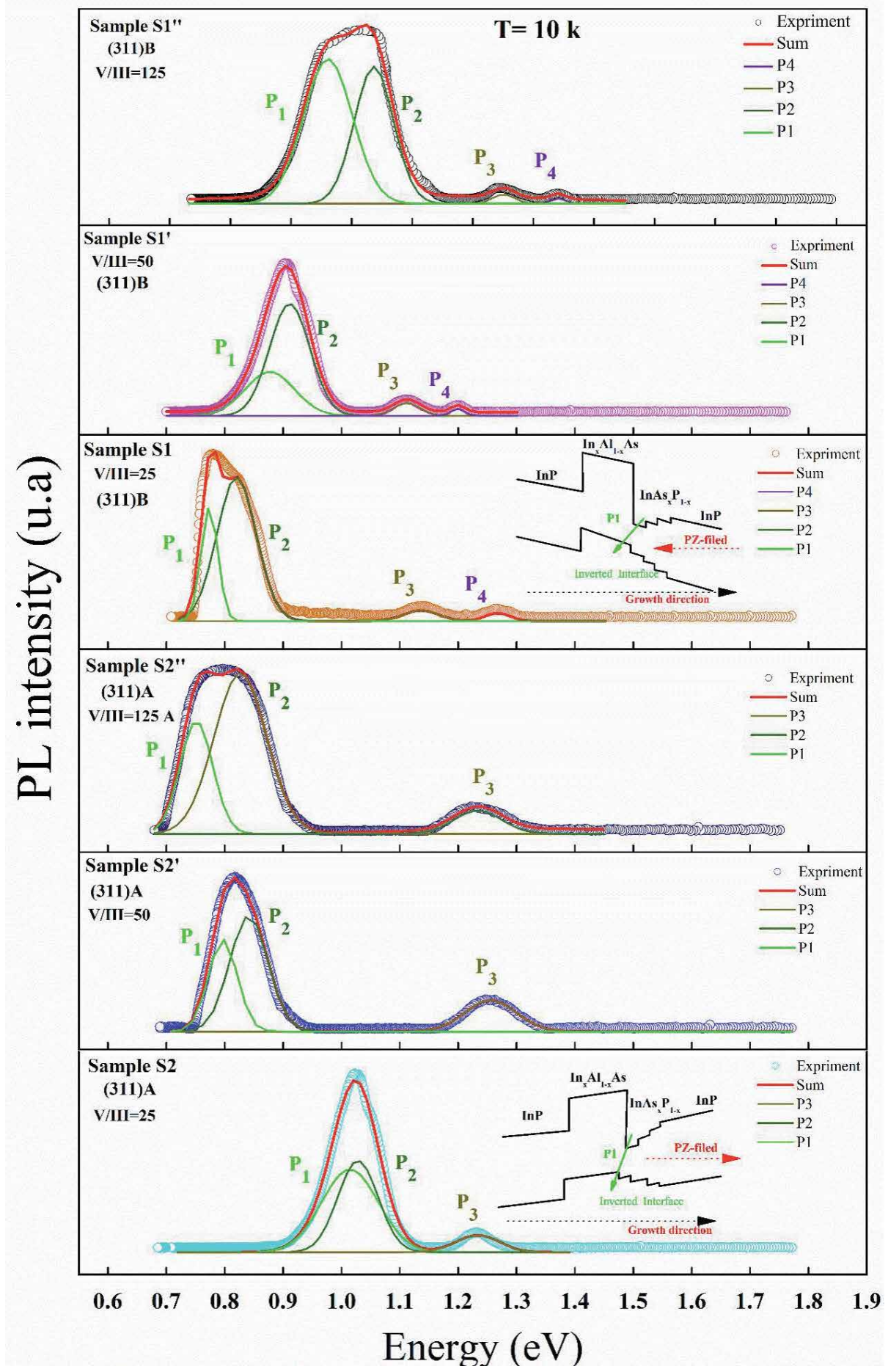

Figure 3.

Low-temperature (10 K) PL for samples $S_{1}, S_{1}{ }^{\prime}, S_{1}{ }^{\prime \prime}, S_{2}, S_{2}{ }^{\prime}$ and $S_{2}{ }^{\prime \prime}$. In order to identify the different emission peaks, the spectra are normalized and deconvoluted by a multiple Gaussian curve fitting. Inset shows the schematic band diagram showing the radiative transitions from the type II transition $\left(P_{1}\right)$. 
identify the different emission peaks. The spectra show four significant emission peaks marked as $\mathrm{P} 1, \mathrm{P} 2, \mathrm{P} 3$ and $\mathrm{P} 4$ for the (311) B sample. The P1 position is attributed to the type II emission across the interface between the two-dimensional electron states in thin $\operatorname{InAs}_{\mathrm{x}} \mathrm{P}_{1-\mathrm{x}}$ graded layer at the inverted interface and holes located on top of the InAlAs valence band [1]. The intermediate layer of InAsP consists of the higher coefficient of incorporation of As compared to $P[1,5,18]$. Numerous investigations have shown that the type II emissions in InAlAs-InP heterostructures observed in the $0.8-1.25 \mathrm{eV}$ range $[1,20,22]$. This very broad variety conceals some general patterns that appear to be related to conditions of growth. Whereas, the peak P2 emission is associated with a mixed type I-II transition and else P3 peak can be related to the interfacial defects in InAlAs/InP emission [1]. Finally, the P4 peak situated in the PL spectrum at a greater energy side for (311) B samples can be ascribed to acceptor-band recombination [5]. See our first part above for more information.

On the other hand, for (311) A plane, the peak $\mathrm{P} 4$ of all samples is not observed in PL spectra, which may be attributed to the existence of composition modulation in the epitaxial layer of InAlAs with varying molar ratio and substrate polarity. Sayari et al. [23] performed a Raman research study of the impact of the V/III flux ratio in heterostructures of $\mathrm{InP} / \mathrm{InAlAs} / \mathrm{InP}$. The research demonstrates that the quantity of clustering is envisaged to depend on conditions of development such as substrate polarity, V/III ratio, etc.

Figure 4 exhibits the variation in the type II transition energy as a function of the V/III molar ratio for samples cultivated on (311) A and (311) B. For the (311) orientation, the peak position depends upon whether the substrate is In terminated (A-face) or P terminated (B-face). The variation of type II transition energy (P1) was acting differently with the V/III ratio, according to the substrate polarity. For the B-face (samples S1, S1 ${ }^{\prime}$ and $\mathrm{S} 1$ ), the $\mathrm{P} 1$ emission peak energy tends to increase as the V/III ratio decreases [5]. Whereas for the A-face (samples S2, S2' and S2'), we noted a decrease in the P1 emission peak as the V/III ratio increases (i.e. a blue shift is observed for (311) B samples [5], while red shift is noted for (311) A samples). These findings could possibly be clarified by the meaning of a piezoelectric field in InAlAs/InP heterostructures resulting from the difference between the atomic terminated surface $[A$ or $B]$ in InP substrate differences in interface reconstructions [1]. The PL shift can simply be attributed to the type of atoms present on the surface,

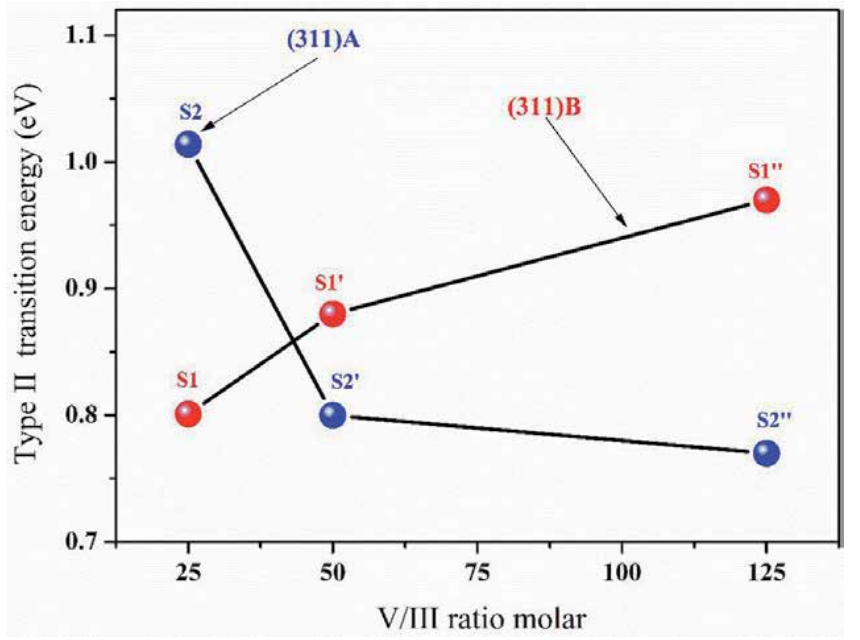

Figure 4.

Variation of the type-II transition energy as a function of V/III ratio for the (311) A and (311) B substrate orientation. 


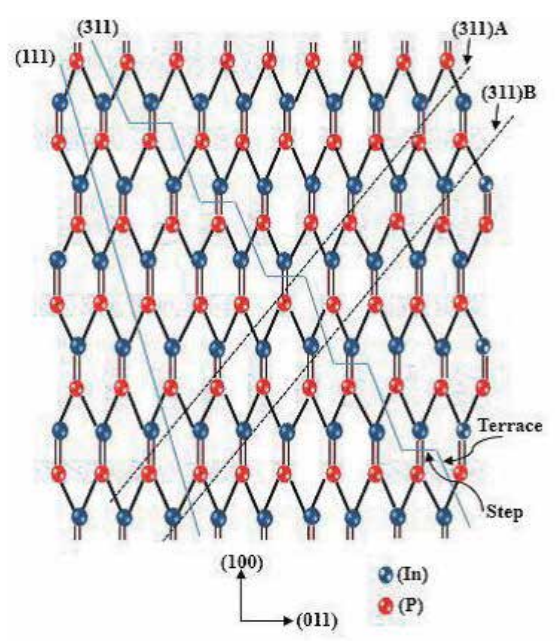

Figure 5.

Illustration of (311) A and (311) B planes of InP.

resulting in different levels of confinement. There are two types of sites on the surface (311): one is the double bond site found in the direction [100] and the other is a single bond site found in the direction [111], their densities being precisely the same [5]. On a (311) A surface, the double dangling bond sites are In sites and the single dangling bond sites are P sites, but on a (311) B surface the double dangling bond sites are $\mathrm{P}$ sites and the single dangling bond sites are In sites (see Figure 5) $[19,24,25]$. These completely distinct configurations of plane bonding are eventually responsible for the meaning of the PZ field on the different planes. Furthermore, the study of the impacts of InP substrate polarity shows that As incorporation (arsenic diffusion) may be improved on (311) A samples but may be decreased on (311) B samples with an increased V/III ratio. It can be shown from Ref. [20] that the increase in arsenic composition (InAsP) will reduce the transition energy of type II.

\subsubsection{Power-dependent photoluminescence (PDPL)}

We studied the evolution of the PL peak energy as PDPL, as shown in Figure 6, to verify these assignments. The energy blue changes with increased excitation

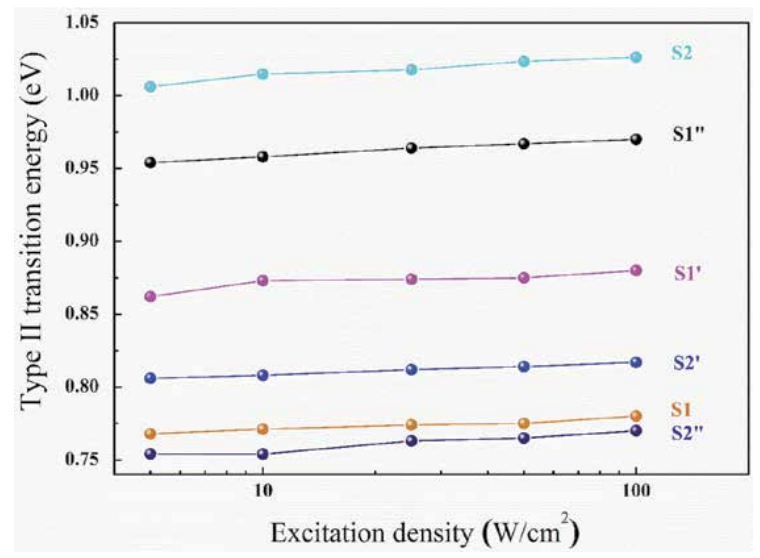

Figure 6.

The PL emission energy for the type II transition of all samples as a function of excitation power densities. 
power intensity for type transition. This conduct is associated with recombination of type II via an interface with other material structures such as GaAsSb/InP [26, 27] and $\mathrm{GaSb} / \mathrm{InGaAs}$ [28].

The following Eq. (1) was used to estimate the nature of the recombination around the InP/InAlAs inverse interface [1]:

$$
\mathrm{I}_{\mathrm{PL}}=\left(\mathrm{P}_{\mathrm{exc}}\right)^{\mathrm{n}}
$$

where $\mathrm{P}_{\mathrm{exc}}$ is the power excitation, IPL is the integrated PL intensity and $\mathrm{n}$ is an exponent.

We found that the exponent $\mathrm{n}$ close to the unity. At higher power of excitation, there is no saturation. This shows that this PL transition is not attributable to impurity or defects, but is intrinsic recombination (band-to-band) [1]. Figure 6 indicates a logarithmic linear dependence of the PL peak energy of the inverse interface with the density of excitation power. Hallara et al. [6] noted this behavior. Additionally, Figure 6 display that the blue-shifted with the increase of the excitation power density. The offset is approximately $7 \mathrm{meV}$. This shows that the change of emission energy with increasing excitation power is also proof to verify our hypothesis (carrier localization) and PZ-filed presence in our structures [18].

A final analysis based on the PL temperature was created to further verify our hypothesis. In both samples, we can gain greater insight into the carrier localization and the mechanism of luminescence.

\subsubsection{Temperature-dependent photoluminescence (TDPL)}

Scientific study shows that two factors are noted for conventional orientation (100), at low temperature $10 \mathrm{~K}$ and with aspect ratio V/III equivalent to 50, two factors are observed: (i) clusters formation and (ii) composition modulation leading to natural superlattice [6]. Some scientists discovered that the latter factor disappears and if the ratio V/III changes $[5,6,22]$, the clustering impact remains only.

It related to the phenomenon of exciton location. Carefully research as a function of PL temperature is conducted to demonstrate our hypothesis.

In both samples (S1 and S2) mentioned above, the PL peak energy-temperature reliance obtained from PL spectra is shown in Figure 7(a) and (b). The PL spectrum (inset Figure 7) is revealed between 10 and $300 \mathrm{~K}$. The so-called S-shaped temperature dependence of emission energy is obviously shown as a successive reform to low-high-low energy. It displays anomalous behavior as a temperature function. This behavior is characteristic of localization effects, and has been already observed in InAlAs alloys on InP [29, 30], GaAsSb/InP layers [31], BGaAs/GaAs layers [32, 33] and In0.21Ga0.79As MQWs on GaAs (311) A [16].

The S-shaped shape in PL peak energy can be separated into three primary intervals of temperature and is interpreted as follows: at the low-temperature range, the excitons should be situated in the levels whose distribution goes into the material's prohibited band. This is called band tails, associated with cluster appearance in the InAlAs [18]. With growing temperature, the excitons get enough thermal energy to reach deeper localized states and recombine primarily from low energy levels, resulting in a dramatic red shift (part I). Indeed, when the temperature increases, the thermal energy becomes adequate for the excitons in the tails to attain the corners of the stripes, where they are delocalized to high levels of energy. A blue shift is noted as a consequence (part II). All carriers are delocalized to the continuum at the high-temperature region, where band-band transitions are favored (part III) [18]. 


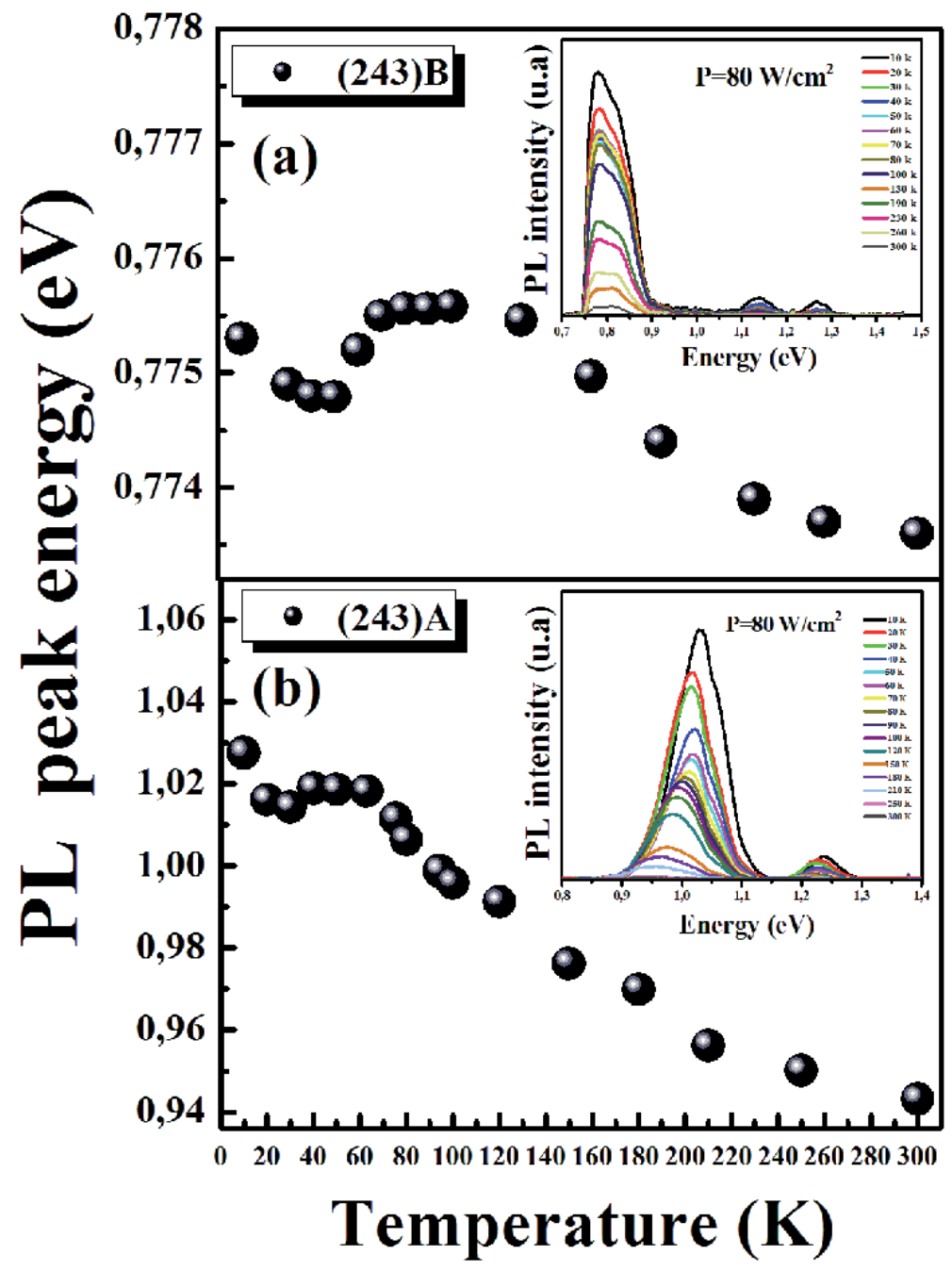

Figure 7.

(a) and (b) experiment data for the temperature dependence of Transition type II emission in Ino.513Alo.487As grown on InP (311) B and on InP (311) A substrates, respectively.

Another main parameter of the presence of the localization phenomenon is the Full width at Half Maximum (FWHM) behavior. Indeed, compared to the classic IIIV semiconductor alloys, it demonstrates atypical behavior. It shows an inverted "N-shape" (decrease-increase-decrease) (see Figure 8). The excitons are localized in the potential minima at cryogenic temperature. The carriers gain more thermal energy at intermediate temperatures to overcome the tiny energy barriers and attain greater energy states. As the temperature increases further, the FWHM broadening is described as the interaction of the electron-phonon. Finally, up to room temperature, the line width decreases continuously. It demonstrates the inverse trend of expanding optical phonons $[1,18]$. This behavior can be clarified by the thermo-activation of the carriers and their transfer between nearby fluctuation potentials induced by the inhomogeneous distribution. Indeed, the carriers that are being thermally activated into a small potential can be further retrapped by the large one [18].

As a function of temperature, the S-shape in PL peak energy is not clear in polarity $\mathrm{B}$ as polarity $\mathrm{A}$. The reason for this distinction can be clarified by 


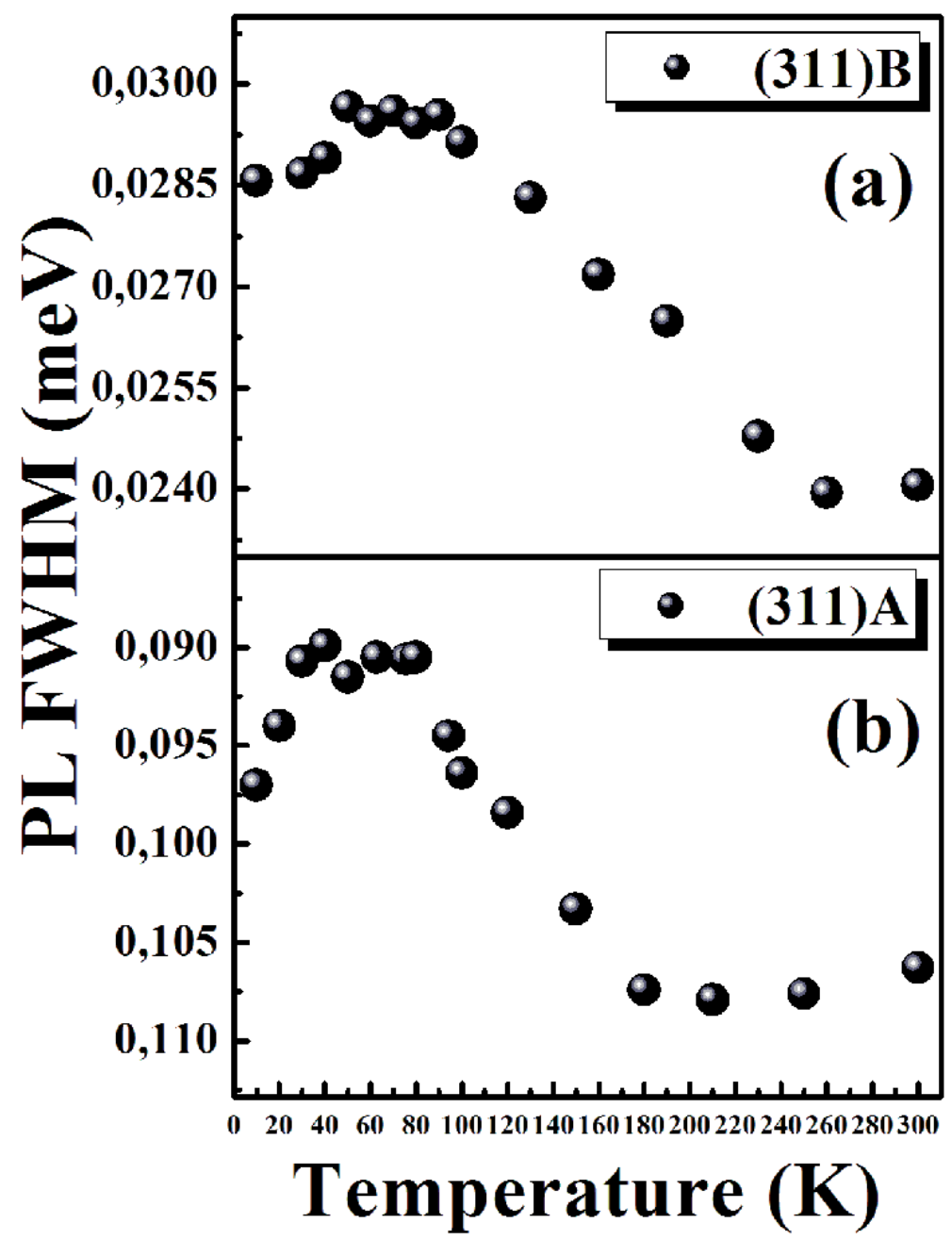

Figure 8.

(a) and (b) Evolution of the FWHM dependence of transition type II emission in Ino.513Alo.487As grown on InP (311) B and on InP (311) A substrates, respectively.

considering the polarity of the surface [In-rich (A) or P-rich (B)] from which the distinction of the PZ field within both structures $[1,18]$. In the growth of the high index plane (311), the PZ field and an internal field are along the same direction (polarity A) [18, 19]. In fact, carriers can be easily delocalized due to the strong phonon coupling carrier, which is an important channel for carrier transfer in-plane hopping effect [24]. In comparison, field direction is opposite in polarity B (see Figure 3) $[25,34]$. Due to the PZ field effect, the impact of localization is affected by interface undulation in this phase. Furthermore, the amount of alloy fluctuations in the composition of the material and the exchange of P-As.

\subsection{Photoreflectance study}

PR measurements were performed at room temperature to explore the evolution of the optical properties of the InAlAs/InP heterostructures during the development phase. The received PR spectra are described in Figure 9, with a distinct V/III ratio and substrate polarity. The PR spectra show a transition varying between 1.18 and $1.21 \mathrm{eV}$ for samples $\mathrm{S} 1, \mathrm{~S}^{\prime}, \mathrm{S} 2$ and $\mathrm{S} 2^{\prime}$. We suggest that this peak is related to the 


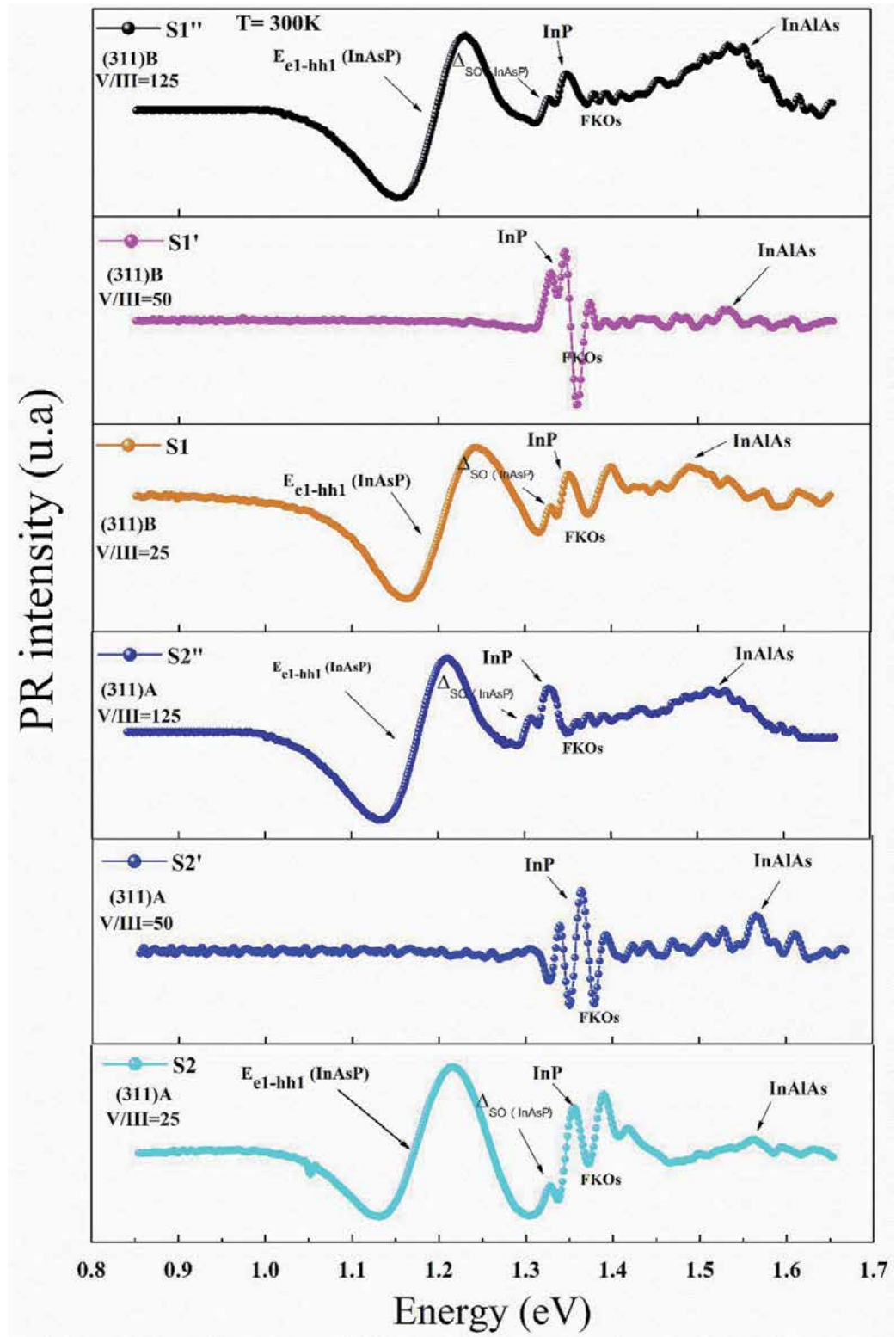

Figure 9.

Room-temperature PR spectra for InAlAs layers grown on (311) B (samples $S_{1}, S_{1}{ }^{\prime}$ and $\left.S_{1}{ }^{\prime \prime}\right)$ and (311) A (samples $S_{2}, S_{2}^{\prime}$ and $S_{2}{ }^{\prime \prime}$ ) InP substrates with different V/III ratio molar.

emission (1e-1h) between electron sub band and light-hole sub-band of quantum well (QW) InAsP [5]. While the spin split-off band of the InAsP layers lies at about $1.32 \mathrm{eV}$. On the other side, for the samples $\left(\mathrm{S}^{\prime}, \mathrm{S}^{\prime}\right)$, these two peaks totally disappear with a V/III ratio of 50 [5]. This result can be related during the growth to the uniformity of the inverse interface between InAlAs and InP. Furthermore, we noted that PR spectra of all samples show an additional above-band-gap ( $\left.E_{0} \mathrm{InP}\right)$ characteristics that are Franz-Keldysh oscillations (FKO), reflecting the presence of a builtin electric field in the InP substrates [5, 35]. Finally, it is possible to attribute the peak exposed between 1.5 and $1.56 \mathrm{eV}$ to the InAlAs band gap transition [19]. The origin of this very wide range is associated with the existence of the clustering phenomenon in the layer $\operatorname{In}_{x} \mathrm{Al}_{1-\mathrm{x}}$ As caused by the fluctuation of the indium composition [19]. 
In order to assess the electrical field quality of each InP substrate, the FKO period is evaluated using an asymptotic expression for the PR spectrum provided in $[36,37]$. In this strategy, InP FKO is indicated by:

$$
\frac{\Delta R}{R} \propto \frac{1}{E^{2}\left(E-E_{0}\right)} \exp \left[-2\left(E-E_{0}\right)^{1 / 2} \frac{\Gamma}{(\hbar \Omega)^{3 / 2}}\right] \cos \left[\frac{4}{3}\left(\frac{E-E_{0}}{\hbar \Omega}\right)^{3 / 2}+\phi\right]
$$

where $E_{0}$ is the transition energy, $\phi$ is an arbitrary phase factor, $\Gamma$ is the linewidth broadening.

According to the asymptotic Franz-Keldysh model [37], the energy of oscillation extrema is given by:

$$
E_{m}=(\hbar \Omega) H_{m}+E_{0}
$$

where $H_{m}=\left[\left(\frac{3 \Pi}{2}\right)(m-1 / 2)\right]^{2 / 3}$ and $m(m \geq 1)$ denotes the $m$ th FKO extremum.

$E_{0}$ is the band-gap energy $(\operatorname{InP})$ and $\hbar \Omega$ is the electro-optic energy expressed as takes after:

$$
\hbar \Omega=\left(\frac{q^{2} \hbar^{2} F i n t^{2}}{8 \mu}\right)^{1 / 3}
$$

Noting that $q$ is the electron charge, $\mu$ is the reduced inter-band effective mass in the electric field direction, $\hbar$ is the reduced Planck constant, and $F_{\text {int }}$ is the internal electric field.

Thus, by plotting $E_{m}$ as a function of $H_{m}$, from linear fitting, we can decide $\hbar \Omega$ and $E_{0}$ from the slope and the intersection with the ordinate at the origin, respectively. Recalling Eq. (4), $F_{\text {int }}$ can be calculated by:

$$
F_{\text {int }}=\sqrt{\frac{8 \mu(\hbar \Omega)^{3}}{q^{2} \hbar^{2}}}
$$

Plotted in Figure 10 is the intercept $E_{0}$ of the best linear adjustments of the variation of $E_{n}$ as a function of $H_{n}$ for all samples [5]. Then, $E_{0}$ was the intercept defined from the best linear adjustment of En ( $\mathrm{Hn})$ plot, whereas $\hbar \Omega$ is simply the slope of the linear fit. Table 2 shows the acquired values of transitions $E_{0}(\mathrm{InP})$ discovered by this fitting procedure and the FKOs analysis method.

From Eq. (3), a plot of $E_{m}$ vs $H_{m}$ is a straight line, and the electric field strength $F_{i n t}$ can be acquired from the plot slope. Figure 10 shows the graphs of $E_{m}$ vs $H_{m}$ for all samples with their corresponding linear fits. The residual strain in (311) A/Boriented samples will generate a PZ field contributing to the $F_{\text {int }}$ electrical field built-in InP. We assumed $F_{\text {int }}$ was the sum of the PZ and built-in electrical fields. So, with regard to built-in electrical fields, the PZ field component predominates. The integrated electric field $F_{\text {int }}$ is therefore equal to the PZ areas [5].

The values acquired from the above-explained FKO evaluation of the built-in electrical field strength are provided in Table 2. We discovered that when the orientation of the substrate changes, the $F_{\text {int }}$ is modified, which suggests $\mathrm{PZ}$ impacts in the samples. The estimated PZ field is of about 36.27, 20.53, 8.11, 25.44, and 32.86 $\mathrm{KV} / \mathrm{cm}$ for samples $\mathrm{S} 1, \mathrm{S1}^{\prime}, \mathrm{S} 1^{\prime \prime}, \mathrm{S} 2$, and $\mathrm{S2}{ }^{\prime}$, respectively. Our findings will show that the PZ field is very susceptible to growth conditions in the InAlAs/InP samples [5].

Figure 11 shows the dependency of the PZ field in the studied samples on the polarity of the InP substrate and the V/III ratio. It is obviously noted that the PZ 
Effect of Piezoelectric Filed on the Optical Properties of (311) A and (311) B Oriented... DOI: http://dx.doi.org/10.5772/intechopen.89441

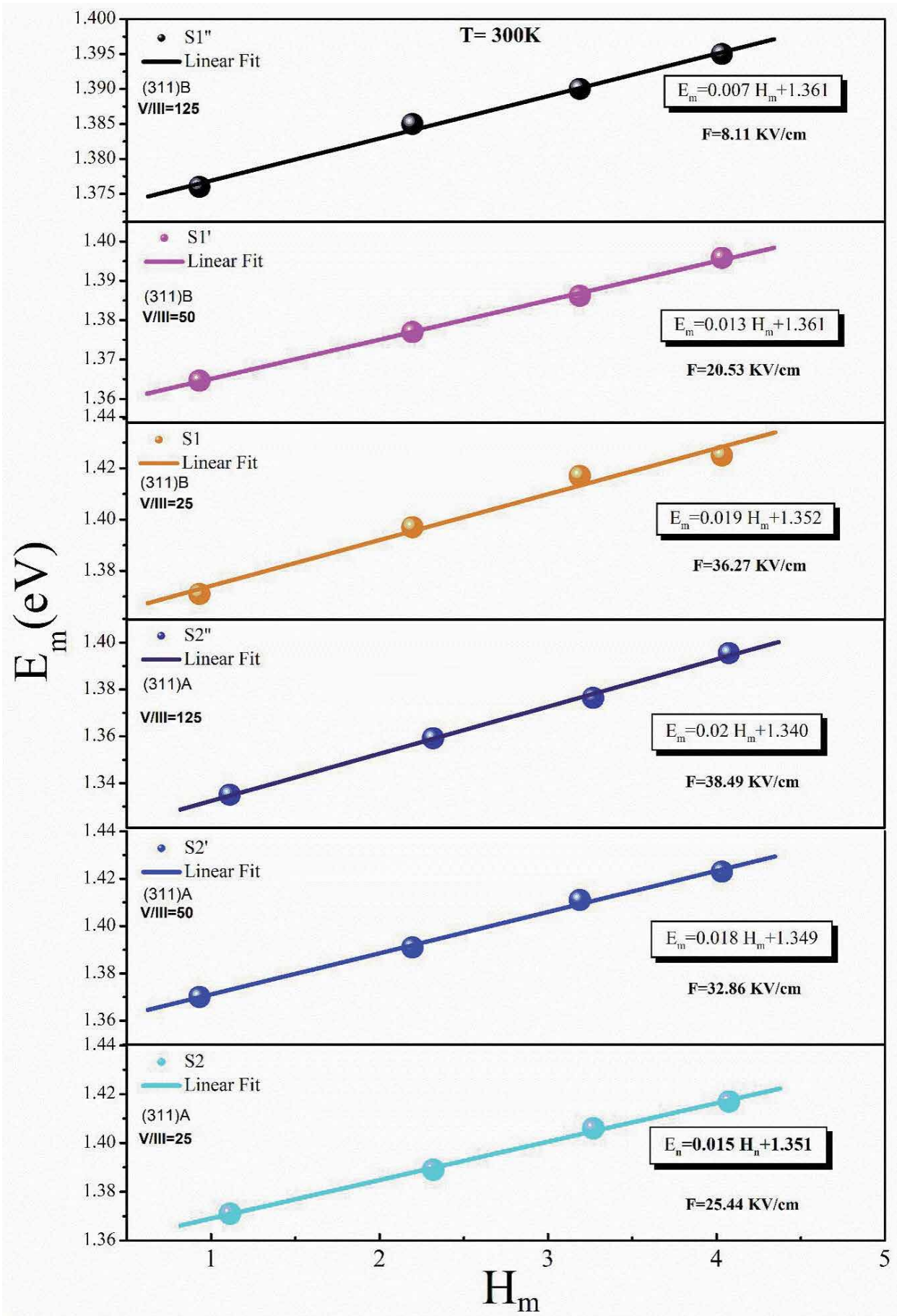

Figure 10.

FKO analysis for samples $S_{1}, S_{1}{ }^{\prime}, S_{1}{ }^{\prime \prime}, S 2, S_{2}{ }^{\prime}$, and $S_{2}{ }^{\prime \prime}$. Symbols are the energies of the FKO Extrema En as a function of $H_{n}=\left[\left(\frac{3 \Pi}{2}\right)(n-1 / 2)\right]^{2 / 3}$. Solid lines are the linear fit using $\mathrm{Eq}$. (2).

field drops with increasing the V/III ratio molar for the orientation (311) B [5]. The $\mathrm{S} 1$ sample indicates a comparatively powerful PZ field. As the V/III ratio increases, the PZ field decreases rapidly to reach $20.53 \mathrm{KV} / \mathrm{cm}$ for sample $\mathrm{S1}^{\prime}(\mathrm{V} / \mathrm{III}=50)$ and $8.11 \mathrm{KV} / \mathrm{cm}$ for sample S1" (V/III = 125). In comparison, for (311) A-surface, we 


\begin{tabular}{lccc}
\hline Samples & Substrate orientation & $\boldsymbol{E}_{\mathbf{0}}(\mathbf{I n P})(\mathbf{e V})$ & $\boldsymbol{F}_{\boldsymbol{i n t}}(\mathrm{KV} / \mathbf{c m})$ \\
\hline $\mathrm{S} 1$ & $(311) \mathrm{B}$ & 1.352 & 36.27 \\
\hline $\mathrm{S} 1^{\prime}$ & $(311) \mathrm{B}$ & 1.396 & 20.53 \\
\hline $\mathrm{S} 1^{\prime \prime}$ & $(311) \mathrm{B}$ & 1.361 & 8.11 \\
\hline $\mathrm{S} 2$ & $(311) \mathrm{A}$ & 1.351 & 25.44 \\
\hline $\mathrm{S} 2^{\prime}$ & $(311) \mathrm{A}$ & 1.349 & 32.86 \\
\hline $\mathrm{S} 2^{\prime \prime}$ & $(311) \mathrm{A}$ & 1.340 & 38.49 \\
\hline
\end{tabular}

Table 2.

Summary of energy gap (Eg) and the piezoelectric field (Fpz) values obtained from photoreflectance PR with different V/III ratio molar.

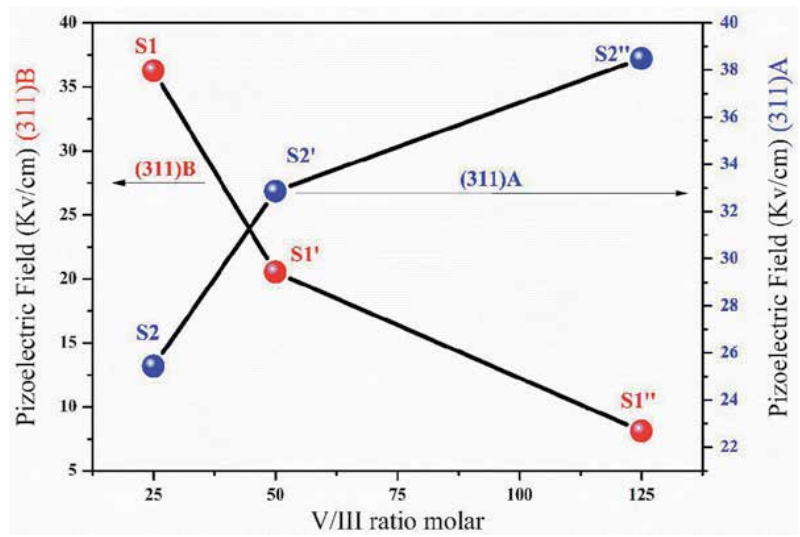

Figure 11.

Piezoelectric field dependence on the V/III ratio molar for (311) A (closed circles blue) and (311) B (closed circles red) substrate orientation.

noted an increase in the PZ field as the V/III ratio increases. It increases from 25.44 to $32.86 \mathrm{KV} / \mathrm{cm}$ when the V/III ratio increases almost twice [5].

From the surface kinetics aspect, it is possible to understand this difference in the variation of the PZ field with the V/III ratio for (311) A and (311) B orientation. The sign of the PZ charge is based on the atomic composition of the interfaces [38-40]. Therefore, it is necessary to distinguish between the substrates In planes, alluded to as (N11) A plane, and the planes P, alluded to as (N11) B-planes. The single-bond sites on a (311) surface are P sites and the double-bond sites are In locations. On the contrary, the surface (311) B has an inverse bonding arrangement as shown in Figure $5[5,19]$. Therefore, for (311) A-face, less As atoms appear to close the cultivated surface at a small V/III ratio. Because of the stronger bond strength of AlAs than In-As, additional As atoms bond to Al statistically, bringing about more AlAs. Therefore, when V/III decreases, the coefficient of incorporation decreases [5]. By contrast, if the V/III ratio increases, though the tendency of As to combining with $\mathrm{Al}$ is still high, because of this oversupply, In atoms have more opportunity to bond with As atoms. In this way, the In incorporation coefficient increases with V/III ratio [5], which creates an increase in the PZ field with V/III ratio for the (311) A-oriented samples ( $\mathrm{S} 2$ and $\left.\mathrm{S} 2^{\prime}\right)$. Furthermore, we noted the opposite phenomenon of what is occurring for (311) B-face. The PZ field in our samples cultivated on (311) A substrate has the opposite direction than for (311) B [5].

We plotted the type II transition energy with the field for both the A and B polarity substrates in order to estimate the role of the PZ field (see Figure 11). The 
shift in energy owing to the PZ field has a distinct impact on the termination of the substrates A and B $[1,5]$. This is because the PZ field may increase or decrease the space separation of the electron/hole that already exists because of the strain [41]. Consequently, the band structures of the InP/InAlAs/InP samples are plotted schematically of the inset of Figure 2. Based on the orientation of the substrate, the band diagrams are shifted to the left side while for (311) A and shifted to the right side for (311) B due to the presence of the PZ field $[1,5]$. Finally, based on the discourse in previous parts, it can be concluded that the red shift in the type II recombination for the (311) A and the blue shift for the (311) B orientation with an increasing V/III ratio is related to the meaning of the PZ field. Another possible raison for the shift of type-II transition is the difference of exchange As/P at the InAlAs/InP interface resulting from a different polarity of $\operatorname{InP}[1,5]$.

\title{
4. Conclusion
}

In summary, InAlAs/InP type-II heterostructures with a varying V/III ratio grown successfully on (311) A or B Fe-doped InP substrates by MOCVD were investigated and the optical properties of the grown structures were examined. The different optical properties of the samples grown on (311) A or B substrates are caused from the difference of their plane-bonding configurations. In particular, the optical properties of InAlAs-InP interface display a significant reliance on $\mathrm{AsH} 3$ overpressure and substrate polarity. PL and PR measurements indicated that substrate orientation and V/III ratio molar have an important impact on the quality of inverted interface. The measurements of excitation power density PL confirm the intrinsic transition of type II. A red shift of the type-II transition was noted at low temperature with an increased V/III ratio of the polarity A samples and a blue shift for polarity B samples. These findings could be clarified by the opposite field of $\mathrm{PZ}$ at the heterostructures of InAlAs/InP resulting from distinct polarity of the InP substrate. We acquired the InP field built-in PZ in the heterostructures from an assessment by the FKO. We have made an explanation of the transition shift from these values. Additionally, the temperature variety shows an anomalous S-shaped dependence that is typical of carrier localization in the material. The optical properties are significantly influenced by the PZ field in our samples. Therefore, the impact of the PZ field on the design and manufacture of greater quality instruments should be taken into consideration.

\section{Author details}

\author{
Badreddine Smiri*, Faouzi Saidi and Hassen Maaref \\ Laboratoire des Micro-optoélectroniques et Nanostructures, Faculté des Sciences \\ Monastir, Université de Monastir, Monastir, Tunisia
}

*Address all correspondence to: badreddinesmiri@gmail.com

\section{IntechOpen}

(C) 2020 The Author(s). Licensee IntechOpen. This chapter is distributed under the terms of the Creative Commons Attribution License (http://creativecommons.org/licenses/ by/3.0), which permits unrestricted use, distribution, and reproduction in any medium, provided the original work is properly cited. (cc) BY 


\section{References}

[1] Smiri B, Fraj I, Saidi F, Mghaïth R, Maaref H. Effect of piezoelectric field on type II transition in InAlAs/InP (311) alloys with different substrate polarity. Journal of Alloys and Compounds. 2018; 736:29-34

[2] Cornet C, Schliwa A, Even J, Doré F, Celebi C, Létoublon A, et al. Electronic and optical properties of InAs/InP quantum dots on $\operatorname{InP}(100)$ and $\operatorname{InP}(311)$ B substrates: Theory and experiment. Physical Review B. 2006;74:035312

[3] Kawamura Y, Kamada A, Yoshimatsu K, Kobayashi H, Iwamura $\mathrm{H}$, Inoue N. Photoluminescence study of interfaces between heavily doped AlInAs:Si layers and InP (Fe) substrates. Institute of Physics Conference Series. 1997;155:129

[4] Yerino CD, Liang B, Huffaker DL, Simmonds J, Lee ML. Molecular beam epitaxy of lattice-matched InAlAs and InGaAs layers on InP (111) A, (111) B, and (110). Journal of Vacuum Science and Technology B. 2017;35:010801

[5] Smiri B, Fraj I, Bouzidi M, Saidi F, Rebey A, Maaref H. Effect of V/III ratio on the optical properties of (311) A and (311) B oriented InAlAs/InP heterostructures. Results in Physics. 2019;12:2175-2182

[6] Hellara J, Borgi K, Maaref H, Souliere V, Monteil Y. Optical properties of InP/ InAlAs/InP grown by MOCVD on (100) substrates: Influence of V/III molar ratio. Journal of Materials Science and Engineering C. 2002;21:231-236

[7] Wang CA, Goyal A, Huang R, Donnelly J, Calawa D, Turner G, et al. Strain-compensated GaInAs/AlInAs/InP quantum cascade laser materials. Journal of Crystal Growth. 2010;312: 1157-1164

[8] Bohrer J, Krost A, Heitz R, Heinrichsdorff F, Eckey L, Bimberg D, et al. Interface inequivalence of the InP/ InAlAs/InP staggered double

heterostructure grown by metalorganic chemical vapor deposition. Journal of Applied Physics Letters. 1996;68:1072

[9] Demir I, Elagoz S. V/III ratio effects on high quality InAlAs for quantum cascade laser structures. Journal of Superlattices and Microstructures. 2017; 104:140-148

[10] Czuba K, Jurenczyk J, Kaniewski J. A study of InGaAs/InAlAs/InP avalanche photodiode. Solid-State Electronics. 2015;104:109-115

[11] Chevallier CB, Baltagi Y, Guillot G, Hong K, Pavlidis D. Application of Photoreflectance spectroscopy to the study of interface roughness in InGaAs/ InAlAsInGaAs/InAlAs heterointerfaces. Journal of Applied Physics. 1998;84:5291

[12] Ajayan J, Nirmal D. A review of InP/ InAlAs/InGaAs based transistors for high frequency applications. Journal of Superlattices and Microstructures. 2015; 86:1-19

[13] Reynolds DC, Bajaj KK, Litton CW, Yu PW, Maselink WT, Rischer R, et al. Sharp-line photoluminescence spectra from GaAs-GaAlAs multiple-quantumwell structures. Journal of Physics. Review B. 1984;29:7038-7041

[14] Kuriharaa K, Takashimab M, Sakatab K, Uedab R, Takaharab M, Ikedab $\mathrm{H}$, et al. Phase separation in InAlAs grown by MOVPE with a low growth temperature. Journal of Crystal Growth. 2004;271:341-347

[15] Vignaud D, Wallart X, Mollot F, Sermage B. Photoluminescence study of the interface in type II InAlAs-InP heterostructures. Journal of Applied Physical. 1998;84:2138-2145

[16] Fraj I, Hidouri T, Saidi F, Maaref H. Carrier localization localization in 
In0.21Ga0.79As/GaAs multiple quantum wells: A modified Passler model for the S-shaped temperature dependence of photoluminescence energy. Superlattices and Microstructures. 2017;102:351-358

[17] Teodoro MD, Dias IFL, Laureto E, Duarte JL, González-Borrero PP, Lourenço SA, et al. Substrate orientation effect on potential fluctuations in multiquantum wells of GaAs/AlGaAs. Journal of Applied Physics. 2008;103: 093508

[18] Smiri B, Hidouri T, Hassen Maaref SF. Carriers' localization and thermal redistribution in InAlAs/InP grown by MOCVD on (311)A- and (311)B-InP substrates. Applied Physics A: Materials Science \& Processing. 2019;125:134

[19] Smiri B, Saidi F, Mlayah A, Maaref H. Effect of substrate polarity on the optical and vibrational properties of (311)A and (311) B oriented InAlAs/InP heterostructures. Physica E: Lowdimensional Systems and Nanostructures. 2019;112:121-127

[20] Abraham P, Monteil Y, Sacilotti M. Optical studies of InP/InAlAs/InP interface recombinations. Journal of Applied Surface Science. 1993;65-66: 777-783

[21] Poças LC, Duarte JL, Dias IFL, Laureto E, Lourenço SA, Toginho Filho $\mathrm{DO}$, et al. Photoluminescence study of interfaces between heavily doped Al0.48In0.52As: Si layers and InP (Fe) substrates. Journal of Applied Physics. 2002;91:8999

[22] Ezzedini M, Bouzidi M, Qaid MM, Chine Z, Rebey A, Sfaxi L.

Comprehensive study of the structural, optical and electrical properties of InAlAs: Mg films lattice matched to InP grown by MOVPE. Journal of Materials Science: Materials in Electronics. 2017; 18:18221-18227
[23] Sayari A, Yahyaoui N, Meftah A, Sfaxi A, Oueslati M. Residual strain and alloying effects on the vibrational properties of step-graded InxAl1-xAs layers grown on GaAs. Journal of Luminescence. 2009;129:105-109

[24] Li Y, Niewcza M. Strain relaxation in (100) and (311) GaP/GaAs thin films. Journal of Applied Physics. 2007; 101:064910

[25] Tromson-Carli A, Patriarche G, Druilhe R, Lusson A, Marfaing Y, Triboulet R, et al. Effect of the $\{$ h11 orientations and polarities of GaAs substrates CdTe buffer layer structural properties. Journal of Materials Science and Engineering B. 1993;16:145-150

[26] Peter M, Herres N, Fuchs F, Winkler K, Bachem K-H, Wagner J. Band gaps and band offsets in strained $\mathrm{GaAs}(1-\mathrm{y}) \mathrm{Sb}(\mathrm{y})$ on InP grown by metalorganic chemical vapor deposition. Applied Physics Letters. 1999;74: 410-412

[27] $\mathrm{Hu}$ J, Xu G, Stotz J, Watkins S, Curzon A, Thewalt M, et al. Type II photoluminescence and conduction band offsets of $\mathrm{GaAsSb} / \mathrm{InGaAs}$ and $\mathrm{GaAsSb} / \mathrm{InP}$ heterostructures grown by metalorganic vapor phase epitaxy. Applied Physics Letters. 1998;73:2799

[28] Shuhui Z, Lu W, Zhenwu S, Yanxiang C, Haitao T, Huaiju G, et al. Lattice parameter accommodation between $\mathrm{GaAs}(111)$ nanowires and $\mathrm{Si}$ (111) substrate after growth via $\mathrm{Au}$-assisted molecular beam epitaxy. Nanoscale Research Letters. 2012; 7(1):109

[29] Ferguson I, Cheng T, Sotomayor Torres C, Murray R. Photoluminescence of molecular beam epitaxial grown Al0.48In0.52As. Journal of Vacuum Science and Technology B. 1994;12:1319

[30] Merkel K, Bright V, Cerny C, Shcuermeyer F, Solomon J, Kaspi R. 
Beryllium ion implantation in GaAsSb epilayers on InP. Journal of Applied Physics. 1995;79:699

[31] Chouaib H, Bru-Chevallier C, Guillot G, Lahreche H, Bove P. Photoreflectance study of GaAsSb/InP heterostructures. Journal of Applied Physics. 2005;98:123524

[32] Saidi F, Hamila R, Maaref H, Rodriguez P, Auvray L, Monteil Y. Structural and optical study of BxInyGa1-x-yAs/GaAs and InyGa1-yAs/GaAs QW's grown by MOCVD. Journal of Alloys and Compounds. 2010;491:45-48

[33] Hamila R, Saidi F, Maaref H, Rodriguez P, Auvray L. Photoluminescence properties and high resolution X-ray diffraction investigation of $\mathrm{BInGaAs} / \mathrm{GaAs}$ grown by the metalorganic vapour phase epitaxy method. Journal of Applied Physics. 2012;112:063109

[34] Hou HQ, Tu CW. Field screening in (111) B InAsP/lnP strained quantum wells. Journal of Electronic Materials. 1996;25:1019-1022

[35] Nukeaw J, Matsubara N, Fujiwara Y, Takeda Y. Characterization of InP $\delta$ doped with Er by FFT photoreflectance. Applied Surface Science. 1997;117/118: 776-780

[36] Bouzidi M, Benzarti Z, Halidou I, Soltani S, Chine Z, Jani BEL.

Photoreflectance investigation of band gap renormalization and the BursteinMoss effect in Si doped GaN grown by MOVPE. Materials Science in Semiconductor Processing. 2016;42: 273-276

[37] Bilel C, Fitouri H, Zaied I, Bchetnia A, Rebey A, El Jani B. Photoreflectance characterization of vanadium-doped GaAs layers grown by metalorganic vapor phase epitaxy. Journal of Materials Science in Semiconductor Processing. 2015;31:100-105
[38] Sanguinetti S, Gurioli M, Grilli E, Guzzi M, Henini M. Piezoelectricinduced quantum-confined Stark effect in self-assembled InAs quantum dots grown on (N11) GaAs substrates. Journal of Applied Physics Letters. 2000;77:1982

[39] Anufriev R, Chauvin N, Khmissi H, Naji K, Patriarche G, Gendry M, et al. Piezoelectric effect in InAs/InP quantum rod nanowires grown on silicon substrate. Journal of Applied Physics Letters. 2014;104:183101

[40] Kwak JS, Lee KY, Han JY, Cho J, Chae S, Nam OH, et al. Crystal-polarity dependence of $\mathrm{Ti} / \mathrm{Al}$ contacts to freestanding $\mathrm{n}-\mathrm{GaNn}-\mathrm{GaN}$ substrate. Journal of Applied Physics Letters. 2001;79:3254

[41] Sacilotti M, Chaumont D, Brainer Mota C, Vasconcelos T, Nunes FD, Pompelli MF, et al. Interface recombination \& emission applied to explain photosynthetic mechanisms for $(\mathrm{e}-, \mathrm{h}+)$ charges' separation. World Journal of Nano Science and Engineering. 2012;2:58-87 


\title{
Static and Dynamic Analysis of Piezoelectric Laminated Composite Beams and Plates
}

\author{
Chung Nguyen Thai, Thinh Tran Ich and Thuy Le Xuan
}

\begin{abstract}
In this chapter, the mechanical behavior analysis of piezoelectric laminated composite beams and plates is influenced subjected to static, dynamic, and aerodynamic loads. Algorithm for dynamic, stability problem analysis and vibration control of laminated composite beams and plates with piezoelectric layers is presented. In addition, numerical calculations, considering the effect of factors on static, dynamic, and stability response of piezoelectric laminated composite beams and plates are also clearly presented. The content of this chapter can equip readers with the knowledge used to calculate the static, dynamic, and vibration control of composite beams, panels made of piezoelectric layers applied in the field different techniques.
\end{abstract}

Keywords: beams, plates, static, dynamic, piezoelectric, composite, stiffened

\section{Introduction}

The content of this chapter is the inheritance and development of the research results of the authors and other authors by published scientific works on composite materials, piezoelectric and structural calculation by piezoelectric composite materials.

\section{Electromechanical interaction of piezoelectric materials}

\subsection{Mechanical-electrical behavior relations}

Let us consider a block of elastic material in an environment with an electric field of zero, the relationship between stress and strain is followed Hooke's law, and written as follows [1, 2]:

$$
\{\sigma\}=[c]\{\varepsilon\},
$$

where $\{\sigma\}$ is the mechanical stress vector, $\{\varepsilon\}$ is the mechanical strain vector, and [c] is the material stiffness matrix of beam.

Mechanical-electrical relations in piezoelectric materials have an interactive relationship, strain $\{\varepsilon\}$ will produce e $\varepsilon$ - polarization, where e is the voltage stress 
factor when there is no mechanical strain. The imposed electric field E produces the -eE stress in the piezoelectric material according to the reverse voltage effect.

Therefore, we have a mathematical model that describes the mechanical-electrical interaction relationship in piezoelectric materials as follows [3-6]:

$$
\begin{gathered}
\{\sigma\}=[\mathrm{c}]\{\varepsilon\}-[\mathrm{e}]\{\mathrm{E}\}, \\
\{\mathrm{D}\}=[\mathrm{e}]^{\mathrm{T}}\{\varepsilon\}+[\mathrm{p}]\{\mathrm{E}\}, \\
\text { or }\{\mathrm{D}\}=[\mathrm{d}]^{\mathrm{T}}\{\sigma\}+[\mathrm{p}]\{\mathrm{E}\},
\end{gathered}
$$

where $[\mathrm{e}]$ is the piezoelectric stress coefficient matrix, [p] is the dielectric constant matrix, $\{\mathrm{E}\}$ is the vector of applied electric field $(\mathrm{V} / \mathrm{m})$, and $\{\mathrm{D}\}$ is the vector of electric displacement $\left(\mathrm{C} / \mathrm{m}^{2}\right)$.

For the linear problem and small strain, strain vector in the piezoelectric structures can be defined as follows:

$$
\{\varepsilon\}=[s]\{\sigma\}+[\mathrm{d}]\{\mathrm{E}\},
$$

in which $[\mathrm{s}]$ is the matrix of compliance coefficients $\left(\mathrm{m}^{2} / \mathrm{N}\right),[\mathrm{d}]$ is the matrix of piezoelectric strain constants $(\mathrm{m} / \mathrm{V})$.

In the field of engineering, piezoelectric materials are used by two types. The first type, the piezoelectric layers or the piezoelectric patches act as actuators, called the piezoelectric actuators. In this case, the piezoelectric layers are strained when imposing an electric field on it. The second type, the piezoelectric layers or piezoelectric patches act as sensors, called piezoelectric sensors. In this case, the voltage is generated in piezoelectric layers when there is mechanical strain.

\subsection{Piezoelectric actuators and sensors}

\subsubsection{Piezoelectric actuators}

Eq. (5) can be written in the matrix form as follows $[4,6]$ :

$$
\{\varepsilon\}=\left\{\begin{array}{l}
\varepsilon_{11} \\
\varepsilon_{22} \\
\varepsilon_{33} \\
\gamma_{23} \\
\gamma_{13} \\
\gamma_{12}
\end{array}\right\}=\left[\begin{array}{llllll}
\mathrm{s}_{11} & \mathrm{~s}_{12} & \mathrm{~s}_{13} & \mathrm{~s}_{14} & \mathrm{~s}_{15} & \mathrm{~s}_{16} \\
\mathrm{~s}_{21} & \mathrm{~s}_{22} & \mathrm{~s}_{23} & \mathrm{~s}_{24} & \mathrm{~s}_{25} & \mathrm{~s}_{26} \\
\mathrm{~s}_{31} & \mathrm{~s}_{32} & \mathrm{~s}_{33} & \mathrm{~s}_{34} & \mathrm{~s}_{35} & \mathrm{~s}_{36} \\
\mathrm{~s}_{41} & \mathrm{~s}_{42} & \mathrm{~s}_{43} & \mathrm{~s}_{44} & \mathrm{~s}_{45} & \mathrm{~s}_{46} \\
\mathrm{~s}_{51} & \mathrm{~s}_{52} & \mathrm{~s}_{53} & \mathrm{~s}_{54} & \mathrm{~s}_{55} & \mathrm{~s}_{56} \\
\mathrm{~s}_{61} & \mathrm{~s}_{62} & \mathrm{~s}_{63} & \mathrm{~s}_{64} & \mathrm{~s}_{65} & \mathrm{~s}_{66}
\end{array}\right]\left\{\begin{array}{l}
\sigma_{11} \\
\sigma_{22} \\
\sigma_{33} \\
\tau_{23} \\
\tau_{13} \\
\tau_{12}
\end{array}\right\}+\left[\begin{array}{lll}
\mathrm{d}_{11} & \mathrm{~d}_{21} & \mathrm{~d}_{31} \\
\mathrm{~d}_{12} & \mathrm{~d}_{22} & \mathrm{~d}_{32} \\
\mathrm{~d}_{13} & \mathrm{~d}_{23} & \mathrm{~d}_{33} \\
\mathrm{~d}_{14} & \mathrm{~d}_{24} & \mathrm{~d}_{34} \\
\mathrm{~d}_{15} & \mathrm{~d}_{25} & \mathrm{~d}_{35} \\
\mathrm{~d}_{16} & \mathrm{~d}_{26} & \mathrm{~d}_{36}
\end{array}\right]\left\{\begin{array}{l}
\mathrm{E}_{1} \\
\mathrm{E}_{2} \\
\mathrm{E}_{3}
\end{array}\right\},
$$

Assuming that the device is pulled along the axis 3, and viewing the piezoelectric material as a transversely isotropic material, which is true for piezoelectric ceramics, many of the parameters in the above matrices will be either zero, or can be expressed through each other. In particular, the non-zero compliance coefficients are $\mathrm{s}_{11}, \mathrm{~s}_{12}, \mathrm{~s}_{13}, \mathrm{~s}_{21}, \mathrm{~s}_{22}, \mathrm{~s}_{23}, \mathrm{~s}_{31}, \mathrm{~s}_{32}, \mathrm{~s}_{33}, \mathrm{~s}_{44}, \mathrm{~s}_{55}, \mathrm{~s}_{66}$, in which $\mathrm{s}_{12}=\mathrm{s}_{21}, \mathrm{~s}_{13}=\mathrm{s}_{31}$, $s_{23}=s_{32}, s_{44}=s_{55}, s_{66}=2\left(s_{11}-s_{12}\right)$. 
Finally, Eq. (6) becomes:

$$
\{\varepsilon\}=\left\{\begin{array}{l}
\varepsilon_{11} \\
\varepsilon_{22} \\
\varepsilon_{33} \\
\gamma_{23} \\
\gamma_{13} \\
\gamma_{12}
\end{array}\right\}=\left[\begin{array}{cccccc}
\mathrm{s}_{11} & \mathrm{~s}_{12} & \mathrm{~s}_{13} & 0 & 0 & 0 \\
\mathrm{~s}_{12} & \mathrm{~s}_{22} & \mathrm{~s}_{23} & 0 & 0 & 0 \\
\mathrm{~s}_{13} & \mathrm{~s}_{23} & \mathrm{~s}_{33} & 0 & 0 & 0 \\
0 & 0 & 0 & \mathrm{~s}_{44} & 0 & 0 \\
0 & 0 & 0 & 0 & \mathrm{~s}_{55} & 0 \\
0 & 0 & 0 & 0 & 0 & \mathrm{~s}_{66}
\end{array}\right]\left\{\begin{array}{c}
\sigma_{11} \\
\sigma_{22} \\
\sigma_{33} \\
\tau_{23} \\
\tau_{13} \\
\tau_{12}
\end{array}\right\}+\left[\begin{array}{ccc}
0 & 0 & \mathrm{~d}_{31} \\
0 & 0 & \mathrm{~d}_{32} \\
0 & 0 & \mathrm{~d}_{33} \\
0 & \mathrm{~d}_{24} & 0 \\
\mathrm{~d}_{15} & 0 & 0 \\
0 & 0 & 0
\end{array}\right]\left\{\begin{array}{c}
\mathrm{E}_{1} \\
\mathrm{E}_{2} \\
\mathrm{E}_{3}
\end{array}\right\},
$$

where $E_{1}, E_{2}$, and $E_{3}$ are electric fields in the 1, 2, and 3 directions, respectively.

\subsubsection{Piezoelectric sensors}

The induction charge equation of piezoelectric sensor layers is derived from Eq. (4) can be written in the matrix form as $[4,6,7]$ :

$$
\{D\}=\left\{\begin{array}{l}
D_{1} \\
D_{2} \\
D_{3}
\end{array}\right\}=\left[\begin{array}{llllll}
d_{11} & d_{12} & d_{13} & d_{14} & d_{15} & d_{16} \\
d_{21} & d_{22} & d_{23} & d_{24} & d_{25} & d_{26} \\
d_{31} & d_{32} & d_{33} & d_{34} & d_{35} & d_{36}
\end{array}\right]\left\{\begin{array}{c}
\sigma_{11} \\
\sigma_{22} \\
\sigma_{33} \\
\tau_{23} \\
\tau_{13} \\
\tau_{12}
\end{array}\right\}+\left[\begin{array}{lll}
p_{11} & p_{12} & p_{13} \\
p_{21} & p_{22} & p_{23} \\
p_{31} & p_{32} & p_{33}
\end{array}\right]\left\{\begin{array}{l}
E_{1} \\
E_{2} \\
E_{3}
\end{array}\right\},
$$

The non-zero piezoelectric strain constants are $d_{31}, d_{32}, d_{15}, d_{24}$, and $d_{33}$, in which $\mathrm{d}_{31}=\mathrm{d}_{32}, \mathrm{~d}_{15}=\mathrm{d}_{24}$. And the non-zero dielectric coefficients are $\mathrm{p}_{11}, \mathrm{p}_{22}$, and $\mathrm{p}_{33}$, where $\mathrm{p}_{11}=\mathrm{p}_{22}$. Eq. (8) becomes:

$$
\{D\}=\left\{\begin{array}{l}
D_{1} \\
D_{2} \\
D_{3}
\end{array}\right\}=\left[\begin{array}{cccccc}
0 & 0 & 0 & 0 & d_{15} & 0 \\
0 & 0 & 0 & d_{24} & 0 & 0 \\
d_{31} & d_{32} & d_{33} & 0 & 0 & 0
\end{array}\right]\left\{\begin{array}{c}
\sigma_{11} \\
\sigma_{22} \\
\sigma_{33} \\
\tau_{23} \\
\tau_{13} \\
\tau_{12}
\end{array}\right\}+\left[\begin{array}{ccc}
p_{11} & 0 & 0 \\
0 & p_{22} & 0 \\
0 & 0 & p_{33}
\end{array}\right]\left\{\begin{array}{l}
E_{1} \\
E_{2} \\
E_{3}
\end{array}\right\},
$$

where $\mathrm{D}_{1}, \mathrm{D}_{2}, \mathrm{D}_{3}, \mathrm{p}_{11}, \mathrm{p}_{22}$, and $\mathrm{p}_{33}$ are the displacement charge, dielectric constant in the 1,2 , and 3 directions, respectively.

Normally, the voltage is transmitted through the thickness of the actuator layers. 


\section{Static and dynamic analysis of laminated composite beams with piezoelectric layers}

\subsection{Displacement and strain}

Based on the first-order shear deformation theory (FSDT), the displacement field at any point of the beam is defined as [1, 2]:

$$
\begin{aligned}
& \mathrm{u}(\mathrm{x}, \mathrm{z})=\mathrm{u}_{0}(\mathrm{x})+z \theta_{y}(\mathrm{x}), \\
& \mathrm{w}(\mathrm{x}, \mathrm{z})=\mathrm{w}_{0}(\mathrm{x}),
\end{aligned}
$$

where $\mathrm{u}, \mathrm{w}$ denotes the displacements of a point $(\mathrm{x}, \mathrm{z})$ in the beam; $\mathrm{u}_{0}, \mathrm{w}_{0}$ are the displacements of a point at the beam neutral axis, and $\theta_{y}$ is the rotation of the transverse normal about the $y$ axis. The bending and shear strains associated with the displacement field in Eq. (10) are defined as:

$$
\{\varepsilon\}=\left\{\begin{array}{c}
\varepsilon_{x} \\
\gamma_{x z}
\end{array}\right\}=\left\{\begin{array}{c}
\frac{d u}{d x} \\
\frac{d u}{d z}+\frac{d w}{d x}
\end{array}\right\}=\left\{\begin{array}{c}
\frac{d u_{0}}{d x}+z \frac{d \theta_{z}}{d x} \\
\theta_{y}+\frac{d w_{0}}{d x}
\end{array}\right\}=\left[\begin{array}{ccc}
\frac{d}{d x} & 0 & z \frac{d}{d x} \\
0 & \frac{d}{d x} & 1
\end{array}\right]\left\{\begin{array}{c}
\mathrm{u}_{0} \\
w_{0} \\
\theta_{z}
\end{array}\right\},
$$

in which $\varepsilon_{\mathrm{x}}, \gamma_{\mathrm{xz}}$ are the normal strain, and shear strain, respectively.

Using finite element method, we consider 2-node bending elements with 3 degrees of freedom per node (Figure 1).

The displacements of the beam neutral axis are expressed in local coordinate system in the form:

$$
\left\{\mathrm{d}_{0}\right\}=\left\{\begin{array}{c}
\mathrm{u}_{0} \\
\mathrm{v}_{0} \\
\theta_{z}
\end{array}\right\}=\left\{\begin{array}{c}
{\left[\mathrm{N}^{\mathrm{u}}\right]\left\{\mathrm{q}^{\mathrm{u}}\right\}} \\
{\left[\mathrm{N}^{\mathrm{v}}\right]\left\{\mathrm{q}^{\mathrm{v}}\right\}} \\
{\left[\mathrm{N}^{\theta_{z}}\right]\left\{\mathrm{q}^{\theta_{z}}\right\}}
\end{array}\right\}=\left[\mathrm{N}^{\mathrm{M}}\right]\left\{\mathrm{q}_{\mathrm{b}}\right\}_{\mathrm{e}},
$$

where $\left\{\mathrm{q}_{\mathrm{b}}\right\}_{\mathrm{e}}$ is the vector of vector of nodal displacements of element, $\left[\mathrm{N}^{\mathrm{M}}\right]$ is the matrix mechanical shape functions:

$$
\begin{aligned}
& \left\{\mathrm{q}_{\mathrm{b}}\right\}_{\mathrm{e}}=\left\{\begin{array}{llllll}
\mathrm{q}_{1} & \mathrm{q}_{2} & \mathrm{q}_{3} & \mathrm{q}_{4} & \mathrm{q}_{5} & \mathrm{q}_{6}
\end{array}\right\}^{\mathrm{T}} \text {, } \\
& \underbrace{\left[\mathrm{N}^{\mathrm{M}}\right]}_{3 \times 6}=\left[\begin{array}{ccc}
{\left[\mathrm{N}^{\mathrm{u}}\right]} & 0 & 0 \\
0 & {\left[\mathrm{~N}^{\mathrm{v}}\right]} & 0 \\
0 & 0 & {\left[\mathrm{~N}^{\theta_{\mathrm{z}}}\right]}
\end{array}\right] \text {, }
\end{aligned}
$$

in which $\left[\mathrm{N}^{\mathrm{u}}\right],\left[\mathrm{N}^{\mathrm{v}}\right],\left[\mathrm{N}^{\ominus \mathrm{z}}\right]$ are, in this order, the row vectors of longitudinal, transverse along $y$, and rotation about $z$ shape functions.

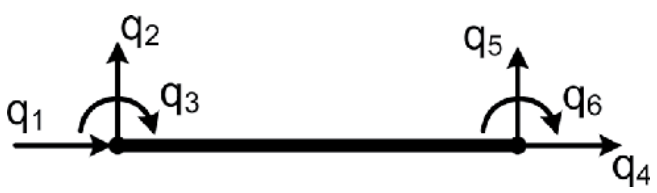

Figure 1.

Two noded beam element. 
Substituting Eq. (12) into Eq. (11), we obtain:

$$
\begin{gathered}
\underbrace{\{\varepsilon\}}_{2 \times 1}=\underbrace{\left[\mathrm{B}_{\mathrm{b}}\right]}_{2 \times 6} \underbrace{\left\{\mathrm{q}_{\mathrm{b}}\right\}_{\mathrm{e}}}_{6 \times 1}, \\
\text { where }\left[\mathrm{B}_{\mathrm{b}}\right]=\left[\begin{array}{ccc}
\frac{\mathrm{d}}{\mathrm{dx}} & 0 & z \frac{\mathrm{d}}{\mathrm{dx}} \\
0 & \frac{\mathrm{d}}{\mathrm{dx}} & 1
\end{array}\right]\left[\mathrm{N}^{\mathrm{M}}\right] .
\end{gathered}
$$

The electric potential is constant over the element surface:

$$
\phi_{k}=\sum_{i=1}^{n} N_{i} \phi_{i},
$$

where $\mathrm{n}$ is the element node number.

A voltage $\phi$ is applied across an actuator of layer thickness $t_{p}$ generates an electric field vector $\{E\}$, such that $[4,8-10]$ :

$$
\left\{E_{k}\right\}=-\nabla \phi_{k}=\left\{\begin{array}{lll}
0 & 0 & E_{k}^{z}
\end{array}\right\},
$$

in which

$$
E_{k}^{z}=-\frac{\phi_{k}}{t_{p k}}=\left[B_{\phi}\right]\{\phi\}=\left[\begin{array}{cccccc}
0 & 0 & \frac{1}{t_{p 1}} & 0 & 0 & 0 \\
0 & 0 & 0 & 0 & 0 & \frac{1}{t_{p 2}}
\end{array}\right]^{T}\left\{\begin{array}{l}
\phi_{1} \\
\phi_{2}
\end{array}\right\},
$$

where $t_{\mathrm{pk}}$ is the thickness of the $\mathrm{k}^{\mathrm{th}}$ piezoelectric layer.

Substituting Eq. (19) into Eq. (18), the electric field vector $\{\mathrm{E}\}$ can also be defined in terms of nodal variables as:

$$
\{\mathrm{E}\}=-\left[\mathrm{B}_{\phi}\right]\{\phi\}_{\mathrm{e}},
$$

Using Eqs. (15), and (20), the linear piezoelectric constitutive equations coupling the elastic and electric fields will be completely determined by Eqs. (2) and (3).

\subsection{Finite element equations}

Using Hamilton's principle, we have [11-13]:

$$
\int_{t_{1}}^{t_{2}}\left(T^{e}-U^{e}-W^{e}\right) d t=0
$$

where $\mathrm{T}^{\mathrm{e}}, \mathrm{U}^{\mathrm{e}}$ are the kinetic and potential energy, respectively and $\mathrm{W}^{\mathrm{e}}$ is the work done by external forces. They are determined by:

$$
T^{e}=\frac{1}{2} \int_{V_{e}} \rho\{\dot{q}\}_{e}^{T}\{q\}_{e} d V,
$$




$$
\begin{gathered}
U^{e}=\frac{1}{2} \int_{V_{e}}\{\varepsilon\}_{e}^{T}\{\sigma\}_{e} d V \\
W^{e}=\int_{V_{e}}\{q\}_{e}^{T}\left\{f_{b}\right\}_{e} d V+\int_{S_{e}}\{q\}_{e}^{T}\left\{f_{s}\right\}_{e} d S+\{q\}_{e}^{T}\left\{f_{c}\right\}_{e},
\end{gathered}
$$

in which $\left\{f_{b}\right\}_{e},\left\{f_{s}\right\}_{e},\left\{f_{c}\right\}_{e}$ are the body, surface, and concentrated forces acting on the element, respectively. $\mathrm{V}_{\mathrm{e}}$ and $\mathrm{S}_{\mathrm{e}}$ are elemental volume and area.

Substituting Eqs. (15), (2), (20), (22), (23), and (24) into Eq. (21), one obtains:

$$
\begin{gathered}
{\left[M_{b b}^{e}\right]\{\ddot{q}\}_{e}+\left[K_{b b}^{e}\right]\{q\}_{e}+\left[K_{b \phi}^{e}\right]\{\phi\}_{e}=\{f\}_{e},} \\
{\left[K_{\phi b}^{e}\right]\{q\}_{e}-\left[K_{\phi \phi}^{e}\right]\{\phi\}_{e}=\{Q\}_{e}}
\end{gathered}
$$

where

$$
\begin{gathered}
\text { Element mass matrix: }\left[M_{b b}^{e}\right]=\int_{V_{e}} \rho\left[N^{M}\right]^{T}\left[N^{M}\right] d V, \\
\text { Element mechanical stiffness matrix: }\left[K_{b b}^{e}\right]=\int_{S_{e}}\left[B_{b}\right]^{T}[H]\left[B_{b}\right] d S,
\end{gathered}
$$

Element mechanical-electrical coupling stiffness matrix:

$$
\left[K_{b \phi}^{e}\right]=\int_{S_{e}}\left[B_{b}\right]^{T}[\bar{e}]\left[B_{\phi}\right] d S,
$$

Element electrical-mechanical coupling stiffness matrix:

$$
\left[K_{\phi b}^{e}\right]=\left[K_{b \phi}^{e}\right]^{T}
$$

Element piezoelectric permittivity matrix:

$$
\begin{gathered}
{\left[K_{\phi \phi}^{e}\right]=-\int_{S_{e}}\left[B_{\phi}\right]^{T}[\bar{p}]\left[B_{\phi}\right] d S,} \\
\text { where }[H]=\left[\begin{array}{cc}
c_{11} & 0 \\
0 & c_{22}
\end{array}\right],[\bar{e}]=\left[\begin{array}{ll}
e_{11} & e_{12} \\
e_{21} & e_{22}
\end{array}\right],[\bar{p}]=\left[\begin{array}{cc}
t_{p 1} p_{11} & 0 \\
0 & t_{p 2} p_{22}
\end{array}\right],
\end{gathered}
$$

$\{f\}_{e},\{Q\}_{e}$ are the applied external load and charge, respectively.

\subsubsection{Static analysis}

In the case of beams subjected to static loads, zero acceleration, from Eqs. (25) and (26), we obtain the static equations of the beam as follows:

$$
\left[K_{b b}^{e}\right]\{q\}_{e}+\left[K_{b \phi}^{e}\right]\{\phi\}_{e}=\{f\}_{e}
$$




$$
\left[K_{\phi b}^{e}\right]\{q\}_{e}-\left[K_{\phi \phi}^{e}\right]\{\phi\}_{e}=\{Q\}_{e}
$$

Assembling the element equations yields general static equation:

$$
\begin{aligned}
& {\left[K_{b b}\right]\{q\}+\left[K_{b \phi}\right]\{\phi\}=\{f\},} \\
& {\left[K_{\phi b}\right]\{q\}-\left[K_{\phi \phi}\right]\{\phi\}=\{Q\} .}
\end{aligned}
$$

where $\left[\mathrm{K}_{\mathrm{bb}}\right],\left[\mathrm{K}_{\phi \phi}\right]$ are the overall mechanical stiffness and piezoelectric permittivity matrices respectively; $\left[\mathrm{K}_{\mathrm{b} \phi}\right]$ and $\left[\mathrm{K}_{\phi \mathrm{b}}\right]$ are the overall mechanical electrical and electrical - mechanical coupling stiffness matrices, respectively, and $\{q\},\{\phi\}$ are respectively the overall mechanical displacement, and electric potential vector.

Substituting Eq. (36) into Eq. (35) yields:

$$
\left(\left[K_{b b}\right]+\left[K_{b \phi}\right]\left[K_{\phi \phi}\right]^{-1}\left[K_{\phi b}\right]\right)\{q\}=\{f\}+\left[K_{b \phi}\right]\left[K_{\phi \phi}\right]^{-1}\{Q\},
$$

Substituting $\{q\}$ from Eq. (37) into Eq. (36), we obtain the vector $\{\phi\}$.

\subsubsection{Dynamic analysis}

From Eqs. (25) and (26), assembling the element equations yields general dynamic equation of motion:

$$
\begin{gathered}
{\left[M_{b b}\right]\{\ddot{q}\}+\left[K_{b b}\right]\{q\}+\left[K_{b \phi}\right]\{\phi\}=\{f\},} \\
{\left[K_{\phi b}\right]\{q\}-\left[K_{\phi \phi}\right]\{\phi\}=\{Q\},}
\end{gathered}
$$

Substituting Eq. (39) into Eq. (38), we obtain:

$$
\left[M_{b b}\right]\{\ddot{q}\}+\left(\left[K_{b b}\right]+\left[K_{b \phi}\right]\left[K_{\phi \phi}\right]^{-1}\left[K_{\phi b}\right]\right)\{q\}=\{f\}+\left[K_{b \phi}\right]\left[K_{\phi \phi}\right]^{-1}\{Q\},
$$

\subsubsection{Free vibration analysis}

For free vibrations, from Eq. (40), the governing equation is:

$$
\left[M_{b b}\right]\{\ddot{q}\}+\left(\left[K_{b b}\right]+\left[K_{b \phi}\right]\left[K_{\phi \phi}\right]^{-1}\left[K_{\phi b}\right]\right)\{q\}=\{0\} .
$$

The beam vibrations induce charges and electric potentials in sensor layers. Therefore, the control system allows current to flow and feeds back to the actuators. In this case, if we apply no external charge $Q$ to a sensor, from Eq. (39), we will have:

$$
\left[K_{\phi \phi}\right]_{s}^{-1}\left[K_{\phi b}\right]_{s}\{q\}_{s}=\{\phi\}_{s}
$$

and $\{Q\}_{s}=\left[K_{\phi b}\right]_{s}\{q\}_{s}$ is the induced charge due to strain.

The operation of the amplified control loop implies, the actuating voltage is determined by the following relationship $[1,10,14]$ :

$$
\{\phi\}_{a}=G_{d}\{\phi\}_{s}+G_{v}\{\dot{\phi}\}_{s},
$$


where $G_{d}$ and $G_{v}$ are the feedback control gains for displacement and velocity.

Substituting Eq. (43) into Eq. (39), the charge in the actuator due to actuator strain in response to the beam vibration modified by control system feedback is:

$$
\left[K_{\phi b}\right]_{a}\{q\}_{a}-\left[K_{\phi \phi}\right]_{a}\left(G_{d}\{\phi\}_{s}+G_{v}\{\dot{\phi}\}_{s}\right)=\{Q\}_{a} .
$$

Substituting (42) into (44) leads to:

$$
\{Q\}_{a}=\left[K_{\phi b}\right]_{a}\{q\}_{a}-G_{d}\left[K_{\phi \phi}\right]_{a}\left[K_{\phi \phi}\right]_{s}^{-1}\left[K_{\phi b}\right]_{s}\{q\}_{s}-G_{v}\left[K_{\phi \phi}\right]_{a}\left[K_{\phi \phi}\right]_{s}^{-1}\left[K_{\phi b}\right]_{s}\{\dot{q}\}_{s} .
$$

Substituting Eq. (45) into (40), we obtain:

$$
\begin{aligned}
{\left[M_{b b}\right]\{\ddot{q}\} } & +\left(\left[K_{b b}\right]+\left[K_{b \phi}\right]\left[K_{\phi \phi}\right]^{-1}\left[K_{\phi b}\right]\right)\{q\}=\{f\}+ \\
& +\left[K_{b \phi}\right]\left[K_{\phi \phi}\right]^{-1}\left(\begin{array}{l}
{\left[K_{\phi b}\right]_{a}\{q\}_{a}-G_{v}\left[K_{\phi \phi}\right]_{a}\left[K_{\phi \phi}\right]_{s}^{-1}\left[K_{\phi b}\right]_{s}\{\dot{q}\}_{s}-} \\
-G_{d}\left[K_{\phi \phi}\right]_{a}\left[K_{\phi \phi}\right]_{s}^{-1}\left[K_{\phi b}\right]_{s}\{q\}_{s}
\end{array}\right),
\end{aligned}
$$

in which $\{\mathrm{q}\}_{\mathrm{s}} \equiv\{\mathrm{q}\}_{\mathrm{a}} \equiv\{\mathrm{q}\}$ is the beam displacement vector, $\left[\mathrm{K}_{\phi \phi}\right]_{\mathrm{a}}=$ $\left[\mathrm{K}_{\phi \phi}\right]_{\mathrm{s}}=\left[\mathrm{K}_{\phi \phi}\right]$ is the piezoelectric permittivity matrix, and $\left[\mathrm{K}_{\phi \mathrm{b}}\right]_{\mathrm{a}}=\left[\mathrm{K}_{\phi \mathrm{b}}\right]_{\mathrm{s}}=\left[\mathrm{K}_{\phi \mathrm{b}}\right]$ is the mechanical-electrical coupling stiffness matrix.

Therefore, Eq. (46) becomes:

$$
\begin{aligned}
& {\left[M_{b b}\right]\{\ddot{q}\}+\left[K_{b b}\right]\{q\}+G_{v}\left[K_{b \phi}\right]\left[K_{\phi \phi}\right]^{-1}\left[K_{\phi \phi}\right]\left[K_{\phi \phi}\right]^{-1}\left[K_{\phi b}\right]\{\dot{q}\}+} \\
& +G_{d}\left[K_{b \phi}\right]\left[K_{\phi \phi}\right]^{-1}\left[K_{\phi \phi}\right]\left[K_{\phi \phi}\right]^{-1}\left[K_{\phi b}\right]\{q\}=\{f\} .
\end{aligned}
$$

In the case of considering the structural damping, the equation of motion of the beam is:

$$
\left[M_{b b}\right]\{\ddot{q}\}+\left(\left[C_{A}\right]+\left[C_{R}\right]\right)\{\dot{q}\}+K^{*}\{q\}=\{f\},
$$

where $\left[C_{A}\right]=G_{v}\left[K_{b \phi}\right]\left[K_{\phi \phi}\right]^{-1}\left[K_{\phi \phi}\right]\left[K_{\phi \phi}\right]^{-1}\left[K_{\phi b}\right]$ is the active damping matrix, $\left[K^{*}\right]=\left(\left[K_{b b}\right]+G_{d}\left[K_{b \phi}\right]\left[K_{\phi \phi}\right]^{-1}\left[K_{\phi \phi}\right]\left[K_{\phi \phi}\right]^{-1}\left[K_{\phi b}\right]\right)$ is the total of mechanical stiffness matrix and piezoelectric, $\left[C_{R}\right]=\alpha_{R}\left[M_{b b}\right]+\beta_{R}\left[K_{b b}\right]$ is the overall structural damping matrix, $\alpha_{R}$, and $\beta_{R}$ are respectively the Rayleigh damping coefficients, which are generally determined by the first and second natural frequencies $\left(\omega_{1}, \omega_{2}\right)$ and ratio of damping $\xi,\{f\}$ is the overall mechanical force vector.

Eq. (48) can be solved by the direct integration Newmark's method.

\subsection{Numerical analysis}

An example for free vibration of laminated beam affected by piezoelectric layers is presented here. The beam is made of four layers symmetrically $\left(0^{\circ} / 90^{\circ} / 90^{\circ} / 0^{\circ}\right)$ of epoxy-T300/976 graphite material with $2.5 \mathrm{~mm}$ thickness per layer, and with one layer piezo ceramic materials bonded to the top and bottom surfaces, $2.0 \mathrm{~mm}$ thickness per layer as shown in Figure 2 is considered $(a=0.254 \mathrm{~m}, \mathrm{~b}=0.0254 \mathrm{~m})$. The material properties of the piezo ceramic layers and graphite-epoxy are shown in Table 1.

The direct integration Newmark's method is used with parameters $\alpha_{R}=0.5$, $\beta_{\mathrm{R}}=0.25$; integral time step $\Delta \mathrm{t}=0.005 \mathrm{~s}$ with total time calculated $\mathrm{t}=15 \mathrm{~s}$. 
Static and Dynamic Analysis of Piezoelectric Laminated Composite Beams and Plates DOI: http://dx.doi.org/10.5772/intechopen.89303

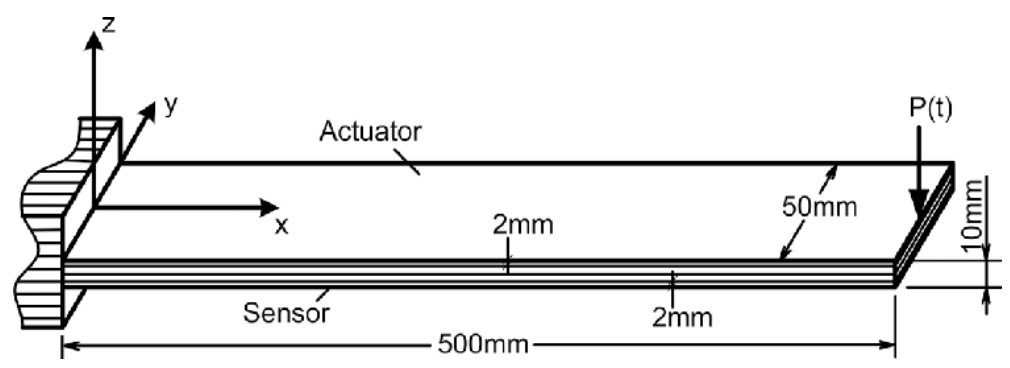

Figure 2.

Piezoelectric composite cantilever beam.

\begin{tabular}{lcc}
\hline Properties & PZT G1195 N & T300/976 \\
\hline $\mathrm{E}_{11}\left[\mathrm{~N} / \mathrm{cm}^{2}\right]$ & $0.63 \times 10^{6}$ & $1.50 \times 10^{6}$ \\
\hline $\mathrm{E}_{22}=\mathrm{E}_{33}\left[\mathrm{~N} / \mathrm{cm}^{2}\right]$ & $0.63 \times 10^{6}$ & $0.09 \times 10^{6}$ \\
\hline$\nu_{12}=\nu_{13}=\nu_{23}$ & 0.3 & 0.3 \\
\hline $\mathrm{G}_{12}=\mathrm{G}_{13}\left[\mathrm{~N} / \mathrm{cm}^{2}\right]$ & $0.242 \times 10^{6}$ & $0.071 \times 10^{6}$ \\
\hline $\mathrm{G}_{23}\left[\mathrm{~N} / \mathrm{cm}^{2}\right]$ & $0.242 \times 10^{6}$ & $0.025 \times 10^{6}$ \\
\hline$\rho\left[\mathrm{kg} / \mathrm{m}^{3}\right]$ & 7600 & 1600 \\
\hline $\mathrm{d}_{31}=\mathrm{d}_{32}(\mathrm{~m} / \mathrm{V})$ & $254 \times 10^{-12}$ & - \\
\hline $\mathrm{P}_{11}=\mathrm{P}_{22}(\mathrm{~F} / \mathrm{m})$ & $15.3 \times 10^{-9}$ & - \\
\hline $\mathrm{P}_{33}(\mathrm{~F} / \mathrm{m})$ & $15.0 \times 10^{-9}$ & - \\
\hline
\end{tabular}

Table 1.

Relevant mechanical properties of respective materials.

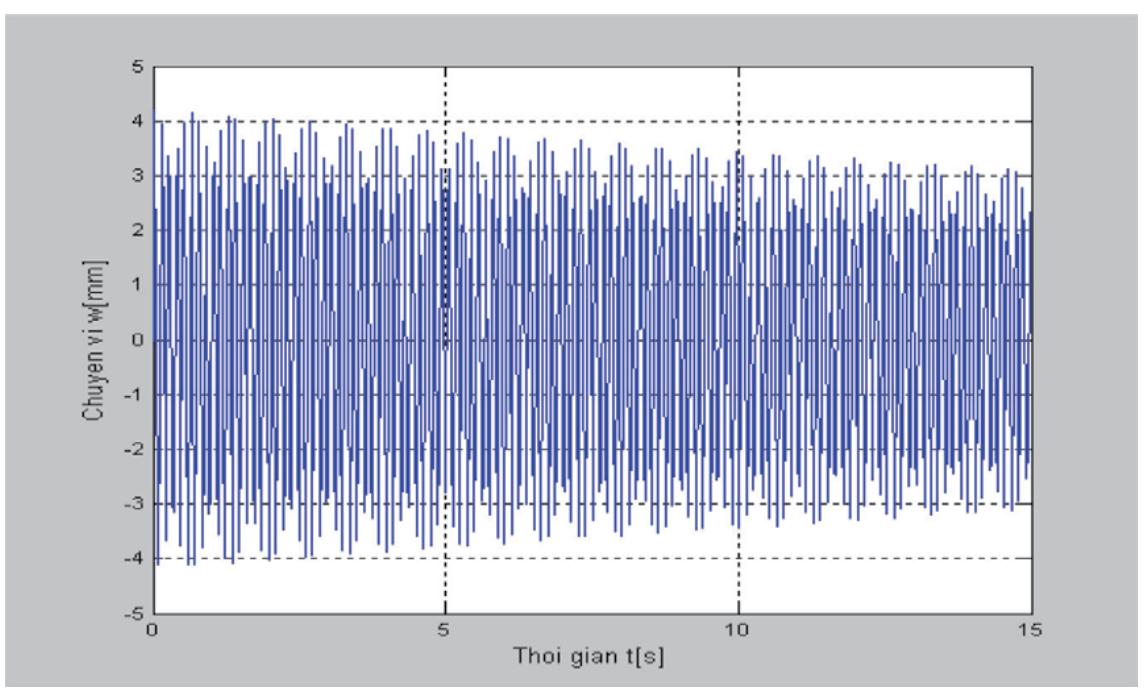

Figure 3.

Vertical displacement response $\left(G_{v}=0, G_{d}=0-\right.$ Case 1$)$.

Figures 3 and 4 illustrate the vertical displacement $\mathrm{w}$ at the free end of the beam for two cases:

Case 1: With structural damping, and without piezoelectric damping $\left(G_{v}=0\right.$, $\left.G_{d}=0\right)$. 


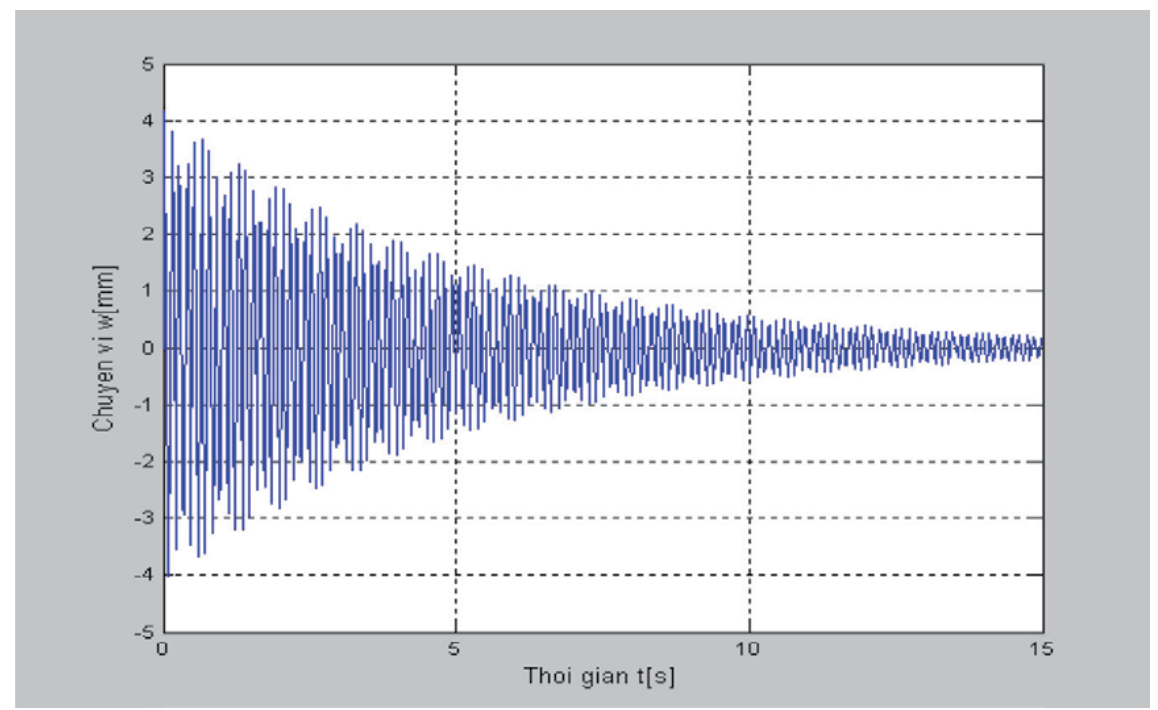

Figure 4.

Vertical displacement response $\left(G_{v}=0.5, G_{d}=30-\right.$ Case 2).

Case 2: With structural damping, with piezoelectric damping $\left(G_{v}=0.5\right.$, $\left.G_{d}=30\right)$.

\section{Dynamic analysis of laminated piezoelectric composite plates}

\subsection{The electromechanical behavioral relations in the plate}

Consider laminated composite plates with general coordinate system $(\mathrm{x}, \mathrm{y}, \mathrm{z})$, in which the $\mathrm{x}, \mathrm{y}$ plane coincides with the neutral plane of the plate. The top and bottom surfaces of the plate are bonded to the piezoelectric patches or piezoelectric layers (actuator and sensor). The plate under the load acting on its neutral plane has any temporal variation rule (Figure 5).

Hypothesis: The piezoelectric composite plate corresponds with ReissnerMindlin theory. The material layers are arranged symmetrically through the neutral plane of the plate, ideally adhesive with each other.

\subsubsection{Strain - displacement relations}

Based on the first-order shear deformation theory, the displacement fields at any point in the plate are $[7,8]$ :

$$
\begin{aligned}
& u(x, y, z, t)=u_{0}(x, y, t)+z \theta_{y}(x, y, t), \\
& v(x, y, z, t)=v_{0}(x, y, t)-z \theta_{x}(x, y, t), \\
& w(x, y, z, t)=w_{0}(x, y, t)
\end{aligned}
$$

where $\mathrm{u}, \mathrm{v}$ and $\mathrm{w}$ are the displacements of a general point $(\mathrm{x}, \mathrm{y}, \mathrm{z})$ in the laminate along $\mathrm{x}, \mathrm{y}$ and $\mathrm{z}$ directions, respectively. $\mathrm{u}_{0}, \mathrm{v}_{0}, \mathrm{w}_{0}, \theta_{\mathrm{x}}$ and $\theta_{\mathrm{y}}$ are the displacements and rotations of a midplane transverse normal about the $y$-and $x$ axes respectively. 


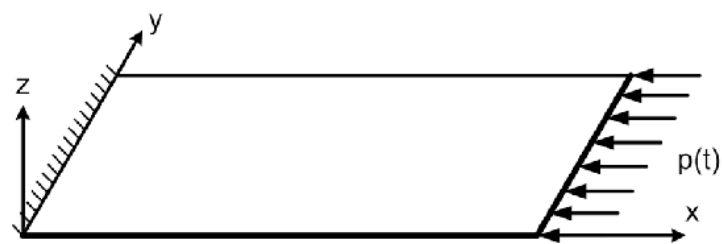

a)

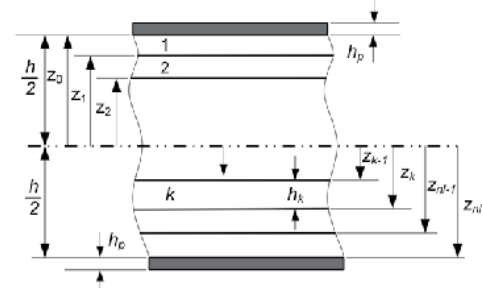

b)

Figure 5 .

Piezoelectric composite plate and coordinate system of the plate (a), and lamina details (b).

The components of the strain vector corresponding to the displacement field (49) are defined as:

For the linear strain:

$$
\begin{aligned}
& \varepsilon_{x}=\frac{\partial u}{\partial x}=\frac{\partial u_{0}}{\partial x}+z \frac{\partial \theta_{y}}{\partial x}, \varepsilon_{y}=\frac{\partial v}{\partial y}=\frac{\partial v_{0}}{\partial y}-z \frac{\partial \theta_{x}}{\partial y} \\
& \gamma_{\mathrm{xy}}=\left(\frac{\partial u}{\partial y}+\frac{\partial v}{\partial x}\right)+\frac{\partial \mathrm{w}}{\partial x} \cdot \frac{\partial \mathrm{w}}{\partial y}=\left(\frac{\partial u_{0}}{\partial y}+\frac{\partial v_{0}}{\partial x}\right)+z\left(\frac{\partial \theta_{y}}{\partial x}-\frac{\partial \theta_{x}}{\partial y}\right), \\
& \gamma_{\mathrm{xz}}=\frac{\partial \mathrm{u}}{\partial z}+\frac{\partial \mathrm{w}}{\partial x}=\frac{\partial \mathrm{w}_{0}}{\partial x}+\theta_{y}, \gamma_{\mathrm{yz}}=\frac{\partial \mathrm{v}}{\partial z}+\frac{\partial \mathrm{w}}{\partial y}=\frac{\partial \mathrm{w}_{0}}{\partial y}-\theta_{x}
\end{aligned}
$$

or in the vector form:

$$
\begin{aligned}
& \left\{\begin{array}{c}
\varepsilon_{x} \\
\varepsilon_{y} \\
\gamma_{x y}
\end{array}\right\}=\left\{\begin{array}{c}
\varepsilon_{x}^{o} \\
\varepsilon_{y}^{o} \\
\gamma_{x y}^{o}
\end{array}\right\}+z\left\{\begin{array}{c}
\kappa_{x} \\
\kappa_{y} \\
\kappa_{x y}
\end{array}\right\}=\left[\begin{array}{cc}
\frac{\partial}{\partial x} & 0 \\
0 & \frac{\partial}{\partial y} \\
\frac{\partial}{\partial y} & \frac{\partial}{\partial x}
\end{array}\right]\left\{\begin{array}{l}
u_{0} \\
v_{0}
\end{array}\right\}+z\left[\begin{array}{cc}
-\frac{\partial}{\partial y} & 0 \\
0 & -\frac{\partial}{\partial x} \\
-\frac{\partial}{\partial y} & \frac{\partial}{\partial x}
\end{array}\right]\left\{\begin{array}{l}
\theta_{x} \\
\theta_{y}
\end{array}\right\}= \\
& =\left[D_{\varepsilon}\right]\left\{\begin{array}{c}
u_{0} \\
v_{0}
\end{array}\right\}+\left[D_{\kappa}\right]\left\{\begin{array}{c}
\theta_{x} \\
\theta_{y}
\end{array}\right\}=\left\{\varepsilon_{0}\right\}+z\{\kappa\}=\left\{\varepsilon_{b}^{L}\right\} \text {, } \\
& \left\{\begin{array}{l}
\gamma_{x z} \\
\gamma_{y z}
\end{array}\right\}=\left[\begin{array}{ccc}
\frac{\partial}{\partial x} & 0 & 1 \\
\frac{\partial}{\partial y} & -1 & 0
\end{array}\right]\left\{\begin{array}{c}
w^{o} \\
\theta_{x} \\
\theta_{y}
\end{array}\right\}=\left[\begin{array}{lll}
\left\{{ }^{w} D\right\} & -\left[I_{s}\right]
\end{array}\right]\left\{\begin{array}{c}
w_{0} \\
\theta_{x} \\
\theta_{y}
\end{array}\right\}=\left\{\varepsilon_{s}\right\} .
\end{aligned}
$$

and for the nonlinear strain:

$$
\begin{gathered}
\left\{\begin{array}{c}
\varepsilon_{x} \\
\varepsilon_{y} \\
\gamma_{x y}
\end{array}\right\}=\left\{\varepsilon_{b}^{L}\right\}+\left\{\varepsilon^{N}\right\}=\left\{\varepsilon_{b}^{N}\right\}, \\
\left\{\begin{array}{l}
\gamma_{x z} \\
\gamma_{y z}
\end{array}\right\}=\left\{\varepsilon_{s}\right\},
\end{gathered}
$$


where $\left\{\varepsilon^{N}\right\}=\frac{1}{2}\left[\begin{array}{cc}\frac{\partial w_{0}}{\partial x} & 0 \\ 0 & \frac{\partial w_{0}}{\partial y} \\ \frac{\partial w_{0}}{\partial y} & \frac{\partial w_{0}}{\partial x}\end{array}\right]\left\{\begin{array}{c}\frac{\partial}{\partial x} \\ \frac{\partial}{\partial y}\end{array}\right\} w_{0}$ is the non-linear strain vector, $\left\{\varepsilon_{b}^{L}\right\}$ is the linear strain vector, $\left\{\varepsilon_{s}\right\}$ is the shear strain vector.

\subsubsection{Stress-strain relations}

The equation system describing the stress-strain relations and mechanicalelectrical quantities is respectively written as $[8,14]$ :

$$
\begin{aligned}
& \left\{\sigma_{b}\right\}=[Q]\left\{\varepsilon_{b}^{N}\right\}-[e]\{E\}, \\
& \left\{\tau_{b}\right\}=\left[Q_{s}\right]\left\{\varepsilon_{s}\right\}, \\
& \{D\}=[e]\left\{\varepsilon_{b}^{N}\right\}+[p]\{E\},
\end{aligned}
$$

where $\left\{\sigma_{b}\right\}=\left\{\begin{array}{lll}\sigma_{x} & \sigma_{y} & \tau_{x y}\end{array}\right\}^{T}$ is the plane stress vector, $\left\{\tau_{b}\right\}=\left\{\begin{array}{ll}\tau_{y z} & \tau_{x z}\end{array}\right\}^{T}$ is the shear stress vector, [Q] is the ply in-plane stiffness coefficient matrix in the structural coordinate system, $\left[\mathrm{Q}_{\mathrm{s}}\right]$ is the ply out-of-plane shear stiffness coefficient matrix in the structural coordinate system. Notice that $\left\{\tau_{\mathrm{b}}\right\}$ is free from piezoelectric effects.

The in-plane force vector at the state pre-buckling:

$$
\left\{N^{0}\right\}=\left\{\begin{array}{lll}
N_{x}^{0} & N_{y}^{0} \quad N_{x y}^{0}
\end{array}\right\}^{T}=\sum_{k=1}^{n} \int_{h_{k-1}}^{h_{k}}\left\{\begin{array}{c}
\sigma_{x}^{0} \\
\sigma_{y}^{0} \\
\tau_{x y}^{0}
\end{array}\right\}_{k} d z
$$

\subsubsection{Total potential energy}

The total potential energy of the system is given by:

$$
\Pi=\frac{1}{2} \int_{V_{p}}\left\{\varepsilon_{b}^{N}\right\}^{T}\left\{\sigma_{b}\right\} d V+\frac{1}{2} \int_{V_{p}}\left\{\varepsilon_{s}\right\}^{T}\left\{\tau_{b}\right\} d V-\frac{1}{2} \int_{V_{p}}\{E\}^{T}\{D\} d V-W,
$$

where $\mathrm{W}$ is the energy of external forces, $\mathrm{V}_{\mathrm{p}}$ is the entire domain including composite and piezoelectric materials.

Introducing $[A],[B],[D],\left[A_{s}\right]$, and vectors $\left\{N_{p}\right\},\left\{M_{p}\right\}$ as $[8]$ :

$$
\begin{aligned}
& ([A],[B],[D])=\int_{-h / 2}^{h / 2}\left(1, z, z^{2}\right)[Q] d z, \\
& {\left[A_{s}\right]=\int_{-h / 2}^{h / 2}\left[Q_{s}\right] d z,\left(\left\{N_{p}\right\},\left\{M_{p}\right\}\right)=\int_{-h / 2}^{h / 2}(1, z)[e]\{E\} d z,}
\end{aligned}
$$

where $\mathrm{h}$ is the total laminated thickness and combining with (5), (6) the total potential energy equation (8) can be written 


$$
\begin{aligned}
\Pi= & \frac{1}{2} \int_{\Omega}\left\{\varepsilon_{0}\right\}^{T}[A]\left\{\varepsilon_{0}\right\} d \Omega+\frac{1}{2} \int_{\Omega}\{\kappa\}^{T}[D]\{\kappa\} d \Omega+\frac{1}{2} \int_{\Omega}\left\{\varepsilon_{s}\right\}^{T}\left[A_{s}\right]\left\{\varepsilon_{s}\right\} d \Omega+ \\
& +\int_{\Omega}\left\{\varepsilon^{N}\right\}^{T}\left([A]\left\{\varepsilon_{0}\right\}-\left[N_{p}\right]\right) d \Omega-\int_{\Omega}\left\{\varepsilon_{0}\right\}^{T}\left[N_{p}\right] d \Omega-\int_{\Omega}\{\kappa\}^{T}\left[M_{p}\right] d \Omega-W
\end{aligned}
$$

where $\Omega$ is the plane xy domain of the plate.

\subsection{Dynamic stability analysis of laminated composite plate with piezoelectric layers}

\subsubsection{Finite element models}

Nine-node Lagrangian finite elements are used with the displacement and strain fields represented by Eqs. (49), (53), and (54). In the developed models, there is one electric potential degree of freedom for each piezoelectric layer to represent the piezoelectric behavior and thus the vector of electrical degrees of freedom is $[6,14]$ :

$$
\left\{\phi^{e}\right\}=\left\{\cdot \cdot \phi_{j}^{e} \cdot \cdot\right\}^{T}, j=1, \ldots, N P L^{e},
$$

in which NPL ${ }^{e}$ is the number of piezoelectric layers in a given element.

The vector of degrees of freedom for the element $\left\{q^{e}\right\}$ is:

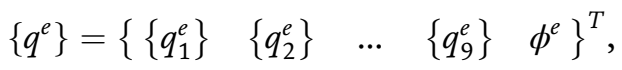

where $\left\{q_{i}^{e}\right\}=\left\{\begin{array}{lllll}u_{i} & v_{i} & w_{i} & \theta_{x_{i}} & \theta_{y_{i}}\end{array}\right\}$ is the mechanical displacement vector for node i.

\subsubsection{Dynamic equations}

The dynamic equations of piezoelectric composite plate can be derived by using Hamilton's principle, accordingly, the vibration equation of the membrane (without damping) with in-plane loads is:

$$
\left[M_{s s}\right]\left\{\ddot{q}_{s s}\right\}+\left[K_{s s}\right]\left\{q_{s s}\right\}=\{F(t)\} .
$$

The equation of bending vibrations with out-of-plane loads is:

$$
\begin{aligned}
& {\left[\begin{array}{cc}
{\left[M_{b b}\right]} & {[0]} \\
{[0]} & {[0]}
\end{array}\right]\left\{\begin{array}{c}
\left\{\ddot{q}_{b b}\right\} \\
\{\ddot{\phi}\}
\end{array}\right\}+\left[\begin{array}{cc}
{\left[C_{R}\right]} & {[0]} \\
{[0]} & {[0]}
\end{array}\right]\left\{\begin{array}{c}
\left\{\dot{q}_{b b}\right\} \\
\{\dot{\phi}\}
\end{array}\right\}} \\
& +\left[\begin{array}{cc}
{\left[K_{b b}\right]+\left[K_{G}\right]} & {\left[K_{b \phi}\right]} \\
{\left[K_{\phi b}\right]} & -\left[K_{\phi \phi}\right]
\end{array}\right]\left\{\begin{array}{c}
\left\{q_{b b}\right\} \\
\{\phi\}
\end{array}\right\} \\
& \quad=\left\{\begin{array}{c}
\{R\} \\
\left\{Q_{e l}\right\}
\end{array}\right\},
\end{aligned}
$$

where $\left[\mathrm{M}_{\mathrm{ss}}\right],\left[\mathrm{K}_{\mathrm{ss}}\right]$ are the overall mass, membrane elastic stiffness matrix respectively, and $\left\{q_{s s}\right\},\left\{\dot{q}_{s s}\right\},\left\{\ddot{q}_{s s}\right\}$ are respectively the membrane displacement, velocity, acceleration vector. $\left[\mathrm{M}_{\mathrm{bb}}\right],\left[\mathrm{K}_{\mathrm{bb}}\right]$ and $\left\{q_{b b}\right\},\left\{\dot{q}_{b b}\right\},\left\{\ddot{q}_{b b}\right\}$ are the overall mass, bending elastic stiffness matrix and the bending displacement, velocity, acceleration vector; $\left[\mathrm{K}_{\mathrm{G}}\right]$ is the overall geometric stiffness matrix; $\left(\left[\mathrm{K}_{\mathrm{G}}\right]\right.$ is 
a function of external in-plane loads); $\{\mathrm{F}(\mathrm{t})\}$ is the in-plane load vector, $\{\mathrm{R}\}$ is the normal load vector, $\left\{\mathrm{Q}_{\mathrm{el}}\right\}$ is the vector containing the nodal charges and in-balance charges.

The element coefficient matrices are:

$$
\begin{gathered}
{\left[K_{G}^{e}\right]=\left[K_{G x}^{e}\right]+\left[K_{G y}^{e}\right]+\left[K_{G x y}^{e}\right],} \\
{\left[K_{G x}^{e}\right]=\int_{A_{e}} N_{x}^{0}\left[N_{x}^{\prime}\right]\left[N_{x}^{\prime}\right]^{T} d A_{e},} \\
\text { where }\left[K_{G y}^{e}\right]=\int_{A_{e}} N_{y}^{0}\left[N_{y}^{\prime}\right]\left[N_{y}^{\prime}\right]^{T} d A_{e}, \\
{\left[K_{G x y}^{e}\right]=\int_{A_{e}} N_{x y}^{0}\left[N_{x}^{\prime}\right]\left[N_{y}^{\prime}\right]^{T} d A_{e},} \\
\frac{\partial \mathrm{w}}{\partial \mathrm{x}}=\left[\frac{\partial \mathrm{N}}{\partial \mathrm{x}}\right]\left\{q_{b b}^{e}\right\}=\left[N_{x}^{\prime}\right]\left\{q_{b b}^{e}\right\}, \frac{\partial \mathrm{w}}{\partial y}=\left[\frac{\partial \mathrm{N}}{\partial y}\right]\left\{q_{b b}^{e}\right\}=\left[N_{y}^{\prime}\right]\left\{q_{b b}^{e}\right\} \\
{\left[K_{G}\right]=\sum_{n e}\left[K_{G}^{e}\right]}
\end{gathered}
$$

\subsubsection{Dynamic stability analysis}

When the plate is subjected to in-plane loads only $(\{R\}=\{0\})$, the in-plane stresses can lead to buckling, from Eqs. (63) and (64) the governing differential equations of motion of the damped system may be written as:

$$
\begin{aligned}
& {\left[M_{s s}\right]\left\{\ddot{q}_{s s}\right\}+\left[K_{s s}\right]\left\{q_{s s}\right\}=\{F(t)\},} \\
& {\left[M_{b b}\right]\left\{\ddot{q}_{b b}\right\}+\left[C_{R}\right]\left\{\dot{q}_{b b}\right\}+\left(\left[K_{b b}\right]+\left[K_{G}\right]\right)\left\{q_{b b}\right\}+\left[K_{b \phi}\right]\{\phi\}=\{0\},} \\
& \quad\left[K_{\phi b}\right]\left\{q_{b b}\right\}-\left[K_{\phi \phi}\right]\{\phi\}=\left\{Q_{e l}\right\} .
\end{aligned}
$$

Eq. (70) is rewritten as:

$$
\begin{aligned}
& {\left[M_{s s}\right]\left\{\ddot{q}_{s s}\right\}+\left[K_{s s}\right]\left\{q_{s s}\right\}=\{F(t)\},} \\
& {\left[M_{b b}\right]\left\{\ddot{q}_{b b}\right\}+\left(\left[C_{A}\right]+\left[C_{R}\right]\right)\left\{\dot{q}_{b b}\right\}+\left(\left[K^{*}\right]+\left[K_{G}\right]\right)\left\{q_{b b}\right\}=\{0\} .}
\end{aligned}
$$

The overall geometric stiffness matrix $\left[K_{G}\right]$ is defined as follows:

- In the case of only tensile or compression plates ( $w=0)$ : Solving Eq. (71) helps us to present unknown displacement vector $\left\{\mathrm{q}_{\mathrm{ss}}\right\}$, and then stress vector:

$$
\left\{\sigma_{s s}\right\}=\left[A_{s}\right]\left[B_{s}\right]\left\{q_{s s}\right\},
$$

where $\left[A_{s}\right]$ and $\left[B_{s}\right]$ are the stiffness coefficient matrix and strain-displacement matrix of the plane problem.

- In the case of bending plate $(\mathrm{w} \neq 0)$, the stress vector is:

$$
\begin{aligned}
& \left\{\sigma_{s b}\right\}=\left\{\sigma_{s s}\right\}+\left\{\sigma_{b b}\right\}, \\
& \left\{\sigma_{b b}\right\}=\left[A_{b}\right]\left[B_{b}\right]\left\{q_{b b}\right\},
\end{aligned}
$$


where $\left[A_{b}\right]$ and $\left[B_{s}\right]$ are the stiffness coefficient matrix and strain-displacement matrix of the plane bending problem.

Stability criteria [14]:

- In the case of plate subjected to periodic in-plane loads and without damping, the elastic stability problems become simple only by solving the linear equations to determine the eigenvalues.

- In case of the plate under any in-plane dynamic load and with damping, the elastic stability problems become very complex. This iterative method can be proved effectively and the following dynamic stability criteria are used:

- Plate is considered to be stable if the maximum bending deflection is three times smaller than the plate's thickness: Eq. (71) has the solution $\left(w_{i}\right)_{\max }$ satisfying the condition $0 \leq\left|\mathrm{w}_{\mathrm{i}}\right|_{\max }<3 h$, where $w_{i}$ is the deflection of the plate at node number $i$.

- Plate is called to be in critical status if the maximum bending deflection of the plate is three times equal to the plate's thickness. Eq. (71) has the solution $\left(w_{i}\right)_{\text {max }}$ satisfying the condition $\left|\mathrm{w}_{\mathrm{i}}\right|_{\max }=3 h$.

- Plate is called to be at buckling if the maximum deflection of the plate is three times larger than the plate's thickness: Eq. (71) has the solution $\left(w_{i}\right)_{\max }$ satisfying the condition $\left|\mathrm{w}_{\mathrm{i}}\right|_{\max }>3 h$.

The identification of critical forces is carried out by the iterative method.

\subsubsection{Iterative algorithm}

Step 1. Defining the matrices, the external load vector and errors of load iterations.

Step 2. Solving Eq. (71) to present unknown displacement vector, $\left\{\mathrm{q}_{\mathrm{ss}}\right\}$ and the stress vector is defined by (72), updating the geometric stiffness matrix $\left[\mathrm{K}_{\mathrm{G}}\right]$.

Step 3. Solving Eq. (71) to present unknown bending displacement vector $\left\{q_{b b}\right\}$, and then testing stability conditions.

- If for all $\left|\mathrm{w}_{i}\right|=0$ : increase load, recalculate from step 2;

- If at least one value $\left|\mathrm{w}_{i}\right| \neq 0$ :

+ In case: $0<\left|\mathrm{w}_{i}\right|_{\max }<3 h$ : Define stress vector by Eq. (73), update the geometric stiffness matrix $\left[\mathrm{K}_{\mathrm{G}}\right]$. Increase load, recalculate from step 2;

+ In case: $0 \leq \frac{\left.|| \mathrm{w}_{i}\right|_{\max }-3 h \mid}{\left|\mathrm{w}_{i}\right| \max } \leq \varepsilon_{D}$ : Critical load $\mathrm{p}=\mathrm{p}_{\mathrm{cr}}$. End.

\subsubsection{Numerical analysis}

Stability analysis of piezoelectric composite plate with dimensions $\mathrm{a} \times \mathrm{b} \times \mathrm{h}$, where $\mathrm{a}=0.25 \mathrm{~m}, \mathrm{~b}=0.30 \mathrm{~m}, \mathrm{~h}=0.002 \mathrm{~m}$. Piezoelectric composite plate is composed of three layers, in which two layers of piezoelectric PZT-5A at its top and bottom are considered, each layer thickness $h_{p}=0.00075 \mathrm{~m}$; the middle layer material is Graphite/Epoxy material, with thickness $\mathrm{h}_{1}=0.0005 \mathrm{~m}$. The material properties for graphite/epoxy and PZT-5A are shown in Section 5.1 above. One short edge of the plate is clamped, the other three edges are free. The in-plane half- 
sine load is evenly distributed on the short edge of the plate: $\mathrm{p}(\mathrm{t})=\mathrm{p}_{0} \sin (2 \pi \mathrm{ft})$, where $\mathrm{p}_{0}$ is the amplitude of load, $\mathrm{f}=1 / \mathrm{T}=1 / 0.01=100 \mathrm{~Hz}(0 \leq \mathrm{t} \leq \mathrm{T} / 2=0.005 \mathrm{~s})$ is the excitation frequency, voltage applied $\mathrm{V}=50 \mathrm{~V}$. The iterative error of the load $\varepsilon_{\mathrm{D}}=0.02 \%$ is chosen.

Consider two cases: with damping $\left(\xi=0.05, G_{v}=0.5, G_{d}=15\right)$ and without damping $\left(\xi=0.0, G_{v}=0.0, G_{d}=15\right)$. The response of vertical displacement at the plate centroid over the plate thickness for the two cases is shown in Figure 6.

The results show that the critical load of the plate with damping is larger than that without damping. In the two cases above, the critical load rises by $6.8 \%$.

Analyze the stability of the plate with damping when a voltage of $-200,-150$, $-100,-50,0,50,100,150$ and $200 \mathrm{~V}$ is applied to the actuator layer of the piezoelectric composite plate.

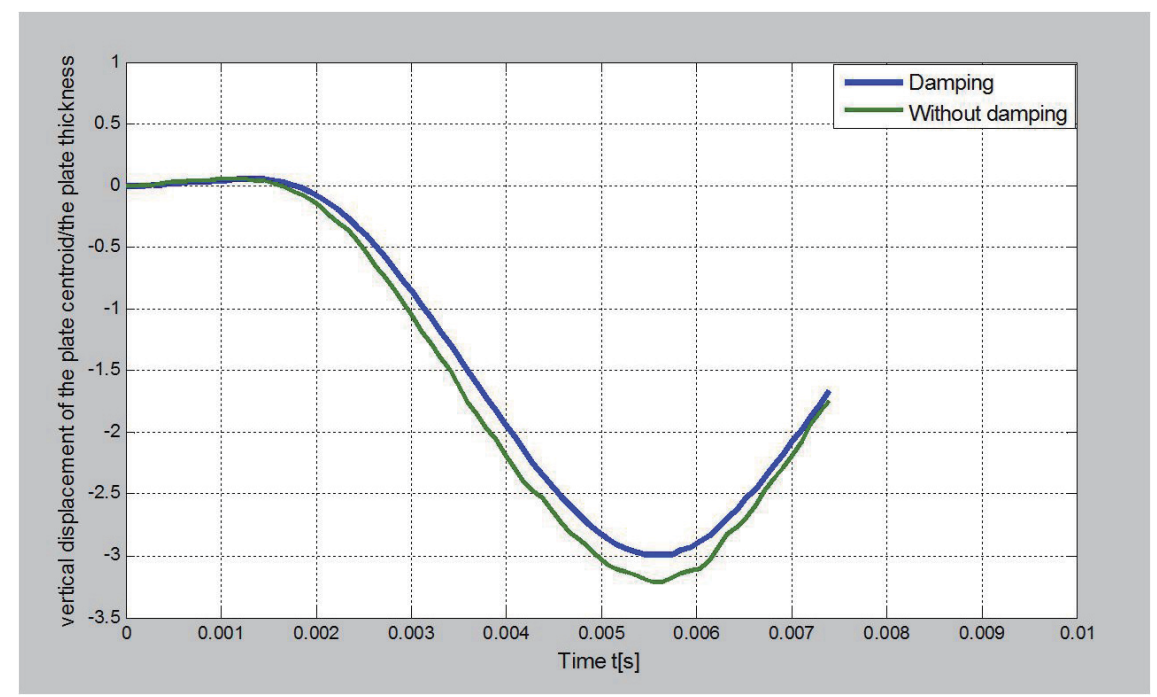

Figure 6.

Vertical displacement response at the plate centroid over the plate thickness.

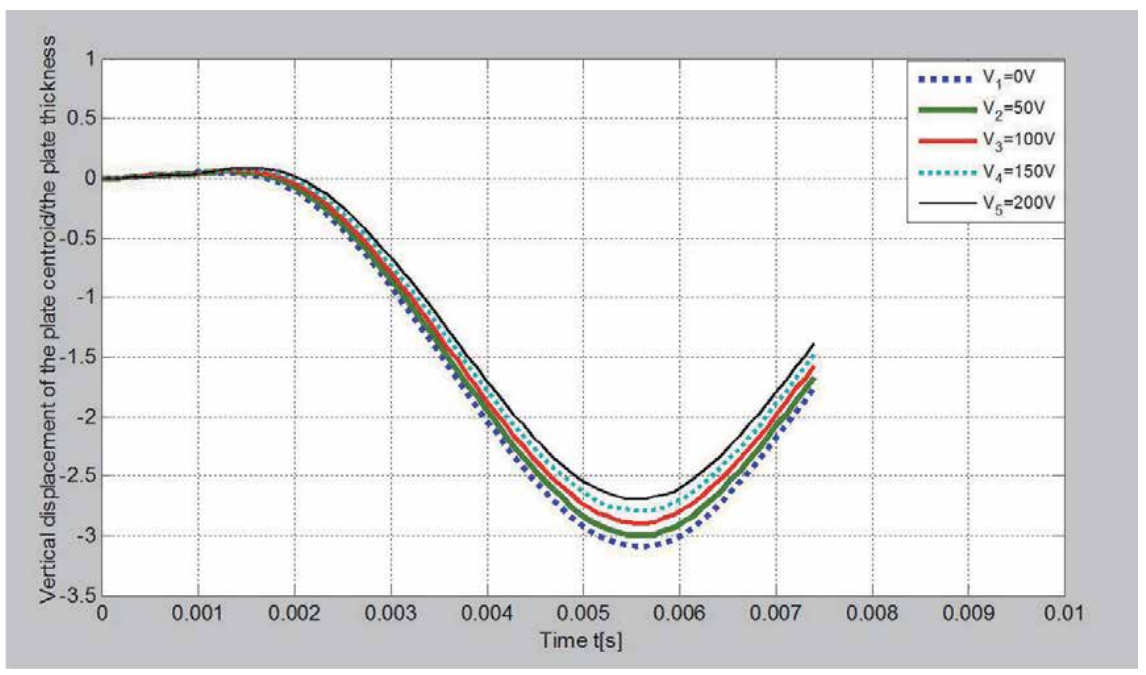

Figure 7.

Vertical displacement response at the plate centroid over the plate thickness. 
Figure 7 shows the time history of the vertical displacement at the plate centroid over the plate thickness when a voltage of $0,50,100,150$ and $200 \mathrm{~V}$ is applied. The relation between critical load and voltages is shown in Figure 8.

The results show that the voltage applied to the piezoelectric layers affects the stability of the plate. As the voltage increases, the critical load of the plate also increases.

When the amplitude of the load changes from $0.25 \mathrm{p}_{\mathrm{pr}}$ to $1.5 \mathrm{p}_{\mathrm{cr}}$ (where $\mathrm{p}_{\mathrm{cr}}$ is the amplitude of the critical load), a voltage of $50 \mathrm{~V}$ is applied to the actuator layer of the plate.

The results show the time history response of the vertical displacement at the plate centroid over the plate thickness as seen in Figure 9.

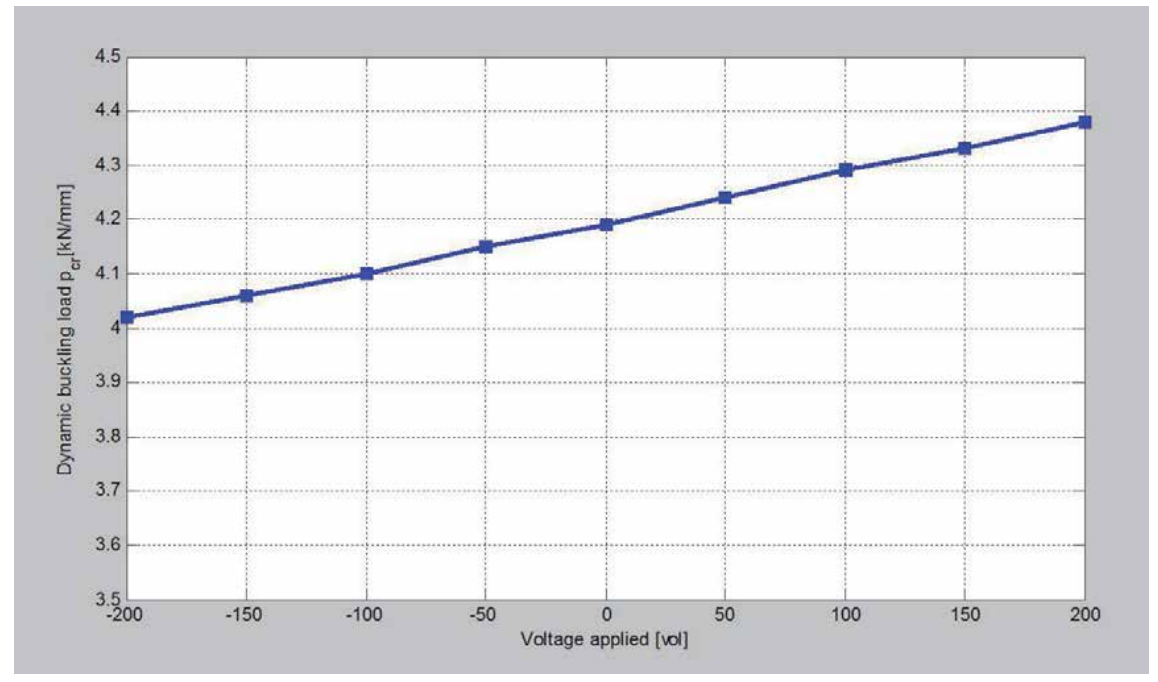

Figure 8.

Critical load-voltage relation.

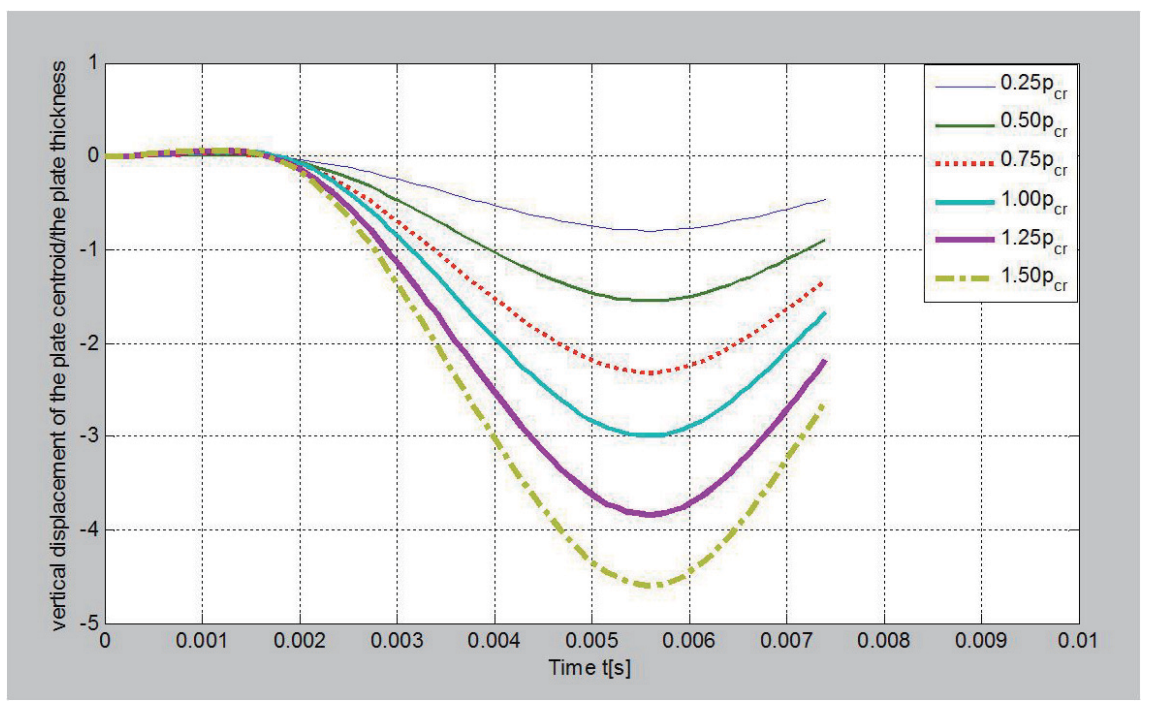

Figure 9.

Time history of the vertical displacement at the plate centroid over the plate thickness when $p_{0}=0.25 p_{c r}, 0.5 p_{c r}$, $0.75 p_{c r}, 1.0 p_{c r}, 1.25 p_{c r}$, and $1.5 p_{c r}$ 


\subsection{Dynamic analysis of piezoelectric stiffened composite plates subjected to airflow}

Consider isoparametric piezoelectric laminated stiffened plate with the general coordinate system $(\mathrm{x}, \mathrm{y}, \mathrm{z})$, in which the $\mathrm{x}, \mathrm{y}$ plane coincides with the neutral plane of the plate. The top surface and lower surface of the plate are bonded to the piezoelectric patches (actuator and sensor). The plate subjected to the airflow load acting (Figure 10).

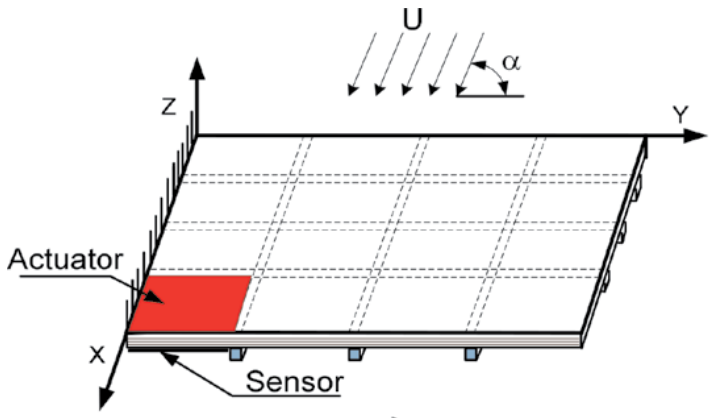

a)

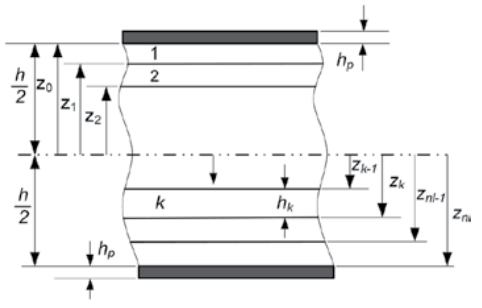

b)

Figure 10.

Smart stiffened plate subjected to airflow. (a) Smart stiffened plate and coordinate system and $(b)$ Lamina details.

The dynamic equations of a finite smart composite plate are written as follows:

$$
[M]_{e}\{\ddot{u}\}_{e}+\left[C_{A}\right]_{e}\{\dot{u}\}_{e}+\left(\left[K_{b b}^{\mathrm{nn}}\right]_{e}+\left[K_{b b}^{\mathrm{n} l}\right]_{e}+\left[K_{A}\right]_{e}\right)\{u\}_{e}=\{f\}_{e}^{m},
$$

where $\left[K_{b b}^{\ln }\right]_{e}=\int_{V_{e}}\left[B_{b}^{\ln }\right]^{T}[Q]\left[B_{b}^{\ln }\right] d V$, and $\left[K_{b b}^{n l}\right]_{e}=\int_{V_{e}}\left[B_{b}^{n l}\right]^{T}[Q]\left[B_{b}^{n l}\right] d V$ are the element linear mechanical stiffness and nonlinear mechanical stiffness respectively, $\{f\}_{e}^{m}$ is element external mechanical force vector.

\subsubsection{Formulation of Stiffener:}

\subsubsection{Formulation of $x$-Stiffener}

$$
\begin{aligned}
& U_{x s}(x, z)=u_{0}(x)+z \theta_{x s}(x), \\
& W_{x s}(x, z)=w_{x s}(x) .
\end{aligned}
$$

where $\mathrm{x}$-axis is taken along the stiffener centerline and the $\mathrm{z}$-axis is its upward normal. The plate and stiffener element shown in Figure 11.

If we consider that the $\mathrm{x}$-stiffener is attached to the lower side of the plate, conditions of displacement compatibility along their line of connection can be written as:

$$
\left.u_{p}\right|_{z=-t_{p} / 2}=\left.u_{x s}\right|_{z=t_{x s} / 2},\left.\theta_{x p}\right|_{z=-t_{p} / 2}=\left.\theta_{x s}\right|_{z=t_{x s} / 2},\left.w_{p}\right|_{z=-t_{p} / 2}=\left.w_{x s}\right|_{z=t_{x s} / 2}
$$

where $t_{p}$ is the plate thickness and $t_{x s}$ is the $x$-stiffener depth.

The element stiffness and mass matrices are defined as follows [2, 15]:

$$
\left[K_{x s}\right]_{e}=\int_{l_{e}}\left[B_{x s}\right]^{T}\left[D_{x s}\right]\left[B_{x s}\right] d x,
$$




$$
\left[M_{x s}\right]_{e}=\int_{A_{e}}\left[P\left(\left[N_{u^{0}}\right]^{T}\left[N_{u^{0}}\right]+\left[N_{w}\right]^{T}\left[N_{w}\right]\right)+I_{y}\left(\left[N_{\theta_{x}}\right]^{T}\left[N_{\theta_{x}}\right]\right)\right] d A,
$$

with $\left[\mathrm{B}_{\mathrm{xs}}\right]$ is the strain-displacement relations matrix, $\left[\mathrm{D}_{\mathrm{xs}}\right]$ is the stress-strain relations matrix and $l_{e}$ is the element length, $\left[N_{u^{0}}\right],\left[N_{w}\right]$ and $\left[N_{\theta_{x}}\right]$ are the shape function matrices relating the primary variables $\mathrm{u}_{0}, \mathrm{w}, \mathrm{x}$, in terms of nodal unknowns, $\mathrm{I}_{\mathrm{y}}$ is the area moment of inertia related to the $\mathrm{y}$-axis and $P=\sum_{k=1}^{n} \int_{h_{k-1}}^{h_{k}} \rho_{k} d z$, with $\rho_{\mathrm{k}}$ is density of $\mathrm{k}^{\text {th }}$ layer.

\subsubsection{Formulation of $y$-Stiffener}

The same as for $\mathrm{x}$-stiffener, the element stiffness and mass matrices of the $y$ stiffener are defined as follows:

$$
\begin{gathered}
{\left[K_{y s}\right]_{e}=\int_{l_{e}}\left[B_{y s}\right]^{T}\left[D_{y s}\right]\left[B_{y s}\right] d y} \\
{\left[M_{y s}\right]_{e}=\int_{A_{e}}\left[P\left(\left[N_{u^{0}}\right]^{T}\left[N_{u^{0}}\right]+\left[N_{w}\right]^{T}\left[N_{w}\right]\right)+I_{x}\left(\left[N_{\theta_{y}}\right]^{T}\left[N_{\theta_{y}}\right]\right)\right] d A,}
\end{gathered}
$$

\subsubsection{Modeling the effect of aerodynamic pressure and motion equations of the smart} composite plate-stiffeners element

Based on the first order theory, the aerodynamic pressure $l_{h}$ and moment $m_{\theta}$, can be described as [15-17]:

$$
\begin{aligned}
& l_{w}=\frac{1}{2} \rho_{a}(U \cos \alpha)^{2} B\left[k H_{1}^{*} \frac{\dot{\mathrm{w}}}{U \cos \alpha}+k H_{2}^{*} \frac{B \dot{\theta}}{U \cos \alpha}+k^{2} H_{3}^{*} \theta\right]+\frac{1}{2} C_{p} \rho_{\mathrm{a}}(U \sin \alpha)^{2}, \\
& m_{\theta}=\frac{1}{2} \rho_{a}(U \cos \alpha)^{2} B^{2}\left[k A_{1}^{*} \frac{\dot{\mathrm{w}}}{U \cos \alpha}+k A_{2}^{*} \frac{B \dot{\theta}}{U \cos \alpha}+k^{2} A_{3}^{*} \theta\right],
\end{aligned}
$$

where $k=b \omega / U$ is defined as the reduced frequency, $\omega$ is the circular frequency of oscillation of the airfoil, $U$ is the wind velocity, $B$ is the half-chord length of the airfoil or half-width of the plate, $\rho_{\mathrm{a}}$ is the air density and $\alpha$ is the angle of attack.

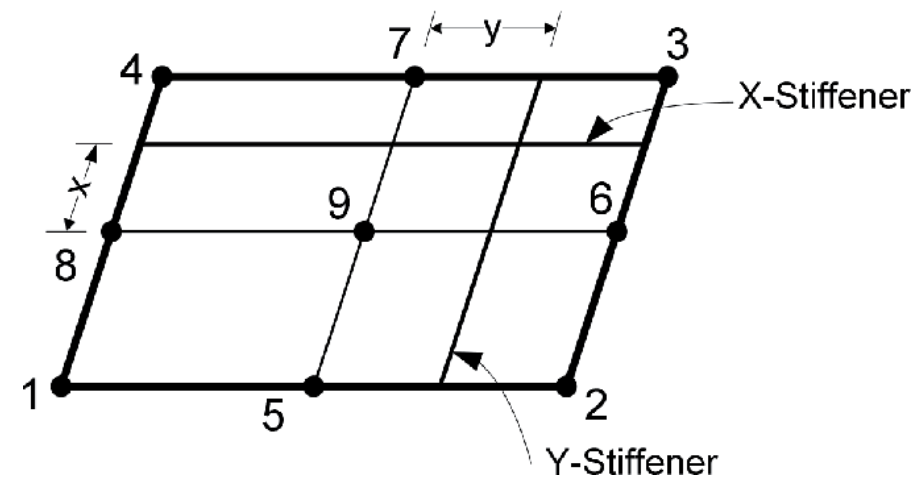

Figure 11.

Modeling of plate and stiffener element. 
The functions $A_{i}^{*}(K), H_{i}^{*}(K)$ are defined as follows:

$$
\begin{aligned}
& H_{1}^{*}(K)=-\frac{\pi}{k} F(k), H_{2}^{*}(K)=-\frac{\pi}{4 k}\left[1+F(k)+\frac{2 G(k)}{k}\right], \\
& H_{3}^{*}(K)=-\frac{\pi}{2 k^{2}}\left[F(k)-\frac{k G(k)}{2}\right], A_{1}^{*}(K)=\frac{\pi}{4 k} F(k), \\
& A_{2}^{*}(K)=-\frac{\pi}{16 k}\left[1-F(k)-\frac{2 G(k)}{k}\right], A_{3}^{*}(K)=\frac{\pi}{8 k^{2}}\left[\frac{k^{2}}{8}+F(k)-\frac{k G(k)}{2}\right],
\end{aligned}
$$

where $F(k)$ and $G(k)$ are defined as:

$$
\begin{aligned}
& F(k)=\frac{0.500502 k^{3}+0.512607 k^{2}+0.2104 k+0.021573}{k^{3}+1.035378 k^{2}+0.251293 k+0.021508}, \\
& G(k)=-\frac{0.000146 k^{3}+0.122397 k^{2}+0.327214 k+0.001995}{k^{3}+2.481481 k^{2}+0.93453 k+0.089318} .
\end{aligned}
$$

Using finite element method, aerodynamic force vector can be described as:

$$
\{f\}_{e}^{a i r}=-\left[K^{a i r}\right]_{e}\{u\}_{e}-\left[C^{a i r}\right]_{e}\{\dot{u}\}_{e}+\{f\}_{e}^{n},
$$

with $\left[K^{a i r}\right]_{e},\left[C^{a i r}\right]_{e}$ and $\{f\}_{e}^{n}$ are the aerodynamic stiffness, damping matrices and lift force vector, respectively

$$
\begin{aligned}
& {\left[K_{e}^{a i r}\right]=\rho_{a}(U \cos \alpha)^{2} B k^{2} \int_{A_{e}}\left[H_{3}^{*}(k)\left[N_{w}\right]^{T}\left[N_{\theta x}\right]+B A_{3}^{*}(k)\left[\frac{\partial N_{\theta y}}{\partial x}\right]^{T}\left[N_{\theta x}\right]\right] d A,} \\
& {\left[C_{e}^{a i r}\right]=\rho_{a}(U \cos \alpha) B k\left[\begin{array}{l}
\int_{A_{e}}\left(H_{1}^{*}(k)\left[N_{\mathrm{w}}\right]^{T}\left[N_{\mathrm{w}}\right]+B H_{2}^{*}(k)\left[N_{\mathrm{w}}\right]^{T}\left[N_{\theta \mathrm{x}}\right] d A\right) \\
+\int_{A_{e}}\left(B A_{1}^{*}(k)\left[\frac{\partial N_{\theta \mathrm{y}}}{\partial x}\right]^{T}\left[N_{\mathrm{w}}\right]+B^{2} A_{2}^{*}(k)\left[\frac{\partial N_{\theta \mathrm{y}}}{\partial x}\right]^{T}\left[N_{\theta \mathrm{x}}\right] d A\right)
\end{array}\right],} \\
& \{f\}_{e}^{n}=C_{p} \rho_{\mathrm{a}}(U \sin \alpha)^{2} \int_{A_{e}}\left[N_{w}\right]^{T} d A
\end{aligned}
$$

where $A_{e}$ is the element area, $\left[\mathrm{N}_{\mathrm{w}}\right],\left[\mathrm{N}_{\theta}\right]$ are the shape functions.

From Eqs. (74) and (84), the governing equations of motion of the smart composite plate-stiffeners element subjected to an aerodynamic force without damping can be derived as:

$$
\left[M^{*}\right]_{e}\{\ddot{u}\}_{e}+\left[C_{A}\right]_{e}\{\dot{u}\}_{e}+\left(\left[K^{*}\right]_{e}+\left[K_{A}\right]_{e}+\left[K^{a i r}\right]_{e}\right)\{u\}_{e}=\left\{f^{*}\right\}_{e}^{m},
$$

where $\left[M^{*}\right]_{e}=[M]_{e}+\left[M_{x s}\right]_{e}+\left[M_{y s}\right]_{e},\left[K^{*}\right]_{e}=\left[K_{b b}^{\mathrm{nn}}\right]_{e}+\left[K_{b b}^{\mathrm{n} l}\right]_{e}+\left[K_{x s}\right]_{e}+\left[K_{y s}\right]_{e}$, $\left\{f^{*}\right\}_{e}^{m}=\{f\}_{e}^{m}+\{f\}_{e}^{n}$.

\subsubsection{Governing differential equations for total system}

Finally, the elemental equations of motion are assembled to obtain the open-loop global equation of motion of the overall stiffened composite plate with the PZT patches as follows: 


$$
\left[M^{*}\right]\{\ddot{u}\}+\left(\left[C_{R}\right]+\left[C_{A}\right]\right)\{\dot{u}\}+\left(\left[K^{*}\right]+\left[K_{A}\right]+\left[K^{\text {air }}\right]\right)\{u\}=\left\{f^{*}\right\}^{m},
$$

where $\left[C_{R}\right]=\alpha_{R}\left[M_{b b}\right]+\beta_{R}\left(\left[K_{b b}^{\mathrm{ln}}\right]+\left[K_{b b}^{\mathrm{n} l}\right]\right)$.

The solution of nonlinear Eq. (89) is carried out by using Newmark direct and Newton-Raphson iteration method.

\subsubsection{Numerical applications}

A rectangle cantilever laminated composite plate is assumed to be $\left[0^{\circ} / 90^{\circ}\right]_{\mathrm{s}}$ with total thickness $4 \mathrm{~mm}$, length of $600 \mathrm{~mm}$ and width of $400 \mathrm{~mm}$ with three stiffeners along each direction $\mathrm{x}$ and $\mathrm{y}$. The geometrical dimension of the stiffener is $5 \mathrm{~mm}$ of high and $10 \mathrm{~mm}$ of width. The plate and stiffeners are made of graphite/epoxy with mechanical properties: $\mathrm{E}_{11}=181 \mathrm{GPa}, \mathrm{E}_{22}=\mathrm{E}_{33}=10.3 \mathrm{GPa}, \mathrm{E}_{12}=7.17 \mathrm{GPa}$, $\nu_{12}=0.35, \nu_{23}=\nu_{32}=0.38, \rho=1600 \mathrm{~kg} \cdot \mathrm{m}^{-3}$. Material properties for piezoelectric layer made of PZT-5A are: $d_{31}=d_{32}=-171 \times 10^{-12} \mathrm{~m} / \mathrm{V}, \mathrm{d}_{33}=374 \times 10^{-12} \mathrm{~m} / \mathrm{V}$, $\mathrm{d}_{15}=\mathrm{d}_{24}=-584 \times 10^{-12} \mathrm{~m} / \mathrm{V}, \mathrm{G}_{12}=7.17 \mathrm{GPa}, \mathrm{G}_{23}=2.87 \mathrm{GPa}, \mathrm{G}_{32}=7.17 \mathrm{GPa}$, $\nu_{\mathrm{PZT}}=0.3, \rho_{\mathrm{PZT}}=7600 \mathrm{~kg} \cdot \mathrm{m}^{-3}$ and thickness $\mathrm{t}_{\mathrm{PZT}}=0.15876 \mathrm{~mm}, \xi=0.05, \mathrm{G}_{\mathrm{v}}=0.5$, $\mathrm{G}_{\mathrm{d}}=15$. The effects of the excitation frequency and location of the actuators are presented through a parametric study to examine the vibration shape of the composite plate activated by the surface bonded piezoelectric actuators. The iterative

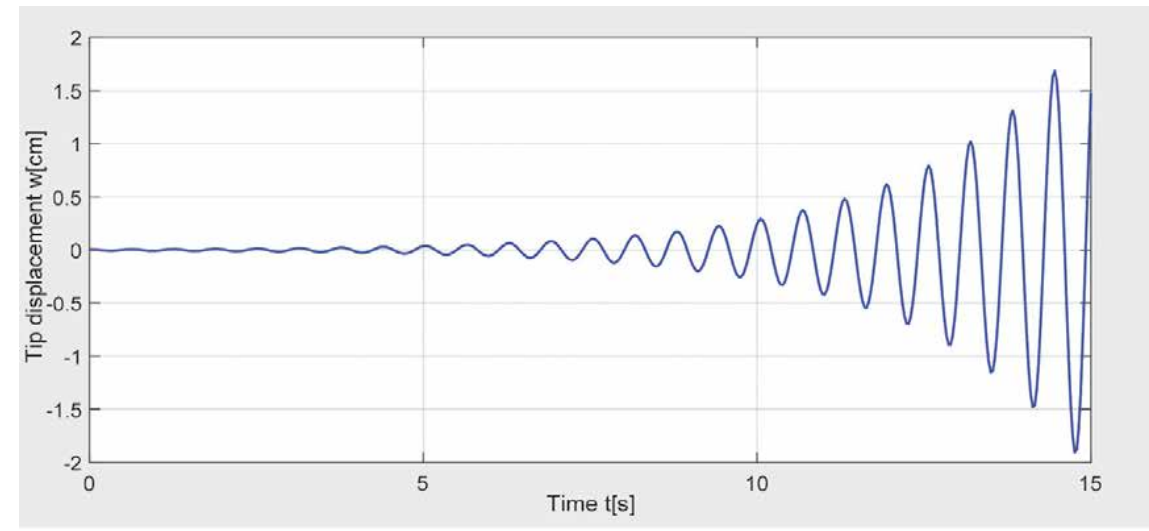

a)

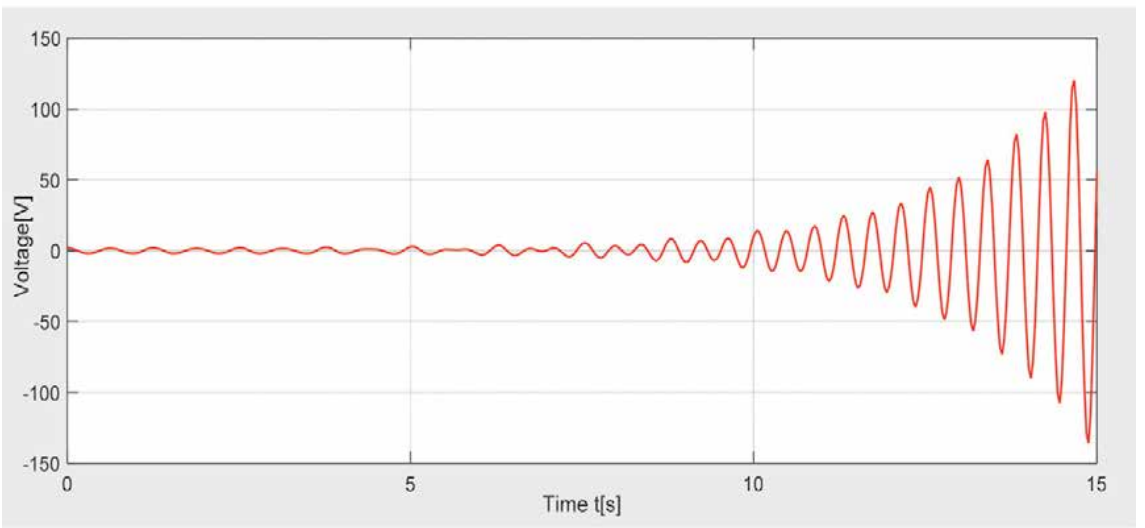

b)

Figure 12.

History of the plate at a critical airflow velocity $U_{c r}=30.5 \mathrm{~m} / \mathrm{s}$. (a) Displacement response and

(b) Piezoelectric voltage response. 
error of the load $\varepsilon_{D}=0.02 \%$ is chosen. The piezoelectric stiffened composite plate is subjected to the airflow in the positive $\mathrm{x}$ direction as shown in Figure 10a.

Dynamic response of the piezoelectric stiffened composite plate is shown in Figure 12.

\section{Author details}

Chung Nguyen Thai ${ }^{1 *}$, Thinh Tran $\operatorname{Ich}^{2}$ and Thuy Le Xuan ${ }^{1}$

1 Le Quy Don Technical University, Hanoi, Vietnam

2 Hanoi University of Science and Technology, Hanoi, Vietnam

*Address all correspondence to: chungnt@mta.edu.vn

\section{IntechOpen}

(C) 2020 The Author(s). Licensee IntechOpen. This chapter is distributed under the terms of the Creative Commons Attribution License (http://creativecommons.org/licenses/ by/3.0), which permits unrestricted use, distribution, and reproduction in any medium, provided the original work is properly cited. (c) BY 


\section{References}

[1] Chung NT. Basic Finite Element Method and Programming ANSYS in Mechanical Engineering. Publishing house of the Vietnam Ministry of Defence; 2016. ISBN: 978-604-51-1959-4

[2] Bathe KJ, Wilson EL. Numerical Method in Finite Method Analysis. New Delhi: Prentice Hall of India Private Limited; 1978

[3] Haskins JF, Walsh JL. Vibration of ferroelectric cylindrical shells with transverse isotropy. The Journal of the Acoustical Society of America. 1957; 29:729

[4] Tiersten HF. Linear Piezoelectric Plate Vibrations. New York: Plenum Press; 1969

[5] Adelman NT, Stavsky Y. Vibrations of radially polarized composite piezoceramic cylinders and disks. Journal of Sound and Vibration. 1975;43:37

[6] Yang J. The Mechanics of Piezoelectric Structures. World Scientific Publishing Co. Pte. Ltd.; 2006

[7] Vinson JR. Plate and Panel Structures of Isotropic, Composite and Piezoelectric Materials, Including Sandwich Construction. Springer; 2005. ISBN: 1-4020-3110-6 (HB)

[8] Reddy JN. Mechanics of Laminated Composite Plates and Shells Theory and Analysis. 2nd ed. CRC Press; 2004

[9] Toupin RA. Piezoelectric relation and the radial deformation of polarized spherical shell. The Journal of the Acoustical Society of America. 1959; 31:315

[10] Thinh TI, Ngoc LK. Static behavior and vibration control of piezoelectric cantilever composite plates and comparison with experiments. Computational Materials Science. 2010; 49:S276-S280
[11] Meirovitch L. Methods of Analytical Dynamics. Vol. 2003. New York: Dover Publications Inc.; 2003

[12] Chopra AK. Dynamics of Structures Theory and Applications to Earthquake Engineering. 2nd ed. Pearson Education Asia Limited and Tsinghua University Press; 2004

[13] Advanced Dynamic of Structures, NTUST - CT 6006. 2006

[14] Chung NT, Luong HX, Xuan NTT. Dynamic stability analysis of laminated composite plate with piezoelectric layers. Vietnam Journal of Mechanics. 2014;36(02):95-107

[15] Chung NT, Thuy NN, Thu DTN, Chau LH. Numerical and experimental analysis of the dynamic behavior of piezoelectric stiffened composite plates subjected to airflow. Mathematical Problems in Engineering. 2019;2019: 2697242. DOI: $10.1155 / 2019 / 2697242$

[16] John DH. Wind Loading of Structures. New York, NY:

Simultaneously Published in the USA and Canada; 2003. p. 10001

[17] Dowell EH. Aeroelasticity of Plates and Shells. Leyden: Noordhoff International Publishing; 1975. ISBN: 90-286-0404-9 


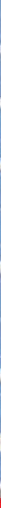

\section{Edited by Someshwar Pola, Neeraj Panwar and Indrani Coondoo}

Investigating in the area of perovskite materials and the fabrication of devices for properties in optoelectronics, we have presented a brief outline of perovskite materials.

The authors present a fairly comprehensive arrangement of this very active area of research, with its past changes and present position and outlooks. Discussions are presented regarding photocatalysis, fabrication of solar cell devices and their stability, lead-free materials, as well as thermoelectric and piezoelectric applications. In view of the present status of perovskite materials, I am assured that each chapter of the book will be of boundless encouragement for researchers, scientists, and academicians working in this field. 